INTERNATIONAL SERIES OF MONOGRAPHS ON

\title{
PURE AND APPLIED BIOLOGY
}

MODERT TRENDS IN PHYSIOLOGICAL SCRENCES 



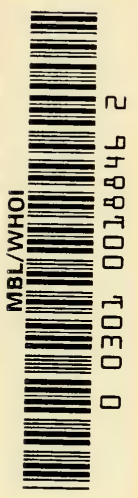



INTERNATIONAL SERIES OF MONOGRAPHS ON PURE AND APPLIED BIOLOGY

Division: MODERN TRENDS IN PHYSIOLOGICAL SCIENCES

General Editors: P. Alexander and Z. M. BacQ

Volume 12

KERAT I N

A ND

K E R A T I N I Z A T I O N 


\section{MODERN TRENDS \\ IN PHYSIOLOGICAL SCIENCES DIVISION}

Vol. 1. Florkin - Unity and Diversity in Biochemistry

Vol. 2. BRAchet - The Biochemistry of Development

Vol. 3. Gerebtzoff - Cholinesterases

Vol. 4. Brouna - Physiology in Industry

Vol. 5. BACQ and AlEXANDER - Fundamentals of Radiobiology

Vol. 6. Florkin (Ed.) - Aspects of the Origin of Life

Vol. 7. Hollaender (Ed.) - Radiation Protection and Recovery

Vol. 8. KAYSER - The Physiology of Natural Hibernation

Vol. 9. FrançON - Progress in Microscopy

Vol. 10. CHARLIER - Coronary Vasodilators

Vol. 11. Gross - Oncogenic Viruses

Vol. 13. Heath - Organophosphorus Poisons

Vol. 14. Chantrenne - The Biosynthesis of Proteins

Vol. 15. Rivera - Cilia, Ciliated Epithelium and Ciliary Activity

\section{BIOCHEMISTRY DIVISION}

Vol. 1. Pitr-Rivers and TATA - The Thyroid Hormones

Vol. 2. BusH - The Chromatography of Steroids

Vol. 3. ENGEL - Physical Properties of Steroid Hormones

\section{BOTANY DIVISION}

Vol. 1. Bor - Grasses of Burma, Ceylon, India and Pakistan

Vol. 2. Turill (Ed.) - Vistas in Botany-Volume 1

Vol. 3. Schultes - Native Orchids of Trinidad and Tobago

Vol. 4. Cooke - Cork and the Cork Tree

\section{PLANT PHYSIOLOGY DIVISION}

Vol. 1. Sutcliffe - Mineral Salts Absorption in Plants

Vol. 2. Siegel - The Plant Cell Wall

\section{ZOOLOGY DIVISION}

Vol. 1. Raven - An Outline of Developmental Physiology

Vol. 2. RAvEN - Morphogenesis: The Analysis of Molluscan Development

Vol. 3. SAVORY - Instinctive Living

Vol. 4. KERKUT - Implications of Evolution

Vol. 5. TARTAR - The Biology of Stentor

Vol. 6. JENKIN - Animal Hormones

Vol. 7. Corliss - The Ciliated Protozoa

Vol. 8. GEORGE - The Brain as a Computer

Vol. 9. ARTHUR - Ticks and Disease

Vol. 10. RaVEN - Oogenesis

Vol. 11. MANN - Leeches (Hirudinea) 


\section{KERATIN \\ AND}

\section{KERATINIZATION}

An Essay in Molecular Biology

E. H. Mercer, D.Sc., Ph.D.

Chester Beatty Research Institute:

Institute of Cancer Research, Royal Cancer Hospital, London

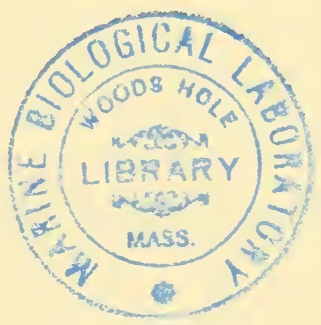

PERGAMON PRESS

NEW YORK - OXFORD - LONDON • PARIS 
PERGAMON PRESS INC.

122 East 55th Street, New York, 22, N.Y. 1404 New York Avenue N.W., Washington 5 D.C.

PERGAMON PRESS LTD.

Headington Hill Hall, Oxford

4 \& 5 Fitzroy Square, London, W.1

PERGAMON PRESS S.A.R.L.

24 Rue des Écoles, Paris, $V^{\bullet}$.

PERGAMON PRESS G.m.b.H.

Kaiserstrasse 75, Frankfurt am Main

Copyright @ 1961

PERGAMON PRESS LTD.

Library of Congress Card No. 60-53516

Set in Imprint 11 on 12 pt. and printed in Great Britain by BELI AND BAIN LTD., GLASGOW, SCOTLAND 
This book is dedicated to the memory of W. T. A S T B U R Y 



\section{Contents}

Preface $\ldots$ xi

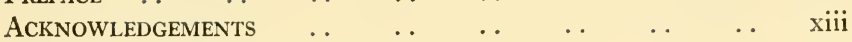

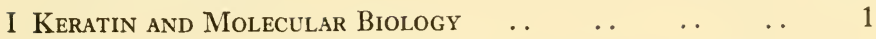

$\begin{array}{llllllll}\text { Macromolecules and biology } & \ldots & \ldots & \ldots & \ldots & \ldots & 1\end{array}$

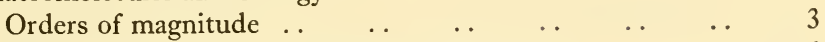

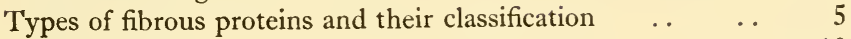

$\begin{array}{lllllllll}\text { Birefringence } & . & \ldots & \ldots & \ldots & \ldots & \ldots & \ldots & 10\end{array}$

$\begin{array}{llllllll}\text { X-ray methods } & \ldots & \ldots & \ldots & \ldots & \ldots & \ldots & 11\end{array}$

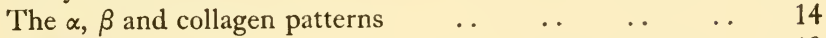

$\begin{array}{lllllllll}\text { Stabilization } & \ldots & \ldots & \ldots & \ldots & \ldots & \ldots & \ldots & 19\end{array}$

$\begin{array}{lllllllll}\text { Ecdysis . . } & \ldots & \ldots & \ldots & \ldots & \ldots & \ldots & \ldots & 22\end{array}$

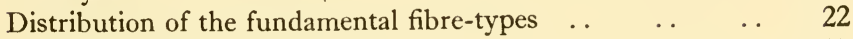

Some difficulties in defining a keratin $\ldots \begin{array}{lllll}\text {. . } & \ldots & \ldots & \ldots & 27\end{array}$

The significance of the variable amino acid composition .. 31

$\begin{array}{lllllll}\text { The fine structure of cells } & \ldots & \ldots & \ldots & \ldots & \ldots & 34\end{array}$

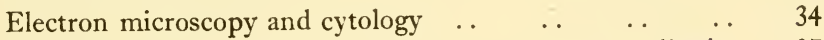

The cell surface, its specializations and intercellular adhesion $\quad 37$

$\begin{array}{lllllll}\text { The cell membrane . } & \ldots & \ldots & \ldots & \ldots & \ldots & 37\end{array}$

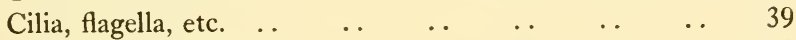

$\begin{array}{lllllll}\text { Surface invaginations } & \ldots & \ldots & \ldots & \ldots & \ldots & 40\end{array}$

Specializations of opposed surfaces. Cell contacts .. 40

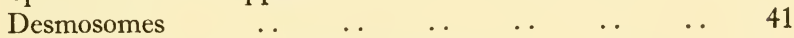

Interdigitation of confronted membranes . . $\quad \ldots \quad$. . 43

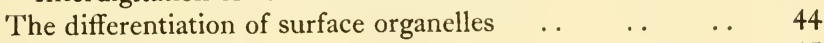

$\begin{array}{lllllll}\text { Cytoplasmic structures } & \ldots & \ldots & \ldots & \ldots & \ldots & 45\end{array}$

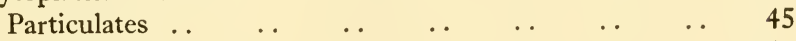

$\begin{array}{lllllll}\text { Membrane systems } & \ldots & \ldots & \ldots & \ldots & \ldots & 46\end{array}$

$\begin{array}{llllllll}\text { The nucleus } & \ldots & \ldots & \ldots & \ldots & \ldots & \ldots & 48\end{array}$

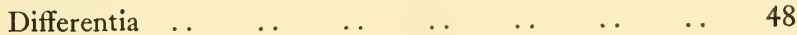

$\begin{array}{lllllll}\text { The phylogeny of keratinization } & \ldots & \ldots & \ldots & \ldots & 49\end{array}$

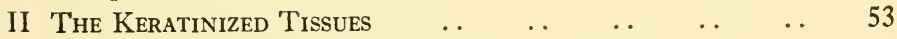

Generalized histology of the vertebrate epidermis $\quad \ldots \quad$. . 53

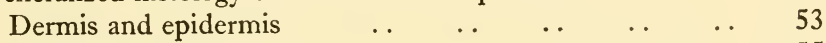

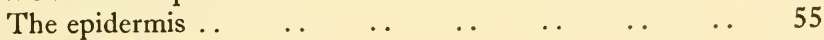

$\begin{array}{llllllll}\text { The epidermal family of cells } & \ldots & \ldots & \ldots & \ldots & \ldots & 57\end{array}$

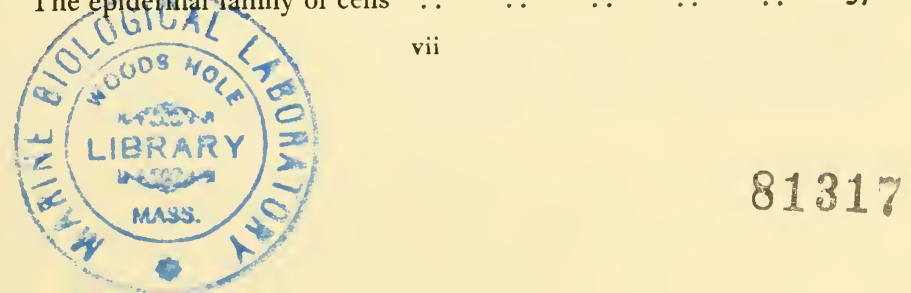


$\begin{array}{lllll}\text { The differentiation of epidermal cells } & \ldots & \ldots & \ldots & 60\end{array}$

$\begin{array}{lllllll}\text { Hard and soft keratins } & \ldots & \ldots & \ldots & \ldots & \ldots & 64\end{array}$

$\begin{array}{llllllll}\text { The specialized appendages } & \ldots & \ldots & \ldots & \ldots & \ldots & 66\end{array}$

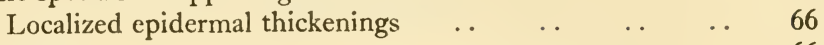

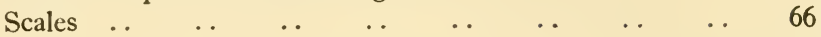

$\begin{array}{llllllllll}\text { Horns } & \ldots & \ldots & \ldots & \ldots & \ldots & \ldots & \ldots & \ldots & 67\end{array}$

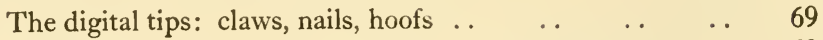

$\begin{array}{llllllllll}\text { Feathers } & \text {. } & \ldots & \ldots & \ldots & \ldots & \ldots & \ldots & 69\end{array}$

$\begin{array}{llllllllll}\text { Hairs } & \ldots & \ldots & \ldots & \ldots & \ldots & \ldots & \ldots & \ldots & 71\end{array}$

$\begin{array}{lllllll}\text { The phylogeny of hair } & \ldots & \ldots & \ldots & \ldots & \ldots & 73\end{array}$

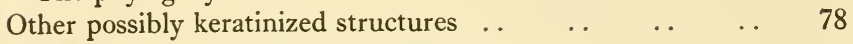

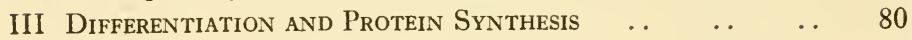

$\begin{array}{lllllll}\text { The cytology of keratinizing cells } & \ldots & \ldots & \ldots & \ldots & 80\end{array}$

$\begin{array}{llllllll}\text { The basal layer cells } & \ldots & \ldots & \ldots & \ldots & \ldots & \ldots & 80\end{array}$

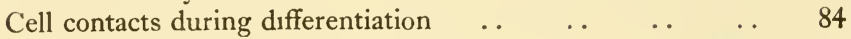

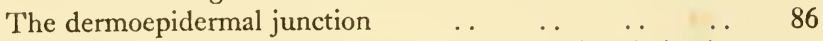

The development of basal membranes and their role in the

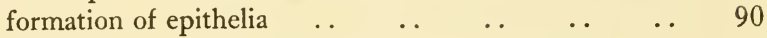

The differentiated layers and the variety of cell products $\quad . .94$

$\begin{array}{llllllll}\text { The epidermis .. } & \ldots & \ldots & \ldots & \ldots & \ldots & \ldots & 94\end{array}$

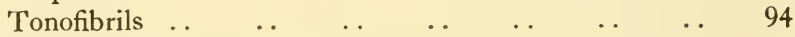

$\begin{array}{lllllllll}\text { Keratohyalin . . } & \ldots & \ldots & \ldots & \ldots & \ldots & \ldots & 95\end{array}$

$\begin{array}{llllllll}\text { The hair follicle } & \ldots & \ldots & \ldots & \ldots & \ldots & \ldots & 95\end{array}$

Intracellular differentiation in the hair bulb $\quad \ldots \quad \ldots \quad 100$

$\begin{array}{llllllll}\text { The feather follicle } & \ldots & \ldots & \ldots & \ldots & \ldots & \ldots & 101\end{array}$

$\begin{array}{lllllll}\text { The avian secreted keratins } & \ldots & \ldots & \ldots & \ldots & \ldots & 107\end{array}$

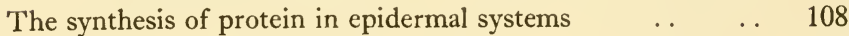

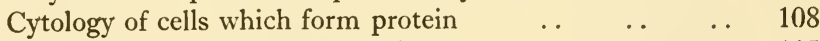

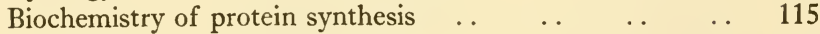

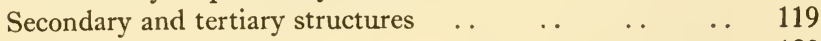

$\begin{array}{llllll}\text { Synthesis in retaining systems } & \ldots & \ldots & \ldots & \ldots & 120\end{array}$

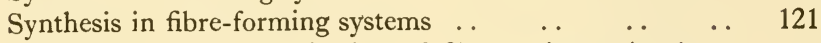

The supermolecular organization of fibrous tissues (tertiary

$\begin{array}{lllllllll}\text { structure) } & \ldots & \ldots & \ldots & \ldots & \ldots & \ldots & \ldots & 123\end{array}$

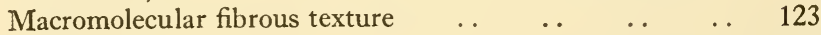

$\begin{array}{lllllllll}\text { Fibrogenesis } & \ldots & \ldots & \ldots & \ldots & \ldots & \ldots & \ldots & 125\end{array}$

$\begin{array}{llllllllll}\text { Collagen } & \ldots & \ldots & \ldots & \ldots & \ldots & \ldots & \ldots & 128\end{array}$

$\begin{array}{llllllll}\text { Fibrous insulin } & \ldots & \ldots & \ldots & \ldots & \ldots & \ldots & 127\end{array}$

Silk fibroin-aggregation of the molecule after unfolding 129

$\begin{array}{llllll}\text { Organization of fibrous tissues } & \ldots & \ldots & \ldots & \ldots & 129\end{array}$

$\begin{array}{lllllllll}\text { Epidermal fibrils } & \ldots & \ldots & \ldots & \ldots & \ldots & \ldots & 131\end{array}$

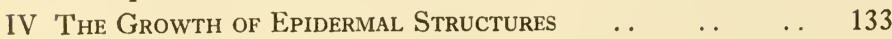

The epidermis as a growing organ $\quad \ldots \quad$. 
$\begin{array}{llllllll}\text { Mitosis in the basal layer } & \ldots & \ldots & \ldots & \ldots & \ldots & 135\end{array}$

$\begin{array}{llllllll}\text { General theories of growth } & \ldots & \ldots & \ldots & \ldots & \ldots & 138\end{array}$

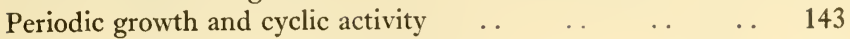

$\begin{array}{llllllll}\text { Control of epidermal growth } & \ldots & \ldots & \ldots & \ldots & \ldots & 146\end{array}$

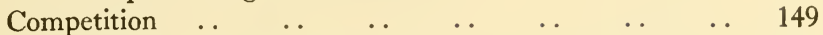

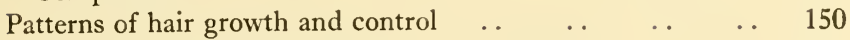

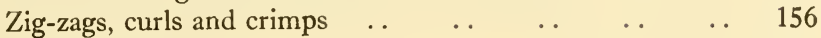

$\begin{array}{lllllllll}\text { Allometric growth } & \ldots & \ldots & \ldots & \ldots & \ldots & \ldots & 159\end{array}$

V Molecular and Macromolecular Structure $\quad \ldots \quad \ldots l 161$

The present status of the chemical structure of the keratins. . 161

$\begin{array}{lllllllll}\text { End groups } & \ldots & \ldots & \ldots & \ldots & \ldots & \ldots & \ldots & 161\end{array}$

$\begin{array}{llllllll}\text { Molecular structure } & \ldots & \ldots & \ldots & \ldots & \ldots & \ldots & 163\end{array}$

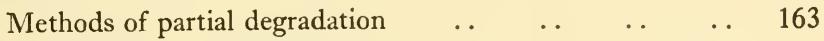

Non-destructive methods

$\begin{array}{llllllll}\text { X-ray diffraction } & \ldots & \ldots & \ldots & \ldots & \ldots & \ldots & 164\end{array}$

$\begin{array}{lllllll}\text { The low-angle pattern } & \ldots & \ldots & \ldots & \ldots & \ldots & 165\end{array}$

The wide-angle pattern $\quad \ldots \quad$.

$\begin{array}{lllll}\text { The elastic properties and the structure of hair } & \ldots & \ldots & 172\end{array}$

$\begin{array}{lllllll}\text { Current crystallographic analysis } & \ldots & \ldots & \ldots & \ldots & 176\end{array}$

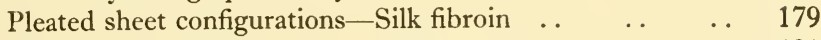

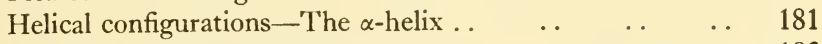

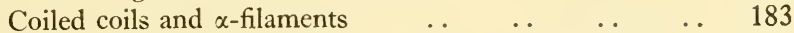

The organization of $\alpha$-filaments into larger structures $\quad . \quad 187$

The $\alpha-\beta$ transformation in terms of the $\alpha$-helix $\quad \ldots \quad \ldots \quad 188$

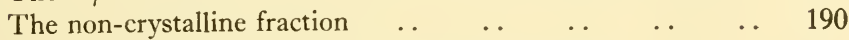

Other methods of determining chain configuration $\quad$. $\quad$.. $\quad 194$

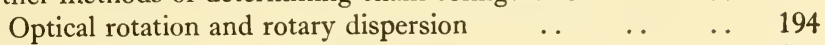

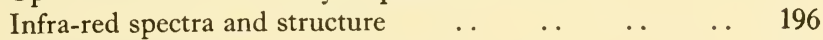

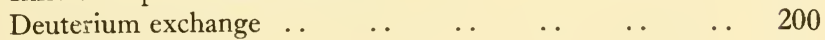

$\begin{array}{llllllll}\text { The cross- } \beta \text { pattern } & \ldots & \ldots & \ldots & \ldots & \ldots & \ldots & 200\end{array}$

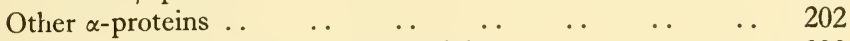

Some $\alpha$ - and $\beta$-proteins of insect origin $\quad \ldots \quad \ldots \quad \ldots 203$

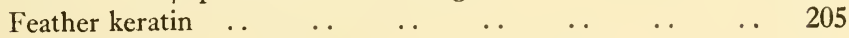

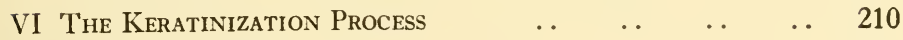

$\begin{array}{llllllll}\text { The hard keratins } & \ldots & \ldots & \ldots & \ldots & \ldots & \ldots & 210\end{array}$

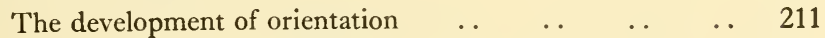

$\begin{array}{lllllll}\text { The development of stability } & \text {. } & \ldots & \ldots & \ldots & 214\end{array}$

Thiol and disulphide groups during keratinization $\quad \ldots 217$

$\begin{array}{lllllll}\text { Nucleic acids and synthesis } & \ldots & \ldots & \ldots & \ldots & \ldots & 219\end{array}$

$\begin{array}{llllllll}\text { Metabolic enzymes } & \ldots & \ldots & \ldots & \ldots & \ldots & \ldots & 221\end{array}$

$\begin{array}{lllllllll}\text { Glycogen } & \text {. } & \ldots & \ldots & \ldots & \ldots & \ldots & \ldots & 221\end{array}$

Acid mucopolysaccharides and Schiff-reactive substances 221

$\begin{array}{lllllllll}\text { Phosphatases } & \text {. } & \ldots & \ldots & \ldots & \ldots & \ldots & \ldots & 222\end{array}$ 
$\begin{array}{lllllllll}\text { Lipids } & \ldots & \ldots & \ldots & \ldots & \ldots & \ldots & \ldots & \ldots\end{array}$

$\begin{array}{llllllll}\text { Water content .. } & \ldots & \ldots & \ldots & \ldots & \ldots & \ldots & 222\end{array}$

The fine histology of the hair follicle in the keratinization zone ..

The cortex

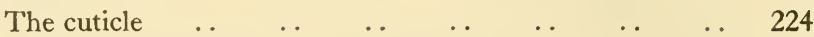

$\begin{array}{lllllll}\text { The inner root sheath } & \ldots & \ldots & \ldots & \ldots & \ldots & 226\end{array}$

$\begin{array}{llllllll}\text { Soft keratinization } & \ldots & \ldots & \ldots & \ldots & \ldots & \ldots & 228\end{array}$

$\begin{array}{llllllll}\text { The epidermis .. } & \ldots & \ldots & \ldots & \ldots & \ldots & \ldots & 228\end{array}$

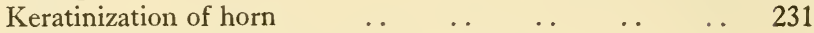

Follicular nutrition and the entrance of sulphur $\quad \ldots \quad$. 232

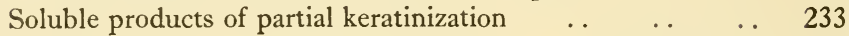

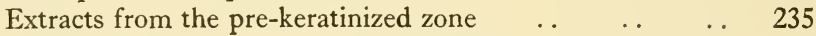

Soluble derivatives of keratinized tissues $\quad \ldots \quad$. . $\quad$. . 236

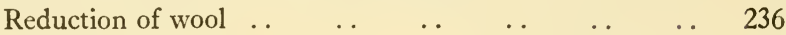

$\begin{array}{llllllll}\text { Oxidizing agents } & \ldots & \ldots & \ldots & \ldots & \ldots & \ldots & 238\end{array}$

$\begin{array}{lllllll}\text { Thioglycollate extracts } & \ldots & \ldots & \ldots & \ldots & \ldots & 240\end{array}$

Soluble derivatives of feather and other keratins _. 243

The location of the cystine residues $\quad \ldots \quad$.

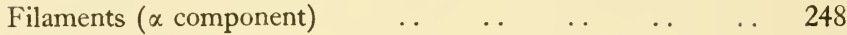

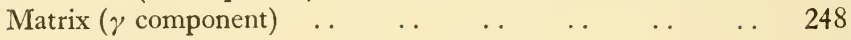

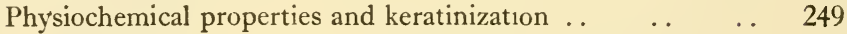

$\begin{array}{lllllllll}\text { Salt linkages } & \ldots & \ldots & \ldots & \ldots & \ldots & \ldots & \ldots & 251\end{array}$

$\begin{array}{llllllll}\text { Disulphide bonds } & \ldots & \ldots & \ldots & \ldots & \ldots & \ldots & 252\end{array}$

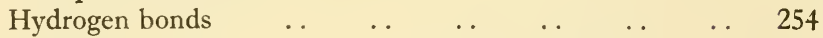

Molecular configuration in the supercontracted state $\quad .258$

$\begin{array}{llllllll}\text { The setting of hairs } & \ldots & \ldots & \ldots & \ldots & \ldots & \ldots & 259\end{array}$

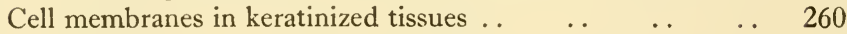

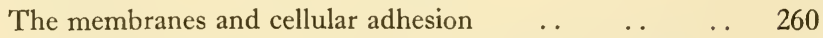

The morphology of the membranes of keratinized tissues.. 262

The fate of the intracellular apparatus during keratinization 263

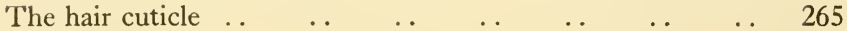

$\begin{array}{llllllllll}\text { The medulla } & \ldots & \ldots & \ldots & \ldots & \ldots & \ldots & \ldots & 268\end{array}$

The residues remaining after the chemical extraction of

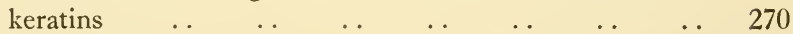

Uneven keratinization and its histological distribution $\quad . \quad 272$

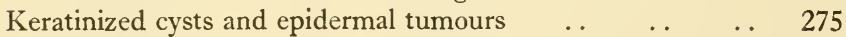

$\begin{array}{lllllllll}\text { Pigmentation } & \ldots & \ldots & \ldots & \ldots & \ldots & \ldots & \ldots & 276\end{array}$

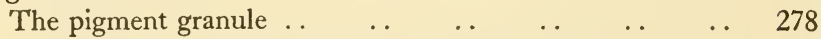

$\begin{array}{lllll}\text { The chemistry of melanization } & \ldots & \ldots & \ldots & 279\end{array}$

$\begin{array}{llllllllll}\text { References } & \ldots & \ldots & \ldots & \ldots & \ldots & \ldots & \ldots & 283\end{array}$

$\begin{array}{lllllllll}\text { Author Index } & \ldots & \ldots & \ldots & \ldots & \ldots & \ldots & \ldots & 300\end{array}$

$\begin{array}{lllllllll}\text { Subject Index } & \ldots & \ldots & \ldots & \ldots & \ldots & \ldots & \ldots & 306\end{array}$ 


\section{Preface}

An explanation of the sub-title of this work may not be out-of-place since it will explain at the same time the treatment of the main theme. The term molecular biology has gained currency recently; its use implies an attitude towards biology, an acceptance of the belief that biological phenomena can be related to the interaction of the molecules found in biological systems. In practice it means that the primary emphasis is placed on the determination of molecular structure by any of the means available.

In its pretensions molecular biology is a generalized science; it aims to provide a common, integrating background to such special sciences as, for example, biochemistry, histology and physiology, by rendering them alike explicable in terms of molecular interaction. The name but not the subject matter is new. In the pre-war period such writers as K. H. Meyer and H. Mark, W. T. Astbury, J. D. Bernal, W. J. Schmidt, F. O. Schmitt and A. Frey-Wyssling, to mention only a few, had the objective well in mind. What is new, however, is the tempo of achievement in the postwar years; detailed structures of key biological macromolecules have been obtained and a complete coverage of cellular contents of all dimensions is possible now by means of microscopy. These achievements have lifted the dream of relating structure and function out of speculation to become a problem capable of experimental investigation.

That it is still largely a project one realises quickly enough in setting out to give an account of even a simple, relatively uniform group of tissues such as the epidermis and its appendages. Thus, while this book attempts to pose the problems of keratinization consistently in molecular terms and to avoid concepts not stateable in such terms, it rather quickly degenerates into an outline of unsolved problems. Keratinization will be regarded as a development of certain primitive cellular traits adapted to serve the end of providing a protective coating to a multicellular organism. The traits emphasised are intercellular adhesion and the proliferation and stabilization of cytoplasmic protein filaments. We shall be concerned for the most part with the structure and synthesis of these filaments, with the structure of cell membranes and the nature of intercellular adhesion.

I am grateful to Professor A. Haddow, F.R.S., Director of the Chester Beatty Research Institute, where I have found the opportunity to 
continue this work and to associate with many stimulating colleagues. My particular thanks are due to: Mr. M. S. C. Birbeck with whom I have collaborated in much of the experimental work described here; to Mrs. Rex Dadd who prepared the typescript; to Mr. K. R. Moreman and Mr. M. Docherty for their careful attention to the photographic material; to Dr. P. Alexander for reading the manuscript and for his encouragement; to Dr. K. M. Rudall for reading the page proofs and for pointing out many shortcomings; to the staff of Pergamon Press Ltd., in particular Mr. B. J. Adams, for their unfailing attention during the preparation of the work for publication.

E. H. Mercer 


\section{Acknowledgements}

My thanks are due to the following who have very kindly provided me with original photographs for plates: Mr. H. J. Woods (Plates 1 and 2), Dr. K. M. Rudall (Plate 3), Mr. M. S. C. Birbeck (Plates 5B and 10B), Dr. G. E. Rogers (Plate 16), Dr. I. Brody (Plate 17) and Dr. I. Heiger (Plate 22A).

The following publishers and societies have granted permission to reproduce figures from their publications:

The Royal Society

(Proceedings of the Royal Society, Philosophical Transactions of the Royal Society)

The Textile Institute

(Fournal of the Textile Institute)

Rockefeller Institute fOR Medical Research

( Journal of Biophysical and Biochemical Cytology, Fournal of General Physiology)

Macmillan \& Co.

(Nature)

Elsevier Publishing Co.

(Biochimica et Biophysica Acta)

North-Holland Publishing Co.

(Mechanical Properties of Fibres)

Cambridge University Press

(Fournal of Endocrinology, An Introduction to Comparatice Biochemistry (3rd edition)

Athlone Press

(Lectures on the Scientific Basis of Medicine)

Society of Dyers and Colourists

(Fibrous Proteins)

Almouist and Wiksell

(Proceedings of the International Conference on Electron Microscopy, Stockholm 1956)

Society Cosmetic Chemists, London

(Fournal of the Society of Cosmetic Chemists) 
Radio Corporation of America

(Radio Corporation of America Scientific Instrument News)

New York Academy of Sciences

(Annals of the New York Academy of Sciences)

Academic Press Inc.

(Advances in Protein Chemistry, Fournal of Ultrastructure Research) SPRINGER-VerLaG, BERLIN

(Proceedings of the 4th International Congress on Electron Microscopy)

C.S.I.R.O. Australia

(Australian Fournal of Biological Sciences, Proceedings of the International Wool Textile Conference, Australia 1956)

American Chemical Society

(Fournal of the American Chemical Society)

Royal Society of Edinburgh

(Transactions of the Royal Society of Edinburgh)

SOCIETY FOR EXPERIMENTAL BIOLOGY

(Symposium of the Society for Experimental Biology) 


\section{Keratin and Molecular Biology}

\section{Macromolecules and biology}

Biological processes, whatever the organism, plant or animal, are inseparably associated with macromolecules. Some of these form the solid frameworks which support and protect organisms; others as moremobile particles effect the reactions with each other and with smaller molecules which we recognize as ultimately characteristic of life; others again form the material basis of inheritance. It is no more than the truth to say that the way of life of an organism is determined by the nature of the large molecules synthesized by its cells. The familiar difference between the higher plants and animals furnishes an example as illustration. Animals are able to move about or to move their parts because certain of their cells have the ability to synthesize contractile muscle proteins. Plant cells have largely lost this power; on the other hand they are able to form and secrete quantities of rigid encrusting substances, such as cellulose, which permit of a very different anatomy and mode of life.

This statement is indeed equivalent to saying that animals have muscles and plants have woody cell walls; but the emphasis is different. In drawing attention in the first place to the macromolecular content of the cell and the organism as the basic factor determining behaviour, we are led to ask a particular kind of question, of which the following are examples:

(a) What are these macromolecules and what is their molecular structure?

(b) Can we predict their biological function from their structure ?

(c) How are these molecules synthesized and what factors control their synthesis ?

(d) When do they appear in the course of embryonic development (molecular ontogeny) and how do they influence this development?

(e) How and when were the various molecular types evolved (molecular phylogeny) and how has their appearance influenced the course of evolution?

Attempts to answer these questions are already engaging much attention and undoubtedly theoretical biology of the future will base itself largely 
on the answers arrived at (Perutz, 1959 and Schmitt, 1960). Even today, when only partial and tentative answers can be given, the effort is made to pose the problem in a form which envisages an answer in terms of the physical chemistry of the molecular constituents of the system. Thus, while it may be admitted at the outset that the treatment is foredoomed to be incomplete, it is in this spirit that we shall approach the study of keratin and keratinization. The discussion of keratin is not in itself an unfavourable theme for the purpose of systematizing parts of the already considerable amount of information on macromolecular biology since, largely as a result of the work of Astbury, this protein and others closely related to it have been shown to occupy a central position in any such discussion.

The keratins form a class of resistant, insoluble proteins found in the vertebrate epidermis and its appendages: hairs, feathers, claws, horns, etc., and in small amounts in certain of the internal epithelia. The name keratinization is given to the process by which these tissues are rendered tough and insoluble. Together with the dermis, which is in effect a closelyknit fibrous meshwork of the protein collagen, the epidermis and its derivatives constitute the protective integument. Everyone is familiar in a general way with the properties of the keratins: their insolubility, their toughness combined with elasticity, the enormous variety of forms assumed. Our object will be to try to correlate these properties with their molecular basis and to sketch (for that is all we can do at present) their development. Attention will be mainly directed towards cellular and subcellular structures for, although the macroscopic anatomy of these tissues is of great variety and interest, it would carry us beyond the present intention to attempt a detailed description. For convenience, the salient facts of their anatomy will be recalled; for the details reference may be made to the specialized texts referred to on p. 80 .

Current research emphasizes the role of two classes of macromolecules in biology: the nucleic acids and the proteins. The genetic functions of cells devolve on the nucleic acids which, it is currently believed, alone exercise the power of self-replication and of controlling the replication of other vital molecules (Crick, 1958); the proteins are components of most of the other working parts of cells and of many extracellular deposits. On the basis of their degree of aggregation two classes of proteins are distinguished: the corpuscular proteins, that is proteins normally carrying out their function in a particulate form in solution or adsorbed on surfaces. Examples of this class are the respiratory proteins and most enzymes. The other class is that of the structural proteins, which carry out their function, often in part mechanical, by virtue of their property of forming large aggregated, often fibrous masses. Among these we place, for example, the contractile muscle proteins and the various reinforcing or protective proteins including the keratins. 
The special suitability of the proteins for these various roles is attributed to their being high molecular weight, long chain polypeptides:

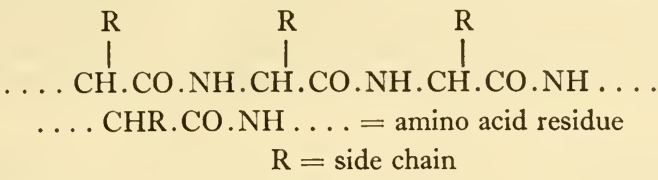

with a large number of reactive side chains which, as a result of the folding of the main chain, may form a variety of surface patterns permitting specific interactions with other molecules, large and small, and with other proteins (Frey-Wyssling, 1953). The fibre-forming habit, common among the structural proteins, is seen as a special example of protein-protein interaction which can lead by association to macromolecular aggregates. Here we are concerned more with the structural proteins, so named because they are major constituents of the large-scale structures of cells and tissues; in their mass they are readily visible in the light microscope and obviously are related to the function of the organ in which they occur. Resistant structural proteins are produced by introducing various crosslinkages between the polypeptide chains.

It is a basic assumption that structure and function are interrelated and for this reason the determination of structure is the primary concern of macromolecular biology. Two other problems, which relate macromolecular structures immediately to main themes of biology, morphogenesis and differentiation, are the biosynthesis and the appearance of the molecules in the course of individual development. Further, since biological macromolecules were developed in the course of a long biochemical evolution, there are phylogenetic as well as ontogenic aspects to the problem of their adaptation to a biological role. In these terms then, keratinization may be looked upon as a particular phenomenon characterized by the appearance in epidermal tissues of certain macromolecules giving rise to gross structures capable of a protective function.

\section{ORDERS OF MAGNITUDE}

The objects which are the concern of molecular biology range in size from small molecules of diameter a few Ångstrom units to massive materials which can be examined with the unaided eye. It is helpful when attempting to form a conception of the vast range of size involved to have some visual aid to hand and for this purpose reference may be made to Fig. 1.

Orders of magnitude are indicated on the right by a logarithmic scale and various levels of organization: molecular, macromolecular, cytological and histological are distinguished. The formations at the higher levels of organization are constructed from the smaller macromolecular and 
molecular elements most easily studied by electron microscopy or X-ray diffraction.

A brief description of these and other specialized techniques will be given in the course of the text when it is necessary to make the discussion clear, but for an adequate account of the methods reference must be made

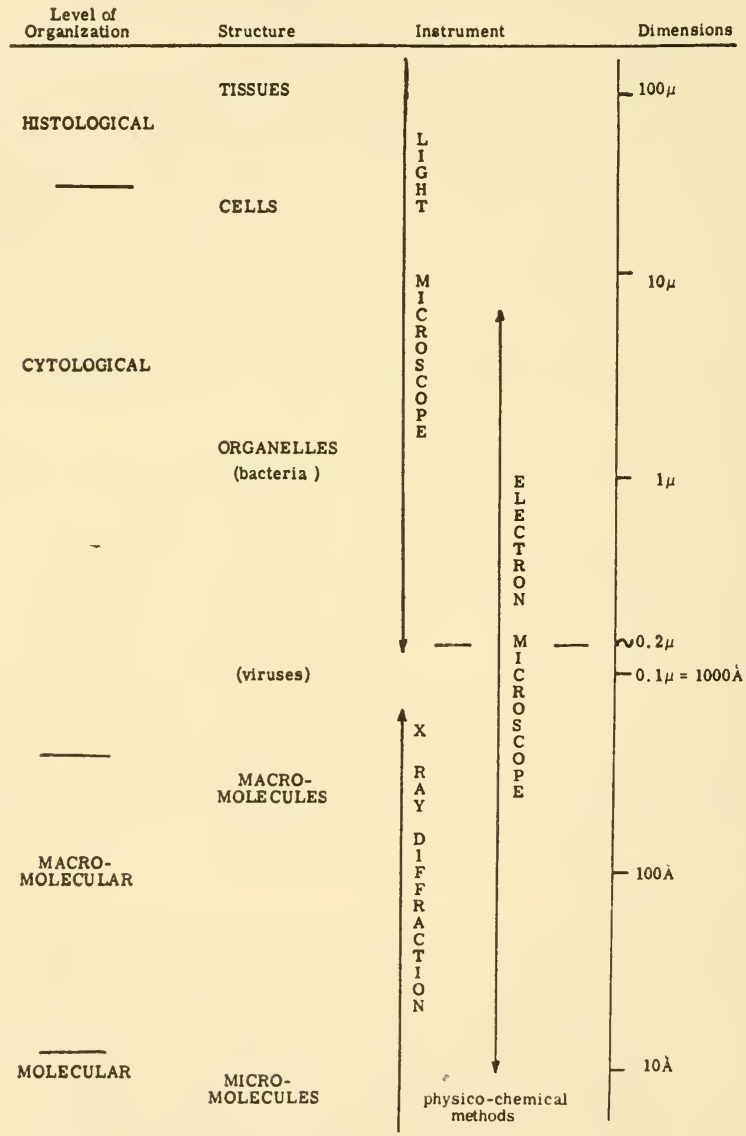

FIG. 1. Levels of organization of biological structure and range of instruments. 
to the various texts available (Bunn, 1946; Hall, 1953; Schmidt, 1924; and Oster and Pollister, 1955-56).

It will be seen from Fig. 1 that with present-day instrumentation we possess the means to investigate all levels of biological significance. Thus it is now feasible to hope to obtain the information necessary to permit of a stepwise reconstruction of a tissue beginning with elements of a molecular size. Already the results of comparative fine-structure studies have indicated the nature of the basic structural elements found in all cells and developed to different degrees in different types of cells. Its particular endowment of these structural elements gives a cell its characteristic cytology and, in turn, from the special association of these cells arises the histology of the tissue. For example the keratinized tissues themselves achieve their primary function as the toughened outposts of the protective integument by virtue of an enhancement principally of two structural features common to all Metazoan cells. In the first place all cells contain greater or lesser amounts of structural fibrous proteins; in keratinized cells there is a great increase in these proteins which are subsequently subjected to chemical changes which stabilize them. Secondly, the cells of most tissues must adhere and in the purely cellular tissues, such as the epithelia, this means that the surfaces of the cells themselves must stick together. In the specialized protective epithelia including the keratinized tissues, clearly this intercellular adhesion must be enhanced. Thus our discussion of keratinization will be found to be concerned largely with these two aspects of the tissues: the nature of the stabilized proteins within the cells and the manner in which the cells themselves are held together. We shall accordingly begin by giving a general survey of the types of fibrous proteins found in tissues and follow this with an account of the fine structure of cells with emphasis on the structural devices associated with their surfaces which may be concerned with intercellular adhesion.

\section{Types of fibrous proteins and their classification}

The classification of the proteins has been made on the basis of several grounds all more or less arbitrary. We have mentioned the convenience of the division into structural and particulate proteins, which is a useful distinction when one is concerned with insoluble proteins forming structures extending far beyond the molecular level. Traditionally the soluble, particulate proteins are classified on the basis of their solubilities in various aqueous media, a means of distinction which dates back to a time when the principal preoccupation of the protein chemist was the separation of definite individuals from mixtures.

The basic chemical character of a macromolecule (protein, polysaccharide, nucleic acid) may be established by the chemical analysis of a 
sample after complete hydrolysis. In this way the familiar fibrous proteins, collagens, keratins and silks were early shown to be distinct proteins, characterized by the different amounts of amino acid residues forming different patterns (Fig. 2). The common polysaccharides, cellulose and chitin, yield glucose and acetoaminoglucose, respectively.

There have been numerous analyses made of keratins beginning with

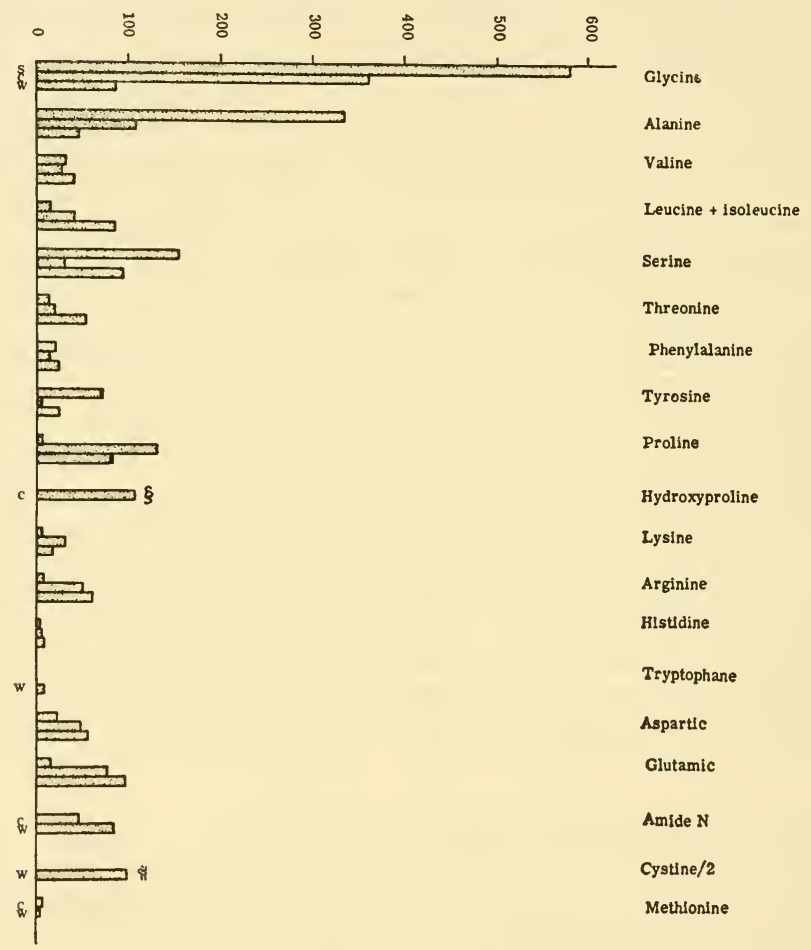

FIG. 2. Number of amino acid residues in $10^{5} \mathrm{~g}$ protein in three fibrous proteins from Tristram's data (1953).

(1) Silk fibroin (s)

(2) Collagen (c)

(3) Wool (w)

$\S$ Hydroxy-proline is only found in collagens and is regarded as " diagnostic."

If The cystine is given as "half cystine" (HS. CH. $\left.\mathrm{NH}_{2} \mathrm{COOH}\right)$. 
those made by Abderhalden and Voitinovici in 1907. Most of these are today of little more than historical interest, but they served to sketch out the broad outlines of the amino acid pattern of the group and to establish a family unity. No purpose would be served here by reproducing these data. Reference may be made to the paper by Simmonds (1955) in which a table of all the results will be found.

Table 1. Percentage Weights of Amino Acids

IN Wool

\begin{tabular}{|c|c|c|c|}
\hline Amino acid & $a^{*}$ & $\mathrm{~b} \dagger$ & $\mathrm{c} \ddagger$ \\
\hline glycine & $5 \cdot 16$ & $5 \cdot 5$ & $5 \cdot 26$ \\
\hline alanine & $3 \cdot 71$ & $4 \cdot 3$ & $3 \cdot 73$ \\
\hline valine & 4.96 & $5 \cdot 7$ & $5 \cdot 78$ \\
\hline leucine & $7 \cdot 63$ & 8.9 & $7 \cdot 69$ \\
\hline isoleucine & $3 \cdot 07$ & $3 \cdot 7$ & 3.79 \\
\hline serine & $9 \cdot 04$ & $9 \cdot 9$ & $7 \cdot 15$ \\
\hline threonine & 6.55 & $5 \cdot 56$ & $6 \cdot 58$ \\
\hline phenylalanine & $3 \cdot 43$ & $4 \cdot 0$ & $3 \cdot 40$ \\
\hline tyrosine & $6 \cdot 38$ & $5 \cdot 5$ & $4 \cdot 10$ \\
\hline tryptophane & $2 \cdot 10$ & 0.94 & - \\
\hline proline & $7 \cdot 28$ & $6 \cdot 8$ & $6 \cdot 58$ \\
\hline hydroxyproline & - & - & - \\
\hline lysine & $2 \cdot 82$ & $3 \cdot 3$ & $3 \cdot 15$ \\
\hline hydroxylysine & 0.68 & - & - \\
\hline arginine & $10 \cdot 49$ & $9 \cdot 8$ & $9 \cdot 03$ \\
\hline histidine & 0.90 & $1 \cdot 2$ & - \\
\hline aspartic acid & $6 \cdot 69$ & 6.8 & $6 \cdot 29$ \\
\hline glutamic acid & 14.98 & $14 \cdot 5$ & $12 \cdot 8$ \\
\hline ammonia & $1 \cdot 42$ & - & - \\
\hline cystine and cysteine & $11 \cdot 3$ & $10 \cdot 3$ & $11 \cdot 0$ \\
\hline methionine & 0.69 & 0.56 & 0.55 \\
\hline
\end{tabular}

* Simmonds (1955).

$\dagger$ Corfield and Robson (1955).

$\ddagger$ Quoted by von Bergen (1954).

The estimations in $\ddagger$ were made by microbiological methods which may not be as accurate as those used by the other authors.

At the present time we possess reliable analyses by modern methods, in which the greater part of the nitrogen of the proteins has been accounted for, only of wool and feather. For purpose of reference we reproduce here the analyses reported by Simmonds (1955) (Table 1 (a)), by Corfield 
and Robson (1955) (Table 1(b)) and those quoted by von Bergen (1954) (Table 1 (c)) all of samples of wool, and by Schröeder and Kay of feathers (1955) (Table 2). Although it contains older and less reliable data, Table 3, taken from Ward and Lundgren (1954), is valuable in that it permits a comparison between several varieties of keratin. The question of the significance of the differences between the figures given, even for an apparently homogeneous material such as wool, will be discussed later (p. 31).

Table 2. Amino acid Composition of White Turkey feather Parts and of Goose Feather Barbs and Goose Down.*

(Values are in terms of $\mathrm{g}$ of amino acid per $100 \mathrm{~g}$ of moisture- and ash-free material.)

\begin{tabular}{|c|c|c|c|c|c|c|}
\hline Amino acid & $\begin{array}{l}\text { Turkey } \\
\text { barbs } †\end{array}$ & $\begin{array}{l}\text { Turkey } \\
\text { calamus }\end{array}$ & $\begin{array}{l}\text { Turkey } \\
\text { medulla }\end{array}$ & $\begin{array}{l}\text { Turkey } \\
\text { rachis }\end{array}$ & $\begin{array}{l}\text { Goose } \\
\text { barbs }\end{array}$ & $\begin{array}{c}\text { Goose } \\
\text { down }\end{array}$ \\
\hline glycine & $7 \cdot 25$ & $9 \cdot 60$ & 8.90 & $10 \cdot 14$ & $8 \cdot 38$ & $7 \cdot 26$ \\
\hline alanine & $4 \cdot 01$ & $7 \cdot 12$ & $5 \cdot 89$ & $7 \cdot 66$ & $4 \cdot 10$ & 3.96 \\
\hline valine & $8 \cdot 60$ & $8 \cdot 43$ & $8 \cdot 59$ & $8 \cdot 65$ & $7 \cdot 34$ & $7 \cdot 89$ \\
\hline isoleucine & $4 \cdot 98$ & 3.94 & $3 \cdot 96$ & $3 \cdot 90$ & $4 \cdot 58$ & $4 \cdot 76$ \\
\hline leucine & $7 \cdot 26$ & $8 \cdot 85$ & 8.07 & $9 \cdot 37$ & $7 \cdot 68$ & $7 \cdot 74$ \\
\hline serine & $12 \cdot 90$ & $15 \cdot 09$ & $12 \cdot 37$ & $14 \cdot 09$ & $12 \cdot 53$ & $12 \cdot 38$ \\
\hline threonine & $4 \cdot 68$ & $4 \cdot 73$ & $4 \cdot 35$ & $4 \cdot 51$ & 4.94 & $5 \cdot 42$ \\
\hline phenylalanine & $4 \cdot 96$ & $5 \cdot 76$ & $5 \cdot 59$ & $5 \cdot 75$ & $4 \cdot 04$ & $3 \cdot 80$ \\
\hline tyrosine & $2 \cdot 32$ & 3.97 & $3 \cdot 81$ & $2 \cdot 91$ & $4 \cdot 46$ & $3 \cdot 69$ \\
\hline proline & $10 \cdot 50$ & $10 \cdot 98$ & $10 \cdot 87$ & 10.97 & $10 \cdot 05$ & $9 \cdot 81$ \\
\hline lysine & $1 \cdot 23$ & 0.98 & $1 \cdot 32$ & $0 \cdot 88$ & $1 \cdot 30$ & $1 \cdot 41$ \\
\hline arginine & $6 \cdot 44$ & $6 \cdot 69$ & $6 \cdot 57$ & $6 \cdot 18$ & $6 \cdot 04$ & 6.52 \\
\hline histidine & $0 \cdot 39$ & 0.59 & $0 \cdot 78$ & $0 \cdot 34$ & 0.44 & 0.33 \\
\hline aspartic acid & $6 \cdot 55$ & 7.09 & $7 \cdot 01$ & $7 \cdot 41$ & $7 \cdot 47$ & $7 \cdot 26$ \\
\hline glutamic acid & $9 \cdot 08$ & $8 \cdot 74$ & $8 \cdot 60$ & $8 \cdot 84$ & 8.99 & $9 \cdot 06$ \\
\hline ammonia & $1 \cdot 85$ & $1 \cdot 43$ & $1 \cdot 35$ & $1 \cdot 49$ & $1 \cdot 91$ & $1 \cdot 89$ \\
\hline cystine & $8 \cdot 68$ & $8 \cdot 29$ & $8 \cdot 10$ & $8 \cdot 48$ & $10 \cdot 75$ & $11 \cdot 31$ \\
\hline methionine & $0 \cdot 36$ & $0 \cdot 34$ & $0 \cdot 44$ & $0 \cdot 39$ & $0 \cdot 25$ & $0 \cdot 32$ \\
\hline
\end{tabular}

* Data taken from Schröeder and Kay (1955).

$\dagger$ For an explanation of the parts of a feather see p. 30 .

Even when the chemical composition of a fibrous macromolecular material is known, its detection and characterization in cells and tissues may offer difficulties in routine histochemistry. For some materials reliable histochemical tests have been developed (see p. 29); others are recognized more or less negatively, simply by their fibrous character and their intractable behaviour towards the usual stains and reagents. 


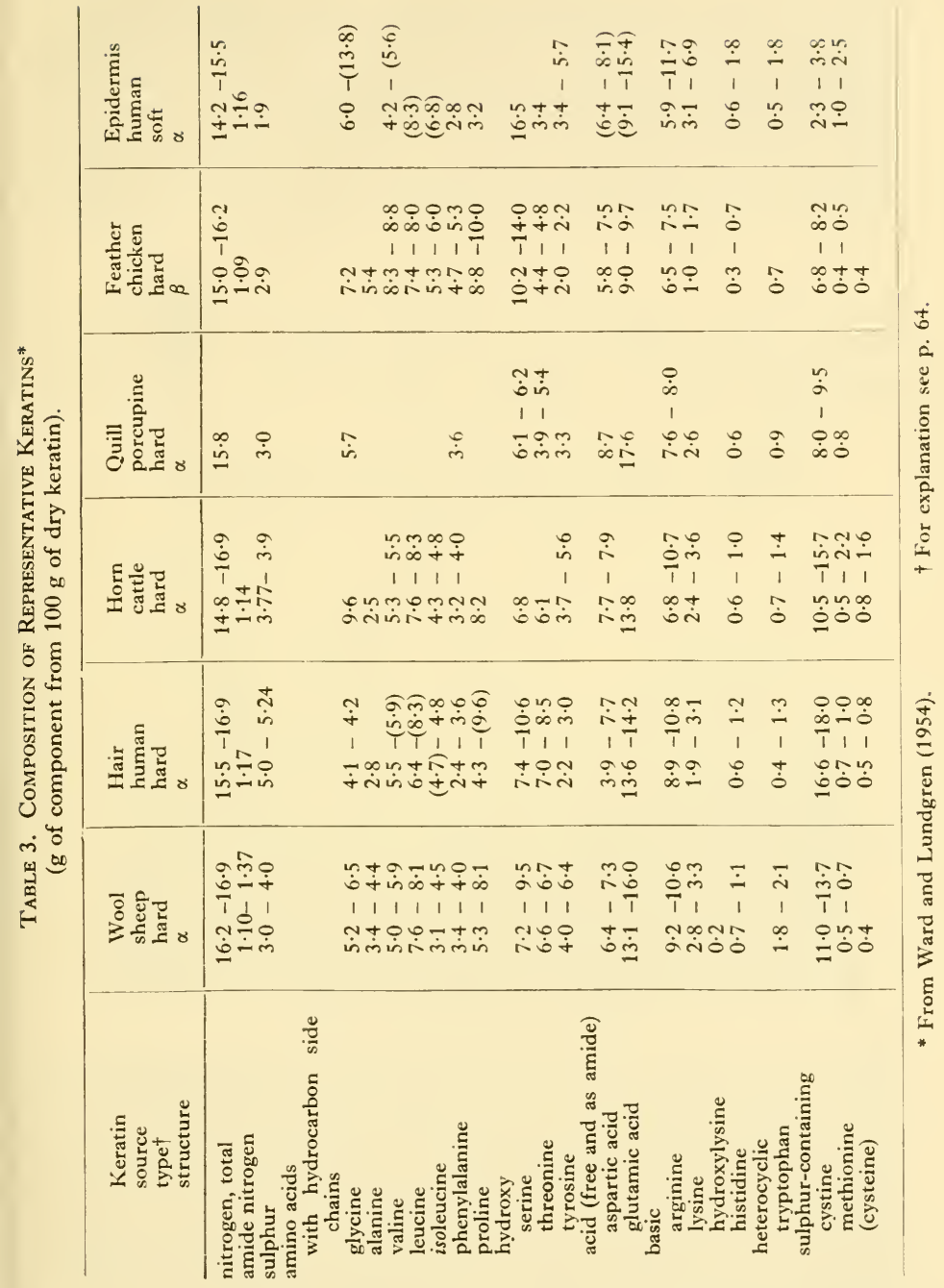




\section{BIREFR INGENCE}

The presence of oriented material, either fibrous or membranous, is most conveniently recognized by its optical properties using the polarizing microscope (Schmidt, 1924). The method is extremely sensitive, but yields no information concerning molecular structure.

All major biological fibres and, in particular, the keratinized epidermal tissues are birefringent (double refracting) and the direction of maximum refractive index coincides with the fibre axis as inferred from microscopic inspection. 'The birefringence is quantitatively defined as:

$$
\Delta \eta=\eta_{11}-\eta_{1}
$$

when $\eta_{11}$ and $\eta_{1}$ are the refractive indices in a direction parallel to the fibre and at right-angles to it. In practice, usually a retardation $R$ is measured for a sample of material of thickness $d$ and $\Delta \eta=R / d$. Some typical values for fibres are given in Table 4.

Table 4. Optical Constants of Hair and Related Fibres: $\eta_{\|}$Refractive Index Parallel to Fibre Axis;

$\eta_{\perp}$ Refractive Index NoRmal to Fibre Axis

$$
\left(\Delta \eta=\eta_{11}-\eta_{\perp}\right) \text {. }
$$

\begin{tabular}{l|l|l|l}
\hline \multicolumn{1}{c|}{ Fibre } & \multicolumn{1}{c|}{$\eta_{\text {II }}$} & \multicolumn{1}{c|}{$\eta_{\perp}$} & \multicolumn{1}{c}{$\Delta \eta$} \\
\cline { 1 - 2 } wool (dry)* $^{*}$ & 1.5633 & 1.5494 & $0.0139 \ddagger$ \\
fibroin (silk) $\dagger$ & 1.584 & 1.529 & 0.057 \\
cellulose (ramie) $\dagger$ & 1.599 & 1.532 & 0.067 \\
nylon (polyamide) $\dagger$ & 1.580 & 1.520 & 0.060 \\
\hline
\end{tabular}

* Barnes (1933).

† Frey-Wyssling (1953).

¥ The value for wool taken as a typical, non-medullated, keratin with a thin cuticle (i.e. consisting largely of oriented fibrous keratin) is regarded as a maximum value. Other observers quote figures for $\Delta \eta$ nearer to 0.01 . Not infrequently lower values $(\sim 0.008)$ can be measured, but this may be presumed to be due to deterioration. Whether $\Delta \eta$ approaches a quite definite figure, characteristic of pure, dry, undamaged fibrous keratin has not been established.

The actual values of $\Delta \eta$ are not greatly used for identifying fibre-types since, being influenced by many factors (such as swelling, imbibition of liquids and tension), they vary greatly and further, although the retardation is easily observed and measured with a compensator, for microscopic objects in cells the thickness is not so easily determined. Nevertheless the polarizing microscope is a most sensitive instrument for the recognition of oriented material and for detecting changes in orientation produced by deformation, chemical effects, heat, etc. It can be particularly useful when studying the degree of stabilization of an oriented material. 


\section{X-RAy Methods}

With the growing hope of actually determining the molecular structure of proteins by means of X-ray diffraction, or at least of obtaining certain experimental criteria of structure even when this may not be determinable in detail, the possibility arises of devising a rational system of classification based on molecular structure. The simple procedures based on X-ray

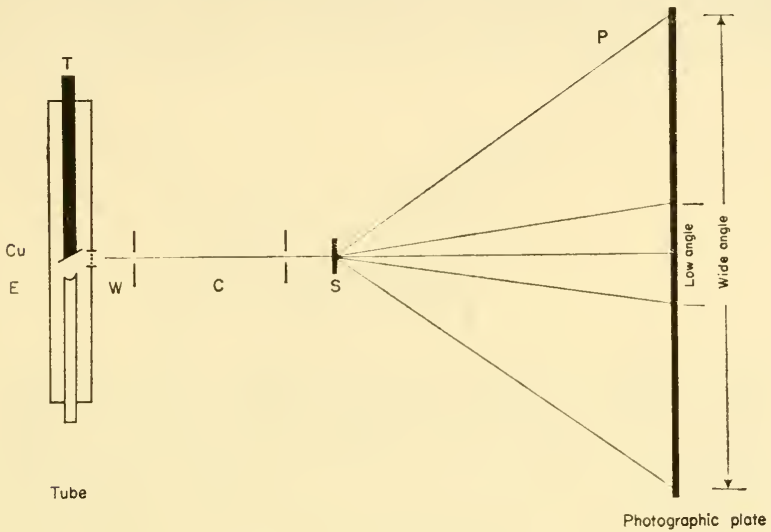

FIG. 3. The principal features of an X-ray diffraction apparatus. $\mathrm{T}, \mathrm{X}$-ray generator consisting of a copper target on which impinge high-speed electrons emitted in vacuo from the heated cathode $\mathrm{E}$; W is the window from which the rays emerge and are collimated into a narrow beam at $\mathrm{C}$ to fall on the specimen mounted at $\mathrm{S}$. The diffracted $\mathrm{X}$-rays are recorded on a flat photographic plate placed at right angles to the beam at P. Other forms of plate may be used but the flat plate is commonly employed in fibre studies. The diffracted rays within a few degrees of the plate centre are referred to as the low-angle pattern; the more widely-scattered reflections constitute the wide-angle pattern used to characterize the fibre-type.

diffraction have been of value for the rapid survey of material as is required in comparative biochemistry replacing the laborious chemical methods. Another advantage is that the material is not destroyed and need not necessarily be isolated pure. Its biological applications were pioneered by Astbury and developed in particular by Rudall. It has played a great part in the study of keratins.

The first X-ray patterns of biological materials were in fact obtained from natural fibres, among them hairs. The commonly-occurring fibrous materials have each been shown to give characteristic X-ray patterns of 
the fibre-type when examined by monochromatic X-rays (Astbury, 1933). It is significant and, at first sight, surprising in view of the astronomical numbers of stereoisomers possible for a polypeptide chain, that, judging from X-ray patterns, very few fundamental arrangements of chains are actually found in nature.

The material in a suitable fibrous form is mounted at right-angles to a narrowly-collimated beam of $\mathrm{X}$-rays (usually $\mathrm{Cu} K_{a}$ radiation) and the diffraction pattern is recorded on a photographic plate as shown in Fig. 3.

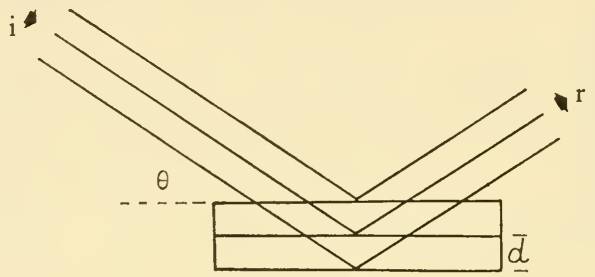

FIG. 4. Illustrating Bragg's law for the reflection of an X-ray beam. $i$ is the incident beam and $r$ the reflected beam. The crystal consists of many parallel planes containing atoms which can scatter the incident radiation. The set shown consists of planes a distance $d$ apart. When the crystal planes are so oriented with respect to the incident beam that the angle $\theta$ satisfies the Bragg equation: $\mathrm{n} \lambda=2 d \sin \theta$, reinforcement of the scattered radiation occurs giving a definite reflected beam. In any natural fibre a great many crystallites having the appropriate direction will occur.

The diffraction pattern is immediate evidence of a characteristic arrangement of the atoms in the specimen, but we need not pause at this point to discuss its interpretation (see Chapter 5). It is sufficient for our present purposes to accept each pattern as a sort of "finger print" revealing the presence of the fibre-type in question. The method is simple and, when a positive result is obtained, reliable. It has the drawback that it tends to overemphasize the crystalline fibrous components, which alone give recognizable patterns, and to overlook non-crystalline components which may contribute importantly to the properties of the material. Patterns are recognized partly on sight, using standard examples such as are reproduced in Plates 1,2 and 3 as guides. The positions of a limited number of characteristic reflections are also measured and a corresponding lattice spacing calculated from the Bragg equation. Crystals consist of a large number of parallel planes containing atoms each of which may scatter $\mathrm{X}$-rays from an incident beam. It was shown by Bragg, that only when certain geometrical conditions are satisfied, is a definite reflected beam of $\mathrm{X}$-rays obtained. This condition may be understood from Fig. 4. 
The equation which must be satisfied by the angle $\theta$ between the incident radiation and a given set of planes is the Bragg law:

$$
n \lambda=2 d \sin \theta
$$

Where $n$ is any whole number (the order of the reflection), $d$ is the spacing between the set of planes and $\lambda$ the wavelength of the $\mathrm{X}$-rays. Thus, from the photographic plate, the angle $\theta$ is determined from the distance of any reflection ("spot") from the central spot due to the undeflected beam, $\lambda$ is known from the target used (in biological work it is often copper

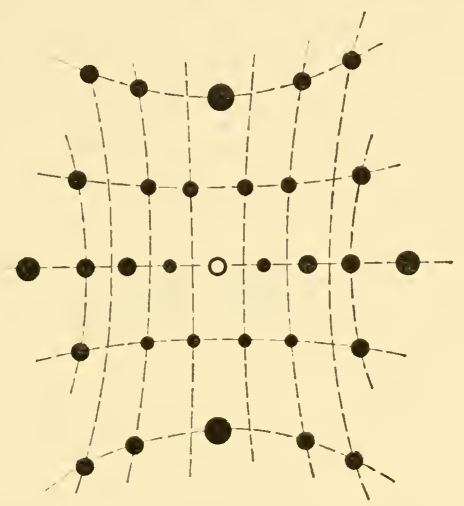

Fig. 5. Idealized diagram of a typical "X-ray fibre photograph" taken with the X-ray beam perpendicular to the fibre-axis and to the photographic film (fibre axis vertical). The reflections are seen to lie along a series of hyperbolae referred to as layer lines. Redrawn from Astbury and Bell (1939).

giving a wavelength of $1.54 \AA$ for copper $\mathrm{K} \alpha$-rays) and thus the distance $d$ between the planes can be calculated. Notice that the smaller $d$ is, the greater the distance the reflection occurs from the centre. For this reason the smaller spacings $<10 \AA$, arising from close-packed atoms and characteristic of the molecular level of organization, are found at wide angles $\left(>10^{\circ}\right)$ and constitute the characteristic "wide-angle patterns".

With single crystals of pure substances the practice is to rotate the crystal in the beam to give all sets of planes in it a chance to pass through the Bragg angle and thus register themselves photographically. In natural high-polymeric materials in the fibrous form, this is not necessary because these consist of innumerable small crystals (or crystallites) with one principal direction parallel to the fibre axis, but randomly arranged in 
other senses. Thus when the fibre is mounted at right-angles to the beam (Fig. 3) every possible orientation will be present and reflections from all sets of planes will be possible without rotating the fibre. Such patterns are called "fibre-type patterns" (Astbury, 1933) and from them the most important characteristic of a fibre, the distance along the axis at which the molecular pattern repeats, can be immediately calculated. The diffracted rays emerge from a fibre (or a rotated crystal) on a series of cones about the fibre axis, and since these intersect a flat photographic plate, mounted as in Fig. 3, in a series of hyperbolae, we find the spots lying on these hyperbolae (Fig. 5) which are referred to as layer lines (Bunn, 1946).

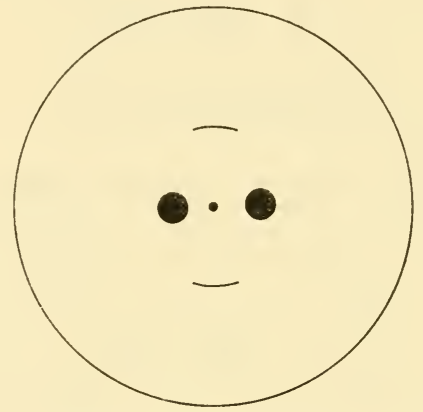

Fig. 6. Diagnostic reflections of the $\alpha$ pattern.

The patterns given by biological fibres are not usually very sharp and as detailed as those of well-formed crystals. This is in part due to the smallness of the crystallites and in part to their imperfect orientation which has the effect of drawing each spot into an arc. These defects render a strict crystallographic determination of structure well nigh impossible. but in no way hinder the use of the patterns for recognition purposes.

\section{The $\alpha, \beta$ and Collagen Patterns}

The principal features of the main, fibre-type, wide-angle $\mathrm{X}$-ray patterns, which constantly recur in discussions on biomolecular structure, are summarized below. The classification and characterization are largely due to Astbury (Astbury and Bell, 1939).

(a) The $\alpha$-pattern (Plate 1A, Fig. 6). Type material, mammalian hair. Characteristic features:

(i) the axial repeat spacing appears as a strong sharp meridional arc corresponding to $5 \cdot 1 \AA(5 \cdot 05-5 \cdot 15 \AA)$. 
(ii) two very strong diffuse extensive areas of reflections symmetrically disposed on the equator and centred about $10 \AA$ referred to as "side spacings";

(iii) a sharp meridional reflection at $1.5 \AA$ not usually recorded on flat photographic plates with an arrangement such as that of Fig. 3 ;

(iv) a strong diffuse halo centred about $4.2 \AA$ forms a background to the sharper fibre pattern.

A definite feature of a material giving an $\alpha$-pattern is that by stretching, pressing, or heating in water, it can be transformed into an isomer giving a $\beta$-type $\mathrm{X}$-ray pattern (see below).

The number of proteins capable of giving an $\alpha$-pattern is large and includes, in addition to all types of mammalian keratins, actomyosin, the

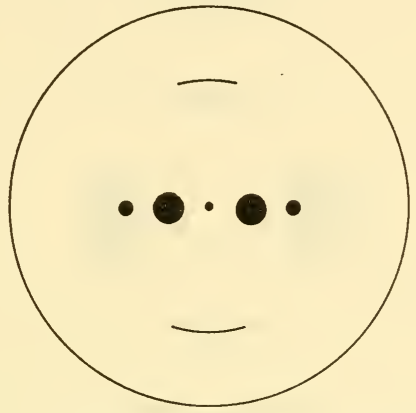

FIG. 7. Diagnostic reflections of the $\beta$ pattern.

contractile muscle protein, the blood proteins, fibrinogen and its insoluble form fibrin, bacterial flagella, etc. There is reason to suppose that most intracellular fibrous proteins are of the $\alpha$-type. Astbury refers to the group as the kmf proteins (keratin-myosin-fibrinogen).

(b) The $\beta$-pattern (Fig. 7 and Plate 1B). Type materials, silk fibroin, stretched hair. Characteristic features:

(i) axial repeat spacing about $3.5 \AA$;

(ii) strong side-chain reflections (in the keratin-type) centred around $10 \AA$ resembling those of the $\alpha$-form. In the simpler silks the spacing may be much less $(3-5 \AA)$;

(iii) strong broad symmetrically disposed spots on the equator at $4.5 \AA$ called the "back-bone spacing ".

(iv) a diffuse halo about $4.2 \AA$ apparently identical with that of $\alpha$ patterns, but less well developed in the well-crystalline silks.

The numerous silks are usually, but not always, of the $\beta$-type. 
(c) Avian $\beta$-keratin pattern (Plate 2A). This pattern is related to the normal $\beta$-pattern in having most of its characteristics. It is distinct in having a shorter (apparent) axial repeat spacing of $3.1 \AA$ and in being generally far more elaborately developed. Type material, bird feather calamus or rachis.

(d) The collagen pattern (Fig. 8). Type material, rat tail tendon. Characteristic features:

(i) axial repeat $\sim 2.8 \AA$;

(ii) diffuse side-chain spacing at $\sim 12 \AA$. Unlike the corresponding spacing at $10 \AA$ in the $\alpha$-patterns, the side spacing of the collagens is sensitive to hydration and may increase to $15 \AA$ and more in swollen materials.

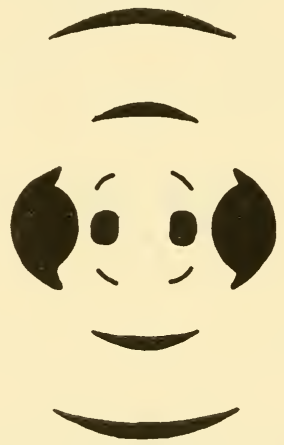

FIg. 8. Principle features of the collagen diagram.

On stretching, collagen fibrils usually break at about 10 per cent extension without any change in the type of X-ray pattern yielded. The reflections are considerably sharpened (Randall, 1953). Heating, swelling and other destructive influences likewise do not change the nature of the pattern although reducing its intensity. Collagen fibres are common in vertebrate connective tissue and have a wide distribution elsewhere (see later).

Notes. Numerous other reflections are visible in all patterns and are shown for $\alpha$ - and $\beta$-patterns in Figs. 9 and 10; they have been variously described and are indexed in Astbury and Bell (1939). They are usually less strongly developed and may not be detectable. When the specimen is poorly oriented the various reflections are drawn out into arcs and are less readily recognized. The completely disoriented condition is not uncommonly met with, particularly in artificial preparations and the corresponding patterns may be recognized as follows:

(e) Disoriented $\alpha$-pattern. Type example, regenerated precipitates of 
$\alpha$-keratins. Only two obvious spacings can be recognized corresponding to the $10 \AA$ side spacing and the original $4 \cdot 2 \AA$ halo, which here appears with a dense and sharper inner circumference due to the presence of the definitive $5 \cdot 1 \AA$ spacing.

\section{$\overline{040}$}

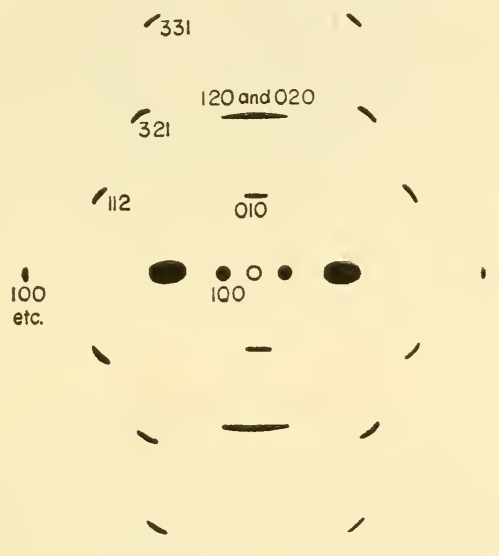

Fig. 9. An early attempt by Astbury and Bell (1939) to indicate the many other reflections visible in an $\alpha$-pattern. The " indexing" (see Bunn, 1946) in terms of an orthorhombic cell: $a=27 \AA, b=10 \cdot 3 \AA$, $c=9.8 \AA$, would not be accepted by all. See also Fig. 68, p. 166.

(f) Disoriented $\beta$-patterns (Fig. 11). Type example, boiled egg white. The characteristic and often very sharp ring due to the $4.5 \AA$ backbone spacing appears overlaid on the diffuse halo. The side-chain spacing is sometimes less well defined.

(g) Non-crystalline protein pattern. Many proteins when dried yield a very vague pattern consisting of two diffuse haloes centred around $10 \AA$ and $4.2 \AA$ which is distinguishable from the unoriented $\alpha$ - and $\beta$-patterns by the absence of either a $5 \cdot 1 \AA$ or $4 \cdot 5 \AA$ reflection. A close inspection at the inner edge of the outer halo may be necessary to recognize the absence of the $\alpha$-spacing.

It should be emphasized that these $\mathrm{X}$-ray patterns are not indicative of single specific proteins but of families of proteins which have in common 
$\widehat{030}$

$1220 \quad 020$

1410<smiles>[GeH3]</smiles>

11

I

1400

200000

1

I
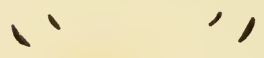

,

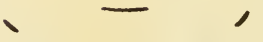

FIG. 10. The $\beta$-pattern indexed in terms of an orthorhombic unit cell $a=9.3 \AA, b=6.66 \AA$ and $c=9.7 \AA$. The 001 and 200 reflections are those ascribed to reflections from the two main dimensions (side and backbone) of the polypeptide chain. The assignment of indices shown in Figs. 9 and 10 should be regarded as tentative only. Many of the weaker reflections indicated in the drawings (Figs. 9 and 10) are often obscured by the extensive halo pattern also present and are rarely measured (Plate 1B). Figures 9 and 10 have been redrawn from Astbury and Bell (1939).

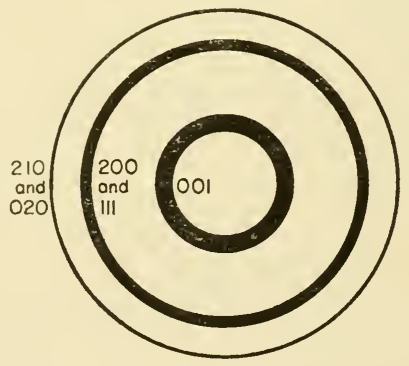

Fig. 11. Idealized X-ray diagram of disoriented $\beta$ and denatured proteins. 


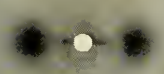

A
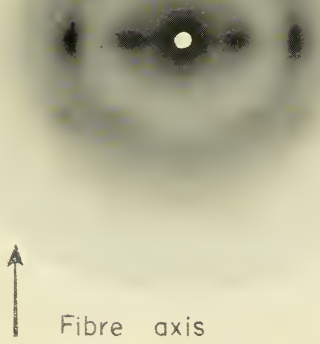

B

Plate 1 (Captions overleaf) 


\section{Plate 1}

A. The $\alpha$-type X-ray diffraction pattern. Material mohair, fibre to plate distance $4 \mathrm{~cm}, \mathrm{~K} \alpha$ radiation. For a description see pp. 14 and 17 . The strong meridional arcs corresponding to $5 \cdot 1 \AA$ on the vertical axis and the very strong reflections $(\sim 10 \AA)$ on the equator are the two most characteristic features. Strong spacings may be seen on the equator corresponding to $27-30 \AA$ and on the meridian a series of faint "long spacings" can be seen. Fibre axis vertical.

B. The $\beta$-type $\mathrm{X}$-ray pattern given by stretched and set fibrous keratins and other $\alpha$-proteins (stretched Lincoln wool). The $5 \cdot 1 \AA$ reflection of $\alpha$-keratin is missing and a further arc $(3 \cdot 5 \AA)$ has appeared. On the equator strong reflections at $4 \cdot 5-4 \cdot 6 \AA$ are present. Other reflections on the layer lines may be made out.

Both fibre patterns are overlaid by a wide diffuse halo centred about $4 \cdot 2 \AA$.

Photographs kindly lent by Mr. H. J. Woods. 
A

\section{(o)}

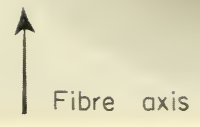

B

Plate 2 (Captions overleaf) 
Plate 2

A. Pattern of sea gull quill, the most detailed yielded by any keratin. It is of the $\beta$-type (Fig. 7) as shown by the meridional arc at $3.3 \AA$ and by the strong spacings $(\sim 10 \AA$ and $4.5 \AA)$ on the equator. For a list of the spacings see Tables 10 and 11.

B. The cross $-\beta$ pattern obtained from Lincoln wool by treatnent with a solution of urea containing bisulphite and restretching (see p. 200). The $4.6 \AA$ formerly on the equator now appears as an arc on the meridian. See Fig. 84 and compare with Plate $1 \mathrm{~B}$.

Figures kindly provided by Mr. H. J. Woods. 


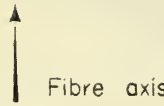

\section{PLATE 3}

A more elaborate version of the $\alpha$-pattern obtained from the protein of the mantid oötheca, see p. 204.

Figure kindly provided by Dr. K. MI. Rudal!. 

certain structural regularities arising from the configurations of their main polypeptide chains. For example, $\alpha$-type patterns, very nearly identical at large angles, are given by a whole class of proteins, including keratin, myosin, fibrin, etc., which, for this reason, is referred to as the kmf etc. . . . family. The specific differences between proteins, which determine their function, concern superficial chemical groups whose presence usually affects little the main chain structures yielding the patterns described above.

More recently it has been found that some fibres have a characteristic fine structure visible in the electron microscope and this may be used to identify them. Of particular value in this respect is the appearance of some kinds of collagen fibril which typically display a longitudinal spacing of $640 \AA$. A recent demonstration of collagen in the neural tissues of certain insects was based on their electron-microscopic appearance (Gray, 1959 and Hess, 1958) and confirmed the earlier reports by Rudall (1955) based on X-ray evidence.

It is currently believed now that the fundamental configurations of the main chains of polypeptides are determined in advance by the stereochemical characteristics of the component residues (see Chapter 5), which permit a limited number of stable configurations, and that these are spontaneously assumed in solution by free chains if circumstances permit it. Since an almost unlimited variety of side-chain composition is compatible with the main-chain configurations, these stereochemical demands place little limitation on the functional possibilities of proteins.

\section{STABILIZATION}

The fibrous macromolecules forming the protective coatings of organisms are usually subjected to a stabilizing process which may take various forms and be developed to varying degrees. As a result of this process the protective layer is hardened and insolubilized. The simplest means of effecting changes of this sort is by crystallization between the long polymer chains. When the chains are of a simple character, or of such a regular shape that they readily fit together, localized crystallization may develop with the formation of crystallites, which, when stabilized by sufficiently large energy of crystallization, virtually lock the chains together and thus render the network insoluble. The effectiveness of this device is apparent in such materials as silk or cellulose which are very insoluble; yet when sufficient of the hydroxy groups of the pyranose rings of cellulose are methylated to prevent crystallite formation, readily soluble methylcelluloses result.

A further very common method of insolubilizing proteins is the chemical process known as tanning (Gustavson, 1956) in which covalent chemical cross-linkages between the polypeptide chains are introduced by a reaction 
with an accessory molecule usually an aromatic polyphenol (Q) (Hackman, 1953 and 1959):

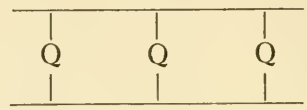

The cross-linked protein may, in turn, embed a fibrous meshwork of a different character. For example, in the arthropod cuticle, a chitinous meshwork of fibrils, themselves stabilized by crystallite formation, is embedded in a tanned $\beta$-type protein (-s) named arthropodin (Fraenkel and Rudall, 1947). The result is a very rigid exoskeleton whose inflexibility has had much to do with the evolution of these animals.

Brown (1949a and b and 1950) has discussed the various chemical and histochemical methods for recognizing the presence of a tanned protein. There is no entirely satisfactory method. A hard, insoluble, darkened material is usually presumed to be tanned. The actual bond between the quinone and the polypeptide is still in dispute. Pryor's original proposal (1940) that the link involved the amino end groups of the polypeptides:

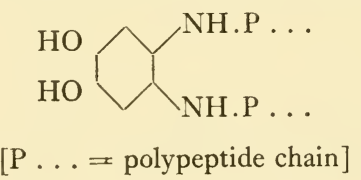

and an 0 - or $p$-diphenol is not now accepted. If the material is not dissolved by keratinolytic agents, i.e. those breaking disulphide bonds and hydrogen bonds (see pp. 234 and 236) but is dissolved by sodium hypochlorite solution (an unknown reaction) it may be tanned. The demonstration of diphenols in the secreting cells is strong support.

Hardness may also be influenced by the deposition in the protein matrix of inorganic materials, usually calcium salts or silica. For example, the crustaceans mix calcium salts with their chitinous cuticles. Probably for reasons of weight, insect cuticles are usually free of calcium salts although oxalates may be deposited in their egg cases (Rudall, 1955). The vertebrates uniformly lay down calcium salts in association with their connective tissue protein, collagen, to form bone as a rigid endoskeleton. Such mineral deposits are rare in keratinized tissues. Pautard (in press) has demonstrated by means of X-rays and by electron microscopy the presence of apatite in "whalebone," which is not bone, as the name implies, but an extensive horny proliferation of the oral epithelium in certain whales. The enamel of teeth may also contain a keratinous component. See p. 78.

Although calcium has many important biological roles its concentration within cells is normally lower than that in the surrounding fluids. Never- 
theless in almost all animal and plant groups deposits of calcium salts may be found intracellularly as well as extracellularly or on the cell membrane. The salts appear usually in association with a protein matrix and it is supposed that some spacial relationship exists between the salt molecules (or ions) and sites on the surface of the protein molecules. In the vertebrates the greater part of the calcium found in bone is always associated with collagen (Fig. 12) and mucopolysaccharides. In this case, the macromolecular form of the collagen is also important since, of the several arrangements of the collagen molecules (see p. 128) which can be formed in vitro, only that having the naturally occurring spacing of $640 \AA$ appears able to initiate salt deposition. The fact that cells containing keratin seem rarely to accumulate calcium may also mean that the special arrangements of surface groupings required is lacking in the keratin molecule (Bachra et al., 1959).

A third method of hardening proteins is keratinization which resembles tanning in that covalent cross-links are established between protein chains; but these are of a special type, the sulphur bridges made possible by the linking of cysteine residues in adjacent chains. Keratinized proteins are also in part stabilized by crystallite formation as is shown in fact by the existence of the $\alpha \mathrm{X}$-ray pattern.

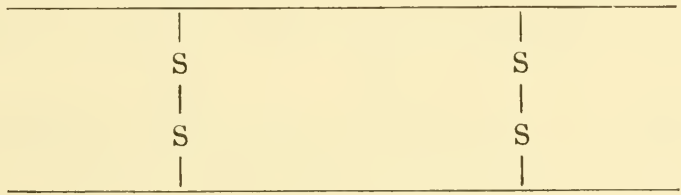

$-\mathrm{CH}_{2}-\mathrm{S}-\mathrm{S}-\mathrm{CH}_{2}-$

the cystine " bridge " or disulphide link

All these methods of insolubilizing and toughening natural polymers have their analogies in the chemistry of artificial polymers and much understanding of the natural process has come from a study of these.

Valuable information concerning the stability of a fibrous system, which is particularly relevant to our present interest in stabilized, protective proteins, can be obtained directly by X-ray means simply by taking a photograph after a tissue has been subjected to some disorienting influence such as heating in water. If the structure has been disrupted by the treatment, the fibre-type pattern of distinct spots or arcs is replaced by a pattern of diffuse, circular haloes. A loss of birefringence may also be detected by means of a polarizing microscope (Fig. 109, p. 213). The X-ray pattern may also change its character. An $\alpha$-keratin may frequently be converted into a $\beta$-type keratin by heating in water (Rudall, 1946) and 
the new pattern may be that of disoriented $\beta$-crystallites, which may be oriented by stretching and then give an oriented $\beta$-pattern.

Ecdysis. One consequence following from the fact that the chemical changes involved in stabilization are irreversible, is that provision must be made for the removal of the hardened layer to permit further growth. This may take the form of moulting or casting when an entire covering of feathers, scales, shell, etc., may be lost or, in the case of the keratinized cellular epidermis, superficial cells may be constantly shed and a covering of constant thickness maintained - a procedure which has the advantage of not exposing its owner to a period of vulnerability such as follows the moulting of a rigid covering. The factors governing ecdysis are obscure; they seem to be hormonal and are often geared to seasonal changes and, in turn, to other cyclic hormonal-controlled activities, such as sexual display, in which the external coverings may play a conspicuous part (pp. 133 et seq.) (Turner, 1960).

\section{Distribution of the fundamental fibre-types}

There are two distributions to be considered: the first is the distribution of the different macromolecular types among the parts of any particular kind of organism, which raises questions of ontogeny; the other is the sharing-out of molecular types between the entire range of organisms and here there are problems of phylogeny.

The first of these distributions is linked with the problem of the differentiation of organs during embryogenesis which is usually recognized and defined by the appearance of the typical histology of the various tissues as seen in the light microscope. These changes at a relatively large-scale level are in fact partly the consequence of the appearance and accumulation in the developing tissues of the characteristic macromolecules under discussion. Differentiation may thus be described in terms of the macromolecules on whose presence the future function of the tissue depends.

In very generalized terms, the fertilized egg is biochemically and structurally omnipotent; it is potentially capable of synthesizing all the products later appearing in its descendant cells. As development proceeds, these potentialities are shared out among the organ systems, each of which finally makes a limited range of substances required for their special function. Thus in the adult, many of the tissues may be characterized by the fact that they contain a limited range of structural macromolecules of which a few (perhaps only one) associated with the tissue's function, greatly predominate in amount.

This is well illustrated by the sharing out of the fibre-forming potentialities in the vertebrates (Fig. 12). Certain groups of cells in the middle 
layers of the embryo come to produce and to retain intracellularly the contractile muscle protein (actomyosin) and thus become muscle cells; others of a similar origin become fibrocytes and by secreting the fibrous protein collagen help to build the connective tissues of the organism and the lower layer of the integument. The superficial cells, on to which devolves the special task of enclosing the whole system, commence to differentiate early and lay down intracellular keratin.

The second distribution, that of the macromolecules among the different phyla, is a fascinating problem with far-reaching implications for their

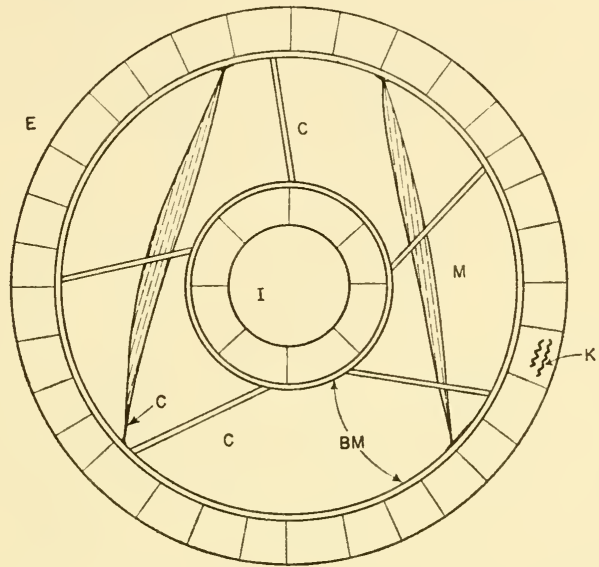

FIG. 12. Cross-section of an hypothetical vertebrate body to show distribution of fibrous proteins. The three major divisions are shown schematically. E, the external cellular epidermis (ectoderm) containing keratin fibrils $\mathrm{K}$; the internal epithelia I (endoderm) are separated by the middle layers (mesoderm) containing the musculature $M$ and the connective tissue (including dermis) containing collagen fibrils $\mathrm{C}$. Basement membranes BM (see p. 86) separate the epithelia from the mesodermal layers.

evolutionary development. It would seem that the power to make protein and/or polysaccharide materials which can be hardened by various chemical devices is a primitive and persistent cell property (Fig. 16). Organisms can call upon this property if and when necessary to form integuments, egg cases and other hard parts. Thus no necessary correspondence between phylogenetic relationships and the distribution of hardened parts can be insisted on. Nevertheless in the event some persistent trends exist. 
The very convenient $\mathrm{X}$-ray method, supplemented by chemical and histological data, has permitted a fairly extensive survey of the types of structural proteins and of their distribution among the various groups of animals and plants. The results of this survey show that in structural matters, as in biochemical matters in general, organisms are conservative for, in the whole course of evolution, only the very limited number of basic macromolecular structures described above have appeared and have been adapted to the necessary variety of uses by introducing variations in the side-chain composition. Given the limited range of biological polymers: proteins, polysaccharides and nucleic acids, the number of solutions at the molecular level to the problem of forming a protective integument is seen to be severely limited. In a similar way the chemical devices available to render proteins and polysaccharides more stable and tough are also limited by the chemical possibilities of these polymers.

There has, nevertheless, been a degree of biochemical evolution in the usage of fibre-types and the several great branches of living organisms are endowed to different degrees with the various possibilities. Cellulose is, for example, the typical structural support in plants, although a material morphologically very similar (but chemically distinct) is found in the tunics of the tunicates-creatures (protochordates) a long way from plants and related to our vertebrate ancestors, and perhaps even in vertebrates (Jeffery and Cruise and Keech, 1959; Cruise and Jeffery, 1959). Higher plants lack the fibrous proteins associated with movement in animals. Insects and crustaceans typically contain chitin in their hard parts (Rudall, 1949 and Lotmar and Picken, 1950). They possess contractile muscle proteins, but apparently little collagen (see Fig. 13).

The vertebrates, a comparatively uniform group, have most of their protein fibres in common: all the land-dwelling forms harden their epidermis with a keratinized protein and support the epidermis with a dermis containing collagen. One remarkable difference distinguishes the reptiles and birds on the one hand from the mammals on the other. The mammals, in this sense at least the more conservative, have taken over and keratinized an $\alpha$-type protein probably similar to that occurring primitively in cells and still found in the partly-keratinized skins of lower vertebrates; in birds and reptiles a similar $\alpha$-keratin is found in the softer regions of the skin, but in their more characteristic hard parts, feathers, scales and claws, an entirely different keratin of the $\beta$-type, referred to as feather keratin, is found. This discovery by Astbury and Marwick (1932) was one of the early triumphs of the X-ray method of detecting and classifying proteins.

The production of proteins having a particular type of polypeptide configuration is undoubtedly a consequence of a genetically-controlled 
activity in the cells forming the protein. Thus the appearance of a new type of protein, such as feather keratin, would seem to imply a mutation, which in this case must have occurred in the reptilian stem-line after the mammal-like reptiles had branched off, but before the birds separated from the main stream (Heilmann, 1926) (Fig. 13). An appropriate new protein appearing in the epidermal cells would presumably be subjected to the same processes of keratinization as its predecessor. In the instance of feather keratin, its utilization by reptiles, the earlier forms, would seem

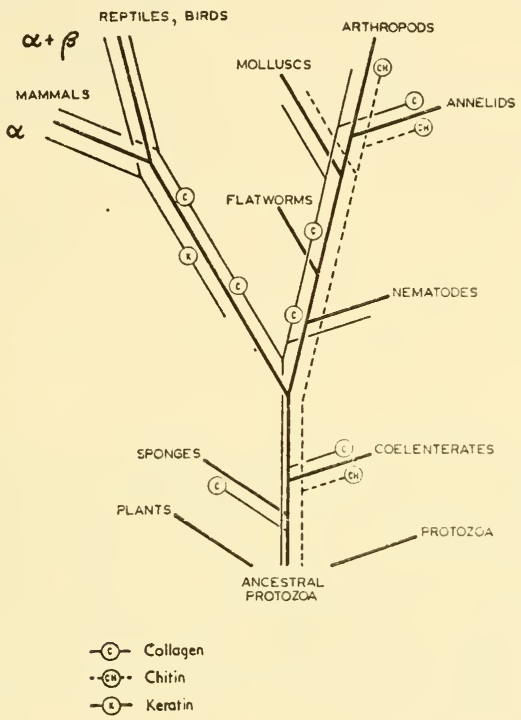

FIg. 13. Distribution of collagen, chitin and keratin shown on a conventional phylogenetic tree.

to be a simple replacement of the commoner $\alpha$-type keratin conferring no advantage obvious to us. In birds the situation is very different. The $\beta$-type molecule, as will be shown in Chapter 5 , is inextensible and inflexible in contrast to the (extensible) $\alpha$-type molecule (also the basis of muscle) and forms an admirable structural foundation for the economical construction of feathers which must be both light and stiff. The successful invasion of the air is thus partly based on the exploitation of this new type of epidermal keratin.

With a sufficient knowledge of the distribution of types of macromolecules among existing animals and plants, combined with a knowledge of the actual evolutionary descent of organisms, it would seem possible 
to construct a geneological table for the major macromolecular species. As soon as this is attempted, however, it becomes plain that, as stated above, in its grand features biochemical evolution has been small relative to structural. The major phyla are distinguished certainly by their characteristic spectra of macromolecules (Fig. 13) but very clearly this

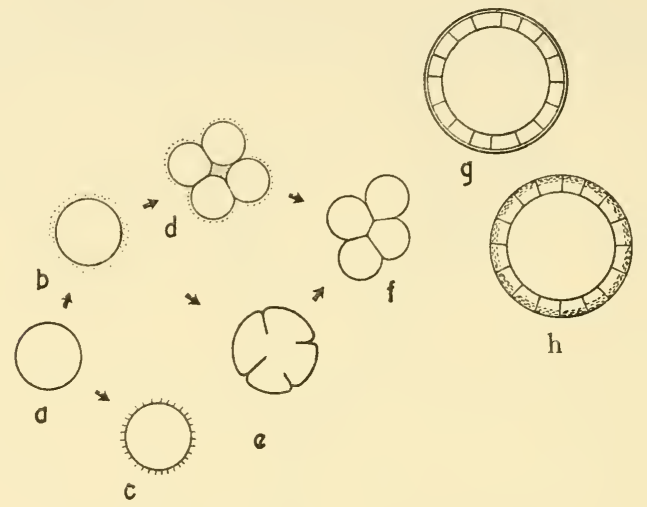

FIG. 14. 'The surface specializations of cells and their role in tissue building and in the formation of an integument. (a) Generalized cell; (b) isolated cell surrounded by a layer of mucin which may form a pellicle; (c) isolated cell with surface covered with motile cilia; $(d, f)$ one theory of the formation of a multicellular organism. Failure of cells to separate after division owing to the adhesions of the superficial coats; $(e, f)$ alternative view of the origin of multicellularity, the cellularization of a large cell (Hadži, 1954). This procedure is followed in the subdivision of the egg. (g) and (h) The two types of protective integument: (g) the extracellular cuticle or pellicle secreted by the surface epithelium. This must be shed to permit growth; $(\mathrm{h})$ an integument formed by the surface layer of cells themselves which have synthesized a fibrous intercellular material; this is the case in the keratinized tissues.

sharing-out of molecular types took place at a time anteceding anything of which we have fossil evidence. The structural evolution of which we have evidence has represented a shuffling of possibilities presented by a more-or-less constant molecular endowment. Nothing may give a more vivid impression of the unity of life than the recognition throughout its manifestations of similar molecular species and of similar biochemical devices based upon them (Florkin, 1960).

Since the distribution of macromolecular types occurred so early, a consideration of this distribution and its relation to function should offer a promising pathway to investigate the obscure field of primordial life and 
its dispersion into phyla. What is of particular interest here, since it is our object to discuss the integument, is the possibility that the molecular basis, both for the appearance of multicellularity and for the separation of a limited number of well-defined types of organism, is to be sought for in the nature of the substances present on the surface of cells (Fig. 14). These substances determine in the first place the intercellular adhesion, the essential basis of the existence of cells in colonies (see Fig. 14(d), (e) and (f)) and, in the second place, the types of material from which superficial cells construct their protective layers, is correlated with the divergent lines of evolution in such a way as to suggest the choice represents a major cause of this dispersion.

The chemical processes and materials responsible for toughening tissues are also distributed in a phylogenetically significant way. For example, among plants we normally find the fibrous, polysaccharide cellulose embedded in various encrusting substances such as pectins and lignins; among the arthropods, we find the polysaccharide, chitin, embedded in a tanned $\beta$-type protein (Richards, 1951). Tanning is more or less universally distributed among animals and thus must be judged the more primitive device for cross-linking protein chains. According to Mason (1955) its wide distribution is related to that of the ubiquitous enzyme systems, phenolases, which catalyse the formation of $o$-quinones from phenols. Tanning seems to be the rule in invertebrates; in vertebrates the process is found mainly in one kind of cell, the pigment producing melanocyte, where pigment granules are darkened and hardened by the formation of tanned melanoproteins (see p. 276). A fundamental difference in body plan with related mechanical consequences thus arises between the invertebrates, with their rigid, tanned exoskeleton on the one hand, and the vertebrates, with a rigid endoskeleton of collagen and calcium salts and a more flexible keratinized epidermis on the other. Some further consideration of the phylogeny of keratinization itself among the vertebrates will be given on p. 49 .

The relationship, between certain fundamental structural " inventions " and the molecular bases on which they rest, is set out in tabular form in Fig. 16.

\section{Some difficulties in defining a keratin}

The definition of a keratin assumed above is that it is a hardened and insolubilized protein found within the epidermal cells of vertebrates. This definition covers almost all the proteins which will be discussed in this work, but it certainly does not cover all those which might, on one ground or another, be considered as having a claim to the name. Therefore it is desirable to consider alternative definitions based on some characteristic molecular structure or chemical feature. The mammalian 
ป⿱艹
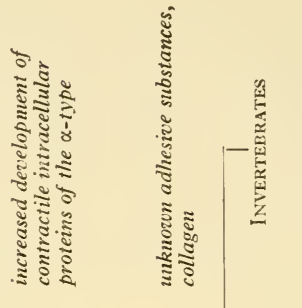

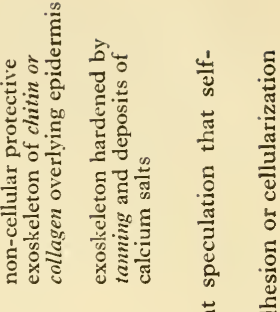

总

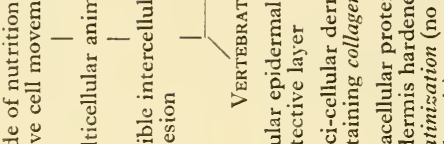

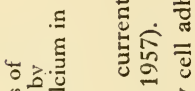

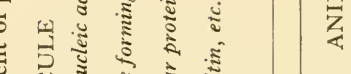

¿

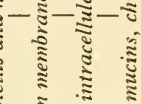

:

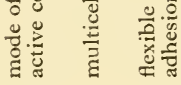

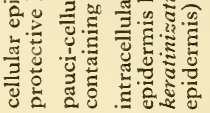

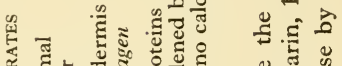

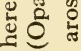

뭉

- $4 \frac{\pi}{3}$

४ 0

芯 莺

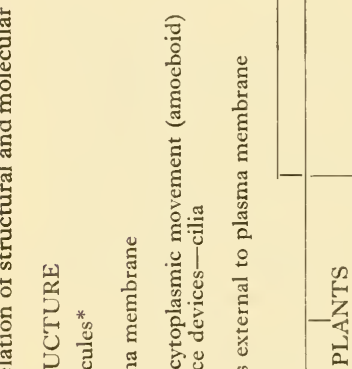

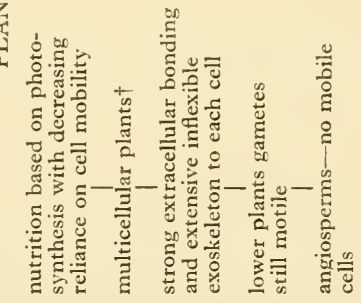

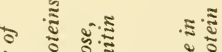

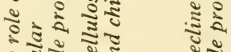

ॠ.

要

ॠ

₹

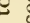

蛋

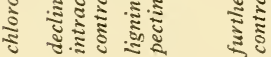

क क

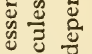

$\div \frac{0}{\circ} . \frac{9}{0}$

¿.

थ

$\leftarrow$ हू हี

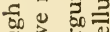

ว.

至导 气

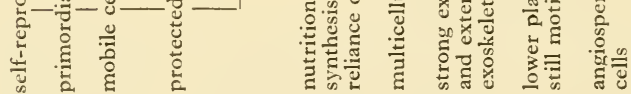

연

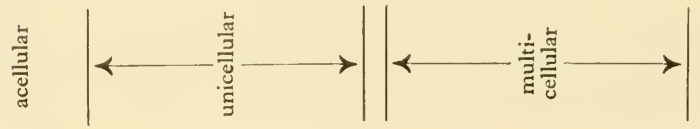


keratins are all of the $\alpha$-type and they owe their stability and insolubility primarily to the covalent cross-links formed between their polypeptide chains by the disulphide bridges of the amino acid cystine. The proteins in the epidermal hard parts of reptiles and birds are also stabilized by disulphide bonds; on the other hand, having $\beta$-characteristics, they are of a quite different molecular structure from the mammalian forms. Mammalian keratins on being stretched can also assume the $\beta$-form as a kind of stereoisomer. Thus a definition of a keratin must include both $\alpha$ - and $\beta$-forms. Further in some special situations, e.g. the hair cuticle (p. 265), a peculiar, very insoluble and highly cross-linked amorphous keratin is found. Thus a more inclusive definition might be forced to place less emphasis on the molecular character of the crystalline form and define a keratin simply as a protein stabilized by disulphide cross-linkages. Emphasis needs to be placed on the fact that the cystine cross-linkages produce insolubility as well as stabilization or such soluble proteins as insulin (12.5 per cent cystine) would be included.

Block's definition (Block and Bolling, 1950; Block, 1931; Block and Vickery, 1931) sums up a traditional view in placing emphasis on the insolubility: "A keratin is a protein which is resistant to digestion by pepsin and trypsin, which is insoluble in dilute acids and alkalis, in water and in organic solvents." He adds, however, a further criterion based on the molecular ratio of the basic amino acids found by hydrolysis, which is not now admissible (pp. 31 and 32 ).

The routine histological tests for "keratin" assume that its presence is to be inferred from the presence of protein-bound disulphide bonds. They are based either on the oxidation of the cystine bridge to produce the very acidic $-\mathrm{SO}_{3} \mathrm{H}$ group:

$$
\mathrm{P}-\mathrm{S}-\mathrm{S}-\mathrm{P} \rightarrow 2 \mathrm{P}-\mathrm{SO}_{3} \mathrm{H}
$$

which is then detected by the increased basophilic at low pH (Pearse's method, 1951 and 1953); or by reduction of the bridge to sulphydryl groups:

$$
\mathrm{P}-\mathrm{S}-\mathrm{S}-\mathrm{P} \rightarrow 2 \mathrm{P}-\mathrm{SH}
$$

and the detection of these by the nitroprusside test, the Prussian blue test (Chèvremont and Frederic, 1943) or most specifically by coupling them to the Bennett reagent: 1-(4-chloromercuriphenylazo)-naphthol-2:

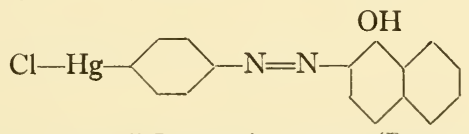

“mercury orange," Bennett's reagent (Barrnett, 1953)

to yield a coloured dye. 
According to this definition various fibrous proteins of the internal epithelia of vertebrates (oesophagous, vagina, etc.) would be keratins and are generally so called. A purely epidermal location cannot therefore be insisted on. Certain extracellular exudates in birds, such as the horny linings of the gizzard and proventriculus, are hardened by the presence of cystine bonds and are therefore also keratins (Broussy, 1932). The so-called "ovokeratin," which forms, along with mucin, the fibrous membranes of birds' eggs, is a more ambiguous case. Some analyses (Calvery, 1933) stress a resemblance to wool keratin in composition (cystine content 3.7 per cent); on the other hand X-ray photographs suggest a protein of the collagen type (Champetier and Fauré-Fremiet, 1938). The fibrils are quite unlike those of other keratins; they consist (as seen electron microscopically) of a core of osmiophilic material enclosed in a sheath of less-stained material (Mercer, unpublished. Plate 18B). They may thus consist of two distinct substances of which one could be cystine-stabilized.

With such a broad definition the keratins cannot be considered as exclusively limited to the vertebrates since there are instances reported of cystine-stabilized proteins of invertebrate origin (Brown, 1949). Krishnam $(1953,1954)$ has shown in a scorpion that the proteins of the epicuticle (analogous morphologically to the tanned structures of other arthropods) are stabilized by disulphide bonds and give an X-ray pattern not identical with either the $\alpha$ - or $\beta$-patterns of keratins or of the $\beta$-pattern of arthropodin. The cuticle of Limulus is reported by Lafon (1943) to be similar. Brown $(1949,1950)$ has cited other instances of invertebrate proteins stabilized by disulphide bonding although she is not inclined to call all these keratins. 'There is even some suggestion that quinone crosslinking may occur along with cystine cross-linking in some instances, or perhaps the quinone may link directly to the thiol of a cysteine residue (Hughes, 1959). Thus tanning and keratinization may become interchangeable or even mixed as a means for the insolubilization and stabilization of proteins.

It may be claimed also that the spindle fibre protein, which plays an important role in the mitotic cycle in animal cells, is a keratin. Mazia and Dan (1952) have succeeded in isolating the mitotic apparatus from seaurchin eggs and found that to redissolve the isolated fibres it was necessary to reduce them with thioglycollic acid (see p. 240) or to use stronglyallkaline or strong solutions of urea. The mitotic cycle, involving an appearance and disappearance of the fibrils, could thus be based on a "reversible keratinization" effected perhaps by an oxidation-reduction cycle between the protein-bound $\mathrm{SH}$ groups and cellular glutathione. The spindle fibres might be formed by an aggregation of particles efiected by disulphide bonds. 
It is evident that, unless a very narrow definition based on distribution is insisted on, the keratins are far from being a homogenous body of proteins. Neither by morphology, by molecular structure nor by overall amino acid composition can they be classed together. This is in great contrast to the collagens, for example. These proteins are widely spread yet always characterized by the same high angle X-ray pattern (Fig. 8), usually, but not invariably, by a low-angle X-ray pattern and electron microscopic appearance derived from the presence of a master period of $\sim 640 \AA$, and by an amino acid composition in which one residue in three is always glycine (a feature known now to be necessarily associated with the molecular structure which gives rise to the X-ray pattern) and quantities of the amino acid hydroxyproline are always present (see Fig. 2).

In fact it may not be far from the truth to say that keratinization is a fate which could befall any of a number of kinds of protein, provided they contain enough cysteine or are mixed with a cysteine-rich accessory (see p. 248) in a biochemical milieu where the cysteine can be oxidized to cystine. Thus it is only the process of keratinization for which a distinction is evident and the conditions for its occurrence seems to be found mainly in epithelia.

\section{The Significance of the Variable Amino Acid Composition}

Before proceeding it is desirable to give some further consideration to the question of the variable composition of these proteins as revealed by the analyses quoted. How many keratins are there ? And are they unique substances with a constant composition or not ? From what will be said below concerning the morphology of the various epidermal tissues, it will be clear that any keratinized tissue is a mixture of numerous chemical species among which a variety of keratin predominates in amount. The analysis of such tissue is not therefore the analysis of a single definite chemical substance and we can envisage the possibility, apparently confirmed by actual analysis, that the composition may vary from animal to animal, and from time to time, and from site to site even in a single animal. The keratinized tissues are in this respect far less constant in composition than other tissues of the body. Block and Bolling (1950) have shown that the total amino acid composition of most tissues is remarkably constant. This finding probably reflects the constancy both of the cytoplasmic apparatus and of the specialized cell products in the tissue. Of the tissues studied by Block, the keratins showed by far the widest spread in their amino acid pattern.

These early analyses of Block and Bolling are not as complete as those now available, nevertheless for comparative purposes they form a basis of comparison, since the same procedures were followed in each case. Block believes, however, that the hard keratins (eukeratins in his nomenclature) 
show a significant constancy in the molecular ratios of histidine to lysine to arginine $(1: 4: 12)$, and thought that this pointed to some underlying structural feature that was quite characteristic of the group.

More recent and more accurate analyses appear to cast doubt on the integral nature of the ratios. Their approximate values are, however, useful in distinguishing analytically between the hard keratins $(1: 4: 12)$ and the soft keratins $(1: 4: 4)$ (see p. 65) and between keratins and other insoluble and ill-characterized proteins.

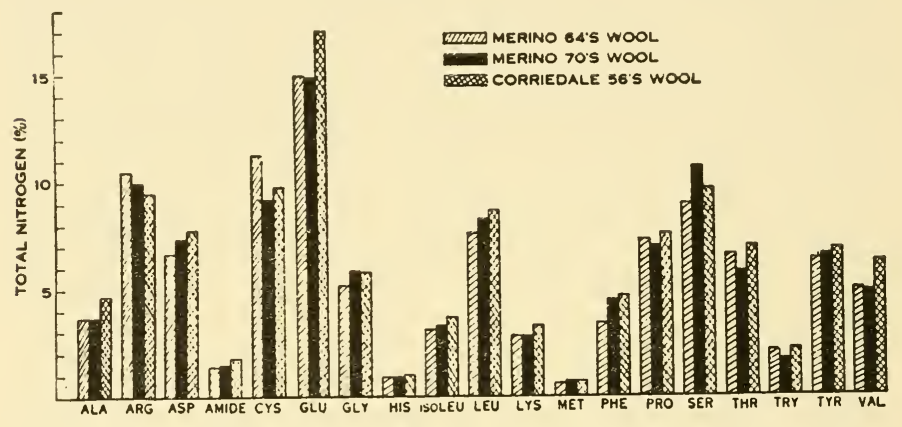

FIG. 15. Amino acid composition of one hydrolysate from each of Merino 64's, Merino 70's and Corriedale 56's quality wools. From Simmonds (1955). The figure shows in graphical form the analyses by Simmonds of three different wools in which a considerable variation in composition is apparent.

When the best of the more recent analyses are compared (see Tables 1 and 2) it seems clear that: (a) very considerable differences exist between some keratins and others and (b) smaller but definite differences exist between different samples of similar materials, e.g. between different wools (Fig. 15). Differences in cystine content are frequently reported even when other acids were not determined. In the case of big differences, e.g. between wool (hair), feather and epidermis, this can only mean that we are dealing with rather different proteins although all may be keratinized. The difference between feather and the other tissues is also revealed by its different X-ray pattern (p. 16). For the $\alpha$-type keratins we have accurate figures only for several wools and hairs. These reveal closer similarities, but even apart from cystine, no exact identity. Is there a unique $\alpha$ keratin? None of the analyses suffices to prove such a protein exists.

The thorough study of the amino acid composition of the protein found in the various morphologically distinct parts of feather: calamus, rachis, 
barbs and medulla (see Fig. 30, p. 70) made by Schroeder and Kay and their associates (1955) shows that the various parts differ in composition and that species differences also exist. We may conclude that feather keratin, like wool keratin, also has no precisely fixed composition. The two keratins, wool and feather, are, however, quite distinct. In particular, feather keratin reveals itself as peculiar in having 10 per cent of the amino acid proline, a circumstance which must profoundly affect the configuration of the polypeptides. Forty per cent of residues are the small residues glycine, alanine and serine. These features of the feather polypeptides have influenced the model proposed by Krimm and Schor (p. 208).

There may be technical reasons for small variations since these analyses are difficult to perform and, further, they are usually carried out on whole tissues, not on isolated purified proteins. As commonly practised the materials are usually simply extracted successively with aqueous and lipid solvents and, without further fractionation, are submitted to various degradative processes as a preliminary to analysis. Since the keratinized fibrous protein itself usually forms the predominate constituent, the analytical findings will give a good idea of its composition. However, it would be a mistake to regard the findings as representing precisely the composition of "keratin" and to draw far-reaching inferences from their exact values. Ideally the several components of the tissue ought to be separated, purified and individually analysed as is obligatory in the case of the soluble proteins. This is a counsel of too much perfection when dealing with insoluble hardened products and little effort has been made to comply with it in the case of the keratins. Nevertheless, since keratin remains as an insoluble residue, when the tissues containing it are simply digested with trypsin, it is regrettable that analysts have not attempted at least this degree of purification before beginning an elaborate and lengthy investigation.

Undoubtedly methods of solubilizing the proteins of a keratinized tissue and of extracting pure individual proteins will be perfected sooner or later. When these have been attained, is it possible to anticipate that definite individual "keratins" will be distinguished? It is difficult to answer this affirmatively. Rather from the biological point of view a variable composition might well be expected, for it is clear that the demands made upon the epidermis and its derivatives are widely variable and, if an adaptive response is to arise, a variation in the nature of its composition might be expected. The interesting idea, discussed by Tristram (1953) and more fully by Colvin et al. (1954) and Fox (1953), that proteins exhibit a certain "spread" in their composition, may well be applicable to structural proteins of the keratin-type even if it proves untenable in other instances. The fact that the replacement of a single amino acid residue may impair the function of a haemoglobin molecule 
(Ingram, 1957) may seem a serious objection to the universal application of this proposal. On the other hand residues not involved in forming the precisely-patterned topography of the active patches of a protein molecule may perhaps be exchanged with greater impunity (Tristram, 1953). The probability that the function of a keratin does not demand the same detailed specificity of structure, as for example an enzyme, may be relevant here. Keratinized tissues have a mechanical function and a role as water barriers. The properties required are a certain insolubility and toughness combined with elasticity. For this a precise sequence of amino acids does not seem immediately in demand; numbers of polymeric networks having an appropriate balance of hydrophobic and hydrophilic side chains might be envisaged with similar properties. Further, a case could be made out for supposing that the ability of the germinal cells to differentiate into cells producing proteins of a variable composition could be the basis of adaptation. Normally, we could suppose, the pattern of synthesis is dominated by the site, e.g. producing hair keratin, horn keratin, etc., in special sites, but, if the synthetic mechanism were also capable of continuous adjustment to (say) mechanical demands, the system would be adaptive as in the epidermis it seems to be.

The biochemical mechanism of such an "adaptive synthesis " would have to be sought in a selective pressure brought to bear on the population of RNA molecules which emerges from the nucleus during the course of synthesis (see p. 110). Since the mechanism by which external influences are fed back through the cytoplasm to influence nuclear activity is one of those phenomena most in need of experimental elucidation, we can carry this speculation no further.

\section{The fine structure of cells}

\section{Electron Microscopy and Cytology}

$\mathrm{X}$-ray diffraction methods are rarely applicable to cell inclusions or to surface structures except when components can be isolated in a suitable form. Chemical analyses and indirect physicochemical methods have proved of more value but at the present time most of our knowledge is coming from electron microscopy. In many cases, as may be appreciated from Fig. 1, this form of microscopy appears the only approach to such minute and irregular detail.

In the last few years following the perfection in the early 1950's of methods of fixing, embedding and sectioning of biological material for the electron microscope, cytology has undergone a veritable revolution. Today we possess a wealth of morphological material covering most cell types expressed largely in terms of the membranes, particles and filaments whose images appear in electron micrographs. The work of recognizing 
the objects visible in the light microscope in terms of these new structures has also made satisfactory progress. Since this information is not yet common currency, it is advisable at this point to name and describe the commonly-occurring fine structural units in terms of which our later accounts of cell structure will be given. These descriptions will be brief and are intended to serve simply as morphological definitions. Fuller accounts of the function and structure of the units will be given later.

To a degree at the present time pure morphology has out-run knowledge of the chemistry and function of cell constituents. It is, for example, not always possible to state with certainty the chemical nature of the materials giving images in electron microscopes. To appreciate the special nature of this problem it is necessary to consider briefly the preparative procedures of electron microscopy. Biological material intended for sectioning is first of all fixed, i.e. subjected to a chemical treatment which kills the cells and converts (more or less effectively) certain of their constituents into derivatives of greater physical and chemical stability. The fixed specimen is then dehydrated, which adds further to its stabilization, embedded in a polymer and cut into suitably thin sections. Certain of the chemicals previously employed by light microscopists, such as osmium tetroxide $\left(\mathrm{OsO}_{4}\right)$ and formaldehyde, have been found useful as fixatives by electron microscopists (Palade, 1952) and others (potassium permanganate) (Luft, 1956) have been introduced. The most commonly used fixative is the buffered ( $\mathrm{pH} 7-8)$ solution of osmium tetroxide introduced to electron microscopy by Palade and it is with the results obtained using it that we are mostly concerned with here. Osmium is an element of high atomic number and there is no question that much of the contrast of osmium fixed material arises from the electron scattering produced by osmium atoms present in the fixed specimen either as lower oxides or as compounds with the organic matrix. Unfortunately for the prospects of a superhistochemistry the nature of the reactions of $\mathrm{OsO}_{4}$ with tissue components is both obscure and hitherto little studied. Bahr (1954), by treating in vitro a series of pure organic compounds with osmium tetroxide, has shown that certain chemical groupings are able to react with osmium tetroxide to produce coloured compounds. It is probably correct to assume that the reaction of osmium tetroxide with a complex molecule such as a protein represents the sum of the reaction with its component reactive groups. Figure 17 taken from Bahr summarizes the known reactivity with osmium tetroxide. Since we are interested primarily in a sulphur-containing protein, it should be noted that the most reactive groups in proteins are the sulphur-containing amino acids.

A further factor of great importance in producing contrast is the compactness of the organic substrate. This influences the amount of osmium 
per cubic centimetre in the fixed state which is the principle factor increasing the contrast. Experiments in vitro have established that objects which exhibit the greatest contrast in osmium fixed material are: phospholipid (or phospholipid plus protein) membranes, compact masses of reactive proteins and lipid inclusions containing unsaturated fats. Nucleic acids, mucopolysaccharides and polysaccharides show little increase in contrast,

FIG. 17. In vitro reactions of osmium tetroxide (Bahr, 1954).

Reaction with $\mathrm{OsO}_{4}$ (appearance of black lower oxides).

$-\mathrm{SH}$

$=\equiv$

$\rightarrow \mathrm{N}$

$-\mathrm{NH}_{2}$

$-\mathrm{S}-$

$-\mathrm{OH}$

$-\mathrm{CHO}$ peptides, proteins, enzymes.

fats, waxes, lecithin, cerebrosides, vitamins, certain hormones, bile acids and other biological substances containing a basic sterol structure.

tert.bases co-ordination, tryptophane.

in terminal positions and not salt-linked.

sulphide sulphur, cystine, methionine.

in terminal positions and on certain carbon chain lengths.

Certain heterocyclic compounds.

Aromatic compounds with at least two hydroxyl groups in suitable positions: plant material, tanning substances.

\section{No reaction with $\mathrm{OsO}_{4}$}

$-\mathrm{COOH} \quad$ acid group.

$-\mathrm{CH}_{2}-\mathrm{CH}_{2}-$ paraffin chain.

$-\mathrm{CO}-\mathrm{NH}-$ peptide bond.

$-\mathrm{COO}^{-} \ldots \mathrm{NH}_{3}{ }^{+}$salt link.

$-\mathrm{HSO}_{3} \quad$ sulphonic acid group.

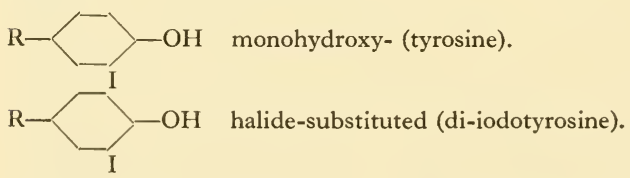

Carbohydrates-Sugars and their polymers such as starch, glycogen, pectin, amino sugars, heparin, hyaluronic acid, lignin.

Nucleic acids-Nucleotides, ribose sugars, sugar phosphates.

Various forms of high and low polymer DNA and RNA.

although compact forms of nucleic acid (see below) are dense because of the phosphate groups they contain. Neutral formaldehyde may give a more complete fixation of proteins; it does not, however, lead to any increase in density (i.e. no staining effect) and is thus not so popular as a fixative since the image is less photogenic. The presence of proteins in formaldehyde fixed material may be demonstrated by staining with 
phosphotungstic acid or lead salts. Useful discussions of these problems have been given by Palade (1952), Sjöstrand (1956), Bahr (1954) and Baker $(1945,1955)$.

The reliability of electron microscopic findings is assessed by comparing them when possible, with those of the light microscopy or X-ray diffraction (see Fig. 1), by the internal evidence of the micrographs, which may suggest deleterious changes and, in the final analysis, by their contribution to the understanding of the problem of the function of the tissue or organism.

We shall treat first, and in greater detail, the cell surface and its specializations, since these are of greater importance for our subsequent discussions; the structures found in the cytoplasm follow next and finally the nucleus.

\section{The Cell Surface, its Specializations and Intercellular ADHESION}

The keratinized epidermal tissues are cellular in the sense that the amount of intercellular material is very small; the cells surfaces are effectively in contact and the whole formation owes its coherence to intercellular adhesion. This is in contrast to the mesodermal tissues where the extracellular material greatly predominates (Fig. 12), and coherence is due in the vertebrate to the meshwork of collagen and other fibres laid down by the cells. The cell membrane, the nature of intercellular adhesion and its modifications in cellular tissues, adapted to withstand mechanical and chemical shock, must therefore be given special consideration in relation to the total phenomenon of keratinization.

The cell membrane. The simplest unicellular organisms possess a boundary, which separates the intracellular domain from the environment, with special properties of permeability and mechanical strength (Fig. 14). This cell membrane or plasma membrane may well have been the first and most primitive organelle of the cell, since its existence without such a definite boundary is difficult to admit. Its earliest function was probably in essentials what it is today: by virtue of its selective permeability it retains, in the neighbourhood of the cellular apparatus, a higher concentration of certain molecules than exists in the surrounding medium. Accordingly the permeability properties of the plasma membrane have been much studied and this work has been summarized by Davson and Danielli (1952) and Danielli (1942). The membrane proves to have predominantly a lipid character, i.e. it is most permeable to substances soluble in non-aqueous solvents, as would be required of a membrane whose principal function is to act as a barrier to substances dissolved in water. This finding is supported by actual chemical analysis of membranes, such as that of the erythrocyte, which can be obtained largely free from 
other constituents. Measurements of the surface tension of free cells show this to be very low $(0 \cdot 1 \mathrm{dyn} / \mathrm{cm})$ which would suggest that the protein is present as an adsorbed surface layer. The chemical analysis (Davson and Danielli, 1952) also shows that proteins are present.

These experimental findings suggest the model (Harvey and Danielli, 1938; Danielli, 1942; and Stoeckenius, 1959) shown in Fig. 18 (a) which pictures the membrane as a continuous lipid-like layer covered on each
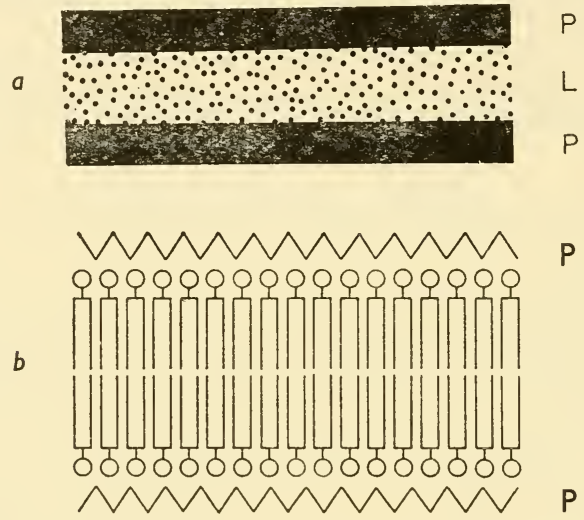

Fig. 18. Two interpretations of the nature of the plasma membrane. Both agree that the membrane consists of a lipid layer covered with protein layers as indicated at (a) the Danielli-Harvey model; (b) shows a somewhat more detailed interpretation in which the lipid is represented as a bimolecular leaflet of lipid molecules covered with monolayers of non-lipid protein.

face by a layer of protein. Its thickness cannot be determined precisely by permeability or impedance measurements, but an order of less than $100 \AA$ is indicated. Direct measurements, using a special device, the leptoscope, made by Waugh (1954) confirm this order of magnitude.

Structures of these dimensions are within the range of the electron microscope and, as mentioned above, our knowledge of membrane structure has been greatly augmented recently by the use of this instrument. Sections cut through the plasma membranes of a variety of fixed cells have shown that its thickness is of the order of $70 \AA( \pm 10 \AA)$ and that it frequently reveals a fine structure consisting of two dense outer surfaces enclosing a less dense inner layer (Plate 4A) (Mercer 1959, Robertson, 1959). This finding may be compatible with the structures of Fig. 18 if we assume that the protein layers, $P$ (and perhaps part of the 
lipid), react with the fixatives (osmium tetroxide or potassium permanganate) more vigorously than the inner layer, $L$, and thus have become "stained."

In some simple organisms the cell membrane is protected by a secretion of slimes or mucins (Fig. 14(b)); in others this takes a more definite form as an external pellicle (Fig. $14(\mathrm{~g})$ ). In these secretions it is perhaps permissible to trace the primitive forerunners of both the adhesive intercellular cements and of the elaborate extracellular coats found in some higher animals. According to Weiss (1933) cells freed from tissues and cultivated in vitro surround themselves with a colloidal exudate.

Cilia, flagella, etc. Cilia and flagella are very similar in internal structure although somewhat different in behaviour. They are long motile protrusions of the membrane and in electron micrographs reveal a complicated

(a)
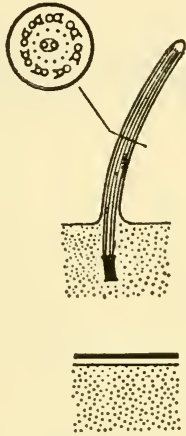

(d) (b)
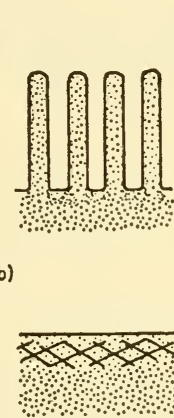

(e)

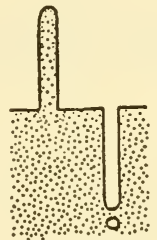

(c)

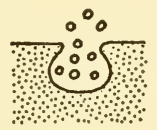

(f)

Fic. 19. Surface membrane specializations. See text.

internal structure (Fig. 19 (a)). (Bradfield, 1955; Fawcett and Porter, 1954; and Manton, 1952). In cross-section they are seen to consist of the enveloping plasma membrane, nine pairs of peripheral filaments, a central pair of filaments of a different character from the nine and an amorphous ground substance. The peripheral filaments in the protozoa are seen to end on a basal body within the cytoplasm which consists of a small short cylinder apparently consisting of nine short rodlets and which may control the organization of the bundle of filaments. The centriole found in many cells of animals appears to consist of an identical object and here too it may assist in organizing cytoplasmic proteins (Porter, 1957; Grimstone, 1961). 
The pattern of $9+1$ pairs of filaments occurs with remarkable persistance throughout the animal and vegetable kingdoms and modified versions of such surface protusions are to be found in such unlikely sites as the rods and cones of the vertebrate eye.

In electron micrographs of these organelles faint indications of a very fine filamentous (diameter $\sim 50 \AA$ ) cytoplasmic component are common (Fig. 19 (e) ). In ciliated epithelia (Fawcett, 1958), for example, and in association with fine pseudopods in other situations, denser deposits beneath the membranes are to be seen and may indicate a region of firmer gelation of the cytoplasm which helps in maintaining the shape of the surface protusions. Little is known of the detailed composition of such filaments. Nevertheless, it is in such ill-defined fibrous proteins of the cytoplasm that we may find primitive precursor of both keratin and the contractile muscle proteins (Fig. $19 \mathrm{~b}$ and c).

Surface invaginations. These occur as long pleats of the plasma membrane forming double surfaced membranes penetrating far into the cell (Fig. 19 (c)). They are particularly common in cells involving water transport. Most elaborate examples are noted in the cells of the stomach which secrete hydrochloric acid.

Temporary invaginations are commonly associated in free living cells with the ingestion of solid material (phagocytosis) and liquids (pinocytosis).

Specializations of opposed surfaces. Cell contacts. In many tissues of the multicellular organism the component cells are closely opposed and at such surfaces of " contact" a variety of specializations has been observed. Most of these seem to be associated with cell adhesion and in their totality they form the devices by which a cellular tissue is held together. Obviously (as mentioned above) in an external, protective, purely cellular layer, such as the vertebrate epidermis, these devices become of great importance; for while an extracellular cuticle may be effectively continuous and sufficiently strong to retain the enclosed cells, a cellular tissue, however its cells may be hardened by intracellular deposits, will be of little protective value unless the adhesion between the cells themselves is of an adequate strength. Certain experiments on the growth of keratinizing cells in tissue culture (McLoughlin, 1959) suggest even that the production of strong intercellular adhesion with the formation of a stratified tissue is in itself an important factor in causing the cells to keratinize.

In electron micrographs of fixed and sectioned tissues it is seen that when two cells are in contact, their dense plasma membranes do not touch or fuse. They remain separated by a space of about 120-200 $\AA$, which appears light by contrast with the darker membranes (Fig. 20 and Plate 5), but which may sometimes be stained with electron-dense materials. We may suppose that this space is occupied by a cellular exudate or 
secretion, of low intrinsic density or poor affinity for the fixatives and stains of current electron microscopy, which normally coats the surfaces of the cells (Weiss, 1960).

A question of nomenclature arises here. The rather definite width of the clear space between cells suggests that the surface coat itself has a definite thickness and might well be included as an element of the plasma membrane itself. This viewpoint is particularly cogent when specialized developments of the cell membrane, such as those forming the myelin sheath of nerve axons (Robertson, 1956 and 1957), are considered. However, for the purely morphological reason, that the double line bounding the

A

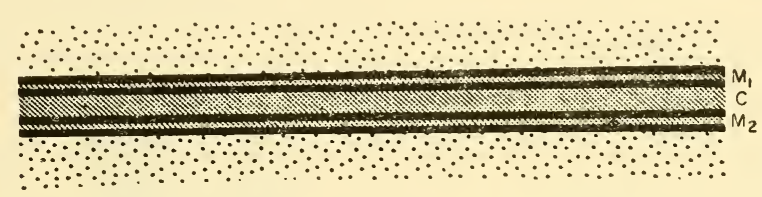

B

FIG. 20. The structure of the surface of contact between two cells $A$ and $B$ (as seen electron microscopically in sections of tissues fixed in osmium tetroxide or potassium permanganate). $\mathrm{M}_{1}$ and $\mathrm{M}_{2}$ are the two plasma membranes which are each seen to consist of three sheets. Between $M_{1}$ and $M_{2}$ is found an intercellular sheet (or cement) C.

cytoplasm is so obvious a feature and definable in all cells, we will refer to it here as the plasma membrane. In this terminology the lighter space is intercellular and, since the material occupying this space is ultimately responsible for sticking the cells together, it may be referred to as an intercellular cement. These definitions are illustrated in Fig. 20.

Desmosomes. In most epithelia even in the lowest of multicellular animals opposed surfaces are studded with small dense areas called desmosomes (Fig. 21). These are most common and conspicuous in stratified squamous epithelia such as the vertebrate epidermis. They have been shown electron microscopically (see Chapter 3 ) to consist of localized thickenings of the membranes produced by the deposition of a layer of amorphous material inside and outside the cell membrane. A similar layer is formed within the opposing cell. In the epidermis these remain localized and appear to resemble a pair of disks about $1 \mu$ in diameter; in other situations, e.g. the columnar epithelium of the intestine (Fig. 21 (a)), the desomosomes may develop into bars or long bands running around the cell near the free surface. There is evidence based on their behaviour when cells are 
stretched (reviewed in Fawcett, 1958) that the desmosomes and terminal bars are effective in holding the cells together and have been referred to as attachment plaques. They are elaborately developed as the intercalated disks in heart muscles where their role is again at least partly mechanical in transmitting tension from cell to cell. Here the contractile fibrils enter the desmosomal deposits when they reach the ends of the cells (Fig. 21 (e)).

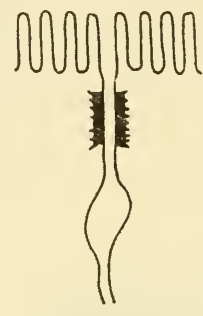

(a)

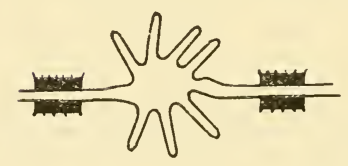

(d)

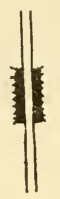

(b)

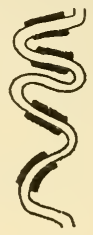

(c)

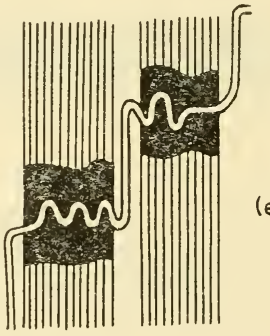

(e)

Fig. 21. Examples of desmosomes. In each case the desmosome is shown as a pair of opposed thickenings of the cell membrane to which may be attached small tufts of fibrils.

(a) The "terminal bar" type of desmosome is found in columnar or cuboidal epithelia where it forms a long band running along near the free edges of the cells. It could assist in preventing the separation of the cells (Plate 5A).

(b) The simple plaque desmosome found in most squamous epithelia. Extracellular sheets may be deposited between the plaques in some situations (see page 92). This type of structure forms the traditional " intercellular bridge."

(c) Desmosomes of type (b) may be regularly spread over the surfaces of contact which may become more or less regularly corrugated to produce a "tongue and groove" effect (Plates 6A and 12C).

(d) When a small duct passes between two cells desmosomes may again form in the positions shown where they could function to contain the duct.

(e) The very elaborate desmosomal development between cells in heart muscle where it is recognized as an "intercalated disk." The contractile muscle filaments end in the dense deposits at the cell boundaries (Fawcett, 1958). 
An important feature of the desmosome, which indeed justifies the special attention given to it, is that it probably represents a point of fixed (quasi-permanent) intercellular attachment. This permanence one deduces from the observation that the structures in one half of the formation, which must have taken some time to form, are mirrored closely by similar structures in the opposing half. The two halves must thus have remained opposed for some time. Elsewhere, over the shared surfaces of contact where one observes interdigitating folds, it would seem that the surfaces, although sticky, can slide laterally over each other, i.e. the intercellular cement has the properties of a viscous liquid. One can readily visualize that a range of viscosity is possible, depending on the degree of crosslinking of the molecules between the surfaces. It is also possible that these deposits may mark the sites where special forms of communication between cells takes place, but definite evidence for this is wanting.

That fine intracellular fibrils, usually ending on desmosomes, occur in many if not all cells has been long recognized (Schneider, 1902 and Schmidt, 1924) and recently Leblond and colleagues (Leblond et al., 1960 and Puchtler et al., 1958) by systematically applying a new staining technique (successive treatment of fixed tissue with tannic acid, phosphomolybdic acid and amido black: "TPA" staining) have demonstrated them in many kinds of cells with exceptional clarity. The geometrical arrangement of these fibrils and their attachment to studs (desmosomes) on the cell membranes suggests a mechanical role in maintaining cell shape and rigidity, i.e. they are literally "tonofibrils" (see p. 94). They stain as basic proteins quite distinct from the extra-cellular collagen fibrils but similar to the first formed fibrils in keratinizing systems. From a comparison of their location and density in several different cells, it is clear that they occur in enhanced amounts in precisely the situations where support is demanded. Epidermal cells and muscle cells show the most marked development of TPA positive fibrils and in these cells their identity with keratin and muscle fibrils respectively is obvious. It would seem possible that all these fibrillar systems are composed of homologous fibrous proteins and that chemical modifications have been developed to fit them for special purposes. Keratinized fibrils, for example, are modified to enhance their strength and stability; muscle fibres show an enhancement of the latent contractility of the polypeptide chain.

Interdigitation of confronted membranes. In some epithelia the opposed cell membranes, while remaining parallel, become greatly convoluted or corrugated and a tongue and groove relation may develop, which has the effect of greatly increasing the area of contact and presumably the adhesion. Desmosomes usually form on such surfaces to add to the adhesion (Fig. 21C and Plate 6A).

In keratinized tissues with their special requirements of strength it is to 
be expected that these devices to increase intercellular adhesion will be elaborately developed. In fact, as will be described later, the cell surfaces become very convoluted, deeply interdigitated and heavily studded with desmosomes. Further the intercellular cement becomes modified in its solubility and chemical stability and forms dense intercellular sheets between the surfaces.

\section{The Differentiation of Surface Organelles}

We referred above to those aspects of differentiation which arise from the appearance within the cell of characteristic macromolecules. An equally important feature is the appearance of the specialized surface organelles which have just been described.

The factors bringing about differentiation are little understood and their investigation is currently a major research preoccupation which will be discussed later. Here we wish merely to refer to a certain antithesis which exists between the specializations found on free surfaces and those on bound surfaces. It seems adequately demonstrated experimentally, by the failure of isolated cells to differentiate or to maintain differentiation (Willmer, 1954) and by the appearance of differentiation when different cell types are cultivated together (Moscona, 1952, 1956, 1957), that differentiation results from the effect of one cell on another. For cells in the interior of an organism the environment is either wholly cellular or consists of solutions containing the products of other cells. On the other hand, cells on the surface are uniquely situated in having at least one face free from the immediate influence of other cells. Their environment on this face resembles that of a free living cell and, in fact, the surface differentia appearing here are identical with those found on free living cells. These special responses to an external situation: the sprouting of cilia, the secretion of mucins, the formation of a cuticle or intracellular fibrils beneath the membrane (see Figs. 14 and 19), may be regarded as a cell's free-surface "repertoire." They appear whenever the surface is free and are repressed on surfaces in contact. Metazoan cells respond to contact by adhering, which implies the secretion of the specialized intercellular cements and the suppression of the free surface repertoire. It may be argued that the property which above all others distinguishes the multicellular organisms from the unicellular (see Fig. 14) is the formation of intercellular adhesives, probably macromolecules among which appeared to be mucopolysaccharides (p. 54) and proteins. Such intercellular cements must have played their part in the evolution of the metazoa and, in the life of each individual, the cells appear to pass from an embryonic condition of poor adhesion to an adult in which, in many organs, strong intercellular attachments are the rule (see Fig. 14). The keratinized tissues carry this process to the extreme. 


\section{Cytoplasmic Structures}

\section{Particulates}

(a) Mitochondria. These oval or elongate objects (diameter $\sim 1 \mu$ ) were recognized by light microscopists and characterized by various staining reactions. Electron microscopically they appear as a small vesicle enclosed by a double membrane, the inner membrane being cast into characteristic folds. This internal structure may now be considered as definitive (Fig. 22a). Mitochondria are extremely common in most cells have been shown by biochemists (p. 115) to be the site of numerous enzyme systems associated with cell metabolism.

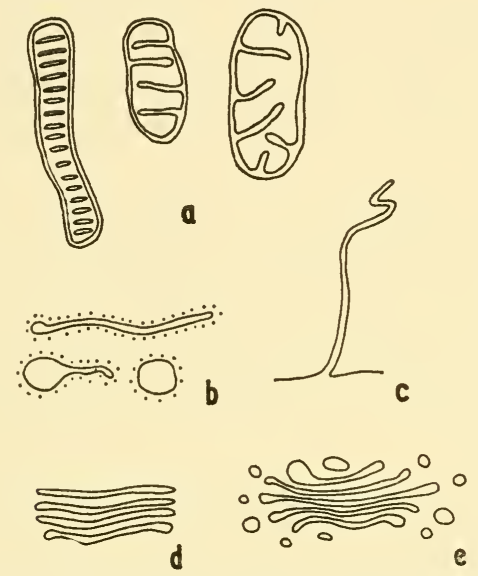

Fig. 22. Intracellular membrane bounded organelles; (a) mitochondria; (b) "particle covered" vesicles and membranes (see Plate 10A); (c) "pleats" of surface (plasma) membrane ( $\gamma$-membrane pairs); (d) and (e) parallel sheets of phospholipid-protein complexes; (e) " Golgi " membranes.

(b) Smaller $(0 \cdot 5-0 \cdot 2 \mu)$ membrane enclosed particles. A variety of small bodies named microbodies, ultramitochondria, small vesicles, etc., scarcely visible in the light microscope, are recognized as distinct on grounds of size, absence of an internal system of membranes (of the mitochondrial type) and the texture of their contents. Their nature is obscure and they are certainly heterogeneous. Some may be virus inclusions, others sacs of special enzymes (lysosomes), small secretory granules, etc. Some vesicles contain phospholipids recognized by characteristic clusters of concentric shells of membranes (see below). 
(c) Small dense particles. Since the electron microscope can resolve macromolecules of diameters as small as $20-30 \AA$, the "ground substance " of cells often presents a fine particulate appearance. Conspicuous among these fine particles on account of its size (100-200 $\AA$ ) and density is a particle shown by Palade (1955) to contain ribonucleic acid (RNA). These particles, ribosomes, may be free or associated with membranes to form an important cell organelle, the basophilic reticulum described below.

\section{Membrane systems}

Membraneous systems are particularly well preserved and "stained" by fixation in osmium solutions and in permanganate and are therefore conspicuous in electron micrographs. Several organelles recognizable in the light microscope are now known to be based on a skeleton of membranes. The mitochondrion described above as a particulate may be regarded as an example. Others less delimited in their extent are described below.

Systems of particle covered membranes (ergastoplasm, basophilic reticulum). The cytoplasm of many cells (see p. 108 et seq.) contains more-orless elaborately developed systems of membranes whose surfaces are covered with small dense particles identical with those described above (Fig. 22 (b)): The membranes exhibit an intricate complexity of profiles when examined in sections, which appear to be views of a geometrically complex and intricately-interconnected reticulum of surfaces. This in effect divides the cytoplasm into two parts: that inside the vesicular system and that outside.

Systems of smooth surfaced membranes. The membranes here are similar to those just described, but their surfaces are not associated with particles. Some are simple "empty" vesicles of uncertain import. Two rather more defined formations are usually described. The first, often well developed in cells associated with the transfer of water, consists of simple pairs of closely opposed parallel membranes which have been traced back and shown to be deep pleats of the plasma membranes of the cell (Fig. 22 (c)). The second is a variable yet always characteristic stack of double membranes (flattened sacs) (Fig. 22 (d) and (e)) which appears in all cells. It is usually assumed to correspond to the Golgi complex of light microscopy, an organelle of uncertain function (p. 110) (Grasse, 1956; Haguenau and Bernhard, 1955; Baker, 1955; Dalton and Felix, 1956). Other rather more regular stacks of flattened sacs associated with the nuclear membrane have also been described.

Concentric membranes, "whorls," myelinic forms. These formations may vary from very perfectly-formed concentric shells of membranes with an intermembrane spacing of the order of $40 \AA$, through more open, concentric-shell formations, to stacks of parallel membranes closely related 
to Golgi clusters. They may be imitated in vitro using preparations of phospholipids extracted from cells (Stoeckenius, 1959; Mercer, 1960). The myelin sheath of vertebrate nerve and the stacks of plates in the retinal receptors are special cases of such structures (Sjöstrand, 1956).

The similar morphology (see p. 37) of all these membranes and the observations on the polymorphic possibilities of phospholipid membranes in vitro, has led to the growing opinion that all intracellular biological membranes have a common molecular basis in consisting of biomolecular leaflets of phospholipids covered with layers of protein, as was proposed many years ago for the plasma membrane itself and described above. The different appearances and functions are thought to be determined by the absorption on their surfaces of various macromolecules. Several systems of nomenclature have already been proposed and, since some confusion is possible, these will be outlined here. Sjöstrand distinguishes three types of membranes: (a) $\alpha$-cytomembranes or membranes associated with dense particles, (b) $\beta$-cytomembranes, smooth surface membranes found in the Golgi region and (c) $\gamma$-cytomembranes, smooth flattened invaginations of the cell membrane. Porter and Palade also recognize these types, but prefer to regard all cytoplasmic membranes as portions of a single membrane system which may become locally specialized for certain functions, e.g. for protein synthesis by becoming associated with RNA particles. The system of particle-studded ( $\alpha$-cytomembranes) is also identifiable with the basophilic ergastoplasm of Garnier (1897) (see Haguenau, 1958) and Bernhard and his associates (Bernhard et al., 1951 and 1954) are inclined to refer to the entire membrane system as ergastoplasm. These several proposals are set out in Fig. 23.

Some authors would go further in an attempt to unify the membrane systems of cells under a single concept, by considering even the external plasma membrane of the cell as part of this system. This view is implicit in Ben Geren's views on the origin of the myelin sheath of nerve fibres as an elaborate involution of the Schwann cell membrane and by the work of Robertson (1959). In some cells, such as amoeba, the formation of many vacuoles by invagination of the external membrane is obvious, and all membranes retain the same fine structure (Mercer, 1959). A common molecular framework forming the basis of biological membranes is also envisaged by the Danielli and Harvey theory of membrane structure already referred to on p. 38 .

A certain lability of membrane structure is indicated by the profusion of forms assumed by the cytoplasmic membranes in vivo and also by the experimentally-produced breakdown of the reticulum and its re-formation as smaller microsome vesicles (p. 111) (Plate 10B). Bacterial membranes, which are membranes of a widely-different origin, also possess a similar property of reforming smaller vesicles on breaking up. 
The nuclear membrane may be considered for the purposes of classification among the cytoplasmic membranes. It consists of two surfaces, whose distance apart is rather variable (500-1000 $\AA$ ), and which touch at intervals to give the impression of a circular pore (Plate 4C). These pores may form a regular pattern over the surfaces of some nuclei and are held by some authors (Watson, 1954) to be genuine pathways permitting nuclear-cytoplasmic interchanges at a macromolecular level.

Fig. 23. Nomenclature of cytoplasmic membranes.

\begin{tabular}{|c|c|c|c|}
\hline Type & Sjöstrand & $\begin{array}{c}\text { Porter and } \\
\text { Palade }\end{array}$ & $\begin{array}{c}\text { Bernard and } \\
\text { Haguenau }\end{array}$ \\
\hline $\begin{array}{l}\text { membranes associated } \\
\text { with dense particles } \\
\text { in } \mathrm{OsO}_{4} \text { fixed material }\end{array}$ & $\alpha$-cytomembranes & $\begin{array}{l}\text { basophilic } \\
\text { endoplasmic* } \\
\text { reticulum }\end{array}$ & ergastoplasm \\
\hline $\begin{array}{l}\text { smooth surfaced } \\
\text { membranes in Golgi } \\
\text { region }\end{array}$ & $\beta$-cytomembranes & $\begin{array}{l}\text { "smooth" } \\
\text { endoplasmic* } \\
\text { reticulum }\end{array}$ & $\begin{array}{l}\text { Golgi } \\
\text { membranes }\end{array}$ \\
\hline $\begin{array}{l}\text { smooth surfaced } \\
\text { membranes linked to } \\
\text { cell surface }\end{array}$ & $\gamma$-cytomembranes & , & - \\
\hline $\begin{array}{l}\text { phospholipid " liquid " } \\
\text { crystals } \\
\text { (inclusions) }\end{array}$ & $-\quad \dagger$ & - & 一 \\
\hline
\end{tabular}

* Occasionally Porter and Palade prefer the less restricted term " reticulum" to cover the entire system of membranes.

$\dagger$ For these the name $\delta$-cytomembranes has been proposed (Schulz et al., 1958).

\section{The nucleus}

Within the volume delimited by the nuclear membrane there are no membrane enclosed objects. One or more dense aggregates of particles appear as the images of the basophilic nucleolus of light microscopy (see p. 80). At the appropriate phases of cell division the nuclear membrane dissolves and chromosomes may appear as denser aggregations of particulate or finely-fibrous material.

\section{Differentia}

Under this name we gather a variety of cell products, which seem distinct from the vital synthetic and respiratory machinery of the cell and represent rather the end results of a specialized path of synthesis. Their presence often gives the cell (and tissue) its characteristic appear- 
ance and function. Examples are numerous secretory granules (enzymes and hormones), pigment granules and fibrils of various sorts including keratin.

It may seem significant that the number of basic structural elements at the macromolecular level is so small. To some the situation would seem to be a strong argument in favour of supposing a common descent from an archetypal cell in which such devices as cilia and mitochondria were already present. To others, and this may be the more austerely biomolecular view, these resemblances in the primary organelles indicate no more than that, given the limited molecular materials available (proteins, phospholipids, polysaccharides, etc.), the number of structural solutions to such problems as: enclosing whole cells, segregating intracellular catalysts and a genetic apparatus, the provision of surface organs of motility and a protective integument, is limited. The actual devices found are effective and perhaps the only solutions to the problems. While we can scarcely hope to discover how these structures came into being, an experimental demonstration of their formation in vitro is certainly conceivable as recent experiments on phospholipids show (Stoeckenius, 1959; Mercer, 1960). In the same way the limited number of fibre-types reflects the few possible ways of folding and packing polypeptide chains. These are limitations at the chemical level. Nevertheless the tendency to produce certain molecular species and to use them in a given situation is inherited and has phylogenetic significance (p. 22).

\section{The phylogeny of keratinization}

In the form found among existing land animals the epidermis is the culmination of a long evolution on dry land, the steps of which can be reconstructed from its histology among these animals (Romer, 1955 and Young, 1950). Obviously this evolution commenced before the dry land was invaded since many fishes deposit intracellular fibrils in their epidermal cells and there are instances, as in the horny teeth of lampreys (Barrnett, 1953), of localized deposits of hardened protein among the lower vertebrates. Also, from their almost universal distribution, there is every reason to suppose that the group of cell responses associated with the surface membrane, which were described in the previous section, can be traced back to the earliest free-living cells. It is not without interest to try to trace back the origin of a specialized epidermis to more remote beginnings, to consider it as part of the more general problem of the evolution of protective layers in organisms. What lends a particular interest to such speculations is the possibility already mentioned that the events at the surface of cells may have played decisive roles in initiating the various lines of evolution.

We have pointed out that the beginnings of such supporting fibrillar 
deposits in the cytoplasm might be found in the cytoplasmic fibrils beneath the plasma membranes in many generalized cells. Possibly too, they share a common molecular ancestor with the contractile muscle proteins and other intracellular fibrillar systems of the same basic molecular type (see p. 22), but these views must remain conjectural in the present state of our knowledge. Such cytoplasmic fibrous proteins are not strictly

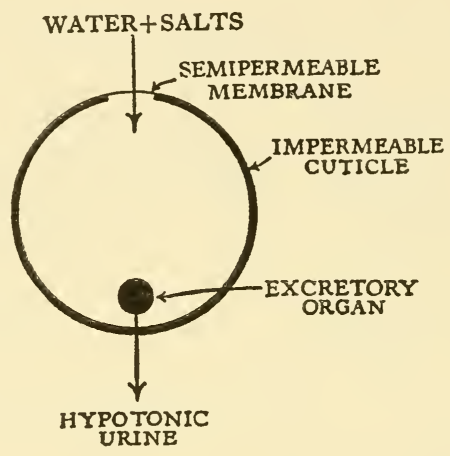

FIG. 24. An osmoregulatory mechanism depending on the development of a water impervious cuticle limiting input and output of water to small areas. From Baldwin (1937) with permission.

keratin in the sense of a definition restricting this term to stabilized fibrils although their role may have been protective. An accepted opinion relates the early specializations of the outer layers to the problem of water control rather than mechanical protection. It is supposed that the need for such control arose in the first place with the colonization of freshwater habitats (Baldwin, 1937). Animals leaving the sea, where the osmotic pressures inside and outside their cells were nearly equal, and entering fresh water, where the salt concentration was less, would be faced with stresses owing to the entry of water into their cells. They would therefore be forced to evolve specialized organs to control the entry and egress of water. At the same time by enclosing themselves in a waterproof coat and thus limiting their water exchanges to a small area (Fig. 24), the work required of the excretory organ would be lessened. At first, if we take the condition in present-day fish as a clue, the epidermal layer was supplemented by the secretion of a slimy mucilagenous layer; this device has, however, tended to lessen in importance as the degree of keratinization of the cells themselves increased. 
If we accept this hypothesis of the origin of the strengthened and waterproofed integument, then we may regard its evolution in fresh water as a fortunate pre-adaptation without which a subsequent colonization of the more unfavourable environment, the dry land, could not have been attempted. However this may be, it was on the land where the full possibilities of keratinization were revealed and the astonishing variety of modified skins, claws and scales were evolved from epidermal thickenings culminating in the appearance of feathers, the distinguishing mark of birds, and of hairs, equally characteristic of mammals. Since these hardened parts may leave fossil imprints and since a sufficient variety of animal types has survived until today, this later evolution is reasonably well documented.

The greatest degree of keratinization was reached in reptiles; in birds and mammals, with the elaboration of feathers and hairs, the thickness and degree of keratinization of the epidermis itself lessens.

In most organisms the waterproofing properties of the toughened framework formed by the structural macromolecules (proteins and/or polysaccharides) are supplemented by the addition of lipid materials.

An increase in the degree of stabilization of the epidermal protein itself appears to have occurred in higher vertebrates, if we may judge from the skins of surviving types. An experimental measure of the degree of stabilization may be obtained by observing the temperature at which an oriented fibrous system contracts on heating due to the shortening of its molecular chains by thermal agitation (see p. 255). Using this method Rudall (1955) has shown more precisely that in the case of the newt, Triturus, the stability of the skin was intermediate between that of a film of myosin, an unstabilized muscle protein of the $\alpha$-type, and human leg stratum corneum. The temperature at which the $\mathrm{X}$-ray pattern became disoriented or was converted into a $\beta$-type pattern was also determined by Rudall and again demonstrated an intermediate degree of stabilization.

The amphibians, in this respect as in others, are "living fossils" and preserve, in the changes which take place in their epidermal cells at metamorphosis, a suggestion of the course of evolution of the keratinized skin of the fully land-dwelling animals. The larval skin contains a variety of cells, some ciliated, some secreting mucins and others containing masses of fine fibrils, from which it is possible to infer that the factors determining complete keratinization are not yet present. Histochemical tests (Barrnett, 1953) based on the demonstration of cystine cross-linkages as stabilizing elements (Hergersberg, 1957) show almost a complete absence of true keratinization; after metamorphosis, when the animals become capable of living out of water, the epidermis becomes keratinized. The change is also provoked by thyroxin which causes premature meta- 
morphosis. Some authors have reported free SH groups in the amphibian stratum corneum.

Obviously the discovery of some method of hardening, reducing the swelling and waterproofing the integument is a prerequisite for life in a dry environment and, in this sense, the process of keratinization is rightly described as one of the key biochemical discoveries on whose exploitation the success of the vertebrates is based. In other land-dwelling forms different macromolecules have been adapted to meet these same needs, and, as mentioned above, the nature of the structural macromolecules found in the integument places certain limitations on the evolution of a phylum. A more fully-developed knowledge of molecular phylogeny may be able to relate the mutations, which gave rise to the various molecular types to the subsequent evolutionary development. 


\section{The Keratinized Tissues}

\section{Generalized histology of the vertebrate epidermis}

\section{DERMIS AND EPIDERMIS}

The integument of vertebrates consists of two quite distinct partsan epidermis and a dermis (Fig. 25) which together form a well-bonded unit for the protection of the organism. The two parts are of very different character and of different origin embryonically. The epidermis is entirely cellular and its most characteristic products are retained within the cells producing them; the dermis is primarily a fibrous meshwork in which are distributed sparsely cells of mesenchymal (mesodermal) origin which secrete a dense feltwork of fibrils of collagen and elastin. The interfibrillar spaces contain, among other constituents, gelatinous mucopolysaccharides. All these materials are found outside the cells in contrast to the intracellular location of the fibrils of the epidermal tissues.

The dermis is continuous with the sub-cutaneous connective tissue and with the other connective tissue of the body and has thus no definite inner boundary such as the definite boundary separating it from the epidermis. This surface separating the two is called the dermoepidermal junction and has long been recognized, but its detailed structure and special character were only revealed by electron microscopy. The considerable early literature on the subject should be read in the light of these newer findings which will be described in detail in the next chapter. Membranes of a similar type seem to separate all the superficial epithelia from the mesodermal tissues (BM in Plates 7, 9 and 23B).

Although conspicuous and predominant, collagen is not the only constituent of the intercellular spaces of the dermis. By means of special staining techniques numerous other substances can be demonstrated in the light microscope and some have been extracted and partly characterized chemically. It is customary to distinguish both formed, fibrillar elements and an amorphous colloidal ground-substance. Collagen, except in a few special situations, constitutes the bulk of the fibrous material. Less conspicuous are elastin fibrils, as yet poorly-characterized but distinguished from collagen by their microscopic appearance and their extensibility, and other finer fibrils called reticulin. 
The work of Meyer (1945, 1951 and 1957) in particular has led to the recognition of the importance of a special class of polymers forming the colloidal ground-substance of the intercellular spaces, the mucopolysaccharides. These are proteins linked to polysaccharides containing hexosamine or glucuronic acid. They are distinguished partly by the amount and type of polysaccharide and by the presence or absence of sulphuric acid, e.g. chondroitin sulphuric acid, contains sulphuric acid and hyaluronic acid does not. Meyer (1957) distinguishes four or five mucopolysaccharides: hyaluronic acid, chondroitin sulphates A B and C differing in their optical rotary power $[\alpha]_{D}$ (see p. 194). Heparin may also be classed among them. The amount and types of mucopolysaccharides vary from site to site which suggests some relation with the overlying tissue. Their importance in tissue maintenance is sufficiently indicated by the dramatic effects produced by cortisone, by the adrenocorticotrophic hormone (ACTH) and by hyaluronidase. The two histochemical tests used to distinguish mesenchymal elements: the periodic acid-Schiff test (PAS) and metachromatic staining are often believed to stain mucopolysaccharides.

Metachromasy is the phenomenon of a change in colour of a dye on becoming associated with a structure, an effect believed to be partly due to the state of aggregation of the combined dye. The commonly used dye, toluidine blue, is blue in dilute solutions and stains (basement membranes) metachromatically to give a purple colour. The PAS test is an application of the well-known test for aldehydes using a bleached solution of basic fuchsin (Pearse, 1953). It may be used histochemically in cases where a chemica ltreatment can cause the release of aldehydes from polysaccharides or sugar-containing complexes. The Feulgen test (p. 80) is based on the fact that mild acid hydrolysis liberates an aldehyde from DNA which restores the colour of the dye. Other polysaccharide complexes yield aldehydes after oxidization by periodic acid and can then be stained by the Schiff reagent. Reticulin and basal membranes are strongly PAS-positive. Formerly the basal membrane was on these grounds said to contain a fine network of reticulin fibrils; probably it contains an amorphous mucopolysaccharide as suggested by its appearance. Leblond and his associates have cast doubt on the interpretation of these tests by showing that extracts of pure components do not always give the expected reactions.

The PAS-positive and metachromatically-staining substances are invariably present in sites of rapid growth (p. 221) and seem therefore associated with the process, but their role remains obscure. They are strongly hydrophilic and could help to retain water and to form a viscous gelatinous scaffolding in advance of more permanent formations. It also seems likely that in a more condensed form they can function as components of intercellular adhesives and the related basement membranes. 
Certain of the polysaccharide fibres from vertebrate skin are said to yield an X-ray diffraction pattern like that of cellulose (p. 24).

The configuration of the boundary between the two layers is much influenced by the presence of specialized appendages-hairs, scales, etc., formed by the epidermis. In the absence of these, it may be smooth and run parallel to the external surface; when present they dip deeply into the dermis. In mammals there is a marked development of small regularlyplaced dermal papillae and ridges which, being more prominent the

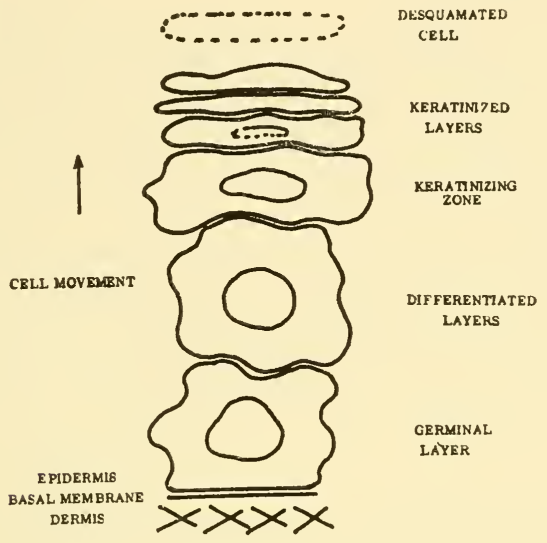

Fig. 25. A generalized, stratified, keratinized, squamous epithelium resting on a basal membrane backed by a collagen-containing dermis. In all keratinized tissues (except the thinnest skins) the several layers shown on the right-hand side may be distinguished.

thicker the epidermis, are related probably to the nutrition of the superficial cells. They have the effect of greatly increasing the area of contact between the two formations and thus should facilitate the transfer of nutrient from the vasculated dermis to the epidermis.

\section{THE EPIDERMIS}

Among the vertebrates the epidermis is always a stratified epithelium consisting of a few to many layers of cells produced by the proliferation of a single basal layer (Fig. 25). Epithelia, more-or-less regular and compact layers of cells, are common tissues and usually cover the external surfaces of an organism or its internal cavities. Their superficial location appears to impose certain common histological features. The cells may 
become progressively more specialized as they near the surface and may show an internal polarization of structure. They are bounded below by the basement membranes separating them from the connective tissues which are vasculated. Being themselves avascular, nutrient materials must reach them by passing from the vessels of the subjacent connective tissue into the interstitial fluid and thence by diffusion across the basement membrane. There may often be a constant loss of cells from the exposed surface (squamous epithelia) which must be made good by the proliferative activity of a germinal layer.

The keratinizing epithelium is normally squamous, its hardened surface cells being shed either continuously or at intervals as a whole in the form of a moult. In its simplest form it may appear to consist of a single layer of living germinal cells and a thin cuticle of keratinized cells as in the mouse. Usually more layers can be defined and the character of the skin is influenced by the number of cells in each layer. An idealized generalized, keratinizing epidermis might be said to consist of at least the following layers: (a) a germinal layer of cells whose proliferation maintains the entire cell population; (b) a differentiating layer in which the protein is synthesized and keratinized; and (c) the dead and hardened layer (see Fig. 25). If the tissue is to be of constant average thickness, the cells formed over a given time must equal those lost by exfoliation in the same time. The thickness varies with the total number of cells in each of the layers and structures with a variety of properties may be produced by variations in the proportion of differentiating cells and hardened cells, i.e. in the relative thicknesses of the softer still hydrated layers and the tough, cornified and relatively drier cells. The ease of exfoliation determines the thickness of the horny layer and obviously a thick, hard layer will result if the exfoliation is slowed down. Further by supposing localized differences in rate of cell formation and loss, it is possible to understand, in principle, the production of the various horny appendages.

It is sometimes said that keratinization is a degenerative phenomenon, a consequence of poor nutrition, of desiccation or other deleterious factors. That this is not true is shown very clearly by observations on cells cultivated in vitro where conditions are under closer experimental control (Fischer, 1924; Miszurski, 1937; Hardy, 1949; and Strangeways, 1931). Skin cultivated in vitro readily undergoes keratinization with the production of histologically-normally keratinized cells. The same sequence of histochemical events as in vivo occurs and the product is also birefringent (Litvac, 1939; Miszurski, 1937; and Hardy, 1949). Feathers have been cultivated to a limited degree. Strangeways and Hardy (Strangeways, 1931; Hardy, 1949) also succeeded in growing hairs and noted even in these abnormal conditions that histogenesis, up to a certain point, and fibrillar orientation proceeded normally. 
Nevertheless keratinization in vitro does not take place in all types of cell regardless of origin; nor, as was shown very definitely by Miszurski (1937), in epithelial cells themselves is it initiated or promoted by poor nutritive conditions or low oxygen tension or lower temperature. The phenomenon is properly to be regarded as the final stage of an intrinsic differentiation of epithelial cells, as well adapted to the function of the tissue as in, for example, the production of collagen by fibrocytes. This is not to say, of course, that it cannot assume an abnormal, perhaps degenerative form.

That the epithelial habit with the potentiality of keratinization is a fundamental type of cell behaviour is shown by the fact that it is one of the forms to which cells revert when cultured in vitro for some time. Willmer (1954) describes three such cells forms: epitheliocyte, mechanocyte and amoebocyte. When conditions are appropriate epitheliocytes adhere laterally and grow to form flat unicellular sheets. The intercellular contacts are of the types described above (p. 40); the cells may secrete mucopolysaccharides (mucins) and may keratinize. Mechanocytes form open meshworks, secrete collagen and a distinct type of mucopolysaccharide. The mobile amoebocytes form no permanent associations with other cells. Recent work (e.g. Puck, 1957) shows that the morphological appearances may be deceptive but the biochemical differences are more persistent and significant.

\section{The epidermal family of cells}

From the ectcderm of the embryo is developed not only the adult epidermis but an entire family of cells which includes the keratinized appendages and numerous glands. The "genealogical tree" of the epidermal family is shown in Fig. 26. The entire population is produced and maintained by the proliferation of the undifferentiated cells of the germinal or Malpighian layer, which everywhere covers the outer surface of the dermoepidermal membrane. These cells not only have the same embryonic origin but, according to some authors (Montagna, 1956), retain everywhere, even in the adult, the potentiality to differentiate into any of the cell types found among their descendants and thus on the biochemical level to produce any of a number of distinct chemical substances, such as keratin (in several forms), sebum, mucin, etc.

The development of glands capable of secreting mucin or lipids is a character of the epidermis as typical as and, phylogenetically speaking, of earlier development $\mathrm{than}$, its keratinizing potentialities. The glands may take the form either of unicellular "glands," i.e. single cells discharging their contents directly on to the surface, or of more elaborate multicellular formations sunk into the dermis and communicating by a duct with the surface. 
The secreted mucins are viscous, shiny substances providing a protective, slippery, extracellular sheath possibly related chemically to the more permanent and condensed cuticles. They are ill-defined chemically, containing a protein and a carbohydrate moiety, but the nature of the association of the two is obscure (Meyer, 1957 and p. 54). The relation between the mucoprotein and other types of protein which may be synthesized by cells arising from the same germinal layer does not seem to

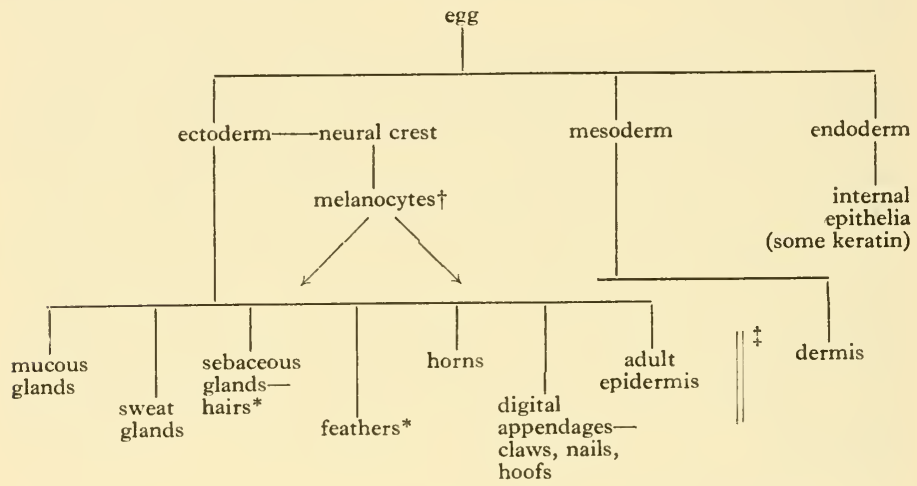

FiG. 26. Genealogical tree of the epidermal family and related tissues.

* It is not implied that all types of appendage (including hair and feathers) appear on a single skin.

$\uparrow$ The pigment-forming melanocytes migrate into the dermis and epidermis where, after attaching to the dermo-epidermal basement membrane, they may pigment the growing epidermal-type cells (p. 276).

$\ddagger$ This symbol is meant to indicate the special anatomical union between dermis and epidermis.

have been explored. Owing to the extremely elongated nature of their molecules and their high negative charge, they raise the viscosity of the secretions and in this way lubricate the surfaces and protect them against mechanical, chemical and perhaps bacteriological injury. The lipids comprise a multitude of compounds having in common a solubility in non-aqueous solvents. They may exhibit species specificity (Hilditch, 1949). The phospholipids are an essential constituent of most biological membranes (p. 37 et seq.).

Mucin secretion is common in aqueous forms or on moist internal surfaces of land forms; lipid secretion is found more among the dry 
skins of land forms where it serves to lubricate and improve the waterproofing of the skin. It is difficult to suggest a reason for the extraordinary variety of compounds found in these secretions.

Birds and reptiles have fewer cutaneous glands than mammals. The heavily-keratinized epidermis of reptiles does not favour their development nor for perhaps the opposite reason does the thin skin of birds. Birds usually possess a uropygial gland opening in front of the tail, the secretion of which, spread over the feathers during preening, helps to waterproof the layer of feathers. Vitamin D is produced from its secretion by the action of sunlight and plays a part in nutrition (Hotta, 1928 and 1929) which seems to demonstrate a special reason for the presence of some lipid molecules.

The mammalian skin is rich in number and has more varied types of glands. The types generally present are: (1) mammary glands which give the phylum its name, (2) sebaceous glands, and (3) sweat glands. The sebaceous glands are usually associated with hair follicles and produce an oily secretion which softens the skin and helps to lubricate the hair. Mammary glands and sweat glands are histologically similar. The small sweat glands or eccrine glands derive directly from the embryonic epithelium, the apocrine (partly sebaceous, larger sweat glands) and the sebaceous glands indirectly via the follicular epithelium of the outer root sheath (p. 96). The latter glands are found usually in association with hairs; the eccrine (sweat) on hair-free surfaces where, as in man, they may play an important role in temperature control. Sebaceous and apocrine glands possibly are related to similar glands in the hairless skins of earlier vertebrates. The very active cutaneous mucinogenic glands of cyclostomes are said to be partly holocrine and may be remotely related (Rothman, 1954). Some cutaneous holocrine glands of reptiles, undoubtedly derived from similar phylogenetic ancestors, are sac-like invaginations of the epidermis producing fatty materials. Certain mandibular cloacal glands of alligators are reported (quoted by Rothman) to produce lipids and keratin simultaneously. The cell peripheries keratinize and the centre produces lipids; the whole is ultimately shed. This is a demonstration of bifunctionality of epidermal cells. It would seem that epidermal cells can still produce lipids, in addition to the structural phospholipid of cell membranes (Table 4, p. 486, Rothman, 1954) indicating that the potentiality is still present (see next section).

There is a sense in which the entire epidermal system may be regarded, as Montagna has put it, as an immense holocrine gland. Most of the "secretion," i.e. the keratinized material, is shed and lost, but certain other constituents may be absorbed either by their producer, its young or associates, and serve further physiological ends. This is most obviously so among the mammals whose milk glands are elaborated sweat glands, 
and whose persistent habits of licking and grooming must introduce many substances of epidermal origin into the alimentary canal. Vitamin I) production is an epidermal function. Among birds the preen gland has been shown to be essential for the well-being of its possessors. An extraordinary example is provided by the aquarium fish (Symphysodon discus) whose young are nourished by the mucous secretion of the parents which covers large areas of their bodies (Hildemann, 1959). Probably much remains to be discovered in this field.

An important anatomical aspect of mammalian skin is the close association of glands and hair follicles. Except in rodents, the central primary follicles and most, but not all secondaries are associated with a sebaceous gland which usually opens into its lumen. This rather constant association of hair follicles and sebaceous glands suggests that the natural functional unit of the mammalian skin is the follicle group and its glands, the "pilosebaceous unit" (Montagna, 1956) (Figs. 34 and 35), which together produce keratinized hairs and the means to lubricate and condition them. In the dry scaly skins of reptiles or the glandless (with the exception of the single preen gland near the tail) skin of birds, it is hard to find an analogue of such a unit.

\section{The Differentiation of Epidermal Cells}

It is a problem of wide interest to determine what are the factors which are responsible for the appearance of the variety of cell-types which we have included in the "epidermal family." Information has been sought principally on the factors determining early embryonic differentiation and on those maintaining the stability of the various cell-types found at different sites on the adult.

The epithelial character of the external cells of an embryo appears very early in life-in effect already in the blastula-and we have mentioned the hypothesis that this is due to the appearance of intercellular adhesion which continues thereafter to play an important morphogenetic role. A further discussion of the role of cellular adhesion in controlling cell differentiation among the persistently embryonic cells of the germinal layer of the epidermis will be given in Chapter 3. Granting that the primary step in the differentiation of epidermal cells is the production of the epithelial habit by intercellular adhesion, there is direct experimental evidence provided by grafting to suggest that in the next phase the factors responsible for localized specializations arise in the underlying mesoderm. For example, using the embryonic chick, by grafting mesoderm from a presumptive foot bud beneath wing ectoderm, one can cause the formation of a claw instead of a wing feather (Cairns and Saunders, 1954). The epidermal cells at this stage may be described as being sandwiched between two environments: the external relatively-free space and the 
underlying mesodermal domain. In the first place the external situation imposes a class of differentiations and secondly the mesodermal organization further limits differentiation and gives rise to site-characteristic developments. As these develop and call into being an appropriate dermal organization to support them, consisting in part of fibrous collagenous depositions and a blood supply, the situation is reversed. The epidermal tissue becomes dominant and grafting now shows that, when sufficient epidermal tissue is transferred, the site characteristics (skin, claws, feathers, etc.) are now preserved (Cairns and Saunders, 1954).
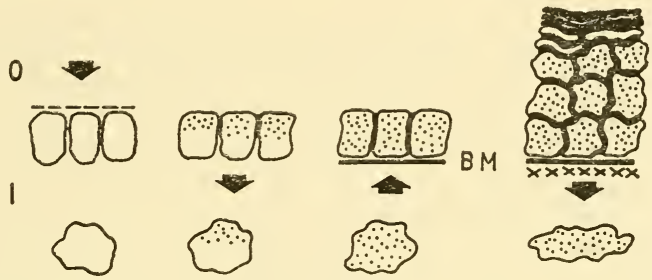

FIG. 27. Diagrammatic representation of the interreaction between epidermis and dermis in successive stages of the establishment of a differentiated epidermis (r.h.s.). O, outside environment, I, internal environment, BM, basal membrane. The arrows indicate the direction of dominant influence and the shading differentiated cells.

From the numerous experiments (Zwilling, 1955; Waddington, rev. 1956; and McLoughlin, 1959) which show that the underlying mesenchymal tissue induces and maintains the different epidermal differentiations, there is a suggestion that the basal membrane itself could be the important factor. It appears to differ in thickness from site to site (200-600 $\AA)$ and there are differences in the types of mucopolysaccharides present. It is easy to picture such a continuous layer of colloids of this type, with their fixed network of charged sites, functioning as ion exchange resins (Meyer, 1956) and exerting a selective effect on the transfer of signal molecules from the blood to the epithelial cell population. The idea is, however, very insufficiently explored experimentally as yet.

If, at some risk, one attempts to summarize the findings in a field as yet imperfectly explored experimentally and in rapid development, it seems possible to distinguish several successive stages in the establishment of the skin in which dominant control in the dermoepidermal partnership swings successively from one member to the other as suggested by the arrows in Fig. 27. In the earlier phase the superficial cells, responding to their exposed position (see also p. 90), begin to stick together and thus 
bring about the epithelial pattern. Their, as yet unstabilized, free, external surfaces produce a selection of responses from what we have termed the " cell's surface repertoire." The establishment of a definite surface layer encloses the other cells in a different environment which diverts them toward synthesizing different products (mesenchymal substances), which in their turn react with the inner surfaces of the basal layer cells to form the basal membrane and to induce local variations in the epidermal layer. By their subsequent development these epidermal variations make return demands for food and support on the underlying layers leading to the building up of a dermal organization of fibrillar scaffolding and supply vessels.

The molecular basis of these events is ill understood and their discussion is often marred by vague concepts which in effect conceal our ignorance. Further discussion of the problem will be found in Chapter 3.

There is much evidence, emphasized by Montagna, Chase and colleagues (Montagna, 1956) and by Billingham (1958), to show that the determination is not irrevocable and that the cells of the germinal layer itself remain effectively multipotential. In many animals, hairs may normally become differentiated from basal layer cells throughout life and Billingham cites the extraordinary case of the hairy velvet covering the growing horns of deer which is in its entirety reformed annually. Further, after losses due to many injuries (wounds, burns, X-radiation) many epidermal elements regenerate from the remaining basal layer cells or from cells of the outer root sheath of the hair follicle (Montagna, 1956; Billingham, 1958).

Other evidence of persistent multipotency is provided by the epithelia of many internal surfaces which may exhibit a cyclic metaplasia under hormonal control with a well defined physiological function. These may be of endodermal origin, but since they may be capable of keratin formation, they are relevant here. The best-known example is that of the vaginal epithelium which oscillates between mucin and keratin production, cells of a contrasted cytology being produced successively by the same basal layer (Nilsson, 1959) (Fig. 59). In other situations keratin production oscillates with glycogen (Hinglais-Guillard, 1959).

Thus it would still seem that the course of differentiation followed by a cell leaving the basal layer is determined by effects emanating from neighbouring cells and from the underlying dermis. That in grafts cells may retain the characteristics of their site of origin could be due to the fact that large numbers of cells are transferred in a graft and, in effect, carry with them their original environment. When a denuded area is merely " seeded" with small groups of cells, the new growths are said to be typical of the new site rather than the old (Montagna, 1956).

In many malignant tumours arising from epithelial surfaces (carcinomas) the normal controls maintaining a stable differentiation seem relaxed and 
latent potentialities of the cells may find expression. Small nests of cells with a well-defined, but aberrant histology, e.g. ciliated borders, mucinsecreting surfaces, etc., may often be observed. That the potentiality of producing both keratin and mucin can exist even in the same cell is indicated by some observations of Glücksmann and Cherry (1956) on mixed carcinomas.

It would seem that the several varieties of epidermal cells are examples of what Weiss (1950) has preferred to term cell modulations which are at first reversible and which require for their maintenance the piesence of other elements of the cellular community. Modulations are to be contrasted with the perhaps irreversible differentiations which accompany embryogenesis and which divide the total cell community into several major families of common descent. Support for such a view is given by direct experimental evidence of cell metaplasia produced by relatively simple chemicals. The work of Fell and Mellanby (1953) (Fell, 1957) and their associates has established that vitamin A disposes the epidermis towards mucin formation. Seven-day old chick embryo ecoderm cultivated in vitro in a normal culture medium undergoes precocious keratinization, the two-layered epithelium being replaced by a stratified layer. When vitamin $\mathrm{A}$ is added to the medium (2000-3000 i.u. per $100 \mathrm{ml}$ ) keratinization is prevented and a mucous secreting, often ciliated, epithelium appears. The change is not stable, for when the layered epithelium was transferred back to a normal medium (i.e. lacking vitamin A) a typical mucous membrane containing ciliated cells and mucous cells at first appeared, but after a time this was replaced by a squamous keratinizing epithelium forming beneath it.

Lasnitzki (1956) showed that the effect on mammalian skin (human embryo) was essentially the same. Embryonic epidermis (3-4 months foetus) in normal medium formed a typical squamous keratinizing epithelium including a keratohyalin layer. In a medium containing vitamin A several layers of large cuboidal cells appeared which contained mucin-like materials. Older skin is less responsive but vitamin A suppressed keratinization.

The vitamin A induced metaplasia was correlated with changes in the uptake of sulphur detected by using radio-active sulphate. In explants of skin treated with $\mathrm{S}^{35}$ (as sulphate), mucous secreting material was intensely active; the keratinizing layers much less so. On the other hand, the uptake of radioactive cystine was greater in the keratinizing epidermis (Fell et al., 1954 and 1956. See also p. 264).

Weiss and James (1955) found that a brief exposure to vitamin A in higher concentration produced the same effect as the continuous administration of lower doses used by Fell and co-workers, and concluded from this that vitamin $\mathrm{A}$ acted as an inductive agent which switched the development 
of the cells along an alternate pathway. A demonstration of induction by " a crucial event of relatively short duration" is of some theoretical importance, but Lasnitzki and Greenberg (Lasnitzki, 1958) have cast doubt on the conditions of the experiment by demonstrating the persistence of vitamin A in cultures treated as were those of Weiss and James. They conclude that the action of vitamin $\mathrm{A}$ is due to its continuous presence.

The effect of vitamin A is most apparent on the germinal layer cells as might be expected, since these are "uncommitted," but cells in the process of keratinization can still be deflected in their course by the vitamin. The mucin forming cells once formed cannot, however, revert when returned to a normal medium but are shed.

The variety of differentiations of the epidermis, its simplicity and its experimental accessibility assure that in the future it will continue to play a part in the investigation of the general problems of differentiation.

\section{Hard AND Soft Keratins}

It is customary to distinguish between "soft" keratins (the epidermis itself) and the " hard " keratins, hair, feather, horn, etc. The classification was put on a well-defined basis by Giroud, Bulliard and Leblond (1934). Primarily the distinction is based on the immediate sensation of hardness or softness, and the fact that soft keratins (epidermis) desquamate while the hard keratins (hair, nails, etc.) persist. These properties were shown to be linked with other differences appearing in the course of keratinization and with the chemical composition of the final product. These are tabulated in Table 5 .

Some of these distinctions, which seem obvious enough at first sight, become less obvious on closer analysis. The difference between the two types is, in fact, only relative and, if pathological material is admitted, a continuous spectrum of tissues between hard and soft exists. Moreover, the same germinal matrix can be made to produce a graded series of tissues of various textures, as is shown by the development of callosities, warts and corns, the thickened skin of Ichthyosis vulgaris and by some effects following radiation (Chase, 1954). However, the classification is useful as representing two extremes of the synthetic potentialities of epidermal cells with obvious adaptive potentialities. Typically where the site and function demand a squamous tissue, soft keratin develops; where a persistent growth is required, we find hard keratins. We know as yet, little of the underlying causes determining the type of keratin produced at any site.

In general terms a structure is hard and coherent when its units are hard and they do not fall apart. Translated into histological terms, this means that the factors underlying the differences between hard and soft 
tissues are to be sought in: (a) the hardness and coherence of the intercellular contents, and (b) the intercellular adhesion. The properties listed in Table 5 give grounds for saying that in hard keratins the fibrous intracellular protein is more plentiful, harder and more completely fused into

Table 5. Properties of " Hard " and " Soft " Keratins

\begin{tabular}{|c|c|}
\hline Soft keratin & Hard keratin \\
\hline soft and pliable* & tough and hard* \\
\hline desquamating & $\begin{array}{l}\text { permanent, non- } \\
\text { desquamating }\end{array}$ \\
\hline $\begin{array}{l}\text { in course of development } \\
\text { cells pass through a kerato- } \\
\text { hyalin layer (p. } 95 \text { ) }\end{array}$ & no keratohyalin phase \\
\hline higher lipid content $\uparrow$ & low lipid content $\nmid$ \\
\hline $\begin{array}{l}\text { lower sulphur content } \\
(<3 \%)\end{array}$ & $\begin{array}{l}\text { higher sulphur content } \\
(>3 \%) \text { and stronger thiol } \\
\text { reaction in course of } \\
\text { hardening (p. } 217)\end{array}$ \\
\hline lower thermal stability & highe thermal stability \\
\hline $\begin{array}{l}\text { ratio of basic amino acids } \\
\text { histidine lysine and arginine } \\
=1: 4: 4(31)_{+}^{+}\end{array}$ & $\begin{array}{l}\text { ratio basic amino acids } \\
1: 4:: 12 \ddagger\end{array}$ \\
\hline less perfect ordering & better oriented \\
\hline
\end{tabular}

* This distinction is more apparent than real and is partly due to the more massive character of the hard keratins.

† The lipids of the softer keratins may act as "plasticizer." When extracted the materials become very tough and hard.

$\ddagger$ This difference in the ratio of the basic amino acids was deduced by Block on the basis of a large number of analyses. Block regards the basic amino acids as forming a characteristic structural element in proteins and he distinguishes sharply between the two classes of keratin prefering to call the soft keratins " pseudo-keratins." Recent analysis seems to show that the ratios are by no means exact integers.

continuous masses and that the adhesion between the cells is more complete and more persistent. In the soft keratins the fibrils may be less completely or less strongly fused. The fibrillar content of soft keratins partly derives from keratohyalin (see p. 95); this substance appears deficient in the covalent cross-linking responsible for cohesion (p. 234) and thus may more readily break up. The thickness of the hardened layers (stratum lucidum 
plus stratum corneum) depends on the coherence of the total formation and such abnormally-thickened areas as corns show a thick clear layerthe most thoroughly-bonded zone. Electron micrographs of desquamating cells of human skin show a simultaneous separation between both cell membranes and between the intercellular fibrillar contents in the body of the cell. The spontaneous exfoliation could thus be due to a failure in both components.

It is evident from Table 5 that the sequence of events in the formation of a soft keratin differs somewhat from that in the formation of a hard. It will be convenient to consider this problem later in Chapter 6, pp. $210-282$.

\section{The specialized appendages}

\section{Localized Epidermal Thickenings}

The epidermis and the relative thickness of its various strata show some characteristic local variations. In thin skins the intermediate layers (Fig. 25) (stratum granulosum and stratum lucidum) may be absent, the transition between the germinal and horny layer being quite abrupt. This condition may have some significance in showing that a granular phase (keratohyalin intermediary) may not be necessary for cornification (p. 94); nevertheless such skins can often be provoked by appropriate stimuli to assume a multilayered appearance and it is possible that, during the actual growth phase, granules are formed.

The thickened areas of the epidermis have a functional purpose, e.g. the horny pads of the digits, and become more thick with use. These thickened areas are genetically determined and display thickening in the embryo before being stimulated externally. What is also inherited is the tendency of the cells in these areas to respond to friction or pressure by a further proliferation, thus leading to an individually-adapted response.

\section{SCALES}

Scales are specialized epidermal thickenings with a characteristically patterned appearance which are strongly developed among reptiles where they form a horny exoskeleton sometimes of considerable thickness (Fig. 28). They are never separate, as in fishes, being simply localized thickenings of an otherwise continuous epidermal layer. The scales of fish are in fact quite distinct structures of dermal origin (p. 75) and are not homologous to epidermal scales. Scales are also found on the legs of birds, revealing their reptilian affinities, and among a few mammals such as rodents, the scaly tails of rats being familiar. The well-developed covering of the scaly ant-eater is said to be a secondary development.

Beaks and bills are horny developments of the jaw margins, often 
associated with a loss or reduction in teeth, and are typical of birds, turtles and even some mammals (platypus).

Embryonically the scale, like the feather, appears as a small outpocketing of the epidermis containing a dermal papilla whose formation, as a denser gathering of cells, precedes the actual proliferation of epidermal cells and is thought to induce and to control the epidermal changes. The flat upper surface of the outgrowth gives rise to the hardened scale (Fig. 28). The lower surface which may be more or less overlapped by the upper scaly surface and constitutes an inter-scale region, usually consists of a softer more normal epidermis, and imparts some flexibility to the entire

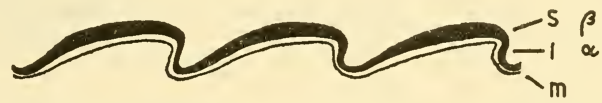

Fig. 28. The reptilian scale structure. The scales $\mathrm{S}$ are thickened epidermis and are not separate. The scaly layer $\mathrm{S}$ yields the $\beta$-type $\mathrm{X}$-ray pattern and the interscale region I an $\alpha$-pattern. $m$ is the germinal matrix.

integument. As mentioned above, the keratin of reptilian scales is of the $\beta$-type which is rather inextensible. Rudall (1949) has found, however, that in the softer, flexible inter-scale region an $\alpha$-type keratin tends to predominate. The production of two molecular types of keratin from neighbouring cells arising from the same germinal epithelium poses some interesting questions of differentiation (p. 104).

A continuous scaly epidermis may be so intensely hardened that it cannot be shed by sloughing or simply worn off, but must be loosened periodically and cast off as a unit. This is effected by a temporary cessation of growth followed by the reformation of an entirely new horny layer beneath the old.

\section{HORNS}

The hollow horns of cattle, goats, sheep, etc., are horny sheaths covering a bony core (Fig. 29 (a), (b)). They are not shed, but as they are worn away they are renewed by the proliferation of a germinal layer. Such horns do not branch although, owing to different rates of proliferation of the germinal layers from one side of the horn to the other, they may grow in graceful curves and spirals ('Thompson, 1942).

The antlers of deer, etc., are not strictly speaking horns, being bony growths forming beneath a covering of hairy skin, the velvet, which dies and is rubbed off leaving the naked bony antler. Pronghorns of certain antelopes are permanent bony antlers extended by a thimble-like sheath of true horn, which in this case is shed periodically like a scale and renewed without loss of the bony core (Fig. 29a). 


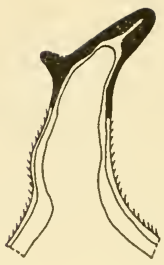

(a)

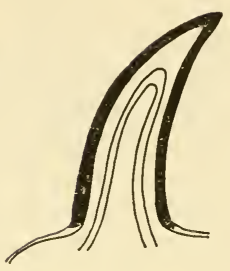

(b)

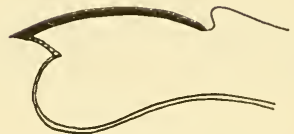

(d)

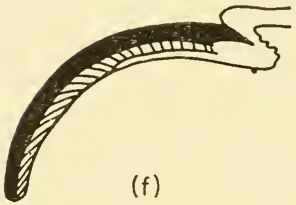

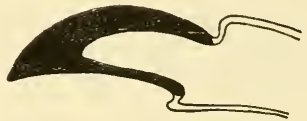

(e)

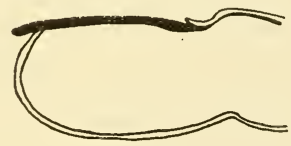

(g)

FIG. 29. The various epidermal appendages containing hard keratin shown black and softer varieties shown stippled.

(a) and (b) are two kinds of horns: (a) is the pronghorn consisting of a " thimble" of true horn capping a "bony horn" covered by hairy skin. The cap is shed annually; (b) is the true horn covered entirely with horn keratin which is not shed. The antlers of deer are not true keratinous horns but consist of bony growths at first covered by a hairy skin. (c) The principal parts of a hoof which consists of an outer covering of hard keratin (the unguis) and an inner subunguis of softer keratin. This combination of a harder and softer keratin recurs in claws (d, e and f). Claws of a carnivore, a bird and a rat. The wearing away of the softer subunguis helps to maintain the sharp cutting edge of the carnivore claw (Le Gros Clark, 1936). (g) The human nail in which only the hard keratin layer remains. 
The Digital Tips: Claws, Nalls, Hoofs

The fingers and toes of all vertebrates above the amphibians are reinforced by horny appendages which are adapted to the way of life of their owners. The homology of all these structures will be apparent from Fig. 29. Claws are structurally similar wherever found and consist of two unequally-developed scale-like surfaces which meet over the end of the digit (Fig. 29 (d), (e) and (f)). The dorsal surfaces are formed of a tougher and more compact keratin than the lower or sole, and this contrivance ensures that where the two meet, a sharp projecting cutting edge will be formed. The orientation of the component cells may be different in the two layers (Fig. 29 (f)). The nails of primates are developed from claws by flattening and losing the underlayer leaving only the compact layer (Clark, 1936 and Horstmann, 1955). The sole is perhaps represented by a small area beneath the projecting nail (Fig. $29(\mathrm{~g})$ ). In hoofs, the sole although softer forms a more important part of the weight-bearing surface, and is encased in a sheath of more compact and harder horn (Fig. 29 (c)). This use of keratins of different degrees of toughness to effect special functional properties is an important aspect of keratinization which will be returned to in Chapter 6.

\section{FEATHERS}

The structure of a typical feather is shown in Fig. 30. The simple down-feather or plumule consists of a cylindrical quill opening into a tuft of barbs and barbules; the filoplume is a fine hair-like feather; contour or flattened flight feathers are more complex, consisting of a quill or calamus and a shaft or rhachis which bears the barbs and barbules.

Feathers, the uniquely-distinguishing mark of birds, are believed on embryological and paleontological grounds, to be homologous with reptilian scales. As in the case of scales the first indication of the site of a presumptive feather is in the gathering of dermal cells beneath the epidermis which then projects to form a papilla containing dermal elements. At this stage, feather and scale "germs" are much alike. Later the whole formation sinks into the skin to form the follicle. Regarded in its simplest ideal form, a feather is a hollow type of cornified epidermis growing from a ring of germinal cells at the bottom of the follicle (Fig. 45, Chapter 3). The development of a plumule (down feather) which consists of a short cylindrical quill opening into a circle of soft barbs and smaller barbules can be understood from Figs. 46 and 47, pp. 103-105. 'The germinal layers at the base of the papilla first form a number of longitudinal columns (seen in cross-section in Fig. 46); these separate and keratinize each to form a barb. The basal part of the growing feather does not separate into columns, remaining as a continuous cylinder to form the 
quill. When the thin skin forming the sheath and covering the feather during growth bursts, the feather opens.

The larger and more elaborate contour feathers in which the barbs project from the sides of a shaft are formed in a more complicated way. Up to a point development is similar to that of a plumule; then the middorsal region of the germinal collar begins to proliferate more rapidly to

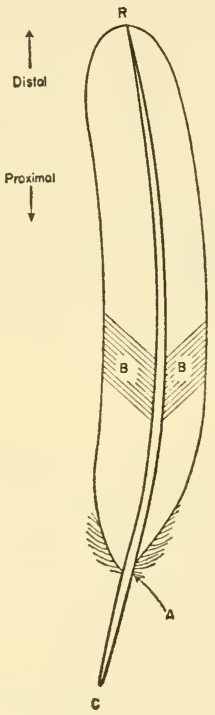

FIG. 30. The parts of a typical flight (contour) feather. CA calamus a simple hollow cylinder, AR the rhachis, and B, barbs. The barbules (not shown) lock the barbs together. X-ray patterns are usually obtained from the calamus or the rhachis.

form the rhachis. As before, the calamus (or quill) grows as a continuous cylinder and fails to split into barbs. This question is returned to in Chapter 3.

Feathers are normally shed in an annual moult and replaced. The first down or nestling feathers are replaced by juvenile feathers which resemble true contour feathers growing from the same follicle. The same follicle is thus capable of growing feathers of different kinds. 
HAIRS

Most of our information concerning the properties of keratin comes from a study of hair and wool which will thus, perforce, form the subject matter of much of the discussion to follow. The reasons for this are partly economic, funds for research being derived from the textile and cosmetic industries, and partly experimental convenience, hairs being easy to obtain in quantity, easy to purify, and their thread-like form lends itself to many physical investigations.

Hairs are as characteristic of mammals as feathers are of birds. When fully grown they consist of a tapering tip, a shaft and a base normally embedded in the skin; in cross-section, a cuticle, cortex and medulla may be distinguished (Fig. 31). A great deal of variety can be produced

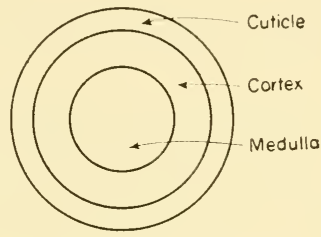

FIG. 31. The parts of a hair seen in cross-section. The proportions may vary greatly, and in fine hairs and wool the medulla may be missing.

with these simple themes, every species of mammal having its own hair style by which it may be characterized.

The diameter and shape of a hair fibre and its parts changes from tip to root. Often these features are characteristic and are employed in the identification of the hair (Lochte, 1938; Hausmann, 1925; Wildman, 1955). Hairs normally cease growing after a more or less definite period, thus achieving a genetically-determined length and, after a further period, are shed usually as the result of a new growth. As with feathers the hairs of successive generations from the same follicle may be different in character.

In what is usually taken as the primitive condition, hairs slope backwards from head to tail and are rather uniform in type and length. This simple pattern is approximated to in rodents; in other mammals great variations involving slope reversals, whorls, tufts, etc., are common. The direction of slope defines the hair-stream which is closely involved with other structures present (scales and glands) and with the organization of the permal fibrils.

As with feathers, a great variety of hairs has been evolved to meet 
peculiarities of the life of their possessors. Only certain broad features distinguishing the main types can be mentioned here. In the classification based on various sources given by Danforth (1932) hairs are primarily divided on the basis of the presence or absence of erectile tissue surrounding their follicles. In effect this division amounts to typing hairs according to their sensory role. The follicles containing erectile tissue are richly enervated (vibrissae, tactile hairs, sinus hairs, whiskers, etc., are common names) and their function is primarily sensory, the possibility of erecting them stiffly adding to their sensitivity. This could represent a more primitive function. Hair follicles without erectile tissue are usually also enervated but their role becomes more purely defensive or protective. Commonly the stiff, longer hairs (guard hairs) are distinguished from an undercoat of finer, softer, and often more curly or crimpy hair, whose function largely is that of heat insulation. Usually the coarser hairs appear ontogenetically earlier than the finer and their follicles are said to be primary; later-formed follicles are termed secondary (Fig. 35).

The much-studied human hair and sheep's wool are each exceptional cases. The long human hair often called "terminal," a variety of guard hair, is of limited distribution on the body which elsewhere is covered by a short fine hair. Wool is largely made up of fine, crimpy secondary hairs and even the primary hairs, although distinguishable, have also become fine and crimped or curly.

The hair grows from a follicle which is an invagination of the epidermis deep into the dermis (Figs. 40 and 43). Embryonically this forms immediately as a downgrowth from the epidermis and is, in this sense, in contrast with the early steps in the formation of a scale or a feather and constitutes a reason for regarding hairs as having a different phylogeny from these (see p. 73).

The fibrous properties of a hair reside in the cortex, a bundle of longitudinally-aligned closely-adhering spindle shaped $(\sim 100 \mu \times 5-7 \mu)$ keratinized cells (Lehmann, 1943, for illustrations). The keratinized residues of these cells, when liberated from the fibre by enzymatic digestion (p. 271) appear fibrous and are birefringent. They are similar, with small differences in length, in all species. Woods (1938) showed that cortical cells paralleled closely, in elastic behaviour, optical and diffraction properties, the properties of the whole fibre.

The cuticle is of a contrasted construction. It consists of thin $(\sim 1 \mu)$ sheet-like overlapping cells forming a protective sheath to the cortex. Whereas the cortex is similar in most hairs, the cuticle is highly variable and its features are much used in fibre identification. The thickness varies owing to the degree of overlapping of the cells, or scales-one to two in sheep, up to twenty or more in some fur hairs (Rudall, 1941; Stoves, 1947). The degree of overlap of free margin, and the shape 
of the free margin, affect the external appearance and are features relied on in identification. The free edges, projecting in a direction away from the skin, make the fibre feel rougher when rubbed towards the skin. This "directional friction" assists in keeping clean and in the grooming of the hairy covering and in freeing it from tangles. However, when the hair is cut from the skin the same property promotes tangling since each individual hair then tends to creep persistently in a rootward direction when the fibre mass is disturbed (Speakman and Stott, 1931). This tendency is made use of in manufacturing felts from wool and fur, but is also the cause of the shrinkage of woollens during washing.

The bulk of the keratinized feather also consists of long, spindle-shaped cells very similar to the cortical cells of hairs. The surface layers are covered with flattened, polygonal cells (Auber, 1955) perhaps analogous to the flattened, cuticular scales of hairs but, unlike the hair cuticle cells, containing within them a lattice-like network of fibrils.

The medulla is remarkably developed in the hair of certain animals, e.g. rodents, where there seems to have been a considerable pressure in the direction of producing a lighter, more bulky and stiffer hair for a given weight of material. Medullary cells are often large and their fibrous contents are concentrated peripherally against the cell wall producing a cavity largely air-filled when the hair is dry. The mechanical problem here (as with feathers too) is similar to that met with in constructional engineering: to obtain maximum stiffness for a given expenditure of material, and in fact many medullated hairs are reminiscent of girders. In such cases, the pattern is genetically determined and is often of use in identifying the hair.

In other types of hair only a feebler disposition towards medullary formation is inherited; its actual manifestation depends upon the sizes of the papilla and follicle and the nutrition of the growing hair. This is the case among sheep where it assumes some economic importance. Rudall's (1956) extensive survey of sheep follicles showed that the papillary dimensions and shape control the appearance of the medulla (p. 150).

\section{The Phylogeny of Hair}

During the early heroic days of the application of the theory of evolution to comparative anatomy, the phylogeny of so distinctive a mammalian character as hair naturally attracted much attention and several theories were advanced. That hairs are homologous at least remotely to feathers and scales is obvious enough. Whereas the likeness between the feather and the scale is close and is supported by their embryology, hairs are sufficiently different to have led paleontologists to suppose that the actual forerunner of the hair may have been some other organ of epidermal origin. Some have found the precursor in teeth, others in cutaneous 
sense organs, and others again in specialized scales. If the problem is less discussed today, this is not so much through lack of intrinsic interest as in the difficulty of obtaining further evidence which might bear upon the question. Because of its importance some aspects of these discussions will be summarized here.

The lining of the mouth is epidermal in origin and character, and the formation of teeth, like that of scales, hairs and feathers, is another example of dermoepidermal co-operation for the production of a superficial organ.

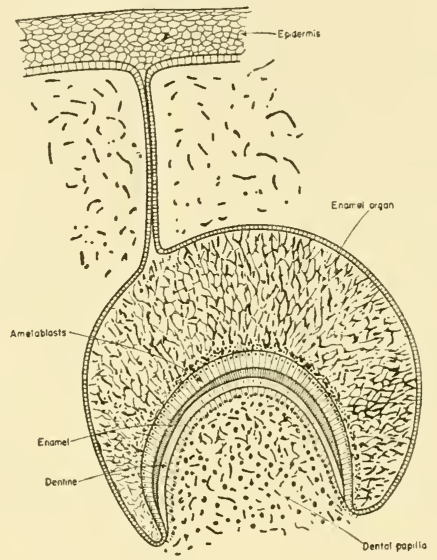

Fig. 32. Diagram of the early development of a tooth to show the dermal and epidermal contribution to its structure. The tooth consists of dentine, secreted by dermal cells of the dental papilla, capped by enamel formed by ameloblasts of the enamel organ derived from the basal layers of the epidermis. The keratinous constituent is found in the enamel. Redrawn from Hyman (1947).

The earliest sign (Fig. 32) of an impending tooth is an epidermal proliferation leading to an infolding of the epidermis, cf. the hair primordium, to form the enamel organ. Beneath the enamel organ a mesodermal papilla now forms and presses into the enamel organ to form a doublewalled cap. The cells of the enamel organ, the ameloblasts, are of epidermal origin and secrete the hard enamel which caps the tooth; the cells of the dermal papilla, or odontoblasts, secrete the dentine, the bulk of the tooth. Thus the tooth, like the hair bulb and papilla, is in origin partly epidermal and partly mesodermal. 
Teeth are certainly homologous with the placoid scales or dermal denticles of elasmobranch fish (Romer, 1955). Embryonically, the formation of these scales follows a similar course although the denticles are said to lack an enamel layer. There are thus similarities between teeth and scales which are similarly distributed as widely over the body of primitive fishes as are hairs in mammals. Brandt, in particular, was led to suppose that, by a degeneration of the dermal element (dentine) and by a concomitant increase in the epidermal contribution, a horny tooth capable of evolving into a hair could be produced. He found support for this in the existence of the genuinely epidermal keratinous teeth of the more primitive cyclostomes (see Danforth, 1932). These teeth are horny caps supported by a cartilagenous pad in an everted dermal papilla. There is nevertheless little resemblance to true teeth, but some to scales and other epidermal thickenings found in amphibians and higher types.

Another group of comparative morphologists has drawn attention to the similarity in early embryogenesis between the hair primordia and those of certain sense organs in fishes and amphibia and has suggested that hairs have developed from these sense organs. A variant of this theory would have that hairs descended from certain tactile spots on the scales of reptiles.

The relationship between hair groups and scales pointed out many years ago by De Meijere (see Noback, 1951) is suggestive in this connexion. Hairs are never distributed uniformly or randomly over the skin; there are regional variations in density and hair-type which are as much a genetically determined morphological feature as any other aspects of anatomy. Even in the hair-bearing areas, the hairs are not randomly distributed, but are arranged in small, well-defined groups (Figs. 33 and 35). De Meijere noted that the basic group seemed to be three hairs with the larger in the centre. When scales were also present (Fig. 34) the hairs emerged from the underside of the scales. This concept of the "basic trio group " as a morphogenetic unit has been accepted as a working hypothesis by most recent workers (Carter, 1943; Carter and Clarke, 1957).

Studies of the course of embryonic appearance of hairs has partly confirmed these views by showing that the first follicles to form (primary follicles) are the central follicles of the trio group and that subsequent follicles differentiate laterally to these to complete the group. The group is not rigidly defined in numbers; some primaries remain solitary, others have only a single lateral. Later other follicles, the secondaries, may develop. The successive generations of follicles are related to the existence in most hairy coats of the two distinct kinds of hairs: a coarser, longer over-hair (guard hair) and a finer (often more woolly) under-hair. The earlier developing follicles produce the over-hair and the later the fine 
undercoat. Many of the differences between different furs and fleeces is to be found in the relative development of the over- and under-hair.

When scales and hairs occur together, as on the tails of rodents, the hair group develops in relation to the scale as mentioned above. The fact that, when scales are absent, the hairs still form in groups suggests that the ancestors of existing mammals may have had a scale associated with each trio group and that in the course of evolution the scale has been lost, leaving the hair group to mark the site. The argument is persuasive,

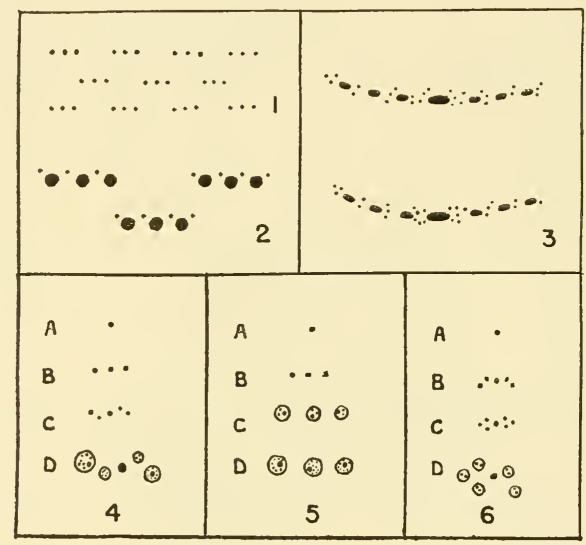

FIG. 33. Various arrangements of hair follicles illustrating the formation of " trio groups" with the suggestion of a relation to an ancestral scale distribution. Reproduced from De Meijere through Noback (1951).

but, when the origin of the hair-scale pattern itself is considered, we are forced further into hypothesis. Two views have been advanced: (1) The early mammals (or pro-mammals) may have been covered with fine scales which diverged into two types, one of which continued to resemble the reptilian form and the other became reduced in size and was converted into hair. It may be significant here that the guard hairs of the primitive platypus terminate in a flat spade resembling a scale. The follicle here at first produces the spade and then turns over to producing a typical hair shaft (Wildman and Hanby, 1938). (2) The hair precursors may have been sense organs on the scales and later moved off into the softer interscale regions where they became true hairs and commenced an independent evolution. Alternatively the scale failed to appear, leaving the hair. 
It would seem that a better knowledge of the factors influencing the relative rates of proliferation of dermal and epidermal elements when these are co-operating to form an organ is necessary. The recent developments in the culture of hairs and feathers in vitro gives promise that this may be obtained.

In the course of their development all these special structures are necessarily related and more light is thrown on their relations by embryology than by an examination of the mature structures and their arrangement. Fleischhauer (1953) has shown, for instance, that regional hair-

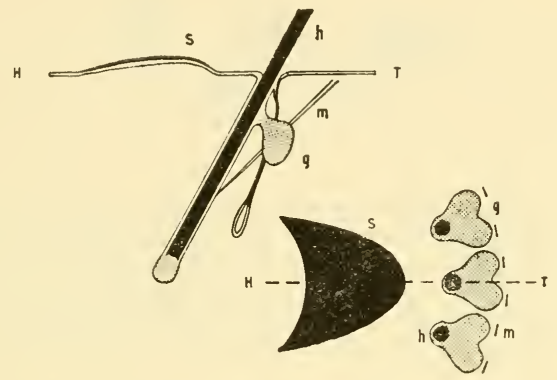

FIG. 34. The hypothetical scale-hair-gland complex. H-T the head-tail line, S, scale; h, hair; g, gland; $m$, muscle. On the lower right-hand side a view looking down on the unit. A further element not shown in this diagram is the " hair disk" found in some cases behind the hair according to Pinkus (1905).

streams can be detected before the hair germs appear. From spreads of foetal human skin he concluded that the first hairs develop at roughly fixed distances from each other in a quasi-hexagonal arrangement. New anlagen appear between these when a critical distance is reached owing to growth. This is a pattern which might be expected theoretically if we regard these primordia as successful centres of proliferation which are able to repress like developments in their immediate neighbourhood. The two lateral hairs completing the "trio group " next appear in a line at right angles to the main body axis. The three hairs already slope backwards. The explanation usually offered for trio formation (above) is that the hairs develop as though they were growing out from under the free edge of a scale as indeed they do on the tails of rodents. There is much to suggest in these facts that the scale, the hair group and its associated glands together form a unit (Fig. 34) which in most mammals has degenerated to the hair group and glands, the "pilosebaceous unit" 
of Montagna. The "scale-hair-gland" unit is more general and plausibly explains some aspects of the mammalian hair pattern, if we suppose that the ontologically-earlier preparations are directed towards the formation of the entire unit and that later the separate single components come to develop to different degrees leading at last to the non-

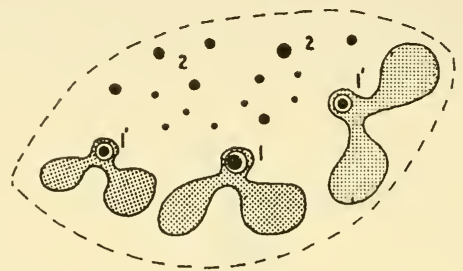

FIG. 35. The basic trio group $\left(11^{\prime} 1^{\prime}\right)$ of primary hairs associated with bilobed sebaceous glands (cf. Fig. 34) and the secondary hairs (22) which appear later arising either de novo or from out-pocketing of the outer root sheaths of the original primaries (Hardy and Lyne, 1956). The entire cluster is marked off from its neighbours by bundles of collagen fibres in the dermis. Redrawn from Carter (1943).

appearance of the scale. Hairs retain a clear sensory function as an accessory mechanical lever for the stimulation of the nerve endings associated with them.

\section{Other possibly keratinized structures}

A small amount ( $\sim 2$ per cent by weight) of a resistant, sulphurcontaining protein, usually referred to as a keratin, is found in the enamel of teeth where it may contribute towards the bonding of this highly crystalline, inorganic material (Scott, 1955). The enamel layer of a tooth is a secretion of modified epidermal cells and thus any keratin it contains would be a secreted protein. The formation of a tooth has already been discussed on p. 74 and its components (partly dermal, partly epidermal) may be seen in Fig. 32 which shows a section of a developing tooth.

The cystine content and basic amino acids of the organic matrix of enamel have been determined (Battistone and Burnett, 1956; Hess, 1953; and Block et al., 1949). The cystine is low for a keratin $(0 \cdot 2$ per cent $)$ and the ratios of the basic amino acids show it to be a pseudokeratin as defined by Block (see p. 31). Hydroxyproline, usually associated with collagen, is reported by Hess et al. (1953) and by Battistone and Burnett (1956). The $\mathrm{X}$-ray pattern is suggestive of keratin rather than of collagen (Pautard, 1961). 
The "neurokeratin," a material remaining when nervous tissue is exhaustively extracted, analysed by Block and found to contain cystine, now appears to be simply a resistant product of decomposition. Histochemists continue to describe a definite, cystine-containing " neurokeratin network."

Keratin-like materials in extracellular situations are found in the linings of the gizzards of gallinaceous birds (see pp. 30 and 107) and in egg-shell membranes. 


\section{Differentiation and Protein Synthesis}

IN THIS chapter we shall discuss some general properties of epidermal cells, such as differentiation and protein synthesis, which they share in common with many other cells of the organism. Their more specialized aspects relating to keratinization will be considered in a later chapter.

\section{The cytology of keratinizing cells}

A brief account of the histology of the epidermis and its derivatives has already been given. For further details reference may be made to the many standard texts (Maximov and Bloom, 1948; Horstmann, 1957; Biedermann, 1926 and 1930; and Cowdry, 1932). 'The description which follows is designed to draw attention to those features at the fine histological and macromolecular level which are particularly relevant to the main themes to be discussed in later sections, or which, as a result of recent electron microscopy, seem to require a redescription in terms somewhat different from those found in the classical works. The cytology of the generalized basal layer cells and the fine structure of the dermoepidermal junction will be dealt with first. Then an account of the more specialized structures of the hair and feather follicles will be given and compared with that of the epidermis.

\section{The Basal Layer Cells*}

The intracellular equipment of the germinal cells in the basal layer is similar wherever these are found, and consists of those elements which are recognized as common necessities for the functioning of all types of cell (p. 34) except those of bacteria and related small forms. The nucleus is large and one or more nucleoli containing dense particles of the order of $120-200 \AA$ in diameter, may be found (Fig. 36 and Plates $4 \mathrm{~B}$ and C, $7,9,10,11$ and 12). The nuclear contents which, except when the cell is dividing, are diffuse and granular at all magnifications (as seen in electron micrographs of thin sections), are strongly basic and stain positively with the Feulgen technique for demonstrating desoxyribonucleic acid (DNA) (Pearse, 1953). The nucleolus is Feulgen-negative, but gives positive tests for ribonucleic acid (Montagna, 1956). The nuclear membrane consists of

* Also called Malpighian cells. 
two distinct sheets and is covered by small circular markings (Plate 4c). Mitochondria are small and not particularly common in the cytoplasm (Fig. 9). They posses a double-layered outer membrane enclosing an inner chamber penetrated by what seem to be invaginations of the inner membrane. According to Montagna (1956), mitochondria are often difficult to see in the light microscope, but no problem arises in observing them electron-microscopically. Numerous small vacuoles, often in clusters, which may be identified with the Golgi apparatus (pp. 46-47) are also visible in electron micrographs (Fig. 22e).

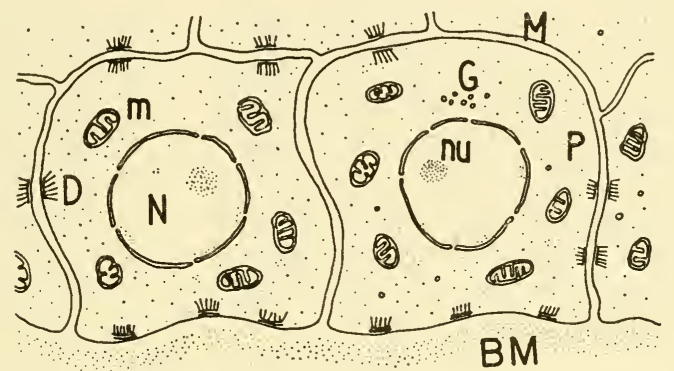

FIG. 36. The cytology of basal layer cells. BM, basal membrane; $\mathrm{N}$, nucleus; $\mathrm{nu}$, nucleolus; $\mathrm{m}$, mitochondrion; $\mathrm{M}$, cell membrane; D, desmosome; $\mathrm{P}$, dense RNP particles.

The cytoplasm of the basal-layer cells is strongly basophilic and rich in ribonucleic acid (Montagna, 1956 and Hardy, 1952). Electronmicroscopically the most striking feature is the presence of large numbers of small (150-200 $\AA$ diameter) dense particles ( $P$ in Plates 7 and 11). In this respect these cells resemble many other kinds of rapidly-growing cells: early embryonic cells in general, tumour cells, etc. Palade (1955) has marshalled the evidence to show that the dense particles are in fact the images of a ribonucleic acid-protein molecule (RNP). This matter is discussed in detail on p. 108 et seq.

The cells rest on and are attached to a basement membrane ( $B M$ in Plates 7, 9 and 14B) the detailed structure of which is considered below. Partly as a result of this attachment on one face and of their close-packed condition, the germinal cells assume a columnar form in some situations. The basement membrane itself is supported by a dense feltwork of collagen fibrils. The follicles of growing hairs and feathers penetrate below the general level of the base of the epidermis and become enclosed in a basketlike network of circular and longitudinal fibrils, which, however, do not 
penetrate within the papillae of the follicles where the epidermal and mesenchymal cells are separated by the single basement membrane. The meshwork of collagen constitutes the "glassy membrane" visible in the light microscope (Horstmann, 1957 and Montagna, 1956).

The one-sided attachment of the cells to the basal membrane also establishes an intracellular polarity which is revealed by the often asymmetrical arrangement of the cell contents. The small cluster of vesicles (Fig. 36, p. 81) referred to as the Golgi complex, tends to lie distal to the nucleus and mitochondria $m$ may be more common nearer the basal membrane.

The germinal cells, by their persistent cell division, maintain the population of keratinizing cells. In this sense they conserve an embryonic character which is emphasized by the generalized nature of their cell contents. The cytoplasm of embryonic cells contains mitochondria, many clusters of smooth-surfaced $\gamma$-cytomembranes (p. 46), vacuoles containing phospholipid and vast numbers of the small dense RNP particles. The more specialized structures, such as the $\alpha$-cytomembranes of secretory cells and the specialized inclusions, which later distinguish differentiated cells, are rare. The similarity of this cytology to that of the germinal cells of the skin is obvious. The surface of attachment of these latter cells is, however, a specialized feature distinguishing them from the earliest embryonic cells.

There are various opinions about the detailed course of the process whereby the basal layer both maintains itself and supplies cells to form the differentiated layers. A common view is that there is some asymmetry in the division of a basal cell in the sense that two unlike cells result; one, referred to as a "stem cell," remaining attached and preserving a generalized character, the other free to move up and enter the stream of differentiating cells. This cell may also be capable of further divisions. Mitoses are to be seen among the matrix cells some distance from the basal layer in the hair follicle and it has been maintained that dividing cells are also to be seen well above the basal layer in the epidermis (Thuringer, 1924). Most observers agree now that nearly all dividing cells in epidermis are found in the basal layers and critical opinion (Hanson, 1947 and Leblond, 1951) holds that the earlier observations were unreliable on the grounds that it is easy to be mistaken when examining oblique sections. It seems more likely that the widely accepted view, that cell division and cell differentiation are mutually exclusive, applies to the epidermis. Cell division appears to cease in cells in which cytoplasmic fibrils have commenced to accumulate, which, on the face of it, means that the cells' synthetic activities have swung over from producing materials needed for division to producing keratin precursors. The factors controlling mitosis are discussed in the next chapter. 

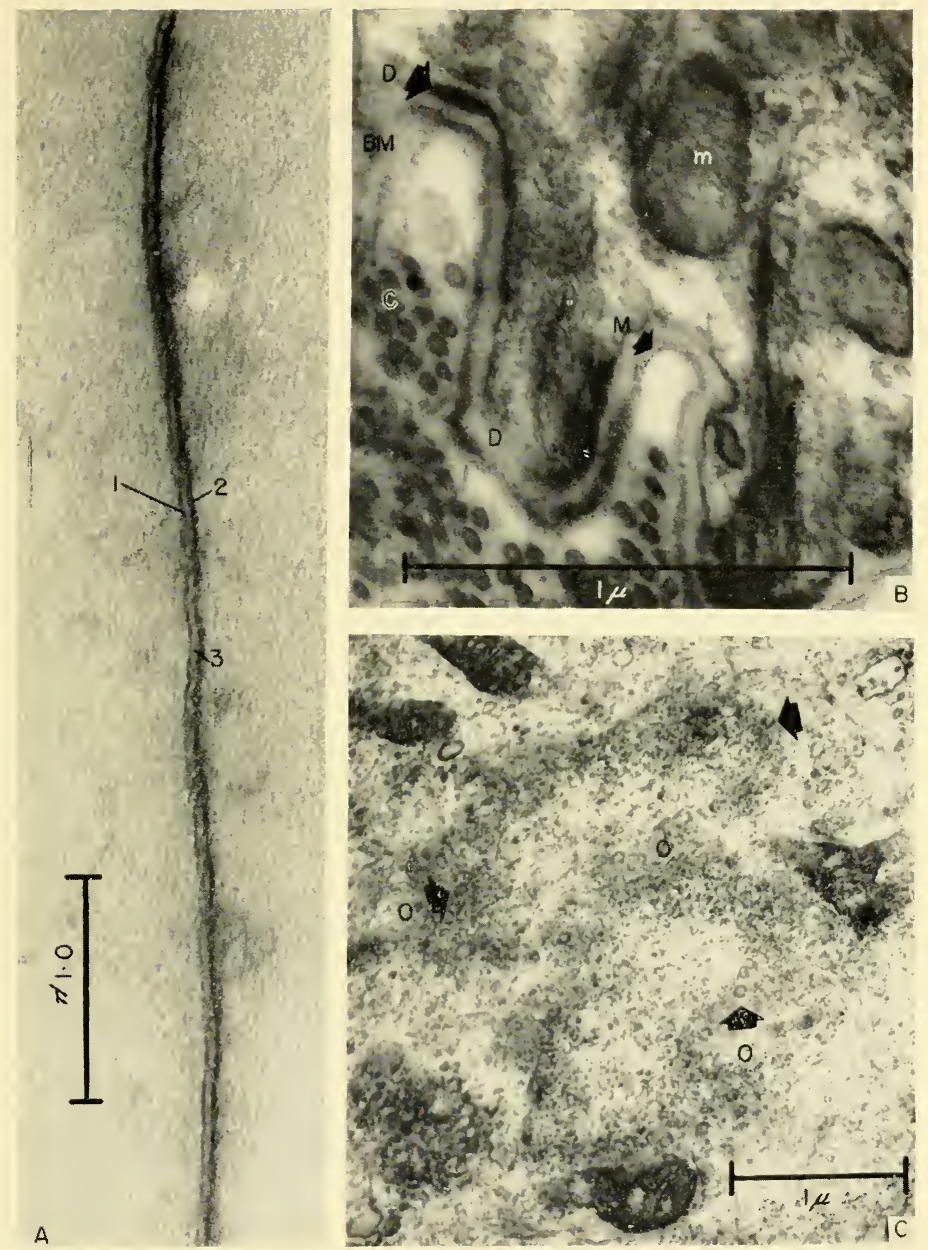

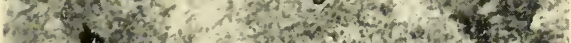

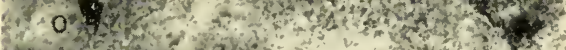

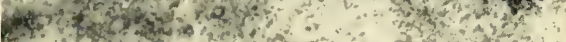

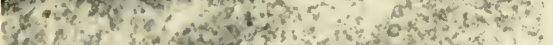

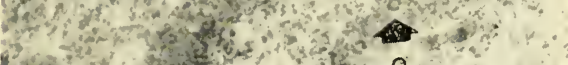

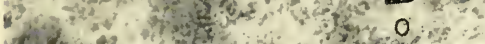

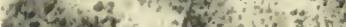

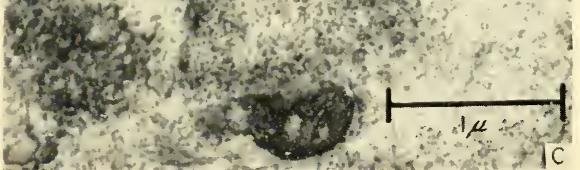

Pl.ATe 4 (Captions overleaf)

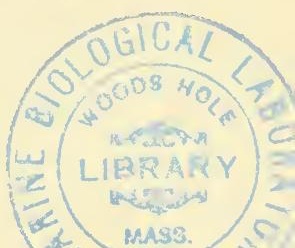




\section{Plate 4}

A. Example of a plasma membrane at high resolution showing the ultrastructure of this membrane. Material: Amoeba proteus, $\mathrm{OsO}_{4}$ fixation. Two dense lines $(\sim 20 \AA$ wide $)$ are seen enclosing a third less-dense layer $(\sim 20 \AA$ ) to form a sandwich-like surface about $60-70 \AA$ thick. For interpretations see Fig. 18, p. 38.

B. The basement membrane as seen in the plantar skin of the rat. PTA stain. $M$, basement membrane; $D$, desmosome; $f$, tuft of desmosomal fibrils (tonofibrils); $C$, cross-sections of dermal collagen fibrils; $m$, mitochondrion.

C. Section passing through the surface of a nucleus in an cpidermal cell of the 12-day-old chick embryo to reveal the "pores," the small circular markings seen at O. PTA stain. 


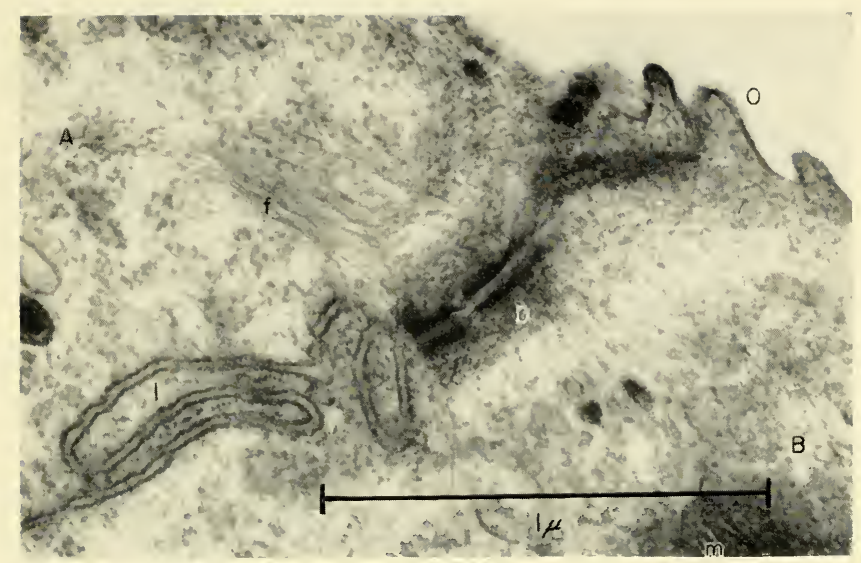

A

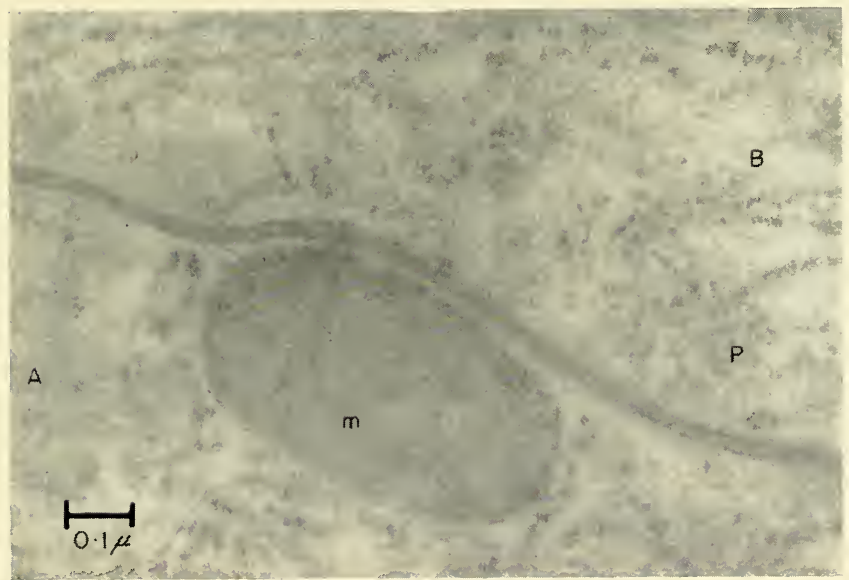

B

Plate 5 (Captions overleaf) 


\section{Plate 5}

A. Examples of the surface of contact between cells. Figure from two ectodermal cells $A$ and $B$, in the surface of a 12-day-old chick embryo. $O$, outer surface; $D$, desmosome; $I$, interdigitated membranes. Note the two dense, parallel plasma membranes and the lighter deposit between them revealing the intercellular cement. $f$ is a tuft of fine fibrils (prekeratin) attached to the desmosome plates. The cytoplasm contains fine dense particles but no particle-covered membranes. Stain: PTA.

B. Another example of an intercellular contact between two rat pancreas cells $A$ and $B$. Again the two contacting membranes appear as dense, parallel lines separated by a dark material, the intercellular cement. $m$ is a mitochondrion, and $P$ are RNP granules attached to the system of cytoplasmic membranes of these granular secreting cells. Stain: lead hydroxide.

Micrograph kindly supplied by M. S. C. Birbeck. 


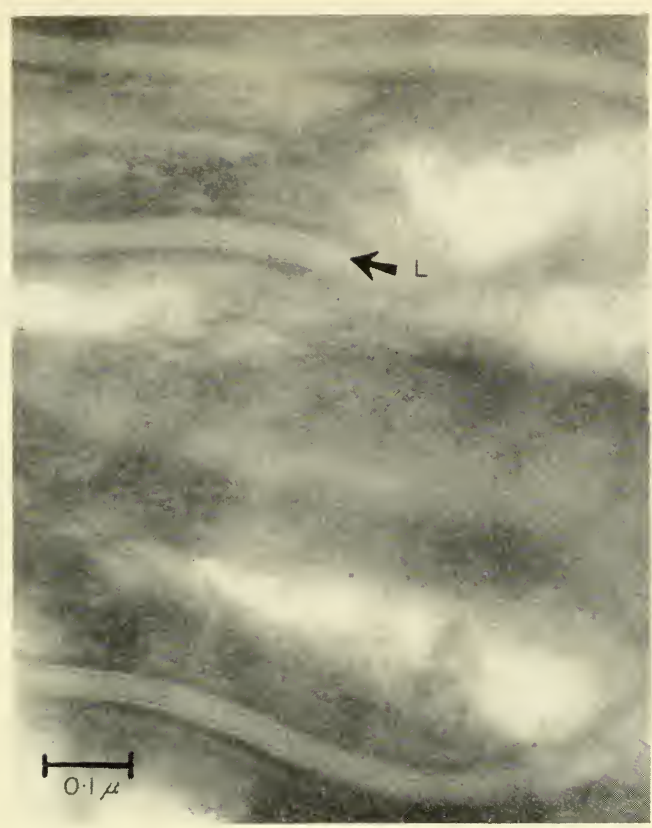

A

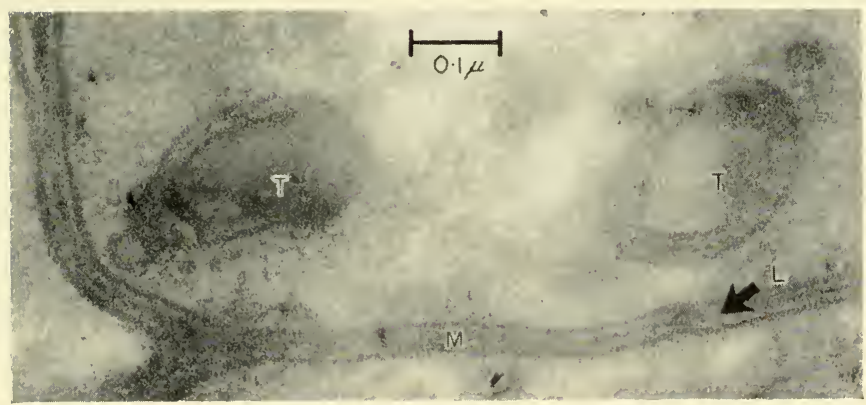

B

Plate 6

A. The convoluted cell contacts in rat skin at high resolution to show the multiple layers $L$ deposited between the dense cell membranes or desmosomes.

B. A further example of a complex membrane development in the hardened cells of the Henle layer of the human hair. Between the dense plasma membranes $M$ is a single dense layer $L$. At $T$ are to be seen sections of two "ball and socket" joints formed by tongues of one cell penetrating into the neighbouring cell. 


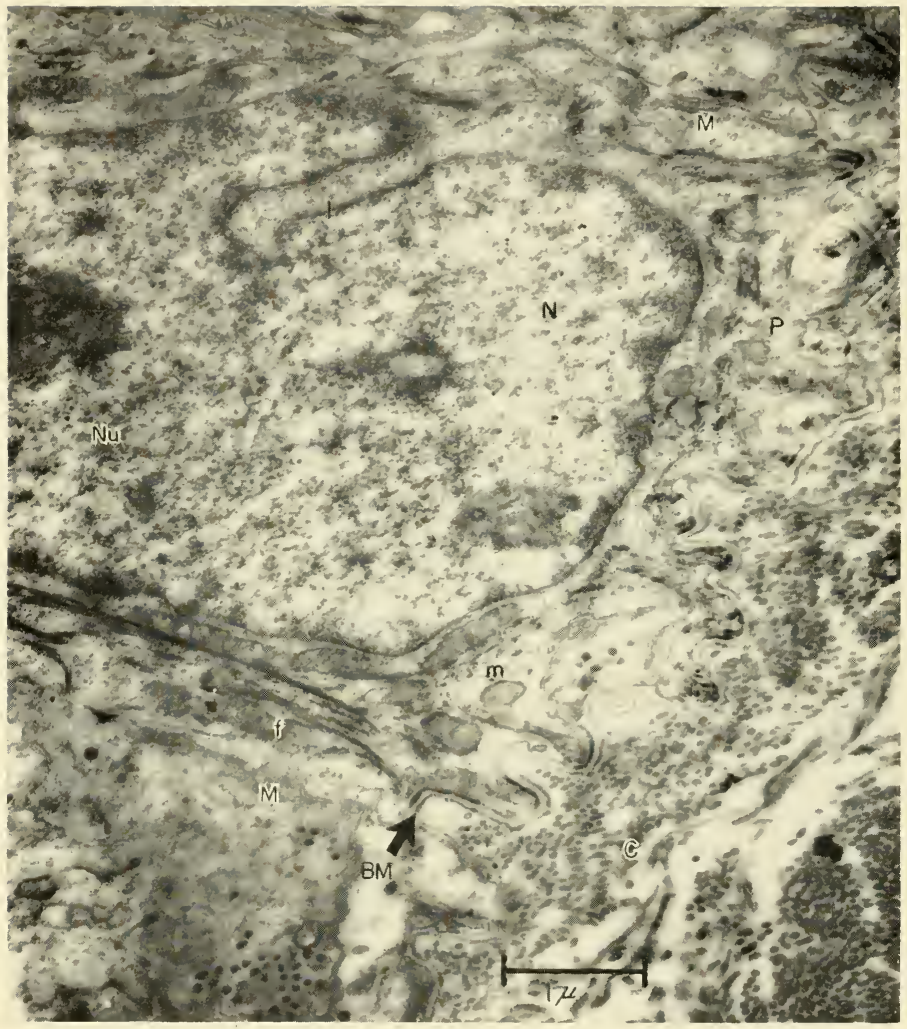

\section{Plate 7}

Portion of the basal layer of rat plantar epidermis and of the basal membrane. $N$, large nucleus of basal layer cell; $N u$, nucleolus; $B M$, continuous basal membrane; $C$, bundles of dermal collagen fibrils beneath the basal membrane; $M$, cell membranes; P, RNP particles and $\mathrm{G}$, Golgi cluster. The curious invagination of the nuclear membrane is common in basal layer cells. 


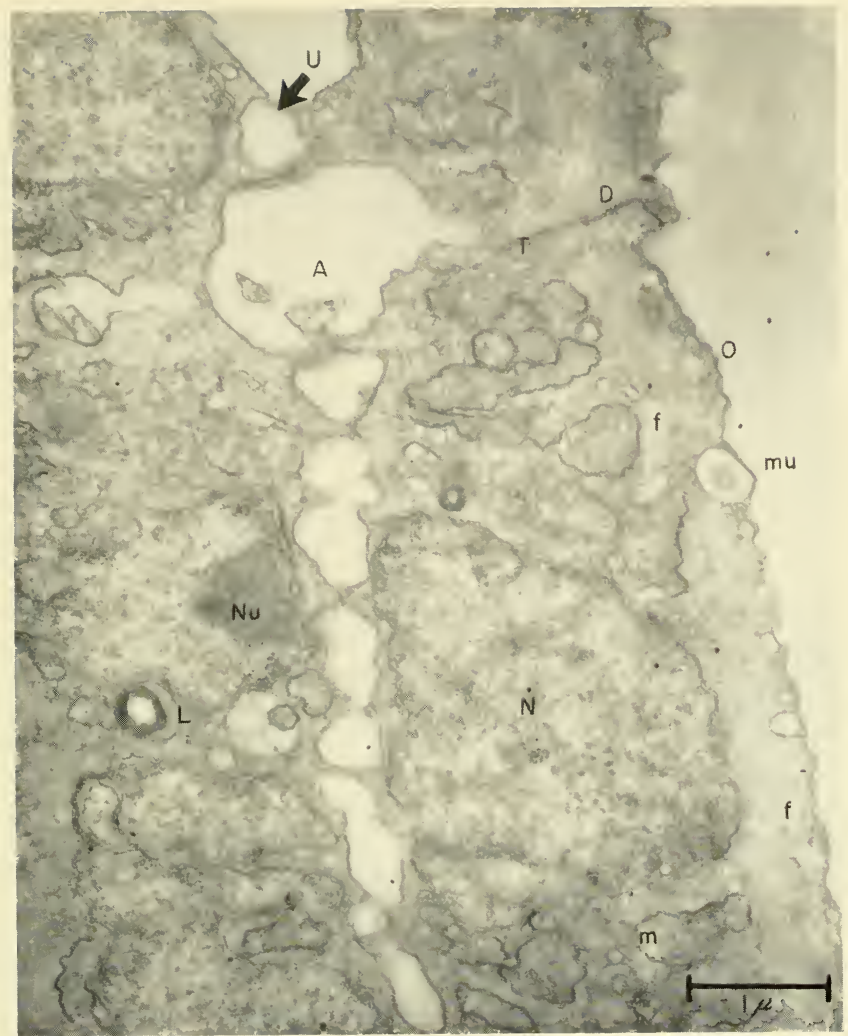

\section{Plate 8}

Xenopus tadpole tail epithelium. Near the end of the tail there are only two layers of cells present and the mesodermal cavity has degenerated to the long gap $A$. No basement membrane lines the inner surfaces of the cells in this site and at this time. $N u$, nucleoleus; $N$, nucleus; $m$, mitochondrion; $L$, phospholipid granule showing concentric whorls; $M u$, mucin pocket on the outer surface $O$ of the cells; $f$, cytoplasmic fibrils underlying the outer surface membrane. The inner surfaces of the cells are poorly adhesive and pseudopods such as $U$ project from the unstabilized inner membrane; at $T$ the outer segment of the surface cells is seen to be forming a close "adhesive contact" and incipient desmosomes $D$ have appeared (see p. 90). 


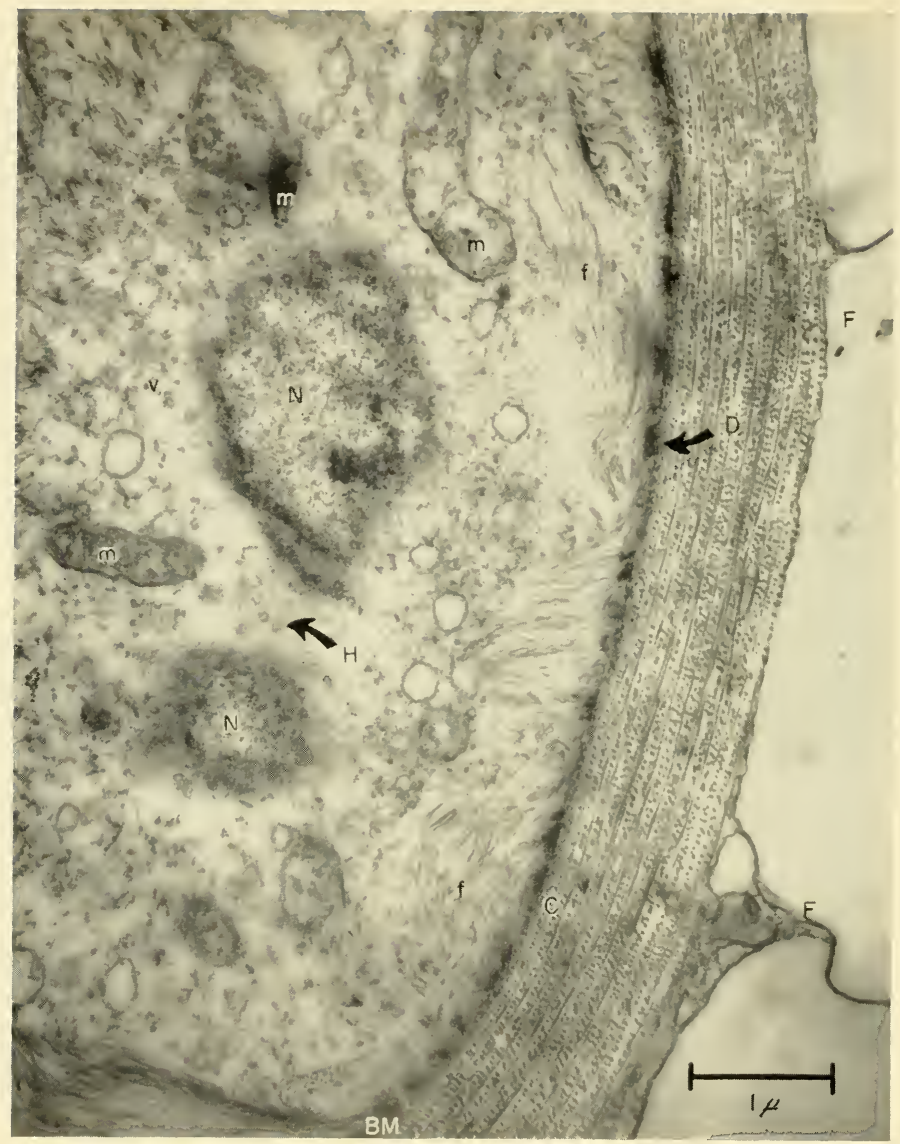

Plate 9

Portion of a basal layer cell of the epidermis of the tail of a frog tadpole (Rana temporaria). Fixation: osmium tetroxide, stain: PTA. $B M$, basal membrane; $D$, desmosomes; $C$, mesh-work of collagen fibrils which support the basal membrane; $F$, base of long process of dermal fibroblast, which applies to and spreads over the surface of the collagen meshwork presumably secreting further fibrils; $M$, mitochondrion; $N$, nucleus; $H$, nuclear " pores"; $v$, small vesicles possibly arising from the pores; and $f$, fibrils of cytoplasmic protein precursors of keratin. 
A further feature of the germinal cells, which is again indicative of their unspecialized nature, is the behaviour of their plasma membranes. These are seen (electron-microscopically in sections) as greatly-convoluted dense lines which may often be separated by gaps of a variable width into which may be thrust small surface protrusions. The pattern suggests that the adhesion of the membranes to each other is not as strong as it later becomes (p. 84).

In the epidermis, but less so in the basal layers of the hard keratins, the cell surfaces are also studded with desmosomes (p. 41). T'he portion of the cell surface facing the basal membrane is covered even more extensively with dense, desmosomal-like deposits (Plate 14B). Between these the plasma membrane appears more free and often small invaginations are to be seen ("blebs") which may well be associated with the entrance of liquid since all the metabolites required by the epidermis appear to enter through this layer (Pillai et al., 1960; Fasske et al., 1959).

Desmosomes have been described earlier (pp. 41-43) but, owing to their importance in epidermal tissues, some further comment is required. As observed electron-microscopically in sections, well-developed examples appear as a pair of very dense, often planar (straight lines in sections) deposits distributed over the cell surfaces. Examples may be seen in Figs. 21, 36 and Plates $12 \mathrm{C}$ and $14 \mathrm{~B}$; their structure is shown diagrammatically in Fig. 21. They may be developed to varying degrees ranging from a mere increase in the density of material immediately adjacent to the cell membranes to large intracellular deposits in which may be embedded tufts of filaments running into the cytoplasm (Plate 6) and associated intercellular (extracellular) deposits. The two halves of a desmosome are usually similar in degree of development. Since they occur in situations where the transmission of mechanical tension from cell to cell seems a reasonable supposition, most authorities think their main purpose is to supplement cell adhesion and to form attachment for fibrils, i.e. the desmosomes permit of an enhanced adhesion, the intracellular deposits providing a sort of supporting backing for the fibrils transmitting tension to other surfaces (p. 95).

The dense deposits on the membrane of the layer of cells facing the dermis are in a sense " half-desmosomes " since, in the absence of a second cell, one half is lacking. Nevertheless here too they seem to increase the adhesion of the cell to the basal membrane and thus to the collagenous meshwork beneath (Fawcett, 1958; Weiss, 1959).

Epidermal desmosomes are visible as small dots (diameter $\sim 1 \mu$ ) in the light microscope. Thus the "desmosome" of the light microscopist would include several distinct elements: the two cell membranes, the dense intracellular deposits backing the membranes, the material between the membranes and the terminations of fibrils in the dense bodies. They are 
therefore not in any sense homogenous and histochemical tests merely inform us of the presence of certain types of material without precisely indicating its location. Wislocki (1951) demonstrated phospholipids and Leblond (1951) describes a positive periodic-Schiff reaction indicating polysaccharides. Protein is undoubtedly present. Possibly the polysaccharide is located intercellularly as the adhesive "cement" and the phospholipid in the thickened cell membranes themselves (see also p. 94). By digesting skin with various enzymes before fixation, Weiss (1958) was able to " dissect" these basal membrane desmosomes. After pancreatic lipase the dense surfaces lost their osmophilia indicating a high lipid content. Amylase also freed the epidermal cell suggesting a polysaccharide constituent.

\section{Cell contacts during differentiation}

The relation of the plasma membranes of cells in close contact in the epithelial type of tissue of ecto- and endodermal origin has been described in Chapter 1 (p. 40). Typically the two dense plasma membranes run closely parallel to each other and are separated by a less-dense layer of material usually of the order of $200 \AA$ thick. We shall refer to such a contact as adhesive. Its strength may, of course, be supplemented by the specialized organs of attachment, the desmosomes. In early embryonic tissues generally, in many tissues of mesenchymal origin and in the germinal layers of constantly proliferating tissues (epidermis, intestinal mucosa, etc.), the adhesive type of contact is less extensively found. In these tissues the cell surfaces are more convoluted, wider and less regular intermembraneous spacings are to be seen (Fig. 37) and the cells seem able to force small protrusions (microvilli, tubular pseudopods) into the intercellular space. The poor adhesion is shown by the ease with which embryonic material may be dispersed into single cells (Weiss, 1958; Moscona, 1952, 1956 and 1957).

Thus it appears that during the progression from embryo to adult there is, in certain organs, an enhancement of intercellular adhesion. A change of this nature could have several consequences which may be factors in influencing the maturation of the tissues taking place in this period. We have given reasons (p. 44) for regarding differentiation as a phenomenon arising from the action of one cell upon another, or in other words, of intercellular communication. This communication must be effected either by the transfer of samples of cell product from one cell to another, or by an effect produced when cells come into contact. In either case a change in the nature and activity of the cell membranes will influence communication. The further possibility that intercellular adhesion can also play an active role in moulding the shape of cells and guiding their movements is suggested by the work of Holfreter (1947 and 1948), Weiss (1958), 
Abercrombie (1947 and 1948) and Moscona (loc. cit.) among many others (De Haan, 1958). That some deterioration in cell adhesion is associated with cancer has been advocated particularly by Coman (1954).

Certain essential notions concerning the activity of cell surfaces and their behaviour when they are brought into contact are best derived from time lapse films of living cells, such as those made by Weiss, and by Abercrombie and Ambrose. From such observations one gains the impression that many isolated cells behave essentially like unicellular protozoa of the amoeboid type. Their surfaces are constantly thrusting

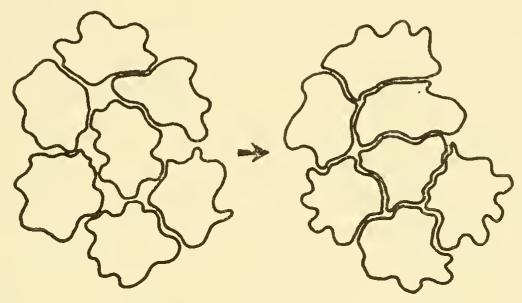

Fig. 37. The increase in intimacy of cell contact in passing from an embryonic to an adult condition. A similar change occurs in the differertiation of epidermal tissues.

forth protrusions which bring about the movement of the entire cell. When like cells are brought into contact the motion of the surface ceases at the point of contact, a phenomenon called contact inhibition by Abercrombie (Abercrombie and Heaysman, 1953 and 1954), and the area of contact may spread zipper-like further immobilizing the cells. On the other hand, unlike cells on meeting do not inhibit each other's movements. Thus, as is shown most clearly in the experiments by Moscona and Weiss, in a mixture of like and unlike cells, the like cells, as a result of their specific adhesion following random contact, will sort each other out (Fig. 37). Under other circumstances, a zipper-like spread of contacts may actually mould the cell formation into columnal epithelium (Fig. 44) or an intricate interdigitated condition as seen in the notocord (Waddington, 1956). These specific intercellular adhesions seem to be effected by a sticky exudate covering the cell surfaces which we have referred to as an intercellular cement.

Little is known of the chemical nature of these cements and, further, little concerning the structural devices involved can be learned from light microscopy since the intercellular spacing is of the order of $200 \AA$, far below the resolving power of the light microscope. However, electron micrographs of thin sections have established the existence of the thin 
plasma membranes and of the less-dense layer of rather constant width which separates them and which must represent in life the site of the postulated intercellular cement. Evidently the intrinsic density (electronscattering power) of this material is low and, further, it does not react with any of the common fixatives (osmium tetroxide permanganate and formaldehyde) to produce a denser reaction product. After a treatment of the fixed material with phosphotungstic acid and/or lead hydroxide (Birbeck, 1959), it becomes visible (Plate 5). These findings merely suggest that the intercellular layer (or exudate) is present in low absolute concentration, and that it consists of chemical substances of an unreactive character. Among the various suggestions compatible with these rather negative requirements, is that it consists predominantly of a polysaccharide, probably with a protein moiety (mucopolysaccharide) responsible for the specificity. We may suppose that during the early stages of embryogenesis it is secreted by or shed from the cell surfaces, each cell type producing its own specific layer. See also the remarks made above concerning the composition of the more specialized desmosome p. 84 .

Since the epidermal cells undergo rapid differentiation when they leave the germinal layer, we are able to find in the stream of cells (Fig. 42), taking its origin in this layer and ending in the fully-keratinized layers, the whole sequence of changes preserved at one time in the correct sequence. It is this circumstance which further recommends the use of epidermal tissues for the study of differentiation. The small volume in which the changes occur makes the material ideal for electron microscopic study which alone permits a visualization of the cell membranes themselves. The results of the electron microscopic study of the developing epidermis are given in the next two sections and of the developing hair follicle on p. 95 .

\section{The Dermoepidermal Junction}

The basal membrane is a structural feature which seems essential to the establishment of an epithelium, for without it there seems no reason why the intercellular adhesion postulated above should produce an orderly, layered structure rather than a ball of interdigitating cells. Its structure and formation therefore require special consideration.

Owing to the difficulty of resolving the fine details of the structure of the dermoepidermal boundary, its nature has been much in dispute. More recently, electron micrographs of a sufficient variety of tissues drawn from amphibian, avian and mammalian sources have clarified the issue and show that essentially the same structure is present in all these classes of organisms (Weiss and Ferris, 1954; Porter, 1956; Jackson, 1954; Selby, 1955; and Mercer, 1958). Proceeding from within a basal-layer cell and moving towards the dermis (Fig. 39), we encounter firstly the plasma 


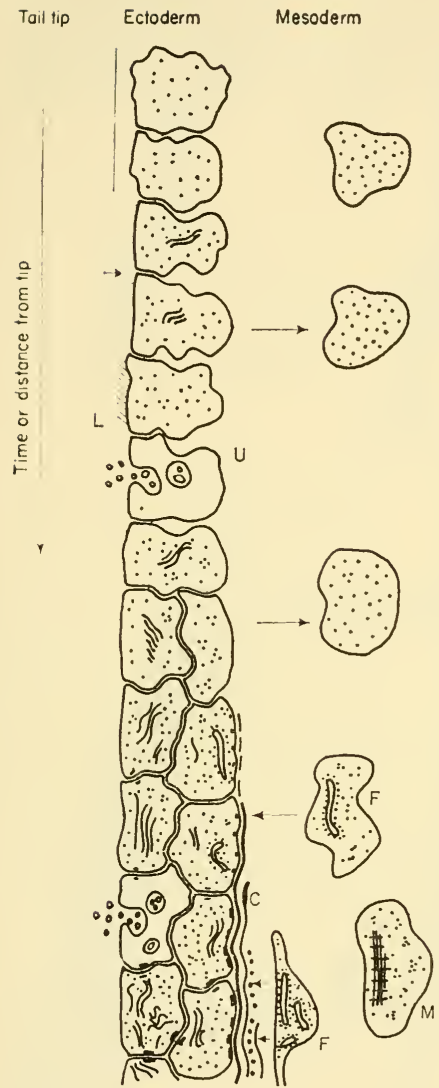

Stage 1. Ectodermal cells showing very convoluted surfaces indicative of poor and impermanent adhesion. No adhesion to or between mesodermal cells.

Stage 2. Formation of close contact between those portions of the cell membranes near to the outer surface. Further in from the surface wide gaps and convoluted surfaces persist. Traces of intracellular fibrils.

Stage 3. Further differentiation of surface cells. Appearance of (1) mucin cells, (2) ciliated cells and (3) definite deposits of intracellular fibrils.

Stage 4. Epidermis increases in thickness and basement membrane appears. Cells in basal layer contain both filaments and endoplasmic reticulum.

Stage 5. Appearance of first collagen fibrils beneath basement membrane. Mesodermal cells increase amounts of organized reticulum.

Stage 6. Rapid build-up of collagen beneath basal membrane, increased synthesis of intracellular fibrils, fading of reticulum in surface cells. Mesodermal cells recognizable as fibroblasts $\mathrm{F}$ (much rough surfaced reticulum) and muscle cells (little rough surfaced reticulum and muscle fibres $M$ ).

Fig. 38. Shows the succession of cell types noted on passing from the extreme tip of a tadpole tail (Xenopus and Rana) towards the head. The succession also represents stages in differentation from a poorly-differentiated condition at the tip to a well-defined state of differentiation further headwards and may thus be regarded as a time sequence also. Six stages are recognizable and are indicated (1)-(6) on the 1.h.s. See Plate 8. 
membrane of the cell about $70 \AA$ thick, the fine structure of which was discussed above (p. 37); next we enter a lighter space (150-200 $\AA$ wide) which is continuous with the lighter space surrounding and separating the cells of the overlying epidermis and is, presumably, of a similar nature. Beneath this layer again we encounter another more dense, diffuse layer
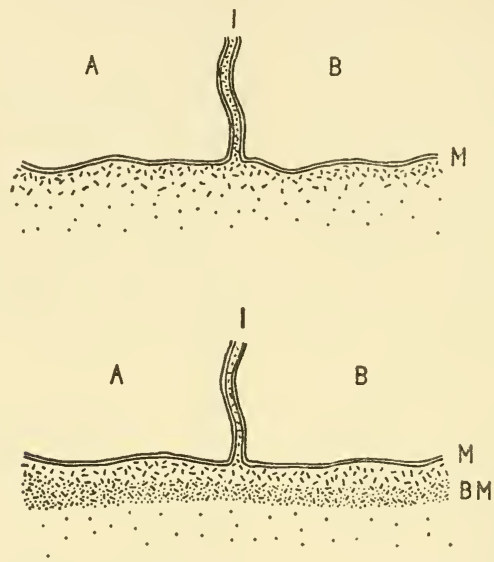

Fig. 39. The fine structure of the basal membrane and an hypothesis to explain its formation.

M represents the cell membrane of two contacting ectodermal (epidermal) cells A and B. Their surfaces are covered with a sheet of intercellular cement which functions as an adhesive (I). On the surfaces facing the mesodermal space a diffuse, denser and thicker membrane (BM) appears which defines the boundary between epidermis and dermis and is named the basal membrane.

It is supposed that this membrane results from an interaction between the fixed ectodermal exudate forming the intercellular cement (I) and a more diffusible component emanating from the mesodermal cells.

which is everywhere continuous (surrounding hair follicles and other epidermal irregularities) and represents a quite definite morphological surface separating the dermis and epidermis. We have reserved for this particular layer the name basal membrane (BM, Fig. 39).

Membranes of this special character, to judge from the limited electronmicroscopic data yet available (Policard and Collett, 1959), appear to form whenever two populations of cells which have followed sufficiently different embryonic pathways, and become differently differentiated, are brought 

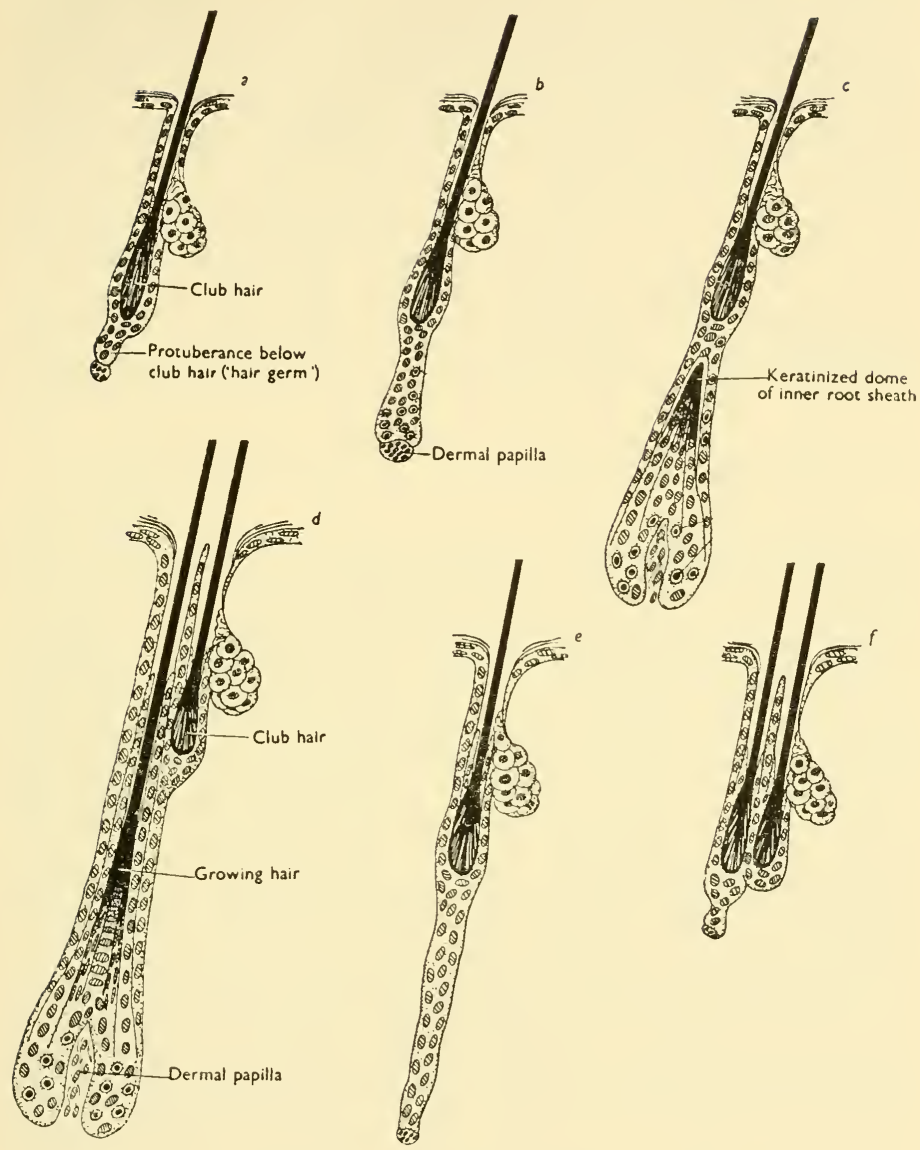

Fig. 40. The hair cycle in the rat: (a) the commencement of growth in a follicle containing a club hair; (b), (c) and (d), stages leading to full growth; (e) and (f), cessation of growth. Reproduced by permission (Johnson, 1957). 
again into physical contiguity. Thus they are found wherever mesodermal tissues contact ecto- or endodermal tissues (Fig. 12, p. 23). They are apparent after various fixation procedures and are more dense after the preparations are stained with such " electron-dense " materials as phosphotungstic acid. Their origin and nature will be discussed further below. Beneath the basal membrane are found bundles of collagenous fibrils, the characteristic product of the dermis (Fig. 25). These fibrils are found more or less regularly arranged in different sites and in different animals. They may form a well-organized orthogonal pattern in amphibian larval skin (Plate 9) which is smooth and free from structural disturbances caused by hair follicles (Weiss and Ferris, 1954 and Porter, 1956) or less well-organized bundles in mammalian skins (Ottoson et al., 1953; Selby, 1955; and Mercer, 1958). For the anatomy of the collagen deposits, see Horstmann, 1959 and Salecker, 1944.

\section{The Development of Basal Membranes and their Role in the Formation OF EPITHELIA}

Since the epidermis (ectoderm) must early take over the task of holding the embryo together, it seems clear that the ectodermal cells develop self-adhesion at an early stage in embryonic development. Thus the study of the establishment of the early epidermis offers a means to investigate these fundamental events. The early work of Holfreter (1947) and the electron microscopy of Weiss and Ferris (1954) and Porter (1956) showed that the larval amphibian skin was useful material for this purpose. These electron-microscope studies have mostly concerned the organization of the dermal collagen fibrils. The present writer has examined earlier stages, commencing before the appearance of the membrane, in the tails of tadpoles of Xenopus and Rana. In a Xenopus tadpole (Stage 19, Nieuwkoop and Faber, 1956) and similarly-developed Rana tadpoles the extreme tip of the tail consists only of a single layer of ectodermal cells enclosing a cavity containing very few cells of mesodermal origin.

The cells are already showing the epithelia habit but, significantly they are only closely adherent at their outside edges, the inner faces being widely separated and highly convoluted (Plate 8 ). There is no basal membrane and no dermal collagen. Later stages (developmental-wise) are found nearer the head. Here the tail skin has acquired a defined epidermal appearance, a basal membrane exists and the deposition of collagen has commenced (Fig. 38) (p. 87). In these more advanced areas the dense basal membrane $(B M)$ everywhere follows the smooth undersurfaces of the epidermal cells (Figs. 38 and 39) at a rather fixed distance. The lighter layer $(C)$ between it and the cell membrane $(M)$ is continuous with the lighter layer extending between the cells. It is probably missing on the free surfaces facing the external environment where significantly may 
be found occasional bunches of cilia, finger-like protrusions or mucincontaining pockets.

The basal membrane once established appears to provide a suitable substratum for the crystallization of fibrils of collagen, the soluble precursor of which (tropocollagen) is thought to be produced by the mesodermal fibroblasts (Fig. 38 and Plate 9).

Essentially similar observations concerning the development of intercellular adhesion between the epidermal cells of chicken embryos have also been made by the present writer.

Thus the key steps in epidermal differentiation appear to be:

(a) The appearance of close contacts between cells which results in the monolayer of surface cells acquiring a coherent epithelial character. Differentiated features (intracellular filaments, cilia and mucin droplets) appear on the outer surface about the same time and recall the normal differentia found in cells with free surfaces (p. 44).

(b) The appearance of a basal membrane-a diffuse sheet underlying the basal layer cells.

(c) At this stage the basal membrane cells are (to judge from their fine cytology) still polyfunctional. They have the dispersed basophilic reticulum of a secreting cell (collagen ?). Collagen in any event rapidly accumulates beneath them; the mesodermal fibroblasts also spread out over the sheet and appear to secrete directly against the collagen meshwork. By this stage the definitive histology is established. The explanation of these events must be speculative at the present time but, with this caution in mind, it is worth while proposing what are in effect workinghypotheses as follows (Fig. 38):

(i) The first differentiations of superficial cells (cilia, mucin formation, synthesis of intracellular fibrils) are similar to those noted in single cells and they appear because the surfaces are free. While this explains nothing, it refers the problem to a larger one not confined to the Metazoa. In these early stages most of the cell surfaces are free and, to judge from their convolutions, in active movement. They are little removed from freeliving cells, as is shown, indeed, by Holfreter's observations on cells, liberated from amphibian embryos by reagents dissolving the weak intercellular adhesive, which were able to assume an amoeboid habit and move about.

(ii) Organized multicellular differentiation is initiated by intercellular adhesion which commences between the contiguous portions of cell membranes facing the external environment and from there travels inwards. It is assumed that this is because the cells at this point start to secrete an intercellular cement; their surfaces become sticky. This would be the decisive metazoan feature. 
(iii) The superficial cells (ectoderm) lead the way in differentiation and it may be supposed that diffusible products from them influence the underlying cells (Fig. 27). Rose (1952) has supposed that the appearance of one form of product in a group of cells will suppress a similar appearance in neighbouring cells and permit a second type of differentiation to arise. Certainly, however this may be, the mesodermal cells henceforth are enclosed in a bag of already-differentiated cells whose products could influence them. Fibroblasts soon are recognizable by their content of

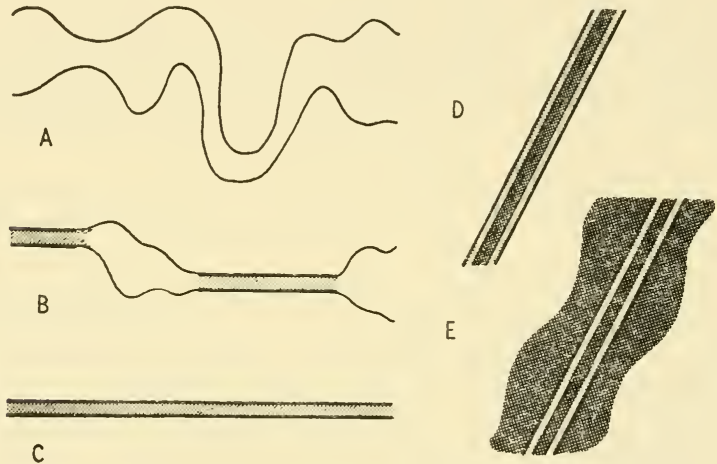

Fig. 41. Types of cell contact seen in the hair follicle. (A) the convoluted cell membranes with irregular intercellular spacing seen in the undifferentiated matrix. (B) localized contacts spreading to give uniform contact. (C) (See also Fig. 43.) The intercellular cement is shown hatched. (D) and (E) Complete intercellular formations noted in the keratinizing levels and higher (from Birbeck and Mercer, 1956 and 1957).

membranes covered with dense particles (RNP). At this stage (Fig. 38) the two races of cells become separated by the appearance of the basal membrane $(B M)$. The peculiarities of this membrane are its fuzzy character, its poor reaction with osmium tetroxide, its staining with phosphotungstic acid (protein), its situation as a further sheet covering the sheet of material already covering the ectodermal cell membranes and which elsewhere acts as an intercellular adhesive. These features combine to suggest that it is a reaction product (precipitate) formed between proteins leaving the ectodermal cell and a more rapidly-diffusing substance emanating from the mesodermal cells, as suggested by Fig. 39.

Henceforth the epithelium is established as a constantly-multiplying and migrating cell population whose cell movement, in an inward direction, is 
constrained by this barrier formed beneath them. The basal membrane, itself becomes the seat of further reinforcement in the form of collagen fibrils. It is therefore proper to emphasize the importance of the basal membrane in the architecture of the tissue; and further, one must pinpoint, as the earlier crucial step in organ construction, the secretion by the epithelial cells of a substance which can (a) link specifically to sites on their surface and become an intercellular cement, and (b) when the cells face the mesodermal space, react with mesodermal products to form a basal membrane to which the cells are anchored.

A further important development which takes place at about the same time as the formation of the basement membrane is the appearance of a patterned arrangement of dense deposits over the surface of the cell facing the basement membrane. These are the dermoepithelial desmosomes (Fig. 36 and Plate 14B). They are not identical in structure with those which form between epidermal cells (epidermal-epidermal desmosomes) or in similar epithelia and which are illustrated in Fig. 21, p. 42 and Plate $12 \mathrm{C}$. That these structures are mechanically attached to the basal membrane was shown by Weiss (1958). The deposit within the epidermal cell often is double-layered (Weiss refers to them as "bobbins") or even multi-layered (Porter, 1956).

The reason for the formation of these localized deposits is not known. They arise evidently in response to some external stimulus - another cell or a basal layer. It could be, adapting an idea due to Weiss (1950), that the localized external stimulus promotes a selective absorption of a cytoplasmic component on the plasma membrane which then provides the site for further depositions. Alternatively, the cells response may be due to the localized entry of some material from without which combines with and precipitates an intracellular component. This is equivalent to saying that the desmosomes mark the sites of porous spots. A section running parallel to and through the neighbourhood of the basal membrane (Fig. 39, Plate 14B) gives the impression of a rather regular arrangement recalling that of the pores in the nuclear membrane (Plate $4 \mathrm{C}$ ). The concept of "membrane pores" is current as an explanation of specific membrane permeabilities (Danielli, 1942). These are not thought of as small " holes" but areas of modified porosity revealing themselves only by the intracellular reaction provoked by an entering molecule.

That the ends of intracellular fibrils become attached to desmosomes may be accounted for by assuming that the dense deposits present favourable sites for the initiating fibril growth. 'The importance of these attachments for the ultimate mechanical function of the cells is obvious (see also p. 95). 


\section{The differentiated layers and the variety of cell products}

\section{The EPIDERMis}

The epidermis is an avascular, stratified, squamous epithelium of variable thickness depending on the number and thickness of the cell layers it contains (Fig. 25, p. 55). It necessarily contains a germinal layer and may, as in the mouse, consist of only one other layer of horny cells (Setälä et al., 1960); in other animals and at other sites, and in some pathological conditions, it may reach an extreme thickness of some millimetres. Although the thin mouse skin does not reveal the usual stratification, this does not mean that the intermediate stages of differentiation are absent or that keratinization follows a different course. Cells representative of the intermediate stages are in fact present, but insufficiently common to constitute distinct layers (Glücksmann, 1945). After various treatments causing hyperplasia, and particularly after the application of carcinogenic hydrocarbons (benzpyrene, methylcholanthene, etc.), the thickened skin appears typically stratified.

When the mammalian epidermis reaches a moderate thickness $(\sim$ ten cells deep) four layers can be distinguished: (a) the germinal or basal layer (stratum germinativum); (b) the stratum granulosum; (c) stratum lucidum; and (d) the stratum corneum (Fig. 25). The development of these stages in differentiation seems inherent in the epidermis and depends on the attainment of a sufficient thickness.

Even the basement layer cells contain loose bundles of dense filaments ( $<100 \AA$ diameter) (Plate 7) showing that synthesis has commenced at this level (Plate 9). These filaments often sprout in bundles from the sense plate-like desmosomes ( $D$ in Plate $4 B$ ) on the plasma membranes. In the layer of cells immediately above the basal layer of the epidermis there is an increase in the number of filaments ending on desmosomes, making them more conspicuous and giving to the cell the appearance of being covered with small short prickles. The layer is for this reason referred to as "the prickle layer".

Tonofibrils. The fine birefringent fibrils visible in the light microscope are often referred to as tonofibrils, particularly in the older literature. The use of this term arises out of the view of the classical light histologists who believed that the fibrils had a particular organization relating them to the mechanical function of the tissue. The word "tonus" (Greek) implies a "brace or support" and the word "tonofibril" expresses the idea that the fibrils run from one face of the cell to another and are thus capable of transmitting tension directly. Some authors, observing the so-called " intercellular bridges," held further that the tonofibrillar system ran continuously from one cell to another. This latter view is now untenable since it is clear that, in several distinct cell types, fibrils certainly 
end on the desmosomes and do not pass across the cell membranes. One of the best established examples of this is found in heart muscle (mentioned above, p. 42) where parallel bundles of myofilaments are seen to enter and fuse with elaborately-developed deposits of the desmosome type which cover the cell membranes and are visible in the light microscope as "intercalated disks".

The situation is not as clear in epithelial cells. Certainly many fibrils end on desmosomes (Porter, 1956; Charles and Smiddy, 1957), but it remains to be shown what proportion of fibrils have both ends attached. In view of the extreme thinness of the sections used in electron microscopy $(<500 \AA)$ proof of continuity is difficult to obtain. From a mechanical point of view it is not necessary to assume that they all do since the whole mass of fibrils is ultimately fused together during keratinization. There is thus a use for both terms "fibrils and tonofibrils" and the latter may be used when an author wishes to assert adherence to the view that the fibrils run from one desmosome to another within the cells. In other cases the less restrictive word "fibrils" will be used here.

Keratohyalin. In the lower layers of the epidermis the synthetic activities of the cell appear to proceed directly to the formation of filaments $(F$ in Plate 9$)$ and in this respect they resemble the cells of the hair cortex to be discussed later. In the stratum granulosum of the epidermis, however, a new product makes its appearance in the form of rounded droplets which, accumulating, give the cells a granular appearance. These droplets consist of a distinct substance called keratohyalin and a great deal of study has been devoted to it, the main point at issue being whether it is, or is not, to be regarded as a precursor of the fibrous keratin of the stratum corneum (see also p. 228). 'The granules disappear in the next cellular layer, the clear, glassy, highly-birefringent stratum lucidum.* The appearance of keratohyalin granules is one of the definitive characteristics of the "soft keratinization" of the epidermis (see Table 5, p. 65) to be contrasted with the keratinization of a hard keratin, such as hair (next section) which proceeds without the formation of a granular layer. In the squamous cells of the inner root sheath of the hair follicle a similar granular layer appears (Plates 22 and 23).

\section{The Hair Follicle}

The hair follicle forms embryonically as a down-growth of the basal layer of the epidermis. The first sign, indicating a locally-increased rate of cell division, is a cluster of smaller crowded cells above the basal

* Ranvier in 1879 introduced the term "eleiden" (from the Greek word for " oil ") as a name for the clear glassy substance of the stratum lucidum. Although this word occurs frequently in the older literature there does not seem to be any need for it in the present description. 
membrane, the primary hair germ. As proliferation continues, the bud projects downwards and penetrates the dermis as a solid cord of cells. Perhaps conditions here favour further division, the cells finding more food and living space. The advancing tip calls forth a response trom the dermis shown by the approach of a number of dermal cells. The two kinds of cells do not mix, since they are already separated by the basement membrane. The advancing epidermal cells spread over the dermal cluster to form a cap, the future hair bulb, and the mesodermal elements within form the future papilla. These events are partly recapitulated each time a new hair develops in a resting follicle (see Fig. 40).

This co-operative relation between dermal cells and epidermal has impressed many observers; moreover, the morphogenetic control which subsequently develops in the follicle is also dependent upon the continued presence of the dermal component. This is shown perhaps most clearly by Hardy's $(1949,1951)$ experiments on the formation of hair in tissue culture and by equivalent experiments concerning the development of feather germs (Lillie, 1942). In the absence of dermal remnants, true follicles never develop in tissue culture; together, dermis and epidermis can produce and maintain follicles with differentiating cells. No other accessory structures, such as blood vessels or nerves, are required. Moreover, the arrangement of the follicle in groups is similar to that of skin growing in situ. This is an important demonstration of the morphogenetic competence of the skin and it emphasizes that the control of differentiation in the epidermis is localized (see p. 146).

After the papilla has formed within the tip of the cells descending from the basal layers of the epidermis, mitosis becomes restricted to the lower half of the follicular bulb. The hair now sprouts up from this matrix and penetrates the originally-solid plug of epidermal cells which thus become the outer root sheath (Fig. 40). The advancing tip forms a cone of cells continuous with those of Henle's layer of the inner root sheath.

Typically follicular activity is cyclic, a growing phase is followed by a resting phase (Montagna, 1956); Fig. 40 depicts, for example, the events in the cycle of growth in rat skin. Cyclic growth is discussed in the next chapter. In a condition of steady growth the hair follicle offers a remarkably compact example of organogenesis. From the mass of undifferentiated cells in the lower half of the bulb (Fig. 42) arises a solid cylinder composed of six concentric cylinders each consisting of cells which become different in shape and which follow different paths in internal development. In terms of their characteristic products of synthesis, the three external cylinders, each a single layer of cells, ${ }^{*}$ which comprise the inner root

* The layer of Huxley may consist of more than one layer in thickness, in asymmetrical hairs (Rudall, 1956). 
sheath, and the central core, the medulla, may be classed together, since they each form a peculiar protein called trichohyalin (probably very similar to the keratohyalin of the skin). It forms as amorphous droplets and is later converted into a fibrous form. Chemically it is distinct from keratin and is unique in containing the amino acid citrulline $(6$ per cent

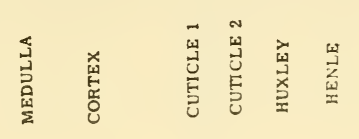

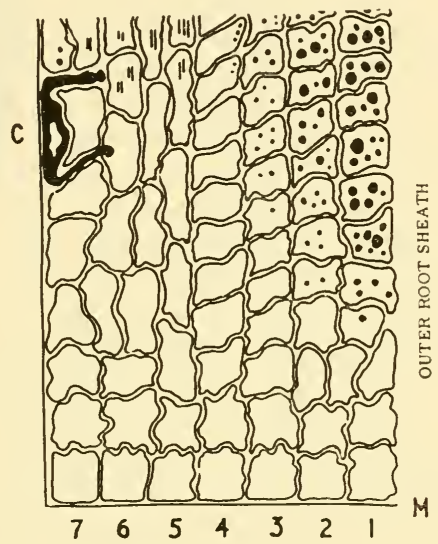

LAYERS FROM OUTER ROO'T SHEATH

FIG. 42. Purely diagrammatic representation of the relations of the several cell streams $(1-7)$ in the hair follicle to the basal membrane $M$ and the outer root sheath to illustrate the " position " theory of differentiation. The intercellular gaps are emphasized. $\mathrm{C}$ is a melanocyte, the papilary space is to the left. The dense dots in the inner root sheath cells (Huxley and Henle layers and cuticle 2) are trichohyalin.

by weight) (Rogers, 1959). The other concentric cylinders form the cuticle and cortex of the hair, each containing a distinct variety of keratin (Fig. 42).

In respect of their developmental history, the cells of the bulb are equipotential and could apparently proceed to synthesize any of the epidermal products. The sole factor, which initially seems to distinguish 

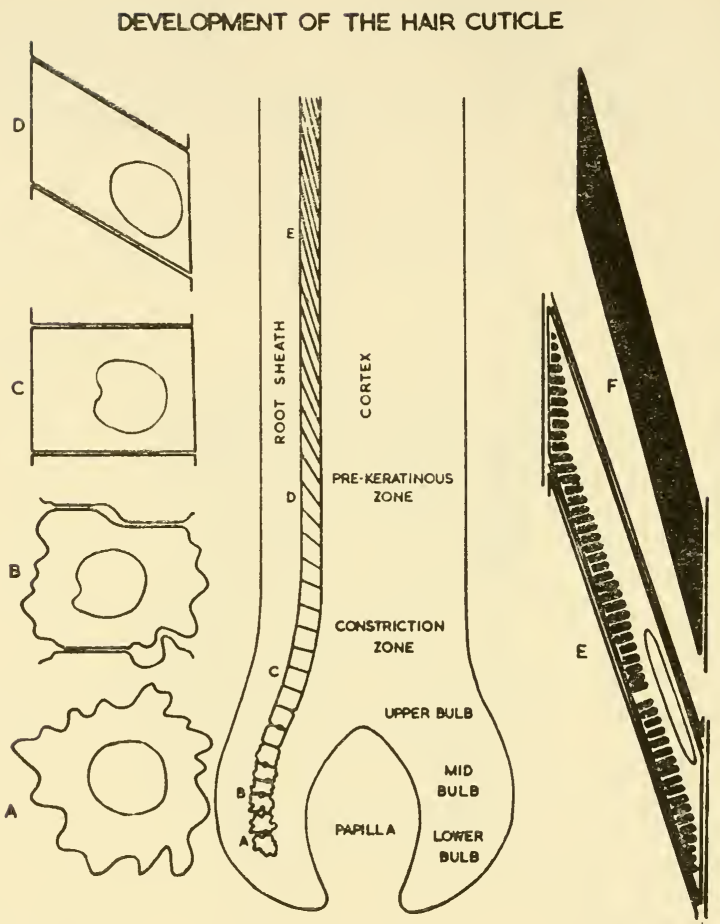

FIG. 43. A hypothesis of the differentiation of the hair cuticle in terms of the development of intercellular adhesion.

(a) A germinal cell with poor intercellular adhesion and convoluted (active) membranes.

(b) The smoothing out and immobilizing of membranes as a result of the appearance of areas of close intercellular adhesion.

(c) and (d) Complete stabilization of membranes followed by a flattening and tilting due to the spread of adhesion " zipping " the cells together (See also Fig. 44).

(e) and (f) the commencement of protein synthesis. (From Birbeck and Mercer, 1957). See also Fig. 41. 
one from another, is their distance from the outer root sheath or from the dermal environment (Fig. 42) which suggests again that some influence from this direction controls differentiation. Differentiation is usually recognized in the light microscope by a change in cell shape or by an alteration in staining properties or cell texture which indicates the accumulation of different chemical products. However, as stated earlier, electronmicroscopically the cell membranes (as defined above) can be clearly

A

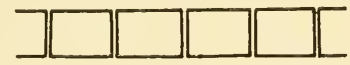

B

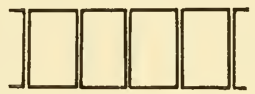

C

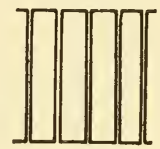

D

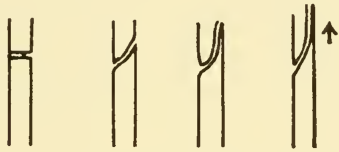

FiG. 44. (a) to (c) The conversion of a flattened into a tall columnar epithelium as a consequence of the spread of intercellular contact. (Refer to Fig. 43.)

(d) The development of "tilting" in a cuticle, for example, owing to the creeping of a cell tip due to enhanced adhesion. (Birbeck, 1957).

distinguished, and their behaviour provides an earlier indication that a change is taking place (see Fig. 41).

Birbeck and the present writer (1956, 1957a and 1957b) have tried to correlate the outset of differentiation with differential membrane adhesion between the several presumptive layers of the hair. The course of events revealed in a longitudinal section of a follicle is illustrated diagrammatically in Figs. 42 and 43. The first cell layers to distinguish themselves from the undifferentiated mass in the lower bulb are those of the cuticles. It may be significant, from the point of view of the penetration of an inductive effect from the dermis, that although these layers are of a variable distance from 
the papilla, they are always the fourth or fifth cell layer from the surrounding basement membrane. Their cells acquire a cuboidal shape, which is easily apparent, since their membranes have drawn together zipper-wise, effacing surface irregularities and becoming somewhat denser. The same events follow in the layers of Huxley and Henle; they occur several cell diameters higher in the central mass of cells destined to become cortical cells. Between these latter cells, openings are observed as high as the papillary tip where they are occupied by processes of the melanocytes (see p. 276).

While it must remain purely speculative in our present state of knowledge, it is possible to suppose that the cuticles lead the way in differentiation because they, in some way dependent on their position relative to the dermis, first begin to form and secrete their specific intercellular cement. The possibility of developing a columnar epithelium by a zipperlike spread of adhesive cell contacts was envisaged by Schmitt (1941) (Fig. 44), and the change in shape of the cuticle cells looks very like an illustration of Schmitt's hypothesis.

\section{Intracellular differentiation in the hair bulb}

The first visible signs of synthesis of intracellular products are observed in the cells immediately in contact with the external root sheath layer. These cells become Henle's layer, the most peripheral layer of the inner root sheath (Fig. 42). Small trichohyalin droplets appear in the cytoplasm of cells at about the middle line of the bulb and rapidly grow in size. Henle's layer hardens, becomes birefringent and clear suddenly at the level of the constriction of the bulb. Its function at this level appears to be largely mechanical; it provides a solid cylindrical support to carry upwards the soft cells within. Its outer surface is said to slide over the surface of the outer root sheath, which in this respect is a static structure and does not move out with the rest of the follicle. The mechanics of these movements are far from being clearly understood (Montagna, 1956; Auber, 1950).

When first formed the trichohyalin droplets are isotropic; at the level where Henle's layer hardens, the cells suddenly become birefringent and the layer itself becomes clear and more difficult to stain. Electron-microscopically fine filaments or ribbons can be seen extending from the tips of the lenticular-shaped droplets of the amorphous precursor (Plate 21). These condense to give a compact highly-oriented mass which is the clear, glassy, birefringent layer visible in the light microscope.

The course of events in Huxley's layer and in the cuticle of the inner root sheath is similar, but the tempo is slower, the cells remaining full. Following Auber (1950) we may suppose that these cells form a firm but tenacious vice to grip and support the softer tissues of the hair itself. 
The synthesis of fibrous keratin commences in the cells of the cortex in the middle and upper bulb. Srrall wispy bundles of filaments are already visible electron-microscopically in the cells of the middle bulb (Plate 12B). There is no obvious accumulation of non-fibrous precursor as occurs in the sheath cells; however, if follicles are fixed for $12 \mathrm{hr}$ in buffered formaldehyde and stained with phosphotungstic acid, a procedure designed to retain more completely the contents of the cells, the cytoplasm is seen to be packed with a structureless protein which may suggest the existence of a precursor in a soluble form.

Synthesis is delayed in the hair cuticle until above the bulb, when droplets of an amorphous keratin separate in peculiar patterns packed against the outer wall of the tilted cuticle cells (Fig. 43) (Plate 20).

The characteristic products of the hair follicle, fibrous keratin, cuticular keratin and fibrous trichohyalin, are thus formed in distinctly different ways which will be more fully discussed in a subsequent section (p. 223). Returning for a moment to consider the cells of the epidermis, it would seem that there the two methods of forming fibrils are actually to be found in the same cell, although at different times, for filaments are built up directly in the basal and prickle-cell layers and from keratohyalin in the stratum granulosum. The conversion of the granular layer into the clear birefringent stratum lucidum involves a transformation of the isotropic keratohyalin granules into a fibrous form apparently analogous to that found in the inner root sheath cells. The fibrils of both origins seem to fuse into a common formation in the stratum corneum. This problem is further discussed in Chapter 6.

\section{The Feather Follicle}

A brief account of the feather follicle has already been given (p. 69). The feather grows out from a germinal matrix (Fig. 48) at the bottom of the follicular shaft consisting of an ectodermal wall and a relatively large mesodermal core called the feather pulp (Figs. 45, 46 and 47). The feather cylinder itself comprises three layers: an external one forming a protective sheath to the developing feather, a thicker middle layer from which the feather itself is derived and an inner layer next to the pulp. The external and internal layers may be likened to the root sheaths of the growing hair. All three layers are produced by the proliferation of the generalized cells of the matrix, called the collar, at the base of the follicle (Fig. 48).

In its growing phase the feather is a hollow, pointed cylinder set like a cap on the core of mesoderm; it simply elongates owing to the addition of cells by division at its lower end, the "collar.". Some details of this process including barb formation are illustrated in the simplified drawing Fig. 45. Here at (a) a portion of the growing cylinder is seen from its ventral side. $R$ is the rhachis found on the dorsal side, the barbs $B$, greatly reduced in 
number, are indicated by their distance from the central rhachis. The collar is at $C$. The barbs form a subdivision of the cylinder by clefts as may be seen more clearly in (b) where following Lillie the cylinder is shown cut along its ventral side, spread out flat, and viewed from inside the follicle. Cell movement is everywhere vertical, i.e. parallel to the rhachis, and, in the area of the collar giving rise to the rhachis, is continuous; in those

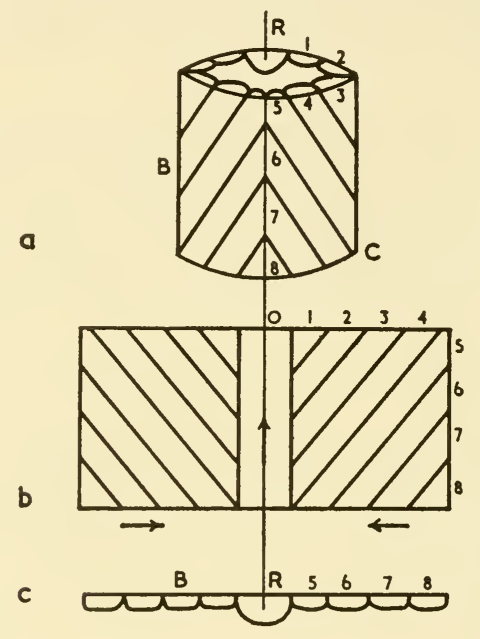

FIG. 45. Illustrating rhachis and barb formation in the feather follicle. (a) A short length of the feather cylinder adjacent to the collar, (b) the cylinder opened out, (c) a section cut across (b). The rhachis R grows steadily upwards. The bases of the barbs move inwards to meet the rhachis as a result of the migration of the growing areas but cell movement is always vertical.

regions giving rise to barbs, however, growth is restricted to the areas shown in (c), separated from each other by clefts which thus give rise to a series of ridges on the inner face of the cylinder. In nesting feathers the ridges ran parallel to the rhachis (itself here only a barb) and the feather when open appears as tube with a slotted end. The growing areas of the collar are here stationary relative to the rhachis. In forming adult (contour) feathers the discrete growing areas generating barb ridges migrate towards the rhachis (Figs. 46 and 47) and ultimately meet it and join the barb to the rhachis. Only the sites of growth migrate inwards, the movement of cells remains vertical, and the effect is to produce the series of spiral grooves inside the cylinder as shown in Fig. 45, each completed barb describing a 


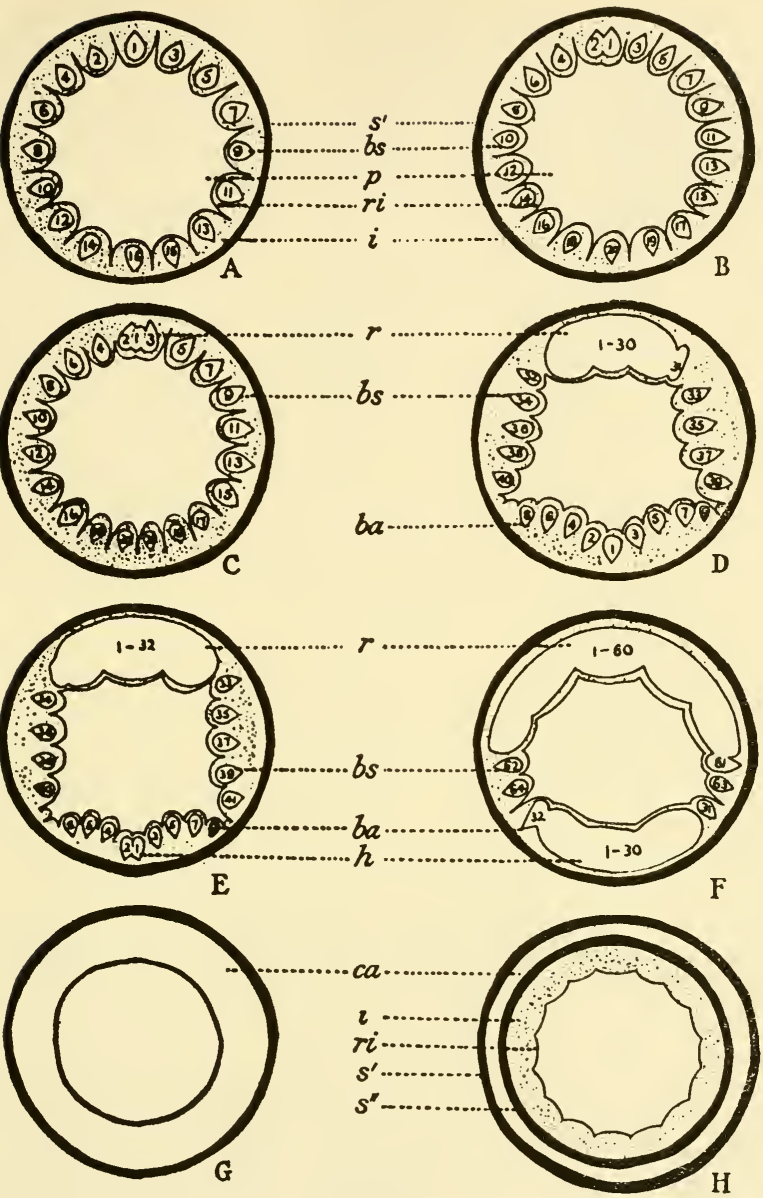

Fig. 46. Diagrams of cross-sections of a feather to show the dorsal fusion of barbs to form the rhachis (A-F); the fusion of barbs ventrally to form the hyporhachis (D-F); the lateral fusion of rhachis and hyporhachis to form the calamus $(F)$; the structure of the calamus $(G)$ and the tip of the new feather forming within the base of the calamus of its predecessor (H); ba, barb of aftershaft; bs, barb of shaft; ca, calamus; h, hypo. rhachis; $i$, intermediate cells (i.e. collar); $p$, pulp; ri, ridge; $s$ ', sheath of old feather; $s^{\prime \prime}$, sheath of new feather. (Redrawn from Hosker (1936).) 
half spiral in the cylinder. During the formation of the continuous cylinder of the calamus, which completes the feather, growth is continuous around the entire ring of the collar.

This description follows the classical accounts due to Strong (1902) and Davies (1889). More recently somewhat different views were advanced by Lillie and Juhn (1932 and 1938) which envisaged an actual migration of growing tissue tangentially along the collar to enter the mounting rhachis as suggested by the drawing Fig. 47. Their view has been contested by Hosker (1936) and 'Espinasse (1939) in particular, and it seems it cannot be held in its extreme form. Lillie and Juhn make the point that the rhachis appears an independent growth to which barb material secondarily becomes attached (Lillie, 1942). Certainly surgical experiments prove that the capacity to generate rhachis is a special differentiation of the dorsal portion of the collar. Following removal of the ventral half of a follicle the entire feather may be regenerated; on the other hand the removal of the dorsal half leads to the regeneration of a feather lacking a rhachis. The actual relevance of some of these data to Lillie and Juhn's theory is not immediately apparent. An interesting discussion of these questions will be found in Waddington's book (1952).

According to the concrescence theory the rhachis is formed by a process of concrescence of the continually-growing right and left halves of the collar, the levels from apex to base being formed successively (Fig. 47). The forming barbs are carried along with the constantly-streaming halves of the collar to their definitive positions at the sides of the shaft with consequent change of orientation. As the series of barbs move dorsally (nos. 1-15, Fig. 47D), new barbs (nos. 16-25, Fig. 47E) take their origin in the space thus provided at the ventral surface of the collar.

The cells of the germinal collar closely resemble in their cytological features those of the hair bulb (Mercer, 1958). The basement membrane is typical and the basal layer cells, which abut it, form a columnar-like epithelium. They are strongly basophilic and their cytoplasm abounds in clusters of small dense particles of the same kind as described in the germinai cells of hair and skin. Differentiation becomes apparent in the cell layers immediately above the basal layer with the appearance of wispy filaments in the cytoplasm. Although these filaments are known to consist of a $\beta$-type keratin (p. 16), their appearance, their manner of formation and the cytology of the cell, seem exactly similar to the cells of the hair follicle forming filaments of an $\alpha$-keratin (Plate 12A).

The production of the $\beta$-keratin type of structure in the epidermis of birds and reptiles poses some interesting questions, which have been considered by Rudall (1949). Both $\alpha$ - and $\beta$-type keratins are produced in these structures by cells which originally belonged to the same primary ectoderm; the later development of two cell-types, distinguished 


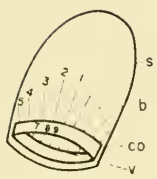

A

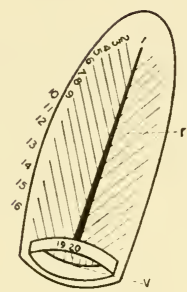

B

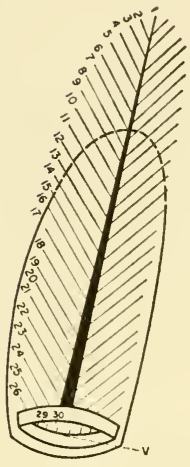

C

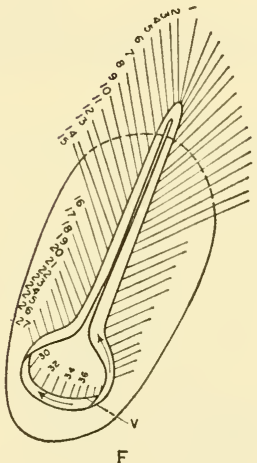

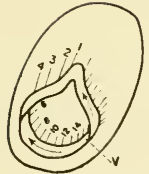

D

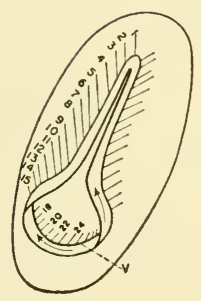

E

FIG. 47. Diagrams to contrast the development of a feather according to the fusion of barbs or classical theory $(\mathrm{A}, \mathrm{B}, \mathrm{C})$ and the concrescence theory of Lillie and Juhn (D, E, F). In the classical theory the whole feather arises from a ring of enbryonic cells (i.e. the collar) surrounding the base of the feather germ. According to the concrescence theory the barbs, nos. 1-9, arise from the collar and as more cells are added to the barbs from the rapidly-dividing collar cells, it will follow that they will gradually approach the mid-dorsal line, and fuse with the dorsal-most barb or rhachis. This of necessity becomes broader and takes on the definitive shape of the rhachis. $b$, barb; co, collar; $r$, rhachis; $s$, sheath; v, ventral (from Hosker, 1936). 
by the type of protein fibril contained, may be regarded as a modulation although little is known about the stability of cell-type in the basal cells supplying the different streams of cells. In the feather follicle the intermediate cells (Fig. 48) produce feather keratin exclusively; the outermost layers of the stratum corneum and the stratum cylindricum produce $\alpha$-keratin with little or no feather keratin. The several streams of cells advance in parallel and the situation is not unlike that found in the hair follicle in which several parallel streams also occur. In the hair follicle the weight of evidence seemed to support the idea that the cells of the germinal matrix
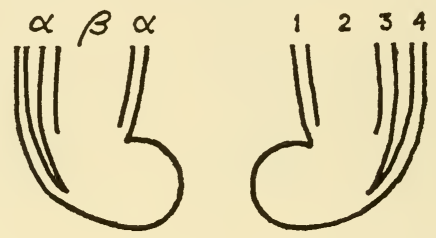

FIG. 48. The germinal collar at the base of the feather follicle showing the several cell streams $(1,2,3,4)$ arising from it: (1) is the layer adjacent to the dermal papilla which gives rise to the medullary caps; (2) is the feather proper; (3) the feather sheath; and (4) the epidermal lining of the papilla. The distribution of protein types is shown on the left-hand side.

were all similar and that their position determined their subsequent development. In the feather system a more fundamental difference develops: a difference in basic chain-type between the proteins produced in the contiguous streams. No histochemical or electron-microscopical feature distinguishes two classes of germinal cells. On his evidence Rudall is unable to decide whether the factors for synthesizing the two kinds of keratin are segregated into two classes of cells but considers the possibility of cells producing both kinds of keratin in a mixed form.

In snake scales the hard horny outer layer is feather keratin and the less compact inner layers give an $\alpha$-pattern (Rudall, 1947). Here it would seem that the same germinal matrix produces both types of protein in series. Unfortunately, again a certain ambiguity remains as to whether precisely the same cells in the matrix contribute to both layers. There seems good reason to believe that growth rhythms (perhaps diurnal) occur in the feather follicle. 'The regularity of the barb structure suggests this clearly, the presence of growth bars and of the succession of bands of radioactive sulphur deposited in the calamus (Lüdicke, 1959) following injection of radioactive sulphur compounds are further indication. The relation of rhythmic growth to the whole phenomenon of feather formation has not yet been explored in detail. 


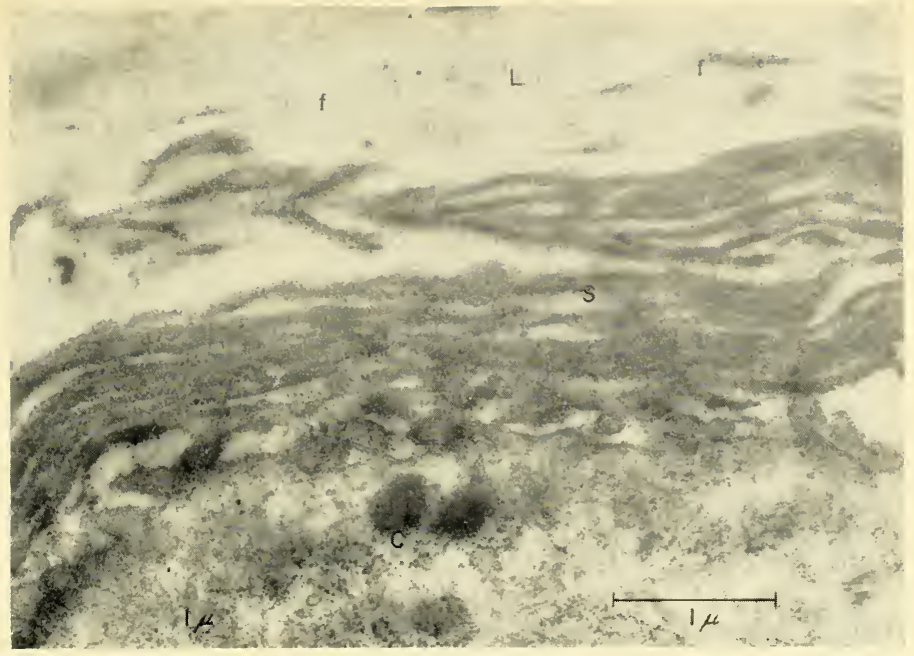

A

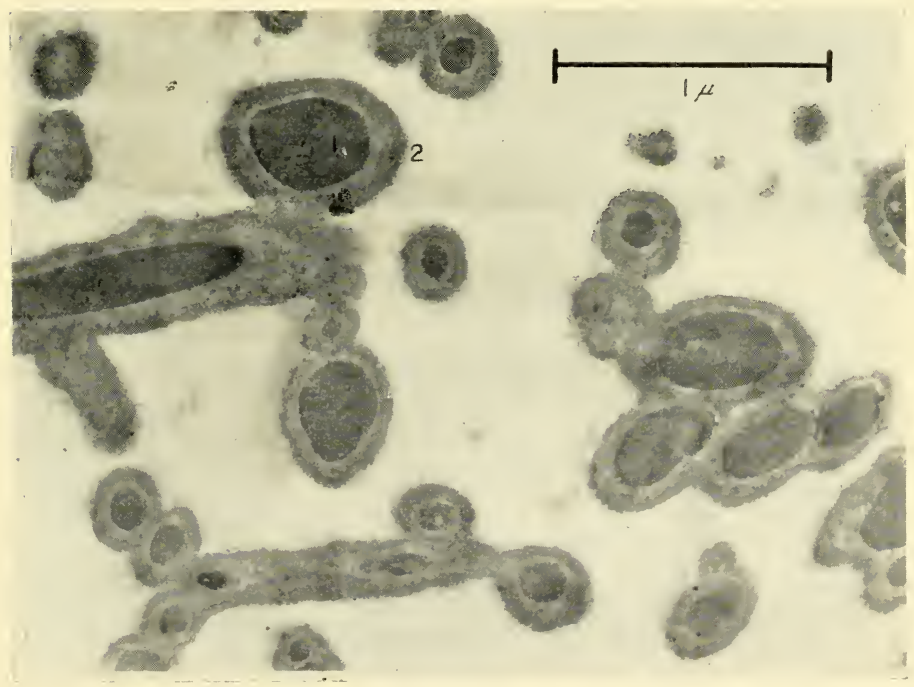

B

Plate 18 (Captions overleaf) 


\section{Plate 18}

A. Section of gizzard of 17-day-old chicken embryo showing threads of keratin $\mathrm{S}$ passing down the lumen $\mathrm{L}$ of the secreting epithelium. The secretion gives a positive test for disulphide groups and appears to form by the coalescence of fine filaments $f$.

B. Cross-section through an egg-shell membrane of the chicken egg. In the light microscope the membrane is seen to consist of fibrils roughly hexagonally arranged parallel to the egg surface. In e.m.gs. the fibrils are cut in various directions and each is seen to consist of two components ( 1 and 2 ); the inner component has reacted more strongly than the outer with the osmium fixative and may thus be the keratin component (see p. 107).

C. Part of a secreting cell. 


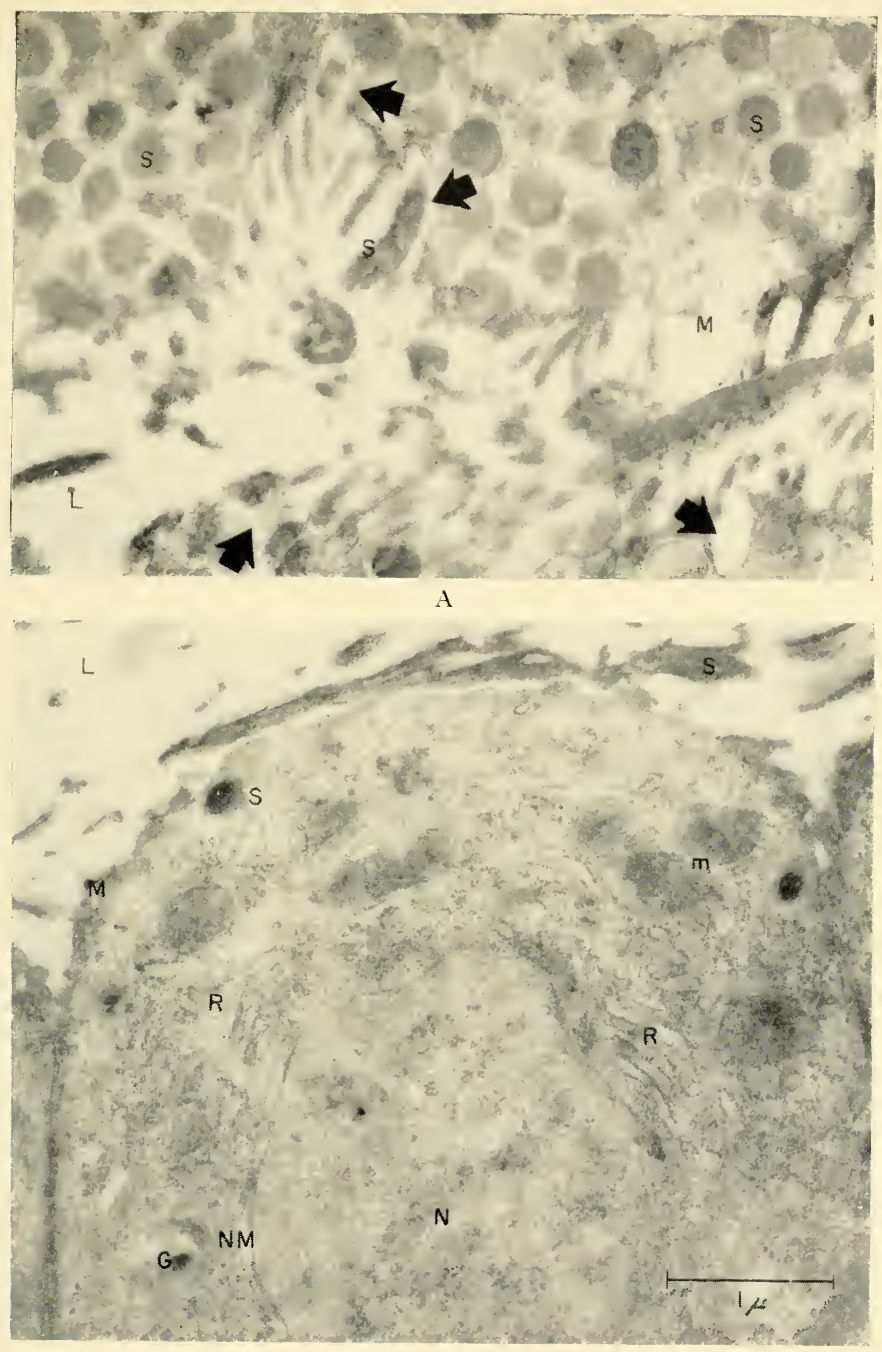

B

Plate 19 (Captions overleaf) 
Plate 19

The cells secreting keratin to form the lining of the gizzard in a 17-day-old chicken.

Top: $S$, secretory granules; $L$, lumen of glandular pocket; $M$, cell membrane. The arrows indicate secretion escaping from the cells between the villi.

Bottom: A cell not filled with secretion granules in which particlecovered membranes $R$, mitochondria $M$ and Golgi apparatus can be seen; $S$ is the secretion in the lumen $L$. $N$, nucleus; $N M$, double nuclear membrane. 


\section{'The Avian Secreted Keratins}

The secreted keratins, the horny lining of birds' gizzards and the eggshell membranes, have not been as extensively studied as their unusual extracellular location would seem to warrant. We lack an exact knowledge of their structure and mode of formation. Even the type of fibrous protein found in egg-shell membranes is disputed. According to Champetier and Fauré-Fremiet (1938) the material gives a collagen pattern. On the other hand, the chemical analysis of purified membranes reported by Calvery (1933) shows it to be of the keratin type. The purification involved an enzymatic digestion aimed at removing constituents less resistant than keratin (mucin, collagen, etc.) which may account for the different findings. The present writer has made electron micrographs of sections of hen's egg membranes (Plate 18B) which revealed a felt-work of fibrils each of which consists of two distinct components. By staining methods and the light microscopy two separate fibrillar systems are usually distinguished (Moran and Hale, 1936): mucin and keratin. It would appear that actually the fibrils of one system (probably keratin from its stronger reaction with osmium tetroxide) are enveloped by a deposit of a less reactive material (mucin?).

No study has been made of the fine cytology of the cells whose secretion coats the eggs with their membranes during their passage through the oviduct.

The tough, thick lining of the gizzard, which protects the cellular surfaces of this muscular mill against wear and tear, consists of coherent sheets of protein mixed with mucin. The material gives a strong and specific reaction for disulphide groups and is accepted as a keratin (Broussy, 1932). The layer is birefringent, but irregularly oriented. Longley (1950) reports that it gives an X-ray pattern consisting of three haloes corresponding to spacings of $10,4.7$ and $3.7 \AA$ which would suggest it is a $\beta$-keratin (feather keratin?).

The layer is secreted by the cells of a columnar epithelium resting on a typical basement membrane. The secretion collects as rounded liquid-like inclusions in the apex of the cells and escapes to flow down a typical glandular lumen to join the overlying material (Broussy, 1932 and Fell (private communication)). An electron microscopic study of these cells in 21 and 17-day-old chicken embryos made by the writer shows their fine cytology to resemble that of epidermal cells. RNP granules are common but a basophilic (particle covered) reticulum is also present (Plate 19). Clusters of smooth membranes ( $\gamma$-cytomembranes) were conspicuous. A structureless basal membrane underlies the epithelium; there are few desmosomes and no intracellular tonofibrils. In the body of the cytoplasm and towards the cell apex the secretion appears as structureless rounded droplets of various sizes (Plate 19) which stain strongly with PTA 
indicative of protein rather than mucin. The free surfaces of the cells are covered with stubby villi between which appear pockets containing granules in the act of escaping. On leaving the cell (or even before it) the granules commence a transformation. They lose their homogeneous appearance, break into a cluster of coarse granules, then into finer granules which appear to open into masses of short fine filaments (Plate 18A) whose coalescence produces thick, tapering rodlets and then the horny layer.

This sequence of changes seems at first sight unlike that found in the formation of other keratins. However, there is a likeness to keratohyalin. In each case an amorphous precursor is produced which accumulates as droplets or granules. In the epidermis the keratohyalin granules transform into fibrils within the cell; in the gizzard on the other hand, the secretion escapes from the cell before the transformation into the fibrous form, which then occurs extracellularly. The problem clearly requires closer study. Since the horny lining is incompletely soluble in keratinolytic solvents and is coloured, the possibility of tanning must be considered. Mucins are produced by the same (or neighbouring) cells but the relation between the two secretions is obscure.

The organic matrix of tooth enamel may be a keratin (p. 78) and recent micrographs of Watson (1960) show that it is extracellular and that the cytoplasm of the ameloblasts has the basophilio (particle covered) membranes characteristic of secretory cells (see p. 113).

\section{The synthesis of protein in epidermal systems}

\section{Cytology of Cells which form Protein}

It is desirable to consider at first in greater detail the cytology and biochemistry of proteogenic cells. Protein synthesis, in the sense of the initial synthesis of a high molecular weight polypeptide having a specific structure, occurs only within cells; certain other processes which, as we shall see later, are important in the assembly and organization of fibrous structures may, however, occur extracellularly. Epidermal cells as a class are notably active in protein synthesis and, on account of their often simple geometrical arrangement in such organs as skin or hair, they lend themselves to microscopic study. On the other hand, the number of cells available is not usually large and this does not favour biochemical techniques. Thus the wealth of morphological detail is offset by a lack of biochemical information based directly on a study of epidermal cells themselves and we are forced to accept as a working basis the general conclusions of the course of synthesis worked out on more convenient systems, such as the mammalian liver or micro-organisms. It would be impossible to do more than present a sketch of this work which is developing rapidly at the present time. 
Glandular cells, such as those of the pancreas or thyroid, have a high rate of protein synthesis and it has long been known that their cytoplasm stains deeply with basic dyes (Caspersson, 1950 and Brachet, 1957). When it was shown that this basophilia was due to nucleic acids a connexion between these acids and synthesis seemed certain. There are two kinds of nucleic acids distinguished, among other things, by the sugars they contain: desoxyribonucleic acid (DNA) containing the sugar desoxyribose, and ribonucleic acid (RNA) containing ribose. The view that nucleic acids were somehow related to synthesis was argued some years ago by Caspersson (1950) who used ultra-violet absorption methods to detect them and Brachet (1957) who used specific dyes. Brachet more definitely urged the participation of RNA. Since that time direct chemical analysis has confirmed the presence of nucleic acid and an enormously diverse amount of cytochemical evidence based on all sorts of organisms has been accumulated to show that DNA is invariably present in the nucleus of all cells, and that RNA is always present in the cytoplasm of proteogenic cells (Brachet, 1957).

Caspersson at first suggested a scheme, based largely on the distribution of ultra-violet absorption material, in which it was supposed that genes (DNA) located on the chromosomes controlled the synthesis of histone-like (basic) proteins which accumulated first as a nucleolus, and later passed through the nuclear membrane and, entering the cytoplasm, provoked the formation of RNA and the specific proteins. This view cannot now be sustained in full. The presence of basic proteins is questioned and the movements of RNA are thought to be different.

Current research is centred on the interrelations of the genetic DNA, RNA and protein and both experiment and speculation are very active. Almost all authors accept the view that genetic information is carried by molecules of DNA (Crick, 1958) and that these molecules must therefore be duplicated at each cell division. The DNA of the nucleus in its genetic role is said to contain all the information to ensure its own replication and to form the various other materials of the cell. It is a basic assumption (a "central dogma " according to Crick) that only nucleic acids possess this peculiar property of conserving information and using it to guide synthesis.

Isolated DNA has been shown by physicochemical methods and by electron microscopy to be an extremely long molecule (several microns) with a molecular weight of several million. A combination of X-ray crystallography and chemical analysis shows that it consists of two helicallyintertwined chains (Watson and Crick, 1953) which are complementary in shape to each other. If the two unwind and separate each might serve as a "template" for the assembly of a new complement or for the formation of other nucleic acids (RNA) as "copies." Current literature abounds with hypothetical schemes for this replication process. In the chromosome 
the nucleic acid is probably combined with basic proteins to form a nucleoprotein, probably also a helix with the polypeptide chain closely linked to the DNA helix (Wilkins et al, 1959).

Except in very general outline it must be admitted that nucleo-cytoplasmic relations remain very obscure. A current hypothesis is illustrated

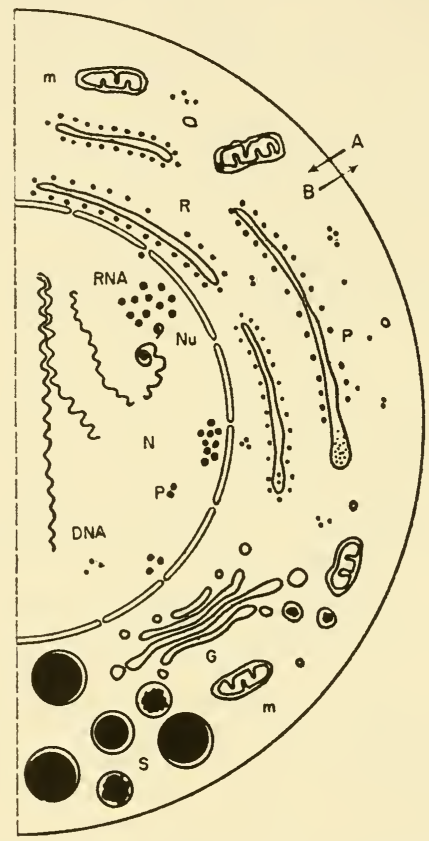

FIG. 49. Principle cytological features of a cell synthesizing protein for secretion. $\mathrm{N}$, nucleus; $\mathrm{Nu}$, nucleolus; $\mathrm{m}$, mitochondrion; $\mathrm{R}$, reticulum (basophilic); $P$, an RNA-containing particle; $\mathrm{G}$, Golgi apparatus; $\mathrm{S}$, secretory granule. The arrows $A$ and $B$ symbolize the exchange of control between cell and environment.

in Fig. 49. Working copies in the form of RNA molecules are formed from segments of the DNA molecules (master copies) of the chromosomes. The gene is here pictured as a copiable segment of the DNA thread. These RNA molecules fold up with protein into corpuscles and accumulate first as a nucleolus (perhaps in this phase some nuclear synthesis occurs): they then pass through the nuclear membrane either to join with a cytoplasmic membrane to form part of the basophilic reticulum of a secretory cell 
or to form small clusters in a cell which retains its products (see later).

The demand for a particular product of synthesis by other cells, or its repression by cells already in production (Rose, 1952) which may be the factor determining the course of differentiation by stabilizing a pattern of synthesis, is symbolized by the feed-back arrows at $A$ (environment to cytoplasm) and $B$ (cytoplasm outwards). The presence of soluble unused products in the cytoplasm may ultimately suppress the production of the RNA copies required for their formation: used products (i.e. secreted or rendered insoluble or "wrapped up" in the cell) will not provoke this kind of feed-back against themselves.

Epidermal cells may be freer of this kind of control than other cells ( $p$. 146); their characteristic differentiated products seem to be produced because they possess free surfaces which face the environment. The various surface induced responses have been mentioned (p. 37), but it must be admitted we have little idea of how the results are produced.

We approach firmer ground when the problem of RNA participation in cytoplasmic synthesis is considered. The greater cytological detail made possible by electron microscopy has, on the whole, supported and extended the views of earlier microscopists.

When thin sectioning for electron microscopy first developed, glandular cells were immediately examined (see Haguenau, 1958, for review). The RNA-containing nucleolus and the basophilic deposits against the nuclear membrane appeared granular, the nuclear membrane itself doublelayered. A more important finding was that the basophilic areas of the cytoplasm contained a system of membranes covered with small dense particles of diameter 120-200 $\AA$ (Plate 10A). Palade (1955) surveyed a large number of cell-types and established the widespread distribution of these particles. Essentially similar findings were reported by Sjöstrand and Hanzon (1954) and Bernhard (Bernhard et al., 1951; see also Haguenau, 1958).

Most of the authors referred to above, recognized at once that the system of membranes-plus-particles must be related to protein synthesis and to be the origin of the cell fragments called microsomes. Palade and Siekevitz (1956) gave a clear proof of this by showing that on mechanical disintegration of liver cells the membrane system broke down into microsomes known to be rich in RNA and lipid and which consisted of smaller particlestudded vesicles (Plate 10B). They further showed that after removal of much lipid, the remaining material richer in RNA consisted largely of the dense particles. Their participation in protein synthesis has been further demonstrated by Zamecnik et al. (1956) and Simkin (1959) (Simkin and Work, 1958) who showed that the particles were common in preparations which most actively incorporated amino acids (see below). 
The internal structure of the microsome particles is not known. They contain both proteins and RNA but their X-ray diffraction patterns show little resemblance to the patterns given either by RNA or by mixtures of protein and RNA, which can only mean that the RNA is bound into the particles in a form different from what it assumes when free. This may be contrasted with the fact that the structure of isolated DNA is similar to its structure in vivo where it also exists in a DNA-protein complex. The microsomal particles resemble viruses in size, composition and in some respects function (it has been suggested that viruses may be microsomal particles gone wrong!), and the protein moiety dominates the structural picture in some plant viruses.

After fixation by freeze-drying, which may be considered to introduce a minimum of chemical change and a maximum of retention of material, the particles are less visible among the protein adjacent to the membranes (Hanzon et al., 1959). This suggests that in their dense, compact form, as seen after osmium fixation, the particles are an artifact. Their original form may be a more diffuse and extended particle, a condition which might render their function as linear templates more understandable.

Some details of a possible means by which the basophilic material, now identified as the granular, RNA-containing material seen in micrographs, might reach the cytoplasm were revealed by Watson (1954) who drew attention to the existence of small circular markings (diameter $500 \AA$ ) on the double-layered nuclear envelope. He suggested these markings were openings or "pores" in the double membrane which might permit nucleocytoplasmic interchanges. The particles may pass through the pores and, after associating with the external sheath of the nuclear membrane, enter the cytoplasm together with it to form a typical particlestudded membrane (Fig. 49).

The above description applies to glandular cells, i.e. to cells which produce protein for secretion. The account given above of the cells of the epidermis shows that protein synthesis may be associated with a different type of cytology from that of secretory cells, as was first clearly pointed out by Birbeck and Mercer (1957). The cells of the hair cortex, for example, contain vast numbers of dense particles of the same size and appearance as those noted in the pancreas and elsewhere; but they are not associated with a membrane system (Fig. 36) (Plate 11). They appear to be scattered in small, often well-defined clusters, throughout the cytoplasmic space. The cytoplasm is uniformly basophilic in these cells due to RNA, and there is no reason to doubt that here, too, the particles contain RNA and participate in the synthesis (see also p. 120). The cytoplasmic distribution of the two kinds of nucleic acid in the hair follicle is admirably demonstrated in Hardy's work illustrated in Fig. 90, p. 220. In the lower bulb, the germinal region, the DNA of the nuclei is obvious; at higher levels where 
cytoplasmic synthesis is active, the diffuse cytoplasmic RNA increases. (See also Braun-Falco (1958).)

The nuclear membrane of epidermal cells also is double-layered and exhibits pores (Plate 4C). There is perhaps a difference in the mechanism by which the RNA particles reach the cytoplasm since clearly they are not

\section{Type of cell \\ Disposal of protein}

Lifetime of synthetic phase

\section{Cytoplasmic*} features

Examples

\section{Secreting}

passed through cell membrane usually as a granule $S$

long, periodic

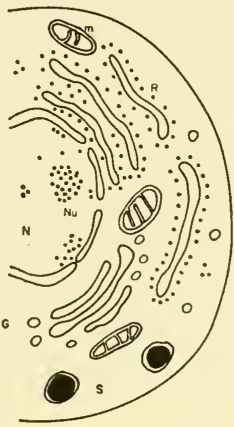

mitochondria $m$ Golgi cluster $G$, reticulum $R$ and bound particles $P$

Pancreatic and thyroid cells, etc., silk gland cells

\section{Retaining}

retained within

cell $(f)$

short, single phase

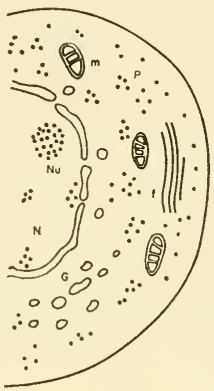

mitochondria $m$ Golgi cluster $G$ little reticulum, many dense free particles $P$

Epidermal cells, myogenic cells, early embryonic cells, anaplastic tumours

FIG. 50. Comparative characteristics of cells forming protein (*see also Fig. 49).

shed along with the external nuclear membrane to form a particle-studded cytoplasmic membrane as pictured for glandular cells. They may simply diffuse through the pores which are larger than particles or they may leave in the small vesicles $V$ to be seen in Plate 9.

There are many other cell-types with these same characteristics, and some, along with examples of cells of glandular cells are mentioned in Fig. 50. It will be seen that two classes of protein-forming cells are to be distinguished 
by: (a) their differences in fine cytology and (b) by the manner in which they dispose of their synthesized protein. In one case the protein simply accumulates within the cell until, perhaps by a kind of mass action or a simple physical exhaustion of space, synthesis is arrested. They appear capable only of a single burst of activity. We shall term such cells retaining cells (Fig. 50). In the other case, the product is discharged from the cell after which another cycle of activity and secretion is initiated. Such cells may continue their synthetic activities for a more or less prolonged period. (Birbeck and Mercer, 1961).

Secreting cells possess the more complex cytology since, in addition to their often elaborately-developed membrane system, they may possess specialized devices for the temporary accumulation, transfer and for the removal of protein from the cell. Since both types of cell synthesize proteins, the capacity to do this must reside in their common structural feature, the RNA particles of the cytoplasm. The membrane system is therefore secondary and appears to be associated with the prolonged activity and removal of secretion. Obviously for a cell to be able to produce many times its weight of secretion, some elaboration of structure is required to facilitate the entrance, transport and removal of material. An organization analagous to the production line of a factory might be expected, and in fact, such cells are typically polarized. One portion of their surface, the basal region, usually adjacent to the sources of raw material (blood vessels) becomes specialized as an input area; the opposite aspect, the apex, where secretion granules may collect and which usually abuts a storage space or lumen, is the output area. These requirements are the basis of the familiar histological pattern of glandular cells. Internally, as Porter has emphasized (1954), a system of interconnected membranes is admirably suited for the channelled diffusion of metabolites to and from active sites and also for the collection of the products of synthesis.

According to Porter and Palade the total cytoplasmic membrane system should be regarded as a unit, a definite cell organelle which may assume a variety of forms: flattened interconnected sacs, cisternae, canaliculi and isolated vesicles, for which the name endoplasmic reticulum is proposed. The qualifying adjective " endoplasmic" was suggested by observations on whole cells in tissue culture (Porter, 1954). Since subsequent observations on cells in sections have shown that membranes may spread throughout the cytoplasm, the simpler name " reticulum " may finally be adopted as more accurate. For nomenclature, see Fig. 23, p. 48.

Such a system of membranes enormously increases the internal surface available as sites for catalysts and is, in fact, precisely the sort of "cytoskeleton" long demanded by biochemists (Peters, 1937) as a structural support for an organized array of enzymes. The mitochondrion provides a similar, even more compact, bundle of membrane-supported sites. 


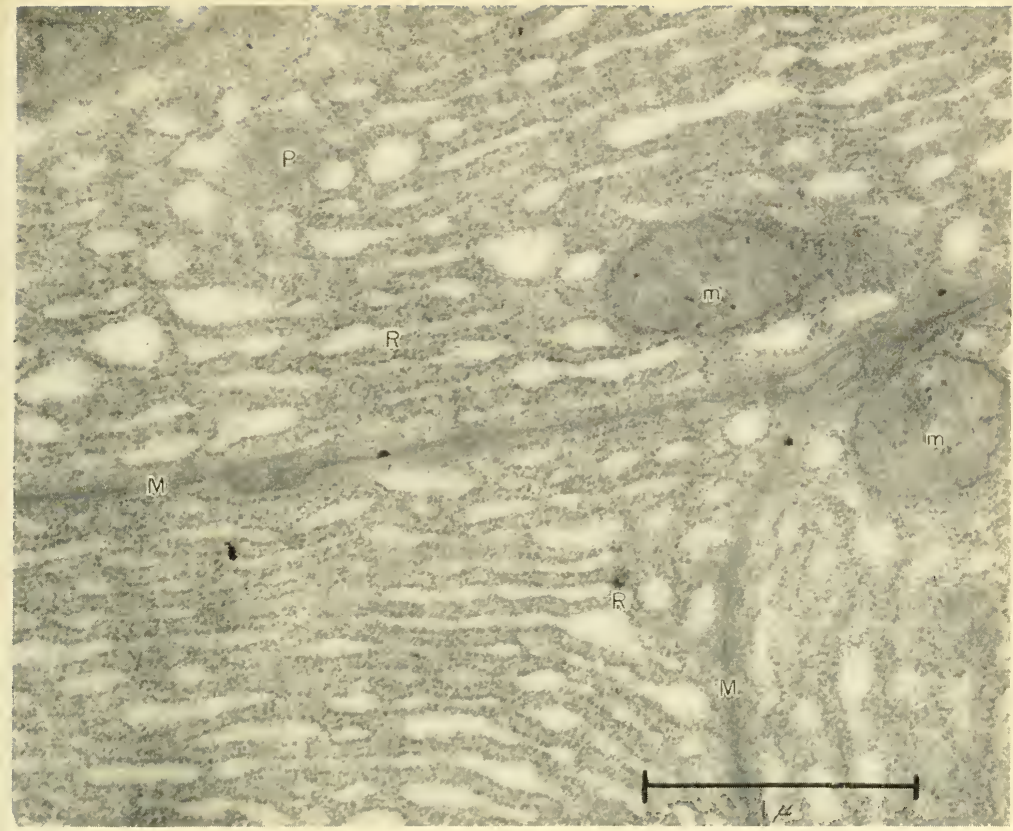

A

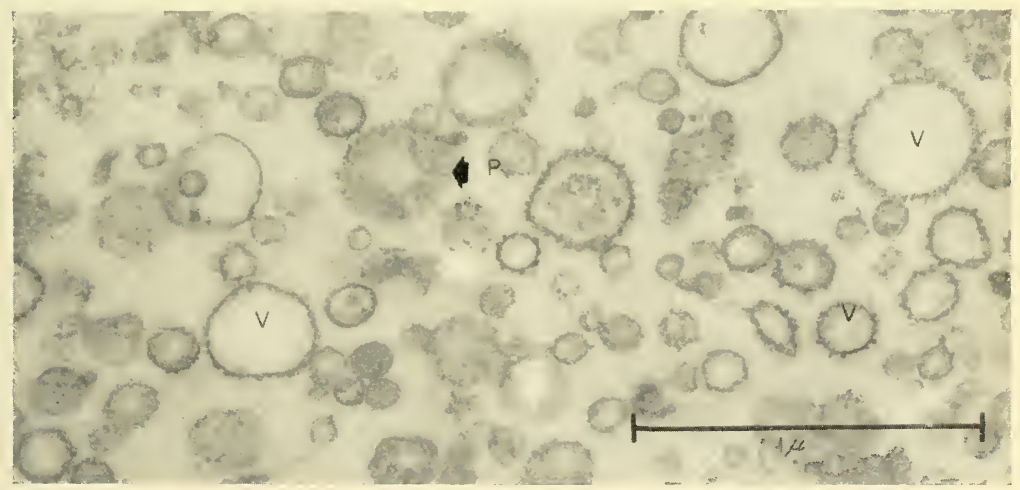

B

Plate 10 (Captions overleaf)

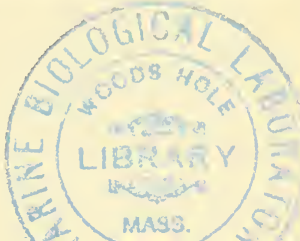




\section{Plate 10}

A. Portion of cytoplasm of a rat pancreas cell. A typical secreting cell (p. 110). $R$, reticulum of particle covered membranes; $P$, are RNP particles; $M$, cell membranes; $m$, mitochondrion.

B. Microsomes from rat liver cells homogenized and fractionated by centrifugation. Note many small, particle-covered vesicles derived from the break-up of more extensively-developed, particle-covered membranes in the original cells. $I$, vesicles, and $P$, particles.

Micrographs kindly supplied by M. S. C. Birbeck. 


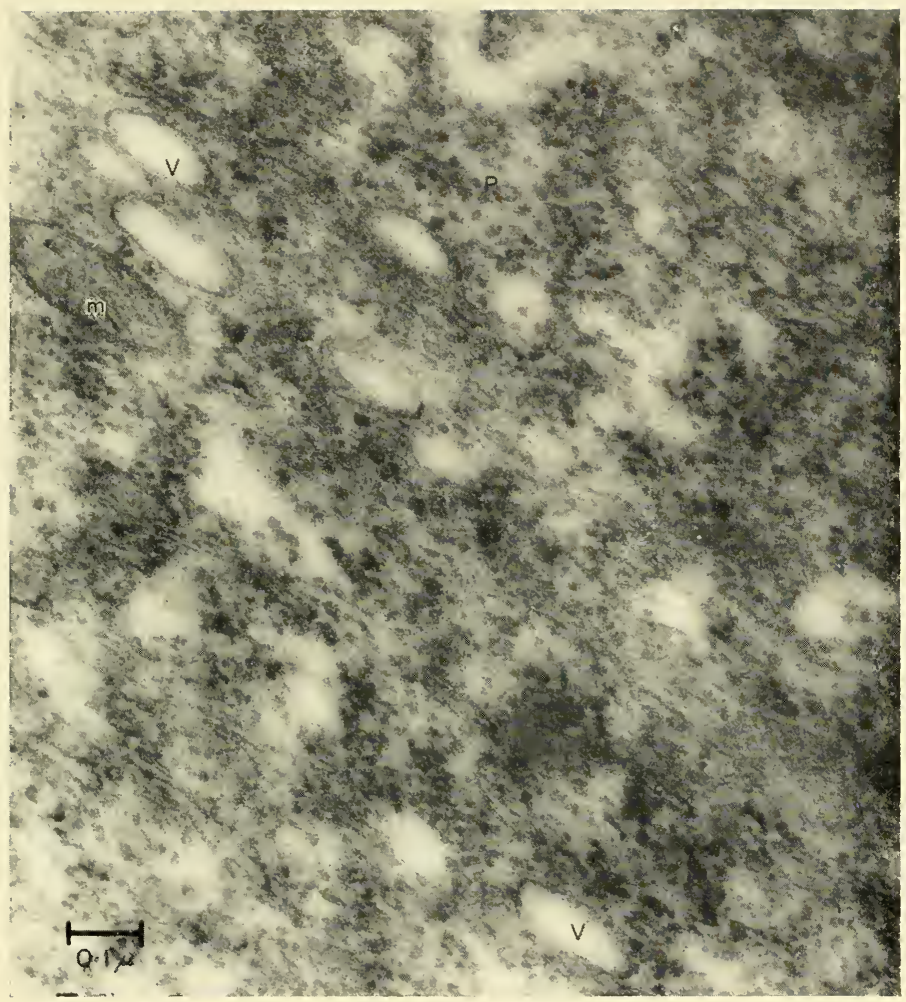

\section{Plate 11}

Portion of the cytoplasm of a cell in the bulb of the hair follicle before filaments of keratin have commenced to accumulate. The picture is typical of a retaining cell. There are small numbers of mitochondria, $(m)$, small vesicles, $V$, not obviously organized as a definite Golgi apparatus, and vast numbers of dense (RNP) particles $\mathrm{P}$ which are apparently free in the cytoplasm and not associated with membranes (Fig. 50, p. 112). 


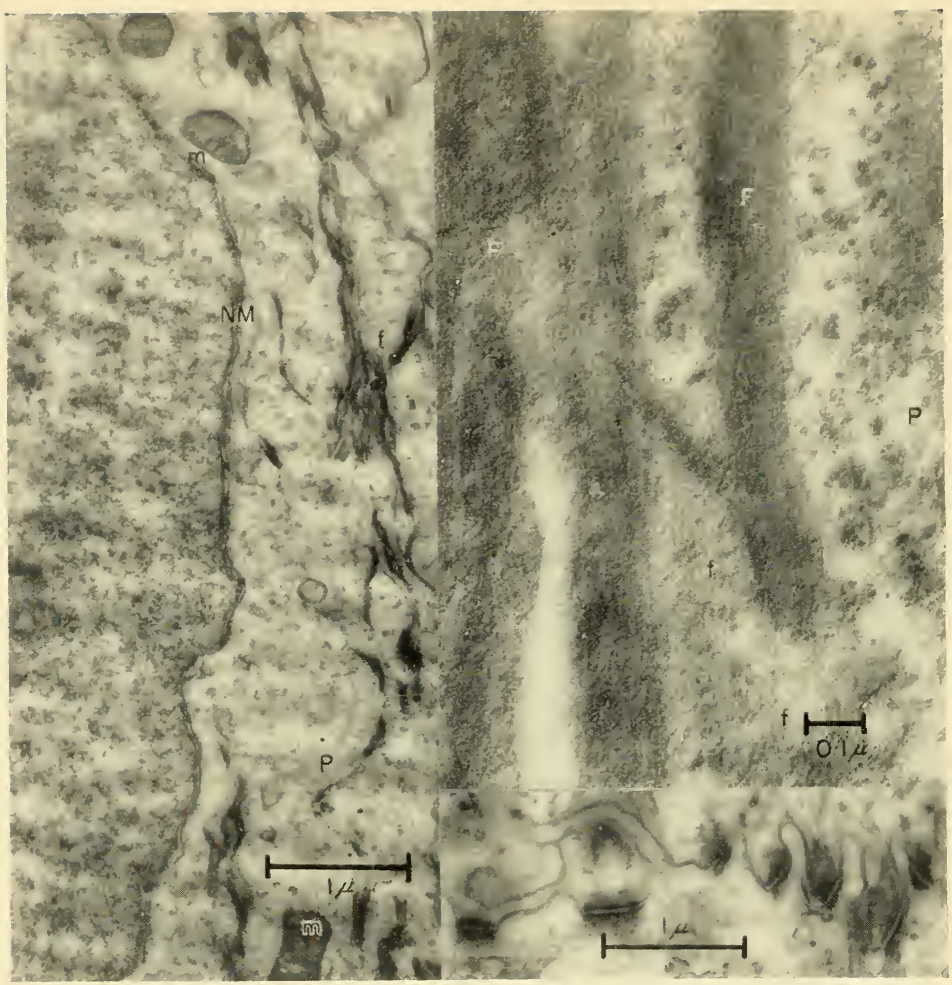

\section{Plate 12}

A. Portion of a typical epidermal cell forming a hard keratin, in this case from the feather follicle, showing the edge of a nucleus (1.h.s.), the double nuclear membrane, $N M$, mitochondria, $m$, and $f$, fibrils of fibrous keratin. The nucleus contains short lengths of filaments and the cytoplasm contains large numbers of dense particles $P$, not associated with membranes.

B. Fibrils, $F$, of fibrous keratin in the bulb region of the human-hair follicle. Each fibril is seen to be composed of fine filaments, $f$. Note the dense particles, $P$, which crowd the cytoplasm.

C. A number of desmosomes distributed along a very convoluted contact between two cells in the germinal layer of rat skin where the desmosomes are not yet associated with cytoplasmic filaments. Each desmosome consists of a paired thickening of the plasma membranes backed in each cell by a dense amorphous deposit. 
Retaining cells do not possess a reticulum in association with RNA particles, but numerous smaller vesicles with smooth-surfaced membranes, i.e. membranes not associated with dense particles, are observed (Plate 11). In secreting cells such vesicles also occur, often with an elongated flattened profile, in compact clusters located adjacent to and distal to the nucleus (Fig. 49). This location is that of the Golgi apparatus of the classical histologists, who have long disputed its structure and function (Baker, 1955); very probably this group of smooth membranes is the electron microscopic image of the Golgi apparatus. A certain unity of organization and coherence is suggested by the fact that the clusters can be separated from homogenized cells (Dalton and Felix, 1956), but the greater structural detail revealed by electron microscopy has not finally clarified their function. No connexion with protein synthesis has been demonstrated, though the opinion prevails that they are associated with the secretory phase and probably it is the cell centre for membrane assembly when these are required for special functions.

\section{Biochemistry of Protein Synthesis}

The combination of the results given by the various forms of microscopy has yielded a sufficiently-detailed and usable picture of the intracellular structures associated with at least one pathway of protein synthesis. Unfortunately, in the present state of histochemistry, it is impossible to investigate the composition and activity of the cellular organelles with the same degree of resolution. For most biochemical work, a considerable weight of any cell derivative is required and this has led to the need to separate from large numbers of cells a sufficient weight of well-defined, selected cellular constituents, such as nuclei or mitochondria for analysis. The most commonly-employed procedure introduced by Claude (1938) is that of homogenizing and fractioning a mass of cells, i.e. of rupturing the cell membranes mechanically and fractionating the homogenate by centrifugation. Most of the work on mammalian tissues has been carried out on the liver, since this large organ is easily homogenized, and nothing comparable has been attempted with an epidermal tissue.

When liver is homogenized mechanically and the product suspended in a sucrose solution $(0 \cdot 25-0.88 \mathrm{M})$ four fractions are conventionally recognized in a fractional centrifugation: (a) a nuclear fraction, (b) a mitochondrial fraction, (c) a microsome fraction, and (d) a supernatant. Such fractions form the basis of most biochemical studies on the activity of the intracellular elements recognized microscopically. The fractions are by no means pure, nor do they consist necessarily of single components. It is preferable to control their composition by electron-microscopical methods (e.g. Palade and Siekevitz, 1956).

A study of the mitochondrial fraction has shown that many of the 
enzymes associated with the oxidative degradation of sugars, etc., are located here. The internal organization of the mitochondrion (Fig. 22a, p. 45) seems admirably designed as a site for the organized array of these enzymes which seem necessary to effect the sequence of reactions envisaged in multi-enzyme reactions.

The mitochondrion is also a seat for the synthesis of low molecular weight key substances such as adenosine triphosphate (ATP) which, because of the so-called " high energy bonds" they contain, are able to effect many biochemical reactions demanding an expenditure of energy. Probably among these reactions is the formation of the peptide bonds linking amino acids in the polypeptide chains in proteins.

Fischer's original suggestion that proteins were polypeptides containing peptide bonds formed by the reaction:

\section{$\mathrm{R}_{1} \cdot \mathrm{CH}\left(\mathrm{NH}_{2}\right) \cdot \mathrm{COOH}+\mathrm{R}_{2} \cdot \mathrm{CH}\left(\mathrm{NH}_{2}\right) \mathrm{COOH}$$$
=\mathrm{R}_{1} \mathrm{CH}\left(\mathrm{NH}_{2}\right) \cdot \mathrm{CO} \cdot \mathrm{NHCH}\left(\mathrm{R}_{2}\right) \mathrm{COOH}+\mathrm{H}_{2} \mathrm{O}
$$

has been amply confirmed both by degradative and synthetic methods. The free energy necessary for the synthesis of a peptide bond has been determined from thermodynamic data on reactants yielding peptides or compounds containing peptide links. It lies in the range of $2000-4000 \mathrm{cal} /$ mole. From the corresponding equilibrium constant, it may be calculated that this value requires in equilibrium $99 \%$ of the material on the side of hydrolysis. Obviously energy must be introduced into the system for synthesis to approach completion.

A method of moving the equilibrium in the direction of synthesis by selecting reactants which would yield insoluble products was devised by Bergmann and Fraenkel-Conrat (1937). In the presence of a hydrolytic enzyme such reactions will move in the direction of synthesis as the product is removed from solution. At the present time a synthesis catalysed by proteolytic enzymes is not thought probable. Nevertheless, in the formation of an insoluble protein such as keratin, the reaction could conceivably be assisted by the removal of the product in the form of insoluble fibrils, etc. That peptide-bond synthesis requires energy has been repeatedly emphasized on biochemical grounds (Borsook, 1955). Siekevitz (1952) showed that the uptake of "tagged" amino acids in homogenates is more closely linked to phosphorylation than to direct oxidation. Zamecnik and Keller (1954) showed that incorporation of amino acids into microsomes proceeds only in the presence of an ATPgenerating source. In the cell ATP generation is a function of the mitochondria and they are thus shown to be indirectly involved in peptide formation. Borsook (1955) showed that for each peptide bond formed one molecule of ATP is broken down.

Nevertheless the amount of energy involved is not large in comparison 
with that required for certain forms of mechanical work, e.g. that required by muscles or for transferring substances across membranes against a concentration gradient, and in fact one finds that the number of mitochondria in muscle cells or in the proximal tubular cells of the kidney is much greater than that in protein-forming cells.

The observation that ATP is implicated in peptide-bond formation suggests that an activated amino acid is probably the intermediate in the synthesis. Haogland et al. (1957) have obtained evidence of enzymes which could effect activation. Enzymes which catalyse carboxyl activation of at least two amino acids (tryptophane and tyrosine) are now known, and probably there is an enzyme for each amino acid, i.e. according to Crick (1958) twenty in all. The product of the reaction between the amino acid, the activating enzyme and ATP is an amino acid-adenosine monophosphate anhydride and it is supposed that compounds of this type are intermediates (see Fig. 51).

The reasonable expectation that the long peptide chains would be proceeded by the formation of short peptide sequences seems to be negatived by several experiments. Small peptides are rarely found and ingested peptides cannot be directly utilized, but are first broken down to amino acids. There is, however, some confusing evidence on this point which cannot be considered finally settled.

Many efforts have been spent tracing the fate of labelled amino acids when these are injected into animals or fed to micro-organisms. The newly-formed protein is customarily isolated as a trichloracetic acidsoluble fraction from homogenized cells. If the liver, for example, is examined very soon after the administration of a labelled acid, the isotope is found predominately in the microsome fraction. Siekevitz (1952) found that incorporation of radioactive amino acids also occurred in cell-free homogenates and that the radioactive label was again predominantly in the microsome fraction. Further, a microsome preparation is effective when cell sap is added, or if even a partially-purified mixture or activating enzymes providing an energy source (ATP or GTP) is also added.

The work of Zamecnik et al. (1956) shows further that the amino acid is activated by the specific enzymes and ATP before incorporation and it is probable that the activated acids are first transferred to a soluble RNA (S-RNA). The transfer of this amino acid from soluble RNA to the RNA particles requires the presence of guanine triphosphate. The microsomal particles contain protein and the labelled acids are linked by true peptide bonds. These steps are summarized in Fig. 51, p. 118.

Other experiments have shown that the material bound to microsomal particles can be dissociated from it and appears in new protein. Rabinovitz and Olson (1957) prepared reticulocytes containing radioactive leucine and incubated the microsomes isolated from these with fresh sap. In the 
presence of ATP the radioactivity was partially transferred to the haemoglobin fraction. Hendler (1957), and Simkin and Work (1958) have reported similar findings. Fig. 51, adapted from Stephenson et al. (1959), summarizes the several stages of protein synthesis as outlined above.

1. Activation of Amino Acids

$$
\mathrm{AA}+\mathrm{ATP}+\mathrm{E} \rightleftarrows[\mathrm{AA} \sim \mathrm{AMP}]-\mathrm{E}+\mathrm{PP}
$$

2. Addition of Nucleotide End Group to S--RNA

$$
-\mathrm{RNA}+\mathrm{ATP}+\mathrm{CTP} \rightleftarrows[\mathrm{RNA}-\mathrm{pCpCpA}]+\mathrm{PP}
$$

3. Binding of Amino Acid to this RNA

$$
\mathrm{AA}+\mathrm{ATP}+[\mathrm{RNA}-\mathrm{pCpCpA}] \rightleftarrows[\mathrm{RNA}-\mathrm{pCpCpA}-\mathrm{AA}]+\mathrm{PP}
$$

4. Transfer of Amino Acids to RNP Particles, Binding to RNA of Particles Followed by Polymerization of Amino Acids

$$
\begin{gathered}
{[\mathrm{RNA}-\mathrm{pCpCpA}-\mathrm{AA}]+\mathrm{ATP}+\mathrm{GTP}+\mathrm{RNP}} \\
\quad \text { (Particles) }+ \text { Soluble fraction (?) } \\
\rightarrow \text { [polypeptide chain - particle }]
\end{gathered}
$$

5. Release of Bound Polypeptide and Completion of Protein by Crosslinking and Secondary Bonding

AA amino acids

E enzyme (many specific enzymes known)

PP pyrophosphate

CTP Cytosine triphosphate

S-RNA “ soluble " RNA

$\mathrm{pCpCpA} \mathrm{nucleotide} \mathrm{end} \mathrm{group}$

[ ] units linked as complex

Fig. 51. A current scheme showing possible steps in protein synthesis.

A special problem, arising in cells which are secreting their formed protein, is the relation of the microsomal RNA particles to the system of membranes found in these cells (see Fig. 49). Probably the microsomal protein is released to the membranes and accumulates within them (Palade and Siekevitz, 1956) as can often be noted in micrographs. In keratinforming cells and other retaining cells, membranes are not involved and the newly-formed protein appears to be released directly into the cell sap (Plates 9 and 11). 
Secondary and Tertiary Structures

While a partial answer to the problem of peptide formation seems in sight, a more formidable problem yet to be solved is that of specificity, i.e. the formation of precise sequences of amino acids in the polypeptide chain and the folding of these chains into equally precise three-dimensional structures. Whether keratin, a protein whose function is a more or less passive mechanical one, will actually prove to have a specificity as clearly defined as an enzyme has not yet been proved by an actual determination of an amino acid sequence. It is, however, antigenic (Pillemer et al., 1939 and 1938) and in well-crystalline forms (feather and porcupine quili) it yields an X-ray pattern suggesting a complexity not inferior to that of soluble proteins (Chapter 5).

Following a suggestion by Haurowitz and by others, it seems reasonable to think that the final assembly of amino acids takes place in two steps: (1) the formation of the definite polypeptide sequence (or sequences) on a template and (2) the folding of the polypeptide to form a three-dimensional molecule (Fig. 51, Steps 4 and 5). The attack on this problem is at the moment largely speculative. Most writers assume that the sequence of bases along a nucleic-acid helix somehow ultimately determines the sequence of amino acids, and attempts to solve the problem, ranging from biochemical experiments to abstract considerations based on coding theory, are being made. The general feeling is that the microsomal RNA is the most likely candidate for a template on which to assemble the amino acids in the correct order and Crick has advanced further arguments to show that an "adaptor molecule" is also necessary to hold the activated amino acid on the template. In the absence of experimental evidence it is not easy to carry this discussion further, but reference may be made to Crick's article (1958).

The problem of the second step, the folding of the long polypeptide chain into a specific configuration and the overall shaping of the molecule, has been illuminated experimentally by the work on the lability of protein configurations in solution and the dependence of both synthetic and natural polypeptides on the interaction between the solvent and the chain. This work will be returned to later (p. 194). What is important is that this step seems to require no enzymatic or nucleic acid intervention; it depends simply on the energy relations of the interactions between side chains of the macromolecule and the molecules of the solvent or other associated molecules Since the side-chain composition of a polypeptide chain is determined in the primary act of synthesis (assembly on a template) the configuration ultimately assumed by the molecule in a given medium (cell sap) will be determined at the same time and by the same means. 
Experimentally, the fact that no special intracellular apparatus is necessary to induce the polypeptide chains to adopt the specific foldings which are responsible for the characteristic fibre patterns, is evident since both proteins and synthetic polypeptides (Bamford et al., 1949, 1956) form the structures spontaneously in solutions in vitro. Newer methods of demonstrating the presence of specific structures in solution (p. 194) have shown that only portions of the chains may adopt the folded form. This probably also occurs in vivo and, surviving into the solid state, is probably one of the sources of the non-crystalline fraction (Chapter 5).

\section{Synthesis in Retaining Systems}

Most of the evidence described above relating to protein synthesis has been obtained from secretory cells; retaining cells such as epidermal cells have been less studied. Their cytoplasm is, however, as rich in RNA and, clectron-microscopically, it is crowded with dense particles (Plates 7, 9, 11 and $12 \mathrm{~A}$ and $\mathrm{B}$ ) apparently identical with the RNA-containing particles of secretory cells. It is perhaps desirable to mention the experimental evidence which shows that in cells with this cytological pattern that synthesis also involves RNA and up to a point is identical with the process in secretory cells. Reticulocytes, which synthesize and accumulate haemoglobin becoming erythrocytes, are retaining cells and in them the course of synthesis seems to follow the lines indicated above. Bacterial cells, although less easily classified, resemble in some respects the retaining cells of higher organisms and in them protein synthesis also follows the same course (Loftfield, 1957).

The same general picture of diffuse cytoplasmic basophilia in the light microscope and of vast numbers of dense particles with a scanty development of membranes, when seen in the electron microscope, is found in the cells of certain anaplastic tumours. These again lend themselves more easily to biochemical study and make it possible to confirm that synthesis is associated with the particulate elements of the cytoplasm. Littlefield and Keller (1957) for example, using ascites tumour cells showed that the most rapid incorporation of radioactive leucine occurred in the cell fraction containing the dense, ribonucleic acid-containing particles.

There is then no reason to doubt that protein synthesis in retaining cells in general follow the same course as in the more commonly studied secreting cells. But whereas in secretory or glandular type cells protein synthesis continues more or less indefinitely in a succession of cycles, the products being (continuously or on demand) removed from the cell, in accumulating cells there is a single phase, the product is retained within the cell and synthesis comes to a hait as the cell fills, possibly as a result of mass action or the simultaneous production of an inhibitor. Such cells then 
enter on a more-or-less prolonged life in which their activity is of a different order. They perform some specialized function based upon the properties and behaviour of their accumulated products, e.g. muscle cells accumulate actomyosin and their subsequent functional behaviour is based on the contractile properties of this substance; the reticulocyte becomes the mature erythrocyte filled with haemoglobin and enters the blood stream as an oxygen carrier; the cells of the stratum corneum harden and form a protective layer to the organism. In this phase their RNA content (dense particles) falls.

\section{Synthesis in Fibre-Forming Systems}

No comparable biochemical studies have been made on cells whose function is the formation of protein fibres. Nevertheless, a survey of the cytology of such cells reveals their essential similarity to that of cells which produce soluble, non-fibrous proteins (Mercer, 1958). The same distinction exists between cell systems secreting a precursor of the fibre, which then forms extracellularly, and systems in which the fibres form intracellularly. The cells secreting fibres have as before elaboratelydeveloped particle-studded membranes of which perhaps the most developed examples are the cells of the silk gland of the silk worm (Bombyx mori) (Mercer, 1958). The cells of the hair cortex are, of course, the typical retaining cells. The dense particles found in all fibre-forming cells have the same range of dimensions $120-200 \AA$ as those found in cells forming soluble proteins, and may be identified on the same grounds as particles containing RNA. Histochemical tests confirm this picture (see p. 220).

Further, no special cytological features are found to be associated with the production of fibres having the different basic types of molecular structure indicated by X-ray analysis of fibres. The $\alpha$-type proteins occur intracellularly as keratin or myosin (Astbury, 1947) in retaining cells, and extracellularly as fibrinogen (Bailey et al., 1943). The secreted fibres (collagen-type) and fibroin ( $\beta$-type), originate in cells having the familiar pattern of membranes and particles of the secreting cell. Thus there would seem every reason again to associate the RNA system only with the original link-up of amino acid in polypeptide chains and to consider that the specific folding determined by this sequence occurs after the release from the RNA particle. The subtle, genetically-determined differences between an RNA particle producing a collagen sequence and one producing an $\alpha$-keratin sequence remain unknown.

The novel feature about a cell, which produces a protein capable of forming a fibre, is not then to be sought in the basic machinery used to effect the synthesis which is common to all, but rather in the geneticallydetermined instructions or information associated with the RNA particle. 
These instructions determine the sequence of amino acids and this sequence determines the sort of intermolecular interaction which follows. Some types of sequence produce strong intramolecular chain association and a corpuscular type molecule with weak forces of intermolecular association; these may form proteins soluble as discrete particles. Other sequences may lead to the stronger form of intermolecular association we recognize as fibrous. The several means by which protein fibres are synthesized are classified in Fig. 52.

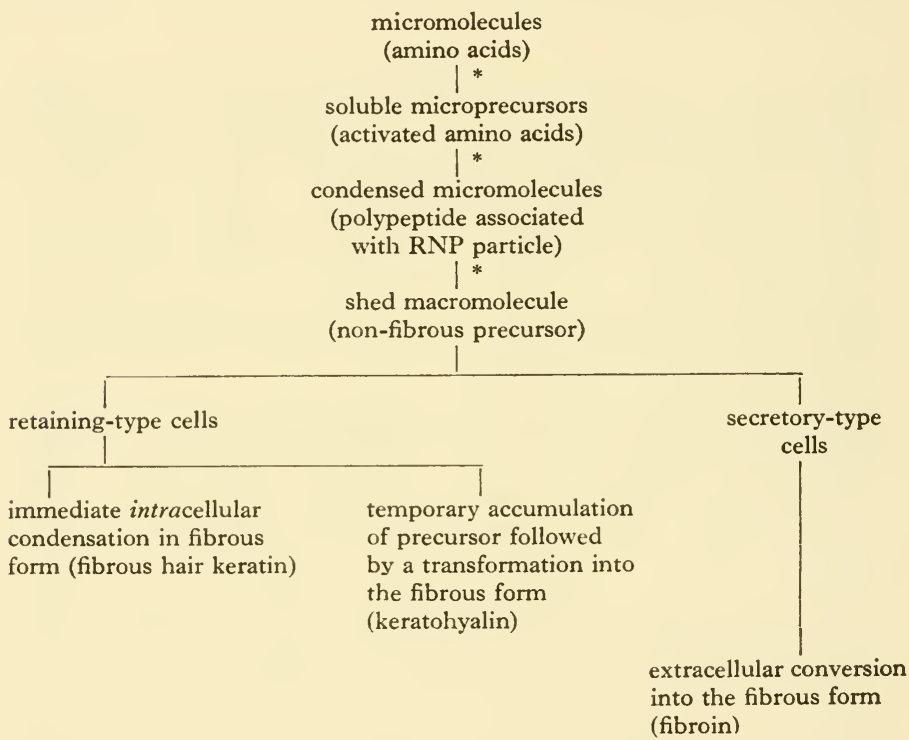

Fig. 52. Classification of types of protein fibre synthesis.

(*See also Figure 51.)

Epidermal cells provide examples of most of these methods of forming fibres. In the hair cortex and in the feather, filaments seem to appear directly without the accumulation of substantial amounts of macromolecular precursor; in the inner root sheath of the hair follicle trichohyalin accumulates as a precursor and undergoes a transformation into fibrils. Skin cells may employ both methods at different times (p. 228). The hair-cuticle cells are peculiar in that the keratin appears as amorphous droplets and condenses without changing into fibrils. These cells remain non-birefringent and yield no defined X-ray pattern. Among birds, 
keratin may even be secreted. The cells lining the gizzard produce a horny lining of keratin (p. 108) and the oviduct covers the egg with a mixed mucin and keratin layer which forms the tough membranes immediately underlying the shell (Plate 18B).

\section{The supermolecular organization of fibrous tissues (tertiary structure)}

\section{Macromolecular Fibrous Texture}

We have up to this point been considering those aspects of the synthesis of fibrous proteins which they share in common with the soluble proteins. However, the special characteristic of fibres is the supermolecular aggregates which they form whose dimensions may extend from the molecular to the histological level and beyond. These aggregates often display a remarkable and intricate structure at several levels and it is a further problem to give an account of the genesis of this larger-scale organization.

Fibres usually perform a mechanical function; i.e. they transmit tension, strengthen membranes or provide against impact, and their organization within tissues is normally related to these functions. In fact some of the most striking examples of biological adaptation are provided by the fibrous tissues. It is more useful in this connexion to speak of a fibrous texture rather than of fibres and thus to focus attention on the microscopic rather than the macroscopic elements of structure.

In a purely geometrical sense we may list the possible varieties of fibrous texture as in Fig. 53.

Organisms exploit all these possible arrangements and it is not usually difficult to relate the textures to the mechanical function. The difficulty is to account for the origin of the structure in molecular terms, particularly in instances where it appears apparently in advance of function.

When studying a complex organism in its structural or functional aspects, it is convenient to distinguish a hierarchy of levels of organization. At each level new possibilities of organization are introduced and the study of the structure as a whole is facilitated if the events at each of the several levels can be considered separately. Fibrous tissues lend themselves to this form of analysis. They exhibit, as Astbury has put it, " patterns within patterns". In this sense we shall distinguish here three levels: the moiecular, the macromolecular and the microscopic level, which happen also to be those which correspond to structures of orders of magnitude most easily studied by X-ray crystallography, electron microscopy and light microscopy respectively (Fig. 1); but, although convenient, there is no significance in this correspondence, which is in any case inexact and not lasting, since the domains of X-ray diffraction analysis and electron microscopy increasingly overlap. The structures existing at each level are 
produced by causes which we might group together generally as directive activities operative at that level and the structures themselves are a record of these activities from which we may hope to infer something of their nature.

\section{Texture}

Tangles (threedimensional)

\section{Possible function}

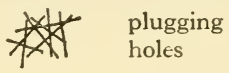

holes

\section{Example}

blood clots (fibrin)

epidermis

Sheets with rodlets in one plane and disordered in other senses

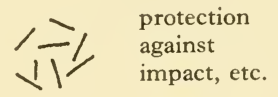

as membranes

protective

membranes, retaining basket (allows movement by distorting mesh)

membrane rigid against distortion

transmission of mechanical tension

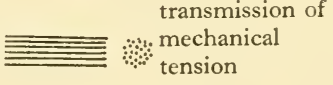

occurs only transiently

earthworm cuticle (Reed and Rudall, 1948), amphibian dermis (Plate 14B)

peritrophic membrane of insects (Mercer and Day, 1952)

hairs, tendons, muscles, etc. in three dimensions (various arrangements in cross-section)

Fig. 53. Types of fibrous texture.

An apt analogy is often made between fibres, yarns and fabrics on the one hand, and molecules, fibrils and tissues on the other. In this vein we may speak of the spinning and weaving of molecular yarns and fabrics from the raw material provided by the primary synthesis at the molecular level. This primary synthesis we have already discussed, our problem here is the spinning and weaving of the yarns and fabrics which we shall term fibrogenesis and fibrillar organization (Fig. 54). More precisely we wish to consider firstly the formation of the long fibrils (or ribbons) which are used as the constructional units of the tissue and, secondly, the forces which organize (or weave) these fibrils into more complex formations and stabilize them. 
In histological systems, unlike those found in the textile mill, " spinning and weaving" usually occur together in time and space, i.e. the fibrils appear precisely where and when they are required to enter into the structure of the growing fabric. However, it may be possible to separate the two processes artificially if the precursor of the fibrous system can be

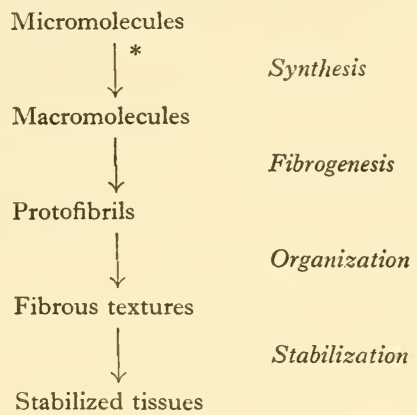

FIG. 54. Formation of secondary and tertiary fibrous structure (*see also Fig. 51 for this step).

isolated, either before it has been converted into fibres or by reversing the process of fibre formation. In this case it may be possible to study the formation of fibrils in vitro and, by artificially orienting these in imitation of the natural system, to gain some insight into the processes which must be operating to orient them in vivo.

\section{FibRogenesis}

Fibrogenesis (Fig. 54) is by definition the formation of an elementary elongated unit from which the more complex formations are constructed. An appropriate name for such a unit, which indicates that it is the first fibrous unit in a hierarchy of such structures, is the protofibril. Electron microscopy definitely established the existence of protofibrils in many fibre tissues although their presence had earlier been inferred from X-ray diffraction and polarized-light studies. Protofibrils form the structural basis of some fibrous keratins; their appearance and condensation into macrofibrils and more massive formations has been described in the hair and feather follicle. Unfortunately, valuable though these morphological observations are, in the case of keratin they cast little light on the physicochemical processes involved. Information concerning these processes could certainly be obtained if it were possible to study the phenomenon 
in vitro. To do this it would be necessary to isolate a precursor in a form still capable of forming fibrils, either from the germinal tissues of the epidermis or from the fibres themselves, by reversing the process of fibrogenesis. Unfortunately, largely as a result of the chemical reactions involved in keratinization (Chapter 6), it has proved impossible to redissolve keratin without gross modifications of its structure. Further, it has also proved impossible to separate the unaltered precursor from the germinal cells.

For these reasons our views on fibrogenesis in keratin must be based on reasonable inferences drawn from a study of more tractable systems and checked against a background of direct observation of cellular events.

Logically we may distinguish two methods of fibrogenesis: either (a) a macromolecular precursor is formed first and subsequently aggregated to form a fibril; or (b) micro-units, e.g. amino acids are directly built into the growing fibril and no macromolecular precursor is involved. In many fibrous systems we know experimentally that (a) is the actual course followed and it may well always be the case. No macromolecular precursor can be demonstrated for the fibrous hard keratins; but while admitting that we do not know precisely how polypeptides are formed, we know that it involves the participation of other large molecules, such as RNA particles, and it is difficult on spatial grounds to see how these large bodies-larger in diameter than the filaments themselves-can be brought into position at the growing points of the filaments. Furthermore, no close association of particles and fibrils is in fact observed in the cells of the hair follicle (Plate 11). Thus it may be concluded that a soluble precursor exists transiently. 'The considerable quantities of amorphous protein demonstrable electron-microscopically after special staining may, in part, represent this precursor.

In a general sense, we can anticipate that the nature of the aggregation is likely to be much influenced by the shape of the precursor molecule, "interaction profile" (Hodge, 1960), or by modifications in its shape which accompany fibrogenesis. In some systems a more-or-less isodiametric molecule may simply aggregate without marked internal change, and the process is then very similar to crystallization (see also Rees, 1951). In others, preliminary modifications of structure precede aggregation as is the case with the fibrinogen-fibrin system (Lorand, 1952; Lorand and Middlebrook, 1952). Other unexpected, even bizarre events should not be ruled out. For example, Rudall (1955-6), in attempting to trace out the development of the fibrous ribbons of the egg case of a mantid, discovered that lumps of precursor are first formed into vacuolated droplets which are thinned, flattened and drawn out to yield the fibrous ribbons. It is obviously necessary to treat each case as a special case, if this is at all possible. We can only review very briefly a few examples of systems which 
have been extensively studied and draw what inferences we can about the formation of keratin.

Collagen. By far the best understood case is that of collagen. As was well demonstrated by Nageotte (1927), collagen is an admirable protein for research on fibrogenesis since its solution in weak acids can very readily be made to reform fibres. Today largely owing to the work of Schmitt and Bear and their associates (1955 and 1960) and of Randall and Jackson (1956) (Randall and Robinson, 1953) it has become the prototype model for studies of morphogenesis at the macromolecular level. Not only may it be readily dissolved and regenerated in fibrous form; it also yields, when conditions are changed, a remarkable variety of fibrous fabrics some of which are not found in nature (Hodge, 1960). The precursor is synthesized by fibroblasts and secreted as a soluble molecule into the intercellular space where it proceeds to form fibrils and fabrics which are exquisitely adapted to the demands of the mechanical forces operative at that point (see Plate 23B).

Soluble collagen, or tropocollagen, has been shown by light scattering (Boedtker and Doty, 1956; Cohen, 1955) to have a long $(3000 \AA)$ thin $(13.6 \AA)$ molecule composed of three helically coiled chains, stronglyH-bonded, of molecular weight about 345,000 in Stainsby, 1958). This long molecule is able to aggregate, principally by lateral adhesion, to yield a variety of structures in addition to that normally found in native collagen fibres and each is recognized electron-microscopically by its banded structure. For recent summaries, see Bear (1952), Robinson (1953), Randell et al. (1953) and Hodge (1960).

The possible arrangements of both intact and partially fragmented molecules have been very fully worked out and are shown diagrammatically in Fig. 55. It is characteristic of the collagen type of lateral aggregation of long thin precursors that there should be large well-marked longitudinal repeat spacings detectable by $\mathrm{X}$-rays or electron microscopy, and an absence of side spacings larger than that corresponding to the molecular diameter. The existence, in the X-ray patterns (see Chapter 5) of wellformed examples of fibrous keratin, of strong long-spacings on the equator, i.e. side spacings (Tables 9 and 10, p. 167), and the absence of the lower orders of the main longitudinal repeat pattern, suggests that the collagen model is not immediately applicable to keratin. Electronmicroscopically, the keratin protofibrils are seen to be thicker $(60 \AA)$ than the collagen unit, and of indefinite length (at least $2000 \AA$ long), and no marked longitudinal spacing is visible electron-microscopically, although long spacings expressible as orders of a major spacing of $198 \AA$ (hair) and $98 \AA$ (feather) are found in X-ray diffraction patterns (Chapter 5).

The aggregation of filaments to form muscles seems in principle to be very like that involved in collagen formation (Hodge, 1959 and 1960). 
Fibrous insulin. An example of fibre formation from a more nearly spherical type of molecule is provided by fibrous insulin ( $\mathrm{F}$-insulin) which has been extensively investigated by Waugh (1954). Insulin molecules are small and display a marked tendency to form small aggregates in solution. When acid solutions are heated, the insulin separates out in the form of long stiff fibrils (Farrant and Mercer, 1952); the suspension displays strong birefringence and may gel. Oriented fibre-type X-ray patterns can be obtained (Bear, 1955). The reaction is reversible in alkaline media and soluble, biologically-active insulin may be regenerated. Since the insulin molecule is cross-linked internally by disulphide bridges, it is unlikely to be grossly distorted when entering the fibril. All these observations suggest a simple aggregation of the insulin units.

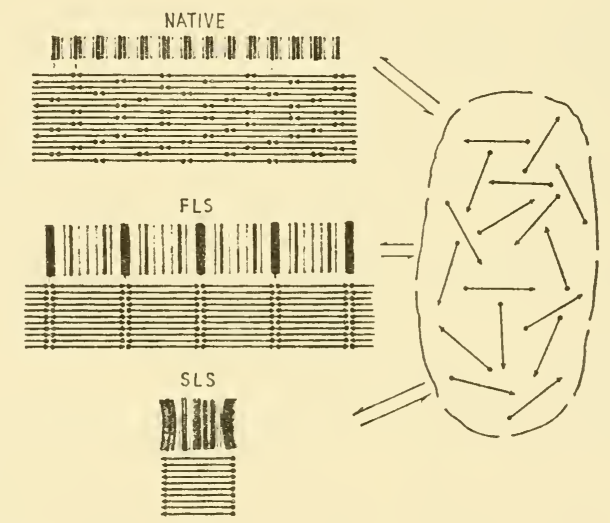

FIG. 55. Diagrammatic illustration of patterns of aggregation of tropocollagen macromolecules in native, FLS and SLS types. Polarization of macromolecules indicated by arrow. (After Schmitt, 1958).

There is an important general geometrical principle, pointed out by Crane (1950) that the structures which result from the successive addition of asymmetrical units are always helices. The linear aggregate formed by the addition of large molecules should conform to this principle, and accordingly we may expect to find that many protofibrils are helices. Pauling (1953) has described F-insulin as a helix of this type and it would seem not unlikely that some of the coiled-coiled models proposed for keratin, which appear as fine filaments in electron micrographs, have such an origin (Chapter 5). 
Silk fibroin-aggregation of the molecule after unfolding. Another model for fibrogenesis, suggested by its analogy with spinning, supposes that aggregation is preceded by an unfolding of the molecular chains composing the precursor particle. 'This process seems probable in cases where fibreformation is produced by a mechanical process of extrusion and drawing, as when artificial fibres are manufactured from viscous solutions of cellulose derivatives or of dissolved keratin derivatives. Nevertheless, it seems unlikely that it can occur generally in biological systems where fibrils may appear and disappear reversibly with a slight change in variables. Often a mechanical factor analogous to drawing is absent. While drawing may orient fibrils once formed, i.e. in fibrillar organization, it seems to play no role in fibrogenesis itself.

The formation of silk fibre (fibroin) by silkworms seems at first sight an example of fibre formation by drawing. A soluble precursor of the fibrous form is produced in cells, which are rich in RNA and contain an extraordinary development of particles and membranes (Mercer, 1957); it is stored as a strong viscous solution in a dilated portion of the silk gland, and is converted into a thread by being extruded through a fine spinerette. Nevertheless, if the contents of the silk gland are diluted with water and allowed to stand for some hours, masses of fine fibrils separate spontaneously from the solution (Mercer, 1951c). Fibrogenesis thus again seems to be a spontaneous phenomenon of aggregation requiring no mechanical assistance, but the extrusion and drawing occurring during spinning are responsible for the orientation of the protofibrils.

\section{Organization of Fibrous Tissues}

Assuming that the basic fibril has been formed, we have now to consider the means by which this is used as a unit for the construction of higherordered structures. The geometrical form of the structures is most easily discovered with the electron microscope, although it may be deducible from X-ray photographs or even with the light microscope. The problem is to find the factors, mechanical or otherwise, which organize it. There is often a relation between the mechanical function of the fibrous system and its structure, which may provide clues.

Some examples of the possible arrangement of fine fibrils are shown in Fig. 53. Tangled "brush heaps" arise in the absence of orienting influences, such as in a fibrin clot. Fibres in which all the elementary filaments are parallel have been subjected to an orienting influence either during or after the formation of the filaments. The most obvious influence is the shear due to flow, which probably initiates the orientation of silk thread. Drawing after extrusion may improve the orientation.

Some of the more interesting structures are to be found among the fibrous membranes. Collagen in skin, and certain cuticles are often found 
arranged into parallel sets of fibrils forming approximately a right angle with a similar set above and below it (Weiss and Ferris, 1954). The ultimate orienting influence is clearly the surface of the animal, which ensures that the whole formation lies parallel to it; the immediate controlling influence is not so obvious (Plate 14B). In other instances the geometry is even more complex and sometimes of surprising regularity.

Such sheets may be formed by exfoliation from a surface composed of the cells that secrete the precursor. Here we can conceive of two kinds of organizer: (a) a pattern or "die" on the cell surface or (b) the existing pattern of the preceding sheet, which acts as a template for the assembly of its successor. The peritrophic membrane lining the mid-gut of insects could be an example of the first suggestion (Mercer and Day, 1952). Here the secreting cells are covered with projecting microvilli (the brush border of histology) with a cross-sectional diameter about the same size as the holes in the membrane. We could imagine the filaments forming in the grooves between the studs on the surface. Sections of the cell surface, although showing the pattern of microvilli, and the layers of shed membrane, have not yet provided an example of a membrane in the act of formation; therefore decisive evidence is still wanting.

The collagen meshworks in skin, and in earthworms (Rudall and Reed, 1948) seem to form some distance from the cell surfaces, which are covered with amorphous material. The "self-template" seems more likely here. We can form a conception of how this could operate by supposing that the upper surfaces of fibrils have " studs " on them which fit into "holes" in other fibrils when these are laid across then at right angles.

This discussion of fibrogenesis and organization has been limited to the special case in which the elementary fibrous unit is a fine filament (or ribbon). While this is applicable to many systems, we must expect that other devices will be found such as Rudall has described in the secretion of the colleterial gland of a mantid (p. 126).

Tactoids, familiar from their occurrence in tobacco mosaic virus (TMV) solutions, have been proposed as fibre-forming elements (Bernal, 1940). The rodlets of TMV are not unlike the protofibrils under consideration here and it seems quite probable that, in some instances, e.g. the bundles of fine filaments of keratin in hair cells, the same forces which maintain tactoids are operating. Spherulites and sheaves, e.g. in F-insulin seem to result from growth by aggregation from a single, or a group of centres in the absence of external orienting forces. The filaments must also be supposed to possess little lateral attraction.

As mentioned in Chapter I calcium salts may be deposited in association with fibrils of collagen to form bone. The deposition seems to be initiated at definite sites in the $640 \AA$ banded collagen fibril and the earliest 
crystals are randomly oriented (Jackson, 1954). As they grow an orientation develops apparently directed by the oriented fibrillar matrix in which the crystal forms. Only the naturally-occurring type of fibril with the $640 \AA$ period seems able to initiate crystal deposition (Bachra et al., 1959).

\section{Epidermal fibrils}

Unfortunately it has not yet proved possible to obtain a soluble precursor of keratin or keratohyalin which will produce fibrils spontaneously in vitro. Our information concerning fibrogenesis is thus limitep to what can be obtained from the microscopy of the tissues themselves combined with any applications of general principles we can infer from a study of other fibre-forming systems such as those just described. From what has been said it is evident that the formation of fibrous keratin has no exact parallel in other systems. A complicating factor is that essentially the same final system (compare Plates 16 and 17) appears to be arrived at by two different courses: (a) in the epidermis partly through the formation of a non-fibrous intermediate form, keratohyalin and (b) in the hard keratins without the appearance of this intermediate form. The isolation of keratohyalin, its analysis and its behaviour in vitro would greatly help to clear up this obscurity. Keratohyalin and trichohyalin after accumulating as droplets of isotropic precursor are converted into the fibrous form (Plate 21) in a manner which has about it something akin to crystallization. The orientation of the fibrous form of trichohyalin in the cells of the inner root sheath of the hair follicle is strictly parallel to the axis of the follicle. Here we may suspect that the slight shear affecting the cells of the bulb as they approach the follicular constriction, which orientates the elongated mitochondria and nuclei, also orientates the initial small formations of fibrous trichohyalin and thus directs the subsequent massive transformation. In the epidermis the transformed, fibrous keratohyalin of the stratum lucidum runs approximately parallel to the stratum corneum, at right angles to the prevailing fibrillar orientation in the germinal layer. The cells at this level are already somewhat flattened and probably here too the shear produced during the change in cell shape controls the direction of orientation (Plate 22).

Many fine filaments are seen attached to desmosomes in the cells of the lower layers, where they seem to provide suitable sites for initiating fibrogenesis, and this attachment may help, by holding on to one end of a tuft of filaments, to orient it when the cell is deformed (Charles and Smiddy, 1957).

Rather less can be asserted about the origin of orientation in hair, feather, horn and nails. No precursor accumulates and filaments, when they appear, are already oriented. The particulate contents of the cells, long nuclei and mitochondria, seem to be oriented by the flow due to cell 
deformation in the hair follicle and it is in this polarized matrix that the first filaments are assembled. Tentatively it would seem that the earliestformed filaments are oriented by the same flow and that the orientation of subsequent deposits is determined in turn by these "seeds".

The opinion sometimes expressed that the narrowing neck of the follicle acts like a spinerette, is certainly not correct except in the sense that it supplies the initial orientation to the "seeds". Thereafter fibrillar growth itself is oriented. 


\section{The Growth of Epidermal Structures}

\section{The epidermis as a growing organ}

The growth of epidermal structures is a subject of interest and importance in itself; however, it gains a wider importance since, for reason of the ease with which superficial changes can be observed, the epidermis is often the tissue chosen for the investigation of the factors governing growth in general. Obviously an account of such an enormous subject would be impossible here. Nevertheless, the peculiar suitability of the epidermal structures for these studies and the probability of their future use, makes a limited discussion of some points desirable.

The sum total of the epidermis and its appendages constitutes an organ of a quite definite morphological and functional character, as well defined as that of the internal organs. Its pattern, in considerable detail, is characteristic of the species, with usually a male and female variation under the supplementary control of the sex hormones. The fact that it is a superficial organ, with much easily-observed structural detail some of which, such as feather or hair, is amenable to quantitative evaluation, is the reason for its use by taxonomists, geneticists, experimental physiologists and others. Its pathology provides valuable signs and symptoms of less-readily observed disorders. The feather, hair or nail is in fact a permanent record in chronological order of the synthetic events which led to its formation.

In the adult, the cells in most organs divide infrequently; growth has practically ceased, and the residual divisions are probably those required to make good "wear and tear." In certain situations, however, e.g in the seminal vesicles, the intestinal mucosa, the bone marrow and in the epidermis itself (Leblond and Storey, 1956), cell loss is a normal physiological process and cell division continues as part of the normal activity of the tissue. In exposed situations superficial cells are simply shed or scraped off and obviously their loss must be made good. The entire range of epidermal derivatives is maintained by the proliferation of the cells of the continuous germinal layer underlying the whole system, a population of apparently-uniform and interchangeable cells (p. 57). Thus the problem of the growth of the whole formation resolves itself into the question of what factors control cell division in the germinal layer and what determines the course of differentiation of the cells after leaving this layer. 
There is no difference here between the coherent hard keratins and the soft, which spontaneously exfoliate. Both varieties are subject to wear and more-or-less continuous growth is required for replacement. Nails, claws and epidermal horns seen, however, to be continuously produced irrespective of demand and their growth in excess of needs may even become a nuisance. Feathers and hairs on the other hand have a quite distinct unity and grow to a defined shape. Plucking is tollowed by regrowth, but only in a remote sense can we speak of this as a renewal in response to wear.

In the epidermis a steady state normally prevails in which cell loss is balanced by cell replacement. The renewal time at any site is defined as the time taken for the replacement of an amount of material equal to the amount present in the layers above that site. It is also the time taken for a cell to pass from the germinal layer to the surface where it is shed, and is analogous to the growth period for hairs and feathers. Since growth may not be continuous over short periods of time (see p. 135) the steady state is only an average state maintained over a more-or-less extended period. Leblond and others have determined the renewal time for a number of proliferating tissues. Some examples are given in Table 6. The methods employed are based on the direct counting of nuclei in division over an extended period of time or on determining the number of nuclei arrested in metaphase by colchicine in this time.

The existence of a definite equilibrium thickness of the epidermis differing from site to site and of the definite shapes of feathers and hairs shows at once that some sort of overall control must exist throughout the epidermal system. In this respect the epidermis is no different from other organs whose forms and cellular composition are also strictly controlled. In fact the entire cellular community constantly maintains a state of homeostasis in which its numbers and composition are kept in balance with each other and with the environment.

The factors likely to affect the growth of a tissue have been sought both by direct observation of normal growth and from the results of experimental interference. In this way a large amount of information has been gathered concerning the growth of whole animals and organs, which although often of immediate practical value, is not easily related back to the activity of the individual cells. A number of growth factors and of hormones influencing growth are known, but their effects are invariably complex when whole tissues are considered. For reviews, see Thomas (1956).

Undoubtedly many hormones also affect the behaviour of epidermal cells, but their action is complex and far from clearly defined. Oestrogens definitely stimulate cell division according to Bullough (1953). The cyclic changes of the vaginal epithelium, the cells of which oscillate mucin 
production and keratin production, are under the control of the sex hormones (p. 144). The effects of various hormones on hair growth have been described by Mohn (1958) and on feather by Lillie (1942).

The most obvious effect of the sex hormones is on hair and feathers. The action of the male hormone appears to affect directly the length of the growth period of certain follicles so that longer and stouter hairs (or

Table 6. Some Renewal Times for Epidermal Tissues.

\begin{tabular}{|c|c|c|}
\hline Tissue & Animal & $\begin{array}{c}\text { Renewal time } \\
(\text { days })\end{array}$ \\
\hline $\begin{array}{l}\text { ear (Malpighian layer)* } \\
\text { abdomen (Malpighian layer) } \\
\text { forearm (Malpighian layer) } \\
\text { hypothmar (Malpighian layer } \\
\text { plus corneum) } \\
\text { foot pad (Malpighian layer) } \\
\text { plus corneum) } \\
\text { foot pad (Malpighian layer) }\end{array}$ & $\begin{array}{l}\text { mouse } \\
\text { human } \\
\text { human } \\
\text { human } \\
\text { guinea pig } \\
\text { rat }\end{array}$ & $\begin{array}{c}28 \\
100 \\
13 \\
30-36 \\
40-50 \\
19 \cdot 1\end{array}$ \\
\hline
\end{tabular}

* Taken from Leblond and Storey (1951 and 1956).

\begin{tabular}{l|c|c}
\hline \multicolumn{1}{c|}{ Tissue } & Animal & $\begin{array}{c}\text { Renewal time } \\
\text { (days) }\end{array}$ \\
\hline skint & guinea pig & 82 \\
ear & guinea pig & 143 \\
metatarsal pad & guinea pig & 85 \\
prepuce & guinea pig & 28 \\
tongue & guinea pig & 8.4 \\
& & \\
\hline
\end{tabular}

$\dagger$ From Platt (1960), other figures will be found in Hooper (1956),

Price (1958), Meyer et al. (1960) and Scheving (1959).

feathers) are produced, although the type may also be affected. That other quite extraneous substances may act as stimulants is shown, for example, by the marked effect of scarlet fever toxin (Heyningen, 1950).

\section{Mitosis in the basal layer}

Although very little that is not hypothetical can be said about the control of the overall patterns of growth, there is better experimental evidence concerning the mechanisms of short period fluctuations.

While the average rate of cell replacement in the epidermis is relatively steady, Bullough has established the presence of diurnal cycles in the 
mouse, and these probably occur elsewhere. The maximum epidermal mitotic activity occurs during sleep and the minimum during muscular exercise. Among humans the maximum of mitosis occurs at night, again the period of rest.

Epidermal cells need a supply of energy for mitosis and division, for the synthesis of their specialized products and for keratinization when this occurs. Carbohydrates are the main source of energy and these are probably supplied as glucose and stored as glycogen. Glycogen is not found in the germinal layers, but may occur in the prickle cell layers (Bradfield, 1951) and is stored in quantity in the outer root sheath of the hair. The energy of the glucose probably becomes available in anoerobic glycolysis through the agency of the tricarboxylic Krebs acid cyclic (Bullough and Johnson, 1951; Bullough, 1952). Many of the intermediate substrates of the Krebs cycle can be utilized by skin and have been found in hair roots (Bullough, 1958). Rothman (1954) believes there may be other pathways specific to skin. Bullough (1952) found that many of the intermediate substrates of the Krebs cycle will support cell division, and was thus led to suppose that mitosis required energy and could only occur when the cells are able to absorb adequate amounts of carbohydrates and oxygen. That is, the special necessities for mitosis are stored in some form during antephase and are syphoned off when division commences. Were this the case muscular activity may well lead to short supplies in the epidermis and delay preparations for division. The diurnal cyclic activity of the epidermal cells is thus seen as an indirect consequence of the cylic muscular activity induced by diurnal fluctuations of light. The cycle is absent in skin cultivated in vitro, thus clearly demonstrating its dependence on extracellular factors. More recently Bullough and Laurence appear to have abandoned this opinion (Bullough and Laurence, 1958). Further, having found that, in the skin of starved rats in which the number of mitoses is very much reduced there is a dramatic burst to several times the normal number when skin is removed and transferred to saline (in absence of oxygen and glucose), Bullough and Laurence (1961) conclude that epidermal cells are always able to complete preparations for division, but that some factor inhibits the process in early prophase. They give reasons for believing that it is the high adrenalin levels associated with muscular activity which inhibit these cells. Thus the epidermal rhythm is linked with the rhythmic changes in adrenal activity (see p. 144).

Bullough's general conclusion, that glucose and its subsequent conversion to yield energy is a critical factor controlling mitosis, has not been accepted without question. In a series of papers Gelfant (1958, 1959a, $1959 \mathrm{~b})$ has confirmed the participation of glucose, but insists that an adequate supply of glucose and oxygen alone will not stimulate mitosis in intact mouse ear epidermis; the mitogenic factor may be the cutting. This 
finding would not necessarily clash with Bullough's present views involving control of inhibitors (p. 149). However, Gelfant's work does imply that some of Bullough's experiments were carried out in conditions that were "sub-optimal " for mitosis and that his conclusions may not be valid under the optimal conditions which may be assumed to prevail in vivo.

Cycles with a $24 \mathrm{hr}$ period, ultimately linked to the diurnal fluctuations in illumination (Reinberg and Ghata, 1957) are common (see p. 146). For example in animals, the body temperature, a measure of muscular activity, the glucose concentration of the blood, the concentrations of water, glycogen, fat and protein of the liver all show such variations. The calcification of teeth, a dermoepidermal function, is also cyclic.

There is no diurnal cycle in the hair follicle of Rodents-the only case examined. To account for this relative immunity of the hair follicle from the fluctuations caused by alternations of rest and activity, it is assumed that the follicle has its own independent source of food supply and, in fact, large amounts of glycogen are found in the cells of the outer root sheath (Montagna, 1956; Hardy, 1952). Glycogen is reduced in amount or is absent when there is no hair growth (Montagna, 1956; Montagna et al. 1952). Possibly when required, the glycogen is mobilized as glucose and transported by the network of blood vessels surrounding the shaft of the follicle to the bulb. In support of this it may be noted that the vascular network of the follicle of growing hairs is remarkably developed (Durward and Rudall, 1949 and 1958; Ryder, 1956). Ryder (1958) injected radioactive glucose into mice and found that in $1 \mathrm{hr}$ there was isotope in the bulb, and also in the outer sheath where it increased over the next 24 hrs. The rapid uptake in the bulb could be due to the glucose which provides energy for mitosis and the slower accumulation in the sheath to the storage of glycogen.

No such detailed information exists concerning the other long-growing, hard keratins, nails, claws, etc., but it is not unlikely that their continuous growth is sustained in a similar way.

The question of the mitotic rate and location of mitoses in the epidermis has occasioned much discussion which has to some extent been cleared up by the realization that there are diurnal variations in the rate in the skins of rats, mice and humans, which provided the bulk of the material (see also p. 146). Further, on hair-bearing skins subject to cyclic variations in hair growth, the activity of the epidermis is linked to that of the adjacent hair follicles. In the mouse and rat, the skin thickens in the early phases of hair growth and relapses again before hair growth ceases.

The mitotic rate is not the only factor which determines the thickness of the total epidermis. Clearly this depends on the renewal time, the time a cell takes to reach the surface and be shed. Ebling (1954) showed, for example, that oestradiol while increasing the number of mitoses four 
times actually decreased the total thickness of the whole layer. The rate of differentiation and of exfoliation, about which less is known, influences the thickness of the intermediate layers and of the horny layer, respectively.

\section{General theories of growth}

More recently attempts have been made to refer the problem of growth to a more fundamental basis by examining directly the behavioural patterns of the individual cells. These investigations take two forms: one is experimental, the direct observation of cellular activity in tissue culturesome of this work has been already referred to-the other is theoretical and attempts to develop an adequate general theory, which would relate the growth of the whole organism to the growth rates of the constituent cells and, further, would be competent to infer the characteristic stability and homeostasis from the properties and interaction of these cells. At the present time only tentative solutions of this problem have been made, but it seems worthwhile to mention them here, not only in an attempt to inquire how far epidermal growth may be included within the scope of a more general discussion but also because it seems that the future development and experimental verification of these theories will involve further experiments on the epidermis.

If the initial special events of cleavage and the blocking out of the early embryo are omitted, and growth considered only after the point where the embryo increases in weight, the bald facts demanding an explanation according to Weiss and Kavenau (1957) are:

(a) The increase in number of cells.

(b) Their divergence into organ systems containing differentiated cells.

(c) The quantitative facts of the growth curve, i.e. the sigmoid shape of the plot of total weight of cell mass against time (Fig. 56).

(d) The steady state which the organisms approach as the adult size is achieved in which the various organs exist in equilibrium with each other and with the external environment. In this state the several organs have become specialized in the functions which they perform on behalf of each other. The equilibrium is dynamic in the sense that it is maintained by a constant intercellular communication to which considerations of a cybernetical order are applicable.

Observations made on cells isolated and cultivated in the absence of other cells show that in these conditions cells gradually cease to produce their characteristic products and assume a more generalized character. Further, when mixed cell populations are grown together differentiation again takes place. These very general findings are sufficient to prove that differentiation is maintained by restraints exerted by one cell type on another, either by direct contact or by exchange of their products through the medium of their common humoral pool (p. 61). 
Such a community of interest functioning in terms of an economy of supply and demand might well maintain a constant ratio of cell-types while permitting an unlimited growth of the total population. However, the fact is ((c) above), that in all organisms the adult size is a rather well-defined limit and the growth curve follows a characteristic sigmoid course (Fig. 56).

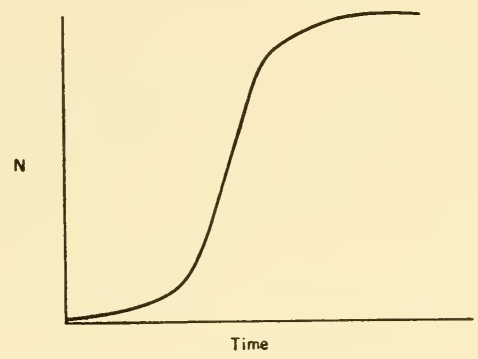

Fic. 56. The sigmoid growth curve. $\mathrm{N}$ is the number of individuals (cells). The population $\mathrm{N}$ early passes through a phase of approximately exponential growth, later a logistic law is a better fit.

It is evidently necessary to suppose that a further control mechanism exists which maintains this size and is responsible for the shape of the growth curve.

That many natural populations follow what is referred to as the logistic law:

$$
\frac{\mathrm{d} N}{\mathrm{~d} t}=\epsilon N-h N^{2}
$$

(Where $N=$ number of individuals, $\epsilon$ and $h$ are constants) has long been known. A population obeying such a law tends towards a limit $W=$ $\epsilon / h$. When $h$ is negligible, $\mathrm{d} N / \mathrm{d} t=\epsilon N$ and the population increases exponentially, $N=N_{0} \mathrm{e}^{\epsilon t}$. This type of increase may be observed in cellular populations during a limited period (the "log phase") when conditions are favourable, but sooner or later limiting factors appear, the growth rate declines and a logistic law is more applicable. The problem is usually to identify the limiting factors.

The sigmoid shape of the growth curve can be simulated in a formal sense by a number of physicochemical models, e.g. by the autocatalytic monomolecular reaction, by systems which make demands in proportion to their mass $\left(L^{3}\right)$ and are able to accumulate (or lose) in proportion to their surface areas $\left(L^{2}\right)$. Such physicochemical models have a broad 
general validity (Rashevsky, 1948), but it is one of the consequences of multicellularity that they cannot be applied in a simple direct form.

It seems more probable to many that each organ system itself produces changes which automatically lead to its limiting its own proliferation. Such a view is in harmony with modern theories of self-controlled mechanisms which envisage control, in very general terms, as being effected by a " feedback" of information which introduces a limiting factor proportional to the deviation from a norm.

Physiological evidence that the entire population of cells in an organ controls its own size is obtained from a variety of experiments in which part of the population is removed. Partial hepatectomy is followed by a burst of mitotic activity in the remaining tissue leading to a restoration in size. That the influence causing the mitotic wave is carried by the blood is shown by experiments in which hepatectomy is practised on one of two rats whose blood supplies have been joined (parabiotic union). Mitosis occurs in the second undamaged liver. These effects might be ascribed to a stimulating substance (wound hormone), but the loss of an inhibitor is indicated by the observations that mitosis can be induced in normal livers if the blood is diluted by saline, and that regeneration itself is slowed up by increasing the plasma concentration (Glinos, 1958). Weiss (1955) has reviewed experiments in which the removal of one member of a paired organ, e.g. the kidney, is followed by an increase in size of the remaining member. Perhaps the best demonstration of the existence of control by inhibitor productions is found in the experiments of Bullough on growth control in the epidermis itself which will be described in the next section.

Certainly numerous other factors, among them well-recognized hormones, affect growth as is made clear in the reviews of Abercrombie (1957 and 1958) and Swann $(1955,1957$ and 1958). Nevertheless, the possibility exists that primarily control is based on hormones of the inhibitor-type and that other hormones could operate by secondarily affecting the cells' response to inhibition. These problems are returned to again below (p. 146).

The logistic growth curve is obtained from the exponential-type curve simply by the addition of a further negative term (see above) which here might be regarded as the " negative feed-back" term. The total growth curve may be considered as the sum of the separate organ sigmoids. Several proposals of this sort have been made, for example, by Morales and Kreautzer (1945) and by Sock and Morales (1945). Weiss (1955) has attempted to give these concepts a more definitely-biological basis and recently with Kavenau (1957) has obtained solutions of a growth equation which are sufficiently precise to be put to a quantitative test. They suppose that each specific cell reproduces itself by a mechanism in which certain key compounds act as catalysts (templates). The growth rate is proportional 
to the concentration of these intracellular templates which constitute the generative mass. This mass is being constantly converted into a nonreproductive mass which is the differentiated product. These reactions are further supposed to be accompanied by the formation of inhibitors (antitemplates) which block the templates, and thus may potentially act as

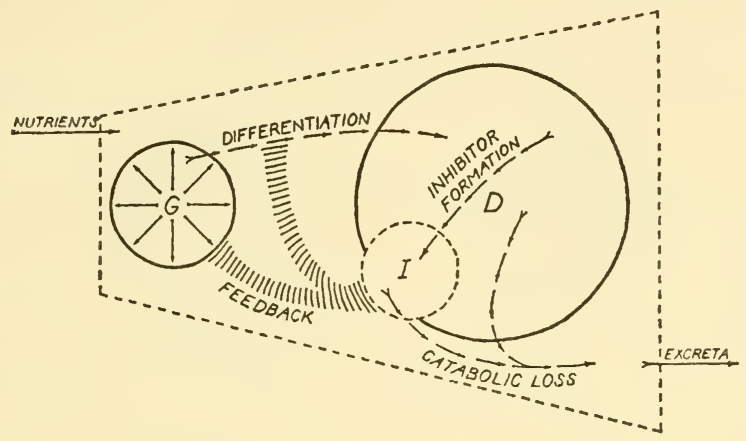

Fig. 57. Illustrating Weiss and Kavenau's model (1957) for a cell showing control of growth by the production of an inhibitor. The cell contents are divided into generative mass $\mathrm{G}$ and its product the differentiated mass D. In this example the inhibitor I is supposed to be produced by $\mathrm{D}$ and its action is fed back to control the processes of growth and reproduction (reproduced by permission).

growth regulators by an intracellular negative feed-back (Fig. 57). In order to effect a control over the whole distribution of a cell type, Weiss and Kavenau assume (a) that the inhibitor molecules diffusing from the cells enter the common humoral pool and thus reach other cells, (b) carry a " tag " or "label " enabling them to be recognized by other cells of the same type and (c) that they have a normal rate of degradation or loss which, in equilibrium, balances their rate of production (Fig. 58).

These assumptions are biologically acceptable and sufficiently general and simple to permit of mathematical expression. It is clear, without attempting to formulate and solve the growth equations, that since they contain negative feed-back terms they will lead to a system which will automatically regulate its own size. Weiss and Kavenau set up differential equations for their system and applied a solution of these to the case of the growth of a chicken. The quantitative agreement between theoretical and experimental data is surprisingly close.

One feature of systems stabilized by feed-back should be pointed out. Since a delay in time occurs between the despatch of a signal from one part 
of a system to the part controlled by the signal, the possibility of oscillations arises (p. 143). Weiss and Kavenau believe these will arise, for example, when organs regenerate after partial removal. These may be manifested also at the cellular level and possibly account for some of the fluctuations in cellular activity commonly noted (see also p. 148).

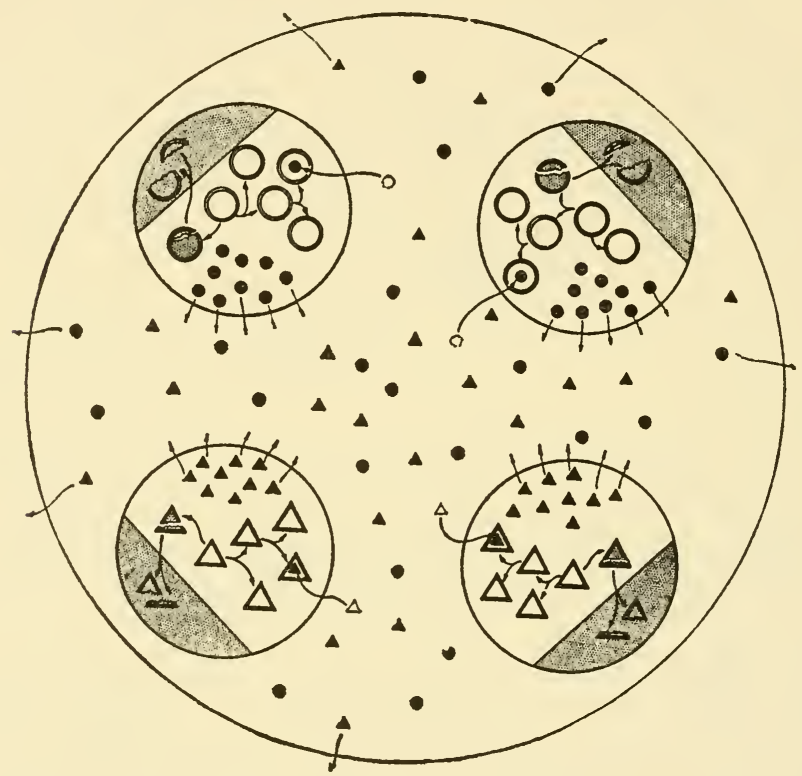

Fig. 58. Further aspects of W'eiss' theory of intercellular control by the exchange in inhibitor molecules. Two kinds of differentiated cells are distinguished by their differentiated products, open circles and triangles. Two kinds of inhibitor molecules, black circles and triangles, are released into the common humoral pool. The rate of production of the two kinds of products is controlled specifically by concentration of their specific products in the pool (reproduced by permission).

In its present state of development the theory does not give a place to interactions between different cells, which are probably in part effected by the exchange of samples of the differentiated mass, and thus does not attempt to account for the appearance of differentiation and its maintenance. A differentiating system can be devised, following Rose (1952) if one supposes that the inception of a certain reaction in one group of cells 
suppresses this development in adjacent cells and permits a second reaction to arise in these cells. The products of the first reaction diffusing from the "dominant" group of cells thus "induce" a second and different reaction in neighbours. Possible fine structural evidence of this form of induction in the epidermis and dermis has been described above (p. 90). It would not be difficult to generalize the growth equations by introducing terms expressing the interaction between cells assuming that the anti-templates (or secreted differentiating mass) in a dominant earlymaturing group of cells can suppress similar development in less-advanced cells. See also Waddington (1948).

Also, no necessary place has been given to the fact that tissues are organized in a cellular form. Certainly, although cell division introduces a discontinuity in the output of a single cell, these irregularities would be smoothed out when the output of a large non-synchronously-dividing population is considered. Moreover, if adequate arrangements exist for transport to and from sites of synthesis, the cellular habit does not in itself seem essential for continued synthesis. For example, in insects relatively enormous differentiated cells are common. Probably cell division is an inherited act, originally developed to permit of replication and dissemination of the genetical apparatus, that occurs normally when the DNA is duplicated and the cell has synthesized adequate amounts of the materials required to provide the apparatus of division. These latter activities could involve paths of synthesis distinct from those involved in the formation of specialized products.

That all authorities do not yet accept the necessity of control by inhibitor production is evident from a recent discussion on the growth of proliferating tissues (Price, 1958). Obviously the possibility that stability is maintained throughout multicellular organisms by the circulation of inhibitors is a conception of far-reaching consequences. It implies, in effect, the existence of a whole system of hormones which has escaped notice. The already-known hormones and other growth influencing agents would seem to effect the sensitivity of the cells to the circulating inhibitors or act to influence the dispersal and disappearance of these. It is highly desirable that an attempt be made to isolate these postulated inhibitors and that their mode of action on cells be determined. It is evident that the theory must be regarded as unproven until some of the postulated inhibiting substances have been isolated and the mode of action on the cells observed directly (Bertalanffy, 1960).

\section{Periodic growth and cyclic activity}

Even in the adult when the size has become more or less constant, certain organs undergo a periodic fluctuation in size and activity. Conspicuous among these are the sexual organs and with their changes are 
linked equally-marked changes in the entire endocrine system (Burrows, 1949; Bullough, 1951). The often striking periodic changes in the plumage of birds and the hairy covering of mammals are clearly linked both to the sexual and seasonal cycles. In exhibiting these changes it seems likely that the integument responds to the general endocrine situation, that is to say, its periodicity arises indirectly from periodic changes in the concentration of circulating hormones.
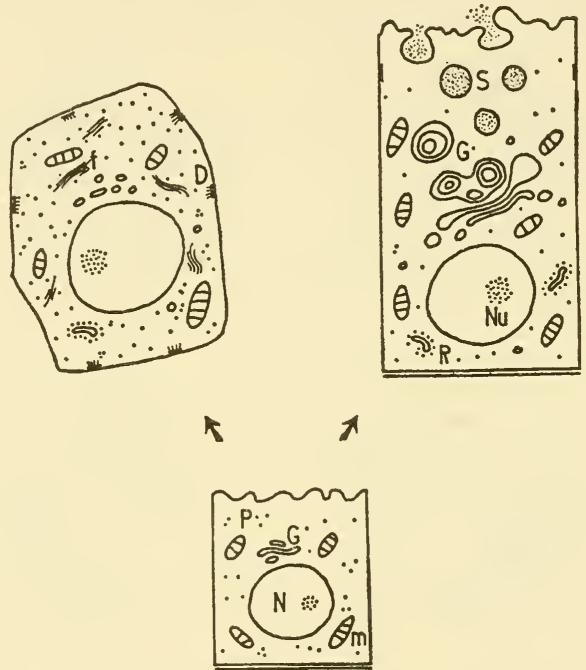

FIG. 59. The production of two cells of contrasted type from the same germinal layer: upper l.h.s. a keratinizing cell, upper r.h.s. a mucin forming cell. The metaplastic change may be effected by hormonic means or by such additions as vitamin A (p. 63).

Obviously cyclic activity, which ranges from short period oscillations, such as the heart beat, to the slow oscillations, which gear organisms to the daily and annual changes in their physical environment, must be regarded as one of the most important biological phenomena. The cyclic changes may be reflected not only in size, but in cell function which is revealed in cyclic histological and cytological changes (metaplasia). For example, in the not uncommon metaplastic cycle between a mucinproducing and a keratinizing epithelium, the same germinal layer gives rise to two types of cells of contrasted cytology: (a) keratinizing cells (1.h.s. Fig. 59) with many RNP granules and a poorly-developed system 
of cytomembranes; and (b) mucous cells with an elaborate system of $\gamma$-cytomembranes and few RNP particles (r.h.s. Fig. 59) (Burgos and Wislocki, 1958; Nilsson, 1959; Schulz et al., 1958). Cyclic behaviour of this character is in effect a cyclic change in differentiation. Like differentiation in the more stable sense, it is a consequence of the interaction between one group of cells (organ) upon another. It was precisely this interaction which was omitted for simplicity in the simplified theory of growth outlined above (p. 138). When interaction is permitted, periodic phenomena can arise spontaneously from the effects of intercellular communication when there is a time lag between the transmission of a stimulus from one organ and the return of a counter stimulus from the other. Such questions are currently discussed under the heading of cybernetics.

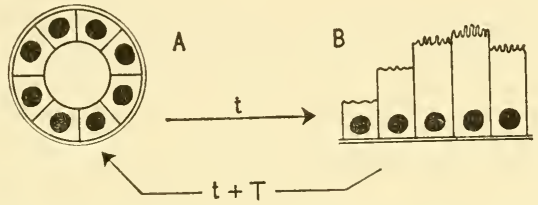

FIG. 60. Illustrating the possibility of generating cyclic activity by a feedback link between two organs A and B. A signal from A stimulates the development of $\mathrm{B}$ whose secretion returns with phase lag $\mathrm{T}$ to repress the activity of $\mathrm{A}$ (see text).

The possibility of oscillations can be understood most easily from a consideration of the ideal situation depicted in Fig. 60. We suppose two organs $A$ and $B$. The secretion $S A$ of organ $A$ stimulates the growth of organ $B$, whose secretion $S B$ is capable of inhibiting the activity of $A$. Suppose the secretion $S A$ reaches a threshold value at a time $t$ and initiates a growth phase in $B$, which after a further time $T$ leads to the release of $S B$ by the organ $B$. This inhibitor $S B$ in turn now acts on and suppresses the action of $A$; with the consequent fall in $A$ 's activity, the stimulus to $B$ falls off and its activity declines again, leading to a fall in the inhibitor $S B$ and the recommencement of activity in $A$. Thus cyclic activity is set-up both in $A$ and $B$ with a difference in phase introduced by the time lag $T$ (Fig. 60).

In the language of cybernetics, we say that the two organs are controlled by a feed-back linkage, and that the oscillation is made possible because the feed-back signal from $B$ to $A$ is out of phase with that from $A$ to $B$. The feed-back from $B$ to $A$ is negative; that from $A$ to $B$ is positive.

As described, the feed-back signals $(S A$ and $S B)$ are " hormones" and the lag between them is introduced by the time of maturation of a target organ $B$. This illustration was chosen because it corresponds, in the type 
of change and in the times likely to be involved (hours or days), with the actual endocrine changes with which epidermal changes are linked. But the argument is general: $A$ and $B$ may be parts of the same organ, $S A$ and $S B$ may be nervous or mechanical signals and $T$ may be very short. Actual situations are never as simple as that of Fig. 60. Usually several cyclic systems interact and at some point a " sensitive " element responsive to the environment may introduce a signal which "gears" the entire system to the diurnal and annual cycles.

To explain this further it is necessary to introduce another important idea, that of adaptive oscillations. The frequency of the cycle $A \rightleftarrows B$ (Fig. $60)$ is capable of great variation because of variations in the time of maturation of the cells in $B$ (and/or $A$ ). Thus when the cycle $A \rightleftarrows B$ is linked to others $C \rightleftarrows D, E \rightleftarrows F$, etc., the possibility of coupled oscillations with resonance arises, because the frequencies of the separate systems can change until all have the same frequency (or multiples of this) when they will resonate at this frequency with phase differences determined by the time lags in the various feed-back loops. It is possible to say that the appropriate matching frequencies evolve from the possible range of frequencies by a kind of natural selection-the best-adapted frequency survives and increases its amplitude. This mechanism in essentials was proposed by Pringle (1951) in developing a theory of the activity of the brain.

By such a feed-back train a system of cycles could be " geared " to the diurnal and annual astronomical cycles and an organism's total activity adapted to the physical environment. In these complex events the integument seems, as far as is known, to follow the lead of the endocrine system. It is a target organ of graded sensitivity, but there is little proven evidence of its returning a control stimulus to the deeper tissues. However, this may seem so largely because of our ignorance; the possibility certainly exists that epidermal products can be fed back into the organism, thus constituting yet another closed cycle. For example, the grooming habits of both birds and mammals are so persistent that considerable quantities of epidermal material must re-enter the organism through the mouth (see p. 59). Further, from the wider viewpoint of the enormous system of communication which constitutes ecology (Hutchinson, 1948), the integrative function of the integument as a signalling system to predators, to congeners, and to sexual partners can only be mentioned here, emphasized and left.

\section{Control of epidermal growth}

When we come to consider the epidermis in terms of such general theories, we see at once, as has been emphasized already, that because of its position on the outside of the cell system, it constitutes a special case among the organs. Moreover, it is non-vascular, unevenly enervated 
(Arthur and Shelley, 1959) and its cells grow outwards. Its cells have evidently only a limited possibility of communicating with each other and the rest of the system. The existence of "fleece mosaics" in sheep has recently assumed some importance as a proof of local autonomy. A fleece is said to be mosaic when it comprises two distinct types of wool grown on different areas of the same skin. The areas may be adjacent and it is clear that the follicles concerned, although producing different types of fibre, enjoy the same environment externally and internally, thus demonstrating beyond doubt the over-riding control of local, non-systemic factors on the kind and quantity of fibre formed. It is assumed that mosaics arise from a somatic mutation, i.e. from a mutation occurring in a cell subsequent to the first division of the egg which initiated development. The change will be apparent only in the line of cells issuing from the mutated cell. Thus an area of skin, producing aberrant type of wool, is assumed to be a colony of cells arising from a single cell in which a somatic mutation has occurred. The actual local histological factors have not yet been fully explored, but the existence of the phenomenon proves the genetic control of localized epidermal structures which in turn determine the nature of the product quite independently of systemic factors.

There is in the epidermis a vertical integration but clearly only a rather limited lateral one. By postulating the same intracellular features, generative mass, differentiated mass (keratin, mucin, etc.) and inhibitor production, the possibilities of control in a simple stratified epithelium may be considered. Division is largely confined to the basal layer and synthesis of specialized products takes place in more distal layers. The inhibitor molecules, produced in the stream of outwardlymoving cells during these later reactions, are largely lost when these cells are shed, and can only feed-back to the germinal layer by back diffusion, and only by crossing the dermoepidermal junction can they reach the general circulation and thus be carried to distant parts of the system.

Certain possibilities may be made clear by considering a cell which has just been produced by division. Since division has occurred we may suppose that inhibition is minimal. The cell leaves the germinal layer and, at a higher level, begins to differentiate, to synthesize both the differentiated product and also the inhibitor. The following conditions may arise:

(a) Before sufficient inhibitor is produced or diffuses back, the cell in the germinal layer again divides. This would be a condition permitting of uncontrolled, continuous growth.

(b) Before division can occur again, sufficient inhibitor diffuses back to prevent it. After a further time the concentration falls again (with the decrease in synthetic activity in the differentiated layer and the decay of inhibitor molecules) and division again occurs. We have here a condition of periodic division under the control of the events in the differentiated 
layer. The time elapsing between divisions will depend on the rate of synthesis (i.e. also the rate of formation of inhibitor) in the differentiated layers and the rate of loss or decay of inhibitor at the germinal level. Since a certain lateral diffusion from any cell is possible, a synchrony could develop in the germinal layers owing to the overlapping of the effects of several adjacent cells. This synchronization may be favoured also by the

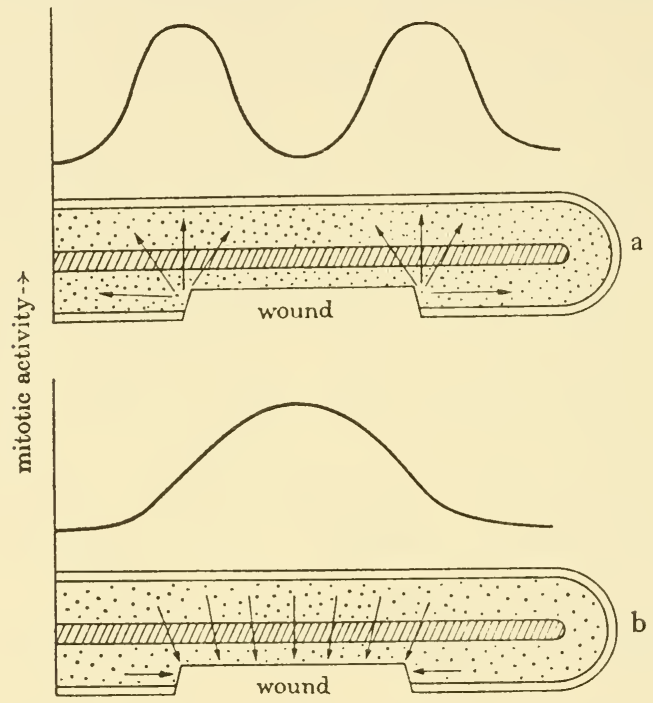

FIG. 61. Diagrams to show the regions of high epidermal mitotic activity expected in undamaged ear epidermis opposite to an area $3 \mathrm{~mm}$ square from which the epidermis and superficial dermis have been removed on the assumption (a) that a stimulating "wound hormone " is produced by damaged epidermis, and (b) that the concentration of epidermal inhibitor is reduced in the neighbourhood of a wound (from Bullough and Laurence, 1960).

possibility that a general systemic stimulation reaching a group of cells, in which the inhibitor concentration is already low, may cause them to enter division together. The diurnal variations in mitotic activity noted by Bullough, may be an instance of this type of control (p. 136).

(c) Stimulation of growth may be caused by activities which facilitate the fall in inhibitor concentration. Experimentally, Pinkus (1951) found an increased mitotic rate following the stripping off of the upper layers of the skin with adhesive tape. Mechanical trauma, such as piercing or cutting 
the skin, are known to be sufficient to provoke regrowth in quiescent areas of skin in rabbits and rodents (Slen, 1958). In animals such as sheep with constantly-growing follicles, an increased blood supply has been thought to increase the rate of growth of wool (Ferguson et al., 1949). Perhaps one of the most characteristic properties of the epidermis, its adaptive response to the effects of hard work, may be produced by the dissipation of inhibitor resulting from friction and pressure. Thorium-X plaster acts similarly.

The epidermis in health fits its bearer snugly. Obviously some control adjusts lateral growth so that the area of the covering increases or decreases with the volume contained. Possibly the factor here is mechanical, a tension or compression arising from the expansion or contraction in volume of the tissues beneath. Stretching would thin the covering layers and reduce the amount of inhibitor diffusing back to the germinal cells which would then divide and replace the overlying layers.

Recently by means of an ingenious experiment, Bullough and Laurence (1960) claim to have shown that inhibition rather than stimulation is the real growth controlling factor. Following a wound, mitosis and growth are initiated in a limited area (approx. $1 \mathrm{~mm}$ wide) of the epidermis surrounding the wound. This could be described, and usually was, as the result of the liberation of a "wound hormone" stimulating growth. Using the fact that the skin on a mouse's ear is less than $1 \mathrm{~mm}$ thick, Bullough removed the epidermis on one face making a wound more than $1 \mathrm{~mm}$ wide (Fig. 61) and observed the effect on the epidermal layer on the other side of the ear. If the diffusion of a stimulant to a radius of the order of $1 \mathrm{~mm}$ was the stimulating factor, a limited area of mitoses opposite the edges of the wounds should be seen. If the removal of inhibitor was the cause, mitoses should be seen over the whole area lying beneath the wound, which was the condition actually observed (Fig. 61).

\section{COMPETITION}

Another factor, which almost certainly plays a part in controlling growth rates and through them morphogenesis, is competition between cells and organs and parts of organs for some essential requirement for cell growth. Here again data obtained from the observation of epidermal growth have been extensively used to demonstrate the actual operation of competition. We have mentioned that a competition between the primordia of follicles may account for their appearance in a hexagonal pattern on certain skin areas (p. 77) and that inter-organ competition within a "scale-hair-gland unit" may be appealed to for an explanation of the suppression of some structures in favour of others.

The very extensive quantitative data concerning the growth rate and dimensional properties of wool fibres available from Australian sources have been used particularly by Fraser (Fraser and Short, 1960) in an 
attempt to demonstrate quantitatively the effects of competition between follicles, An interfollicular competition for nutrient substance is immediately suggested by the most striking feature which emerges from the comparative study of the fleeces of the many domesticated sheep: viz. the fact that the denser the fleece (fibres per $\mathrm{cm}^{2}$ ) the finer and shorter are the individual fibres. For quantitative data see Carter and Clarke (1957). Fraser and Short (1960) have demonstrated also a negative correlation between fibre size and the diameter and distance of adjacent fibres. See also Ryder (1957). Fraser (1951) originally used the concept of competition to explain the differences in the shape of the tip curl between fibres formed by central and lateral primary follicles (Fig. 35), (p. 78). The number of crimps in a periodic function of time (Norris, 1931, p. 156) and a regular crimp of constant curvature will result if the growth rate is constant. If the rate decreases, following on the initiation of adjacent new follicles which compete for fibre forming substances, the curvature would decrease and a sickle shaped tip would result, as is found on the primary central fibre of a trio group.

Fraser's original proposals (1951) were clear-cut and offered plausible suggestions relating growth rates, tip shapes and order of appearance of wool fibres during development. The subsequent attempt to evaluate these theories quantitatively by statistical analysis of the data has led to some secondary elaboration which makes it difficult to test them exhaustively as once envisaged. Such concepts as: competition for space in early development and variations in a genetically-determined "efficiency of competition" on the one hand, and actual histological findings of secondary follicles having different origins-directly from the epidermal surface and by budding from existing follicles on the other-have introduced complexities. Fraser and Short (1960) are now of the opinion that more information concerning the performance of the individual follicle must be obtained. In fact, Rudall (1956) (p. 73) has already shown that it is the dimensional structure (diameter, surface area and height) of the papilla which is most strongly correlated with the follicle output and the dimensions of the fibre. Presumably it is these papillary dimensions which are influenced directly by competition and other controlling factors. Considerations of this sort, which promise to relate follicle output to the activity of the actual cells covering the papillary surface by supplementing the statistical approach, are likely to lead to a clearer understanding of the growth process in general. See also Burns and Clarkson (1949).

\section{Patterns of hair growth and control}

Hair growth and replacement varies in different animals and is a species characteristic; within a species the sex hormones in particular determine a masculine and feminine pattern which disappears after gonadectomy. The 
same is true of birds' feathers. Since much is known about hair patterns and the factors controlling them, we shall consider them in terms of the theory developed above.

Three different types of follicular activity may be distinguished:

(a) Periodic activity with neighbouring follicles not in phase, i.e. each follicle behaves independently with periods of growth followed by periods of rest. This may be referred to as "mosaic growth" and probably represents the basic pattern of isolated follicular activity.

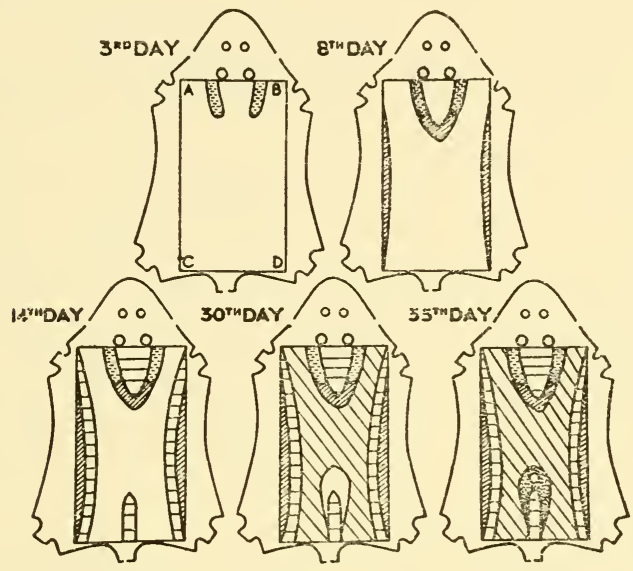

Fig. 62. The growth waves on rat skin demonstrated by the dyeabsorption of growing follicles. Regeneration of hair at $3,8,30$ and 35 days after shearing the area of A, B, C, D. Similarly-hatched areas are those in which the hair is growing at the same time and in which the alloxazine pigmentation would develop in response to administration of the compound at about that time. (Reproduced with the kind permission of Professor Haddow and the Editor of Nature).

(b) Continuous activity as found in sheep and on the human head. This is a special case of (a) with exceptionally long growing phases and a short resting phase.

(c) Periodic activity with neighbouring follicles in phase as is found in rats and mice (Butcher, 1934; Haddow et al., 1945; Fraser and Nay, 1953). At any time most of the follicles are quiescent and activity is confined to small areas which may form recognizable wave-fronts (Fig. 62).

Intensive studies have been made of hair growth in man, sheep, some rodents and fur-bearing animals. Quasi-continuous activity (b) is rare: 
in most animals hair of a well-defined species-characteristic, site-characteristic length is produced during a growth phase and a more-or-less lengthy resting phase follows (Chase, 1954 and 1955). Shedding may be continuous or exhibit a seasonal dependence (Fig. 40, p. 89).

The pattern is essentially similar in the mouse and the rat. Important dermal changes occurring (Durward and Rudall, 1949; Montagna, 1956, Review) in correlation with the growth cycle are (a) an increased vascularity beneath the growing area and (b) increased deposits of hypodermal fat. It has been debated whether these changes cause or are caused by the epidermal activity. Durward and Rudall proved the absence of nervous control of the growth wave in the rat by severing all nervous connexions. They demonstrated that the growth proceeded largely under local control and independent of systemic control by exercising portions of skin rotating them and grafting. The pattern of growth (and also that of pigmentation) is quite unrelated to the distribution of nerves or blood vessels. Butcher earlier had reached different conclusions but Durward and Rudall believe that, by choosing to study young animals, he may have failed to distinguish the characteristic behaviour of the mature skin.

Durward and Rudall conclude that, in very general terms, the growth wave is a consequence of a "resting stage inertia and a stimulus provided by neighbouring vascular activity." The vascular activity probably arises in response to the demand of the cells in follicles already actively growing and synthesizing keratin. Special modifying systemic factors such as hormones or food supply (p. 135) certainly exist but they are assumed to remain constant in these experiments.

Montagna and Chase, using the mouse, have reached the same conclusions. Chase (1954) in particular has tried to trace out in greater detail the interconnexion of the various elements of the skin. He emphasizes the morphological continuity of the basal layer of the epidermis, the external root sheath and bulb of the hair follicle and the peripheral cells of the sebaceous gland and their functional interdependence. These elements act as a unit (the pilosebaceous unit) sometimes centred on a single follicle, sometimes on a group of follicles and their associated glands as described earlier (Fig. 63).

The control diagram, Fig. 63 taken from Chase and simplified, is an attempt to bring out the morphological continuity and to indicate the possible lines of communication which transmit the control from one unit to another in the skin, and effect the integration of the whole.

Essential conditions required for prolonged growth are themselves probably provided by the geometry of the follicle, a long thin cylinder penetrating deeply into a potentially well-vasculated region, the dermis and hypodermis, where growing cells may satisfy their demands for food and where, at the same time the rate of accumulation of inhibiting 
molecules is reduced. In isolation from other follicles, a follicle might be expected to continue in production until the accumulation of inhibiting molecules reaches a critical concentration, when growth would cease. It could recommence when the concentration fell again to a lower critical threshold. Local histological pecularities probably control the rate of

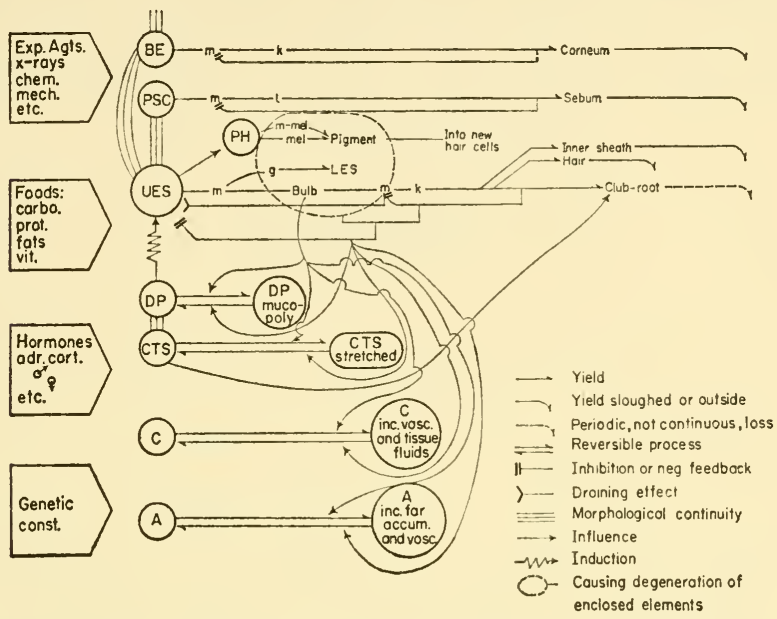

Fig. 63. Chase's attempt to illustrate the factors which operate to integrate the pilosebaceous unit (hair follicle plus sebaceous gland).

$\mathrm{BE}$ basal layer of epidermis.

PSC peripheral cells of sebaceous gland.

UEC upper, permanent outer root sheath.

DP dermal papilla.

C'TS connective tissue sheath.

C corium.

A adipose layer.

accumulation and dissipation of the inhibitors; and, as an important distinction from the general systemic control mechanism described above, it is supposed that the inhibitor molecules which enter general circulation have a short life and that, therefore, little control over distant sites exists. Since there are no diurnal variations in growth rate, as is found in skin (p. 136) we must assume an adequate supply of nutrition (p. 137).

Co-operative behaviour becomes possible according to Chase in a population of follicles when the individuals are close enough $(c a .1 \mathrm{~mm}$ 
apart) to share the same diffusion fields. It is in such conditions that growth is transmitted in a wave-form. This view is essentially that of Durward and Rudall if we equate their " resting stage inertia" with "accumulation of inhibitor." In these terms the development of growth

A
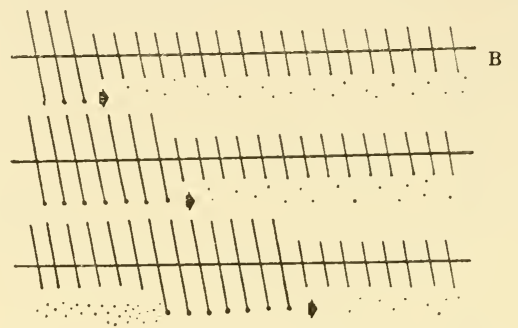

(a)

(b)

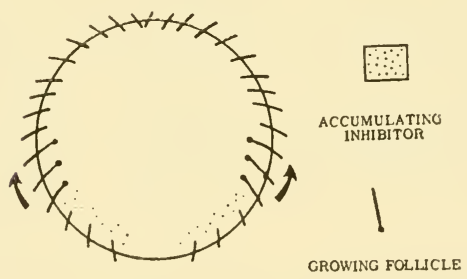

FIG. 64. (a) The generation of a growth wave in a population of follicles whose growth is accompanied by the build-up of inhibitor molecules (small dots) which on reaching a critical concentration cause growth to regress. In the top line growth is advancing towards the right as follicles recommence growth. After growth has persisted for sufficient time, sufficient inhibitor accumulates to cause the follicle to cease growth (bottom line). Growing follicles are indicated by a line with an enlargement at the end, and non-growing follicles as a shorter line.

(b) The lower illustration is an indication of how a typical growth wave, commencing on the ventral surface of an animal, can travel dorsally.

waves can be explained as follows. We consider a population of resting follicles and suppose that at $A$ the concentration of inhibitor falls below the threshold value permitting growth to recommence (Fig. 64). In response to the demands of the growing cells, which initially include cells of the adjacent epidermis, and outer root sheath, the vascularity beneath $A$ increases. If now the concentration of inhibitor in the neighbour of 
follicles adjacent to $A$ is also approaching the critical value, and if the follicles are sufficiently close to one another, the increased blood flow will initiate growth in these follicles. By the same process further follicles will be stimulated and growth will travel towards $B$ as a wave. Behind the front, growth will continue until the inhibitor again accumulates to the critical valve when the follicles will again enter the resting state together.

On a simple cylinder (Fig. 64b) (model of the body of an animal) if growth commences along a ventral line, waves will travel dorsally on lines parallel to the initial line as is observed. The extremities, the legs, ears,

DORSAL

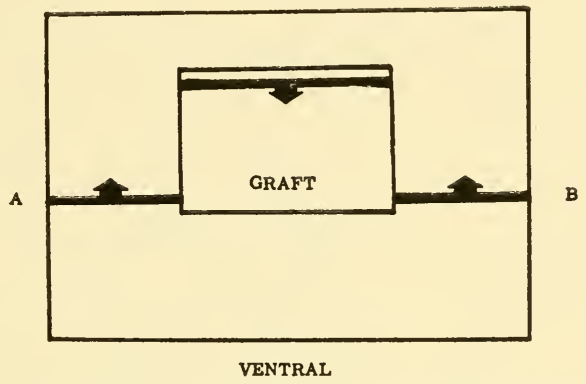

Fic. 65. The result of rotating an area of skin through an angle of $180^{\circ}$ and regrafting it as described by Ebling and Johnson (1959). When a growth wave moving dorsally reaches the level $\mathrm{AB}$, hair commences to grow at the dorsal edge of the rotated graft (as if this were not displaced) and travels ventrally. This is contrary to the predictions of a theory based on simple control by accumulated inhibitor.

etc., pose special conditions which will break the uniformity of the pattern but growth should still proceed on fronts. Inhibitor theory is thus able to describe qualitatively the appearance of waves, but it cannot yet be developed quantitatively.

Ebling and Johnson (1959) claim to throw doubt on this explanation. They severed areas of rat skin entirely from their dermal connexions and regrafted them after rotation through $180^{\circ}$. On such grafts the growth wave normally proceeding in a ventral-dorsal direction (see Fig. 65) commenced on the dorsal side of the graft and travelled downwards. That is to say the follicles in the graft behaved exactly as they would have if they had not been rotated, whereas the wave transmission theory would predict that the wave on reaching $A B$ would advance dorsalwards across the graft. Ebling and Johnson infer that the follicles are actually independent and the wave advances simply because there is a ventral dorsal gradient 
in the sensitivity of the follicle to some periodic systematic stimulant which is perhaps brought about by a graded difference in the rate at which the growth inhibitors are dissipated. Whitely's (1958) findings are similar.

Periodic growth is also apparent in feathers, in the formation of growth bars and perhaps daily variations also occur (Lillie and Wang, 1940; Lüdicke, 1959).

\section{Zig-Zags, Curls and Crimps}

In the rat and mouse, the fine hairs are not straight, they divide into short lengths separated by narrow nodes to form " zig-zags" (Dry, 1926 and 1928). This phenomenon seems to point to the existence of small localized fluctuations in inhibitor concentration which affect more the finer hairs, less deeply embedded, often more closely clustered, and able to exert less command over supplies and/or with less chance to dissipate inhibitor concentration than the stouter more deeply-seated primary hairs.

Zig-zags are probably related to crimps and curls. Histologically these latter modifications are associated with curved follicles and it seems not unlikely that the basic cyclic activity of the bulb is linked to cyclic changes in a curved and asymmetric follicle which co-operate to stabilize the emerging wave-form, i.e. the period of the bulb becomes related to the time taken for the cells to pass through the curved tube formed by the upper part of the follicle. The remarkable regularity of the emerging wave-form would suggest that some kind of feed-back, possibly mechanical, must integrate the entire follicular activity.

With stout hairs the simple existence of a follicle curved in the zone of hardening (Chapter 6) would seem sufficient to produce a hair of more-orless constant curvature, i.e. a helix or simple curl, since the emerging hair would retain the shape of the "mould" in which it was set (Fig. 66). The curly locks usually found in the fleece or pelt are of extra-follicular origin and seem to result from the tendency of the hairs after emergence to adhere laterally to each other in clumps or bundles (Horio and Kondo, 1953).

Crimps are more nearly planar wave-forms and often of impressive regularity (Norris, 1931). They develop typically in fine flexible hair and there is a close correlation between the wavelength of the crimp and the ease of bending the fibre (diameter) which is taken advantage of in the practical method of judging fibre diameter by eye. The variable curvature, which gives rise to " sickle tips," is discussed on p. 150 .

The periodicities of crimped wool which comprise not only wavelength but rhythmic changes in diameter, shape and chemical composition (Mercer, 1954) force one to suppose that the various steps in its formation are interconnected in such a way that information concerning the portion of a wave already produced is fed back to the keratinizing and germinal layers to control current production. We appeal here to a broad principle 
of cybernetics, that such a regularity of form in changing conditions could only be maintained by information transfer. It remains, however, to identify the links in the feed-back train.

The histology of the wool follicle has been much studied and a very complete account given by Auber (1950). Problems relating to crimp formation are discussed by Wildman (1932), by Rudall (1936) and also by

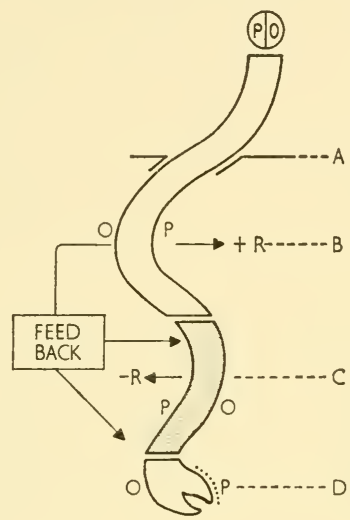

FIg. 66. Theory of crimp formation in a wool follicle (see text). The following structural elements are distinguished:

(a) The point of emergence of the fibre from the skin. The fixed orifice.

(b) The upper reaches of the follicle which contain a set and permanently curved segment of fibre with radius of curvature R.

(c) The keratinization zone. The "mould" of curvature - R (opposite to that in segment B) in which the growing fibre is "set" with a curvature $-R$.

(d) The bulb, the origin of the rodlet of protein which enters the zone C. For the purposes of clearer illustration, the geometry of the follicle has been simplified and idealized. The situation depicted shows the follicle in an extreme position in which $\mathrm{C}$ is shown in its extreme extent to the right. $\mathrm{O}$ and $\mathrm{P}$ indicate the locations of the two types of keratin orthoand para- (see p. 273).

Auber (1950). We shall return in Chapter 6 to special questions relating to keratinization in curved follicles. For our present purposes the structural elements likely to be involved in crimp production and maintenance are: the deflected bulb, the curved follicle and the (usually) asymmetric placing of the hair structures within the outer root sheath (Fig. 114). 
It can be ascertained by passing a wire, bent into the form of a sine curve, through a short length of flexible tubing held at the end from which the wire emerges, that the movement leads to an oscillation in the curvatures of the free end. Thus in the case of the crimped fibre, the passage of the hardened hair through the upper portions of the follicle (fixed end) will tend to cause an oscillation in curvature of the softer, lower portions of the follicle (the analogue of the free end) (Figs. 66 and 67). A feed-back

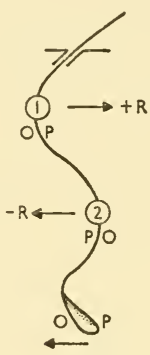

(a)

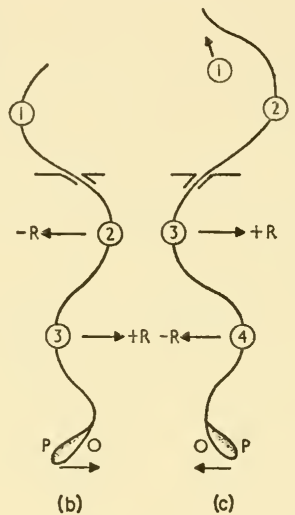

(b)

(c)

FIG. 67. Successive stages in the production of a full crimp wave.

(a) A segment 1 of curvature $+\mathrm{R}$ is passing along the neck $\mathrm{B}$ and emerging through the fixed orifice. At the lower end of $\mathrm{B}$ a segment 2 of radius $-R$ is about to enter $B$.

(b) The segment 2 has entered $\mathrm{B}$ and has deflected the neck $\mathrm{B}$ into the opposite curvature $-\mathrm{R}$ by the time it is about to emerge. By deflecting $\mathrm{B}$ into this shape it has deformed the setting zone $\mathrm{C}$ into the reverse curvature $+R$ so that the length of fibre 3 being hardened at this point will now have the reversed curvature.

(c) Segment 3 has now entered the upper levels swinging them over to the original shape $+\mathrm{R}$ and the effect on the lower levels is to reverse the curvature there.

Thus the curvature in the "setting zone" is maintained in an out-ofphase condition relative to the upper zone by the mechanical feed-back link. Growth conditions on the inner face of the curved zone $\mathrm{C}$ of Fig. 66 and the adjacent zone of the bulb D are assumed to be less favourable than on the outer face and to result in less rapid growth on that side. Assuming further that growth in the bulb is also periodic, the rate, quality (o or $\mathrm{p}$ ) and size of outer root sheath also swing from side to side. This oscillation may also resonate with the mechanical vibration in zones $\mathrm{B}$ and $\mathrm{C}$, the asymmetric growth giving rise to a curved segment to enter in phase with the curvature of the zone C. 
to the germinal level could thus be effected through this mechanical link. A control over growth in the bulb could be produced by the movement of the bulb into regions differing in inhibitor content or simply by mechanical deformation. Three periods are involved: (a) the period of oscillation produced by the curved fibre passing through the upper levels; (b) the time for the fibre to pass through the zone of hardening; and (c) the period of the growth cycle of the bulb. All these periods are variable within limits and it is likely that the coupled oscillation, which evolves and is stabilized by feed-back, results from a selected resonance between all three (p. 146).

The successful transfer of control from the curved fibre in the upper reaches of the follicle back to the lower levels required that the fibre be stiffened before it passes into the curved upper levels. A soft fibre would not produce the postulated oscillation. In confirmation of this, Marston (1946) has shown that in the event of incomplete keratinization, as occurs in sheep deficient in copper, the wave-form is poorly developed and its frequency (number of waves produced per unit time) is lower as would be expected from a weaker fibre. The above explanation of crimp formation has some features in common with that given by Auber (1950) and Wildman (1932). However, Auber's assumption on which his explanation rests must be rejected. He supposes that the $\alpha$-structure of the fibrils is produced by an actual contraction in length produced during hardening and that the contraction is greater on the more highly-keratinized inner face (para) of the fibre. There is ample experimental evidence (p. 211 et seq.) that the $\alpha$-structure is present in the fibrils as originally formed and owes nothing to the subsequent chemical changes occurring during keratinization.

A characteristic relationship between crimp form and tip shape in the various classes of wool fibres was first described by Dry (1926 and 1928) and more recently discussed by Fraser (1951) (p. 149) who has tried to explain it in terms of interfollicular competition. Fraser develops the idea of a competition between follicles for the materials needed for growth, and suggests that their efficiency in this competition depends in part on the time of origin of the follicle. In these terms he gives an explanation of the formation of the first few curves at the tip of a wool fibre which precede the establishment of the regular crimp form. The concepts of competition and follicular efficiency seem to overlap and supplement those based on inhibition; both ideas stand in need of further analysis and testing (see also p. 149).

\section{Allometric growth}

When the different parts of an organism are compared it is usually found that they grow at different rates and that the proportions of the organism thus change as life continues. A formula which has often been found to 
relate the amounts of growth in different parts is the allometric equation:

$$
y=b x^{\alpha}
$$

where $x$ and $y$ are the sizes of two parts and $\alpha$ and $b$ are constants. The equation gives expression to the idea that each part grows by self-multiplication (giving the exponential law p. 139), but that for reasons depending on their "appetites", their command over or access to food supplies, etc., the exponential factors are not equal in the two separate growth equations (Reeve and Huxley, 1945; Richards and Kavanagh, 1945).

That the law applies to epidermal growth vis $\dot{a}$ vis that of the whole organism is suggested by the often noted fact that horns tend to be relatively larger, the larger the animal, but no data really adequate to test it exist. In any case much epidermal growth is strictly accretionary, i.e. a fixed amount of growing tissue is constantly adding to a store of dead material which itself, since it is no longer contributing to the growth, should be subtracted from the measured size before testing the equation as in the modified version proposed by Robb (see Reeve and Huxley, 1945): $y=b x^{\alpha}+c$, where $c$ is the "dead weight". The existence of cyclic growth, ecdysis and changes in relative rates due to changes in hormonal patterns following crises such as puberty (equivalent to discontinuous changes in $\alpha$ ) are further complications. 


\section{Molecular and Macromolecular Structure}

\section{The present status of the chemical structure of the keratins}

The chemical composition and constitution of a protein may be considered established when the following are known: (a) the number of separate polypeptides composing the molecule and the nature of any covalent cross-linkages uniting them; (b) the amino acid sequence in each of the polypeptides; (c) if a prosthetic group is present, its relation to the polypeptide moiety.

Complete solutions to (a) and (b) are available for three proteins; for the keratins, which are far more complex, there is no immediate prospect of even partial solutions. There is no evidence, however, to show that there is anything in the nature of a prosthetic group to complicate the position further. Essential information concerning a protein is provided by a knowledge of its total amino acid composition and its end-group composition, i.e. the groups which terminate the main polypeptide chains. For many keratins we have adequate, although not complete, determinations of both end-group and total amino acid composition from which may be inferred a general picture of the overall chemical reactivity of the molecular complex (Table 7). For purposes of reference, in Tables 1, 2 and 3 (Chapter I) will be found the amino acid composition from a number of determinations as reported by various authorities. The most studied material is wool and the data relating to it are considered to be of a high order of accuracy although, it is clear that precise agreement between independent analysts has not been reached. Table 7, devised by Ward and Lundgren (1954), presents a summary of the amounts and kinds of chemical groups to be found in several keratins as calculated from their amino acid composition. Assuming all these are accessible to reagents the Table enables a fair prediction to be made of chemical behaviour. Similar Tables will be found in Tristram's extensive compilation (1953) from which the data for Fig. 2 (Chapter I) were taken.

\section{End Groups}

Information concerning molecular weight, the minimum number of polypeptides, the presence of branched or cyclic chains and amino acid composition may be obtained by determining the amino acids which form 
Table 7. Keratins as Chemically-Reactive Substances* (gramme equivalents per $10^{5} \mathrm{~g}$ of keratin).

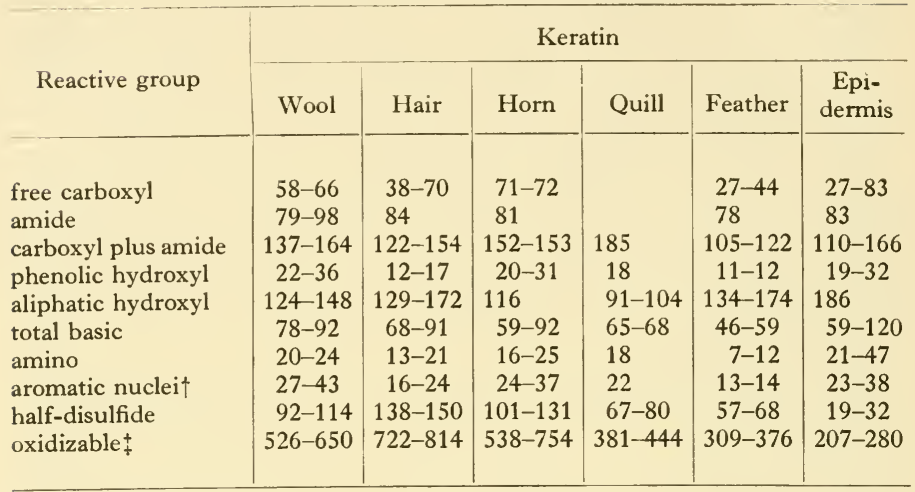

* Taken from Ward and Lundgren (1954).

† Aromatic nuclei available for coupling with diazonium salts : tyrosine plus histidine.

$\ddagger$ Oxidation capacity calculated as twice the number of oxygen atoms rapidly consumed from performic acid.

Table 8. N-Terminal Amino Acids of Hair and Wool*.

\begin{tabular}{l|c|c|c|c|c}
\hline & \multicolumn{5}{|c}{ Material } \\
\cline { 2 - 3 } \multicolumn{1}{c|}{$\begin{array}{c}\text { Amino } \\
\text { acid }\end{array}$} & $\begin{array}{c}\text { Lincoln } \\
\text { wool }\end{array}$ & $\begin{array}{c}\text { Romney } \\
\text { wool }\end{array}$ & $\begin{array}{c}\text { Merino } \\
\text { wool }\end{array}$ & $\begin{array}{c}\text { Wool } \\
\text { (fine) }\end{array}$ & $\begin{array}{c}\text { Human } \\
\text { hair }\end{array}$ \\
\hline & & & & & \\
glycine & 5.2 & 4.5 & 4.7 & 3.8 & 3.9 \\
alanine & 1.3 & 1.2 & 1.2 & 1.1 & 1.0 \\
valine & 2.4 & 2.4 & 2.4 & 1.0 & 4.0 \\
serine & 1.3 & 1.2 & 1.2 & 1.05 & 1.0 \\
threonine & 4.8 & 4.9 & 4.9 & 3.2 & 4.0 \\
aspartic & 0.6 & 0.6 & 0.6 & 0.5 & 0.5 \\
glutamic & 1.3 & 1.2 & 1.2 & 0.9 & 1.0 \\
& & & & & \\
\hline
\end{tabular}

* See references on page 163 . 
the terminal residues of the chains. The end with a free $-\mathrm{NH}_{2}$ is referred to as $\mathrm{N}$-terminal; that with a free $\mathrm{COOH}$ as a $\mathrm{C}$-terminal.

$\mathrm{N}$-terminal groups are determined by reacting the protein with Sanger's reagent dinitrofluorobenzene (DNF) which attaches to the free amino group producing a substituted amino acid easily identified after hydrolysis by its yellow colour. The $\mathrm{C}$-terminal groups are found by reacting the protein with hydrazine $\left(\mathrm{N}_{2} \mathrm{H}_{4}\right)$ when the chain residues are converted into hydrazides with the exception of the C-terminal acid.

Several workers have examined wool and are in good agreement as to which acids form the end groups although estimates of numbers vary. Blackburn (1950) reports the same groups for a N.Z. coarse wool as Kerr and Godin (1959) for human hair and horse hair. The latter refrain from citing their quantitative findings, accepting Thompson's (1957) criticism that these methods are not reliable for materials such as solid keratins.

Middlebrook (1951) rounded off his figures to give the following twentyseven $\mathrm{N}$-terminal groups: glycine 8 , alanine 2 , valine 4 , serine 2 , threonine 8 , aspartic acid 1 , and glutamic acid 2 in $10^{6} \mathrm{~g}$ wool keratin. He calculates a chain weight of the order of 60,000 and thus a total molecular weight for "wool keratin" of $27 \times 60,000 \sim 1,600,000$ assuming homogeneity. Estimates for chain weight from the figures in Table 8 vary from 50,000 90,000 approximately.

The C-terminal groups of wool were also found by Blackburn and Lee (1954) to be glycine, alanine, serine, threonine, aspartic and glutamic acid. These same six residues were found by Kerr and Godin in hair. Alexander and Smith (1956) have determined the end groups of three fractions $\alpha, \beta$ and $\gamma$ derived from wool oxidized by peracetic acid (see p. 238) and found the same groups in somewhat different proportions.

The N-terminal end groups of a soluble derivative of feather keratin have been determined by Woodin (1954a and b, 1955 and 1956). The same groups as for wool and hair were found in very small quantities indicating a deficiency of $\mathrm{N}$-terminal groups which Woodin thinks may mean that feather keratin is a cyclic polypeptide. Krimm and Schor (Schor, 1958), who favour a much larger unit (see p. 208) than Woodin's monomer (m.w. 10,000), think that in solublizing his feather, he hydrolyses the peptide bonds linking the frequently-occurring proline residues and thus produces shorter chains with proline end groups which are not then detected by the procedure used.

\section{Molecular structure}

\section{Methods of Partial Degradation}

Amino acid analyses and other analytical procedures show that keratin consists of polypeptides and that, if constituents other than amino acids are present, they are there in very small quantities. Methods of partial 
degradation are resorted to in the attempt to discover the existence of macromolecular units of an intermediate range of size. The study of the problem of how many polypeptides participate, the composition and length of these chains and their sequence of amino acids has not progressed far. The formidable difficulties of the problem are obvious. Most methods of studying proteins have been developed for the soluble proteins and, to apply these, we have first to develop methods of obtaining soluble derivatives of keratin by procedures which will permit the structure of the insoluble original to be inferred from that of the soluble derived fragments. The solutions must be fractionated into their constituent polypeptides and the location of the constituents in the original solid complex established.

Most of the work of this nature will be described in Chapter 6 and is only mentioned here for reasons of formal completeness. Most workers, accepting the view that the insolubility of keratin is due to two factors: (a) the interchain cross-linking by the sulphur bridge of the cystine residues, and (b) the presence of numerous hydrogen bonds, have attempted, either in a single stage or in successive stages, to rupture both classes of bonds and thus to obtain a preparation of the free, constituent polypeptides (p. 233). A considerable number of preparations have in fact been made, often with a view to obtaining a product of some commercial value, but the few of these that have been examined in detail have not proved promising sources for the extraction of pure proteins or their derivatives. It is in the purification of these mixtures that a very substantial contribution towards further progress is expected. For the present we may summarize the results for wool as follows: all the materials examined contain several polypeptide species probably differing in composition. There is some evidence to suggest that the derived polypeptides can be grouped into two classes probably related to two distinct morphological components, filaments and matrix, visible in electron micrographs (Chapter 6). The proteins derived from the filaments have a higher molecular weight $(50,000-80,000)$ than those of the matrix $(\sim 10,000)$; the latter contain a greater amount of the cystine residues. None of the many components have been prepared in a state of complete purity and little progress is possible at the moment towards forming a detailed picture of the place of each in the original insoluble hair. In the case of feather Woodin's $10,000 \mathrm{~m}$.wt. unit has some claims to be regarded as a definite monomer.

\section{Non-Destructive Methods}

\section{$X$-ray diffraction}

Although the chemical analysis of a complex material of biological origin may have only a limited value unless pure components are first extracted, the $\mathrm{X}$-ray analysis of the same material may bear immediate 
fruits if a single dominant component is sufficiently crystalline to yield a recognizable diffraction pattern (Chapter I). The method, as it were, cuts through the tangle of secondary structures and minor constituents and yields immediate information concerning the arrangement of the atoms in the crystalline regions. Further, since it leaves the material unharmed the same sample may be used for other tests. Fortunately all the keratinized mammalian tissues, as was discovered by Astbury and his associates (see Chapter I), give substantially the same pattern, proving that they contain crystallites of similar molecular structure. Feather keratin gives a $\beta$-type pattern, one of the most detailed yielded by any protein fibre.

The formal analysis of the X-ray diffraction patterns of a well-crystalline material can lead to the exact placing of the atoms (other than hydrogen) in the structures and a growing number of organic compounds of biological importance have been determined in this way. The proteins, whose molecules may contain thousands of atoms, offer enormous difficulties but great success has been achieved in recent years (Perutz, 1959).

The low-angle pattern. The total $\mathrm{X}$-ray pattern yielded by biological fibres is separated conventionally into two parts (Fig. 3, p. 11) described as the wide-angle pattern and the low-angle pattern. The dividing line is arbitrary, but reflections corresponding to a Bragg spacing of less then $20 \AA$ are referred to the wide-angle pattern, those corresponding to longer spacings belong to the low-angle pattern. As described in Chapter I, the number of wide-angle patterns is small-whole groups of fibrous proteins being characterized by the same pattern. In contrast to this simple situation among the wide-angle patterns, a considerable variety of small-angle patterns are found even among proteins which are classified together on account of their similar wide-angle patterns. These low-angle $\mathrm{X}$-ray patterns of the protein fibres are often of great complexity, and indicate the existence of elaborate structures of macromolecular dimensions which are as yet ill-understood. The relation of these structures to the smaller molecular formations responsible for the large-angle patterns we have just been considering, is also obscure. The "long spacings", which may be either meridional or equatorial in placing in the X-ray patterns, can be measured with some accuracy. If the reflection is sharp, a lattice spacing corresponding to it is usually calculated by means of the Bragg equation. When they are diffuse this procedure is difficult to apply; but the totality of the pattern is characteristic of the protein and may undergo changes when the material is chemically altered. In this respect the low-angle pattern is more characteristic of a particular protein than are the wide-angle patterns.

Sketches of two low-angle $\alpha$-patterns are given in Figs. 68 and 69, and in Table 9 are listed the long spacings of the keratins as measured by 
Macarthur (1943), Bear (1943) and Bear and Rugo (1951). Table 10 by Schor (1958) summarizes the various reflections reported for the more elaborate feather diagram. Discussion of this is postponed until later ( $p$. 208). In $\alpha$-keratin the longitudinal spacings can be regarded as orders of a master spacing of $198 \AA$ and the equatorial spacings as orders of $80-90 \AA$. In feather the main longitudinal spacing is usually given as $95 \AA$ but Schor prefers $189 \AA$.

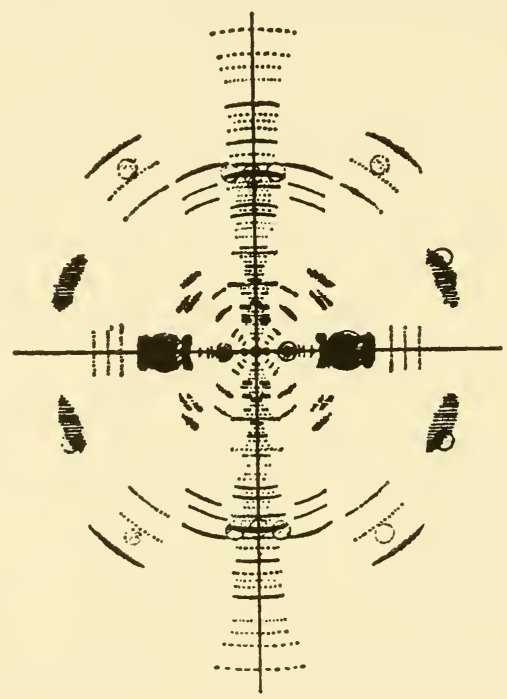

Fig. 68. Composite chart of $\alpha$-keratin diffraction pattern given by porcupine quill. Fibre axis vertical; plane plate. (To $D=3 \AA$.) Taken from Macarthur (1943).

A certain independence between the long spacing (macromolecular level) and the short spacings (molecular level) is suggested by the fact that the large-scale order may be destroyed without affecting the order at the molecular level. This is commonly observed in the regenerated keratins, i.e. materials which have been dissolved and reformed into fibres. These may give either $\alpha$ - or $\beta$ - or cross $\beta$-patterns at high angles (Astbury, 1947; Rudall, 1946 and 1952; Mercer, 1949a) indistinguishable from the original patterns, but they lack long spacings proving that although the small-scale structures in the $\alpha$-crystallites have been regenerated the 
larger-scale structures have not reformed. On the other hand, experimental procedures, such as the "heat-moisture treatment" of Bear and Rugo (1951), also exist by which the small-scale molecular order may be deranged while leaving the macromolecular pattern largely intact.

The long spacings revealed by diffraction $(30-700 \AA)$ overlap those which can be resolved in the electron microscope (50 $\AA$ and longer) and in some instances the two methods give results in good agreement. For

Table 9. Small-Angle Diffractions of $\alpha$-Keratin Fibres $\dagger$

Meridional and near meridional reflections.

\begin{tabular}{c|c|c|c|c|c|c}
\hline & \multicolumn{2}{|c|}{ African porcupine quill } & \multicolumn{3}{|c}{ Human hair } \\
\cline { 2 - 7 }$k$ & $\begin{array}{c}d \\
(\AA)\end{array}$ & $\begin{array}{c}b_{0} \\
(\AA)\end{array}$ & $I$ & $\begin{array}{c}d \\
(\AA)\end{array}$ & $\begin{array}{c}b_{0} \\
(\AA)\end{array}$ & $I$ \\
\hline 3 & 66 & 198 & 6 & 65 & 195 & 6 \\
4 & $49^{*}$ & 196 & 1 & $49^{*}$ & 196 & 1 \\
5 & 39 & 195 & 2 & & & \\
7 & $27 \cdot 4$ & 192 & 4 & $27 \cdot 6$ & 193 & 1 \\
8 & $24 \cdot 5$ & 196 & 2 & $24 \cdot 3$ & 194 & 4 \\
9 & $22 \cdot 0$ & 198 & 2 & & & \\
10 & $19 \cdot 8$ & 198 & 4 & $19 \cdot 4$ & 194 & 2 \\
11 & $18 \cdot 1$ & 199 & 3 & & & \\
13 & $15 \cdot 2$ & 198 & 1 & $15 \cdot 0$ & 195 & 2 \\
15 & $13 \cdot 2$ & 197 & 1 & & & 2 \\
16 & $12 \cdot 4$ & 198 & 4 & $12 \cdot 1$ & 194 & 2 \\
19 & $10 \cdot 4$ & 198 & 3 & & & \\
& & & & & & \\
\hline
\end{tabular}

Equatorial reflections

\begin{tabular}{c|c|c|c|c|c|c}
\hline $\begin{array}{c}h \\
\text { (?) }\end{array}$ & & $\begin{array}{c}a_{0} \\
\text { (?) }\end{array}$ & & & $\begin{array}{c}a_{0} \\
(?)\end{array}$ & \\
\hline 1 & 83 & 83 & 10 & 90 & 90 & 10 \\
2 & $45^{*}$ & 90 & 6 & $47 *$ & 94 & 6 \\
3 & $28 \dagger$ & 84 & 4 & $29 \dagger$ & 87 & 4 \\
\hline
\end{tabular}

The $d$ columns contain the measured spacings which, when multiplied by the assigned $k$ or $h$ indices, yield the large fibril period, $b_{0}$, or the large transverse fundamental spacing, $a_{0}$, of $80-90 \AA$. The $I$ columns indicate in rough fashion the relative intensities of the diffractions.

* Overlaid with faint, poorly-oriented rings, probably due to lipid.

$\dagger$ From Bear and Rugo (1951) 
example, in the case of collagen which shows a well-marked long spacing of $640 \AA$, the electron-microscopic image reveals the same fundamental period.

Yet even in this instance, in which a relatively-large amount of data from many sources is available and the basic arrangements of the polypeptide chains as a triple chain helix are precisely known (see p. 127), the nature of the major spacing and the many subspacings visible electronmicroscopically is still under discussion (Hodge, 1960).
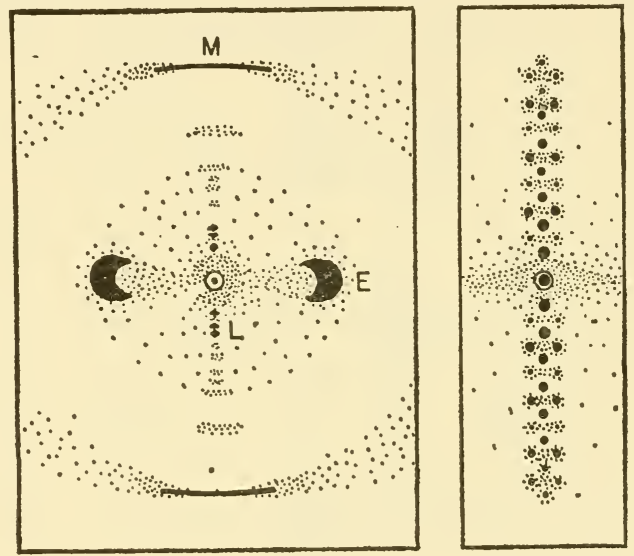

FIG. 69. Drawings of the respective wide-angle and pinhole small-angle patterns of clam (Venus) muscle. The fibre-axis direction in all patterns is vertical. $M$ is the prominent composite meridional arc at $5 \cdot 1 \AA$, E the equatorial diffraction at $9.6 \AA$, these being the characteristic $\alpha$-pattern diffractions at wide angles referred to in the text. $\mathrm{L}$ in the left diagram marks the small-angle series of diffractions which are indicated in greater detail in the right figure. Adapted from Bear (1951).

With keratin the situation is much less satisfactory. Convincing electron-microscopic observation of the longitudinal spacings is wanting. Filaments can be observed both in growing hair and in disintegrated wool which have diameters of the expected order of 60-100 $\AA$ and the packed array of these may be responsible for the lateral spacings (Birbeck and Mercer, 1957a; Rogers, 1959) (Plates 15 and 16). Fibrils in disintegrated wool (Farrant et al., 1947; Jeffrey, Sikorski and Woods, 1956) and in extracted sections of skin (Porter, 1956) have a quasi-regular nodular appearance which does not possess a periodicity of the expected order 


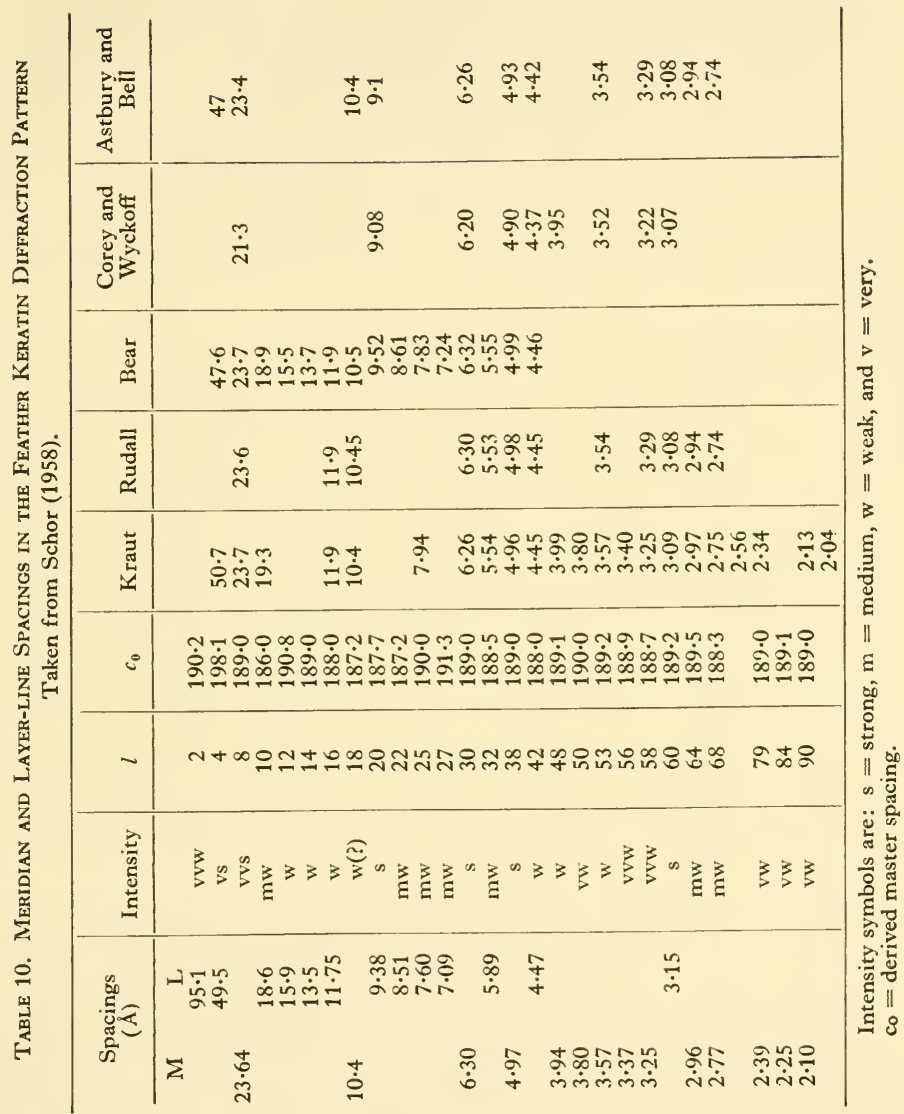


calculated from the X-ray patterns ( $198 \AA$ ). In thin sections even wellformed materials, such as feather and porcupine quill, fail to show unambiguous evidence of longitudinal spacings although the expected values, $96 \AA$ and $198 \AA$ respectively, are well within the instrumental range. This further emphasizes the different organization of the collagens and keratins. The system of long equatorial side spacings could very likely arise as scattering from the quasi-regular packing of filaments embedded in a matrix of different scattering power (p. 247). Significantly, Fraser et al. (1957 and 1959) have observed intensity changes in this group of spacings when fibres are treated with osmium tetroxide in a manner known by electron microscopy to lead to strong deposits of osmium compounds in the matrix (p. 248). The $84 \AA$ lateral spacing probably corresponding to the interlayer spacing of filament and matrix is strongly enhanced.

Speculation on the nature of these long meridional spacings has tended to take the form of either of two extreme theories which may be called (a) long chain theories, and (b) corpuscular-aggregate theories. In the first theory the larger fibril is pictured as being built up from many parallel chains (or other thin linear elements) and the periodicities along the macroformation arise from the repetition of structure in the basic chain. This mode of construction seems to apply to collagen. Many of the electronmicroscopical images can be accounted for in terms of such a unit (Hodge, 1960). Solutions of both types have been proposed at one time or another for the keratins. The elaborate equatorial reflections given by feather suggested an aggregation of corpuscles to Astbury and Marwick (1932). Macarthur (1943) considered the possibility of the long-chain model; and the nodular appearance of the fibrils released from reduced wool by enzymatic digestion suggested a linear aggregation of corpuscles to Farrant et al. (1947). There is other evidence that many other fibres are formed by the aggregation of particles (Astbury, 1949 and 1958; Jeffrey et al., 1956).

The wide-angle patterns. The wide-angle patterns yielded by the fibrous proteins (see Chapter 1) contain far less information in the crystallographic sense than those now available from crystalline proteins. Nevertheless, the first progress towards an understanding of the arrangement of the polypeptide chains in the solid state came from a study of mammalian hair and silk fibroin. This progress was made possible by an ingenious integration of data derived from a variety of sources: chemical composition, X-ray diffraction, physicochemical and elastic behaviour. We owe the development of these methods principally to Astbury (1933) and Astbury and Woods (1933), and in spite of an enormous increase in the precision of the crystallographic side of the work, the principles of the methods remain essentially as devised then. See also Kendrew (1954). 


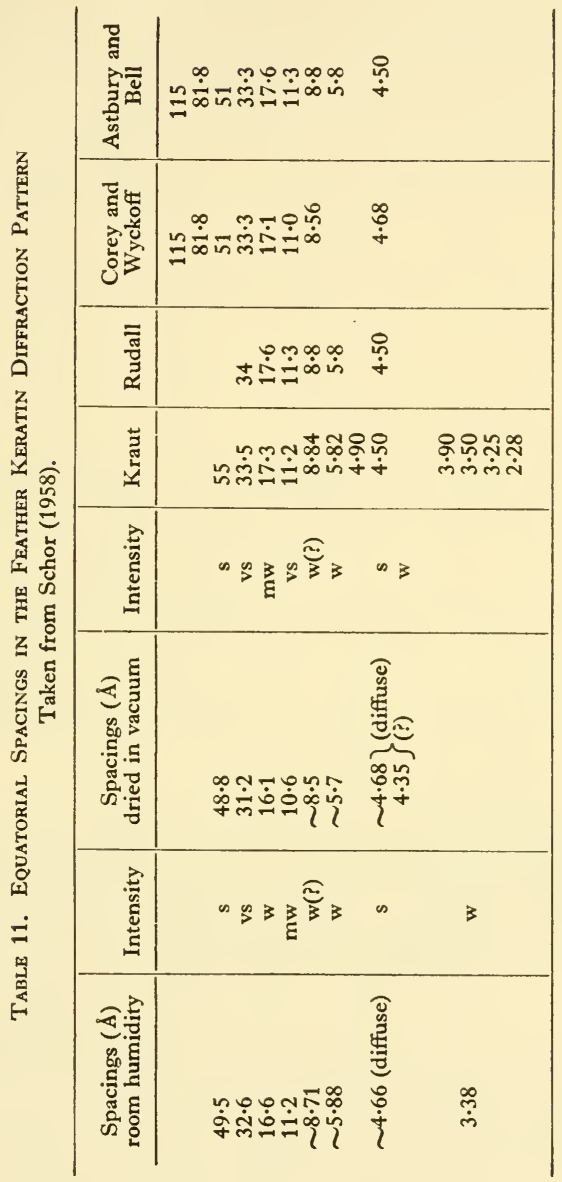


The first and most essential experimental step was the discovery by Astbury and Street (1931), that stretched hair gave a different pattern from unstretched hair, a pattern of the $\beta$-type similar in fundamentals to that yielded by silk. Meyer (Meyer and Mark, 1930) had given a broadly satisfactory interpretation of the structure of silk in terms of a model structure composed of a bundle of parallel straight polypeptide chains oriented parallel to the fibre axis. Astbury recognized that, allowing for the differences in amino acid composition between the rather simple fibroin and the more complex keratin containing large bulky amino acid groups, the same type of model would account for the major features of the $\beta$-type keratin pattern. This close similarity between silk fibroin and $\beta$-keratin still forms the basis of present-day proposals for the structure of the latter. The structure of fibroin is now known with great precision, (Marsh, Pauling and Corey, 1955) and accordingly the proposed structures for $\beta$-keratin are formulated with equal precision and are thus more capable of being experimentally checked. These will be described later.

Astbury's second step was the far-reaching proposal that the $\alpha$-structure developed from the shortening by folding of the straight polypeptide chains of $\beta$-keratin. This idea was suggested by a comparison with rubber, the elasticity of which was currently being explained in terms of the coiling up of the polyisoprene chains and by the proposal of Meyer and Mark (1930) that other forms of " protoplasmic " elasticity, such as muscle contraction might rest on chain coiling. In Astbury's hands this concept of polypeptide chain folding was extensively exploited and today forms the basis of most ideas about protein structure and of polypeptide behaviour.

\section{The elastic properties and the structure of hair}

If $\alpha$-keratin is a shortened form of $\beta$-keratin, then it would seem possible that the degree of folding of the polypeptide chains in $\alpha$-keratin could be inferred from the increase in length necessary to produce the $\alpha-\beta$ transformation. This possibility led Astbury and Woods (1933) to an extensive analysis of the elastic properties of wool and hair.

A description of some aspects of the complex rheological behaviour of hair and wool will be given later (p. 249). Here we are concerned with one particular problem: the relation between molecular extensibility and the change in length of the whole fibre, and the possibility of inferring the extensibility of the molecular chains from the extension of the whole fibre. For this purpose it is necessary to analyse in greater detail the sequence of events which occur during the stretching of a hair. Fig. 70 is an idealized version of a stress-strain curve for a wool fibre or hair as described by Speakman (1928) and by Astbury and Woods (1933). It will be seen that it appears to divide into several distinct steps:

$O A(0-2 \%)$ : In this section the curve is closely linear and is for this 
reason referred to as the Hooke's law region. Here the fibre is behaving like a conventional elastic solid and the axial molecular-lattice spacings, as found by X-ray photographs, show a reversible increase of the same order of extension as the fibre. The value of Young's modulus and the amount of the Hookian extension decrease as the humidity and temperature are increased.

$A B(\sim 2-\sim 20 \%)$ : At the point $A$ the fibre yields suddenly and there is a rather rapid extension of the fibre to about $20 \%$. During this extension the X-ray photograph remains of the $\alpha$-type but becomes less

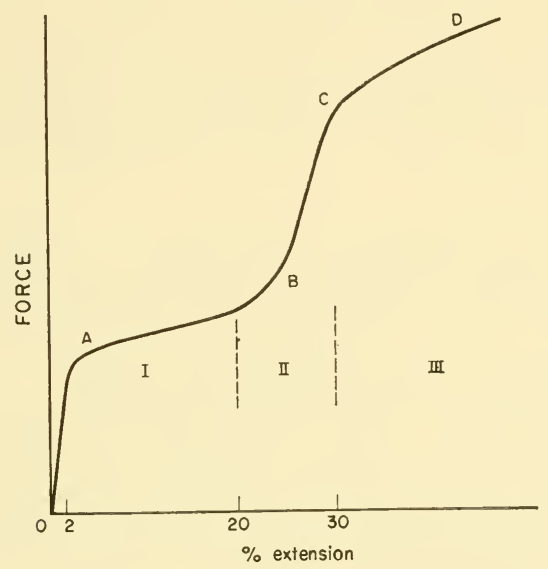

Fig. 70. Idealized stress-strain curve for a keratin fibre (wool or hair) stretched in water at room temperature: OA (0-2\%) Hooke's law region; AB $(2-20 \%)$ extension of amorphous phase (phase I) $\alpha$-fades and $\beta$ appears; BC $(20-45 \%)$ extension of crystalline phase (phase II); CD $(45 \%)$ extension of phase III non-crystalline (adapted from Astbury and Woods, 1933).

perfect as extension increases. Dry fibres break usually at about $20 \%$ extension. At $B$ a shoulder develops showing that the fibre is becoming more difficult to stretch; the $\alpha$-pattern rapidly fades and $\beta$-reflections appear. This shows that in this region the $\alpha$-crystalline phase is being destroyed and that a new configuration is appearing.

$C D(>\sim 45 \%)$ : The fibre again becomes more easy to stretch. The transformation of the $\alpha$-crystallites into the $\beta$-modification seems complete. Extension rarely exceeds $60-70 \%$ in cold water, but is greater in hot 
water or in steam. It is supposed that at these higher extensions a further non-crystalline fraction must extend.

An important feature of these extensions and the X-ray changes that accompany them is that (excluding the phenomena of set, p. 249) they are reversible and that the type of $\mathrm{X}$-ray pattern is closely connected with the degree of extension.

Astbury and Woods suggested that the step-like nature of the curve $O A B C D$ was due to the presence in the fibre of three "phases" of keratin which differed in their ease of extensibility and which were effectively in series. On stretching a fibre each extended in turn: at $A$ the " transformation tension" of phase I was reached, at $B$ phase II, etc. Since no marked change in X-ray pattern occurs in the range $A B$ or beyond $C$ these portions of the curve must correspond to the extension of noncrystalline material (phases I and III); the range $B-C$ where the $\alpha-\beta$ transformation occurs must be where the crystalline phase extends (phase II).

Since the steps in the curve are not sharp it was assumed that the separate phases were not simply in series but that, as a result of restrictions exerted by one phase on another, they were also partly in parallel. With the assumption of three extensible elastic elements, with different resistance to extension, this model has sufficient variables to provide a fit even for the infinitely varied responses of the wool fibre.

Woods (1938) met the possible criticism that, since hairs were histologically complex, certain features of the step-curve might be due to the extension of various histologically-recognizable components joined partly in series and that the extension of these parts (including the crystalline region) might not be the same as that of the whole fibre. He stretched wool fibres to various percentage extensions, "set " them at these lengths by steaming and, by means of tryptic digestion, isolated the stretched and set, keratinized contents of the cortical cells. By plotting the percentage increase in length of the whole fibre against that of the cell residues, he showed that the cell contents parallelled very closely the extension of the fibre as a whole. A slight lag was interpreted as due to either a loss of set during the isolation of the cells or, as is most likely the case, to the extension of the component which links the cells together, i.e. in current histological terms, to the cell membranes and intercellular cement. It is thus possible to say that the elastic properties of the fibre are mainly those of the keratinized contents of the cortical cells.

In water at ordinary temperatures extensions of the order of $70 \%$ are possible; at elevated temperatures (steam) or in solutions of dilute caustic soda a limiting extension of the order of $100 \%$ was found by Astbury and Woods (Fig. 71). They assumed from this that at $100 \%$ extension all the polypeptide chains were fully extended. By making a 
further assumption that all parts of the fibre (and their component molecules) were equally extensible, they were thus able to conclude that the fully extended molecular chain $(\beta$-form) was twice as long as the folded chain $(\alpha$-form). This was equivalent to assuming that the molecular configurations in the non-crystalline fractions (phases I and III, Fig. 70) were essentially similar to those prevailing in the crystalline phase II, but for various steric reasons were unable to assume the perfection necessary for crystallization.

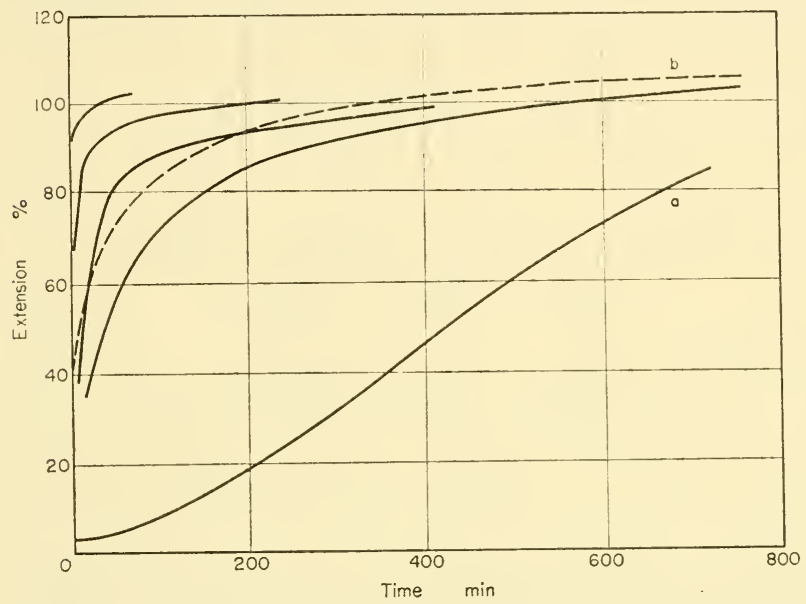

Fig. 71. Creep curves for Cotswold wool under constant load in steam (continuous line) and in a $1 \%$ aqueous solution of caustic soda (broken line). The timescale has been contacted by $\mathrm{X} 2$ for curve (a) and expanded by X10 for curve (b) (Astbury and Woods, 1933).

On the basis of models of polypeptide chains and the assumption that the $\alpha$-form is half the length of the $\beta$-form, Astbury and his associates advanced successively two definite proposals for an $\alpha$-keratin structure. The first of these (Astbury and Woods, 1933) was shown by Neurath (1940) to be too tightly folded to accommodate the side groups. The second (Fig. 72) (Astbury and Bell, 1941) gives more ample opportunities of intrachain H-bond formation, appeared to satisfy most of the requirements. Even without a completely detailed description of atomic positions this broad concept of a folded polypeptide chain linked by a variety of side chains to other main chains proved, in the hands of the 
Leeds School, capable of co-ordinating a great deal of physicochemical data. Recent discussions of the elastic properties of fibres are given by Feughelman (1959), Peters and Woods (1956), Skertchly and Woods (1960).

The conclusion that the $\alpha$-keratin chain was half as long as the $\beta$-chain was not universally accepted and other models were also proposed. Notably Ambrose and Hanby (1949) and Zahn (1947 and 1949) proposed a fold, which yielded the required axial periodicities and gave an $\alpha \rightarrow \beta$ extensibility of $\sim 33 \%$. This appeared to accord with the observation that the actual transformation of the $\alpha$-crystallites into the $\beta$-form took place between fibre extensions of $20-45 \%$ (phase II, Fig. 70) which could be

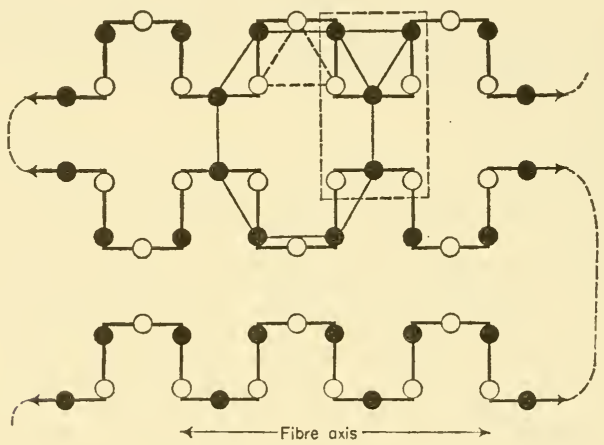

FIG. 72. The Astbury model for $\alpha$-keratin: arrows represent the direction of the main chain; represents a side chain pointing up from the plane of the diagram; $O$ represents a side chain pointing down from the plane of the diagram. From Astbury and Bell (1941).

interpreted to mean that the crystallites themselves were fully extended by a change of length from 1.2 to $\sim 1 \cdot 5$, i.e. of the order of $25-30 \%$. Ambrose and Hanby also claimed that the less-folded structure fitted in better with their observations on the average orientation of $\mathrm{H}$-bonds inferred from absorption spectra of polarized infra-red radiation (p. 197). Other proposals stemmed from Huggins (1943). It is to be noted that none of these earlier models appears capable of yielding an axial periodicity of $1.5 \AA$ which is now recognized as being fundamental to the $\alpha$-structure (p. 182). All yield the $5 \cdot 1 \AA$ (axial) and $10 \AA$ side spacing.

\section{Current crystallographic analysis}

A decisive step in the approach to the problems of the structure of the fibrous proteins was ushered in by the proposal of helical structures by 
Pauling, Corey and Branson (1951). Up to this time it had seemed difficult to propose structures which would be capable of quantitative test. However, stimulated by the precise structures proposed by Pauling and Corey, a new attack opened, with the result that at the present time probable structures for most of the fibrous proteins are known.

These impressive developments stemmed in the first place from methods which were in principle the same as those originally applied by Astbury to the keratin problem. That is, from the study of a number of small and simple compounds, such as amino acids and peptides, which permit of a full crystallographic analysis, accurate information about the bond lengths and angles found in unit structural elements occurring in proteins is obtained. From these data models of the structural units are constructed, and from these the probable conformations of peptide chains are inferred. In this way Pauling and Corey, using the very accurate data relating to bond lengths and angles which had accumulated in their hands by 1953 , predicted the existence of two helical structures, which could serve as models of folded chains in fibrous proteins, and of other structures built from extended chains which could form the basis of models for $\beta$-type structures.

A later important development, increasing the purely crystallographic element in this work, was the publication by Cochran, Crick and Vand (1952) of a theory of X-ray diffraction from helical structures which greatly facilitates the interpretation of the patterns. The most fruitful result of this theory was the elucidation of the structure of DNA (p. 109). It has, however, also allowed a detailed comparison to be made between the observed X-ray pattern of several synthetic polypeptides, prepared as simpler models of proteins, and that predicted on the basis of helices, with the result that the structures of these polypeptides are no longer in doubt.

The basis of any model of the polypeptide chain is the amide unit and originally Pauling, Corey and Branson (1951) formed their conclusions concerning its structure on these three compounds: $N$-acetyl glycine, $\beta$-glyclyglycine and diketo-piperazine. Since that time several more glycylpeptides have been solved and also the tripeptide, glutathione. These analyses combine to give as the probable value for the $\mathrm{C}-\mathrm{N}$ distance $1.32 \AA$ and show that the amide group is planar with the $\mathrm{CO}$ and $\mathrm{NH}$ groups in the trans-configuration. These features now figure in all models of polypeptides and proteins (Fig. 73).

The structural principles formulated by Pauling, Corey and their associates (Pauling, Corey and Branson, 1951; Pauling and Corey, 1951a and b; Pauling and Corey, 1953; Pauling, 1958) as necessary conditions for the precise prediction of stable polypeptide configurations are as follows:

(1) The amide group ( $-\mathrm{NH}-\mathrm{CO}-$ ) (Fig. 73) is planar. 
(2) The following bond distances and angles found in small peptides, etc., will occur in polypeptides:

$$
\begin{array}{ll}
\alpha \mathrm{C}-\mathrm{C}^{\prime} & =1.53 \AA \\
\mathrm{C}^{\prime}-\mathrm{O} & =1.24 \AA \\
\mathrm{C}-\mathrm{N} & =1.32 \AA \\
\mathrm{N}-\alpha \mathrm{C} & =1.47 \AA(\text { all } \pm 0.01 \AA) \\
\mathrm{N}-\alpha \mathrm{C}-\mathrm{C}^{\prime} & =110^{\circ} \\
\alpha \mathrm{C}-\mathrm{C}^{\prime}-\mathrm{N} & =114^{\circ} \\
\mathrm{O}-\mathrm{C}^{\prime}-\mathrm{N} & =1.25^{\circ} \\
\mathrm{C}-\mathrm{N}-\alpha \mathrm{C} & =123^{\circ}
\end{array}
$$

These dimensions are illustrated in Fig. 73 taken from Pauling and Corey. The planarity of the amide group and the shortening of the $\mathrm{C}-\mathrm{N}$ distance to $1 \cdot 32 \AA$ is attributed to resonance.

(3) Hydrogen bonds $\mathrm{NH}-\mathrm{O}$ will form where possible with an $\mathrm{N}-\mathrm{O}$ distance of $1 \cdot 79 \pm 0 \cdot 1 \AA$ and the $\mathrm{O}$ lies close to the $\mathrm{NH}$ axis.

(4) The trans-configuration of the amide group is significantly more stable than the cis-configuration.

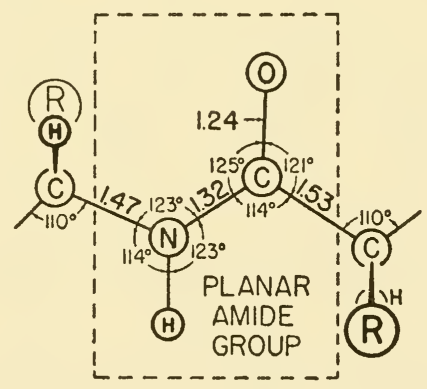

FIG. 73. The planar amide group according to Pauling and Corey (1955).

This structural information was systematically employed to construct polypeptide models and two types of structure were derived depending on whether the attempt was made to form the H-bonds by intrachain or, on the other hand, by interchain association. The first led to helical, folded chains, the second to sheets of associated polypeptides.

Not all crystallographers accept the Pauling-Corey conditions. Huggins (1958) in particular, points out that a departure of $30^{\circ}$ from planarity of the amide group does not involve a marked instability and might well 
exist if other structural or energetic requirements were better satisfied. There would seem to be reason to expect that structural irregularities arising from this and other causes will be found in the polypeptide chains in the amorphous regions.

\section{Pleated-Sheet Configurations-Silk fibroin}

Figure 74 (a) is a view parallel to the planes of the planar amide groups in a polypeptide in which all of the side chains ( $\beta$-carbons) project away from the amide groups. The planes of the amides slant alternatively to right and left and thus may be fitted on to a " pleated sheet " with the pleats running out from the paper. Successive chains may be placed on the sheet in either

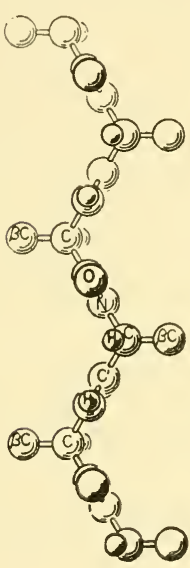

(a)

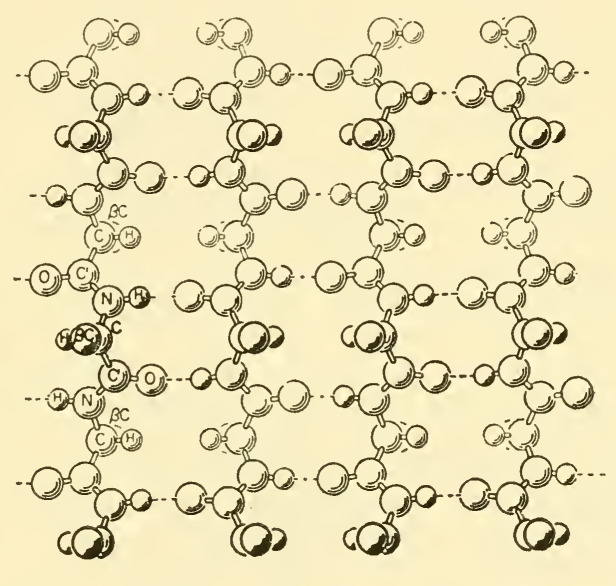

(b)

FIG. 74. The structure of silk fibroin: (a) view parallel to the planes of the planar amide groups; (b) the pleated sheet.

of two ways depending on whether adjacent chains are parallel or antiparallel in direction. By making small adjustments of the parameters of the atoms in order to achieve near straight $\mathrm{H}$-bonds, it was found that two configurations had significantly different identity distances along the polypeptide chains: $7 \cdot 0 \AA$ for the antiparallel sheet and $6.5 \AA$ for the parallel pleated sheet. Thus the two sheets might well serve as models for silk fibroin (axial repeat distance $7 \AA$ ) and $\beta$-keratin $(\sim 6.5 \AA$ ), respectively. Among the fibrous proteins the silk fibroins are the most promising for 
structural analysis. Chemically they are rather simple. Bombyx silk and Tussah silk fibroin are alike in that glycine, alanine, serine and tyrosine comprise over $90 \%$ of the amino acids. The classical studies of Meyer and Mark and Astbury, which have been referred to above, made it clear that in certain fibres, yielding the $\beta$-type pattern with a fibre period of $7 \AA$, the polypeptide chains must be almost fully extended. The completely extended chain is calculated to have a repeat spacing of $7.27 \AA$ which is significantly greater than that actually found.

Marsh, Corey and Pauling (1955) have made a thorough comparison between the diffraction data and the predictions based on the anti-parallel sheet model and have established beyond question that this structure exists in Bombyx silk. In their model the chains of fibroin consisting of (in the main) alternate glycine and alanine residues, are arranged so that the $\mathrm{CH}_{3}$ groups of the alanines stick out entirely on one side of the sheets. Pairs of sheets are thus separated alternately by distances of $3.5 \AA$ and $5 \cdot 6-7 \AA$, spacings which figure prominently in the X-ray patterns. There are minor points still disputed. Peptides comprising the bulk of the protein and containing, apparently, long sequences of glycyl-alanine pairs (Lucas et al., 1956 and 1958) and sequences in which serine substitutes for glycine or alanine residue (Waldschmidt-Leitz and Zeiss, 1955) have been separated from digest of Bombyx fibroin. These glycyl-alanine clusters probably join together to form the crystallites. The solution of Warwicker (1954) is essentially the same.

There is no place in these models for the more bulky side chains, such as that of tyrosine, which together constitute some $18 \%$ of the protein. It is supposed therefore that they are inserted at intervals (regularly or at random) in the chains, thus buckling the sheets and causing some of the diffuse reflections always present. Since in the case of keratins the residues with larger side chains form a far greater part of the protein, distortions arising from such causes must be far more common and perhaps account for the larger amount of diffuse scattering from these materials.

Tussah silk yields a $\beta$-pattern distinct from Bombyx silk and is isomorphous with polyalanine (Bamford et al., 1954). It contains more alanine than Bombyx and thus, assuming a similar antiparallel-sheet arrangement, both faces must contain methyl groups and all pairs of sheets must be similarly spaced at $5 \cdot 3 \AA$. Kay et al. (1956) have shown the existence of "cores" of peptides containing long sequences of alanine.

No attempt has yet been made to discuss rigorously the diffraction data of the $\beta$-keratins of stretched hair and wool in terms of the pleated-sheet models and it would seem, in view of the small amount of information to be obtained from the actual patterns themselves, that the attempt would not be immediately fruitful. The general similarity of all these patterns leaves little doubt, however, that in the crystalline regions of the materials 
yielding them, structures based on pleated sheets are present. There are other silks with somewhat bulkier side-chain composition and hence larger side spacings which approach more closely to the actual conditions prevailing in the keratins. A knowledge of the particular peptide sequences in keratins which participate in crystallite formation is still wanting.

Helical Configurations-The $\alpha$-Helix

Helical structures were first proposed by Huggins (1943) and Taylor (1941); those described by Pauling, Corey and Branson are, however, more precisely specified and are thus capable of quantitative evaluation.

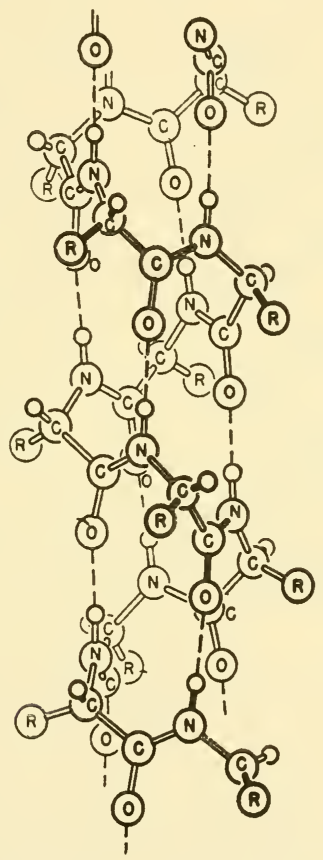

Fig. 75. Diagram of the $\alpha$-helix of Corey and Pauling (reproduced by permission).

They discovered two helical ( $\alpha$ and $\gamma$ ) arrangements, and since that time Low and Baybutt (1952) have described another. Only the $\alpha$-helix has been extensively studied since it does seem to provide the required structure. 
The $\alpha$-helix is based in the first place on geometrical requirements deduced from the known structures of small peptides (see above) (Fig. 73). When a model of a single polypeptide chain, built according to these requirements, is formed into a spiral, it is found that one very compact formation results, in which there are 3.6 amino acid residues per turn of $5.4 \AA$ so that each residue occupies $1.5 \AA$ of the length of the helix (Figs. 75 and 76). The stability of the helix is assured by the formation of multiple internal H-bonds, and Donohue (1953) has shown that of the several possible helices the $\alpha$ is the most stable.

\section{DIMENSIONS OF AN $\propto$ HELIX}

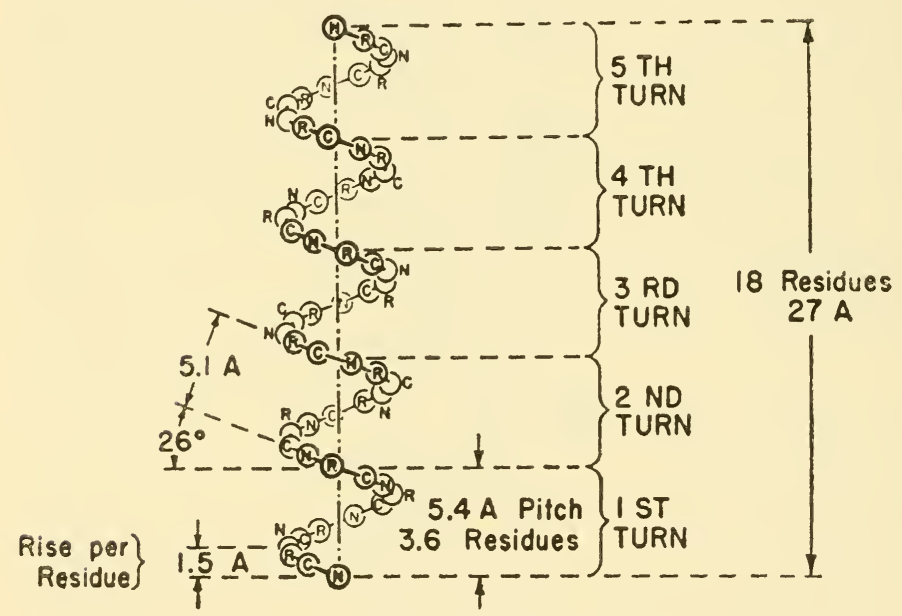

Fig. 76. Diagram of an $\alpha$-helix indicating the $1.5 \AA$ rise per residue which gives rise to the characteristic axial $\mathrm{X}$-ray spacing and the relation between the pitch $5 \cdot 4 \AA$ ( 36 residues) and the $5 \cdot 1 \AA$ spacing. (After Corey and Pauling.)

The $\alpha$-helix was put forward in the first place as a proposal. As a direct experimental test for the presence of such a helix, Perutz (1951) pointed out that such a structure should give a strong meridional reflection of $1.5 \AA$ corresponding to the segment of the helix occupied by one residue (Fig. 76). He found that, in fact, this reflection was present in the patterns given by hair, quill, muscle, various other proteins and polypeptides (Perutz and Huxley, 1951). This reflection had also been recorded earlier by Macarthur from porcupine quill (1943). Since no other of the chain configurations proposed for the $\alpha$-proteins can give a $1.5 \AA$ reflection, the 
occurrence of this particular spacing has become diagnostic for the $\alpha$-helix. Astbury et al. (1959) proved that the $1.5 \AA$ reflection arose from the same structure as the $5 \cdot 1 \AA$ by showing that both reflections were similarly altered by the small $(2 \%)$ reversible extensions of fibres.

Very detailed comparisons have been made between the observed X-ray patterns of certain synthetic polypeptides and that predicted from the $\alpha$-helix by Bamford et al. (1956), Yakel (1953) and Brown and Trotter (1956) and they have demonstrated that the $\alpha$-helix forms the structural basis of these polypeptides. The patterns given by fibres of these materials are often of remarkable perfection and far superior to those of the natural fibrous proteins. It seems probable that the study of a synthetic polypeptide, based more closely on the naturally-occurring sequence of residues in the crystalline regions of the keratins may lead most easily to further advances in the understanding of the natural structures.

When the patterns of the $\alpha$-synthetic polypeptides (see Bamford, Elliott and Hanby, 1956) are compared with those of keratin and muscle, the resemblance is striking and leaves little doubt that the natural structures are based on the helix. Differences are equally striking, and these have been emphasized by Bamford and Hanby (1951). The main characteristics of the $\alpha$-pattern are the strong meridional arcs of $5.15 \AA$ and $1.5 \AA$, and a group of spacings centred around $10 \AA$ at or near the equator (Astbury and Woods, 1933; Macarthur, 1943). When these observational facts are compared with the pattern to be expected from a crystal of hexagonallypacked (Fig. 78) $\alpha$-helices parallel to the axis two difficulties appear:

(1) The $\alpha$-helix would give a strong layer line at $5 \cdot 4 \AA$ but the intensity on the meridian would be zero. In fact we find the strong $5 \cdot 15 \AA$ arc on the axis.

(2) If the centre of the broad equatorial reflection $(9 \cdot 8 \AA)$ is taken as the $(10 \cdot 0)$ reflection of a simple hexagonal lattice the calculated density for $\alpha$-keratin is too low.

\section{Coiled coils and $\alpha$-filaments}

An attempt to resolve these difficulties by suggesting that the whole helix (minor helix) might be twisted into a super helix (major helix) or coiled coil, has been made by Pauling and Corey (1953a), and by Crick (1952). Both proposals involve tilting the $\alpha$-helices to form the super-helix or a coiled coil (Fig. 77) with a pitch angle of about $18^{\circ}$ giving a projection on the axis with a periodic variation in density at intervals of $5.4 \cos 18^{\circ}=$ $5 \cdot 1 \AA$. According to Crick the reason for the deformation may be found in the difficulty of packing the side chains projecting from the non-integral helix. If a simplified model in which the side chains are represented by knobs is taken and, on a piece of paper wrapped around it, the position of the knobs is marked, a pattern is found on unwrapping into which a second 
helix can only fit when it is tilted $\sim 20^{\circ}$ to the first. Crick suggests a three-strand rope to give the $198 \AA$ longitudinal period but this does not account for the strong $27 \AA$ reflection on the equator. The high density $\left(1 \cdot 3 \mathrm{~g} / \mathrm{cm}^{3}\right)$ remains a difficulty.

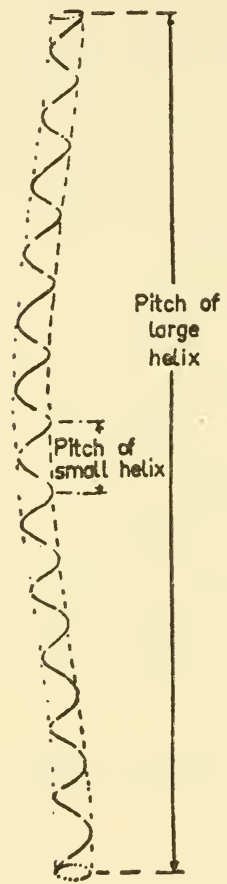

(a)

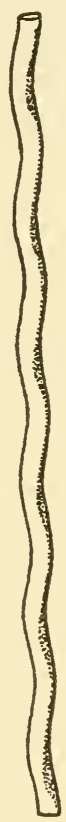

(b)
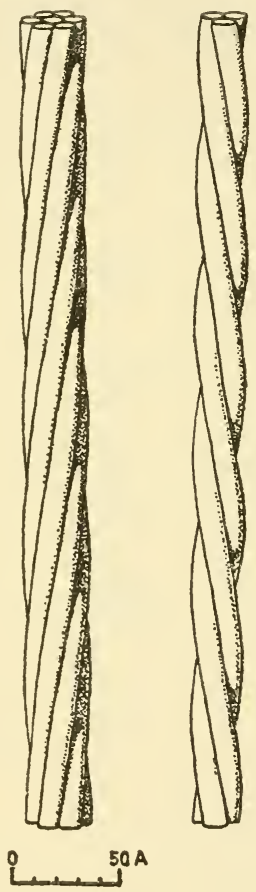

(c) (d)

FIG. 77. Illustrating the curving of the $\alpha$-helix into a super helix (a) and (b) and two possible combinations of super helical structures to give compound helices (coiled coils), (c) the seven-strand cable and (d) a threestrand coiled coil (Pauling and Corey, 1956. Reproduced with permission).

Pauling and Corey reached a similar conclusion on the grounds that an $\alpha$-helix composed of a repetition of different amino acids in a regular pattern would not have a straight axis but one distorted into a large helix. They suggest that a radius of $10 \AA$ for the $\alpha$-helix would permit six 
molecules to twist around a seventh straight $\alpha$-helix to form a "sevenstrand cable" (Fig. 78). Further, a closely-filled structure of hexagonallypacked seven-strand cables with individual $\alpha$-helices occupying the interstices, would improve the fit to the density (Fig. 78). This solution gives a good fit for the many strong reflections (Pauling and Corey, 1956) but is very much a crystallographers' solution and lacks support from other directions. A subfilament of three $\alpha$-helices with irregularities about $27 \AA$ apart ("segmented three-stranded cable") is indicated by the most recent analysis of the keratin diffraction data (Fraser and MacRae, 1961).

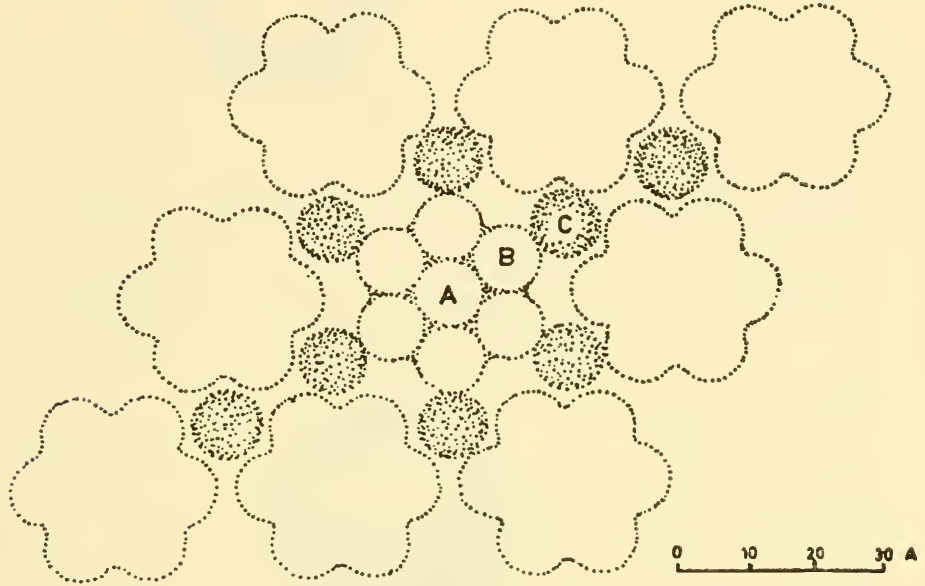

FIg. 78. A suggestion by Pauling and Corey for the macromolecular structure of $\alpha$-keratin in terms of hexagonally-packed seven-stranded cables in the interstices of which are packed single helices $(\mathrm{C})$ to correct the density deficit.

The $\alpha$-helix of diameter $(c .10 \AA)$ is a structural element rather on the small side for current electron microscopy. With the concept of superhelices we enter a domain of dimensions which should be accessible to microscopy. The diameter of the seven-strand cable is $20-30 \AA$. Such a dimension has not yet been observed in cross-sections of keratin in hair and wool. The actual cross-section of what seem to be elementary filaments of keratin in hair and skin is of the order of $60 \AA$ (Figs. 79 and 80) and for myosin a similar value is reported. These figures would seem to demand much more than seven-component spirals, the width of the cylindrical filament being approximately that of six $\alpha$-helices suggesting a rope of between twenty and thirty component helices as indicated in Fig. 79. 
When models of structures of this size are attempted either by forming concentric shells of $\alpha$-helices or by arranging these on helical sheets, difficulties are met in effectively filling the centre of the filament without some disorder (Fig. 79). Perhaps an indication that the nature of the

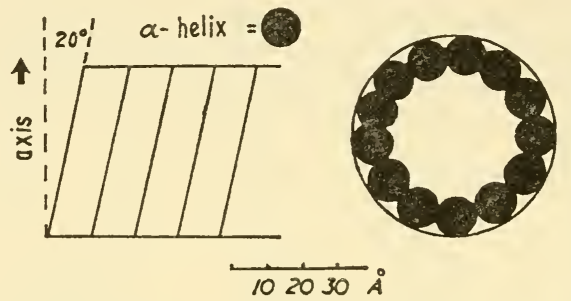

FIG. 79. Relation between the $\alpha$-helix and the $\alpha$-filament. The $\alpha$-helix (diameter $10 \AA$ ) is the structural unit deduced from model building and from the data afforded by actual X-ray patterns. The $\alpha$-filament l.h.s. (diameter $\sim 60 \AA$ ) is the smallest structural unit observed electronmicroscopically. The filament could be a twisted yarn of $\alpha$-filaments with a prevailing angle relative to the axis of about $20^{\circ}$ but the internal arrangements are obscure.

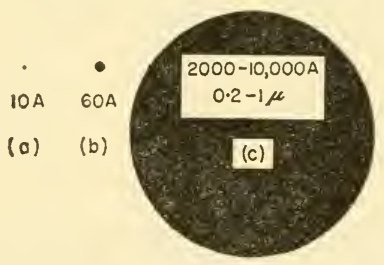

Fig. 80. Relative dimensions in cross-section of the molecular, macromolecular and histological units of hair keratin. (a) the $\alpha$-helix of diameter $10 \AA$ (molecular level); (b) the $\alpha$-filament diameter $\sim 60 \AA$ whose internal structure in terms of $\alpha$-helices is not known (macromolecular level); (c) the fibril visible in the light microscope composed of large numbers of filaments (histological level). Cross-sections of fibrils are to be seen in Figs. 102 and Plates 15 and 16.

packing alters (or that an element of disorder enters) is given by the fact that the centres of the filaments seen electron-microscopically are somewhat more stained than their peripheries (Rogers, 1959). There seems no structural principle that we can appeal to limit the number of helices in a filament, yet the evidence is that the filaments are a uniform and definite structural element. 
The flagellum protein of certain flagellated bacteria is of the $\alpha$-type and each single flagellum is a whip-like thread whose dimensions are of the same order as those of an $\alpha$-filament in hair of which it may be regarded as a structural analogue. Burge (1960) has inferred from the X-ray pattern of isolated flagella (see Fig. 86) that the hexagonally close-packed bundles of $\alpha$-helices must consist of only a small number (3-7) of helices. There must then be several of such bundles in parallel to form a structure as large as the whole flagellum $(\sim 100 \AA)$ suggesting again the existence of sub-filaments within a main $\alpha$-filament.

\section{The Organization of $\alpha$-Filaments into Larger Structures}

On passing from the molecular level to the macromolecular and histological levels we enter the domain (see Fig. 1) where microscopy is able to provide definite answers to structural problems. While these matters will be more fully considered in the next chapter it will be useful here to discuss some geometrical aspects of the larger structures. It is perhaps advisable to emphasize the succession of structures of increasing size with which we are concerned. In hair, for example, $\alpha$-helices $(\sim 10 \AA$ diameter $)$, $\alpha$-filaments (60-80 $\AA$ diameter) and fibrils $(0 \cdot 05-1 \mu$ diameter $)$. These are depicted diagrammatically in cross-section in Fig. 80.

Various and somewhat speculative schemes can be advanced to explain the packing of the $\alpha$-filaments as it is actually observed in the fibrils of hair (Fig. 102, p. 247), wool and skin (Fig. 98, p. 225). The geometry of the packing is very variable: in skin cells, an extreme case of irregular packing, the filaments cluster in irregular-sized sheaves with no constant orientation relative to each other but on the whole tending to lie in the plane of the flattened cell (Fig. 99, p. 229). In hair, wool and quill, we find definite macrofibrillar bundles of filaments oriented parallel to the fibre axis (Fig. 102 , p. 247). In cross-section these show some variety of appearances ranging from good hexagonal packing to spirals (see Plates 15 and 16) the latter appearing more common in the middle stages of development of the hair. Stacks of flat sheets may occur in the $\alpha$-protein of the mantis oötheca (see p. 205).

If an $\alpha$-filament (diameter $<100 \AA$ ), the constructional unit of these formations, is not circularly symmetrical but is polarized as suggested in Fig. 81, the energy conditions governing the recruiting of new members to an already-formed aggregate might well favour the development of flat sheets, Fig. 81 (b) or (a) helical sheets. The stacking of one sheet against another could be governed by conditions of the sort discussed by Crick in connexion with the association of the smaller units, the $\alpha$-helices, to form filaments. That is, we may assume that the filaments themselves also have helically-fluted surfaces (or lines of special attraction) in this case arising from their construction as multi-stranded cables of $\alpha$-helices, and 
that compact arrangements can result either from flat sheets lying over each other at a suitable angle (Fig. 87) as Rudall has proposed for the oötheca protein (p. 205), or as in hair, where they lie on the circumference of concentric circles (or helically-wound sheets) with an appropriate increase in the angle of pitch between successive layers.

The degree to which these initial aggregations would persist into the finally-hardened state could easily depend on the speed with which the final changes are effected. For, if we regard the cystine-rich matrix protein (p. 248) as an interpolation between the filaments (or as an addition to

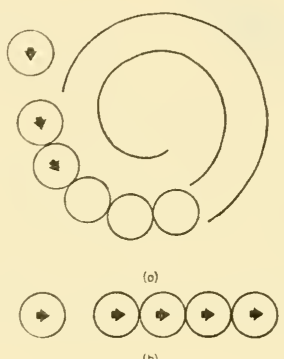

(b)

Fig. 81. Illustrating the lateral aggregation of rodlets ( $\alpha$-filaments) to give (a) a spiral compound or (b) a sheet. The rodlets are here shown in cross-section.

their surfaces) this could, by rapidly cross-linking, maintain an earlier condition; on the other hand, were hardening more slow, it would facilitate a rearrangement of the filaments into the most compact condition, hexagonal packing. This may be pictured more plausibly by thinking of the filaments as being separated by a viscous but fluid medium which both reduces the close interfilamentous contacts responsible for the earlier packing and also permits the movements of readjustment. Fraser et al. have appropriately likened the matrix in keratin to the interfilamentous water of other fibrous systems (1959). In skin we may suppose these changes occur too rapidly to permit of much readjustment. Rogers (1959b) has shown that hexagonal packing prevails in the more stable fraction of wool (paracortex).

\section{The $\alpha-\beta$ transformation in terms of the $\alpha$-helix}

One of the commendable features of the $\alpha$-helix is that, while it was developed primarily to satisfy structural principles derived from a study of simple peptides, etc., by crystallographic means, it has also, on being 
stretched into a straight-chain configuration, an extensibility of the right order $(\sim 120 \%)$ to satisfy the requirements of the $\alpha-\beta$ transformation as envisaged by Astbury (p. 174). No detailed proposal of the nature of this transformation in terms of the helix has been given and there are certainly formidable unsolved problems relating to side-chain movement and of inter- and intra-chain cross-links to be considered. These become increasingly difficult when multi-strand cables are introduced.

An inspection of models shows that when two helices of the same sense are joined at more than one point uncoiling is physically impossible without

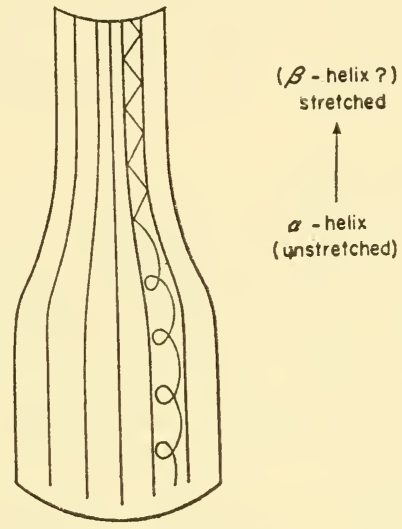

FiG. 82. Difficulties encountered in extending a bundle ( $\alpha$-filament) of $\alpha$-helices to yield a $\beta$-fibril. The $\alpha$-helices must be supposed to uncoil in some manner and to reform to yield a $\beta$-type structure in the stretched filament. If rotation is restricted in any way this would seem geometrically incompatible with the filament retaining its identity.

rupture. If segments in which the sense changes from right to left-hand are permitted, as Lindley (1955) has proposed for insulin, taking advantage of the bends introduced by proline residues, straightening may be possible. In multi-stranded filaments, it would seem that the strand structure must persist during extension and again permanent cross-links must be severely restricted or the individual helices will lack the mobility to open up. Those who are convinced that coiled helical structures exist in keratin may find in this a further argument for excluding sulphur cross-linkages from the helical (fibrous) phase.

Figure 82 has been constructed to emphasize these difficulties assuming that the electron-microscopically visible filaments are bundles of $\alpha$-helices. 
Individual helices must have freedom to uncoil and the $\beta$-form remains a multi-stranded structure in which the $\beta$-chains may be conceived to form stacks of pleated sheets which are slightly twisted to form concentric, closed cylindrical pleated sheets ( $\beta$-helices) such as has been recently proposed for feather keratin (p. 209).

It would seem that the structure at the macromolecular level of the forms of $\alpha$-proteins found in hair is far from settled. The purely theoretical discussion given by Lindley (1955) on model making and the packing of helices shows that the possibilities are far from being exhausted. Lindley's models are based strictly on the dimensional criteria of Corey and Pauling but, by skillfully exploiting the possibilities of helices of left-hand and right-hand sign and the discontinuities introduced by the presence of such residues as proline and cystine, he showed that a number of unexpected packings could be achieved.

\section{The non-crystalline fraction}

The structures we have been discussing up to this point are those of the crystalline material occurring in a keratinized tissue, i.e. that part of the tissue in which the molecules are sufficiently well arranged to yield a definite X-ray diffraction pattern in the form of discrete spots. There is no question but that a large part of these tissues is not in such a well-organized form and that, moreover, many of the important properties of the materials, such as their elastic and chemical behaviour towards mild reagents, is influenced by this fraction. It is not very useful to regard the keratins as perverse molecules which may some day be persuaded to assume a perfectly crystalline form and give the crystallographers their chance. Ordered crystalline regions certainly exist in the large masses of hardened protein, and for these precise structures may be described; but equally certainly disordered non-crystalline regions exist too, and these imperfect regions must be regarded as an essential part of the whole formation since they confer on it certain properties required for a performance of its biological role. For these reasons it is necessary to consider as a separate problem the type of structure which prevails in these regions and to estimate what fraction it forms of the entire tissue. For this purpose there are available in addition to X-ray diffraction techniques, various other methods of a physicochemical nature. It should be emphasized that we are not considering here the non-keratinous constituents (p. 270) but rather that portion of the keratin itself which, since it does not contribute to the fibre-type X-ray pattern, may be referred to as " non-crystalline", a term more exact than " amorphous".

The amount of this material, usually expressed as a crystalline/amorphous ratio, may well vary from cell to cell and from tissue to tissue. The estimates of its value as found in the literature are mostly for wool or hair 
and vary widely. 'The methods employed do not in theory all measure the same quantity and even when the actual measurements themselves are precise, their theoretical bases are much in dispute. An attempt has been made to make sense out of this unsatisfactory situation by Alexander and Hudson (1954) and reference may be made to their discussion since it would carry us far beyond the present intention to attempt to review the work here. The broad conclusion of the physicochemical work based on the penetration of various molecules into fibres is that only a small proportion of the fibre (10-30\%) is inaccessible to small molecules and can in this sense be regarded as crystalline. "Inaccessibility" is a concept not necessarily equivalent to "crystallinity" in the X-ray sense, since crystallites capable of giving a Bragg reflection may be partly or wholly penetrated by some reagents.

From a suitable $\mathrm{X}$-ray pattern an estimate of the crystalline/amorphous ratio may also be calculated from measurements of the amount of radiation scattered in the form of discrete reflections and that scattered into diffuse reflections. In favourable cases, e.g. rubber, this theory has been used to determine the ratio in the stretched and unstretched state, and has helped to confirm that the long-range elasticity is based on the stretching of randomly-coiled chains which may then crystallize when held in the stretched condition. A similar study carried out on a suitable keratin fibre would be of great value. However, the vague patterns render the project well-nigh impossible since there is considerable overlap of diffuse and discrete reflections.

An inspection of a typical $\alpha$-pattern (Plate 1 ) reveals the presence of considerable amounts of diffuse scattering due to amorphous material. This is seen principally as a broad diffuse halo centred about an average spacing of 4.5 $\AA$ (Astbury and Street, 1931; Astbury and Woods, 1933). No quantitative estimate of the amount of this reflection has been made. Qualitatively it appears considerable and seems to suggest that more than half of the fibre substance is amorphous. During the stretching of a hair, the appearance of the pattern is little affected in the range of $0-20 \%$ extension; after that certain reflections specifically associated with the $\alpha$-type structure fade, although there is no perceptible change in the distribution and intensity of the diffuse scattering. When a fibre which has been stretched beyond $50 \%$ is steamed, in order to induce recrystallization of the $\beta$-form, the $\beta$-pattern appears against a background of diffuse scattering very similar in intensity to that noted in the unstretched fibre. This shows that stretching does not itself produce an increased amount of the fibre substance in an ordered form $(\alpha$ or $\beta)$ and suggests that the same well-ordered regions give rise both to the $\alpha$ - and the $\beta$-pattern according to their extension.

Measurements of the birefringence of hairs (Barnes, 1933; Mercer, 
1949c) (p. 10) show that the original value is of the order of $N_{\|}-N_{\perp}=$ 0.01 , and that it increases in the range of stretching from 0 to $40 \%$ suggesting an increased ordering of the structure. It reaches its highest value in fibres stretched to $40-50 \%$ extension and held under tension, and falls when these are steamed to relax the tension and to produce the $\beta$-forms causing " set." From this it may be deduced that set involves a decreased average molecular alignment parallel to the axis of main chains plus side chains and that the $\beta$-form itself is not necessarily more birefringent than the $\alpha$-form.

All methods combine to suggest that a very considerable fraction of the material is in a somewhat disordered form. Such non-crystalline regions could be pictured in various ways. Astbury and Woods (1933) originally suggested that the non-crystalline regions approximate to the crystalline in structure and thus that conclusions drawn from the diffraction pattern of the crystallites are applicable broadly to the whole keratin system. This assumption necessarily underlies their interpretation of fibre elasticity (p. 174) since all " phases " must be capable of the same ultimate extensibility of $100 \%$ if one is to infer molecular extensibility from whole fibre extensibility. The sequence of events during extension is still under investigation by X-rays. (Bendit, 1957; Skertchly and Woods, 1960).

Whatever the solution may be, it seems not unlikely that, as in the fibroins, the poorly-organized regions will be associated with side chains which are difficult to pack. Fibroin crystallites contain predominately the shorter side chains which pack readily; the acids with longer chains seem to be excluded from these crystalline clusters and their interpolation elsewhere impairs the chain alignment and thus introduces a noncrystalline region. There are many more amino acids with bulky side chains in keratin, a fact which might be associated with the lower crystalline/ amorphous ratio, and the crystalline regions might well be those where clusters of the smaller acid residues (glycine, alanine, leucine, serine) occur. The isolation from a natural keratin of peptides containing such sequences, as has been analogously effected with fibroin, would be a valuable indication. Large side chains do not in themselves preclude crystallization. Among the synthetic polypeptides containing $\alpha$-helices, excellent ordering is possible even with large side chains since the homogenity favours packing even when the side chains are long. The helices are spaced further apart in this event (Bamford et al., 1956). Certain natural silks contain crystalline regions with long side chains (Warwicker, 1959).

The presence of certain residues in the crystalline region may be doubted on other grounds than size. Model building shows that most residues have no special importance in the sense that their side chains do not influence the possible configurations of the main chain. Four residues-those 
derived from proline and hydroxyproline, cystine and glycine-have, however, special consequences. Proline actually constrains the shape of the polypeptide chain by introducing a " bend." Thus a chain containing a proline residue cannot be straight and an $\alpha$-helix cannot be maintained at segments where a proline or hydroxyproline residue is inserted; although Lindley (1955) has pointed out that, in conjunction with appropriate neighbours, it can change the sense of the helix from r.h. to 1.h., a feature which could be of importance. Glycine is unique in having no side chain. It is a "space saver" and may permit a more compact packing of adjacent chains. It is common in collagen (a frequency of 1 in 3 ) and its smallness plays a special part in the construction of the triple helix assumed by that protein. Cystine, by virtue of its power to form intra- or inter-chain cross-links, would reduce drastically the mobility of any polypeptide system.

It is significant that most chemical reactions with wool and hair have little effect on the X-ray diffraction pattern unless they are of the type that leads to a dissolution of the H-bonds. Reactions not markedly affecting the pattern include: absorption of water, neutralization of acid or basic side chains, nitration, iodination, reduction and substitution of the cystine bridges. It would seem then that many residues are either excluded from or are to be found only on the surfaces of the crystallites.

The picture originally (1933) presented by Astbury and Woods of the crystalline and amorphous regions, stretching partly in series and partly in parallel when the fibre is stretched, still commands general acceptance in spite of a considerable evolution of views concerning the actual $\alpha$ structure itself. There have been other proposals based mainly on different interpretations of the course of events in stretching (see p. 176). For example, Peters and Speakman (1949) and Burte and Halsey (1947) have envisaged the possibility, even at zero extension, of an equilibrium between portions of the molecular chains in the $\alpha$ - and $\beta$-configurations in the non-crystalline region and have developed this concept to give a quantitative description of limited aspects of the elastic behaviour of swollen wool. The existence of any considerable fraction of the molecules in an extended form at zero extension would invalidate the basic assumption of Astbury and Woods that ultimate extensibility of the whole fibre $(100 \%)$ is also that of the component molecular chains. According to Elliott (p. 199) there is evidence from infra-red absorption spectra of $\beta$-configurations in unstretched hairs.

In terms of the " matrix-plus-filament" model developed in Chapter 6 a certain amount of the diffuse scattering of $\mathrm{X}$-rays must be produced also by that fraction of the material described as $\gamma$-keratin, which is considered to exist outside of, and to envelop the fibrillar component proper. The polypeptides in this region may be pictured as being so heavily cross-linked 
by the frequently-occurring disulphide bridges (a frequency of from 1 in 2 to 1 in 3 ) that crystallization is impossible and that, as in liquids, a diffraction pattern of diffuse haloes is produced simply because certain interatomic spacings occur with a higher frequency. This "liquid-like order " of the non-crystalline fraction is to be distinguished from an unoriented $\beta$-arrangement. The $\beta$-form is a well-defined structure maintained by H-bonds of a perfectly definite length. The $\gamma$-structure is pictured as being structurally amorphous since the packing of the chains in either $\alpha$ - or $\beta$-forms is hindered by either cross-links or awkwardlyshaped side chains. A certain amount of the non-crystalline material within filaments themselves may also be pictured either in this form or as a disordered $\alpha$-structure, since all $\alpha$-type protein patterns are characterized by the same broad halo of average spacing of $4 \cdot 5 \AA$ irrespective of their fine histological structure. 'This is the case for natural fibres, keratinized or not, and also for regenerated protein fibres devoid of histological or fine structure. When no crystalline material is present, as in some regenerated fibres, only the broad haloes appear. It seems probable that, to a degree limited by the steric hindrance of side chains and cross-links, short segments in the $\alpha$-fraction approximate more-or-less closely to the $\alpha$-helix favoured on energetic grounds.

The radial distribution patterns to be expected from the unoriented $\alpha$-helix have been calculated by Donohue (1954) and some comparison with the experimental scattering curves made by Arndt and Riley (1955), but according to Kendrew and Perutz (1957) the radical distribution function is not a sensitive test of configuration.

There appears to be some connexion between crystallinity and density. The densities of horn, wool and porcupine quill (1.28, 1.302 and 1.32) are in order of the degree of crystallinity; but human hair, far less crystalline than quill, has much the same density $(1 \cdot 317)$. This discrepancy may rise from the rather higher cystine content of the hair (Fraser and MacRae, 1957).

\section{Other methods of determining chain configuration}

\section{Optical Rotation and Rotary Dispersion}

During the last few years there has been a renewed interest in the measurement of optical rotation $[\alpha]_{\lambda}$ and its dependence on wavelength (rotary dispersion) as an added tool for the evaluation of configurational changes and for estimating the "helical content" of protein preparations. While little work has been attempted on keratin preparations, a good deal of information has been gathered concerning proteins in general and of various well-characterized $\alpha$-type fibrous proteins which is relevant to the keratin problem. 
The theoretical basis for the interpretation of the measurements is still developing (Leach, 1959) but with the increase in data sound, partlyempirical methods now exist. The specific rotation $[\alpha]$ is defined as:

$$
[\alpha]_{\lambda}^{T}=\frac{100 \alpha}{C \rho L}
$$

where $\alpha$ is the angle of rotation of the plane of polarized light, $C$ the concentration in grammes of solute in 100 milliequivalents of solvent, $\rho$ the density, and $L$ the path length. The change of $[\alpha]_{\lambda}$ with $\lambda$ is referred to as dispersion and is described by the classical equation of Drude:

$$
[\alpha]_{\lambda}=\frac{k}{\lambda^{2}-\lambda_{c}^{2}}
$$

( $k$ and $\lambda_{c}$ constants of the system).

The Drude equation is followed at high wavelengths in solutions of proteins; at low wavelengths the dispersion becomes " anomalous " and the anomalous contribution is thought to be supplied by the portion of the molecular chains folded into helices.

Anomalous dispersion is usually described by a modification of the Drude equation due to Moffitt (1956):

$$
[\alpha]_{\lambda}=\left(\frac{100}{M_{0}} \frac{n^{2}+2}{3}\right)\left[\frac{a_{0} \lambda_{0}^{2}}{\lambda^{2}-\lambda_{0}^{2}}+\frac{b_{0} \lambda_{0}^{2}}{\left(\lambda^{2}-\lambda_{0}^{2}\right)^{2}}\right],
$$

$M_{0}$ is the residue weight, $a_{0}, b_{0}$ and $\lambda_{0}$ are constants. The second term in square brackets represents the anomalous contribution due to the helical structure. It is customary to plot $1 /[\alpha]$ against $\lambda^{2}$ to test the fit to the Drude equation which is usually good for protein solutions at longer wavelengths and allows an estimate of $\lambda_{c}$ and $k$. Various methods exist for estimating the amount of folded material. In general globular proteins in aqueous solution have specific rotations ( $\mathrm{Na} D$-line) of the order of from $-25^{\circ}$ to $-80^{\circ}$ which increases to $-80^{\circ}$ to $-120^{\circ}$ when unfolded by urea. Assuming that the change in $[\alpha]_{D}$ on unfolding is a linear function of the number of residues unfolded, an estimate of the percentage of folded form is possible if the values of $[\alpha]_{D}$ in the fully helical and fully unfolded conditions are known. These values have been obtained from polymers and certain proteins where independent means of knowing the helical content exist.

The estimated helical content of most globular proteins is only $15-50 \%$. For certain $\alpha$-type fibrous-muscle proteins (Review by Leach, 1959) the figure is higher $(50-90 \%)$. This high figure is thought to be due to their low content of proline residues and disulphide bonds which restrict the formation of the $\alpha$-helix. 
Woods (1959) found that the "low sulphur extracts" of wool resemble the synthetic polypeptides in their optical behaviour. In $8 \mathrm{M}$ urea the molecule is in a completely random form with $[\alpha]_{D}=-105^{\circ}$; in some organic solvents $[\alpha]_{D}$ is approximately zero, i.e. the molecule is largely helical. In aqueous solution $[\alpha]_{D}$ is of the order of $-60^{\circ}$ which, according to some forms of calculation, would mean a helical content of $30-40 \%$. Undoubtedly the helical content of a keratin will be restricted by its proline and cystine content. When there is more than $8 \%$ proline distributed along a polypeptide chain (wool keratin $9.5 \%$ and feather keratin $10 \%$ ) it is possible that there will be no segments long enough to be stable in the helical configuration. Very high proline contents may favour another structure such as that proposed for feather by Krimm and Schor (p. 208).

\section{InFRA-RED Spectra ANd Structure}

Infra-red spectra arise from changes in the vibrational energy of molecules produced by the absorption of infra-red radiation. Their value in structural studies is due to the fact that the absorption effects the movement of nuclei in the field of the interatomic binding forces, and the examination of large numbers of substances of known structure has shown that certain frequencies are associated with particular valency bonds whose presence in other compounds of unknown structure may thus be deduced from their spectra. Fortunately for protein studies, bonds of hydrogen atoms with other atoms give characteristic absorptions and their study provides a method of investigating associations between groups which are mediated by $\mathrm{H}$-bonds.

When the molecules are oriented as in crystals or in fibres it is also possible to obtain information concerning the direction of some valency bonds by using polarized infra-red radiation (Ambrose and Elliott, 1951 and 1952). The absorption coefficient is proportional to the square of the cosine of the angle between the $E$-vector of the radiation and the direction of the rate of change of the dipole moment of a normal mode of vibration of the molecule. If the bond associated with this mode of vibration is already known, it may be possible to infer its direction in the fibre (see Fig. 83).

The bands in infra-red spectra are usually given as wave-numbers, rather than frequencies, where the wave-number is the reciprocal of the wavelength in centimetres $\left(\mathrm{cm}^{-1}\right)$; and the spectra are presented graphically by plotting wave-number against optical density:

$$
\log _{10} \frac{\text { intensity of incident radiation }}{\text { intensity of transmitted radiation }}
$$

The ratio of the optical density, measured first with the electric vector 
parallel to the fibre and then at right angles, is called the dichroic ratio. Infra-red spectra have proved fruitful in the study of the synthetic polypeptides particularly in the hands of the Courtauld group and reference may be made to their book (Bamford et al. 1956).

The frequencies associated with the $\mathrm{CO}$ and $\mathrm{NH}$ groups may show small variations depending on whether they are $\mathrm{H}$-bonded in an inter- or intra-molecular configuration. The wave-number associated with a group

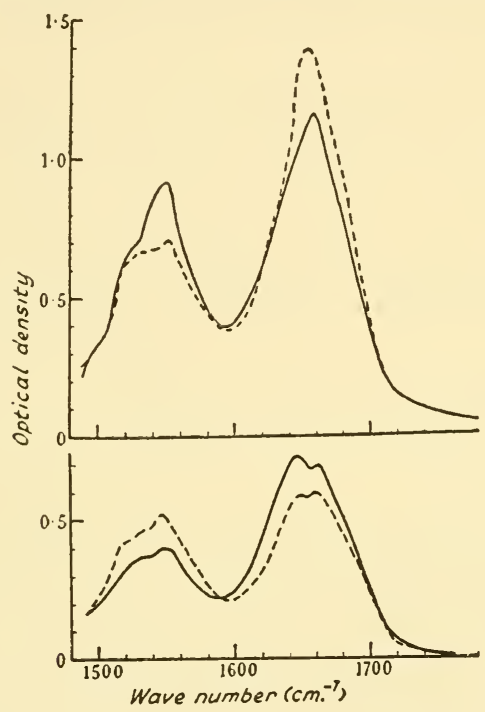

FIG. 83. Infra-red spectra of $\alpha$-keratin (porcupine quill), upper curves, $\beta$-keratin (swan quill), lower curves. Full line, light polarized perpendicular to fibre axis; broken curve, light polarized parallel to fibre axis.

(Ambrose and Elliott, 1952. Reproduced by permission.)

bonded in a $\beta$-configuration is somewhat lower than in the other forms. These relationships have been particularly studied by Ambrose and Elliott (1951 and 1952) who deduced a correlation between structure and absorption from a study of certain synthetic polypeptides known on X-ray grounds to exist in more than one form. In Table 12 some key frequencies as determined by them are given. For example, poly-l-glutamicbenzyl ester shows amide absorption as a single band at $1658 \mathrm{~cm}^{-1}$ and the X-ray diffraction photograph shows it to be in the $\alpha$-form. When the methyl 
ester (normally giving two bands at $1658 \mathrm{~cm}^{-1}$ and $1628 \mathrm{~cm}^{-1}$ ) is cast from formic acid, a pure $\beta$-form results giving only a band at $1629 \mathrm{~cm}^{-1}$.

Generalizing from these observations the Courtauld group proposed that as a simple test an $\alpha$-configuration could be diagnosed, for example, by a carbonyl stretching frequency round about $1665-1660 \mathrm{~cm}^{-1}$ as opposed to the corresponding bond of the $\beta$-configuration at about $1630^{-1}$. Not all authorities accepted this test (Darmon and Sutherland, 1949; Sutherland, 1952) and recent reports by Elliott, Hanby and Malcolm $(1954,1956$ and 1958) show that these workers themselves have abandoned its rigid application.

TABle 12. Wave-Numbers $\left(\mathrm{cm}^{-1}\right)$ OF INFRA-Red ABSORPTION BONDS IN $\alpha$ - AND $\beta$-CONFIGURATIONS.

\begin{tabular}{l|c|c}
\hline & $\alpha$-configuration & $\beta$-configuration \\
\cline { 2 - 3 } CO stretching & $1650-1660$ & $1630(\perp)^{*}$ \\
NH deformation & $1540-1550$ & $1520-1526(\|)$ \\
NH stretching & $3290-3305$ & $3287-3301(\perp)$ \\
\hline
\end{tabular}

* The dichroic sense in the $\beta$-form is indicated by the symbols (II) parallel and $(\perp)$ perpendicular to the fibre axis.

Recently developments (p. 194) have shown that $\alpha$-helices can be diagnosed more certainly by observations on the dispersion of the optical rotation, and Elliott, Hanby and Malcolm, having measured the infrared absorption spectra and the optical rotation of several polypeptides and protein films, have come to the conclusion that the carbonyl bond near $1660 \mathrm{~cm}^{-1}$ is not necessarily associated with the $\alpha$-helix. They describe several polypeptide preparations (alkaline salts of poly-L-glutamic acid and copolymers of L- and D-polyalanine) and a soluble fibroin in which the dispersion of the optical rotation shows conclusively that $\alpha$-helices are absent, but which also have the carbonyl absorption bond near $1660 \mathrm{~cm}^{-1}$ earlier proposed as a test for the presence of the $\alpha$-form. The present position would seem to be that the $\beta$-configuration shows a characteristic absorption at $1630 \mathrm{~cm}^{-1}$ and that almost any departure from this ranging from an $\alpha$-helix to a random coil may show a bond at 1660 $\mathrm{cm}^{-1}$.

The spectra of proteins of course show many other bands to be expected from our knowledge of chemical composition of the proteins, but identification is not always easy (Bellamy, 1954). The measurement of dichroic ratios is valuable in excluding some proposed structures. The dichroic ratios found are small and, when these are found in specimens giving a 
well-oriented X-ray pattern, a low crystalline amorphous ratio is indicated. The spectra in this case may provide data concerning chain configuration in the important amorphous phase which is not accessible to X-ray methods. In particular the $\alpha$ - and $\beta$-configurations may be recognized by their distinct dichroic effects as demonstrated in several polypeptides. Ambrose and Elliott (1951) examined sections of elephant tail hair ( $\alpha-$ keratin) and obtained spectra such as shown in Fig. 83. The $\mathrm{NH}$ and $\mathrm{CO}$ directions seem to be more parallel to the fibre axes (parallel dichroism), although the dichroic ratio is very low. Robinson and Ambrose (1952) claimed that this evidence excludes the Astbury-Bell model.

A structure based on the $\alpha$-helix would have a high parallel dichroism, and the low figure found is therefore only possible if we assume a large admixture of amorphous material. Parker (1955) modified the amorphous regions by replacing the $\mathrm{H}$ atoms by $\mathrm{D}$ atoms and thus displaced the ( $\mathrm{OH}$ and $\mathrm{NH}$ ) bands to much lower wave-numbers enabling the dichroism of the crystallite to be observed alone. The parallel dichroism rose from 1.5 to 4.5 showing more certainly that the low value usually obtained is due to the large fraction of amorphous material and lending support to structures of the $\alpha$-helix type.

Elliott (1952) has also concluded that there are significant amounts of $\beta$-keratin in the amorphous phase of unstretched hair and of the $\alpha$-form in hair stretched $100 \%$. This deduction is now uncertain. It was held that these observations show that care must be exercised when attempting to correlate molecular and whole-fibre extensions. The chief value of these infra-red observations remains now that they allow some investigation of the often large amounts of non-crystalline constituents not revealed by $\mathrm{X}$-ray diffraction and indicate whether or not this is in the $\beta$-form with some measure of its orientation.

The important fact that reversible $\alpha-\beta$ transformations can be obtained from purely synthetic polypeptides was established by the Courtauld group using indications provided by infra-red absorption spectra (Bamford et al., 1956). For examples, fibres of $\alpha$-poly-L-alanine stretched in steam give a highly-oriented $\beta$-pattern and somewhat similar results are obtained with poly- $\alpha$-amino- $n$-butyric acid. Reversible conversions from one form to another are also obtained by immersing specimens in various swelling solvents. Concentrated formic acid often produces a $\beta$-form and chloroform or $m$-cressol may reconvert to the $\alpha$-form.

$$
\begin{gathered}
\text { formic acid } \\
\alpha \rightleftarrows \beta \\
m \text {-cresol }
\end{gathered}
$$

The conversions are reversible and there is no loss or degradation of material. This demonstration of the influence of the solvent on the 
configuration assumed by the polypeptide chains is of considerable interest from the viewpoint of the biosynthesis (Chapter III) of the various polypeptide configurations.

\section{DeUterium Exchange}

A further method of estimating helical content (or at least the content of tightly packed chain segments) is that of deuterium exchange which is based on the finding that, when a protein is dissolved in deuterium oxide $\left(\mathrm{D}_{2} \mathrm{O}\right)$, certain of the hydrogen atoms participating in hydrogen bonding are exchanged with deuterium atoms and are given off again at varying rates when the protein is returned to water. Thus one can distinguish instantaneous, rapidly and slowly exchangeable hydrogens. The slowly exchangeable hydrogens are interpreted by Linderstrøm-Lang (1955) to be the hydrogen atoms in the tight helically-folded segments and thus the helical content can be estimated by the proportion of these to the total $\mathrm{H}$-bond content. The method is being actively applied. Fraser and MacRae (1958) have shown that in fibrous keratin the irreplaceable $\mathrm{H}$ bonds (inaccessible) were in the crystalline phase, i.e. presumably the $\alpha$-helices.
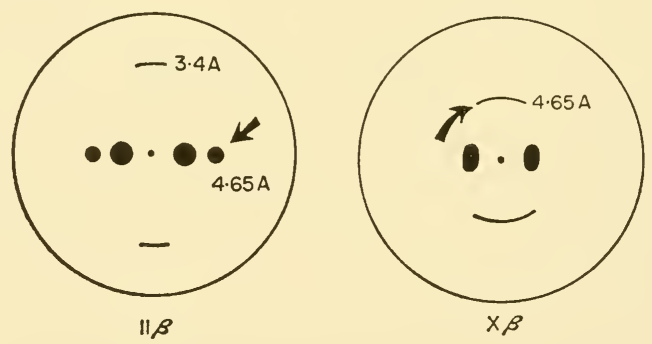

FIG. 84. Characteristic reflections of the parallel- $\beta(\|)$ and the cross $-\beta$ (X $\beta$ ) patterns. The "backbone spacing" $4.65 \AA$ in the $\| \beta$ pattern occurs on the equator; in the cross $\beta$ it is found on the meridian.

\section{The cross $\beta$ pattern}

In X-ray photographs of denatured proteins, such as the muscle proteins and egg white (Astbury et al., 1935) and some contracted keratins (epidermin (Rudall, 1946), wool (Mercer, 1949a; Peacock, 1959)), there may be found a $\beta$-type pattern in which the $(4.65 \AA)$ reflection usually associated with interchain $\mathrm{CO}$... NH linkages is not in its normal place on the equator (see Plate 2B) but is on the meridian (Fig. 84). To distinguish this pattern from the usual $\beta$-pattern, or parallel- $\beta(\| \beta)$, it is referred to, by Rudall and his associates, as the cross- $\beta$ pattern $(\mathrm{X} \beta)$.

The stretching of boiled egg white at first produces an oriented pattern 
which can only mean that the crystallites (or orientable units) contain bundles of polypeptide chains running at right angles to the long axis of the crystallites. While the actual molecular arrangements are still regarded as uncertain, a proposal by Rudall (1946), to the effect that large transverse folds (Fig. 85) such as were originally proposed by Astbury for the supercontracted state, would be likely to lead to the cross- $\beta$ pattern, forms the current working hypothesis.

The discovery that highly-oriented " natural " cross- $\beta$ patterns are given by the egg stalk of the green lace-wing fly Chrysopa (Parker and Rudall, 1957) places this pattern on a more definite basis. The polypeptide chains

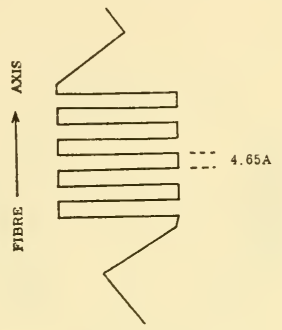

FIG. 85. Illustrating Rudall's proposal for a cross- $\beta$ crystallite which could give rise to reflections from the $4.65 \AA$ spacing on the meridian. The chains are extensively folded with their side spacings parallel to the fibre axis. The crystallite is supposed to be longer in the side-chain direction and stretching converts it directly to the parallel- $\beta$ form without rotating the crystallite.

here undoubtedly run at right angles to the length of the fibre and are folded since a conversion to the $\| \beta$ form takes place on stretching. Long spacings relatable to the transverse folds disappear on stretching.

Astbury holds that a supercontraction resulting from a folding from the $\alpha$-form into a shorter configuration, such as that proposed in the cross $-\beta$ structure, occurs in muscle contraction although it appears most clearly in contracted muscle when this is produced by heating. Strength is given to this opinion by a recent discovery (Astbury et al., 1955) that the cross- $\beta$ configuration normally accompanies the $\alpha$-pattern in bacterial flagella (see Fig. 86) which appear from their helical shape in life to be in a state of equilibrium between a contracted and an extended configuration.

An entirely different proposal to explain the occurrence of the spacing of $4.7 \AA$ on the meridian in the cross- $\beta$ pattern has been made recently by Zubay (1959). He points out that an arc of spacing $4 \cdot 7 \AA$ passing over the meridian could be produced by small microcrystals formed by $\alpha$-helices 
which had been released from the coiled-coil form of the normal $\alpha$-structure by the swelling and warming required to produce the pattern. If the crystals are small and sufficiently disoriented, the two off-meridional spots of the first row line (from the $5.4 \AA$ spacing) will spread and produce a single arc across the meridian which will then appear meridional arising

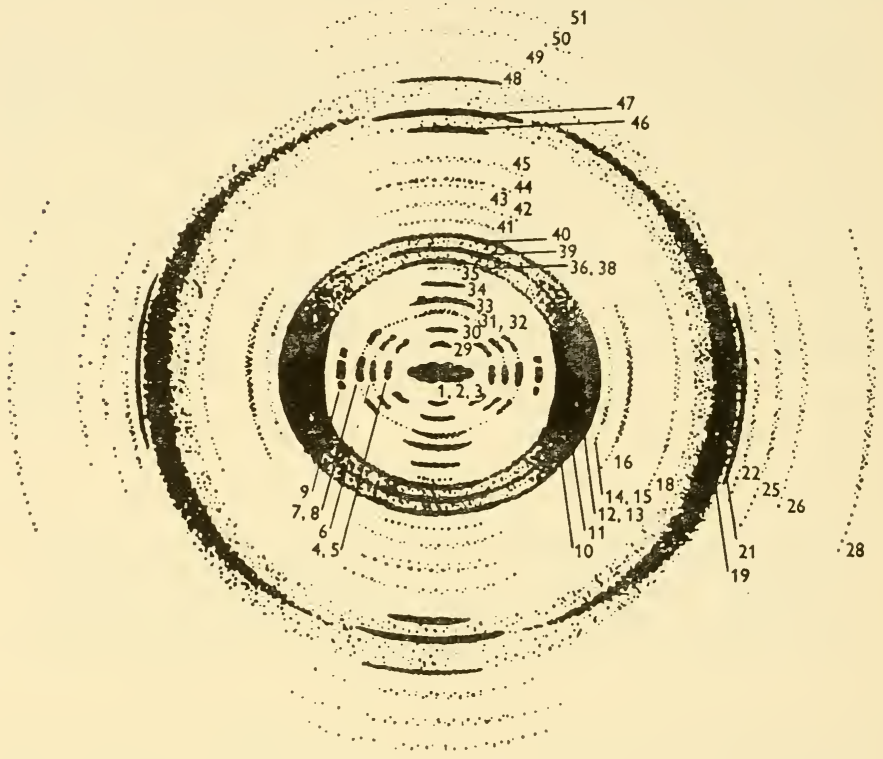

FIG. 86. Composite fibre diagram of X-ray reflections from Proteus vulgaris and Bacillus subtilis. The $\alpha$-characteristics are indicated by reflection 47 and the cross $-\beta$ by 48 . For the other spacings listed, see Astbury et al. (1955). Reproduced by permission.

from a $4.7 \AA$ spacing. He likens the pattern to the completely-disoriented halo pattern with spacings of 4.7 and $10 \cdot 1 \AA$ (see p. 16) given by many unoriented proteins. No trace of the $1.5 \AA$ spacing occurs in the $\mathrm{X} \beta$.

\section{Other $\alpha$-proteins}

The $\alpha$-proteins are among the commonest structural proteins to be found both intra- and extra-cellularly. There is now no doubt that the $\alpha$-helix perhaps in a distorted form is to be found in the compact corpuscular molecules (Perutz, 1959). The significance of this would 
seem to be largely stereochemical: a polypeptide chain tends to assume the tightly H-bonded helix unless other overriding side-chain interaction or interference is present to prevent it. When sufficiently long runs of suitable residues exist the helix may form and may associate with other helical segments to produce an $\alpha$-crystallite which may be detected by $\mathrm{X}$-ray means. While many such forms may thus yield the definitive wide-angle $\mathrm{X}$-ray pattern they may differ greatly in their secondary and tertiary structures. Some of these arrangements, interesting as special problems, may not be immediately relevant to the keratin problem; others, on the other hand, suggest structural possibilities which may be found in the keratins themselves.

An important example of an $\alpha$-protein, important because of its origin and the absence of complicating histological structures, is the material of the bacterial flagellum to which Astbury has given the name "flagellin " (1955). Bacterial flagella are thin $(\sim 100 \AA)$ whip-like threads attached to bacteria and connected with mobility.

Astbury, Beighton and Weibull (1955) have described the rich X-ray pattern obtainable from preparations of flagella from Proteus vulgaris and Bacillus subtilis (Fig. 86). It is clearly of the $\alpha$-type with added reflections indicating an admixture of the cross $-\beta$ configuration. Heating produces a $\beta$-form which may be oriented by pressing. The diagnostic $5 \cdot 1$ and $1.5 \AA$ reflections are present. The numerous meridional reflections appear as orders of a master spacing of $410 \AA$ which is similar to that observed in muscle. The thickness of flagella would suggest that they are structural analogues of the $\alpha$-filaments of hair keratin. Their poor thermal stability and composition show that they are not keratinized and they could therefore be related to the primitive non-stabilized $\alpha$-type proteins of the cytoplasm. Although they are located extracellularly, they are not simple extrusions of the cell wall or capsule. They in fact take their origin from a small basal granule located beneath the protoplast membrane to which they remain attached even when the cell wall has been removed. Thus they remain in direct communication with the cytoplasm and it is permissible then to regard them in this special sense as cytoplasmic filaments.

\section{Some $\alpha$ - and $\beta$-proteins of insect origin}

Keratinization is not known in insects; their sclerotized proteins are normally hardened by tanning with aromatic phenols (Chapter I). The various dermal glands are, however, able to produce some variety of protein-types among which are forms of $\alpha$-type proteins whose study has added importantly to our knowledge of molecular configurations and their interconversions. Silk glands in Bombyx are labial glands and their secretion while in the gland, according to infra-red evidence, has random coil features. It transforms into the insoluble $\beta$-form during 
spinning. The detailed structure of $\beta$-fibroin illustrates clearly how the insolubilization is produced by multiple $\mathrm{H}$-bonding between the carbonyl and imino groups of the peptide bonds. Nevertheless the transformation is not fully understood; there is evidence both for an unfolding of the chains effected by stretching and for an aggregation process which does not obviously involve chain unfolding (p. 129).

Other glands, the colleterial glands, accessory to the female genital system, produce various fibrous secretions, which serve to support or protect the eggs. One example, the egg-bearing stalk of Chrysopa, is important as a naturally-occurring example of a cross- $\beta$ system (see p. 201), i.e. the polypeptide chains in a $\beta$-form are oriented at right angles to direction of stretching of the fibre. Such a configuration is not uncommon in tissue proteins under experimental conditions (see p. 200) but never achieves the perfection of orientation found in these egg stalks. Another extremely interesting structure based on an $\alpha$-form was discovered by Rudall (1955-6) in the egg case of a mantis which forms from the secretion of the colleterial glands. The dried case is tanned and insoluble. However, when freshly formed it may be dispersed by tryptic digestion and is seen to be composed of masses of long, very thin ribbons $(50 \mu \times$ $1-2 \mu$ ) which (electron-microscopically) are crossed by regularly-spaced lines (120 $\AA$ apart) making an angle of about $20^{\circ}$ with the length of the ribbon.

When first secreted from the colleterial gland the protein is a viscous mass of globules which change first into long fibrils and then into ribbons. The air-dried secretion before this change gives a somewhat diffuse X-ray pattern but the definitive $\alpha$-spacings: $1 \cdot 5 \AA$ and $5 \cdot 18 \AA$ are present. The usual, strong, equatorial reflections at $10 \AA$ are missing, the lateral spacing being represented by diffractions near 14.5 and $8 \cdot 3 \AA$. This pattern could be given by rods of diameter $16.5 \AA$ packed in hexagonal array. This same secretion after changing into ribbons yields a remarkably different pattern: the main wide-angle meridional spacings at $1.5 \AA$ and $5 \cdot 1 \AA$ are present, but photographs taken at right angles to the length of the ribbons and parallel to their face are of the $\alpha$-type, and the pattern is dominated by a set of row lines which are orders of $17 \cdot 5-18 \cdot 5 \AA$ (depending on hydration (Plate 3A)).

Parallel to the long axis of the ribbons the diffractions from planes perpendicular to the surface of the ribbons contain one set indicating a period of 31 or $62 \AA$, leading to a picture of rods $10.3 \AA$ in diameter in hexagonal packing (Fig. 87).

On stretching the ribbons in water a $\beta$-form with axial periodicity of $3.33 \AA$ is produced which returns to the $\alpha$-form on releasing the tension. The $\beta$-structure is still double-oriented, i.e. the "backbone spacing" $(4 \cdot 7 \AA)$ is oriented perpendicular to the ribbon surfaces, which would seem to imply that the $\alpha \leftrightarrows \beta$ transformation is effected with the maintenance of 
the double orientation. The devising of a molecular structure capable of accomplishing this change is a difficult problem.

Rudall has offered two interpretations which, however, he finds only partially satisfactory. The first considers straight $\alpha$-helices of diameter $10 \cdot 3 \AA$ hexagonally packed. Spacings of $31 \AA$ and $17 \cdot 8 \AA$ at right angles are thus achieved (Fig. 87). The $\alpha \leftrightarrows \beta$ transformation presents no special difficulty since the helices are straight. The diagonal lines crossing the ribbons (as seen electron-microscopically) are accounted for by supposing a superficial layer of fibrils making an angle of $20^{\circ}$ lying across the main sheets. Crick's argument suggests a good fit would be achieved between

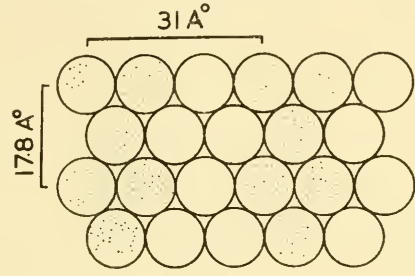

A

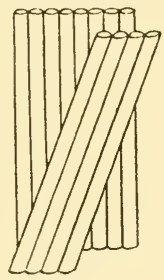

B

FiG. 87. Proposals for the packing of $\alpha$-helices to give rise to the spacings observed in X-ray patterns fron mantis oötheca (Rudall, 1955-56) and to the diagonal lines observed electron-microscopically crossing ribbons of the material at an angle of $20^{\circ}$.

(a) hexagonal packing of straight $\alpha$-helices of diameter $10.3 \AA$;

(b) two superposed layers of $\alpha$-helices inclined at about $20^{\circ}$. Reproduced by permission.

fibrils placed together in this way (p. 183). The other interpretation is based on a two-stranded rope of $\alpha$-helices. A layer of such ropes, in which the pitch of the supercoils is chosen to be $180-190 \AA$, has a pattern of well-marked grooves running parallel across the layer making an angle of $20^{\circ}$ with its length. The similarity to the pattern seen in the ribbons is close, but the grooves in the model are separated by $30 \AA$ while those in the ribbons are separated by about $120 \AA$ indicating an axial period of $360 \AA$ or double that of the model. A model involving coiled helices also meets some difficulty in explaining how a double-oriented condition can be maintained during extension to the $\beta$-form.

\section{Feather keratin}

The X-ray patterns yielded by feathers are much more detailed than those given by any mammalian keratin (Plate 2A). The best patterns are given by the calamus and rachis (Fig. 30, p. 70) and Rudall has shown 
that essentially the same basic features are exhibited by patterns from many hard parts of birds and reptiles (1949). The feather pattern is perhaps more promising for detailed analysis than any other given by a keratin, but a complete solution of the structure has not yet been given.

According to Astbury and Marwick (1932) the wide-angle pattern is typically $\beta$ with the definitive axial repeat of $6 \cdot 2(2 \times 3 \cdot 1) \AA$ and complex side spacings centred around 10 and $4.5 \AA$ (Plate $2 \mathrm{~A})$. Feathers may be stretched by about $7 \%$ of their length before breaking. This does not alter the nature of the pattern, the various longitudinal spacings increasing by the same order; this behaviour contrasts with that of the $\alpha$-keratins. The axial long spacings have been regarded as orders of a main spacing of $95 \AA$, cf. in $\alpha$-keratin $198 \AA$. The existence of definite and strong lateral spacings at low angles distinguished the feather pattern sharply in type from the pattern of collagen. Collagen fibrils give no lateral reflections indicative of structures of greater thickness than the basic monomeric filament. It was the lateral spacings of the feather pattern which early led to the idea that in this material we were concerned with what were essentially long crystals of precisely-constructed protein molecules not essentially different in the detailed nature of their internal structure from molecules of soluble proteins (Astbury and Marwick, 1932). This idea persists in the more recent attempts to elucidate the structure made by Bear and Rugo (1951).

In these attempts, Bear and Rugo (1951), while not proposing a solution of the small-scale structure, have drawn attention to the implication at the macromolecular level of the characteristic manner in which the pattern degenerates when feather is subjected to the disintegrative action of water and heat (" heat-moisture treatment "). After a prolonged treatment many of the details of the pattern become blurred and fade and are replaced by a much simpler pattern (" net-pattern ") which can be derived from a net of the type shown in Fig. 88. What seems to have happened is that the heatmoisture treatment has disturbed the fine-scale structural order to the point that it is no longer capable of coherent reflections leaving only the large particles (now effectively internally amorphous) centred about the nodal points of the net to dominate the scattering. Two arrangements of these large particles (macromolecules), are envisaged by Bear and Rugo, and it will be seen that the major axial-repeating distance of $95 \AA$ may be referred to the length of two or four of the participating particles.

These proposals illustrate very clearly the tendency shown in connexion with the structure of protein fibrils, and already referred to above (p. 165), to separate the X-ray diffraction pattern into two parts: (a) that given by the small-scale molecular spacings (i.e. smaller than $20 \AA$ ), in this case the $\beta$-pattern; and (b) that to be referred to the ordered packing of macromolecular units. 
Evidence for the existence of large macromolecular units of this kind may be sought in the products which result when fibres are dissolved. For feather, as for wool and hair (Chapter VI) unfortunately the solubilization process is drastic and destructive of larger units. Ward, High and Lundgren (1946) using a detergent and reducing agents have dissolved feather and found a molecular weight of a detergent-feather complex of the order of $30-40,000$. They concluded also that the particle was elongate and
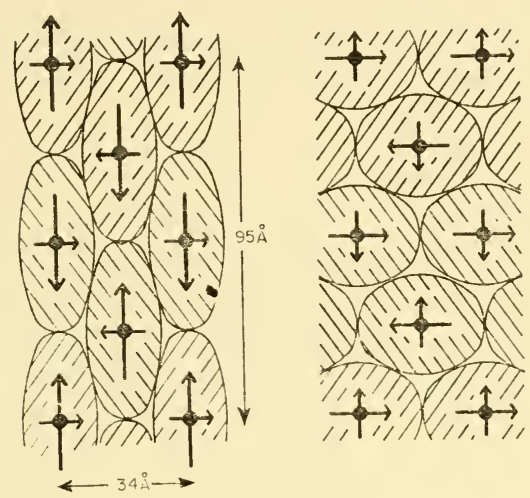

Fig. 88. A proposal due to Bear and Rugo (1951) for the fibrillar structure of feather keratin. Two packings of ellipsoidal molecules which would account for the pattern of long spacings remaining after successive treatments with hot water have disorganised the wide-angle pattern, are illustrated. An example of an " aggregation of macromolecular particles model ". Reproduced by permission.

the mixture polydisperse. Probably the molecules were unfolded by the process. If formed into a sphere, a molecule of the molecular weight found by Ward $e t$ al. would have been of a size as envisaged by Bear and Rugo. More recently Woodin (1954a and b) using a reducing solution containing urea obtained an electrophoretically-homogenous material. Osmotic pressure measurements, viscosity, sedimentation rate and light scattering concurred to give a molecular weight of the order of 10,000 and showed that the particle was rather asymmetric (Woodin, 1955). Rougvie (1954) found the same particle weight in an extract of feather oxidized by peracetic acid (see also p. 163).

The infra-red absorption spectra of feather has provided information about the orientation of hydrogen bonds. The $\mathrm{CO}$ stretching mode 
at $1650 \mathrm{~cm}^{-1}$ reveals a perpendicular dichroism. The $\mathrm{NH}$ stretching mode at $3315 \mathrm{~cm}^{-1}$ is also perpendicularly dichroic. By exchanging the $\mathrm{H}$ of the $\mathrm{OH}$ and $\mathrm{NH}$ groups in the accessible (non-crystalline) regions with deuterium, Parker (1955) removed their absorption from these regions and showed that the perpendicular dichroism of the well-oriented region rose to $4 \cdot 8$.

The absorption bond of the $\mathrm{CO}$ stretching frequency has a double peak (Fig. 83) and, on the basis of their empirical rule (see p. 198), Ambrose and Elliott (1952) concluded that this meant that a mixture of $\alpha$ - and $\beta$-forms were present. We have already indicated (p. 198) that this deduction would not necessarily follow from our present-day views of the meaning of these frequency shifts.

Recently a new examination of the diffraction data has been made by Krimm and Schor (1956) who, while confirming that the structure is of a $\beta$-type, consider that none of the hitherto-proposed arrangements is correct. On the grounds of the difficulty of fitting in all the axial reflections in Tables 10 and 11 as orders of $94.5 \AA$ they consider that the true spacing is $2 \times 94.5=189 \AA$. The $3.07 \AA$ spacing usually regarded as meridional is stated by them to be an off-meridional $3 \cdot 15 \AA$ layer line spacing. A meridional reflection at $2.9 \AA$ is interpreted as the amino acid repeat (or rise) and there are thus sixty-four residues in the master period (189 $\AA$ ).

They have proposed a model which might be described as a modified $\beta$-helix which consists of ten polypeptides, each of sixty-four residues, wrapped helically around a cylinder (Fig. 89). The helices are righthanded and every eighth residue is a prolyl, the side chain being on the inside of the cylinder. The non-proline sequences form a modified $\beta$-pleated sheet. The ten chains aggregate coaxially by hydrogen bonding, the prolyl residues of neighbouring chains coming out at about the same level, and the strong $23.4 \AA$ meridional reflection arises from the planes containing these residues (see Fig. 89). The $\mathrm{R}$ groups project both inside and outside the cylinder. The particles of molecular weight $\sim 10,000$ isolated by Woodin and which appeared to have no (or very few) end groups, they believe arise by a fission of the cylinder adjacent to the prolyl planes thus producing prolyl end groups which are not estimated by the usual methods.

A fairly satisfactory prediction of the strong equatorial reflections at 33.5 11.2 and $55 \AA$ (Schor's figures) was obtained by assuming that compound cylinders, each consisting of seven unit cylinders, were placed in hexagonal array.

It will be seen that the Schor-Krimm model is essentially a return to the idea that the large axial spacings represent distances over which a sequence of amino acids is repeated. In this particular instance the prolyl residues are supposed to recur at intervals of ten residues. What little chemical 
evidence is available is not incompatible with this, the frequency of proline by analysis being 1 in 10 , but direct evidence on the form of isolated peptides is still wanting. It would not seem too difficult to observe electron-microscopically the hexagonal packing of the compound cylinders (diameter $67 \AA$ ) and filaments of a diameter less than $100 \AA$ have in fact been observed by the author (Mercer, 1956). One interesting result of this work is the introduction of the idea of $\beta$-helices.

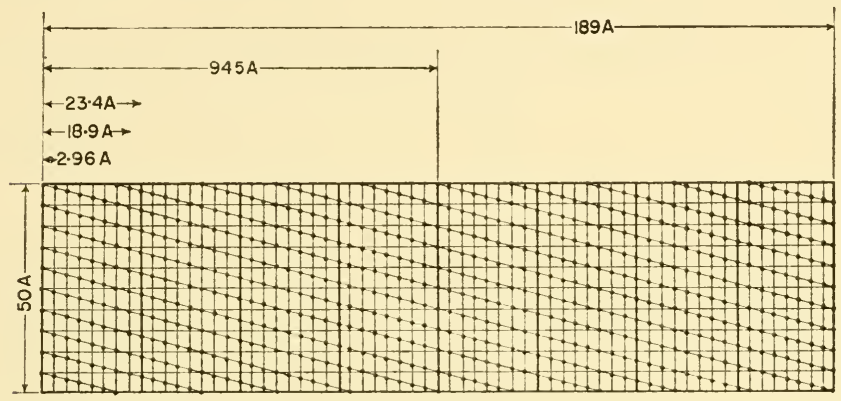

Fig. 89. Illustrating the proposal of Krimm and Schor for a feather keratin structure. The drawing shows a projection on an $8 \mathrm{~A}$ cylinder of one turn of their cylindrical unit $\mathrm{X}=$ propyl residue, $\mathrm{O}=$ non-propyl. The master repeat period is $189 \AA$ and the $94.5 \AA$ is regarded as a pseudo repeat unit (taken from Schor's thesis (1958). Reproduced by permission.

In a recent comment on feather keratin Fraser and MacRae (1959) reject the proposals of Krimm and Schor as being inadequate to account for the intensity distribution of the diffraction pattern and re-assert that some variant of the older $\beta$-type structure will prove the correct solution. Since none of these discussions attempts a strictly crystallographic treatment it is difficult to assess their merits, but it is evident that a final solution of the structure of feather keratin has yet to be proposed. 


\section{The Keratinization Process}

IN THIS chapter we shall discuss in detail the changes by which the living and growing epithelial tissue is converted into a lifeless, tough, insoluble, translucent, fibrous substance. These changes, occurring in a more-or-less clearly-defined zone which follows the zone of differentiation and growth (Fig. 25, p. 55) are characteristic and broadly similar in all instances; by them we recognize and define a keratinized or cornified tissue. In thin or quiescent skins it may not be possible to distinguish a distinct zone of keratinization; in many thicker skins, or in the matrix of the hard keratins, even more subdivisions suggest themselves.

The literature concerning the staining of keratinized tissues is large and many writers have described progressive changes in staining properties of cells during their keratinization. A good historical account with particular reference to wool and hair will be found in Auber's monograph (1950). Recent reviews have been given by Montagna (1956) and Braun-Falco (1958). There is no point in further summarizing these accounts; rather, we shall by selection and omission attempt to co-ordinate the findings into a coherent view of the process.

For all its usefulness in making evident histological detail, much of this work is disappointing in contributing little towards elucidating the process itself because most dyes have little specificity and their reactivity is poorly understood. Histochemical demonstrations of definite substances (or groups) are more rare and more useful. It is fortunate that reliable histochemical methods exist for demonstrating thiol groups (SH).

Since there is some difference between the sequence of events in the formation of a hard keratin and a soft keratin, it will be better to describe these separately, commencing with a typical hard keratin, hair.

\section{The hard keratins}

These include all the coherent appendages: hairs, feathers, nails, claws, horns, etc. Most of the data relating to the hardening of this type of keratin comes again from the hair follicle, but the fewer observations made on other examples (horn, nails, and feathers) are sufficient to show that the changes seen in the hair follicle are general and justify the use we propose to make of it for purposes of illustration. For economy of presentation it will be an advantage to summarize the data in a uniform 
diagrammatic form in one place. The hair follicle, the best-studied and most "diagrammatic" tissue, will be used for this purpose and is shown in the series of Figs. 90, 91 and 97. The illustrations in most cases refer to events in the presumptive cortical cells and each diagram summarizes the findings relative to some property or particular activity. An inspection of these diagrams shows clearly that in the hair follicle several distinct stages in keratin formation, separated in time and in space, may be distinguished. These are indicated in Fig. 90:

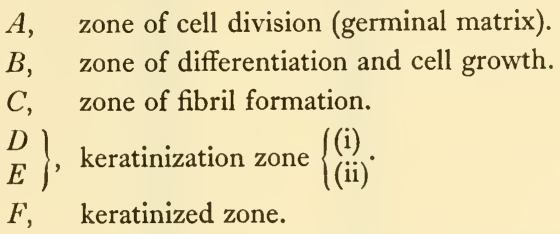

Analogous levels can usually be distinguished in the other tissues.

The diagrams have been devised in terms of the several interrelated themes which can be distinguished in the process of keratin formation:

(1) General cellular phenomena: (a) nucleic acid metabolism and protein synthesis (Fig. 97b); (b) cell metabolism (Figs. 97a and d).

(2) Phenomena peculiar to Keratinisation: (a) orientation (Fig. 91); (b) stabilization (Figs. 91, 92 and 97c).

\section{The Development of Orientation (Fig. 91)}

The fibrous contents of the cells of the hard keratins are usually well oriented and, since this orientation is related to the mechanical function, its development is of special interest. In the upper regions of the bulb (Chapter III) the cells of the presumptive cortex elongate and there is a marked increase in the number of oriented fibrils within the cells. The orientation is most conveniently observed by means of the polarizing microscope (Schmidt, 1924) and its quantitative development may be measured by means of a compensator (Mercer, 1949b). Figure 91 (r.h.s.) shows the growth of birefringence in a follicle plucked from the human head and the 1.h.s. shows the development of birefringence in relation to the anatomy of the follicle. The important feature is the rapid rise at the constriction of the bulb to a value which is almost equal to that of the final hair.

While polarization microscopy provides the simplest method of detecting the existence of an oriented structure, the interpretation of the results is not without ambiguity. It is useful to be able to distinguish between intrinsic birefringence, i.e. birefringence due to an oriented molecular structure, and form birefringence, which arises simply from a 


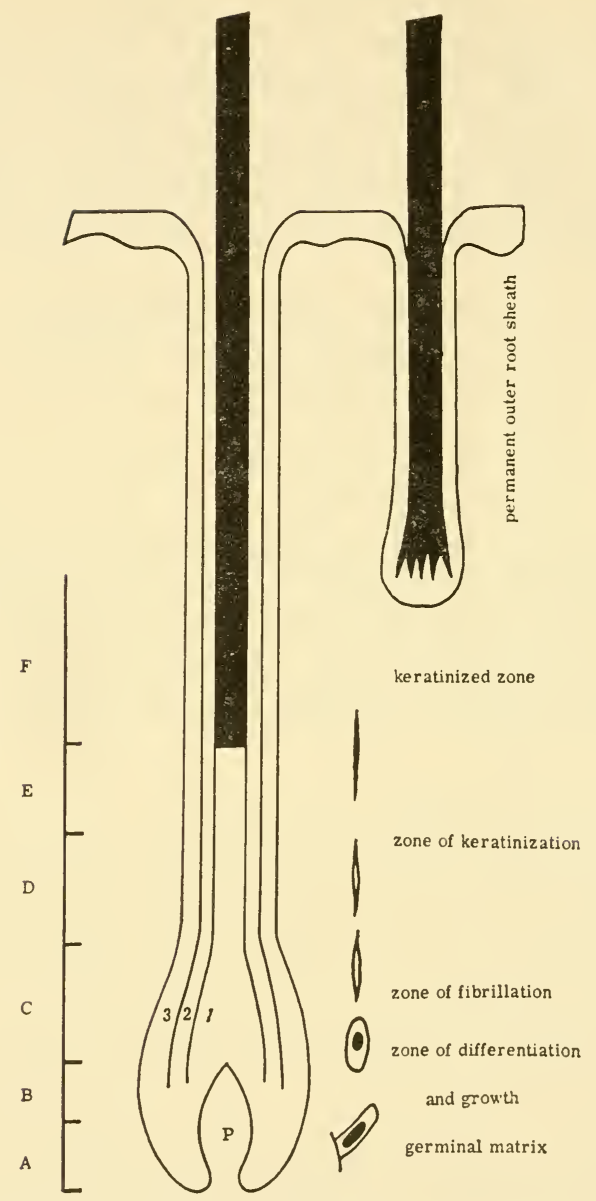

Fig. 90. The location diagram for the series of figures (Figs. 90-97) illustrating the development of keratiaization in the human hair follicle. On the left-hand side are indicated the several zones $\mathrm{A}-\mathrm{F}$ into which the cortex is divided. In the growing follicle (centre) (1), (2) and (3) indicate the cortex (and cuticle), the inner root sheath and the outer root sheath, respectively. The cell shapes are those of the presumptive cortical cells. On the r.h.s. is shown a "club root" or non-growing follicle to indicate the extent to which the growing tissues may be resorbed (see also Fig. 40). 
parallel arrangement of long, thin rodlets, with or without internal anisotropy, when immersed in a medium of a different refractive index (Schmidt, 1924; Schmitt and Bear, 1939). Experimentally the distinction is made by observing the change in the birefringence due to form

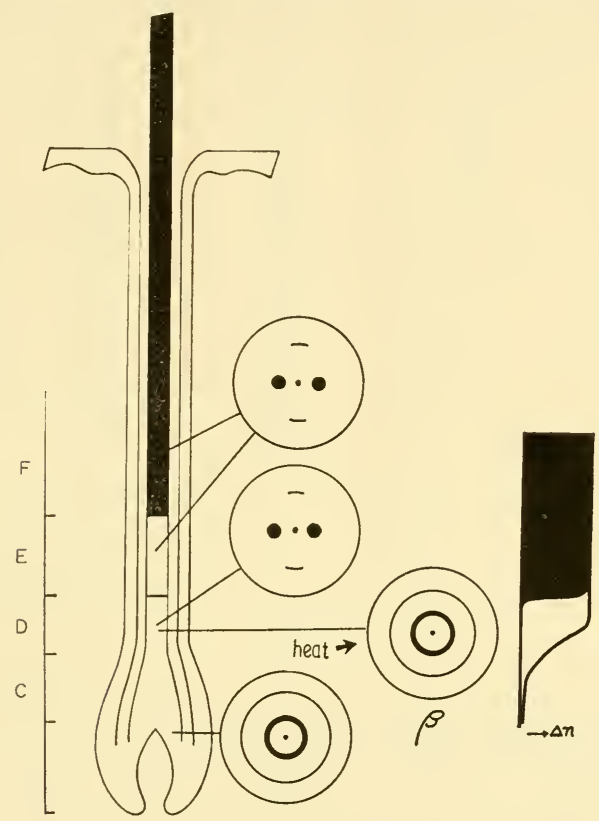

F1G. 91. The development of orientation and its stability. X-ray patterns obtainable from the different levels are shown diagrammatically. The $\alpha$-pattern is obtained immediately above the bulb in zone $\mathrm{D}$ but on warming the unstabilized fibre is disoriented and yields an unoriented $\beta$-pattern. In the zones $\mathrm{E}$ and $\mathrm{F}$ the pattern is stable to heating below $100^{\circ} \mathrm{C}$. On the right-hand side is shown the birefringence $(\Delta n)$ in the cortex and the levels (zone D shown clear) where it is destroyed by heat.

when fluids of different refractive index are allowed to penetrate the object. Unfortunately this method is inapplicable to the hard keratins, such as hair, since by the very process of keratinization the material has become impervious to liquids which do not cause far-reaching disintegration (Barnes, 1933; Mercer, 1949). For this reason the clearest understanding of orientation phenomena comes from X-ray diffraction. 
The appearance of an oriented structure at the molecular level can be demonstrated readily by X-ray diffraction (see Chapters I and V), and the method has the advantage that the type of structure (in the molecular sense) is also demonstrated. From the bulb alone only a diffuse, uncharacteristic diffraction pattern of two rings (Fig. 91) can be obtained. Immediately above the bulb, however, an oriented $\alpha$-type pattern, apparently identical to that of the final hair, is obtained. This finding shows beyond question that at this level there is a considerable amount of oriented protein present with essentially the same crystalline molecular organization $(\alpha$-type) as in mature hair.

This important point, that the appearance of the typical $\alpha$-structure precedes keratinization was demonstrated originally by Derksen, Heringa and Weidinger (1937), using the thickened epidermis of cow's lip, a more amenable material than hair follicles, and by Giroud and Champetier (1936) using the " chestnut" of the horse. Sections cut at different levels up to the fully-hardened layers gave the same $\alpha$-pattern. Similar experiments on a cow's nose were later carried out by Rudall (1946) and by the present writer using the hair root (1949b). After heating in water the lower unstabilized layers become disoriented.

In their totality these experiments prove that the filaments, which can be seen to form in the cells below the keratinizing zone, already possess the typical $\alpha$-structure, and further that, whatever chemical reactions may go on in the later stages of keratinization, they in no way affect the arrangement of molecules in the crystalline regions.

The older histologists, whose work is summarized by Biedermann (1926), recognized that the oriented structures and birefringence arose from fibrils and deduced the existence of smaller invisible anisotropic units from their polarization studies. Various schemes of fibrillar architecture (see Biedermann) were developed which were substantially correct. For this type of research the modern electron microscope is now more convenient (see p. 223) but the polarizing microscope is still much used.

\section{The Development of Stability}

Fully-hardened hair has a high stability towards many chemical reagents and physical conditions and, accordingly, the development of keratinization may be assessed in terms of the action of any of these influences. Owing to its linear arrangement the plucked hair root is a very convenient object on which to make such tests, and the results of several are depicted in Figs. 91 and 92.

All these tests agree in showing that the cortex of human head hair is fully stabilized at a level about one-third of the total length above the bulb. Further, it shows that the keratinization zone itself which extends from the 
constriction to the fully stabilized level may be divided into two distinct parts:

(a) A lower zone (Fig. 90, zone D) in which the synthesis and orientation of the fibrous protein itself seems largely complete, but in which the structure is poorly stabilized.

(b) An upper zone (Fig. 90, zone E) in which stability rapidly develops.

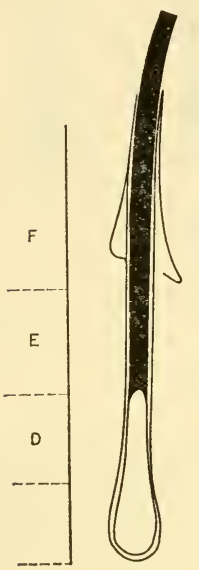

(o)

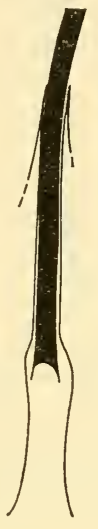

(b)

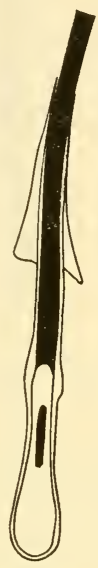

(c)

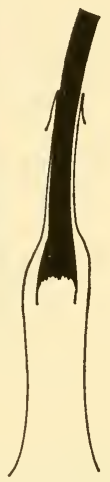

(d)

Fig. 92. Histochemical methods of demonstrating the unconsolidated zone $\mathrm{D}$ of the pre-keratin. Birefringent regions are shown in black. (a) Destruction of orientation in zone D by heating for $30 \mathrm{sec}$ at $90^{\circ} \mathrm{C}$. (b) Result of tryptic digestion of a follicle for some hours. D is removed, the inner root sheath is resistant above the level $\mathrm{B}$ and the earlier hardening of the fibre cuticle is apparent. (c) Destruction of DR due to the penetration of dilute acid. (d) Result of treatment with dilute alkali which acts destructively even on the partly-hardened regions, zone E.

From Mercer (1949b).

These zones are well distinguished by the tests illustrated in Fig. 92. Briefly, many reagents and treatments dissolve only the lower zone $(D$, Fig. 90) of weaker stability; more violent treatment is required to derange the subsequent levels (Fig. 92d). The exact range of these levels is not the same in all types of follicle, but Hardy's work on the mouse follicle shows that the sequence of changes is the same.

In experiments on plucked hair roots the present writer (1949b) showed that the cortical orientation (observed as double refraction) was destroyed 


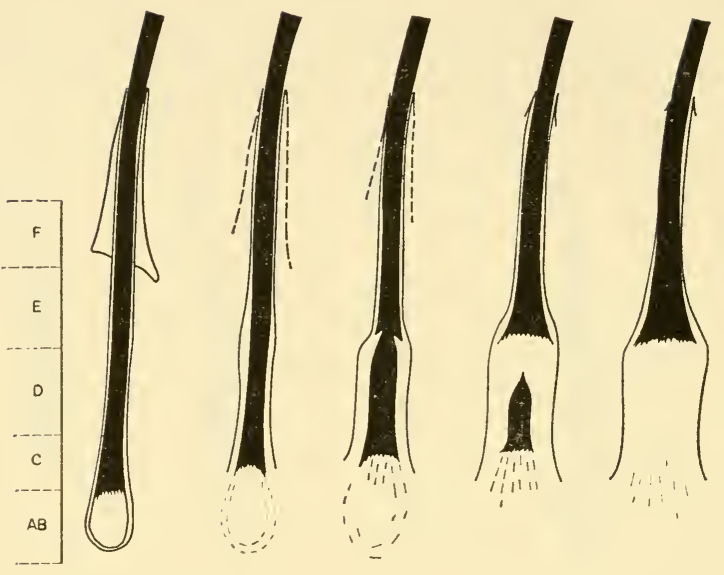

FIG. 93. Progrcssive dispersion of the unconsolidated pre-keratin by saturated urea. Above D the partly-hardened protein swells only and to a diminishing degree as the hardening progresses. Birefringent cortex shown black. The time taken for the whole action is from 5 to $10 \mathrm{~min}$ (from Mercer, 1949d).

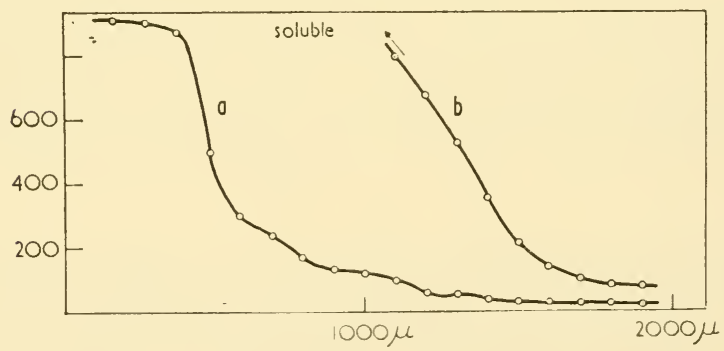

Fig. 94. Swelling (percentage of original area on ordinate) of a follicle in $a$, water, b, saturated urea as a function of the distance from the base of the papilla (Mercer, 1949b). 
in zone $D$ by heating for $30 \mathrm{sec}$ at $90^{\circ} \mathrm{C}$ or by soaking in dilute hydrochloric acid. Trypsin readily digests the same layers $A, B, C$ and $D$. Alkaline reagents are, however, able to destroy the birefringence in the lower parts of zone $E$ (Fig. 92). Perhaps the most spectacular and instructive effects follow the application of saturated urea to the base of a plucked hair follicle (Fig. 93). The lower zone $D$ swells rapidly and is dissolved; the upper half $E$ swells only, and to a diminishing degree, as the follicle is ascended (Fig. 94).

(a)

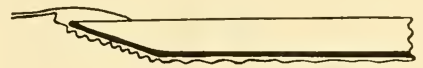

(b)

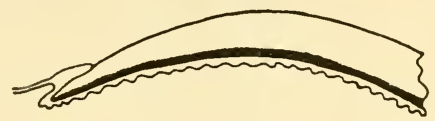

(c)

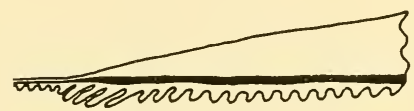

FIG. 95. The location of the keratinization zone $(+\mathrm{SH})$ in three hard keratins, (a) the nail, (b) the claw and (c) a horn (edge only shown). Positive SH shown black. Redrawn from Giroud and Bulliard (1930).

Thiol and Disulphide Groups during Keratinization (Fig. 97(c))

The most important single observation relevant to the chemistry of keratinization is that the keratinizing layers give a positive reaction for thiol $(\mathrm{SH})$ groups and that this reaction fades as the tissues harden. In their important comparative study of numerous keratinizing tissues Giroud and Bulliard (1930) established the existence of an SH-positive layer in every case and distinguished between the hard and soft keratins by the intensity of the reaction. Fig. 95 has been redrawn from their work. Giroud and Bulliard used the nitroprusside reagent for thiol groups. Since that time more permanent and specific reagents have been introduced (Chèvremont et al., 1943; Rudall, 1952; Barrnett, 1953; Barrnett and Seligman, 1952) and the use of these has confirmed the earlier work. The conclusion to be drawn from these results is that the fixed $\mathrm{SH}$, i.e. that resistant to washing by being joined to a protein framework, disappears in the course of keratinization and that therefore one of the reactions underlying keratinization is the oxidation of the $\mathrm{SH}$ groups to produce cystine bridges: (-S-S-)

$$
2-\mathrm{SH}+0=-\mathrm{S}-\mathrm{S}-+\mathrm{H}_{2} \mathrm{O}
$$

When the $\mathrm{SH}$ groups are first blocked by alkylation, and the tissue reduced. 
the oxidation may be reversed and the distribution of new $\mathrm{SH}$ groups will be that of the former disulphide bonds. This distribution is found to be complementary to the SH (Hardy, 1952; Rudall, 1952) as may be seen from Fig. 97(c).

In some pathological conditions oxidation may be incomplete, and a positive thiol reaction persists into the normally-hardened layers. A significant case of this was described by Marston (1946), in sheep reared on a diet deficient in copper (p. 159). This element seems to play a role

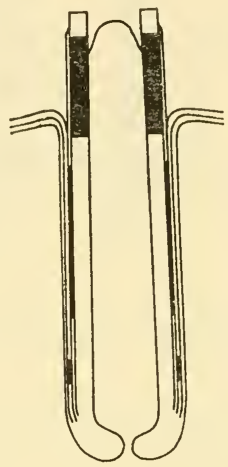

Fig. 96. The positive $\mathrm{SH}$ zones in the growing feather follicle (after Giroud and Bulliard). Compare with figure of the hair follicle (Fig. 90).

as a coenzyme in the oxidation of thiol groups (Flesch, 1949); when deficient in copper the positive-SH reaction persists along the greater part of the follicular shaft, the emerging wool fibre is less thoroughly stabilized and the crimp has a longer wavelength (see also p. 159).

Thus the outstanding fact relating to the progress of keratinization is that it occurs substantially after fibril formation (zones $D$ and $E$ ) and in two steps. The two steps can be satisfactorily correlated with (a) a primary stabilization effected by hydrogen bending (zone $D$ ) and rather readily disorganized (Fig. 90D) followed by (b) a consolidation of the primary structure by the progressive introduction of cystine bridges resulting from the oxidative linking of cysteine $(\mathrm{SH})$ side chains. These chemical changes are accomplished without detectable change in the $\alpha$-type structure which is established and stabilized by hydrogen bonds in the first-formed fibrils. The sudden increase in stability, particularly apparent in the tests depicted in Figs. 92 and 93 (level (E)), seems to coincide with the appearance of a dense material between the filaments seen in the electron microscope and described on p. 224. 
It seems not unlikely that other chemical modifications occur concomitantly. The changes in the affinity of the proteins in zones $C, D$ and $E$ for acidic and basic dyes described by Auber (1950) Odland (1953) and Montagna (1956) suggest that other side chain modifications are occurring. According to Montagna's summary, the first-formed fibrils are basophilic, i.e. they take up basic dyes in a $\mathrm{pH}$ range $4-6$; as the material matures it loses its power to bind basic dyes at lower $\mathrm{pH}$ values although it still binds acid dyes. Evidently some acid groups are lost or modified. This could be due to the conversion of some acid groups $(\mathrm{COOH})$ to amides $\left(\mathrm{CONH}_{2}\right)$. For example, in wool the effective acid and basic groups are closely equivalent when allowance is made for the acids in amide form. Table 13 is adapted from Simmonds (1954 and 1955).

TABLE 13.

(gramme equivalents amino acids in $10^{5}$ g wool.)

\begin{tabular}{|c|c|c|c|}
\hline glutamic acid & $101 \cdot 8$ & lysine & $19 \cdot 3$ \\
\hline aspartic acid & $50 \cdot 3$ & arginine & $60 \cdot 2$ \\
\hline tyrosine $\mathrm{OH}$ & $35 \cdot 3$ & histidine & $5 \cdot 7$ \\
\hline amides & $\begin{array}{r}187 \cdot 4 \\
-\quad 88.6\end{array}$ & unknown & $7 \cdot 1$ \\
\hline total & $98 \cdot 8$ & total & $92 \cdot 3$ \\
\hline
\end{tabular}

The amides may also contribute to the stability by participating in hydrogen-bond formation. A more complete investigation aiming at the localization of amino acid residues other than cysteine and cystine is needed. Ryder has described preliminary tests for several (1959). Gillespie et al. (1960) point out that analysis of the fibrillar ( $\alpha$-component, see p. 240) and the $\gamma$-component, which may enter during the progress of keratinization (see p. 224), differ in their content of basic and acidic amino acids. The matrix proteins ( $\gamma$-keratins) (p. 248) are more basic than the fibrillar $\alpha$-proteins.

\section{Nucleic Acids and Synthesis (Fig. 97 (b))}

The dividing cells of the matrix (zone $A$ ) have prominent Feulgenpositive nuclei and a basophilic cytoplasm containing RNA which is said to increase as the cells differentiate (zone $B$ ) and commence to synthesize protein. The pattern of the nucleic acids: well-developed DNA-containing nuclei, prominent RNA nucleoli and a strongly RNA-positive cytoplasm are compatible with current views of the role of these acids in synthesis as outlined in a previous chapter. 
The disappearance of the nucleic acid from the keratinized hair poses some problems. A nuclear residue persists (see p. 263) but is not Feulgenpositive. It would seem that, even in the upper levels of zone $E$ where it would be thought that the dehydrated and moribund cells would be incapable of further activity, some reactions, which mobilize and remove nucleic acids, can still take place. Perhaps this operation is of value in the hair follicle in that valuable materials (phosphorus) are resorbed. Bolliger and Gross (1952 and 1956) have, however, reported ample quantities of nucleic acid breakdown products in hairs and other keratins.

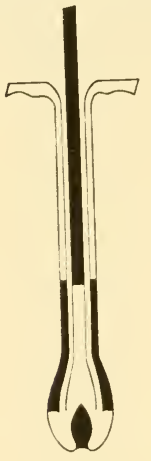

(a)

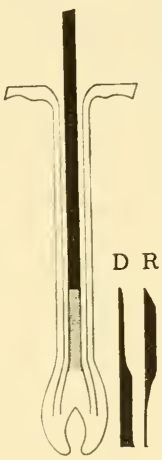

(b)

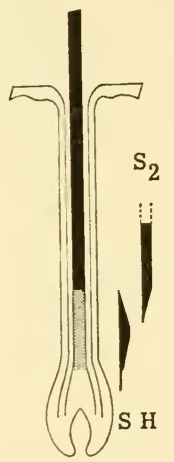

(c)

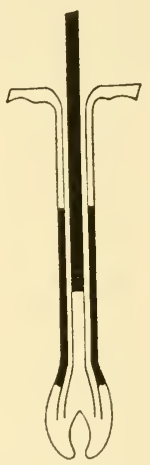

(d)

FIG. 97. The results of some histochemical tests on the human-hair follicle: (a) mucopolysaccharides and/or alkaline phosphatase; (b) the nucleic acids $[\mathrm{D}=\mathrm{DNA}, \mathrm{R}=\mathrm{RNA}]$; (c) thiol $(\mathrm{SH})$ and disulphide sulphur $\left(\mathrm{S}_{2}\right)$; (d) glycogen. In the shaft of the cortex the black areas are the fully-stabilized hair, and the stippled are the pre-keratin = positive thiol regions.

Another unsolved problem is raised by the recent results of Fell, Mellanby and Pelc (1954 and 1956) which revealed an apparent localization of radioactive sulphur in nuclei of the cells of the oesophageal epithelium after injection of labelled crystine. This would seem to imply that some reaction involved in the addition of cystine to the cytoplasmic proteins has its onset in the nuclei. Pelc (1958 and 1959) believes that cystine is exchanged against some DNA constituent which is itself broken down. Such a view would link the final disappearance of the DNA with the late synthetic processes (see also p. 264). However, the actual finding in these experiments is the localization of sulphur in nuclei and this could equally well be explained by an association between nuclear RNA and a sulphurcontaining protein, a view which would accord better with the other views of the participation of RNA in synthesis. 


\section{Metabolic Enzymes}

Certain enzymes concerned with basic cellular metabolism are necessarily present in all cells and show a diffuse cytoplasmic distribution. Rogers (1953) applied the Nadi reagent as a test for cytochrome oxidase to wool roots and found a strong reaction in the bulb extending to the proximal third of the shaft. Montagna (1956) reports similar findings. Dehydrogenase activity has been demonstrated by means of tetrazolium salts which are reduced by the enzymes to yield purple granules (see Montagna, 1956 , for review). Rogers specifically demonstrated succinic, $\beta$-glycerophosporic, lactic and malic dehydrogenases.

The oxidases and dehydrogenases are associated with energy-supplying reactions and their presence in the hair bulb could also be associated with mitosis which Bullough (p. 136) has shown requires energy. The dehydrogenases of the shaft could also be concerned with the energy-consuming process of transport of metabolites from the bulb or of the glycogen from the outer root sheath. A cyclic variation in these enzymes in the epidermis during the hair cycle is described by Carruthers et al. (1959). Presumably also, the energy required for protein synthesis is obtained through similar reactions. Since many of these enzymes are now known to be located in mitochondria, their presence could also be inferred from the mitochondria visible in electron micrographs of these cells.

\section{Glycogen (Fig. 97 (d))}

The most conspicuous deposits of glycogen are in the o.r.s. cells. In the middle third of the follicle the cells are virtually filled with it. The possible role of this glycogen as an energy store, which buffers the follicular system against the fluctuations in glucose content of the systemic blood supply and thus enables a steady rate of mitosis to be maintained in the bulb, has been mentioned in Chapter IV. When the hair follicles of rodents are quiescent no glycogen is present, it increases rapidly when growth recommences (Montagna et al., 1951). In good agreement Ryder (1958) found that radioactive glucose rapidly $(1 \mathrm{hr})$ entered the bulb where it was presumably being utilized directly to sustain mitosis and later was localized (probably as stored glycogen) in the outer root sheath. Bradfield (1951) believed that epidermal cells stored glycogen while in the basal layer and carried it outwards where it supplied energy for protein synthesis in the outer layers.

\section{Acid Mucopolysaccharides and Schiff-Reactive Substances (Fig. 97 (a))}

Metachromatically-staining substances (p. 54) are present in the dermal papilla (Sylvén, 1950 and 1951; Montagna, 1956) and Schiffpositive material seems to occur in much the same situation (Leblond, 
1951). Both materials increase during the growing phase and decrease during rest. Metachromatic staining usually indicates acid mucopolysaccharides and is common in growing mesenchymal tissues elsewhere but its exact role is not clear. Sylvén's suggestion that the sulphur of polysaccharide was transferred to the growing hair through the papilla and was a source of sulphur for keratinization seems to be refuted by Ryder's observations to be described later (p. 232).

\section{Phosphatases (Fig. 97 (a))}

Moog (1946) has summarized the evidence to show that there is commonly an association between alkaline phosphatase and transport of materials. The distribution of these enzymes in the skin and hair follicle (Fell and Danielli, 1943; Montagna, 1956) is such as to suggest that they could be concerned with the transport of metabolites. They occur together with the mucopolysaccharides. From their location it would seem that phosphatases are not directly associated with the processes of keratinization itself. See also Braun-Falco (1958).

\section{LiPIDS (NOT Illustrated)}

According to Montagna (1956) lipids stainable by means of Sudan dyes are not strongly developed in the lower follicle and even less can be demonstrated in the upper levels. This is in contrast to the epidermis, but it seems to show that lipids in themselves have little to do with keratinization at least in the hard keratins. In the epidermis also they are probably accessory, serving to plasticize and waterprocf the formation. Contrary opinions have been expressed (see Rothman, 1954).

\section{WATER CONTENT}

The water content of the cells of the lower bulb is probably of the order of $90 \%$; that of the keratinized hair about $30 \%$. There is clearly a considerable dehydration in the course of the formation and consolidation of the fibre, but it has not been measured with any accuracy. A very similar dehydration occurs in the stratum lucidum and corneum of the epidermis. In the hair follicle the most marked loss of water accompanies the rapid synthesis and coalescence of fibrils in the upper bulb and coincides with the narrowing of the lumen and the rise in birefringence. It would seem that, with the condensation of the cytoplasmic proteins into compact fibrous masses, many hydrophilic end groups are either modified chemically or are utilized in the formation of various bonds internal to the fibrils and thus release the water molecules previously bound by them. The "free" water may then leave the cells for osmotic reasons. When $\mathrm{H}$-bonds are broken in reduced hairs a marked swelling and hydration occurs, which is probably a partial reversal of processes occurring in the follicle in zones $B$ to $C$. 


\section{The fine histology of the hair follicle in the keratinization zone}

In the hair follicle may be found examples of the formation of both hard and soft keratins and for this reason the detailed study of the fine structure of its various layers provides an opportunity to compare and contrast the two modes of development.

As indicated in Chapter III, the separate cell streams, which form the inner root sheath, the cuticle, cortex and medulla of the hair, are clearly differentiated above the middle of the bulb; synthesis and stabilization follow distinct courses in each. They will be described separately.

\section{The Cortex}

Interest here chiefly concerns the further development and consolidation of fibrous keratin. The fine parallel filaments which first appeared as wispy bundles in the cells of the presumptive cortex at a level a little below the tip of the papilla (Fig. 90, zone B) rapidly accumulate in the upper regions of the bulb until, at the level of the constriction of the follicle, the cells appear almost full. The length of the follicle from its constriction to the level where the cortex achieves its definitive form has been called the zone of keratinization (D and E, Fig. 90).

The fine filaments (diameter $\sim 60 \AA$ ) (Plate 12B) also described as the protofibrils (Chapter III) are at first individually distinct and form small clusters which rapidly grow in size. When in the upper bulb, these aggregates become a few tenths of a micron in width, they can be seen in the light microscope and are then described as fibrils (Plates 13, 15 and 16). It is at this same level, a point immediately following the follicular constriction, that birefringence rapidly rises and reaches almost the value of the final fibre and an oriented $\alpha$-type X-ray pattern (Fig. 91), indistinguishable from that of the fibre, can be obtained. These observations show again that the synthesis of the basic fibrous structure is virtually complete at this level and that the chemical changes, which follow and produce hardening and stabilization of the formation, must take place outside the crystalline regions, and probably outside the filaments themselves if we take the further step of identifying the filaments with the "X-ray crystallite".

Some support for the idea that changes external to the filament occur comes from changes in the electron-microscopic appearance of the bundles of filaments as they advance through the zone of keratinization. At first separate filaments are seen in cross-section as clusters of dense dots and their arrangement is rather irregular. Then areas of better order (quasihexagonal packing) appear in which the filaments now appear relatively light against a darker matrix (Plates 15 and 16). It is as if a new and more 
dense component was forming between the filaments and causing them to pack more compactly (Birbeck and Mercer, 1957; Mercer, 1958). At the level of the constriction (Fig. 90) and immediately above it, it is possible to distinguish a population of fairly distinct fibrils (diameters in the range $0 \cdot 05-0 \cdot 2 \mu$ ) each consisting of masses of fine, light filaments in quasihexagonal array, which thus constitute a rather definite level of organization of the fibrous keratin (Fig. 80 and Plate 13); then, as the cells advance, these fibrils rapidly fuse laterally, to produce progressively large, irregularly-shaped aggregates, and finally an almost complete fusion into a solid mass in which only residual, irregularly-dispersed gaps remain as evidence of the earlier existing interfibrillar spaces (Fig. 16). It should be noted that whereas the filaments appear to be perfectly definite structural elements, all having the same diameter, the fibrils, which are aggregates of filaments (Figs. 80 and 102), are not of uniform size although they cluster around an average diameter.

In the later stages of consolidation, the hexagonal arrangement of the filaments within the fibrils becomes distorted, leading to the appearance in cross-section of spirals or fingerprint-like whorls. It is possible that each fibril becomes slightly twisted on its long axis (Plates 14A, 15 and 16).

At the histological level (light microscopy), the course of keratinization in the other hard mammalian keratins seems essentially similar to that of hair. The fine histology has not yet been studied, but there would seem to be every reason to think it will also prove similar to that of hair. In feathers the formation and condensation of fine filaments follows similar lines (Mercer, 1957). There is nothing in the electron-microscopic appearance of the feather cells to show that a $\beta$-type rather than an $\alpha$-type protein is being formed.

\section{The Cuticle}

The presumptive cuticle cells, which form a single layer as they leave the matrix of the bulb, tilt sharply as they approach the bulb constriction and achieve their fully-tilted and overlapping condition at the level of the constriction where Henle's layer turns birefringent. Here they still contain little or no keratin and their contents are no longer symmetrically disposed. Nuclei are usually found towards the basal pole of each flattened cell and a well-developed system of vesicles (Golgi apparatus) lies largely apical to it. The flattened cell is almost vertical and its two surfaces now face towards very different environments, the internal towards the hair cortex, the external towards the sheaths and follicular surroundings. In such circumstances the cell contents develop a stratification parallel to the cell membranes (Plate 20A).

Keratin appears as small $(\sim 300 \AA)$ rounded, dense droplets and moves 
towards the peripheral surface of each cell where it collects in curiously patterned groups (Birbeck and Mercer, 1957). The aggregation continues and with the closure of the gaps a compact layer packs against the cell wall. The cellular apparatus comes to occupy the inner part of each cell and the stratification thus produced persists in the final cell state. Cross-sections of cuticle cells (Plate 20A) show these two layers of distinct texture, but reveal also that within the keratin itself there is also some stratification (Sikorski and Simpson, 1959). When reduced and stained by osmium tetroxide or metal salts, the layer adjacent to the external-facing membrane

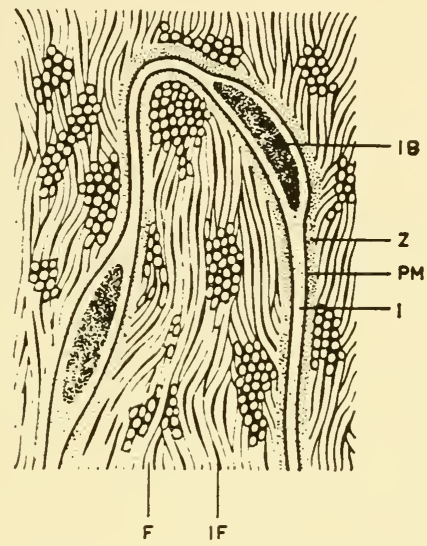

FIG. 98. Schematic drawing of two cells in the stratum corneum of the guinea-pig showing the characteristic keratin pattern with bundles of filaments (F) embedded in an interfilamentous substance (IF). The cell boundary consists of a fairly opaque, broad inner zone $(Z)$ and an opaque, fine outer membrane (PM). The intercellular space is filled with a substance of a fairly low opacity (I). In this substance opaque, homogeneous, diffusely outlined bodies (IB) are observed. From Brody (1959).

is more dense (probably a higher cystine content). Further, the cell membrane facing the cuticle of the inner root sheath is the more dense and conspicuous. This stratified structure explains many of the properties and reactions of cuticles (see p. 265).

The keratin of the cuticle is not fibrous in texture; only traces of fibrils form and there is no transformation into a fibrous form as occurs to the rather similar droplets of trichohyalin in the inner root sheath cells ( $p$. 226). Consolidation seems to be a simple coalescence of the (perhaps sticky) droplets to form the very coherent, continuous layer immediately 
beneath the cell membrane. The reasons for the delay in synthesis and for the different type of keratin produced are not known. It is possible to speculate that the denser and closer opposed membranes of the cuticular formation deprive the cells of some essential factor, and that synthesis proceeds under limiting conditions which tend to produce amorphous peptides richer in cysteine. Similar conditions may prevail in the upper keratinization zone of the cortex where an increase in cystine also occurs. A sufficiently-high frequency of cystine residues in a polypeptide would seem to preclude both the possibility of assuming the regular folding necessary for crystallization and of long-range extensibility.

The birefringence, which can be observed in cross-sections of the cuticle cells, particularly in swollen hairs, may be due either to the fact that the molecular chains of the keratin are compressed into one plane or to the compression of the largely membranous cell apparatus (Schmidt, 1925).

\section{The InNer Root Sheath (I.R.S.)}

The characteristic product of the inner root sheath is trichohyalin, a substance as yet imperfectly characterized. The name is due to Vorner (see historical review in Auber) (1950) who wished to emphasize by it the clear structureless character of the granules and a distinction from similar granules (keratohyalin) found in the skin. It is a protein and when in the fibrous form is strongly birefringent and yields an $\alpha$-type X-ray pattern. It is quite distinct chemically from fibrous keratin, notably in being deficient in cystine. Rogers (1959) has shown further that it is peculiar in containing the amino acid citrulline. The similar keratohyalin occurs in the cells of the epidermis proper where its appearance, as strongly staining granules, gives rise to the name, stratum granulosum. In spite of some differences in stainability (see Rothman, p. $376(1954)$ ) the two substances seem essentially similar in nature. In this sense the inner root sheath is a soft keratin; and, like the epidermis itself, it desquamates half-way along the follicle. It is this event which actually frees the advancing hair shaft from its enveloping sheaths.

The synthesis of trichohyalin commences first and proceeds further in the cells of the layer of Henle, the outer-most layer of the i.r.s. contiguous with the outer root sheath. Small dense granules of trichohyalin appear free in the cytoplasm and grow rapidly. They have no internal structure, are not birefringent, stain heavily (in the electron-microscopic sense) with phosphotungstic acid and with basic dyes as judged by light microscopy. The fact that the granules absorb basic dyes, as does nucleic acid, has led some to think that they contain nucleic acid. This is not now thought to be the case since digestion with ribonuclease has only a small effect on the basophilia (Leuchtenberger and Lund, 1951) and electron-microscopically the trichohyalin droplets are free from the small dense particles usually 


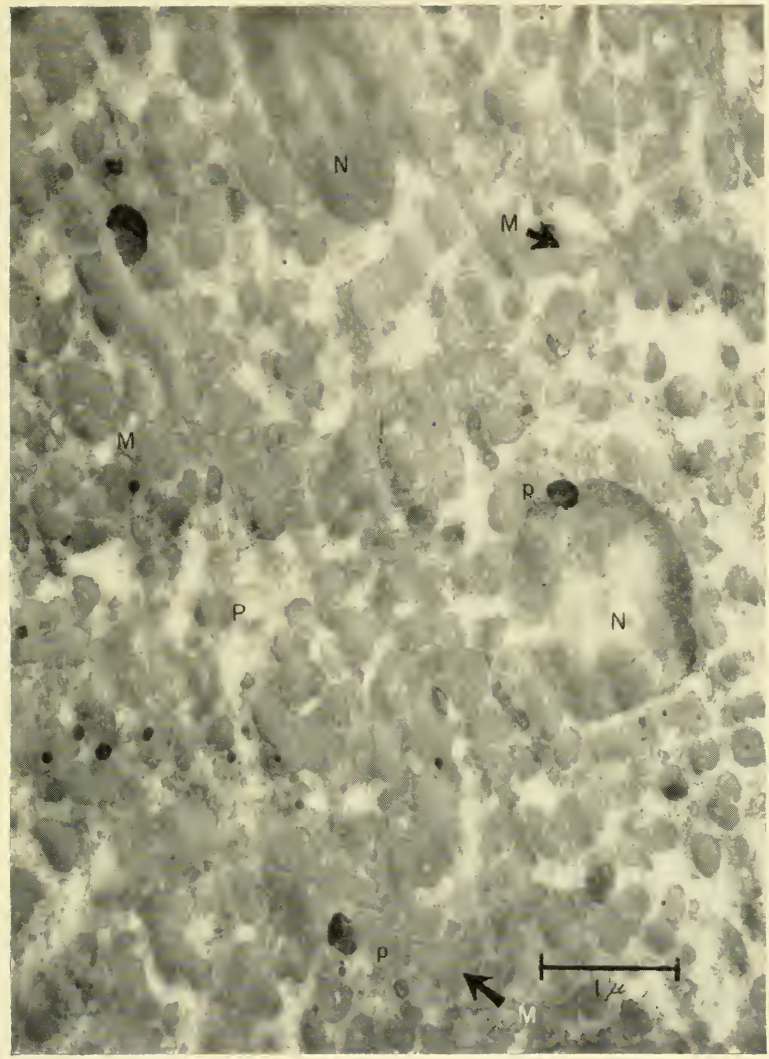

\section{Plate 13}

Cross-section of the cortex of a human hair in the keratinizing zone of the follicle to show the arrangement of fibrils. $N$, nucleus; $M$ (arrows), cell membranes bounding the cortical cells; $p$, pigment granules; $P$, particulate cytoplasmic residue. Higher magnifications of fibrillar keratin appear in Plates $14 \mathrm{~A}, 15$ and 16. 


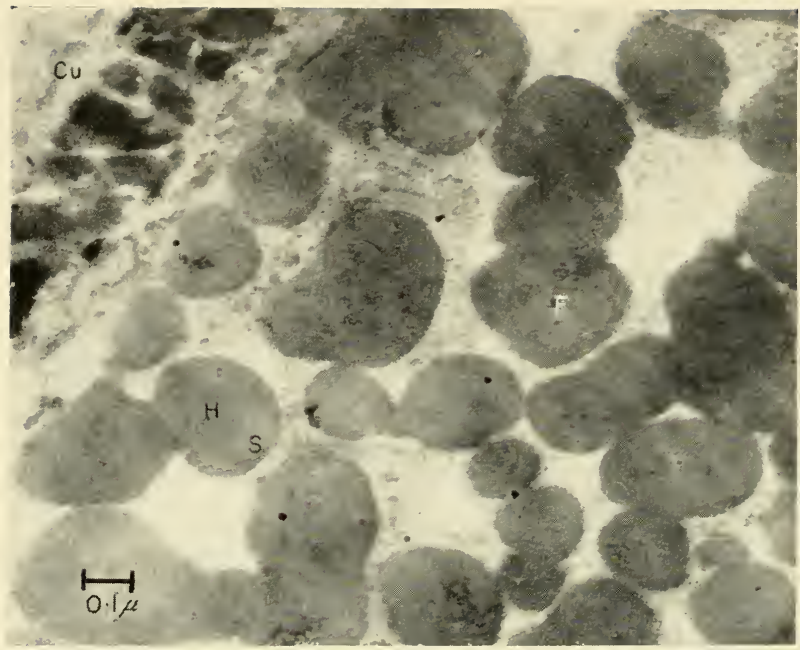

A

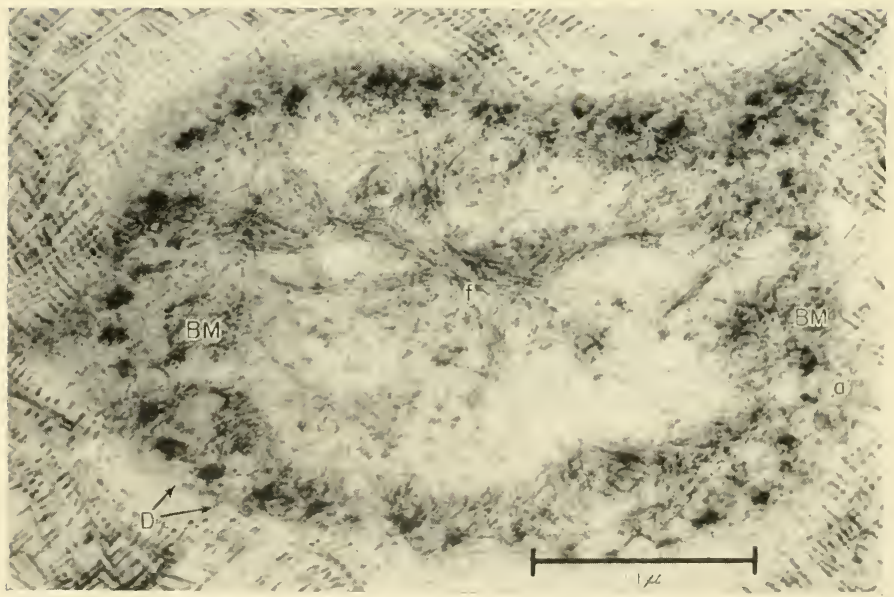

Plate 14 (Captions on facing page) 
A. Cross-section of human-hair follicle in the pre-keratin zone to show the whorl-like arrangement of the fine filaments in the fibrils, $F$. The fine filaments may show a hexagonal packing $(H)$ in the centre of the fibrils which changes towards the fibril periphery to resemble a spiral $(S)$. Notice the filaments are embedded in a denser ground substance. For an enlargement showing this detail, see Plates 15 and 16. At $\mathrm{Cu}$ appears a portion of a cuticle cell containing irregular lumps of cuticular keratin devoid of fine structure.

B. Section running approximately parallel to the basement membrane in the epidermis of a frog tadpole. Centrally it passes into the epidermal cell and whorls of "keratin" fibrils, $f$, are seen; nearer the edges of the picture the section passes obliquely through the basal membrane, $B M$. Note the pattern of dense desmosomal studs $D$ and the finer granular deposits $a$. Surrounding this are seen the dermal collagen fibrils in quasiorthogonal array. For a section at right angles to this, see Plate 9. PTA stain. 


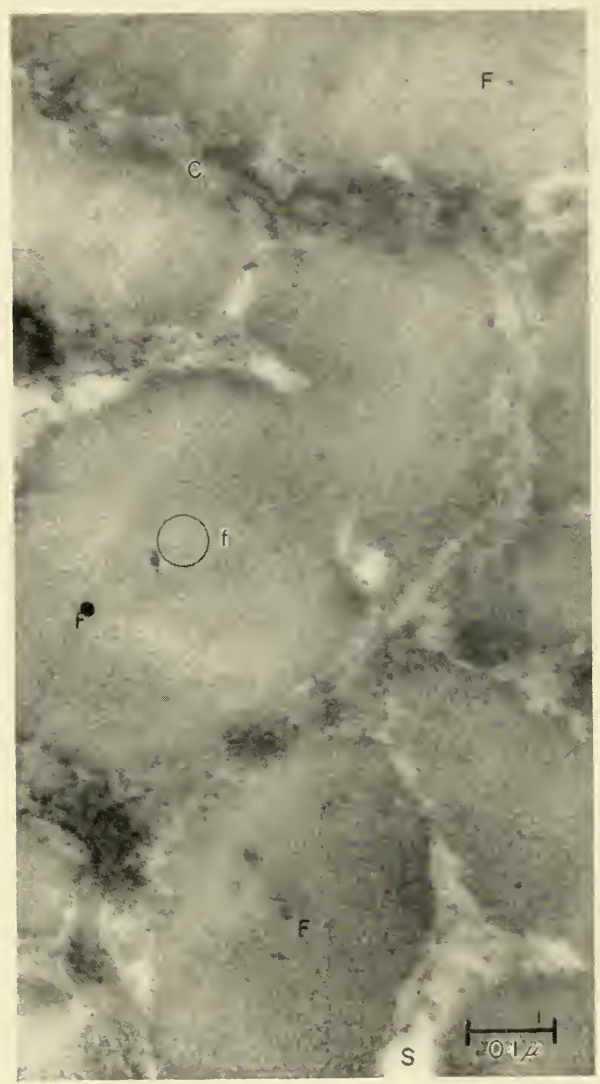

Plate 15

High resolution electron micrograph of several fibrils as noted in cross-section of the hair cortex in the pre-keratinous zone of the humanhair follicle. Compare with Plate 16.

A fibril, $F$, is seen to be composed of fine filaments, $f$ ( $\alpha$-filaments) about $60 \AA$ wide which are embedded in a denser ground substance to produce a characteristic pattern. For other forms, see Plates 16 and 17. A surface of contact between two cells winds across the section and may be seen in detail at $C$. In the intercellular spaces $S$ much cytoplasmic debris persists. Taken from Birbeck and Mercer (1957). 


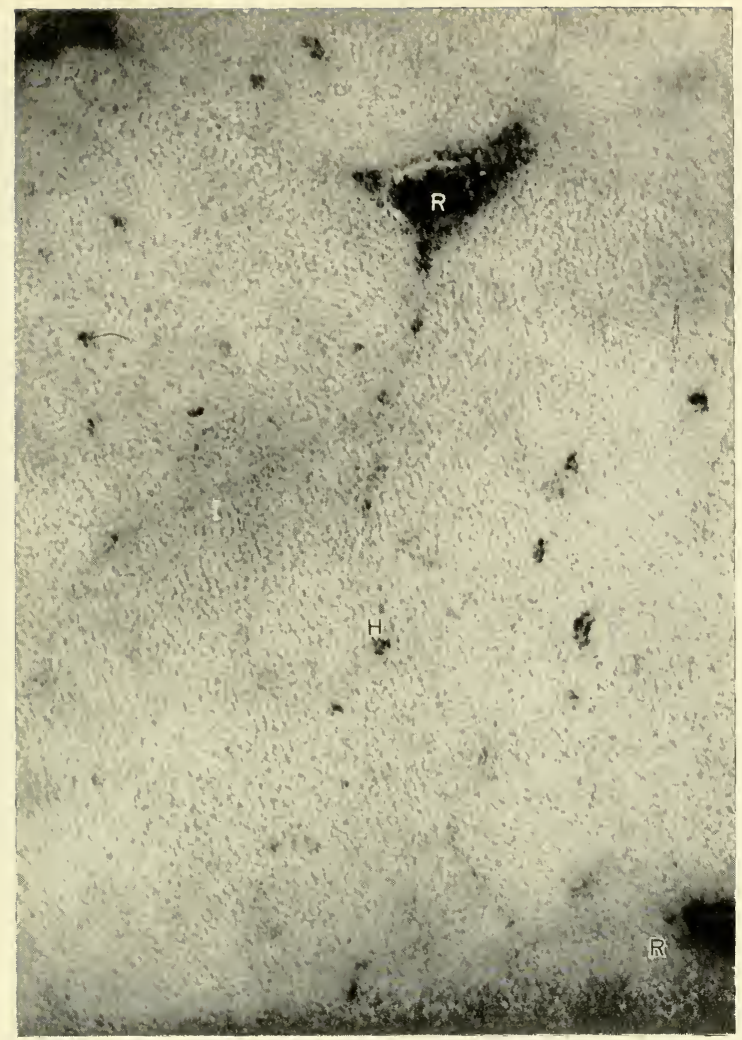

Plate 16

Cross-section of fibrous keratin in the cortex of the fully-hardened wool fibre. Portions of several fibrils are seen with an almost effaced interfibrillar line at (I). At $R$ are seen residues of cytoplasmic debris. The material consists of close packed filaments embedded in a ground substance (matrix), which is denser after reaction with the osmium fixative than the filaments themselves. At $H$ an almost perfect hexagonal packing is visible. For an interpretation of the pattern see Figures in text. Compare with the less well-oriented material found in skin keratin shown in Plate 17. Magnification $\times 100,000$.

Photograph kindly lent by Dr. G. E. Rogers. 


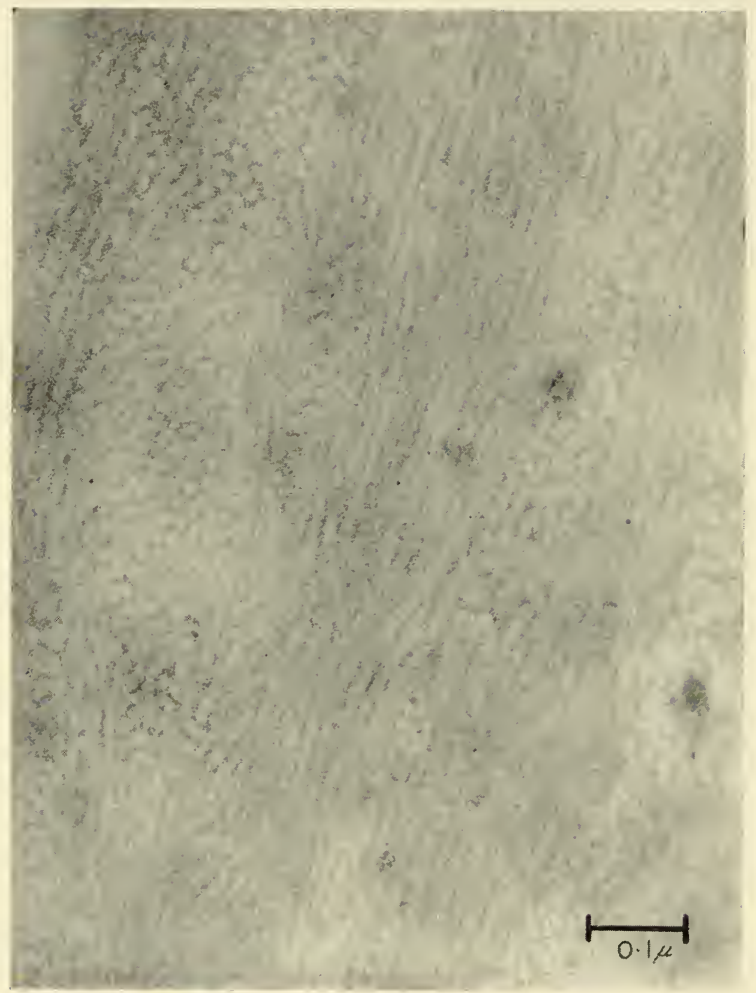

Plate 17

Section of the fibrous keratin in the keratinized cells of the guinea-pig epidermis. The pattern of light filaments embedded in a dense matrix is in essentials identical with that of the well-oriented hair shown in Plates 15 and 16 , but the orientation is here less perfect and filaments seem to run in bundles in various directions.

Photograph kindly lent by Dr. I. Brody. 


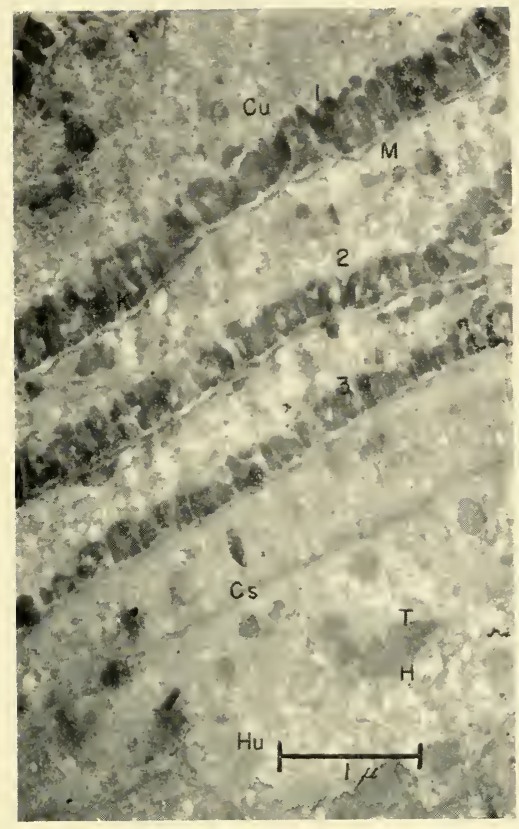

A

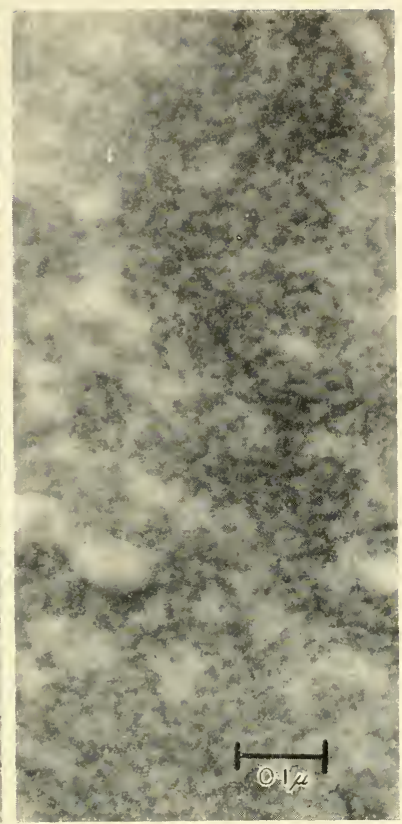

B

Plate 20

A. Cross-section of edge of hair cuticle and the inner-root sheath of a human hair in the hair follicle. Same level as Plate 14A. Sections of three cuticle cells ( $C u$ 1, 2 and 3 ) appear in the upper half and within each cell may be distinguished the cytoplasmic material largely particulate, and the lumps of amorphous keratin, $K$, which are packing against the outer membrane, $M$, limiting each cell. Outside the cuticle (lower half of picture) may be seen the cuticle of the inner root sheath $C s$ and a Huxley-layer cell $H u$. In each of these may be seen sections of granular trichohyalin, $H$, and transformed material, $T$ (fibres seen in longitudinal section, see Plate 20B).

B. Higher magnification of the transformed trichohyalin ( $T$ of $20 \mathrm{~A}$ ). There seem to be here sections of filaments and of ribbons (or coalesced filaments). 

regarded as containing RNA although the adjacent cytoplasm itself is densely packed with these.

All the cells of the i.r.s. produce trichohyalin, but its appearance is the more delayed the further the cells are from the outer root sheath, i.e. it appears last and least abundantly in the cuticle of the i.r.s.

The most characteristic property of trichohyalin (as also of keratohyalin) is its ability to be transformed into a compact fibrous modification. This change is shown vividly in the light microscopy by sudden appearance of birefringence in the cells of Henle at the level of the constriction in the follicle. At the same time these cells become more difficult to stain and the tubular sheath formed by Henle's layer becomes relatively insoluble in strong solutions of urea.

Electron-microscopically the change is equally sudden and remarkable. It is associated with the appearance of bundles of fine filaments which seem to "stream" out of the tips of the now lenticular-shaped droplets of trichohyalin (Plate 21). Birbeck and the writer (Birbeck and Mercer, 1957; Mercer, 1958) consider that this is evidence of a transformation of the accumulated reserves of amorphous trichohyalin and is in some ways analogous to other instances, e.g. that of actin, in which a fibrous modification develops from a non-fibrous precursor (see Chapter III, p. 127). The filaments appear in well-ordered bundles strictly parallel to the axis of the follicle, i.e. to the long axis of the extended cells. Electron micrographs, such as that shown in Plate 21, suggest a likeness to crystallization, the long thin filaments seeming to move out from the droplets as though they were being continuously formed at their surface.

In cross-sections the bundles of trichohyalin are ambiguous in appearance; both dots, i.e. sections of filaments, and wavy lines, sections of sheets or ribbons, are found (Plate 20B).

After the transformation, Henle's layer forms a strongly-coherent sheath not dispersed in urea solutions. There is, however, no sign that the filaments of trichohyalin cross from cell to cell. They continue to the cell membrane and seem to end in close contact with this structure. A very similar picture is found in muscle cells where the filaments also end on extensive desmosomes (p. 42). The actual seat of intercellular adhesion must still be sought in the intercellular cement between the cells. This question has been discussed earlier (p. 84).

The function of the transformation of the trichohyalin into a hard fibrous tube at the level of the follicular constriction seems to be to provide a solid retaining support to carry the still soft tissues contained within it towards the surface of the skin. The somewhat slower transformation in the inner layers of the sheath may permit the layers to remain plastic and to form a more snug attachment between the hair and its sheaths (Auber, 1950). 


\section{Soft keratinization}

\section{The EPIDERMis}

Matoltsy (1958) gives as constituents of stratum corneum of human epidermis: $65 \%$ insoluble keratin, $10 \%$ soluble protein, $10 \%$ dialysable material (largely amino acids), $7.9 \%$ lipid and $5 \%$ cell membranes. The tissue is clearly more heterogeneous than a hard keratin and gives the impression, from its content of soluble protein and low molecular weight substances, that the hardening process is incomplete. On the other hand, a comparison of Plates 16 and 17 shows that, apart from the orientation of the filaments, the fine structure of soft keratin is very similar to that of hard keratin.

There is a large amount of histochemical work on the epidermis. Like that on hair its significance is marred by the lack of specificity of the means employed. The significant tests (summarized in Montagna (1956)) are essentially those already described for hair and their meaning for the understanding of keratinization is much the same. A good deal of attention has been paid to elucidating the nature of keratohyalin granules but without complete success (see p. 226). Staining with fluorescent dyes (Jarrett et al., 1959) demonstrates vividly several phases of keratinization, but the interpretation of the effects is not obvious.

The distinct histological feature of epidermal (soft) keratinization, when several layers are fully developed, is indeed the occurrence of a granular layer due to the deposition of granules of keratohyalin. This substance is in essentials identical with trichohyalin of the inner root sheath of the hair follicle but whereas in the i.r.s. trichohyalin is apparently produced alone and its transformation provides the entire fibrous contents of the hardened cells, the course of formation of the fibrous component of the epidermal cells is more complex and not fully understood. Filaments, as far as can be ascertained, of the same type as those of the cortex of hair follicle, are already to be found in small amounts in the basal layer cells (Plates 7 and 9). Here these are directed predominantly at right angles to the basal layer and a weak birefringence in this direction is detectable (Montagna, 1956; Biedermann, 1926). In the stratum granulosum keratohyalin granules appear as dense amorphous bodies apparently quite independently of the earlier-formed filaments (Plates 22 and 23).

The relation between keratohyalin granules and the fibrous keratin of the stratum corneum has for long been a subject for dispute and it cannot be said that electron microscopy has finally settled the points at issue. There are two main points of view current, both of long standing although it is not always easy to interpret the views of the classical microscopists immediately in modern terms. The first view is that keratohyalin "mixes with " or "spreads over" the fibrils (tonofibrils) already present to yield 
the cornified keratin which thus may be regarded as a mixture. This view is supported in part by the superb micrographs of Brody (1959) (Plate 17) which show that the keratin of the epidermis has essentially the same fine structure as the hair cortex: poorly-stained filaments embedded in a strongly-stained matrix. The filaments here are not strictly parallel as in

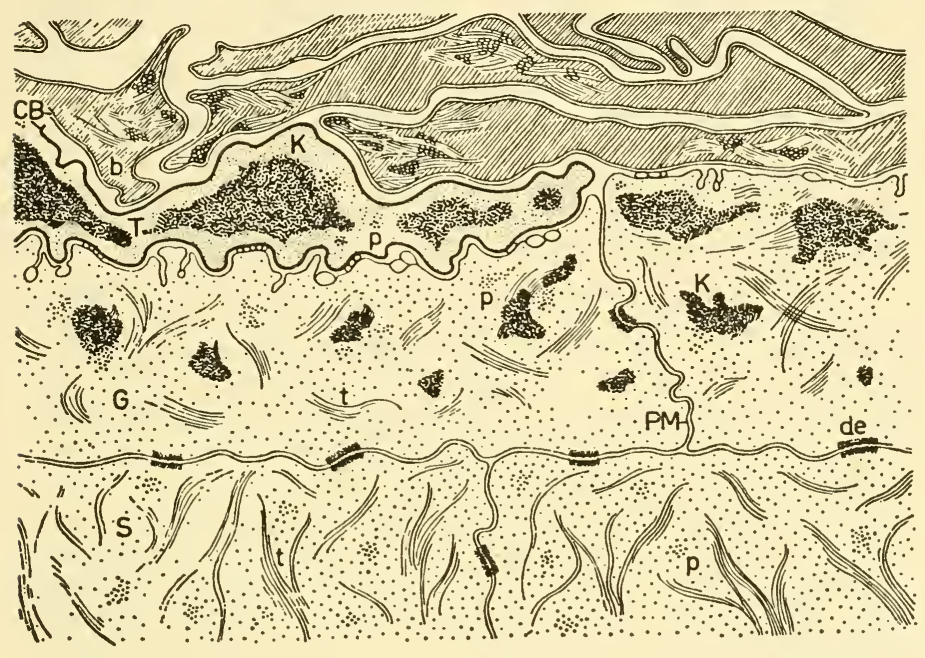

FIG. 99. Schematic drawing of the upper part of the epidermis (guineapig) taken from Brody (1959). The fully-keratinized cells contain the typical keratin pattern of unstained filaments embedded in a stained amorphous matrix as illustrated in the electron micrographs (Plate 17). In the stratum spinosum $\mathrm{S}$ tonofibrils predominate, in the granular layer $\mathrm{G}$ filaments and granules $\mathrm{K}$ of keratohyalin are present. The curious association of granules and filaments is suggested at $T$. In the transitional cell, which follows, the cytoplasm is more condensed, the cell boundaries more dense and keratohyalin predominates. The dense particles $\mathrm{P}$ still persist.

hair cortical keratin; they cluster in somewhat wavy bundles lying more or less in the plane of the flattened cell. Brody suggests from the juxtaposition of granules and filaments in the stratum granulosum that the keratohyalin granules "incorporate" the filaments (Fig. 99). He thus derives the interfilamentous cement from the keratohyalin and on the analogy with the work on the cortex supposes that this material must be rich in cystine. He cites in support the finding of Chèvremont and 
Frederic (1943) of a high concentration of SH groups in the granules. On the other hand, most other observers seem to agree that there is little or no sulphydril or disulphide in the granules (Van Scott and Flesch, 1954; Montagna, 1956; Eisen et al.).

The present writer (Mercer, 1958) has supported the view that the keratohyalin granules are a direct precursor of the fibrous keratin and that something of the same type of transformation occurs as was found in the Henle layer of the inner root sheath of the hair follicle (see Plate 22). Images showing filaments apparently emerging from granules may be found. The amounts of fibrillar material in the cell of the granular layer also appear to be too small to provide the amounts visible in the immediately adjacent cells of the stratum corneum. Thus it seems that this material can come only from the accumulated granules. A conversion of the granules into fibrils running roughly parallel to the skin in the flattened cells could account for the sudden rise in birefringence (with its positive axis parallel to the skin) and for the glassy appearance of these layers (stratum lucidum) which stongly resembles Henle's layer above the transformation level. It would seem that here the two potentialities of synthesis of the basal epidermal cells, which become separated into two distinct cell lines (the cortex and the i.r.s.) in the hair follicle, occur together in the epidermal cell but manifest themselves at different times and levels.

Thus (in this view) the final fibrillar contents of the cells of the stratum corneum would seem to be derived from two sources: (a) a small early contribution of fibrils analogous to the cortical filaments of hair and appearing like them without a precursor, and (b) a larger amount produced later by the transformation of the non-fibrous precursor, keratohyalin. The relation of these two kinds of fibrils to each other and the reasons why the cells switch from one form of production to another are not known.

When in the fibrous form, trichohyalin yields an $\alpha$-type X-ray pattern and its sulphur content is low (Rogers, 1959a). These findings also apply to the keratohyalin of skin, if we accept the claims that the granules contain little or no S. Amounts of $\mathrm{SH}$ and disulphide are demonstrable in the cytoplasm of epidermal cells at all levels with a stronger band near the clear layer. Van Scott and Flesch (1954) report that there is little increase in the total $\mathrm{S}$ in passing from the germinal to the horny layer which might agree with the picture presented above if we assume that the earlier formed fibrils become stabilized by disulphide cross-linking but that keratohyalin on forming fibrils contributes little further disulphide. By measuring the specific absorption of X-rays, Engström and Lindstrom (1947) showed that the concentration ( $\mathrm{S}$ per $\mathrm{cm}^{3}$ ) was many times higher in the stratum corneum. This increase is undoubtedly largely due to cell dessication but may also indicate an absolute increase in S content.

Rothman (1954) has proposed the idea that the cell inclusions may 
dissolve and reform as the final keratin but this is not provable by microscopy. There is some evidence in electron micrographs that the early-formed tonofibrils partly' disappear in the granular layer.

A property of the fibrous form of trichohyalin of the hair follicle, probably connected with the fact that it is not stabilized by disulphide cross-linking, is its tendency to "fall apart " during the disintegration of the inner root sheath. This same property plays a similar role in the desquamation of the epidermis.

One further puzzling feature of epidermal keratinization is that keratohyalin is not invariably present. Many thin skins (e.g. birds) keratinize normally without a granular layer and it is found in a variable degree elsewhere. The view that it represents an alternative pathway of synthesis and may make a contribution to an independently-formed system of fibrils does something to explain these facts. The scaly (hard) keratin of rat tails forms without a granular layer, whereas adjacent perifollicular skin is softer, more flexible and has a granular layer. According to Jarrett and Spearman (1961) treatment (externally) with vitamin A causes the appearance of a granular layer and a softer keratin in the scale regions. Epidermal cells differ evidently in the effect on their keratinization of vitamin A with a range of responses included in the sequence:

$\underset{\text { (no keratohyalin) }}{\text { hard keratin } \stackrel{\text { vit A }}{\longrightarrow} \text { softer keratin } \stackrel{\text { vit } \mathbf{A}}{\text { (keratohyalin) }} \rightarrow \text { mucin }}$

See also p. 63 et seq.

X-ray photographs, optical methods (Matoltsy, 1957) and electron micrographs (Plate 17) all show that the arrangement of the filaments in the stratum corneum is less perfect in soft keratins than in hard. It is also likely then that this imperfect organization contributes to lowering the stability of the formation. It is known in wool (Rogers, 1959b) that in cells where the filaments are less perfectly aligned, the keratin is less stabilized. In skin no fibrils analogous to those of the hair cortex form, the filaments appearing to form simply loose inter-connecting bundles (see Fig. 99).

Recently Swanbeck (1959), on the basis of the scattering of X-rays at low angles, has concluded the existence of scattering unit of diameter of $260 \AA$, which, assuming that the scattering phenomena have been correctly interpreted, would seem to imply a close association of the $100 \AA$ filaments in small groups. This is not immediately apparent in micrographs.

\section{KeRATINiZATION OF HORN}

The special interest of horn lies in the fact that from it one can obtain massive samples particularly suitable for some kinds of experiment. The SH reactivity was examined by Giroud and Bulliard (1930) (Fig. 95) and in further detail by Rudall (1956) who established clearly that there was an 
increase in the intensity of the $\mathrm{SH}$ reaction on passing from the inner mucosum to the outer, suggesting that an increase in the amount of cysteine occurs during the later stages of hardening. This was confirmed by an actual chemical analysis of the several layers which in this material can be separated mechanically and chemically. This conclusion is in good accord with the suggestion to be developed later that cysteine-rich peptides are added during hard keratinization.

\section{Follicular nutrition and the entrance of sulphur}

There seems little doubt on histological grounds that the greater part of the material supplies for the growth of the hair are conveyed by the papilla whose dimensions control the output of keratinized cells. This impression is given a quantitative basis by Rudall's (1956) extensive survey of wool follicles referred to on p. 150.

The elaborate vascularization of the middle region of the shaft (Durward and Rudall, 1949; Ryder, 1958), which fluctuates with the hairgrowth cycle, remains to be explained. Possibly it is involved in the transport of glucose, mobilized from the glycogen of the outer-root sheath to the papilla or it could be associated with keratinization since the evidence (see below) is that the sulphur enters at this level.

At the biochemical level it has been shown by the use of radioactive tracers that methionine and not cystine in the diet contributes its sulphur to the hair of rats (du Vigneaud, 1947; Marston, 1946). It is thought that methionine is converted to homocystine and then linked to l-serine to form the compound:

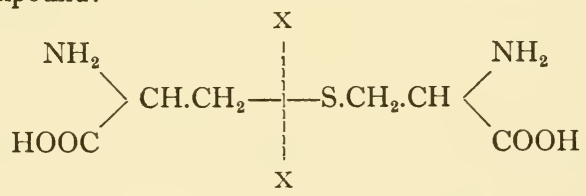

This compound is split in vivo at $\mathrm{X}-\mathrm{X}$ and l-cysteine is produced, the $\mathrm{S}$ having been transferred to the serine to give cysteine. However, in other animals the sulphur metabolism may differ (Ryder, 1958).

The site of entry into the follicle would seem at first sight to be the zone of keratinization since here for the first time SH can be detected (Figs. 95, 96 and 97). It would be possible on this histochemical evidence alone to suppose that below this level the sulphur is present in a concealed (non $\mathrm{SH}$ ) form, but further work on the uptake of radioactive sulphur following injection has dispelled uncertainties. Injection of radioactive cystine is followed by the rapid appearance of radioactivity in the keratinization zone, but not in the bulb (Ryder, 1958; Bern et al., 1955 and 1957). On the other hand labelled carbon and phosphate compounds enter through the 
bulb. This evidence shows clearly that sulphur compounds do not enter through the papilla and advance up the follicle. They must enter in some way through the walls of the follicle at the level of the keratinization zone. The transport of sulphur compounds could be one of the functions of the extensive vascular network surrounding the follicle. There is also evidence of an absolute increase in labelled compounds in the upper levels of the keratinizing levels according to Ryder (1958). Rudall, who separated and analysed the several layers of the growing tissues of horn, established the same fact (1955).

On account of its shape, the hair follicle enables sulphur absorption to be separated from fibrillar growth, thus facilitating interpretation of the phenomena. In the epidermis on the other hand, the sulphur compounds must diffuse upwards from the basal layers into the keratinization layers, and this may have some influence on the lower uptake. Comparable experimental work has not been carried out on other keratinizing tissues.

Sylvén (1950), commenting on the presence of acid sulphate-containing mucopolysaccharides in the papilla (see also Montagna (1956)), believed these might be active in transferring their sulphur to the growing hair. In view of the demonstration that labelled sulphur-containing amino acids enter at the level of keratinization while labelled phosphate enters the papilla, this would now seem unlikely. These acid mucopolysaccharides (substances staining metachromatically with thiazine dyes) are most obvious during active hair growth and seem clearly connected with the proliferation of the bulb, but their sulphur is certainly not transferred to the growing cells at this level.

No definite intracellular structure has been yet shown to be associated with the absorption of the sulphur acids or their change into cystine. Enzymes are of course suspected but not isolated. "Microbodies " (Plate 22 ), single-walled dense bodies, are often common in the keratinizing zone and may contain a special enzyme system.

\section{Soluble products of partial keratinization}

However the process of keratinization is viewed, it certainly involves in the first place the synthesis of one or more polypeptides which subsequently become stabilized by the formation of cystine bridges. Clearly some insight into the nature of keratinization would be obtained if the state of aggregation of the proteins in the cells at various levels in the keratinization zone were known. The concept of the molecular weight of keratin in the hardened tissue is itself meaningless, since the protein is extensively united into large and indefinite heterogeneous formations by covalent cross-linkages. It would, however, be valuable to know in the first place the number, composition and molecular weight of the primary polypeptide chains, i.e. chains containing only peptide links, which 
presumably form the first stage of synthesis. And further, it is meaningful to ask whether or not there exists a hierarchy of definite molecular association of increasing molecular weight whose formation precedes the appearance of the finest filaments which can be seen microscopically (diameter $\sim 60 \AA$ ).

There are two ways in which these problems have been attacked: (a) extracts have been made of the growing tissues with the object of dissolving the proteins before they become keratinized, and (b) attempts have been made, starting with the hardened tissues, to reverse the keratinization process and to obtain soluble macromolecular products from the hardened tissue. This degradation could obviously be continued until small peptides and amino acids were obtained. The full analysis of the complete mixtures resulting from such a partial hydrolysis would clearly do much to elucidate the amino acid sequence of the original polypeptides and it is regrettable that, apart from the pioneering work of Consdon, Gordon, Martin and Synge (Martin, 1946), little has been attempted. For the present we are more concerned with the possible existence of high molecular weight, intermediate polypeptides of a definite character.

From a consideration of the composition and reactions of proteins in general, and of the keratins in particular, the following kinds of bonds might be supposed to participate in the consolidation of an insoluble protein:

(a) Hydrogen bonds, i.e. associations between neighbouring $\mathrm{CO}$ and $\mathrm{NH}$ groups mediated by the hydrogen atom (see Chapter V).

(b) Salt bridges, i.e. salt-like linkages formed between acid groups $(-\mathrm{COOH})$ and amino groups $\left(-\mathrm{NH}_{2}\right)$. Speakman (1934) has amassed evidence to show these are effective in hair and wool. (For a contrary opinion, see Jacobsen and Linderstrom-Lang (1949).)

(c) Weaker and less well-defined forces referred to as Van der Waal's forces.

(d) Disulphide bridges (-S-S-).

(e) Other bonds have been proposed, e.g. between phenolic OH groups and acid groups (Alexander and Hudson, 1954) but are not known to exist for certain.

All these bonds and perhaps others not yet discovered, may play a role in stabilizing insoluble proteins; accordingly, the solvents used to effect a solution or make extracts of keratinized tissues have been chosen because of their specific effect on one or more of these bonds. In keratin the covalent disulphide bonds appear ultimately to prohibit solution and next in importance on account of their number are the hydrogen bonds. These appear particularly to influence the dry hardness and extensibility. The histochemical experiments described above (Figs. 91 and 92) seem to show that the $\mathrm{H}$-bond sustains the structure in the early stages of keratinization 


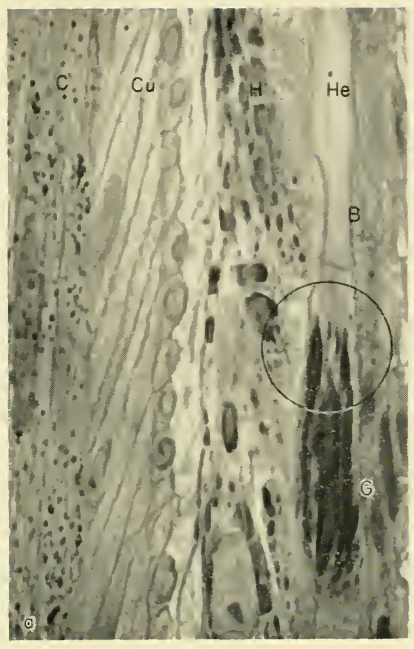

A

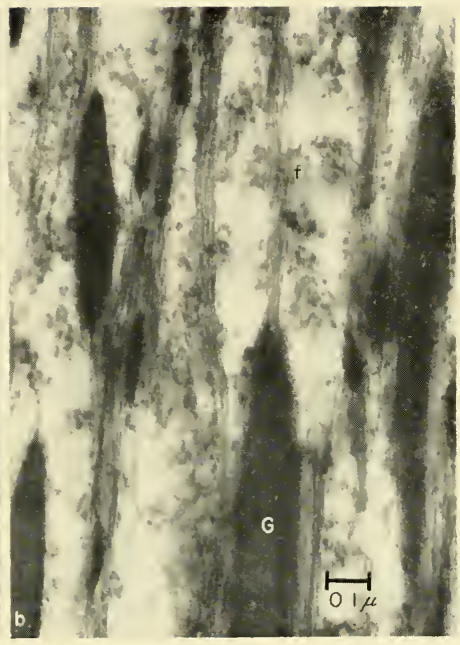

B

Plate 21

The level of transformation of granular trichohyalin into the fibrous form as seen in Henle's layer of the human-hair follicle. On the l.h.s. (A) is a light micrograph in which may be seen the edge of the hair cortex $C$ containing fibrils of keratin and pigment granules, the hair cuticle $\mathrm{Cu}$, the cuticle of the inner root sheath, Huxley's layer containing granular trichohyalin $H$ and Henle's layer $H e$ in which the sudden change from the granular section $G$ into the hyaline birefringent, coherent section $B$ is evident. An electron micrograph of the portion encircled (in an adjacent section) is shown on the r.h.s. (B) where the fibrous strings, $f$, of transformed keratohyalin may be seen extending from the tips of the elongated granules $G$. Dense particles persist in the cytoplasm. PTA stain. Taken from Birbeck and Mercer (1957). 


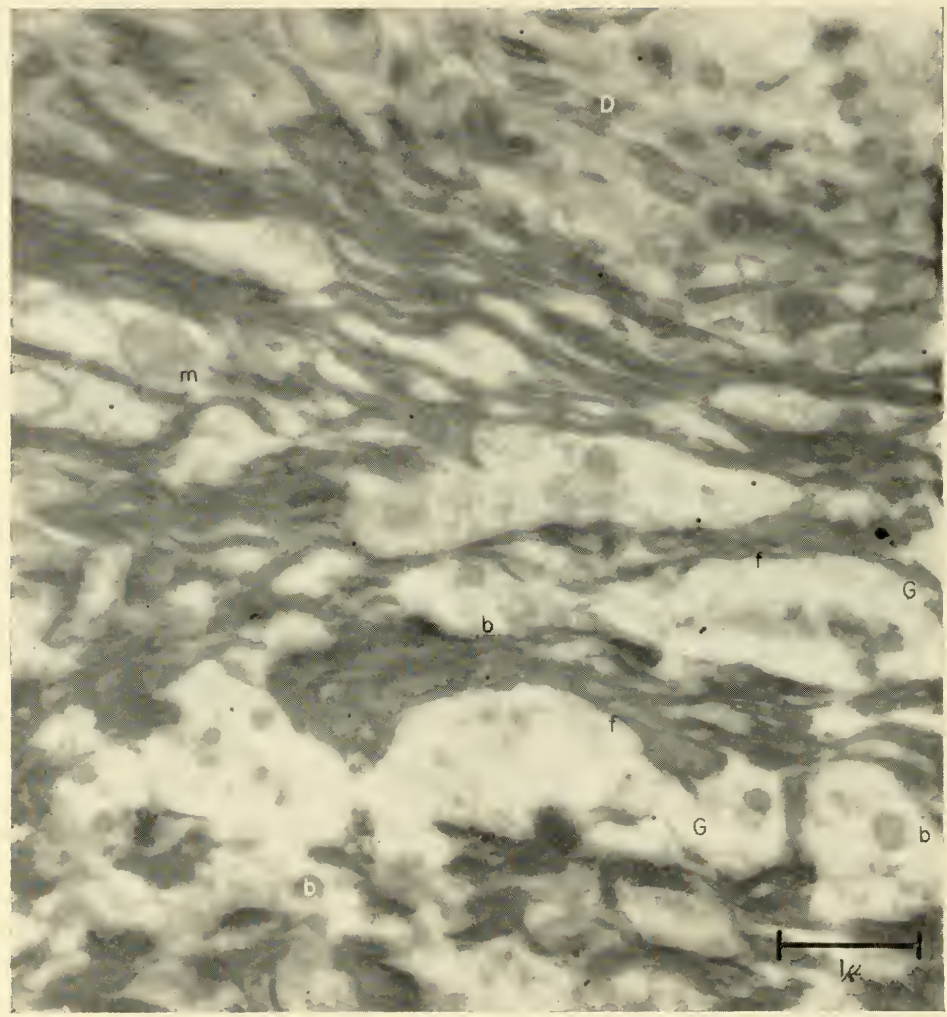

\section{Plate 22}

The transitional zone between granular keratohyalin and fibrous keratin as seen in the upper layers (stratum lucidum) of the plantar epidermis of the rat. $G$, granule of keratohyalin with fibrils, $f$, which appear run out of it in a manner analogous to those seen in Plate 21. $m$, mitochondrion; $b$, microbodies; $D$, desmosomes associated with the convoluted cell membranes. 

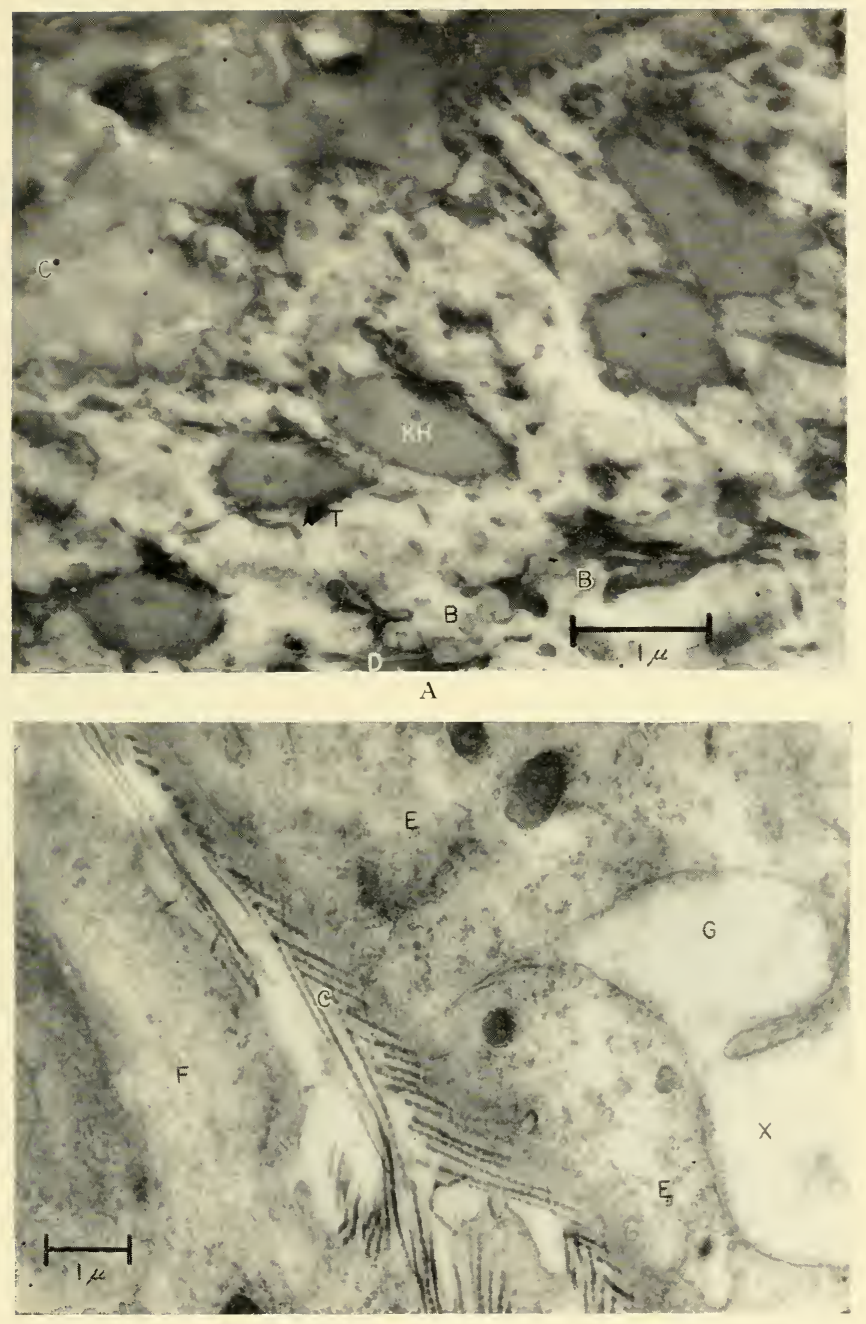

B

Plate 23 (Captions overleaf) 
Plate 23

A. Portion of granular and cornified layers of the plantar skin of a rat showing keratohyalin granules; $K H$, keratohyalin; $C$, "transformed" keratohyalin; $T$, microbodies; $B$, cell membrane $D$.

B. Illustrating the development of the basal-layer cells of the epidermis of a 12-day-old chicken embryo. $C$, collagen fibrils below the basement membrane (here cut obliquely); $F$, fibroblast containing filaments; $E$, epidermal cell; $G$, intercellular gap; $X$, intercellular exudate; $m$, mitochondrion (P'TA stain). 

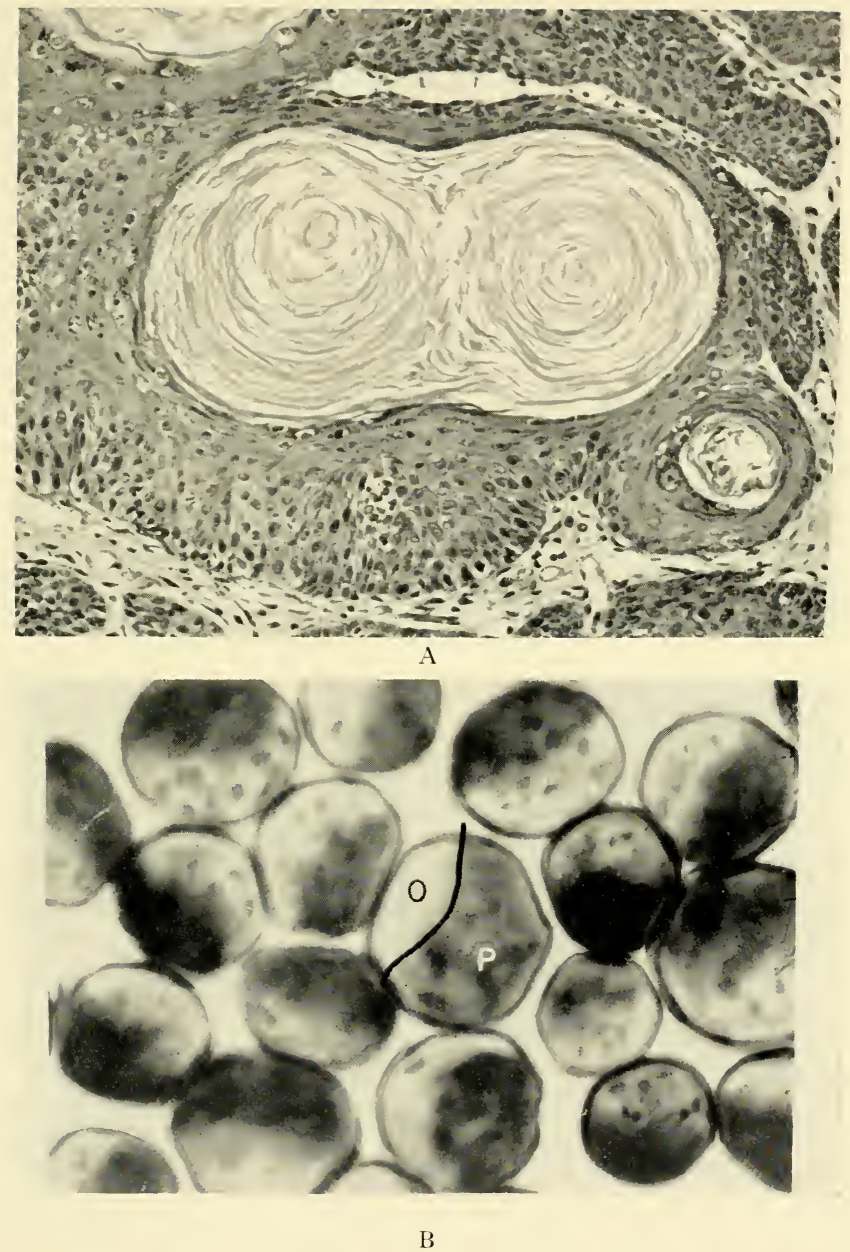

Plate 24 (Captions overleaf) 


\section{Plate 24}

A. A pair of coalescing " keratin pearls", cysts lined with a squamous, stratified, keratinizing epithelium in a tongue of invasive epidermal tissue on the skin of a mouse treated with benzpyrene. Light micrograph, stain: haemotoxylin-eosin. Preparation made by Dr. I. Hieger.

B. Light micrograph of cross-sections of wool fibres to show the distribution of $o$ - and $p$-type cells. The fibre has been oxidized with peracetic acid and stained under acid conditions with methylene blue (Pearse's technique (1951)). The enhanced basiphilia (the dark stain) indicates the regions of higher sulphur content (para-type). Note that although the ortho-para distribution is predominantly bilateral, para-cells may be found among the ortho. 
before the closure of the disulphide bonds. Hydrogen bonds are usually weakened by the introduction of urea into the solvent. The thermodynamic factor involved here is the heat change accompanying the transfer of the $\mathrm{H}$-bond between two peptides to a pair of urea molecules which appears to favour the peptide-urea association. In a sense the urea molecules prise apart the chains and destroy the secondary structures maintained by them. Useful discussions of this problem will be found in articles by Ward and Lundgren (1954) and by O'Donnell and Woods (1956).

\section{Extracts from the Pre-keratinized Zone}

The idea of extracting the proteins from a keratinizing tissue before they harden is attractive since the extract might be expected to contain soluble precursors. Rudall (1946 and 1952) showed, however, that the buffered aqueous solvents commonly used in biochemical extractions removed very little protein from skin. He found, however, that the addition of urea to the solution suffices to dissolve copious amounts of protein from a thick skin such as a cow's nose. He named this protein "epidermin".

When precipitated from solution by ammonium sulphate, epidermin forms a voluminous, white, sticky, curd-like material, easily gathered together and drawn into fibres. These fibres are somewhat elastic when wet, are birefringent and yield an excellent $\alpha$-type X-ray pattern. Stretching produces a fibre giving a $\beta$-pattern. When heated in water $\left(50-60^{\circ} \mathrm{C}\right)$ oriented fibres contract, and the contracted material gives a disoriented $\beta$-pattern. Epidermin thus behaves as an unstabilized keratin, i.e. its basic molecular framework is established but, in the absence of cross-linkages, is readily disorganized.

Rudall (1946) and others (Derksen et al., 1937) have demonstrated the increasing thermal stability of strips of tissue cut from successively higher layers of epidermis. The effect of keratinization can be imitated closely by cross-linking epidermin fibres with formaldehyde and benzoquinone (Rudall, 1946).

Similar extracts may also be made from other keratinized tissues by urea solutions. Here again the simple linear arrangement of the hair follicle enables an exact location of the extracted protein to be determined (Mercer, 1949b). When a plucked human head follicle bearing a papilla is placed in concentrated urea solutions there is an immediate swelling followed by dissolution of the lower half of the keratinization zone (Fig. 93). Above a rather definite level the precortex merely swells and to a decreasing degree the higher the level (Fig. 94). The germinal tissues of the bulb containing little protein are less affected; nuclei are not dissolved. The relation of these events to other histochemical features is best seen in the series of Figs. 91, 92 and 97 . It will be noted that only the lower half 
of the SH-positive zone dissolves which means that disulphide crosslinking becomes effective at a rather definite point. Below this point $\mathrm{H}$-bonding is the major stabilizing element; above it is supplemented progressively by disulphide cross-linking. The addition of reducing agents (thioglycollic acid) to the urea carries the solution to a higher level.

A velocity-sedimentation analysis of epidermin solutions in the ultracentrifuge was made by Mercer and Olofsson (1951a) and revealed the presence of several components (three or more) with sedimentation constants ranging from 1 to 7 . These components were reduced to one by the addition of a reducer which suggests that the heavier components were aggregates of the lighter, held together by disulphide linkages. Epidermin would seem to be a mixture containing some complexes already united by disulphide bonds.

Proteins have also been extracted from the follicle of the wool fibre using the ingenious method of harvesting these in quantity devised by Ellis (1948). Rogers (1959) has reported a detailed investigation on these extracts and reached the conclusion that the larger part of the extract is a fibrous material of low-sulphur content $(<2 \%)$ and that the remainder, less well-defined, has the higher sulphur content $(>4 \%)$. A comparison of the amino acid composition of whole wool and the fibrous low sulphur component is given in Table 14 taken from Rogers.

\section{Soluble Derivatives of Keratinized Tissues}

This approach has attracted more attention for economic reasons. The large quantities of keratinous materials going waste (hair, horns, feathers, etc.) are a potential source of high molecular weight protein possibly of use in the polymer industry (Jones and Mecham, 1943).

On theoretical grounds one might look for a series of soluble derivatives of definite molecular size; in practice, solvents of little specificity must be used and polydisperse mixtures, difficult to analyse, are obtained. Two approaches to rupturing the disulphide bond are open: reduction or oxidation. By using various different $\mathrm{pH}$ values and by adding hydrogen bond breakers, a variety of products can be obtained.

Reduction of wool. Sulphides which combine reducing properties with a high $\mathrm{pH}$ have long been known as keratinolytic agents. Olofsson and Gralèn (1953) found that a sulphide extract of wool contained a mixture of polypeptides of average weight of the order of $8000-10,000$. Since all disulphide bonds were broken, these polypeptides might be regarded as primary although the high $\mathrm{pH}(11)$ may have produced some main chain hydrolysis. Much earlier Goddard and Michaelis (1934 and 1935) used the more specific reagent thioglycollic acid in alkaline solution, and obtained solutions which contained two main components.

Other methods of extraction make use of the simultaneous action of 
hydrogen-bond breakers, and reducing agents at a more moderate $\mathrm{pH}$ (7-8). As reducing agents, thioglycollic acid, bisulphites, sulphides, cyanides, mercaptoethanol, etc., have been used. By using a lower $\mathrm{pH}$ it was hoped that peptide-bond hydrolysis and the conversion of the cystine bridge into the more stable lanthionine bridge $\left(-\mathrm{CH}_{2}-\mathrm{S}-\right)$ could be avoided.

Table 14. Comparison of Amino Acid Composition of Wool and the Low-Sulphur Protein $(\alpha-$ Fraction) of Wool Roots (Rogers (1959)).

\begin{tabular}{l|c|c}
\hline \multicolumn{1}{c|}{ Amino acid } & $\alpha$-Fraction & Whole wool \\
\hline glycine & $5.30 *$ & 5.58 \\
alanine & 4.76 & 4.04 \\
valine & 3.77 & 2.57 \\
leucine & 7.14 & 4.69 \\
isoleucine & 2.89 & 1.62 \\
serine & 5.34 & 7.39 \\
threonine & 3.40 & 4.06 \\
& & \\
phenylalanine & 2.00 & 1.76 \\
tyrosine & 2.37 & 2.54 \\
proline & 2.71 & 5.61 \\
& & \\
lysine & 7.00 & 3.60 \\
arginine & 19.48 & 18.32 \\
histidine & 2.37 & 1.41 \\
aspartic acid & 6.54 & 4.64 \\
glutamic acid & 9.26 & 7.18 \\
ammonia (amide N) & 7.74 & 6.57 \\
cystine & & 6.99 \\
methionine & 3.18 & 0.31 \\
& & \\
\hline
\end{tabular}

* Amino acid $\mathrm{N}$ as $\%$ of total $\mathrm{N}$.

About a quarter of the weight of wool dissolves in $5 \%$ sodium bisulphite $(\mathrm{pH} 8)$ and $10 \mathrm{M}$ urea at $50^{\circ} \mathrm{C}$ in $24 \mathrm{hr}$ (Jones and Mecham, 1934; Mercer, 1949a). The extraction portion is now known to come from the less keratinized orthocortex (see p. 268). When reprecipitated by the addition of ammonium sulphate the extracted keratin derivative is obtained as a white, sticky, coherent curd very closely resembling Rudall's epidermin and Alexander's $\alpha$-keratose (q.v.). Like these it can be drawn into birefringent threads which give an $\alpha$-type X-ray pattern. The attempts made to estimate the molecular weight of extracts in urea are not wholly 
satisfactory owing to a marked polydispersity and a tendency to aggregate with time. Mercer and Olofsson (1951b) reported 84,000; Woods (1952) estimated for a low molecular weight diffusible fraction 10,000 and for the non-diffusible 50,000. For a recent review see Gillespie et al. (1960).

Jones and Mecham (1943) showed that many other keratins give soluble extracts in solutions of urea containing reducing agents but with the exception of feather (see below) little is known of the molecular characteristics of the dissolved material. There is a marked resemblance between these protein extracts and Rudall's epidermin. All precipitate as white, sticky curds on the addition of ammonium sulphate and can be drawn into fibres giving an $\alpha$-type $\mathrm{X}$-ray pattern.

Very similar extracts were obtained from feathers by Lundgren, Ward and associates (Ward et al., 1946; Ward and Lundgren, 1954). More recently Woodin (p. 163) has claimed that better defined "monodisperse " derivatives can be obtained by careful reduction with thioglycollate at $\mathrm{pH} 11$ which would seem to prove that in the case of feather at least a definite macromolecular monomer can be isolated.

Oxidizing reagents. Oxidation of the disulphide bonds with peracetic acid was shown by Alexander and Earland (1950) to be an extremely satisfactory method of obtaining a soluble derivative from keratins. The oxidized keratin is readily soluble in dilute alkalis and can be thrown out of solution on the addition of ammonium sulphate or acids as a white coherent material, again resembling epidermin, which may be drawn into threads yielding well-oriented $\alpha$-type $\mathrm{X}$-ray patterns.

Not all of the material extracted from oxidized wool can be so readily precipitated from solution. A portion remains and has been designated $\gamma$-keratose by Alexander (Alexander and Hudson, 1954). Ultracentrifugal analysis shows that the solution of oxidized wool contains two somewhat polydispersed components, but the physicochemical characteristics of these are still in dispute (O'Donnell and Woods, 1955). This particular method of dissolving keratins has provided nevertheless one of the most valuable insights into the nature of keratinization. It suggested to Alexander the existence in a hard keratin of two main components of very different character: one fibrous of high molecular weight, the other nonfibrous and of a lower molecular weight. This second fraction has the much higher sulphur content (see Table 15). The insoluble fraction, called $\beta$-keratose (less than $10 \%$ ) has been shown to consist principally of the membranes of the keratinized cells (Mercer, 1951d and 1953), see p. 260.

Corfield, Robson and Skinner (1958) have determined the amino acid composition of the oxidized keratin fractions $(\alpha, \beta$ and $\gamma$ keratose $)$ and their results for $\alpha$ - and $\gamma$-keratose are given in Table 16.

It will be seen that $\gamma$-keratose contains much larger amounts of cystine 
(cysteic acid), proline, serine and threonine and smaller amounts of alanine, aspartic and glutamic acids, leucine, lysine and phenylalanine than the original wool. The reverse is true of the $\alpha$-fraction. These figures

Table 15. Protein Fractions Obtained from Oxidized Wool. (Alexander and Hudson, 1954.)

\begin{tabular}{|c|c|c|}
\hline Property & $\alpha$-Keratose & $\gamma$-Keratose \\
\hline solubility & $\begin{array}{l}\text { precipitated from } \\
\text { ammonia extract at } \\
\text { pH c } 4\end{array}$ & $\begin{array}{l}\text { not precipitated } \\
\text { at } \mathrm{pH} 4\end{array}$ \\
\hline appearance & $\begin{array}{l}\text { white sticky curds } \\
\text { of fibrous texture } \\
\text { birefringent as } \\
\text { thread }\end{array}$ & $\begin{array}{l}\text { after drying, } \\
\text { yellow brittle } \\
\text { solid or powder }\end{array}$ \\
\hline mol. weight & $50,000-80,000(?)$ & $\begin{array}{l}3000-10,000 \\
\text { (more poly- } \\
\text { disperse) (?) }\end{array}$ \\
\hline chain length & & \\
\hline $\mathrm{X}$-ray diffraction & $\alpha$-type pattern & $\begin{array}{l}\text { ill-defined } \\
\text { perhaps } \beta \text {-type }\end{array}$ \\
\hline sulphur content* & $\begin{array}{l}2 \cdot 4 \\
2 \cdot 90 \\
2 \cdot 5 \\
1 \cdot 88 \\
1 \cdot 96\end{array}$ & $\begin{array}{l}6 \cdot 1 \dagger \\
-\ddagger \\
6 \cdot 13 \S \\
5 \cdot 84^{* *} \\
4 \cdot 72\end{array}$ \\
\hline $\left.\begin{array}{l}\text { percentage weight } \\
\text { of original sample }\end{array}\right\}$ & $\begin{array}{l}60 \\
56\end{array}$ & $\begin{array}{l}30 \dagger \\
25^{* *}\end{array}$ \\
\hline
\end{tabular}

* S content of original wool averages $3.5 \%$.

$\dagger$ Alexander and Hudson (1954).

$\ddagger$ Earland and Knight (1956).

$\S$ Alexander and Smith (1955).

** Corfield, Robson and Skinner (1958).

- O'Donnell and Thompson, quoted Gillespie (1960) (performic acid).

should be compared with those for " kerateine 2 " (Table 17) and those for the "wool root extract" (Table 14). The higher sulphur content of $\gamma$-keratose shows it to be the more cross-linked and the higher proline is 
of some structural significance in rendering the formation of regular helices more difficult (p. 193).

Other oxidizing reagents which destroy cystine bridges (hydrogen peroxide, chlorine, chlorine peroxide, etc.) have been less used and appear less satisfactory. Das and Speakman (1950) demonstrated a variety of polypeptides in extracts of wool oxidized by chlorine peroxide. The molecular size was of a similar order to that found in sulphide extracts.

Table 16. Analysis of $\alpha$ - And $\gamma$-Keratose (Oxidized WooL). (Corfield et al., 1958.)

(\% of total nitrogen)

\begin{tabular}{|c|c|c|}
\hline Amino acid & $\alpha$-Keratose & $\gamma$-Keratose \\
\hline $\begin{aligned} & \text { glycine } \\
& \downarrow \text { alanine } \\
& \text { valine } \\
& \downarrow \text { leucine } \\
& \text { isoleucine } \\
& \uparrow \text { serine } \\
& \uparrow \text { threonine } \\
& \downarrow \text { phenylalanine } \\
& \downarrow \text { tyrosine } \\
& \uparrow \text { proline } \\
& \downarrow \text { lysine } \\
& \text { arginine } \\
& \text { histidine } \\
& \downarrow \text { aspartic acid } \\
& \downarrow \text { glutamic acid } \\
& \text { ammonia* } \\
& \uparrow \text { cysteic acid }\end{aligned}$ & $\begin{array}{c}5 \cdot 16 \\
4 \cdot 83 \\
3 \cdot 98 \\
7 \cdot 30 \\
2 \cdot 49 \\
6 \cdot 70 \\
3 \cdot 45 \\
1 \cdot 94 \\
2 \cdot 44 \\
2 \cdot 69 \\
4 \cdot 60 \\
20 \cdot 8 \\
1 \cdot 24 \\
6 \cdot 25 \\
10 \cdot 9 \\
10 \cdot 25 \\
3 \cdot 72\end{array}$ & $\begin{array}{c}4 \cdot 97 \\
2 \cdot 58 \\
4 \cdot 15 \\
2 \cdot 55 \\
2 \cdot 14 \\
9 \cdot 70 \\
7 \cdot 46 \\
1 \cdot 15 \\
1 \cdot 41 \\
9 \cdot 85 \\
1 \cdot 03 \\
19 \cdot 0 \\
1 \cdot 57 \\
1 \cdot 79 \\
5 \cdot 87 \\
11 \cdot 05 \\
14 \cdot 5\end{array}$ \\
\hline
\end{tabular}

The arrows indicate an increase $(\uparrow)$ or decrease $(\downarrow)$ of a residue in the $\gamma$-fraction relative to that of the original wool.

* Too high according to Gillespie et al. (1960).

Performic acid, which may have a more specific action, has also attracted some attention (Thompson et al., 1959; Gillespie et al., 1960).

Thioglycollate extracts. The original method of Goddard and Michaelis (1934), who introduced the use of thioglycollic acid as the reducing agent, also pointed to the existence of two different types of protein in extracts of reduced keratin or kerateine. This method is undoubtedly one of the best solubilization techniques since it is in a sense reversible, insoluble disulphide cross-linked powders being obtained by oxidation of kerateines. 
These products are amorphous, the larger structural elements characteristic of the original material not being rebuilt.

This method has been much developed recently by Gillespie and Lennox (1953 and 1955) who have made an extensive study of the electrophoretic behaviour of several kerateines and their alkylated forms (Gillespie, 1960). Their practice is to reduce in thioglycollic acid at $\mathrm{pH}$ 10.5 at $59^{\circ} \mathrm{C}$ to obtain several extracts (extracts $A-E$ ) at this $\mathrm{pH}$, which prove to be very heterogeneous, then to raise the $\mathrm{pH}$ to $12 \cdot 3$ when larger amounts of an electrophoretically pure component "kerateine- 2 " are removed. The extracts are stabilized by blocking the $\mathrm{SH}$ groups by

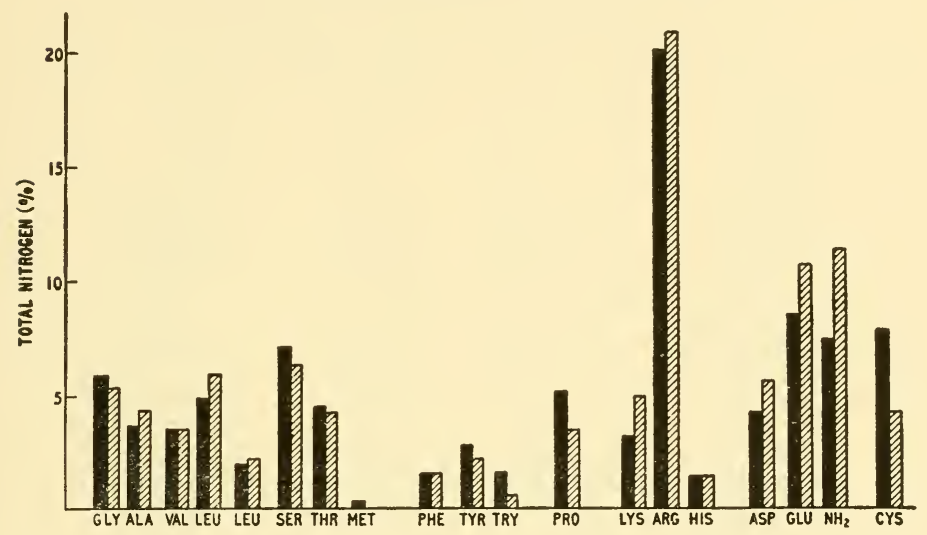

Fig. 100. Comparison of the amino acid composition of a keratin derivative (stippled) with that of merino wool from which it was extracted. Figures represent percentages of total nitrogen. Reconstructed from data given by Simmonds (1954).

alkylation. Simmonds and Stell (1956) have analysed these extracts and demonstrated some striking differences in composition between whole wool kerateine- 2 and extract $A$. Extract $A$ is characteristically higher in cystine than kerateine-2. Figure 100 permits a visual comparison between the composition of kerateine- 2 and the wool from which it was obtained and Table 17 shows the amino acid composition of the extract.

At $50{ }^{\circ} \mathrm{C} 65 \%$ of wool can be dissolved in $0.1 \mathrm{M}$ thioglycollate at $\mathrm{pH} 12.6$ and seven components can be detected electrophoretically. The minor components can be removed by five $20 \mathrm{~min}$ extractions at a lower $\mathrm{pH}$ $(10 \cdot 5)$ leaving the residue from which the major component $(41 \%$ of wool) can be obtained by a further extraction at $\mathrm{pH} 12 \cdot 3$ (kerateine-2). 
In these extractions a number of processes are involved, the rates of some of which are diffusion controlled, the limiting step being either the ingress of the reagent on the egress of the dissolved protein. These rates differ in the two segments ( $o$ and $p$, see p. 268) of the fibre and probably the $o$-segment contributes most of the extracted protein.

The analysis of kerateine-2 by Simmonds ('Table 18) shows that relative to the original wool it contains: increased amounts of the di-amino acids,

Table 17. Amino Acid Composition of Whole Wool and Various Fractions. (Simmonds and Steel, 1956.)

(Amino acid-N as per cent total-N).

\begin{tabular}{|c|c|c|c|}
\hline Amino acid & Extract $A$ & Kerateine-2 & Whole wool \\
\hline glycine & 12.09 & $5 \cdot 27$ & $5 \cdot 80$ \\
\hline alanine & $2 \cdot 75$ & $4 \cdot 22$ & $3 \cdot 51$ \\
\hline valine & 3.47 & $3 \cdot 55$ & $3 \cdot 57$ \\
\hline leucine & 3.92 & $6 \cdot 01$ & $4 \cdot 90$ \\
\hline isoleucine & $1 \cdot 96$ & $2 \cdot 24$ & 1.97 \\
\hline serine & $10 \cdot 04$ & $6 \cdot 47$ & $7 \cdot 25$ \\
\hline threonine & $5 \cdot 56$ & $4 \cdot 44$ & $4 \cdot 61$ \\
\hline phenylalanine & $2 \cdot 40$ & $1 \cdot 72$ & $1 \cdot 75$ \\
\hline tyrosine & $4 \cdot 49$ & $2 \cdot 46$ & 2.97 \\
\hline proline & $6 \cdot 52$ & $3 \cdot 66$ & $5 \cdot 33$ \\
\hline lysine & $1 \cdot 12$ & $5 \cdot 03$ & $3 \cdot 25$ \\
\hline arginine & $14 \cdot 66$ & $12 \cdot 26$ & $20 \cdot 32$ \\
\hline histidine & 1.49 & $1 \cdot 42$ & $1^{\prime} 46$ \\
\hline aspartic acid & $2 \cdot 91$ & $5 \cdot 68$ & $4 \cdot 24$ \\
\hline glutamic acid & $5 \cdot 70$ & $10 \cdot 82$ & $8 \cdot 58$ \\
\hline amide- $\mathrm{N}$ & $5 \cdot 79$ & $11 \cdot 56$ & $7 \cdot 46$ \\
\hline cystine & $13 \cdot 76$ & $4 \cdot 33$ & $7 \cdot 93$ \\
\hline
\end{tabular}

leucine, lysine and ammonia $\mathrm{N}$, and less of cystine, proline, serine and tryptophane. Harrap's (1955) molecular weight determinations by means of a surface balance suggest a magnitude of 30,000. Table 17 shows the change in composition between the first extract $A$ and the major component "kerateine-2". It is evident from the analyses that $\alpha$-keratose and kerateine- 2 are not derived from precisely the same original fraction but there appears to be some "overlap". There is thus reason to think that numerous separate polypeptides exist in the original wool and that the various solubilization procedures sample these differently. 
Soluble derivatives of feather and other keratins. Feather contains less cystine $(6-8 \%)$ than hair $(16-18 \%)$ or wool $(11-13 \%)$ and is more readily dissolved (Jones and Mecham, 1943). About $80 \%$ dissolves in $10 \mathrm{M}$ urea, with $0 \cdot 1 \mathrm{M}$ mercaptoethanol and $0.2 \mathrm{M}$ lithium chloride and Ward et al. (1946) estimated a molecular weight of 10,000. Ward, High and Lundgren (1946) also examined the protein-detergent complex which is dissolved in bisulphite and sodium alkylbenzenesulphonate. They found a number average molecular weight of $40,000(50 \%$ detergent $)$. Woodin reports the

Table 18. Amino Acid Composition of a Keratin Derivative Extracted From Merino 64's Quality Wool.

(From Simmonds (1958). Reproduced with permission.)

\begin{tabular}{|c|c|c|c|c|}
\hline Amino acid & $\begin{array}{l}\mathrm{Wt} . / 100 \mathrm{~g} \\
\text { dry protein }\end{array}$ & $\begin{array}{l}\text { Wt. residues/ } \\
100 \mathrm{~g}\end{array}$ & $\begin{array}{r}\text { No. of res } \\
\text { per } \mathrm{m} . \\
15,00\end{array}$ & $\begin{array}{l}\text { idues } \\
\text { w. } \\
0\end{array}$ \\
\hline $\begin{array}{l}\text { alanine } \\
\text { arginine } \\
\text { aspartic } \\
\text { amide } \\
\frac{1}{2} \text { cystine } \\
\text { glutamic } \\
\text { glycine } \\
\text { histidine } \\
\text { isoleucine } \\
\text { leucine } \\
\text { lysine } \\
\text { phenylalanine } \\
\text { proline } \\
\text { serine } \\
\text { threonine } \\
\text { tryptophane } \\
\text { tyrosine } \\
\text { valine }\end{array}$ & $\begin{array}{r}4 \cdot 01 \\
9 \cdot 72 \\
7 \cdot 81 \\
2 \cdot 02 \\
5 \cdot 67 \\
18 \cdot 79 \\
4 \cdot 14 \\
0 \cdot 77 \\
3 \cdot 21 \\
8 \cdot 54 \\
3 \cdot 70 \\
3 \cdot 86 \\
4 \cdot 59 \\
7 \cdot 62 \\
6 \cdot 59 \\
0 \cdot 79 \\
4 \cdot 50 \\
4 \cdot 76\end{array}$ & $\begin{array}{r}3 \cdot 20 \\
8 \cdot 71 \\
6 \cdot 75 \\
2 \cdot 02 \\
4 \cdot 82 \\
16 \cdot 49 \\
3 \cdot 15 \\
0 \cdot 68 \\
2 \cdot 77 \\
7 \cdot 37 \\
3 \cdot 24 \\
3 \cdot 44 \\
3 \cdot 87 \\
6 \cdot 33 \\
5 \cdot 59 \\
0 \cdot 72 \\
4 \cdot 05 \\
4 \cdot 03\end{array}$ & $\begin{array}{r}6 \cdot 75 \simeq \\
8 \cdot 19 \\
8 \cdot 81 \\
18 \cdot 90 \\
14 \cdot 16 \\
19 \cdot 16 \\
8 \cdot 29 \\
0 \cdot 75 \\
3 \cdot 68 \\
9 \cdot 78 \\
3 \cdot 80 \\
2 \cdot 78 \\
5 \cdot 98 \\
10 \cdot 89 \\
8 \cdot 26 \\
0 \cdot 58 \\
3 \cdot 73 \\
6 \cdot 10\end{array}$ & $\begin{array}{r}7 \\
8 \\
9 \\
19 \\
14 \\
19 \\
8 \\
1 \\
4 \\
10 \\
4 \\
3 \\
6 \\
11 \\
8 \\
1 \\
4 \\
6\end{array}$ \\
\hline
\end{tabular}

isolation of more definite units with molecular weights of the order of 10,000 and of a considerable asymmetry (1954a and b). See also p. 163.

Apart from the work of Jones and Mecham (1943) little effort has been made to examine the other hard keratins.

There is still, in spite of recent efforts, particularly on the part of the Australian group, much that remains to be explained in the results obtained from the various solubilized keratin proteins. In their detailed study in which viscosity, sedimentation and diffusion measurements were made on both oxidized and reduced products, O'Donnell and Woods (1956a and b) 
showed that all these preparations were more polydisperse and unstable than had been previously described. They concluded that possibly the solutions contain monomers in equilibrium with aggregates and that there may be complicating factors in changes of shape and solvation under various conditions. Further, in most cases some peptide bonds are broken as well as disulphide bonds and special precautions must be taken to prevent further hydrolysis with time. Moreover, most solutions show, superimposed on these changes, others due to a progressive aggregation of material with standing. They are not hopeful that the various effects due to aggregation and disaggregation, hydrolysis, changes in shape and solvation can be distinguished. In later articles, Woods (1952) and Gillespie et al. (1960) take a somewhat gloomy view of the possibility of further analysing these solutions, since apart from the actual experimental difficulties, the theoretical interpretation of the results in such systems is also obscure. Nevertheless when the whole of the results obtained from solubilized wool keratin is reviewed it is clear that several significant conclusions emerge:

(a) It is possible to extract from wool quantities (50-60\% of weight) of an $\alpha$-type, fibre-forming protein which are low in cystine and have other significant departures in composition from the original wool. This may be termed the $\alpha$-component; its oxidized form is $\alpha$-keratose with a molecular weight of the order of 50,000-80,000. Kerateine- 2 appears to be a related product. The actual analyses in part support this view.

(b) In addition to the $\alpha$-component, other less well-defined, probably heterogeneous polypeptides, of smaller molecular weight $(3000-10,000)$ and higher in cystine, can be extracted. These are, in their reduced form, contained in part in Lennox and Gillespie's $A-E$ extracts and, when oxidized, Alexander's $\gamma$-keratose. These polypeptides show little tendency to form fibres.

(c) These various polypeptides may be linked by oxidation of their thiol groups to reform higher polymers and insoluble, partly-synthetic keratins.

(d) Even in the absence of the possibility of disulphide cross-linking they still exhibit a marked potentiality for aggregation. 'This property probably arises from the multiple possibilities of interaction between the rich sidechain population of the keratin polypeptides, and may well be of importance in the formation of the initial fibrous aggregates in the follicle prior to the formation of disulphide bonds.

The similarities between $\alpha$-keratose, kerateine- 2 and Lindley's (1947) cetylsulphonic acid (CSA)-soluble extract on the one hand and $\gamma$-keratose and the CSA-insoluble fraction on the other, have also been pointed out by Earland and Wiseman (1959) and are brought out in their table (Table 19). The CSA method is effective although the disulphide bonds remain largely intact. 
Table 19.* The Amino Acid Composition of Hydrolysates of Fractions FROM MERINO 64's WOOL. $\dagger$

\begin{tabular}{|c|c|c|c|c|c|c|c|}
\hline Amino acid & Who & wool & $\alpha$-Keratose & $\begin{array}{l}\text { CSA- } \\
\text { soluble }\end{array}$ & Kerateine-2 & $\gamma$-Keratose & $\begin{array}{l}\text { CSA- } \\
\text { insolubl }\end{array}$ \\
\hline $\begin{array}{l}\text { alanine } \\
\text { amide-N } \\
\text { arginine } \\
\text { aspartic acid } \\
\text { cystine } \\
\text { glutamic acid } \\
\text { glycine } \\
\text { histidine } \\
\text { isoleucine } \\
\text { leucine } \\
\text { lysine } \\
\text { phenylalanine } \\
\text { proline } \\
\text { serine } \\
\text { threonine } \\
\text { tyrosine } \\
\text { valine }\end{array}$ & $\begin{array}{r}3 \cdot 51 \\
7 \cdot 46 \\
20 \cdot 32 \\
4 \cdot 24 \\
7 \cdot 93 \\
8 \cdot 58 \\
5 \cdot 80 \\
1 \cdot 46 \\
1 \cdot 97 \\
4 \cdot 90 \\
3 \cdot 25 \\
1 \cdot 75 \\
5 \cdot 33 \\
7 \cdot 25 \\
4 \cdot 61 \\
2 \cdot 97 \\
3 \cdot 57\end{array}$ & 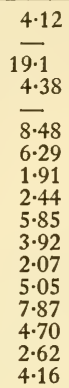 & $\begin{array}{c}4 \cdot 83 \\
10 \cdot 25 \\
20 \cdot 8 \\
6 \cdot 25 \\
3 \cdot 72 \\
10 \cdot 9 \\
5 \cdot 16 \\
1 \cdot 24 \\
2 \cdot 49 \\
7 \cdot 30 \\
4 \cdot 60 \\
1 \cdot 94 \\
2 \cdot 69 \\
6 \cdot 70 \\
3 \cdot 45 \\
2 \cdot 44 \\
3 \cdot 98\end{array}$ & $\begin{array}{l}4 \cdot 0 \\
Z \\
\overline{5 \cdot 20} \\
= \\
\bar{Z} \\
\overline{9 \cdot 2} \\
\overline{1 \cdot 7} \\
\frac{1 \cdot 0}{\bar{Z}} \\
2 \cdot 0 \\
4 \cdot 0\end{array}$ & $\begin{array}{r}4 \cdot 22 \\
11 \cdot 56 \\
21 \cdot 12 \\
5 \cdot 68 \\
4 \cdot 33 \\
10 \cdot 82 \\
5 \cdot 27 \\
1 \cdot 42 \\
2 \cdot 24 \\
6 \cdot 01 \\
5 \cdot 03 \\
1 \cdot 72 \\
3 \cdot 66 \\
6 \cdot 47 \\
4 \cdot 44 \\
2 \cdot 46 \\
3 \cdot 55\end{array}$ & $\begin{array}{c}2 \cdot 58 \\
11 \cdot 05 \\
19 \cdot 0 \\
1 \cdot 79 \\
14 \cdot 5 \\
5 \cdot 87 \\
4 \cdot 97 \\
1 \cdot 57 \\
2 \cdot 14 \\
2 \cdot 55 \\
1 \cdot 03 \\
1 \cdot 15 \\
9 \cdot 85 \\
9 \cdot 70 \\
7 \cdot 46 \\
1 \cdot 41 \\
4 \cdot 15\end{array}$ & $\begin{array}{l}\frac{3 \cdot 3}{15 \cdot 5} \\
\overline{20 \cdot 30} \\
\bar{Z} \\
\bar{Z} \\
3 \cdot 6 \\
\overline{0 \cdot 70} \\
7 \cdot 6 \\
\overline{1} \\
\frac{1 \cdot 3}{3 \cdot 5}\end{array}$ \\
\hline
\end{tabular}

* per cent $\mathrm{N}$ of total-N.

$\dagger$ Earland and Wiseman (1959).

\section{The location of the cystine residues}

The evidence, which has been reviewed up to this point, is adequate to establish that during the later stages of the process of keratinization the thiol groups (SH) of cysteine residues in some polypeptides are oxidized to yield cystine bridges or disulphide cross-linkages (-S-S-). There are, however, two different views as to the location of these cross-linkages. The simpler view is that keratin is a single uniform protein of more-or-less definite composition and that half cystine residues are distributed along the component polypeptide chains in a definite manner as are the residues (Fig. 101) of other proteins. In a physicochemical sense the resulting cross-linked system is closely analogous to the artificial, three-dimensionally cross-linked polymers, such as vulcanized rubber, to which it is often likened. This picture gives an explanation of the major facts: the stability, insolubility and the sensitivity to disulphide rupture. It is compatible with the evidence that the sulphur enters after the establishment of the fibrous structure, if we assume that the original polypeptides contain less cysteine and that other residues (e.g. serine) are later converted into cysteine residues by a topochemical reaction and then cross-linked. This view of the location of the cystine is widely accepted and is adequate for the interpretation of most physical and chemical experiments on intact material.

The second view tries to take into account a number of other observations which suggest that keratin is not a uniform material at the 
macromolecular level, that in fact it consists of two components: one of a fibrous, relatively-crystalline character, the other less well-organized, and containing the larger part of the cystine residues.

This view was implicit in the earlier X-ray work (Astbury and Woods, 1933; Astbury, 1943) which showed clearly that the crystalline fraction of keratin fibres, as judged by the persistence of the $\alpha$-pattern, was unaltered by a variety of chemical treatments which involved the disulphide bridges and the acid and basic side chains of the protein. It was recognized that

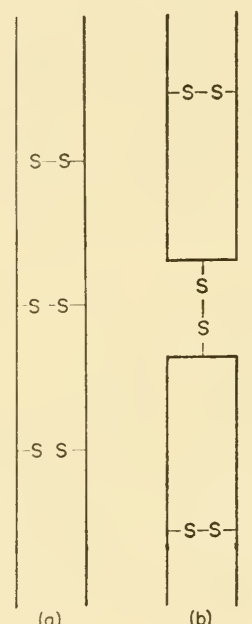

FIG. 101. The classical view of the location of disulphide bonds $\left(\mathrm{S}_{2}\right)$ as cross-linkage between polypeptide chains. In (a) all the linkages are shown as side chains. In (b) the possibility of the linkage occurring in the main chain direction is indicated.

most of these reactions must take place in the " non-crystalline" regions. The size of the various reflections of the X-ray diagram and the greater strength of reflections arising from planes parallel to the fibre axis showed that the crystallites were small ( $<100 \AA$ wide), long and thin with their long axis parallel to the fibre axis. They may well be identical with the filaments seen in electron micrographs.

More direct, morphological evidence was later obtained by examining fragments of disintegrated fibres. A system of filaments (microfibrils) embedded in an amorphous matrix was proposed by Farrant, Rees and 
Mercer (1947) to account for the appearance of fragments of reduced and ethylated wool fibres produced by enzymatic digestion.

The analysis of " solutions" of keratin was not at first productive but with the introduction of peracetic acid as the agent for breaking disulphide bonds (p. 238) clear-cut results were obtained. The separation of a fibreforming $\alpha$-component and a non-fibrous sulphur-rich $\gamma$-component from solutions of oxidized wool led Alexander and Hudson in their book (1954) to propose unambiguously a crystallite-plus-matrix model for keratin.

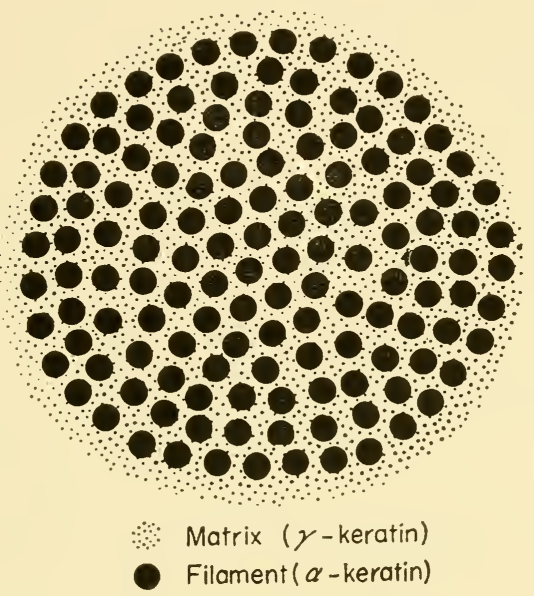

Fig. 102. The "filament plus matrix" model for a fibril of fibrous keratin. The filaments consist of bundles of $\alpha$-helices (see p. 183) and are embedded in an amorphous matrix with a higher cystine content.

See also Fig. 98, p. 225. (Birbeck and Mercer, 1957.)

Strong, directly-morphological evidence from intact material was later forthcoming when it became possible to examine electron-microscopically (see p. 223) sections of developing hairs. Birbeck and Mercer (1957) concluded from the pattern of osmium deposition in the hair fibrils (Plate 15) the existence of a system of filame.ts embedded in a sulphur-rich matrix (Fig. 102). This was confirmed in fully-hardened hair, wool (Plate 16) and quill by Rogers (1959). Brody (1959) (Plate 17) demonstrated a similar pattern in the epidermis.

The existence of a filamentous system prior to, and distinct from, the finally stabilized keratin, is demonstrated by X-ray methods (p. 211), by 
histochemistry (p. 214), by chemical (p. 236) and mechanical separation (p. 231), followed in some cases by analyses proving a low-sulphur content. Alexander has also pointed out that the existence of several distinct methods of producing supercontraction, which are discussed on p. 259 et seq., also shows that keratin is divided into cystine-stabilized and hydrogenbond-stabilized fractions (1951).

A summary of the outstanding properties of the two components of what may be termed the filament-plus-matrix model (Fig. 102) is as follows:

\section{(a) Filaments ( $\alpha$-component)}

Dimensions. About $60-80 \AA$ diameter; length indefinite, at least $>2000$ $\AA$ in wool and hair.

Internal structure. Composed of $\alpha$-type fibrous protein (see Chapter 5) (Figs. 77, 78 and 79).

Composition. Cystine content is less than whole keratin (Table 15). Serine, threonine, proline lower, acidic residues higher than whole fibre.

Macromolecular composition. May contain a primary peptide of molecular weight between $50,000-80,000$. Its relation to the filament is obscure.

\section{(b) Matrix ( $\gamma$-COMPONENT)}

Amorphous, shorter chain polypeptides having a higher content of cystine, serine, threonine and proline.

Molecular structure. Probably not $\alpha$-type, may be irregular. The ratios of $\alpha: \gamma$ is of the order of $1: 1$ in wool and hair to judge from electron micrographs. From the analysis of extracts of oxidized wool it would appear that the $\alpha$-component may amount to $60 \%$ of the total.

It seems reasonable to suppose that all the hard keratins will possess a similar fine structure (Rudall, 1952). Fibrils essentially similar to those noted in wool and hair have been demonstrated in feather both in the germinal layers of the follicle and in the fully keratinized material (Mercer, 1958; Mercer, unpub.). Porcupine quill possesses a most regular structure (Rogers, 1959a and b). In the present state of our knowledge it is possible to suppose that the $\gamma$-matrix protein is an entirely new protein which the cells of the tissue commence to make in the keratinization zone in response to the altered conditions prevailing there and that it secondarily deposits on the bundles of $\alpha$-filaments to form a cementing matrix. Or it may be thought to be synthesized directly on the filaments establishing an actual peptide linkage with existing polypeptides. The first alternative would seem the more probable since the two proteins are separable when the cystine links are severed. It is suggestive that, in the hair cuticle cells at this same level, a very similar amorphous high-sulphur keratin is also synthesized.

With the soft keratin of the epidermis the situation is by no means so clear. This material, composed largely of transformed keratohyalin, has an 
irregular fibrous texture (Fig. 99), in which a definite fibrillar and nonfibrillar component can be discerned. Epidermin, the precursor of skin keratin, shows several components in the analytical ultracentrifuge, none of which can be related with certainty to the $\alpha$ - and $\gamma$-components of hair. Moreover, the lower sulphur content, the sudden nature of the change from amorphous keratohyalin (or trichohyalin) granules into the fibrous form and its consolidation as a resistant birefringent fibrous layer, all are in some contrast with the consolidation of the prefabricated fibrils of $\alpha$-keratin in the hair. If a $\gamma$-component exists it must be smaller in amount and perhaps not so easily distinguished on account of the lesser regularity of the structure. Brody $(1959 \mathrm{~b})$ is of the opinion that a $\gamma$-component is derived from keratohyalin, but the evidence that the granules contain cysteine is not good (see p. 230). Nevertheless it is possible to conclude that in most instances the vertebrate keratins are duplex structures produced by embedding a primary system of filaments (usually $\alpha$-type but, as feathers and claws show, a $\beta$-type is possible) in a matrix of short-chain polypeptides rich in cysteine residues whose conversion into cystine residues stabilizes the formation.

The concept of keratinization as a process subsequent to a primary process of fibril formation harmonizes very well with the broader viewpoint which presents the $\alpha$-type proteins as the common intracellular fibre type which, by secondary modifications, is adapted to a variety of functions.

\section{Physicochemical properties and keratinization}

A great deal has been learned concerning the stabilizing bonds produced during keratinization by the study of the dependence of the mechanical and dimensional properties of the hardened tissue on the physicochemical environment. For the study of these " mechanochemical " properties, to adopt Speakman's useful expression, the tissue chosen needs to have a convenient form, and in fact the greater part of these experiments have been made on hair, wool, feather and horn. Since information concerning the properties of wool and hair is of value to both the textile and the cosmetic industries, the amount of work carried out is enormous and it would be impossible to review it here. Reference may be made to the book by Alexander and Hudson (1954), the review of Ward and Lundgren (1954) and papers by Speakman (q.v.).

When stretched under well-defined conditions hairs yield characteristic stress-strain curves (Fig. 70). Their dependence on temperature (Fig. 104) and water content (Fig. 103) shows that the effect of water and a rise in temperature is to loosen those internal bonds which are opposing extension and to reduce the work of extension. Various theoretical attempts have been made to explain the shape of the stress-strain curve. As already described in Chapter V (p. 172) the normal curve shows several 
steps (Fig. 70) which according to the theory of Astbury and Woods (1933) mark the successive extension of "phases " differing in the force required to stretch them (see p. 174). American workers (Burte and Halsey, 1947), with the complex elastic behaviour of polymers in mind, have been more inclined to regard the curve as being that of a single, uniform cross-linked polymer. No entirely satisfactory quantitative account of the whole range of elastic behaviour has been given, but some success has been obtained in

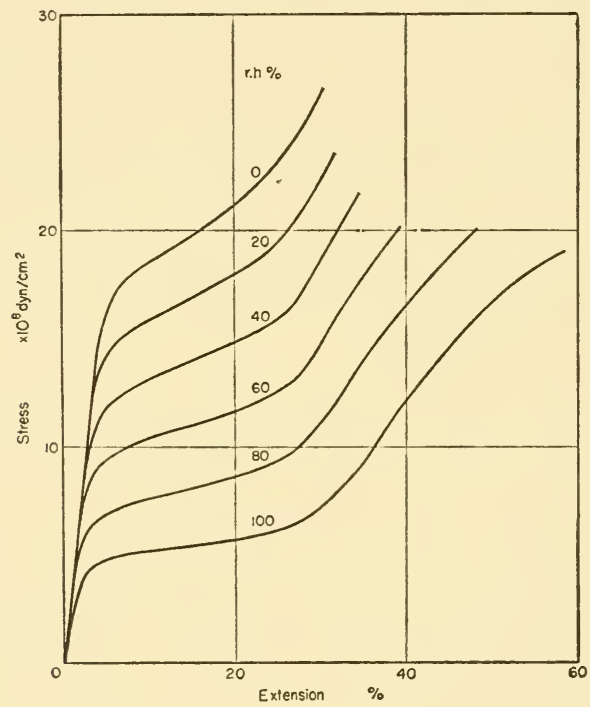

FIG. 103. Influence of relative humidity on the stress-strain curve of wool at $22^{\circ} \mathrm{D}$ with constant rate of loading of $18 \mathrm{~g} / \mathrm{min}$ (Peters and Woods, 1956).

the simpler problem, the description of the elastic behaviour of fibres in which most of the internal molecular restraints have been removed (Burte and Halsey, 1947; Peters and Speakman, 1949).

To assess numerically the effect of a chemical treatment on a keratin fibre, Speakman (1934 and 1947) introduced what may be called the " $30 \%$ work index", which is defined as the ratio of the work required to stretch the treated fibre $30 \%$ to the work required to stretch it $30 \%$ before treatment. The choice of $30 \%$ (or in some instances $20 \%$ ) is based on the experimental fact that the stretching of wool fibres in the range of 
$0-30 \%$ is completely reversible. Beyond this point the fibre may recover its length after stretching, but it is thereafter easier to stretch, showing that irreversible damage has occurred. The smaller the index the greater the reaction between the fibre substance and the reagent.

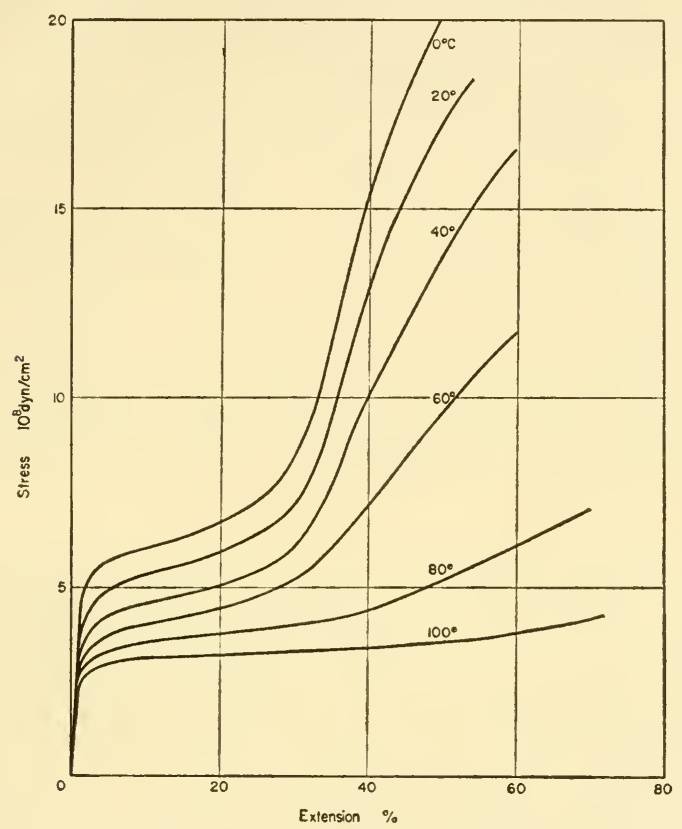

F1G. 104. Stress-strain curves of wool fibres in water at various temperatures. From Peters and Woods (1956) by permission.

\section{Salt Linkages}

All the keratins contain a high proportion of residues of the diamino and dicarboxylic acids, and in wool these are approximately equal in amount (p. 219). In the neutral condition when both types of group are ionized:

$$
\text { P.COO- }{ }^{+} \mathrm{H}_{3} \text { N.P } \quad(\mathrm{P}=\text { polypeptide chain })
$$

there is the possibility of electrostatic attraction between the two which could contribute to the cohesion of the material. This electrostatic force is referred to as a " salt-link". Experimentally the probable existence of such salt link combinations is demonstrated by numerous experiments made by 
Speakman and his collaborators. The dependence of the $30 \%$ index on $\mathrm{pH}$ is shown in Fig. 106 (Speakman and Hirst, 1933; Speakman, 1934) reveals a weakening of the fibre towards the extremes of $\mathrm{pH}$ suggestive of "hydrolysis" of salt-linkages, that is a weakening of electrical attraction following the discharge of ionized groups by combination with $\mathrm{H}^{+}$or $\mathrm{OH}^{-}$. Speakman and Hirst (1933), showed that the reduction in work in acid solutions was proportional to the amount of acid combined with the fibre, i.e. proportional to the number of salt-links put out of action. The amount of acid or base bound as a function of $\mathrm{pH}$ is shown in Fig. 105 and it may be compared to the work-reduction in Fig. 106.

There is room for some difference in opinion over the theoretical interpretation of these findings or even whether the term "salt-link" is really appropriate. Nevertheless, from the point of view of the effects of keratinization, it is significant that the reduction in cohesion of the fibre and its swelling (and its combination with acids and bases which is interrelated with these) are minimal in a broad range of $\mathrm{pH}$ values including neutrality. The material is in a sense "buffered " against environmental changes in the range of variables which, biologically speaking, might well be encountered by the integument.

\section{Disulphide Bonds}

In the literature dealing with the interpretation of the elastic properties of wool the relative importance of disulphide bonds and hydrogen bonds has been much debated. Speakman in particular insisted on the predominant role of the sulphur bridge; Alexander (1951), Elöd and Zahn (1944 and 1949) principally did much to direct attention to the importance of hydrogen bonding. Speakman and collaborators (Speakman, 1934, 1936 and 1947) have demonstrated both the weakening of fibres when the disulphide bond is broken and their recovery when it is reformed.

The existence of disulphide bonds and their effect on fibre properties is revealed most clearly by the series of elegant experiments of Harris and his collaborators (Patterson et al., 1941; Harris and Brown, 1946) who used thioglycollic acid to reduce cystine bridges:

$$
\begin{aligned}
\mathrm{P}-\mathrm{S}-\mathrm{S}-\mathrm{P}+2 \mathrm{HS} . \mathrm{CH}_{2} \cdot \mathrm{COOH} \rightarrow 2 \mathrm{P} . \mathrm{SH}+ & \left.\right|_{\mathrm{S}_{2} \cdot \mathrm{CH}_{2} \cdot \mathrm{COOH}} ^{\mathrm{CH}_{2} \mathrm{COOH}}
\end{aligned}
$$

and investigated not only the properties of the reduced fibres, but also those of the fibres in which the cross-linkages had been rebuilt (a) or blocked (b) by reacting the reduced thiols with alkylhalides:

$$
\begin{aligned}
& 2 \mathrm{P} . \mathrm{SH}+\mathrm{Br} \cdot \mathrm{CH}_{2} \mathrm{CH}_{2} \mathrm{Br} \rightarrow \text { P.S. } \mathrm{CH}_{2} \cdot \mathrm{CH}_{2} \cdot \mathrm{S} . \mathrm{P}+2 \mathrm{HBr} \\
& \text { P.SH }+\mathrm{Br} \cdot \mathrm{CH}_{2} \cdot \mathrm{CH}_{3} \rightarrow \text { P.S. } \mathrm{CH}_{2} \mathrm{CH}_{3}+\mathrm{HBr}
\end{aligned}
$$




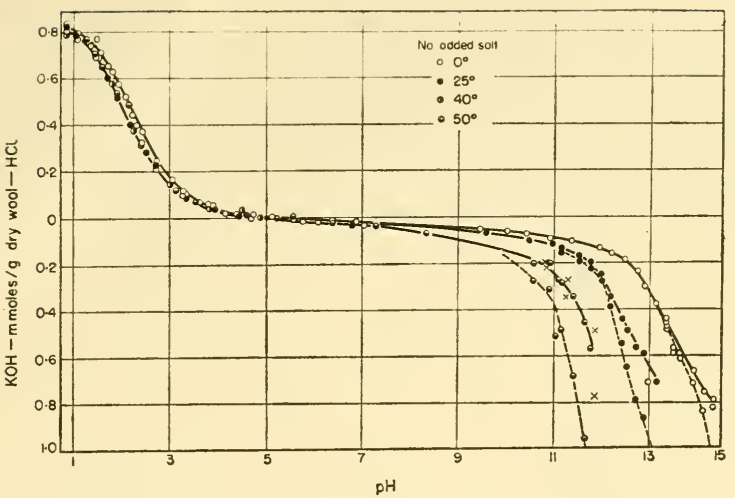

FIG. 105. Combination of wool with hydrochloric acid and with potassium hydroxide as a function of $\mathrm{pH}$ and temperature in the absence of added salt. (The dotted lines show the correction by Harris and Rutherford for the reaction of alkali with cystine.) (Reproduced by permission.)

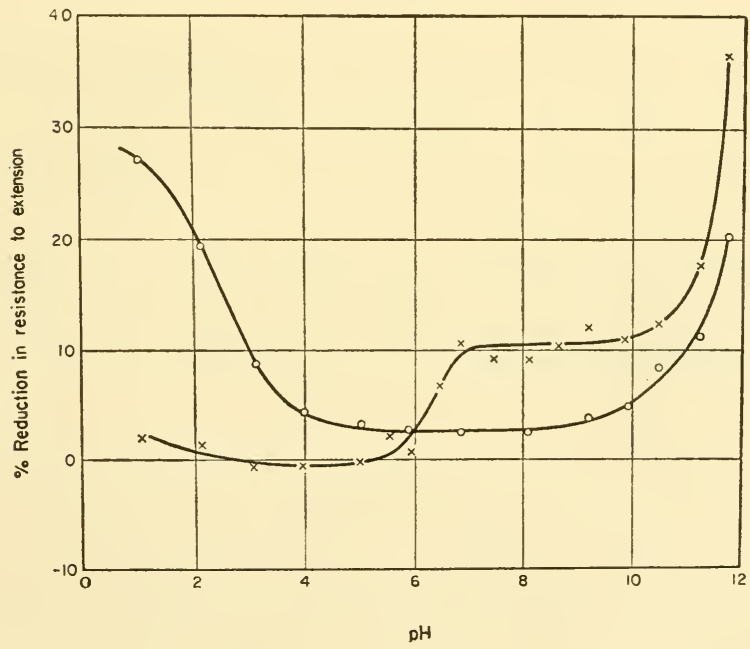

Fig. 106. The effect of $\mathrm{pH}$ on the reduction in work to stretch a fibre by $30 \%$ for normal $(O)$ and deaminated hair $(X)$. (Speakman, 1934.) Peters and Woods, 1956. (Reproduced by permission.) 
The effect of the reduction of the disulphide bonds on the work of extension is shown in Fig. 107. Reduced wool or " blocked wool " (reaction (b)) was found to be more easily stretched, more readily dissolved and less resistant to enzymatic digestion than normal (Geiger et al., 1941 and 1942). Resistance was restored when new cross-linkages were introduced by alkylation (reaction (a)).

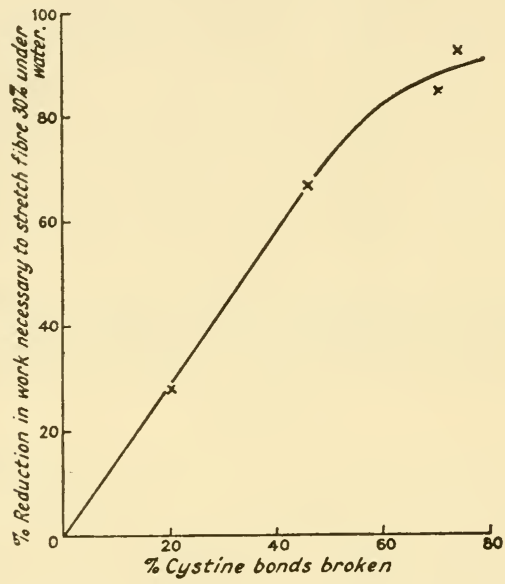

Fig. 107. Relationship between wet strength of wool fibres and the number of disulphide bonds (Harris and Brown, 1946).

These reactions form the basis of current hair-waving treatments in which the hair, softened by thioglycollate reduction, is deformed into the desired shape and held there until cross-linkages have reformed by oxidation (McDonough, 1952).

\section{HydRogen BoNdS}

Even when the greater part of the disulphide bonds are broken, the strength of dry fibres is not greatly reduced and the fibre form may be retained when the fibre is placed in solutions of $\mathrm{pH}<9$ at normal temperature. Above this $\mathrm{pH}$ much of the keratin may enter solution (see p. 240). The stabilization persisting after reduction is attributed to hydrogen bonding, the presence of which is directly revealed by infra-red absorption spectra (Chapter V, p. 196). When steps are taken to break the hydrogen bonding in a previously-reduced fibre, a characteristic contraction in length occurs. Following Astbury and Woods, this is usually called 
supercontraction since it may follow also on the ordinary recovery of length occurring when stretched fibres are released (Fig. 108).

Supercontraction is a property that the keratin fibres share with other systems containing long oriented molecular chains. The most probable configuration of a long, free, flexible molecular chain in solution is a random coil, for in this condition the entropy is a maximum. However, less probable configurations may be assumed as a result of the molecule interacting with other molecules or with other parts of itself. These inter-

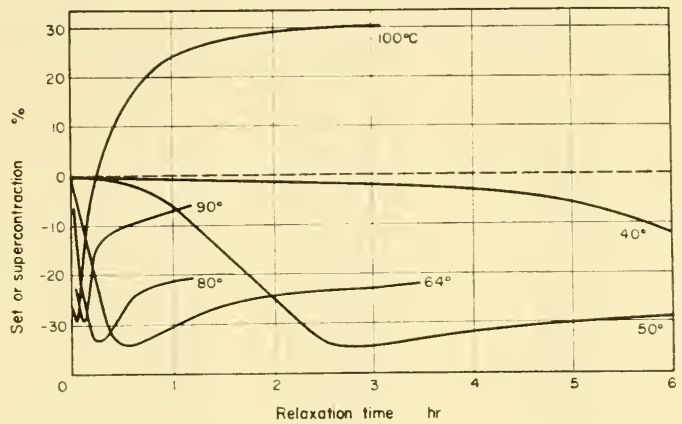

Fig. 108. Recovery of Cotswold wool in steam, after being held at $50 \%$ extension in steam or hot water for given times of relaxation. Times to be multiplied by 3 for the $40 \%$ (Astbury and Woods, 1933).

actions provide the " forces of crystallization " and thermodynamically the reduction in internal energy resulting from these interactions opposes the randomizing effects of entropy. A system of long, flexible molecules with very weak interaction between the chains may constitute a rubber. The form of the molecules and of the bulk specimen in the rubbery state is largely controlled by the entropy factor, i.e. the contractile force opposing extension arises from the tendency of the extended molecules to return to a shorter, more probable configuration.

Among the protein fibres a large contribution to the internal energy factor is provided by hydrogen bonding which can occur between the numerous peptide groups (Fig. 109). In general terms, where steric conditions are favourable and the close packing of portions of many chains is possible, a crystallite stabilized by multiple $\mathrm{H}$-bonds is formed. We may say then that such systems are stabilized by "crystallite cross-links" to distinguish the condition from the single-chain covalent linkages due to cystine bridges. Some protein fibres, collagen and silk for example, owe their insolubility and stability almost entirely to crystallite cross-linking. 
The destruction of the crystallites in such a system, produced either by a solvent able to penetrate the crystallites or by a rise in temperature sufficient to "melt" them, releases the molecular chains which may then assume a more probable, less oriented, and shorter, configuration. This change may be visible as a change in shape of an oriented specimen, and is accompanied by the loss of other signs of orientation, such as birefringence and the fibre-type X-ray pattern (Fig. 109). The contracted

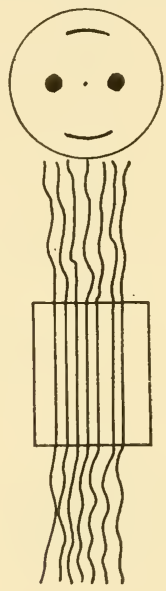

(a)
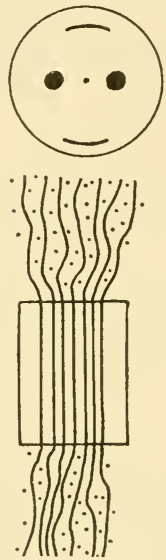

(b)
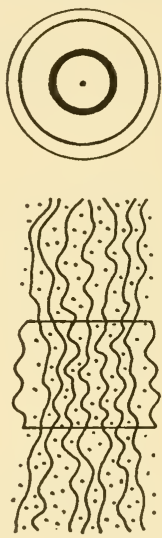

(c)

Fig. 109. To illustrate the relation between the X-ray pattern given by a crystallite in an unswollen fibre (a); a fibre (b) in which swelling molecules have penetrated only the amorphous phase and the X-ray pattern is unaffected and (c) a fibre in which molecules have penetrated the crystallite disorienting the molecular arrangement to give a non-oriented X-ray pattern and causing a further shortening (supercontraction) of the fibre length.

material may also acquire rubber-like properties. Perhaps the most striking example of this is provided by collagen fibres which have less interchain bonding than keratin and may shorten to as much as one-third of their original length when heated above a well-defined temperature in water. This "contraction temperature" is the point at which the crystallites disperse or "melt"; it is not as well defined in the keratins. On cooling, contracted collagen spontaneously resumes its original length and the crystallites reform. Clearly the normal triple helical structure is adequately stabilized by internal energy considerations at normal temperatures. 
The temperature at which contraction commences is a useful measure of the degree of stabilization of a fibre. Most hard keratins require a temperature above $100^{\circ} \mathrm{C}$ in water. It is lowered by treatments which reduce the degree of cross-linking ( $\mathrm{H}$ bonds, covalent bonds or saltlinkages) and the temperature difference is a measure of the reduction in internal stabilization. For comparison of different keratins it is more convenient experimentally to use solutions of substances (phenol, formamide, etc.) which weaken the internal cohesion due to hydrogen bonding and bring the contraction-temperature below $100^{\circ} \mathrm{C}$. Elöd and Zahn (1946 and 1949) and Stoves (1947) examined various hairs in this way and found that the temperature required to initiate contraction increased with the cystine content and the fibre diameter.

The careful analysis of the elastic behaviour of keratin fibres by Bull (Bull and Gutman, 1944; Bull, 1945) and Woods (1946) has thrown much light on the relation between the internal energy factors tending to stabilize the oriented structure and the randomizing entropy factors. It is shown in thermodynamics that, if the tension on a specimen is $P$ and its length $L$, then for reversible changes:

$$
P=\left(\frac{\delta U}{\delta L}\right)_{T}-T\left(\frac{\delta S}{\delta L}\right)_{T}=\left(\frac{\delta U}{\delta L}\right)_{T}+T\left(\frac{\delta P}{\delta T}\right)_{L}
$$

Where $U$ is the internal energy, $S$ the entropy and $T$ the temperature.

In these equations the tension $P$ is expressed as the sum of the two terms: one $P u$ depending on the internal energy changes and the other $P s$ depending on the change in entropy when $L$ is increased. Since the latter can be measured by observing the temperature coefficient of the tension at constant length $(\mathrm{d} P / \mathrm{d} T)_{L}$, the two can be determined separately.

The dominant factor controlling the length was shown by Woods and by Bull to be the internal energy term $(P u)$; even in swollen and relaxed fibres the entropy term remains small but becomes more important in supercontracted fibres in which the crystallites are dispersed and the molecular arrangement more randomized.

The long-range reversible elasticity shown by hairs should not be thought of as a characteristic of keratin per se. It is rather a characteristic of the $\alpha$-type proteins and is related to the $\alpha$-type molecular structure. The definitive feature of a keratin is the stabilization based on cystine crosslinking and this chemical device may be used to stabilize proteins of a different structure and quite different elastic potentialities (see p. 24). Feather cells, for example, are similar in fine structure to the cortical cells of hair and the fibrillar system is also stabilized by cystine cross-linking; the keratin is, however, of $\beta$-type which permits of extensions of only a few per cent. This lack of long range extensibility was one of the direct indications which led Astbury and Marwick (1932) to the view that the 
feather structure was based on more or less fully-extended chains. Somewhat surprisingly the well-oriented $\beta$-configuration of feather does not supercontract in solvents which both reduce disulphide bonds and break $\mathrm{H}$-bonds although it swells and partly dissolves. When regenerated in fibre form, the amorphous threads in water are rubber-like (Ward et al., 1946).

Meyer et al. $(1949,1952)$ have criticized the broad picture developed by Astbury and Woods and asserted that the long range elasticity is really rubber-like. This criticism seems to be based on a misconception. Meyer's experiments were carried out on hairs which were strongly swollen and reduced, i.e. on hairs in which special steps had been taken to reduce the internal energy factors, and under these conditions the entropy term may be expected to predominate. The theories of Astbury and Woods were, in fact, developed to give an account of the elastic behaviour of fibres in which the internal constraints were effective. In their theory the swollen fibre with its reduced interchain bonding and a relative absence of covalent cross-linking should be essentially rubber-like. In fact this condition has been demonstrated experimentally by Meyer and Haselbach (1949).

\section{Molecular Configuration in the Supercontracted State}

The molecular mechanism involved in supercontraction is complex. In terms of the earlier interpretation of Astbury (1933) the shortening in length (about $30 \%$ ) is due to a further folding of the polypeptide chains into a shorter configuration and this view still prevails although no precise picture of how an $\alpha$-helix shortens has been proposed.

Strongly supercontracted fibres usually yield an unoriented $\beta$-type $\mathrm{X}$-ray pattern which is not very helpful. In some instances an oriented $\alpha$-pattern may persist in fibres shortened by $20 \%$, showing that the contraction is occurring in the non-crystalline phase; in others all signs of pattern may vanish. These findings suggest that the mechanism of contraction may not always be the same. The numerous chemical treatments which produce some degree of supercontraction have in common only the property of reducing interchain bonding.

A classification of types of supercontraction is, however, possible on structural grounds. The simplest situation is perhaps the most drastic, e.g. boiling in solutions of bisulphite, which leads to a disoriented $\beta$-type of configuration (Astbury and Woods, 1933; Whewell and Woods, 1946). The explanation here is that as a result of a chemical weakening of interchain interaction, the main chains, acting as mobile individuals under the influence of thermal agitation, are able to assume a shorter and more random configuration. On drying these randomized chains may in part recrystallize in the $\beta$-configuration.

The second type of supercontraction is that which results from hydrogen 
bond rupture and the dispersion of the crystallites without rupture of disulphide bonds. In this form the X-ray $\alpha$-pattern fades and the contracted fibre shows no wide-angle pattern. Characteristically the contraction may be reversed by washing out the reagent; the crystallites reform, the fibre resumes its original length and the $\alpha$-pattern returns. Examples: supercontraction in cuprammonium solution (Whewell and Woods, 1946), lithium bromide (Alexander, 1951), phenols (Zahn, 1947) and formamide (Elöd and Zahn, 1944). A third type of contraction occurs when the disulphide bond system is destroyed and when the conditions of contraction are not too violent $(<20 \%$ contraction). In this case the $\alpha$-pattern may still be elicited from the contracted fibre. Examples: contractions in dilute caustic soda (Whewell and Woods, 1946) or after peracetic acid oxidation (Alexander, 1951). When conditions are more drastic (higher temperature or longer treatments) the crystallites may be affected and the more strongly-contracted fibre gives a $\beta$-pattern.

Astbury and Woods, Whewell and Woods and Alexander all recognized that these results show that, in a sense, the keratin may be divided into two parts which may be induced to contract more or less independently. This separation can now be understood in terms of the filament-plus-matrix model. Disulphide-bond destruction converts the matrix into a viscous material which facilitates chain movement both in itself and in the filaments. If a limited contraction of the non-crystalline chain segments occurs, the fibre shortens and the $\alpha$-crystallites persist. In the other case, when the disulphide cross-linked matrix is intact while contraction is induced by freeing the chains in the crystallites, a contraction results with crystallite destruction which is reversible, because the over-ruling macromolecular organization is preserved by the cross-linked matrix which envelops the filaments.

Another form of supercontraction is that which leads to the formation of a cross $-\beta$ configuration in the contracted fibre. This may be the case when contraction is produced under mild conditions in reagents which both loosen the matrix by reducing disulphide bonds and also disperse the crystallites by rupturing hydrogen bonds, e.g. strong solutions of urea containing bisulphite (Mercer, 1949a). See Fig. 84 and Plate 2B. The cross- $\beta$ pattern has already been discussed on p. 200 et seq.

\section{The Setting of Hairs}

The study of the important phenomenon of set, by which is meant the more-or-less permanent retention of a deformed state, has shown that the same factors which stabilize a fibre in its natural state are also those which operate to maintain a stretched state or set. A hair which is stretched in water, relaxed and dried will partly retain the stretched length. It quickly returns to its original length in water (Fig. 108) and more rapidly in 
solutions of high or low $\mathrm{pH}$. Evidently this set is being maintained only by salt-bonds and hydrogen bonds (Speakman, 1934; Woods, 1933). A more permanent set is induced by relaxation at higher temperatures under conditions in which the crystallites are transformed into the $\beta$-modification (Astbury and Woods, 1933; Woods, 1933). This type of set can be released by strong solutions of urea (Rudall, 1946), and here therefore $\mathrm{H}$-bonds are obviously the factors stabilizing the $\beta$-crystallites. Permanent set is defined as a set which is not relaxed by prolonged steaming or by solutions which rupture hydrogen bonds, i.e. it is a set which is sustained by covalent cross-linkages analogous to the disulphide bonds which are effective at the original length. These cross-linkages may be reformed disulphide bonds (Speakman, 1933) or bridges introduced between reduced disulphide bonds by dihalides (see p. 252) or linkages apparently formed between $\mathrm{COOH}$ groups and amino groups in steamed fibres (Speakman, 1933).

Taken together all these physicochemical methods provide semiquantitative measures of the contribution of the several cross-links: salt-linkages, disulphide and hydrogen bonds, to the stabilization of the keratinized fibres, which are in good agreement with conclusions reached on other grounds.

\section{Cell membranes in keratinized tissues}

\section{The Membranes and Cellular Adhesion}

Up to this point we have concentrated attention on what happens to the intracellular proteins during keratinization. Other constituents of the cells also undergo changes during keratinization. The cell membranes, in particular, play an important role in maintaining the hardened structure. These membranes and their behaviour during the establishment of tissues have been discussed already in Chapter III; that they are also important after keratinization is proved by many experiments.

Electron microscopy has shown that the fibrils of keratinizing cells are wholly intracellular and that no intercellular connexions composed of fibrils cross from cell to cell binding the mass together. Such "bridges" were often described in earlier works, but their true nature is now better understood. For many histologists who feel that cells must be held together by "string " rather than "sealing wax", they had a strong fascination. In fact cells are stuck together, and for this reason the properties of the adhering surfaces, and of the adhesive, are as important in maintaining the whole formation as the hardened cell contents themselves. This is shown very simply by digesting a tough keratin, such as hair, by means of an enzyme (trypsin) which removes the membranes and cement. The tensile strength falls rapidly (Elöd and Zahn, 1946), and in a few days the fibres drop apart. Examination of the residue by a variety of means 
shows that the keratin itself is unchanged (Mercer et al., 1956); sections examined electron-microscopically show that the bundles of keratinized fibrils are of normal appearance and reveal that the components which have been removed are the cell membrane and intercellular cement. The loss in weight is of the order of only $10 \%$ (Elöd and Zahn, 1946) but it represents the vital links connecting the chains of cells.

A further important characteristic of these altered membranes is that their chemical character is complementary to that of the keratinized protein. That is to say, chemical conditions which soften or dissolve keratin have little effect on the membranes. The strength and weakness of keratin itself lies largely in the disulphide bond which is peculiarly vulnerable to reduction, hydrolysis and oxidation. However, the system of membranes resists these actions to a far greater degree. It is found that, when keratin is dissolved (by the methods described above) the insoluble residue consists largely of membranes (Mercer, 1951 and 1953) (Fig. 112) (Lagermalm et al., 1951).

Considering their biological origin the chemical resistance of these membranes is remarkable. They are not dissolved by the following strong reagents, which include both reducing agents and hydrogen bond breakers: $5 \mathrm{~N}$ caustic soda, $8 \mathrm{M}$ urea, $8 \mathrm{M}$ urea containing thioglycollic acid or sodium bisulphite at $\mathrm{pH} 10$, concentrated formic acid, $10 \%$ sodium sulphide, and aqueous peracetic acid followed by $0 \cdot 1 \mathrm{~N}$ alkali. On the other hand, when not protected by being incorporated in a solid, intact tissue, they are rapidly digested by proteolytic enzymes.

These properties show that, while a protein constituent is certainly present, the resistance cannot be due entirely to hydrogen bonds or disulphide bonds of the type found in keratin. It is perhaps permissible to see that a certain biological advantage is gained by enclosing keratin in small sacs which resist dissolution by precisely those reagents most injurious to their contents.

The little known of the composition of biological membranes (p. 37) does not help to explain the changes which could convert them into the singularly-insoluble form they assume in keratinized tissues. That a protein moiety is present is shown by the dissolution by proteolytic enzyme; the several reports (Corfield et al., 1958) of amino acids found in membrane hydrolysates confirm this. Matoltsy (1957) has reported finding the following amino acids in membranes from human skin: glycine, valine, leucine, isoleucine, serine, threonine, aspartic and glutamic acids, arginine lysine, histidine and methionine. Other analyses indicating protein have been made on the particularly-toughened membrane obtained from Allwörden sacs (p. 267) on wool fibres. Corfield, Robson and Skinner (1958) carried out a complete amino acid determination of the residue remaining after oxidized wool is extracted with ammonia (referred to as 
$\beta$-keratose), which consists largely of membranes (p. 270) and found it to resemble whole wool. The few small differences observed are not sufficient to explain the insolubility. All these analyses must, however, be treated with some reserve unless the absence of unremoved keratin in the membrane preparations is demonstrated.

Since disulphide bonds and hydrogen bonds seem inadequate to explain the insolubility (see above), suggestions have been made that some new bond is present, e.g. a type of tanning linkage as found in melanin or the insect cuticle. It may be significant that melanin granules have a similar chemical resistance to the hardened membranes. Residues consisting largely of membranes have been examined by X-ray diffraction and were found to give only an unidentifiable pattern with some $\beta$-characteristics which, after such a chemical treatment, is of little significance.

\section{The Morphology of the Membranes of Keratinized Tissues}

The special nature of these membranes is made clear by examination of their fine structure.

The intercellular spacing commonly found between cell membranes in normal tissues is about $150 \AA$ wide (p. 41) and a spacing of this order is found between cells in the upper bulb of the hair follicle and in all other germinal tissues. As the cells of the presumptive cortex fill with fibrous keratin, this spacing widens and may reach $400 \AA$. At the same time the outlines of the cells become more wavy, so that the surfaces themselves are more interlocked (Birbeck and Mercer, 1957; Rogers, 1959a and b). In material fixed in osmium tetroxide the membranes are dense and the intermembrane material contains light and dark bands. In the final keratinized cortex these relative differences in density still persist. These observations prove that the amount of intercellular cement is increased during keratinization and that it undergoes some chemical modification.

More elaborate changes associated with membranes are visible in the cuticle and the inner-root sheath (Birbeck and Mercer, 1957a and b; Rogers, 1959a and b). The intercellular space between the cuticle cells also widens and in this instance several dense layers, separated by lighter layers, appear transiently and later fuse to give a single broad dense layer. Between the various cells of the sheath, single broad sheets are deposited (Plate 6B). Again while we have no idea of the chemistry behind these appearances, they show that changes are going on in the intercellular spaces and we may assume they they are related to strengthening the adhesion.

Somewhat similar events occur in skin. The localized "studs" or desmosomes have been described (p. 83). In the granular layers and higher, the contacting cell surfaces may become extremely wave-like producing a very considerable degree of quasi-regular interlocking. The 
intercellular space widens (Fig. 98) and bands are deposited (Odland, 1958; Mercer, 1958; Horstmann and Knoop, 1958; Pillai, 1960). When a cell is finally shed the parting may occur either between the cells, i.e. due to failure of the cement, or in the bulk of the keratin due to its separating into fibrils (Plate 6A).

The purely mechanical consequences of the changes in the geometry of the surfaces of contact of the cells deserve special emphasis. Not only is the area of contact and therefore the total adhesion vastly increased by the formation of wavy surfaces, but the interpenetrating crests may develop to the point where they constitute veritable "press-studs", which after the hardening of their contents, literally lock the cells together. The enhancement of these surface irregularities in the hair may be a consequence simply of the continued addition of filaments to centres near the surfaces of the cells. In the epidermis the numerous desmosomes and their associated fibrils appear to cause the buckling of the membranes (Plate 6A).

\section{The Fate of the Intracellular Apparatus during}

KeRATINIZATION

Much of the intracellular apparatus either consists of membranes or is enclosed in membranes. We have mentioned above that there are reasons for supposing that all these membranes have a similar basic constitution and, it is of interest to note that, during keratinization of the cell, a portion of the cellular apparatus undergoes changes in solubility similar to those affecting the external cell membranes and is found along with these among the resistant residues when the keratin is extracted (Figs. 110 and 112).

The exact fate of the cellular apparatus-nuclei, mitochondria, RNA, etc., in keratinizing cells is obscure (see p. 220). In a structural sense the nuclei can be followed through the keratinization zone and remnants can be demonstrated in the emerging hair. They elongate probably passively with the cell in the upper bulb, and are here Feulgen positive (Fig. 97b); but this reaction fades during keratinization although a "structural residue" is still visible electron microscopically. Spier and Van Caneghem (1957) report increased DNA-ase activity in this zone, and presumably the DNA is depolymerized. Bolliger and Gross (1952 and 1956) found many possible low molecular weight breakdown products (pentoses, uric acid and other purines) of nucleic acid in hair. When keratin is chemically extracted from hair, a portion of the insoluble residue consists of long, thin, chemicallymodified remnants of nuclei (Mercer, 1953).

In the light microscope the nuclei are seen to shrink and grow more dense and are said then to be "pycnotic." Electron micrographs show first a thick gathering of dense material beneath the membrane and some signs that material may be being shed into the cytoplasm.

Pycnosis is a degenerative condition recognized by an increase in both 
the acid and base-binding capacity of nuclei accompanied by a decrease in nuclear volume. It is supposed that the basic proteins (histones) normally combined with the DNA become dissociated from it, each then becoming more easily stainable, and that there is also a loss in water-binding power. The total DNA content remains more or less constant.

According to Bern et al. (1954 and 1957) the nuclear changes during keratinization are, however, different from this and are not simply degenerative. The nuclear volume at first increases to be followed by a collapse in the final stages of cell condensation. During the swelling stage the nuclei are less stainable and the DNA content decreases. This fall in DNA continues during the phase of active keratin synthesis. The histone content seems to remain constant and these proteins in an altered form may represent in part the "nuclear remnants" found in the keratinized cell.

A production of masses of keratohyalin by the nucleus or even the actual dissolution of that body has been envisaged by Hinglais-Guillard (1959) as a result of her study of keratinization in the cervical epithelium of women. This tissue undergoes a cyclic change of functional activity in phase with the other sexual tissues (see p.144), the cells oscillating between a keratinizing condition and one in which glycogen is accumulated. In the keratinizing phase the nuclei of the superficial cells reveal a curious clumping of their contents and rather similar lumps of material are to be seen in the adjacent cytoplasm.

These observations are not incompatible with the possibility that the nucleus is playing a different synthetic role in the later stages of keratin formation. Evidence, given below (p. 268), suggests that a peculiar cystine-rich protein may be formed at this stage and its formation may involve the nucleus. The observation of Fell and Pelc, already referred to (pp. 63 and 220), that radioactive sulphur compounds on injection appear first in nuclei may also mean that sulphur-containing amino acids are built into protein precursors in the nucleus. It is known that a small amount of protein synthesis occurs in nuclei in other sites and that it involves energy transfers following pathways not involving the enzymes normally participating in the cytoplasm (Allfrey et al., 1953 and 1957). The situation in the keratinizing cell is admittedly peculiar-it is largely cut off from supplies of metabolites and its life as a synthetic unit is drawing to a close-it would not be surprising then if special mechanisms were called into play. For example, it is compatible with the little evidence we have to suppose that here the DNA molecules participate at first-hand (i.e. not through the intermediary of RNA) in the synthesis of proteins and are themselves consumed in the process.

The RNA granules of the cytoplasm persist into the keratinization zone and are lost from view between the masses of condensing fibrils. Histochemically also the cells of the bulb are strongly RNA positive, and the 
reaction disappears in the lower part of the keratinizing zone (Fig. 97 (b)). We have seen that protein synthesis is substantially complete at this same level.

Mitochondria and various vacuoles (Golgi apparatus) are also lost sight of as distinct structures between the condensing fibrils. Their remnants persist as modified membranes and seem responsible for various gaps which are to be seen in the otherwise almost uniformly-fused mass of keratin fibrils (Plates 15, 16 and 17). It is not known what enzymes are involved in the oxidative closure of the sulphur bridges, although copper is concerned as it is with the oxidases in melanin formation (p. 279).

\section{The hair cuticle}

The peculiar type of amorphous keratin of the hair cuticle cells seems to possess a limited extensibility, since these cells usually part company or split when the hair is stretched more than $50 \%$ (Lehmann, 1943), and when a many-layered cuticle is present as in fur hairs (Stoves, 1947 and 1943) the elastic behaviour of the whole hair is considerably modified. Owing to its fibrous texture the hair cortex may fray and split; against this tendency the cuticle forms a retaining sheath whose laminated structure is adapted to this end. The keratin of the cuticle is, in fact, so different from that of the cortex as to require separate consideration; it represents yet another distinct product of epidermal cells.

Proceeding inwards from the surface of the fibre (Fig. 110) we encounter (a) a thickened and altered external cell membrane, which is strongly attached, through the intermediary of an intercellular sheet, to the membranes of contiguous cells. The whole of this formation of external membranes forms a strong, chemically resistant skin of such distinct character that it is referred to as an epicuticle (Lindberg, 1949). (b) Beneath the epicuticle lies the layer of keratin, its compact amorphous structure being responsible for much of the mechanical protection. This may be called an exocuticle. It is not uniform in texture. A layer immediately beneath the epicuticle stains more deeply after reduction and fixation and thus seems more cross-linked (Sikorski, 1960). (c) A layer of modified cellular residue (p. 270) (3 in Fig. 110) (endocuticle) lies next. Its high resistance to keratinolytic solvents (see pp. 261 and 268) supplements that of the exocuticle and thus increases the total protection against chemical action.

Cuticular keratin is distinct from the other products of the cells of the hair follicle in the following respects.

(a) It is not fibrous. It has rather, during its formative stages (Plate $20 \mathrm{~A}$ ), the appearance of a viscous liquid condensing to give a coherent amorphous lamella closely adhering to the external cell membrane (Fig. 
110). 'The X-ray pattern (Rudall, 1941) shows no orientation and no crystallite formation.

(b) It has a higher sulphur content than whole hair (Geiger, 1944). It forms somewhat later than the mass of fibrils in the cortex, in fact accumulating most rapidly during the later keratinization of the cortex. We have given reasons above to suppose that at this level the cortical cells have
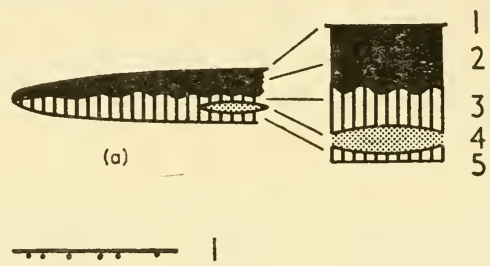

(b)

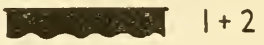

(c)

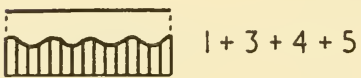

(d)

FIG. 110. The lamellar structure of the hair cuticle cell and the results of selective removal of components.

(a) 1. is the resistant external cell membrane or epicuticle;

2. the layer of amorphous keratin or exocuticle;

3. the inner layer of altered cellular residue;

4. the nuclear residue;

5. the inner cell membrane less altered than 1 .

(b) The epicuticular membrane as released by bursting Allwörden sacs.

(c) The keratinized residue remaining after tryptic digestion.

(d) The "non-keratinous" residue remaining after removal of the keratin (2) by oxidization and extraction.

swung over to the synthesis of a cystine-rich, amorphous protein, $\gamma$-keratin. It is possible that the cuticle cells are producing a very similar protein which here, in the absence of fibrils, simply condenses as an amorphous heavily cross-linked mass.

From the viewpoint of cellular differentiation, it is interesting to find 
that, before commencing definitely to form "droplets" of cuticular keratin, the cuticle cells form small amounts of fibrillar material (Birbeck and Mercer, 1957).

The laminated structure and the differing chemical nature of the several component layers are responsible for some peculiar reactions. When hairs are immersed in acidified chlorine water, which attacks keratin with the production of low molecular weight osmotically-active substances, the resistant surface membranes are inflated into bubbles by the water, which enters the cell (Allwörden's reaction) (Fig. 111). The bubbles may develop
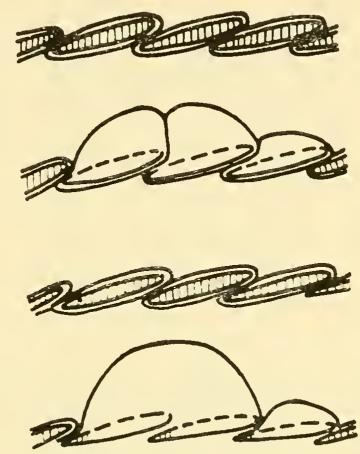

FIG. 111. Explanation of the appearance of bubbles on the surface of hairs immersed in chlorine water (Allwörden's reaction). The external cell membranes are modified chemically and form a very resistant layer, the epicuticle. When chlorine penetrates it and oxidizes the proteins within the cell, lower molecular weight soluble compounds are produced. The membrane is dilated by the entry of water owing to the high osmotic pressure within the cell.

to an extraordinary degree on hairs with elaborate frilly cuticular scales such as bat hair (Müller 1939). The study of this phenomenon first led Müller to recognize the existence of specialized resistant surface membrane. Lindberg (1949) obtained clear pictures of the membrane by mechanically abrading wool fibres covered with well-developed bubbles. Bromine water is less active. Bubbles form, but the skin is thicker and seems to consist of the surface membrane and the more resistant portion of the exocuticle. A variety of other sheaths and tubules can also be obtained from the lamellar cuticular structures of hair which differ principally in the amount of keratin remaining attached to the epicuticle (Mercer et al., 1949; Manogue and Moss, 1953; Lagermalm et al., 1951). 
The surprisingly hydrophobic character of the intact surfaces of hairs and feathers, even after thorough removal of lipids, is presumably due to the chemical inertness of the modified external cell membrane. When this is mechanically or chemically damaged the material becomes wetable and dyes and other large molecules more readily penetrate (Mercer et al., 1949).

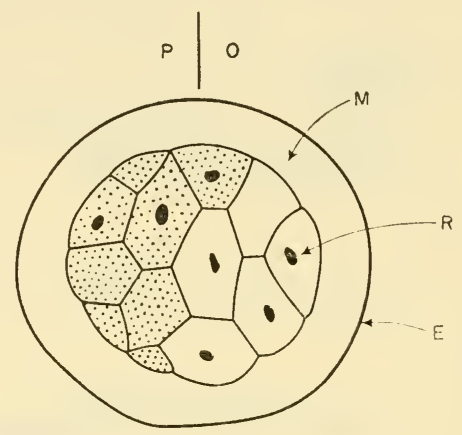

Fig. 112. Illustrating the resistant residues which remain after a wool fibre, oxidized by means of peracetic acid, is extracted with ammonia. The cell membrances and nuclear residues of the cortical cells are at $\mathrm{M}$ and $R$, respectively. The external membrane $\mathrm{E}$ of the cuticle cells encloses the swollen fibre. In the paracortical segment $\mathrm{P}$ some keratin remnants may persist; $O$ is the orthocortex (see text).

\section{The medulla}

As is apparant from the description given by Auber (1950) (Fig. 113), the changes taking place in the medulla of hairs are complex. Keratohyalin granules appear in the differentiated cells and electron micrographs of rodent hairs show that they change into a fibrous form as elsewhere. The total amount synthesized is inadequate to fill the cell cavities and much of the transformed material simply condenses against the cell membranes. During desiccation intracellular gaps appear (Fig. 113c) and the final result may be a rather open girder-like structure, light but stiff.

Chemically the medulla resists alkalis and keratinolytic reagents and may be isolated (undoubtedly altered) by digesting away the keratinized cortex and cuticle. It thus resembles the altered membranes of these structures rather than their keratinized contents and morphologically this seems to arise from a close fusion of fibrous keratohyalin and membranes. That much of the protein of the medulla is of the trichohyalin type is supported by analyses, reported by Rogers (1959a), of the medullary cells 

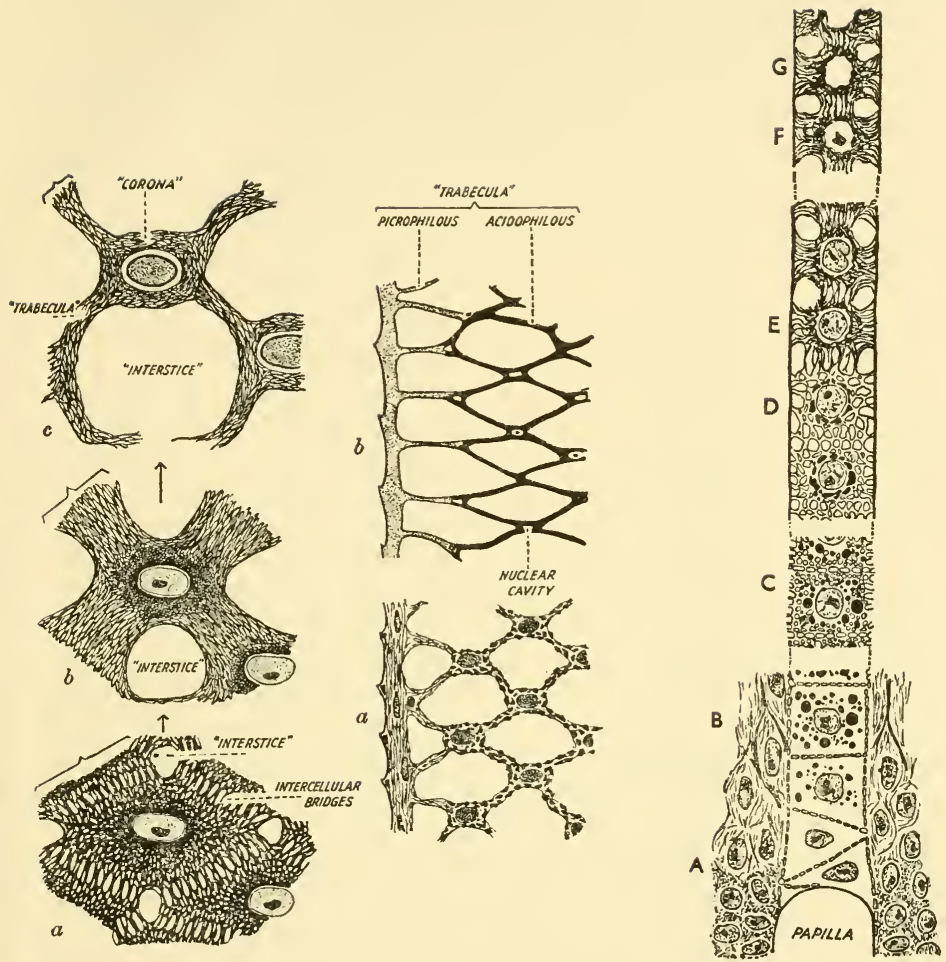

FIG. 113. Structure of medulla. Diagrams to illustrate the detailed formation and structure of the medulla in hair. a, b and c are three stages in the obliteration of a cell and the condensation of its contents. $A-G$ illustrates the full sequence of cell forms in which the appearance and assimilation of trichohyalin (see p. 268) may be followed. Reproduced from Auber (1950) by permission. The transformation of trichohyalin into fibrils and its fusion with the cell membranes is indicated. 
of rabbit fur (Table 20). The cystine content is very low, citrulline is present and half the side chains are either acidic or basic. It thus appears quite distinct from other hard keratins and resembles trichohyalin.

TABLE 20. Composition of RabBit Fur and Medulla.*

( $\mathrm{N}$ as per cent of total-N.)

\begin{tabular}{|c|c|c|}
\hline$\alpha$-Amino acid & Fur & Medulla \\
\hline glycine & $4 \cdot 74$ & 1.42 \\
\hline alanine & $2 \cdot 50$ & $2 \cdot 78$ \\
\hline valine & $2 \cdot 21$ & $1 \cdot 18$ \\
\hline leucine & $4 \cdot 22$ & $7 \cdot 95$ \\
\hline isoleucine & 1.53 & 1.00 \\
\hline serine & $5 \cdot 38$ & $1 \cdot 38$ \\
\hline threonine & 3.02 & 0.86 \\
\hline phenylalanine & $1 \cdot 38$ & 1.45 \\
\hline tyrosine & $1 \cdot 55$ & 0.80 \\
\hline tryptophane & - & - \\
\hline proline & $4 \cdot 49$ & 0.85 \\
\hline citrulline $\dagger$ & $0 \cdot 35$ & $5 \cdot 2 \dagger$ \\
\hline lysine & $4 \cdot 64$ & $15 \cdot 84$ \\
\hline arginine & $17 \cdot 60$ & $11 \cdot 72$ \\
\hline histidine & $3 \cdot 81$ & $1 \cdot 38$ \\
\hline aspartic acid & $3 \cdot 29$ & $2 \cdot 42$ \\
\hline glutamic acid & $6 \cdot 84$ & $21 \cdot 7$ \\
\hline cystine & $9 \cdot 11$ & - \\
\hline methionine & 一 & 一 \\
\hline ammonia & $1 \cdot 32$ & $7 \cdot 02$ \\
\hline
\end{tabular}

* Taken from Rogers (1959a).

$\dagger$ The presence of citrulline may prove to be diagnostic of trichohyalin.

\section{The residues remaining after the chemical extraction of keratins}

Much has been learned concerning the chemical nature of the constituents of keratinized tissues by scrutinizing the residues which remain after subjecting the tissue to the treatments described above designed to remove definite components. For example, the extraction of the proteins, which become soluble after complete disulphide-bond destruction, enables 
a useful distinction to be made between keratinized and non-keratinized components, and to establish what fraction of the tissue actually consists of keratins.

The most satisfactory procedure is to oxidize the disulphide bonds with peracetic acid and to extract thoroughly the oxidized material with dilute ammonia. Thioglycollates at high $\mathrm{pH}(11-12)$ have also been used, but effect a less complete extraction. The undissolved residue contains the following components recognizable in the light microscope:

(a) The ill-defined and obscurely altered remnants of the cell nuclei.

(b) Many small particles distributed throughout the cytoplasmic space which are the remnants of the various cell organelles, mitochondria and Golgi membranes.

(c) The external membranes of the cells which are still held together in a foam-like formation by the intercellular deposits.

Similar residues remain after extraction by other reagents and the findings are the same for all types of keratinized tissues (Mercer, 1953; Matoltsy, 1958). In some cases, when the cells assume a specialized form, as in the hair cuticle discussed earlier, the intercellular remnants may separate during growth from the keratinized protein and thus form a distinct layer within the cell which is visible (Fig. 110 (d)) after extraction.

A picture which is almost the " negative image " of the above is obtained when the material is digested with a proteolytic enzyme (trypsin or papain) which removes everything except the keratinized proteins. The procedure is not very satisfactory with the soft keratin, epidermis; with all the hard keratins (hair, horn, nails, etc.) after prolonged digestion, a clear delineation of the resistant protein results. The tissue falls apart, since the cell membranes are removed, and the residue is often referred to as " cells", which they resemble superficially in shape, although they consist, in fact, only of the keratinized protein, all other components visible in the intact state being removed (p. 261). Such preparations should form the material for analysis of "keratin". More prolonged digestion removes part of the keratin itself, suggesting that this material is not entirely uniform in composition. This suggestion, that the " degree of keratinization " may be variable at a microscopic level, is compatible with the idea that keratinization is a process which takes place gradually and may remain incomplete and patchy.

A "dissection" of the cuticle cell, as effected by various methods of extraction, illustrated in Fig. 110, shows clearly the differing properties of its several layers.

The recognition that hardened tissues contain several components differing in resistance to chemical attack was the basis of an earlier system of classification, introduced by Unna (1926) in which keratins were to be distinguished by the proportions they contained of " keratin A", insoluble 
in nitric acid or in sulphuric acid plus hydrogen peroxide, and "keratin B " which was soluble. The system was little used although it gave rise to the more useful distinction between hard and soft keratins. Nevertheless, it would be valuable when classifying keratins to have measures of two factors, which really were at the root of Unna's ideas: (a) the amount of keratinized protein present in a tissue relative to non-keratinized material, and (b) the degree to which the keratin is insolubilized. From the standpoint of our present-day knowledge, these could be usefully defined as follows:

Ratio of keratins to total weight weight of tissue soluble in $0 \cdot 1 \mathrm{~N} \mathrm{NH}_{3}$ $=$ after oxidizing for $24 \mathrm{hr}$ in $2 \%$ peracetic acid

original weight

Ratio of easily soluble keratin to keratin soluble with difficulty weight of material extracted by $8 \mathrm{M}$ urea $0 \cdot 2 \mathrm{M}$ thioglycollic acid adjusted to $\mathrm{pH} 7$

weight of peracetic acid oxidized material soluble in $0 \cdot 1 \mathrm{~N} \mathrm{NH}_{3}$

In the choice of a solvent for the "softer" keratin in the second ratio, there is, of course, an arbitrary element and other solvents could be used. In textile circles, for example, the " alkaline solubility," is assessed, i.e. the amount of fibre soluble in $0.1 \mathrm{~N}$ caustic soda at $65^{\circ} \mathrm{C}$ in $1 \mathrm{hr}$, and this is found useful as a measure of "damage", where this is defined as a deterioration in the insolubility or stability of the keratin. After allowing for custom and the practical aspects, it would seem nevertheless, better to use a solvent, such as thioglycollic acid and urea, whose action is based on differences in the degree of $\mathrm{H}$-bonding and disulphide cross-linking which are the chemical bases of insolubilisation. The experiments of Lees and Elsworth (1955), Jones and Mecham (1943) and of Ward and Lundgren (1954) on dissolving keratins show that they differ significantly when their solubilities are compared by solvents such as proposed above.

\section{Uneven keratinization and its histological distribution}

The variations in keratinization, shown by such tests as "alkaline solubility" or "urea-bisulphite" solubility or by histological stains, which reveal directly the distribution of disulphide concentration (Plate 24B) in a tissue, may arise in either of two ways. Firstly, since in a hard keratin the consolidation process takes place after the synthesis and organization of the protein into a fibrous structure and requires a certain time, it could easily fail to achieve completion for accidental or systemic reasons. Secondly, 
soft keratin containing transformed keratohyalin may be "mixed" in various proportions with a fibrous keratin of the hard type. These differences in degree of keratinization produce differences in physical and chemical properties having obvious functional value and this has not been overlooked in the evolution of the various epidermal appendages. It takes perhaps its most interesting form when the function of a particular organ is found to depend on differences in keratinization in its several histological parts. The case of the hair follicle has been discussed above. There we find the soft cells of the root sheaths desquamating and freeing the hair; the tough impact-resisting cuticle protecting the fibrous cortex; the light open framework of the medulla providing a rigid girder-like internal skeleton.

The epidermis itself varies in thickness and toughness from site to site and the distribution is clearly related to the special demands made on each site. The thickened epidermis with its marked pattern of papillary ridges found on the palmar surface of the hands and feet is genetically determined as is also the ability to respond to mechanical friction and pressure by further thickening. On the histological level, hard and softer regions in the papillary ridges are said to have the effect of enhancing mechanically the sensitivity to touch (Cauna, 1954).

In the terminal appendages (claws, hoofs, etc.) two parts, the unguis and the softer subunguis, can normally be distinguished (Fig. 29, p. 68) and the different rates of wear of these two parts has much to do with the maintenance of the shape and the functional efficiency of the parts. For example, in the ungulate hoof the hard cylinder of the unguis largely surrounds the softer subunguis which wears the more rapidly and thus maintains a more or less flattened, load-bearing surface. The sharp cutting or piercing tip of a claw is maintained by a difference between the hardness and orientation of the two layers of which the claw is composed. These two layers, the superficial stratum and the deep stratum (unguis and subunguis) may be seen in Figs. $29 \mathrm{~d}$, e and f, and it is obvious how the sharp cutting edge of the claw is maintained automatically by the more rapid wearing of the deeper layer.

Hair also contains parts varying in keratinization in the cortex. An elaborate relationship is found between morphology, physical behaviour and variations in keratinization in fine crimpy or curly hair or wool (Mercer, 1954). This type of hair, already discussed in Chapter IV, p. 156, forms waves which are very nearly uniplanar and it is found that, in terms of stabilization and of chemical reactivity, these fibres, like many of the larger appendages, are also bilateral (Horio and Kondo, 1953). They consist in fact of two hemi-cylinders differing in stability which are twisted together in such a way that the helix of the two hemi-cylinders is always in phase with the crimp wave and that the outside face of the fibre is always less keratinized than the inside face (Horio and Kondo, 1953; 
Mercer, 1953 and 1954; Fraser et al., 1954) (Fig. 114). A bilateral fibre has thus a built-in tendency to maintain its curled shape and the functional value of this in maintaining the insulating properties of the fleece of the animal, which depend on the air trapped by the mass of crimpy fibres, is obvious.
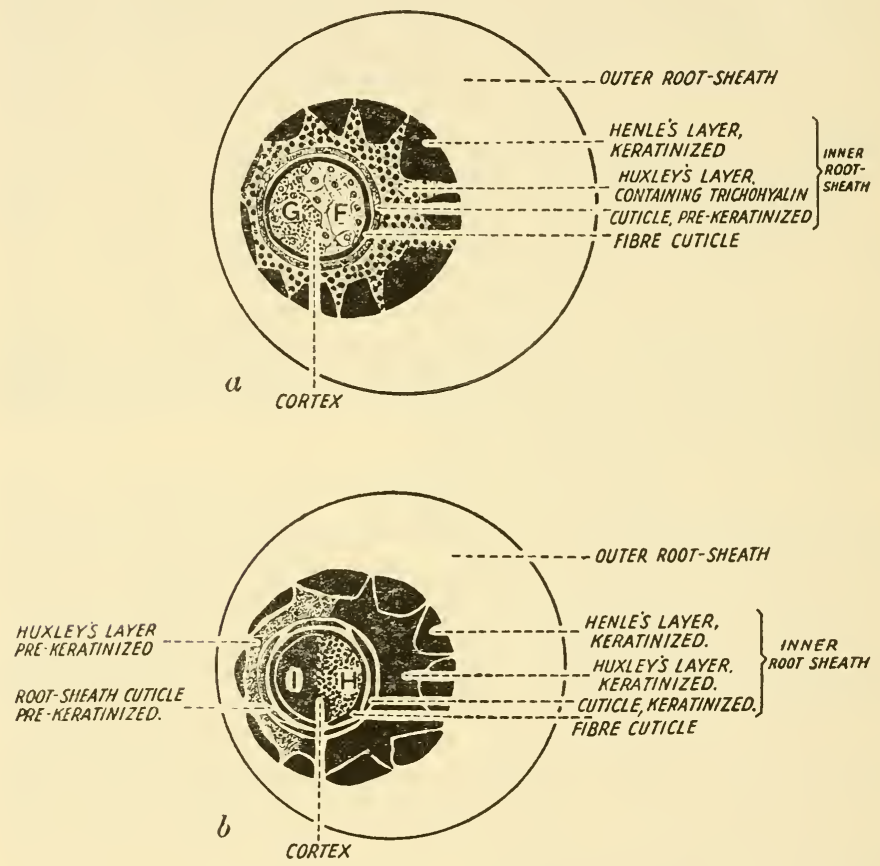

Fig. 114. The development of keratinization in an asymmetrical wool follicle leading to the formation of ortho and para segments (FG and $\mathrm{HI}$ ) (see Fig. 112). From Auber (1950) by permission. The sections pass through the early (D) and late (E) levels of the keratinizing zone. Notice the asymmetrical root sheaths.

Bilateral fibres of this type are widely distributed in the woolly coat (secondary hair) of many animals, and are particularly marked in the sheep. They are less well-defined in coarse hair and in the case of some forms of crimpy negro hair are difficult to demonstrate (Spearman and Barnicot, 1960). The relation between external form and internal structure revealed in this phenomena poses some interesting problems in keratinization which 
are only partly understood and are more properly viewed in the wider context of the general factors controlling the differentiation of the many cell subtypes produced from the epidermal germinal layer.

The problem of crimp formation has already been discussed in Chapter IV and it is obvious that the factors governing keratinization must be related to the other morphogenetic factors involved. Auber (1950) and Rudall (1936) discovered that keratinization is bilateral in follicles producing crimpy fibres, i.e. the hardening of the fibre begins at a lower level on the side which will emerge as the more keratinized (Fig. 114). Auber showed that in such follicles the hair shaft was asymmetrically placed within the cylinder of the outer root sheath and that hardening commenced on the side of the hair nearest the thinner part of the sheath. This immediately suggested that something supplied from beyond the sheath was required for keratinization and could penetrate (or escape) most readily on the thin side. There is ample other evidence implying a close dermo-epidermal co-operation in controlling epidermal differentiation (p. 61). By partly dissolving plucked follicles it has been shown that the difference between the two sides extends below the keratinization zone, i.e. that the cell types are committed early in their course. This again illustrates the interlocking of the events at various depths in the follicle (p. 156).

The chemical basis of the difference between these variants of keratin is not yet clear even in a case as well studied as wool. There is evidence from histochemistry (Dusenbury and Menkart, 1956 (Plate 24B): Menkart and Coe, 1958) and from the analysis of resistant residues to suggest (but not prove) that in wool the resistant fraction (para-type) has a higher cystine content and perhaps differs in other respects. Simmonds (1958) could, however, find no difference between high- and low-crimped material. Rogers (1959b) found that the packing of the $\alpha$-filaments in the para-cells was hexagonal (Plate 16) and that whorls occurred more often on the ortho-side (less resistant). Nevertheless whorls are no less common in human hair which on a basis of its resistance and sulphur content is paratype (Birbeck and Mercer, 1957).

\section{Keratinized cysts and epidermal tumours}

Tumours and cysts arising from epidermal cells are not uncommon and may exhibit interesting, if abnormal aspects of keratinization that deserve notice here. The skin of the mouse is also one of the commonest test objects of experimental carcinogenesis, and figured largely in the classical work of Kennaway and his colleagues which led to the isolation of definite carcinogenic compounds from tar (Kennaway, 1955; Ludford, 1925).

It seems now established that the tumours produced by benzpyrene and other carcinogenic hydrocarbons take their origin from the rather undifferentiated basal cells of the upper outer root sheath of the hair follicle 
(Wolbach, 1951; Borum, 1954) and that the effectiveness of the carcinogen depends on the phase of the hair cycle. When applied to a skin area containing resting follicles the effect is small; however, when the hairs are growing the carcinogen seems able to penetrate the skin via the hair follicles and produces a more profound effect. If the hair papilla cells are killed by the applied chemical, normal follicle reformation becomes impossible and the cyclic regenerative changes associated with growth waves (p. 150) leads to proliferation of the outer root sheath cells with the production of deeply-seated keratinizing cysts or tubes at the centres of which the keratinizing cells, unable to exfoliate in the normal way, build up to form keratin pearls (see Plate 24A) (Glücksmann, 1945). The continued growth forms at first a benign papilloma. According to Wolbach such cells are still responsive to the stimulus of the growth waves passing over the neighbouring normal skin. Genuinely malignant tumours may ultimately develop from such papillomas.

In the so-called "hairless" mouse mutant after the first wave of hair growth, follicles fail to reform normally and very similar keratinizing cysts may form beneath the skin from the upper portion of the root sheath (Grüneberg, 1952).

\section{Pigmentation}

Most epidermal derivatives are pigmented, and the great variety of integumental colours and pattern which can be produced is of immense importance in the life of animals. The various colours ("structural colours", which result from the diffraction of light by regular structures,

Table 21. Epidermal Pigments.

\begin{tabular}{l|l|l}
\hline \multicolumn{1}{c|}{ Property } & \multicolumn{1}{c|}{ Melanin } & \multicolumn{1}{c}{ Pheomelanin } \\
\hline $\begin{array}{l}\text { colour } \\
\text { shape } \\
\text { dimensions } \\
\text { chemical type }\end{array}$ & $\begin{array}{l}\text { brown black } \\
\text { oval to round } \\
0 \cdot 1-3 \mu \\
\text { protein tanned with } \\
\text { melanin polymer } \\
\text { almost insoluble } \\
\text { tyrosine }\end{array}$ & $\begin{array}{l}\text { yellow (red) } \\
\text { round and smaller }\end{array}$ \\
$\begin{array}{l}\text { alkali solubility } \\
\text { precursor }\end{array}$ & $\begin{array}{l}\text { soluble } \\
\text { tyrosine and tryptophane }\end{array}$ \\
\hline
\end{tabular}

excepted) are derived solely from combinations of black, brown or yellow pigment in the form of granules combined possibly with a red non-granular pigment. Table 21, adapted from Fitzpatrick, Brunet and Kukita (1958) summarizes the main facts relating to granular pigments.

Pigment granules are the exclusive product of pigment-forming cells called melanocytes which in the adult animal are found among the basal 
layer cells of the keratinizing system. According to Medawar (1953) the melanocytes comprise from 5 to $15 \%$ of the total cell population of the germinal layers of the epidermis. The epidermis thus really consists of two entirely different classes of cells the members of which have distinct morphologies, functions and embryonic origins. Keratinizing cells arise embryonically from the ectoderm; melanocytes have, however, been traced back largely to the neural crest (Rawles, 1947) (see also Niu, 1959) and they enter the epidermis only after this has been clearly differentiated. They are an amoeboid type of cell with several long arborescent processes called dentrites, and are perhaps best referred to as dentritic cells. In their dispersion from their site of origin they seem impelled by a mutual repulsion which leads them ultimately to colonize the dermis and epidermis and there to adopt a dispersed distribution, each cell occupying a small domain determined by the extreme reach of its dentritic processes. They also accumulate densely in a few other sites, such as the pigmented layer of the eye. Each epidermal melanocyte pigments the small group of keratinizing cells within reach of its dentrites. The granules of pigment are formed in the perikaryon of the cell, pass along the processes and enter the keratin cells. Owing to their situation in the basal layer attached to the basal membrane, pigmentation occurs before the formation of keratin and the subsequently-formed fibrils may lead to an orientation of the granules. The presence of melanocytes seems in no way essential to the well-being of the keratinizing system since some epithelia naturally lack melanocytes and others may be deprived of them, accidently or by experiment, without appearing to be at a disadvantage.

The distribution of pigment cells is under genetic control and, since changes in the integument are easy to observe, much attention has been given to the genetics of skin and hair pigmentation. The value in terms of natural selection of pigmentary patterns is obvious; but for all that, little is known of the underlying causes determining the distribution of pigment cells (Du Shane, 1944).

According to Billingham and Medawar (Billingham, 1948; Billingham and Medawar, 1948 and 1953; Billingham, 1958) not all dentritic cells produce pigment. White skin patches are said to contain a full complement of dentritic cells although special means are required to demonstrate these, since they contain no pigment. The skin of white human beings is said to contain as many melanocytes as that of negroes, for example. It would seem that melanocytes differ in their response to the influences which provoke pigmentary activity. This would be an inherited difference distinguishing different sub-races of melanocytes even on a single skin. Some never fail to begin production once they reach the epidermis; others may be provoked into activity by exposure to actinic radiation; others normally remain latent. The hormonal balance can also cause changes in activity. 
There would appear to be ample opportunity for these variations to arise when the long train of enzymatically-calalysed reactions involved in pigment formation is considered (p. 279).

In their grafting experiments on guinea-pig skin, Billingham and Medawar (1948) have demonstrated an actual diffusion of pigmentary activity from a black graft into a white skin. They proposed an explanation which is of some theoretical importance. They consider that the latent melanocytes of the white skin are actually " infected " by the neighbouring black melanocytes by a process which is related to the normal method of transferring pigment from melanocyte to a keratinizing cell. They have demonstrated microscopically that anastomoses between processes of neighbouring melanocytes do actually occur and suppose that through such contacts one melanocyte may transfer to another a sample of its cytoplasmic apparatus. Electron micrographs of the tips of dentritic processes found in keratinizing cells show that in fact samples of cytoplasmic membranes and particles are transferred along with pigment granules (Birbeck et al., 1956).

Such a transference of a cytoplasmic element capable of permanently modifying the activity of an acceptor cell, if it proves not merely a peculiarity of the system of dentritic cells, could be of importance in the normal developmental history of cell lines. It would imply first that inherited differences between cells could have a cytoplasmic as well as a nuclear basis, and second that a population of cells could establish and maintain uniformity among their members by cytoplasmic exchanges. The actual mechanics of the transfer of pigment from melanocyte to epidermal cell needs further elucidation. Two broad possibilities may be envisaged: the keratin cell may be actively penetrated by the tip of the melanocyte process or alternatively, it may play the active role and phagocytize the tip. The latter process receives some support from electron micrographs which show small processes of the keratinizing cell in various stages of enveloping the pigmented tips.

\section{The Pigment Granule}

The actual pigment granule contains both protein and pigment and is often referred to as a melanoprotein, although almost nothing is known of the nature of the linkage between the two components. It may be formulated as a bipolymer in which polypeptides alternate with melanin, or it may be a tanned protein, i.e. a network of protein chains cross-linked by melanin polymer or other phenols. Whatever its structure, the granules may exhibit remarkable chemical stability as is proved by the fact that their separation from keratinized tissues by violent chemical destruction of the keratin seems to leave them morphologically intact. Electron micrographs by Birbeck demonstrate clearly the existence of a regular framework of 
protein in the pre-melanin granules on which the melanin later polymerizes (Fig. 115 (h) and (i)) (Birbeck and Barnicot, 1949).

The similarity between the types of chemical resistance exhibited by melanin granules and the cell membranes of the keratinized tissue has been mentioned above. It is certainly possible that a similar tanning reaction has cross-linked the proteins of the membranes and the granules-a possibility of some phylogenic interest. Recently, tanned membranes having similar solubility properties have been described by Jones (1958).

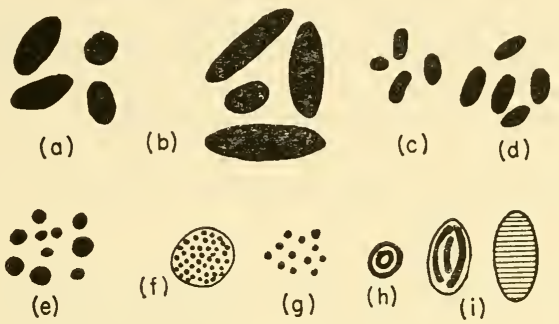

Fig. 115. Varicties of melanin granules. Redrawn to scalc from authors cited. The examples are chosen to show the extreme range of size and type. $\times 10,000$.

(a) Human hair white male (Birbeck, Mercer and Barnicot, 1956).

(b) Human hair negro male (Birbeck, Cuckow and Barnicot, 1955).

(c) Horse hair (Laxer and Whewell, 1955).

(d) Black alpaca (Laxer and Whewell).

(e) Retinal eye pigment (Birbeck, private communication).

(f) Harding-Passey melanoma from section of tumour by the writer.

(g) Squid ink (Birbeck, private communication).

(h) and (i) Human-head hair with indications of internal structure as seen in sections of melanocytes.

The granules vary in size from $0 \cdot 1$ to $3 \mu$ and for this reason their size and shape has been much studied electron-microscopically. This may be done either on isolated granules or in sections of fixed tissue. Fig. 115 shows outline drawings of a number of types of granule and will give some idea of the range of size encountered. Sections of granules often suggest a clear separation between layers of melanin deposits and those of a lighter material, presumably protein.

\section{The Chemistry of Melanization}

Although the protein moiety and its relation to the melanin polymer has been little studied, much attention has been paid to the biochemical steps in the formation of the melanin itself. Melanins, quite apart from 
their occurrence as pigment granules, are very widely distributed in nature, occurring in plants, in vertebrates and invertebrates. They are formed by the action of copper-containing oxidases, known as phenolases, that catalyse the oxidation of mono- and di-hydric phenols to o-quinones (Mason, 1953 and 1955). Phylogenetically the chemical reactions involved in melanization developed before keratinization and quite independently of it. Mason has discussed the wide distribution and varied applications of the "phenolase system" in the different phyla. It constitutes an excellent example of a simple biochemical system, which catalyses essentially the same reactions in plants and highly-organized animals, although with an increased specificity towards substrates as the phylogenetic tree is ascended, and which thus finds expression in widely-different characters at different levels. For example, it is responsible for the browning of plant tissues, the hardening of cuticle of arthropods and the pigmentation of chordates. In the higher animals the site of melanin formation has become limited to the pigment cell or melanocyte.

The biochemical evidence has been reviewed recently by Mason (1955) and Fitzpatrick, Brunet and Kukita (1958). Briefly, the amino acid tyrosine has been shown to be the precursor of the insoluble pigment. The phenolase, tyrosinase, converts tyrosine to DOPA (3:4-dihydroxyphenylalanine) and to "DOPA quinone", which becomes 5:6-dihydroxyindole, the immediate precursor or monomer of the large polymer molecule melanin, which may then be linked to a protein (Fig. 116).

The darkening of a tissue when treated with DOPA (dihydroxyphenylalanine), Bloch's DOPA-oxidase reaction, has long been used to demonstrate cytologically the sites of melanin formation (Block, 1921), but it is now less favoured, since it may be non-specifically oxidized to melanin. Tyrosine is preferred as substrate although it may fail to demonstrate tyrosinase when the latter is in low concentration. Histochemically tyrosine activity has been demonstrated in autoradiographs by using radioactive tyrosine and $\mathrm{C}^{14}$ (see Fitzpatrick et al., 1958).

The small quantities of melanized material from mammalian sources have hindered biochemical research, but by taking advantage of the larger quantities of material available in pigmented tumours (melanomas) this may be overcome. The granules of the Harding-Passey melanoma appear as aggregates of fine dense granules perhaps incompletely supplied with a protein framework (Mercer, unpublished) (Fig. 115(f)).

Something of the cytological structure associated with these syntheses has been revealed by the electron microscope and by the histochemical location of tyrosinase activity. The cell is roughly polarized in a manner similar to glandular cells (see p. 110) with a limited basophilic reticulum at the end proximal to the attachment to the basement membrane (Birbeck et al., 1956). Here presumably the protein of the granule is synthesized. 
Distal to the nucleus is a clear region relatively free of dense melanized particles with large numbers of small, rounded vacuoles (Golgi-type vacuoles) which contain variable amounts of denser material often arranged in concentric shells. The vacuoles on the periphery of this region contain additions of very dense material identifiable as melanin.

On both histological and electron-microscopical grounds there is now little doubt that the intracellular sites for the formation of melanin are these

Fig. 116. Possible courses in the synthesis of melanins and melanoproteins. *

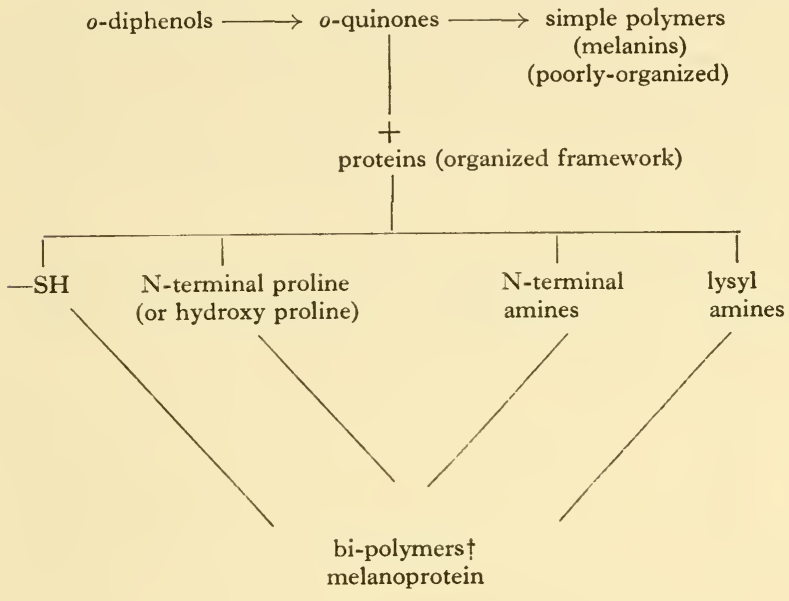

* From Mason (1955) with modifications.

† Morphological evidence would indicate that this is a bipolymer whose components are each of a macromolecular size.

small vesicles. An older opinion, based on similarity of size and staining properties of granules and mitochondria, held that the melanogenic vesicle is really a mitochondrion. Since the granules contain, in addition to tyrosinase at least two other enzymes, cytochrome oxidase and succinic dehydrogenase usually located in mitochondria, some support is given for this view. Morphologically, however, the two organelles are quite distinct although in their remote origins both may possibly be traced back to similar vesicular formations. Recently mitochondria and premelanin granules, characterized by containing tyrosinase and not mitochondrial enzymes, have been separately isolated from melanoma homogenates (Baker et al., 1960). 
The appearance of granules in early stages of formation suggests that a rather regular protein framework (Fig. $115(\mathrm{~h})$ ) is laid down first and is subsequently melanized by a polymerization occurring in its interstices (Birbeck and Barnicot, 1959). The melanoprotein thus seems to be a wellordered particle although little of this is apparent after melanization is complete. Melanocytes in albino hair possess large numbers of small vacuoles containing the protein framework, but no deposition of dense material occurs. In these cases, in the absence of tyrosinase, the melanin polymer is not formed.

Some interest attaches to the control of melanocyte activity in the hair root which is correlated with the hair growth cycle (Montagna, 1956) and with the development of various pigmentary patterns both in hair and in feathers. Montagna's observation that melanocyte activity ceases shortly before hair growth ceases is cited as evidence of the accumulation of a general growth inhibitor (see Chapter IV) affecting first the more sensitive melanocytes.

The yellow-red pigment (pheomelanin) also occurs in granular form, but less is known about the metabolic pathways leading to its formation. The ultimate precursor appears to be the amino acid tryptophane. Reference may be made to the review by Fitzpatrick et al. (1958). 


\section{References}

Abercrombie, M. (1957) Symp. Soc. exp. Biol. 11, 235.

- A Symposium on the Chemical Basis of Development, p. 318. Ed. by McElroy,

W. D. and Glass, B. Johns Hopkins Press, 1958.

Alexander, P. (1951) Ann. N. Y. Acad. Sci. 53, 653.

- and Earland, C. (1950) Nature, Lond. 166, 396.

- and Hudson, R. F. Wool: its Chemistry and Physics. Chapman \& Hall, London, 1954.

and Smith, L. F. (1955) Proc. Inter. Wool Text Conf. Austr. Vol. B., p. 56;

C.S.I.R.O., Melbourne, 1956.

Allfrey, V. G., Daly, M. M. and Mirsky, A. E. (1953) F. gen. Physiol. 37, 157.

- Mirsky, A. E. and Osawa, S. (1957) F. gen. Physiol. 40, 451.

Ambrose, E. J. and Elliott, A. (1951a) Proc. Roy. Soc. A205, 47.

- and Hanby, W. E. (1949) Nature, Lond. 163, 483.

- and Elliotr, A. (1952) Proc. Roy. Soc. A206, 206.

Arndt, U. W. and Riley, D. P. (1955) Phil. Trans. A247, 409.

Arthur, R. P. and Shelley, W. B. (1959) F. invest. Dermat. 32, 397.

Assheton, R. (1896) Quart. F. micros. Sci. 38, 465.

Astbury, W. T. and Street, A. (1931) Phil. Trans. A230, 75.

— and Marwick, T. C. (1932) Nature, Lond. 130, 309.

— and Woods, H. J. (1933a) Phil. Trans. A232, 333.

- Fundamentals of Fibre Structure. Oxford U.P., London, $1933 \mathrm{~b}$.

- (1947) Proc. Roy. Soc. B134, 303 (Croonian Lecture).

- (1943) Advanc. Enzymol. 3, 63.

-_ Dickinson, S. and Bailey, K. (1935) Biochem F. 29, 2351.

— and Bell, F. O. (1939) Tabulae Biologica XVII. part 1, 90.

— and Dickinson, S. (1940) Proc. Roy. Soc. B129, 307.

- and Bell, F. O. (1941) Nature, Lond. 147, 696.

- Dalgleish, C. E., Darmon, S. E. and Sutherland, G. B. B. M. (1948) Nature, Lond. 162, 596.

(1949) Exp. Cell Res. Suppl. 1. 234.

- Beighton, E. and Weibull, C. (1955) Soc. exp. Biol. Symp. 9, 282.

- (1955) Proc. Int. Wool Text. Conf. Austr. Vol. B., p.204; C.S.I.R.O., Melbourne, 1956.

(1958) Disc. Faraday Soc. 25, 80.

Beighton, E. and Parker, K. D. (1959) Biophys. Biochim Acta 35, 17.

Auber, L. (1950) Trans. Roy. Soc., Edinb. 62, 191.

- (1955) Nature, Lond. 176, 1218.

Bachra, B. N., Sobel, A. E. and Stanford, J. W. (1959) Arch. Bioch. Biophys. 84, 79.

Bahr, G. F. (1954) Exp. Cell Res. 7, 457.

- and Moberger, G. (1954) Exp. Cell Res. 6, 506. 
Bailey, K., Astbury, W. T. and Rudall, K. M. (1943) Nature, Lond. 151, 716. Baker, J. R. Cytological Technique. 2nd Ed. Methuen, London, 1945.

- (1955) F. Roy. micros. Soc. 74, 217.

- (1959) F. Roy. micros. Soc. 77, 116.

Baker, R. V., Birbeck, M. S. C., Blaschko, H., Fitzpatrick, T. B., and Seiji, M. (1960) Nature, Lond. 187, 392.

Baldwin, E. An Introduction to Comparative Biochemistry. 3rd Ed. Rep. Cambridge University Press, 1949.

- Perspectives in Biochemistry, p. 99. Ed. by J. Needham and D. E. Green, Cambridge University Press, London, 1937.

Bamford, C. H. et al. (1953) Proc. Roy. Soc. B141, 49.

- Elliott, A. and Hanby, W. E. Synthetic Polypeptides. Academic Press, New York, 1956.

- Brown, L., Elliott, A., Hardy, W. E. and Trotter, I. F. (1954) Nature, Lond. 173, 27.

- Hanby, W. E. and Happey, F. (1949) Nature, Lond. 164, 138. and HANBY, W. E. (1951) Nature, Lond. 168, 340.

BARker, S. G. Wool Quality, H.M. Stationery Office, 1933.

BARnes, R. J. Studies in the Optical Properties of Wool Hair and related Fibres, Ph.D. Thesis, Leeds, 1933.

Barnicot, N. A., Birbeck, M. S. C. and Cuckow, F. W. (1955) Ann. Human Genet. 19, 231.

BarrnetT, R. J. (1953) ₹. Nat. Cancer Inst. 13, 905.

- and Seligman, A. M. (1952) Science 116, 323.

Battistone, G. C. and Burnett, G. W. (1956) Y. Dent. Res. 35, 260.

Bear, R. S. (1943) F. Amer. Chem. Soc. 65, 1784; (1944) Ibid. 66, 2043.

- and Rugo, H. J. (1951) Trans. N.Y. Acad. Sci. 53, 627.

- (1952) Advanc. protein Chem. 7, 69.

Bellamy, L. T. The Infra-red Spectra of Complex Molecules. Methuen, London, 1954.

Bendit, E. G. (1957) Nature, Lond. 179, 535.

Bennet, H. S. (1948) Anat. Rec. 100, 640.

von Bergen, W. Matthew's Textile Fibers. 6th Ed. Ed. by H. R. Mauersberger, p. 484. John Wiley, New York, 1954.

Bergmann, M. and Fraenkel-Conrat, H. (1937) F. Biol. Chem. 119, 707.

BERN, H. A. (1954) Nature, Lond. 174, 509.

Harkness, D. R. and Blair, S. M. (1955) Proc. Nat. Acad. Sci., Wash. 41, 55.

- Alfert, M. and Blair, S. M. (1957) F. Histochem. Cytochem. 5, 105.

Bernal, J. D. in The Cell and Protoplasm, p. 199. Ed. by F. R. Moulton, Wash. Ass. Adv. Sci., 1940.

Bernhard, W., Gautier, A. and Roulller, C. (1954) Arch d'Anat. micros. et de Morph. exper. 43, 236.

- Haguenau, F., Gautier, A. and Oberling, Ch. (1952) Z. f. Zellforsch. u. mikros. Anat. 37, 281.

Gautier, A. and Oberling, Ch. (1951) C. R. Soc. Biol., Paris 145, 566.

Bertalanffy, L. von, in Fundamental Aspects of Normal and Malignant Growth, p. 137. Ed. by W. N. Nowinski, Elsevier, Amsterdam, 1960.

BiedermanN, W. (1926) Ergebn. Biol. 1, 342; (1928) Ibid. 3, 354; (1928) Ibid. 4, 361.

Billingham, R. E. (1948) F. Anat. 82, 93; 83, 109.

— and Medawar, P. B. (1948) Heredity 2, 29. 
Billingham, R. E. and Medawar, P. B. (1953) Phil. Trans. B237, 151.

The Biology of Hair Grozuth. Ed. by W. Montagna and R. A. Ellis. Ch. 9, p. 451, Academic Press, New York, 1958.

Birbeck, M. S. C. (1957) Private communication.

- Mercer, E. H. and Barnicot, N. A. (1956a) Exp. Cell Res. 10, 505. and Mercer, E. H. (1956b) Nature, Lond. 178, 985.

and Barnicot, N. A. in Biology of Hair Growth, p. 239. Ed. by W. Montagna and R. A. Ellis, Academic Press, New York, 1958.

and Barnicot, N. A. Pigment Cell Biology, p. 549. Ed. by M. Gordon, Academic Press, New York, 1959.

and Mercer, E. H. (1957a) f. biophys. biochem. Cytol. 3, 203, 215, 227.

and Mercer, E. H. (1957b) Proc. Int. Conf. Elect. Micros., Stockholm, 1956, p. 156.

and Mercer, E. H. (1961) Nature, Lond. 189, 558.

BLACKBURN, S. (1950) Biochem. F. 47, 443.

- and LEE, G. R. (1954) Biochem. F. 58, 227.

Block, B. (192I) Archiv. Dermatol. u. Syphilis 135, 77.

Block, R. J. (1931) f. F. Biol. Chem. 94, 647.

- and Vickery, H. B. (1931) F. Biol. Chem. 93, 113.

- Horwitt, M. K. and Bolling, D. (1949) F. dental Res. 28, 518.

- and Bolling, D. Amino Acid Composition of Proteins and Foods. Thomas, Springfield, Ill., 1950.

Boedtker, H. and Doty, P. (1956) f. Amer. Chem. Soc. 78, 4267.

Bolliger, A. and Gross, R. (1952) Aust. F. exp. Biol. med. Sci. 30, 181, 399, 395.

- (1956) Aust. F. exp. Biol. med. Sci. 34, 219.

Borsook, H. Proc. 3rd Int. Congr. Biochem., Brussels, p. 92. Academic Press, New York, 1955.

- (1955) Advanc. Protein Chem. 8, 127.

Borum, K. (1954) Acta Pathol. Microbiol. Scand. 34, 521.

BRACHET, J. Chemical Embryology. Interscience, New York, 1950.

—- Biochemical Cytology, Academic Press, New York, 1957.

Bradfield, J. R. G. (1951) Nature, Lond. 167, 40.

- (1955) Soc. exp. Biol. Symp. 9, 306.

Braun-Falco, O. in Biology of Hair Growth. Ch. 4, p. 65. Academic Press, New

York, 1958.

BRody, I. (1959) ₹. Ultrastruct. Res. 2, 482.

- (1959) F. Ultrastruct. Res. 3, 84.

(1960) F. Ultrastruct. Res. 4, 264.

Broussy, J. (1932) Thesis No. 52, Université de Montpellier, Faculté de Medicine. Brown, C. H. (1949) Quoted in L. E. R. PICKen, Phil. Trans. B234, 1.

- Ph.D. Thesis, Cambridge, 1949.

- (1949) Exper. Cell Res. Suppl. 1, 351.

(1950) Nature, Lond. 165, 275.

(1950) Quart. F. micros. Sci. 91, 331.

Brown, L. and Trotrer, I. F. (1956) Trans. Faraday Soc. 52, 537.

Bull, H. B. and Gutman, M. (1944) F. Amer. Chem. Soc. 66, 1253.

- (1945) F. Amer. Chem. Soc. 67, 533.

Bullough, W. S. (1946) Phil. Trans. B231, 453.

- Vertebrate Sexual Cycles, Methuen, London, 1951.

- and Johnson, M. (1951) Proc. Roy. Soc. B138, 562.

- (1952) Biol. Rev. 27, 133. 
Bullough, W. S. (1954) Exp. Cell Res. 7, 176; (1954) Ibid. 7, 186.

- (1955) Exp. Cell Res. 9, 108.

- and Laurence, E. B. (1957) Brit. F. exp. Path. 38, 278.

- and Laurence, E. B. In Biology of Hair Growth, ch. 8. Ed. by W. Montagna and R. A. Ellis, Academic Press, New York, 1958.

- and Laurence, E. B. (1960) Proc. Roy. Soc. B151, 517.

- and Laurence, E. B. (1961) Private communication.

Buns, C. W. Chemical Crystallography, Clarendon Press, Oxford, 1946.

Burge, R. E. (1960) Proc. Roy. Soc. A, 260, 558.

Burgos, M. H. and Wislocki, G. B. (1958) Endrocrinology 63, 106.

Burns, M. and Clarkson, H. (1949) F. Agric. Sci. 39, 315.

Burrows, H. Biological Action of Sex Hormones, Cambridge University Press, 1949.

Burte, H. and Halsey, G. (1947) Text Res. F. 17, 456.

Butcher, E. O. (1934) Anat. Rec. 61, 5.

Cairns, J. M. and Saunders, J. W. (1954) F. exp. Zool. 127, 221.

Calvery, H. O. (1933) F. Biol. Chem. 100, 183.

Calvin, M. (1956) Amer. Scientist 44, 248.

Carruthers, C., Quevedo, W. C. and Woernley, D. L. (1959) Proc. Soc. exp. Biol., N.Y. 101, 374.

- and Santzeff, V. (1953) Physiol. Rev. 33, 229.

Carter, H. B. (1943) Bull. C.S.I.R.O., Australia, No. 164, Melbourne.

— and Clarke, W. H. (1957) Aust. F. agric. Res. 8, 91, 109.

Caspersson, T. O. Cell Growth and Cell Function. W. W. Norton, New York, 1950.

- (1947) Symp. Soc. exp. Biol. 1, 127.

Cauna, N. (1954) Anat. Rec. 119, 449.

Champetier, G. and Faure-Fremiet, E. (1938) C. R. Acad. Sci., Paris 207, 1133.

Charles, A. and Smiddy, M. B. (1957) $\mathcal{F}$. Invest. Dermat. 29, 327.

- (1959) Exper. Cell Res. 18, 138.

Chase, H. B. (1954) Physiol. Rev. 34, 113.

— (1955) F. Soc. Cos. Chem. 6, 9.

- (1959) Ann. N. Y. Acad. Sci. 83, 365.

Chevremont, M. and Frederic, J. (1943) Arch. Biol. Liège 54, 589.

Clark, W. E. Le Gros (1936) Proc. Zool. Soc. 106.

Claude, A. (1938) Proc. Soc. exp. biol. Med. 39, 398.

- (1946) Y. exp. Med. 84, 51.

Cockran, W., Crick, F. H. C. and Vand, V. (1952) Acta Cryst., Camb. 5, 581.

Colvin, J. R., Sмith, D. B. and Cook, W. H. (1954) Chem. Rev. 54, 687.

Coman, C. R. (1954) Cancer Res. 14, 519.

Corey, R. B. and Wyckoff, R. W. G. (1936) F. Biol. Chem. 114, 407.

- and Pauling, L. (1955) Proc. Int. Wool Text. Res. Conf. Austr. Vol. B., p. 249; C.S.I.R.O., Melbourne, 1956.

Corfield, M. C. and Robson, A. (1955) Biochem. F. 59, 62.

- Robson, A. and Skinner, B. (1958) Biochem. F. 68, 348.

Cowdry, E. V. Special Cytology. Hoeber, New York, 1932.

Crane, H. R. (1950) Scientific Monthly 70, 376.

Crick, F. H. C. (1952) Nature, Lond. 170, 882.

- (1953) Acta Cryst., Camb. 6, 685, 689.

- (1958) Symp. Soc. exp. Biol. 12, 138.

- and Watson, J. D. (1954) Proc. Roy. Soc. A223, 80.

Cruise, A. J. and Jefrery, J. W. (1959) Nature, Lond. 183, 677. 
Cruise, A. J. and Keеch, M. K. (1959) F. Roy. micros. Soc. 78, 95.

Dalton, A. J. and Felix, M. D. (1956) F. biophys. biochem. Cytol. 2 Suppl., 79. — and Felix, M. D. (1957) Symp. Soc. exp. Biol. 10, 148.

Danforth, C. H. Special Cytology. Sect. 2, p. 41. Ed. by E. V. Cowdry, Hoeber, New York, 1932.

Danielli, J. F. in Cytology and Cell physiology, p. 1. Ed. by G. H. Bourne, Oxford University Press, 1942.

Darmon, S. E. and Sutherland, G. B. B. M. (1949) Nature, Lond. 164, 440.

Davies, H. R. (1889) Morph. Fb. 15, 560.

Davson, H. and Danielli, J. F. The Permeability of Natural Membranes. Cambridge University Press, London and New York, 1952.

Das, D. B. and Speakman, J. B. (1950) F. Soc. Dy. Col. 66, 583.

Day, M. F. (1951) Aust. F. Sci. Res. B4, 42.

Derksen, J. C., Heringa, G. C. and Weidinger, A. (1937) Acta néerl. morph. norm. et path. 1, 31.

Donohue, J. (1953) Proc. Nat. Acad. Sci., Wash. 39, 470.

- (1954) Proc. Nat. Acad. Sci., Wash. 40, 377.

Doty, P. and YAng, 'T. J. (1956) F. Amer. Chem. Soc. 78, 498.

- (1956) Proc. Nat. Acad. Sci., Wash. 42, 791.

DrY, F. W. (1926) F. Genet. 16, 287; (1928) F. Genet. 20, 131.

Durward, A. and Rudall, K. M. (1949) $\mathcal{~}$. Anat. 83, 327.

- - in The Biology of Hair Growth. Ch. 9, p. 189. Academic Press, New York, 1958.

Dusenbury, J. H. and Menkart, J. (1955) Proc. Int. Wool Text. Cont. Austr. Vol. F, p. 142. C.S.I.R.O., Melbourne, 1956.

Du Shane, G. P. (1944) Quart. Rev. Biol. 19, 98.

Earland, C. and Wiseman, A. (1959) Biochim. Biophys. Acta 36, 273.

Ebling, F. J. (1953) F. Endocrinol. 9, 31, 32.

— (1954) $\mathcal{~}$. Endocrinol. 10, 147.

— and Johnson, E. (1959) F. Embryol. Exper. Med. 7, 417.

Eisen, A. Z., Montagna, W. and Chase, H. B. (1953) F. Nat. Cancer Inst. 14, 341. Elliott, A. (1952) Text. Res. Y. 22, 783.

- (1953) Proc. Roy. Soc. A221, 104.

(1954) Proc. Roy. Soc. A226, 408.

- Hanby, W. E. and Malcolm, B. R. (1954) Brit. F. App. Phys. 5, 377.

- Hanby, W. E. and Malcolm, B. R. (1956) Nature, Lond. 178, 1170.

- Hanby, W. E. and Malcolm, B. R. (1958) Disc. Faraday Soc. 25, 167.

Ellis, W. J. (1948) Nature, Lond. 162, 957.

- Gillespie, J. M. and Lindley, H. (1950) Nature, Lond. 165, 545.

Elod, E. and ZAHN, H. (1944) Kolloid-Z. 108, 94.

- and ZAHN, H. (1946) Melliand Textilber. 27, 68.

- and Z Z $\mathrm{AHN}$, H. (1949) Kolloid-Z. 113, 157.

Engstrom, A. and Lindstrom, B. (1947) Experientia 3, 191.

'Espinasse, P. G. (1939) Proc. Zool. Soc. Lond. Ser. A. 109, 247.

Farrant, J. L., Rees, A. L. G. and Mercer, E. H. (1947) Nature, Lond. 159, 535. and Mercer, E. H. (1952) Biochem. biophys. Acta 8, 355.

Fasske, E. and Themann, H. (1959) Z. Zellforsch. 49, 447.

Fawcett, D. W. Frontiers in Cytology, p. 19. Ed. by S. L. Palay, Yale Univers ity Press, Newhaven, 1958.

- and Porter, K. R. (1954) F. Morph. 94, 221. 
Fell, H. B. and Canti, R. G. (1934) Proc. Roy. Soc. B116, 316.

- (1957) Proc. Roy. Soc. B146, 242.

- and Danielli, J. F. (1943) Brit. F. exp. Path. 24, 196.

- and Mellanby, E. Sir (1953) \%. Physiol. 119, 470.

- Mellanby, E. Sir and Pelc, S. R. (1954) Brit. med. F. 2, 611.

- Mellanby, E. Sir and Pelc, S. R. (1956) F. Physiol. 134, 179.

Feughelman, M. (1959) Text. Res. Y. 29, 223.

Fischer, A. (1924) F. exp. Med. 39, 585.

Fitzpatrick, T. B. and Kukita, A. J. (1956) f. Invest. Dermat. 26, 173.

- Brunet, P. and Kunita, A. The Biology of Hair Growth, p. 255. Ed. by

W. Montagna and R. A. Ellis, Academic Press, New York, 1958.

Fleischauer, K. (1953) Z. f. Zellforsch. 38, 50, 328.

Flesch, P. (1949) Proc. Soc. exp. Biol. Med. 70, 79.

Florkin, M. Unity and Diversity in Biochemistry. Pergamon, Oxford, 1960.

Fox, S. W. (1953) Amer. Nat. 87, 253.

Fraenkel, G. and Rudall, K. M. (1947) Proc. Roy. Soc. B134, 111.

FrASeR, A. S. (1951) Nature, Lond. 167, 202.

- (1952) Austral. F. agric. Res. 3, 419.

- and Short, B. F. (1952) Austal. F. agric. Res. 3, 445.

- (1955) Austal. F. agric. Res. 6, 770.

- and Nay, T. (1953) Aust. F. Biol. Sci. 6, 645-656; (1955) Ibid. 8, 428.

- and Short, B. F. (1958) Aust. F. Biol. Sci. 11, 200.

- and Short, B. F. The Biology of the Fleece. Animal Res. Lab. Tech. Paper

No. 3. C.S.I.R.O., Melbourne, 1960.

Fraser, R. D. B., Lindley, H. and Rogers, G. E. (1954) Biochim. biophys. Acta $13,295$.

— and Rogers, G. E. (1955) Aust. F. Biol. Sci. 8, 289.

- and MacRae, T. P. (1956) Text. Res. F. 26, 618.

- and MAcRaE, T. P. (1957) Text. Res. F. 27, 867.

- and MacRae, T. P. (1957) Nature, Lond. 179, 732.

- and MacRae, T. P. (1958a) Biochim. biophys. Acta 29, 229.

— and MacRae, T. P. (1958b) F. chem. Phys. 28, 1120.

- and MACRAE, T. P. (1959) f. mol. Biol. 1, 387, 1959.

- MacRae, T. P. and Rogers, G. E. (1959) Nature, Lond. 183, 592.

- and MacRae, T. P. (1961) Nature, Lond. 189, 572.

Frey-Wyssling, A. Submicroscopic Morphology of Protoplasm. Elsevier, Amsterdam, 1953.

Garnier, Ch. in Hagenau, F. The Ergastoplasm; (1958) Int. Rev. Cytol.7, 425. Geiger, W. B. (1944) F. res. Nat. Bur. Stand. 32, 127.

- Patterson, W. I., Mizell, L. R. and Harris, M. (1941) f. res. Nat. Bur. Stand. 27, 459.

- Kobayashi, F. F. and Harris, M. (1942) F. res. Nat. Bur. Stand. 39, 381.

Gelfant, S. (1958) Exp. Cell Res. 15, 423, 451; (1959a) Ibid. 16, 527; (1959b)

Ibid. 18, 594.

- (1960) Ann. N. Y. Acad. Sci. 90, 536.

Gillespie, J. M. (1958) Biochim. biophys. Acta 27, 225.

- (1955) Proc. Int. Wool Text. Res. Cont. Austr. Vol. B., p. 35; C.S.I.R.O., Melbourne, 1956. 
Gillespie, J. M. (1960) Aust. F. biol. Sci. 13, 81.

- and Lennox, F. G. (1953) Biochim. biophys. Acta 12, 481.

and Lennox, F. G. (1955) Austr. F. biol. Sci. 8, 97.

O'Donnell, I. J., 'Thompson, E. O. P. and Woods, E. F. (1960) Proc.

Wool Text. Res. Conf., Harrogate, 1960, f. Text. Inst. 51, T703.

Giroud, A. and Bulliard, H. (1930) Arch. de morph. 29, 1.

- and Champetier, G. (1936) Bull. soc. chim. biol. 18, 656.

- and Leblond, C. P. (1951) Ann. N. Y. Acad. Sci. 53, 613.

- Bulliard, H. and Leblond, C. P. (1934) Bull Histol. Tech. micr. 11, 129.

Glinos, A. D. The chemical Basis of development (Mech. of liver growth), p. 813.

Ed. by W. D. McElroy and B. Glass, Baltimore, Johns Hopkins Press, 1958.

Glücksmann, A. (1945) Cancer Res. 5, 385.

- and Cherry, C. P. (1956) Cancer 9, 971.

Goddard, D. R. and Michaelis, L. (1934) f. Biol. Chem. 106, 605.

- and Michaelis, L. (1935) f. Biol. Chem. 112, 361.

Gordon, M. Pigment Cell Growth. Academic Press, New York, 1953.

Grasse, P. P. (1956) C. R. Acad. Sci., Paris 242, 858.

Gray, E. G. (1959) Proc. Roy. Soc. B150, 233.

Grimstone, A. V. (1961) Biol. Rev. 36, 97.

Gross, J. (1956) F. biophys. biochem. Cytol. Suppl. 2, 261.

- Highberger, J. H. and Schmitt, F. O. (1954) Proc. Nat. Acad. Sci., Wash. 40.

(1956) f. biophys. biochem. Cytol. Suppl. 2, 261.

GrünebERG, H. The Genetics of the Mouse, ch. 6. Martinus Nijhoff, The Hague, 1952.

Gustavson, K. H. The Chemistry and Reactivity of Collagen. Academic Press, New York, 1956.

—- The Chemistry of Tanning Processes. Academic Press, New York, 1956.

HaAn DE, R. L. In The Chemical Basis of Development, p. 339. Ed. by W. D. McElroy and B. Glass, The Johns Hopkins Press, Baltimore, 1958.

Hackman, R. H. (1953) Biochem. f. 54, 362, 367, 371.

- (1959) Proc. 4th Int. Cong. Biochem. Vienna (1958). Ed. by O. HoffmanNOSTENHOF, 12, 48.

Haddow, A., Elson, L. A., Roe, E. M. F., Rudall, K. M. and Timmis, G. M. (1945) Nature, Lond. 155, 379.

HADžI, J. in De Beer, G. R. Evolution as a Process, p. 24. Ed. by J. Huxley, A. C.

HARdy and E. B. Ford. Allen \& Unwin, London, 1954.

Haguenau, F. (1958) Int. Rev. Cytol. 7, 425.

Hall, C. E. Introduction to Electron Microscopy. McGraw-Hill, New York, 1953.

Hanson, J. (1947) f. Anat. 81, 174.

Hanzon, V., Hermodsson, L. H. and Toschi, G. (1959) f. ultrast. Res. 3, 216.

HARdy, M. H. (1949) Four. Anat., Lond. 83, 364.

- (1952) Amer. F. Anat. 90, 285.

- (1951) Ann. N. Y. Acad. Sci. 53, 546.

— and Lyne, A. G. (1956) Aust. F. Biol. Sci. 9, 423.

Harkness, D. R. and Bern, H. A. (1957) Acta Anatomica 31, 35.

Harrap, B. S. (1955) Aust. F. Biol. Sci. 8, 122. 
Harris, M. and Brown, A. E. in Fibrous Proteins. Sym. Soc. Dyers and Col., Leeds, p. 203. Chorley \& Pickersgill, Leeds, 1946.

Harvey, E. N. and Danielli, J. F. (1938) Biol. Rev. 13, 319.

Haurowitz, F. Chemistry and Biology of Proteins. Academic Press, New York, 1950.

Hausman, L. A. (1925) Amer. Nat. 59, 529.

Heilmann, G. The Origin of Birds. H. F. \& C. Witherby, London, 1926

Hendler, R. W. (1957) Fed. Proc. 16, 194.

HergersberG, H. (1957) Z. f. Zellf. 45, 569.

Hess, A. (1958) F. biophys. biochem. Cytol. 4, 731.

van Heyningen, W. E. Bacterial Toxins, p. 52. Blackwell, Oxford, 1950.

Hildemann, W. H. (1959) Amer. Nat. 93, 27.

Hrlditch, T. P. Chemical Composition of Natural Fats. Chapman \& Hall, London, 1949.

Hinglais-Guillaud, N. (1959) Bull. du Cancer 46, 212.

Hoagland, M. B., Zamecnik, P. C. and Stephenson, M. L. (1957) Biochem. biophys. Acta 24, 215.

Hodge, A. J. (1959) Rev. Mod. Phys. 31, 409.

- 4th Internat. Congress for Electron Microscopy, 1958. Vol. 2, 119. SpringerVerlag, 1960.

HOLFRETER, J. (1947) F. morphol. 79, 27.

- (1948) Ann. N. Y. Acad. Sci. 49, 708.

Hooper, C. E. S. (1956) J. Hist. Cytochem. 4, 531.

Horio, M. and Kondo, T. (1953) Text Res. F. 23, 373.

Horstmann, E. Handbuch der mikroskopischen Anatomie des Menschen. Haut und

Sinnesorgane. Dritter Band, Dritter Teil. Die Haut., p. 1-276. SpringerVerlag, Berlin, 1957.

- (1955) Z. Zellforsch. 41, 532.

- and KNoop, A. (1958) Z. Zellforsch. 47, 348.

Hosker, A. (1936) Phil. Trans. B226, 143.

Нотта, K. (1928) Chinese F. Physiol. 2, 345; (1929) Ibid. 3, 171.

Huggins, M. L. (1943) Chem. Rev. 32, 195.

- (1958) F. Polymer Sci. 30, 5.

Hughes, T. E. (1959) f. exp. Biol. 36, 363.

Hutchinson, G. E. (1948) Ann. N. Y. Acad. Sci. 50, 221.

Hyman, L. H. Comparative Vertebrate Anatomy. Chicago University Press, 1947.

INGRAM, V. M. (1957) Nature, Lond. 180, 326.

Jackson, S. Fitton (1956) Proc. Roy. Soc. B144, 556.

Jacobsen, C. F. and Linderstrøm-Lang, K. (1949) Nature, Lond. 164, 411.

Jarrett, A., Spearman, R. I. and Hardy, J. A. (1959) Brit. F. Dermatol. 71, 277. - and Spearman, R. I. (1961) Private communication.

Jeffrey, G. M., Sikorski, J. and Woods, H. J. (1955) Proc. Int. Wool Text. Conf. Aust. Vol. F., p. 130; C.S.I.R.O., Melbourne, 1956.

Jennings, M. A. and Florey, H. W. (1956) Quart. F. exp. Physiol. 41, 131.

Johnson, E. (1958) F. Endocrinal. 16, 337.

Jones, B. M. (1958) Proc. Roy. Soc. B149, 263.

Jones, C. B. and Mecham, D. K. (1943) Arch. Biochem. 2, 209; 3, 193. 
Karrer, H. E. (1960) F. biophys. biochem. Cytol. 7, 181.

Kay, L. M., Schroeder, W. A., Munger, N. and Burt, N. (1956) F. Amer. Chem. Soc. 78, 2430.

Kendrew, J. C. Structure Proteins, I. The Proteins. Ed. by H. Neurath and K.

Bailey, Vol. II, Part B. Academic Press, New York, 1954.

- and Perutz, M. F. (1957) Ann. Rev. Biochem. 26, 327.

Kennaway, E. Sir (1955) Brit. Med. J. 2, 1382.

KerR, M. F. and Godin, C. (1959) Canad. F. Chem. 37, 11.

King, H. T. and Nicholls, J. E. (1932) Trans. Faraday Soc. 29, 272.

Krebs, H. A. (1953) Brit. Med. Bull. 9, 92.

Krimm, S. and Schor, R. J. (1956) F. Chem. Phys. 24, 922.

Krishnan, G. (1953) Quart. F. micr. Sci. 94, 11; (1954) Ibid. 95, 371.

Kukita, A. J. (1957) F. Invest. Dermatol. 28, 273.

Lafon, M. (1943) Bull. Inst. Océanogr. Monaco 40, No. 850. Quoted by KRISHNAM, 1953.

Lagermalm, G., Philip, B. and Lindberg, J. (1951) Nature, Lond. 168, 1080.

LASNitzki, I. (1956) Brit. F. Cancer 10, 510.

- (1958) Ann. Rev. Cytol. 7, 79.

Laxer, G. and Whewell, C. S. (1955) Proc. Int. Wool Text. Conf. Austr. Vol. F., p. 186; C.S.I.R.O., Melbourne, 1956.

LeACH, S. J. (1959) Rev. pure appl. Chem. 9, 33.

Leblond, C. P. Dynamics of Proliferating Tissue. Ed. by D. Price, University of Chicago Press, 1958.

- (1951) Ann. N. Y. Acad. Sci. 53, 464.

- Puchtler, H. and Clermont, Y. (1960) Nature, Lond. 186, 784.

and Storey, W. F. (1956) Physiol. Rev. 36, 255.

Lees, K. and Elsworth, F. F. (1954) Proc. Int. Wool Text. Conf. Austr. Vol. C,

p. 363; C.S.I.R.O., Melbourne, 1955.

Lehmann, E. (1941) Melliand Textilber. 22, 145.

Lerner, A. B., Fitzpatrick, T. B., Calkins, E. and Summerson, W. H. (1949)

J. Biol. Chem. 178, 185.

Leuchtenberger, C. and Lund, H. Z. (1951) Exp. Cell Res. 2, 150.

Lindberg, J., Philip, B. and Gralen, N. (1948) Nature, Lond. 162, 458.

- (1949) Text. Res. F. 19, 43.

Lillie, R. F. and Wang, H. (1941) Physiol. Zool. 14, 103.

- (1942) Biol. Rev. 17, 247.

and Juhn, M. (1932) Physiol. Zool. 5, 124 ; (1938) 11, 434.

and Wang, H. (1944) Physiol. Zool. 17, 1.

and Wang, H. (1940) Proc. Nat. Acad. Sci., Wash. 26, 67.

Linderstrøm-Lang, K. Symposium on Peptide Chemistry. Special Publication 2,

The Chemical Society, London, 1958.

and Duspiva, F. (1936) C. R. Lab. Carlsberg Sér. Chim. 21, 53.

Lindley, H. (1947) Nature, Lond. 160, 190.

- (1955) Proc. Int. Wool Text. Conf. Austr. Vol. B., p. 193; C.S.I.R.O., Melbourne, 1956.

Littlefield, J. W. and Keller, E. B. (1957) F. Biol. Chem. 224, 13.

Litvac, A. (1939) Arch. d'anat. microsc. 35, 55.

Lоснте, Th. Atlas der menschlichen und tierschen Haare. Paul Schöps, Leipzig, 1938. 
Loftrield, R. B. (1957) Prog. Biophys. Chem. 8, 347.

Longley, J. B. (1950) Ph.D. Thesis, Cambridge.

Lorand, L. and Middlebrook, W. R. (1952) Biochem. F. 52, 196.

- (1952) Biochem. F. 52, 200.

Losee, F. L. and Hess, W. C. (1949) Ұ. Dental Res. 28, 512.

LOTMAR, W. and PiCKen, L. E. R. (1950) Experientia 6, 58.

Low, B. W. and Baybutt, R. B. (1952) F. Amer. Chem. Soc. 74, 5806.

Lucas, F., Shaw, J. T. B. and Sмiтh, S. G. (1956) Nature, Lond. 178, 861.

- Shaw, J. T. B. and Smith, S. G. (1958) Adv. Prot. Chem. 13, 107.

Ludford, R. J. (1925) Proc. Roy. Soc. B98, 557.

LÜDICKE, M. (1959) Naturwissenschaften 46, 658.

LuFT, J. H. (1956) F. biophys. biochem. Cytol. 3, 799.

Lundgren, H. P. (1945) Text. Res. F. 15, 335.

MACARTHUR, I. (1943) Nature, Lond. 152, 38.

McDonough, E. G. (1952) Chem. Engng. News 30, 5254.

McLoughlin, C. B. Ph.D. Thesis, Cambridge, 1959.

Manogue, B. and Moss, M. S. (1953) Nature, Lond. 172, 806.

Manton, I. (1952) Symp. Soc. Exper. Biol. 6, 306.

Marsh, R. E., Pauling, L. and Corey, R. B. (1955) Biochem. biophys. Acta 16, 1.

Marston, H. in Fibrous Proteins. Symp. Soc. Dyers and Col. p. 207; Chorley \& Pickersgill, Leeds, 1946.

Martin, A. J. P. Fibrous Proteins. Symp. Soc. Dyers and Col. p. 1; Chorley \& Pickersgill, Leeds, 1946.

Mason, H. S. Pigment Cell Biology, p. 563. Ed. by M. Gordon, Academic Press, New York, 1953.

- (1955) Advanc. Enzymol. 16, 105.

Matoltsy, A. G. (1958) in The Biology of Hair Growth. Ch. 7, p. 135. Academic Press, New York, 1958.

Maximow, A. A. and Bloom, W. A Textbook of Histology. 5th Ed. Saunders. Philadelphia and London, 1948.

Mazia, D. (1955) Symp. Soc. Exper. Biol. 9, 335.

- and Dan, K. (1952) Proc. Nat. Acad. Sci., Wash. 38, 826.

Medawar, P. B. (1953) Quart. F. micros. Sci. 94, 481.

Menefee, M. G. (1957) F. Ultrast. Res. 1, 49.

Menkart, J. and Coe, A. B. (1958) Text. Res. F. 28, 218.

MerCER, E. H. (1949a) Nature, Lond. 163, 18.

- (1949b) Biochim. biophys. Acta 3, 161.

- (1949c) Ph.D. Thesis, Leeds University.

(1949d) F. Text. Inst. 40, T640.

- Lindberg, J. and Philip, Bo. (1949e) Text. Res. F. 19, 678.

- and Olofsson, B. (1951a) J. Polymer Sci. 6, 261.

- - (1951b) F. Polymer Sci. 6, 671.

_- (1951c) Nature, Lond. 168, 792.

(1951d) Nature, Lond. 168, 824.

and DAY, M. F. (1952a) Biol. Bull. 103, 384.

(1952b) The Sci. Mon. 75, 280.

(1953) Text. Res. Y. 23, 388.

(1954) Text. Res. F. 24, 39.

- Farrant, J. L. and Rees, A. L. G. (1955) Proc. Int. Wool Text. Conf. Austr. Vol. F., p. 120; C.S.I.R.O., Melbourne, 1956. 
Mercer, E. H. The Biology of Hair Growth, p. 91. Ed. by W. Montagna and

R. A. Ellis, Academic Press, New York, 1958.

(1959) Proc. Roy. Soc. B150, 216.

- Electron Microscopy in Anatomy, p. 100. E. Arnold, London, 1961.

Meyer, J., Medak, H. and Weinmann, J. P. (1960) Growth 24, 29.

Meyer, K. (1945) Advanc. Protein Chem. 2, 249.

- and RAPPORT, M. M. (1951) Science 113, 596.

- (1957) Harvey Lectures 51, 88.

Meyer, K. H. and Haselbach, C. (1949) Nature, Lond. 164, 33.

- and Mark, H. Der Aufbau der hochpolymeren organischen Naturstoffe, Acad.

Verlagages. Leipzig, 1930.

- Wyk, Vander, A. J. A., Gonon, W. and Haselbach, C. (1952) Trans. Faraday

Soc. $48,669$.

Middlebrook, W. R. (1951) Biochim. biophys. Acta 7, 547.

Miszurski, B. (1937) Arch. exp. Zell. forsch. 20, 123.

Moffitt, W. (1956) \%. Chem. Phys. 25, 467.

Mohn, M. P. The Biology of Hair Growth, p. 336. Ed. by W. Montagna and R. A. Ellis, Academic Press, New York, 1958.

Montagna, W. (1950) Quart F. micros Sci. 91, 205.

- Chase, H. B., Malone, J. D. and Melaragno, H. P. (1952) Quart. J.

Microbiol. Sci. 93, 241.

The Structure and Functions of Skin. Academic Press, New York, 1956.

- Chase, H. B. and Hamilton, J. B. (1951) J. Invest. Derm. 17, 147.

Moog, F. (1946) Biol. Rev. 21, 41.

Morales, M. F. and Kreutzer, F. L. (1945) Bull. Math. Biophys. 7, 15.

Moran, T. and Hale, H. P. (1936) F. exp. Biol. 13, 35.

Moscona, A. (1952) Exp. Cell Res. 3, 535.

- (1956) Proc. Soc. exp. Biol. N. Y. 92, 410.

- (1957) Proc. Nat. Acad. Sci., Wash. 43, 184.

(1957) Science 125, 598.

Muller, C. (1939) Z. f. Zellforsch. 29, 1.

Nageotte, J. (1927) C. R. Acad. Sci., Paris 184, 115.

Neurath, H. (1940) F. phys. Chem. 44, 296.

Nieuwkoop, P. D. and FABer, J. Normal Tables of Xenopus laevis. North Holland, Amsterdam, 1956.

Nilsson, O. (1958) Exp. Cell Res. 14, 341.

- (1959) F. Ultrastr. Res. 2, 331, 342.

Niv, M. C. Pigment Cell Biology, p. 37. Ed. by M. Gordon, Academic Press, New York, 1959.

Noback, C. R. (1951) Ann. N. Y. Acad. Sci. 53, 627.

Norris, M. H. (1931) F. Text. Inst. 22, T17.

Odland, G. F. (1953) F. Invest. Dermat. 21, 305.

- (1958), F. biophys. biochem. Cytol. 4, 529.

O'Donnell, I. J. and Woods, E. F. (1955a) Proc. Int. Wool Text. Conf. Austr.

Vol. B., p. 48; C.S.I.R.O., Melbourne, 1956 a.

_- and WooDs, E. F. (1956b) F. polymer Sci. 21, 397. 
Olofsson B. and Gralen, N. (1947) Proc. 11th Inter. Conf. Chem. 5, 151, London, 1953.

Oparin A. I. The Origin of Life on the Earth. 3rd Ed. Translated by A. SYNGE, Oliver \& Boyd, London, 1957.

Oster, G. and Pollister, A. W. Physical Techniques in Biological Research. Academic Press, New York, 3 vols, 1955-6.

Ottoson, D., Sjöstrand, F. S., Stenström, S. and Svaetichin, G. (1953) Acta Physiol. Scand. 29, 611.

Palade, G. E. (1952) f. exp. Med. 95, 285.

- (1955) Y. biophys. biochem. Cytol. 1, 59, 567.

- (1956) \%. biophys. biochem. Cytol. 2, 417.

and Siekevirz, P. (1956) F. biophys. biochem. Cytol. 2, 171.

Parat, M. (1928) Arch. anat. microsc. 24, 73.

Parker, K. D. (1955) Biochim. biophys. Acta 17, 148.

- and Rudall, K. M. (1957) Nature, Lond. 179, 905.

Paschkiss, K. E. (1958) Cancer Res. 18, 981.

Patterson, W. I., Geiger, W. B., Mizell, L. R. and Harris, M. (1941) Y. Res. Nat. Bur. Stand. 27, 89.

Pauling, L., Corey, R. B. and Branson, H. R. (1951) Proc. Nat. Acad. Sci., Wash. 37, 205.

and Corey, R. B. (1951) Proc. Nat. Acad. Sci., Wash. 37, 261.

- - (1951) Proc. Nat. Acad. Sci., Wash. 37, 251.

(1953a) Nature, Lond. 171, 59.

(1953b) Disc. Faraday Soc. 13, 170.

Symposium on Protein Structure, p. 17. Ed. by A. Neuberger, Methuen, London, 1958.

Pautard, F. G. E. (1958) Nature, Lond. 182, 788.

- (1961) Arch. oral Biol. 3, 217.

Peacock, N. (1959) Biochim. biophys. Acta 32, 220.

Pearse, A. G. E. (1951) Quart. F. micros. Sci. 92, 393.

- Histochemistry, Theoretical and Applied. Churchill, London, 1953.

Pelc, S. R. (1959) Exp. Cell Res. Suppl. 6, 97.

- (1958) Exp. Cell Res. 6, 105.

Perutz, M. F. (1951) Nature, Lond. 168, 653.

— and Huxley, H. E. (1951) Nature. Lond. 167, 1053.

- (1959) Research 12, 8-9, 326-334.

Peters, L. in Fibrous Proteins, Soc. Dyers and Col., p. 138; Chorley \& Pickersgill, Leeds, 1946.

— and Speakman, J. B. (1949) f. Soc. Dyers Col. 65, 63.

- and Woods, H. J. in The Mechanical Properties of Textile Fibres, p. 153. Ed. by R. MEREDITH, North Holland, Amsterdam, 1956.

Peters, R. A., Sir in Perspectives in Biochemistry, p. 36. Ed. by J. Needham and D. E. Green, Cambridge University Press, 1937.

Philip, Bo., Lagermalm, G. and Gralen, N. (1951) Biochem. biophys. Acta 6, 497.

Pillai, P. A., Guenin, H.-A. and Gautier, A. (1960) Bull. Soc. Vaud. Sci. Nat. 67, 215.

Pillemer, L., Ecker, E. E. and Wells, J. R. (1938) Science 88, 16; (1939) Y. exp. med. 69, 191. 
Pinkus, F. Die normale Anatomie der Haut. Handbuch der Haut und Geschlechskankeiten. Vol. 1, Part 1. Ed. by JaDassohn S. Karger, Berlin, 1927.

Pinkus, H. (1951) J. Invest. Dermatol. 16, 383.

Policard, A. and Collett, A. (1959) Rev. franc. Etud. clin. biol. 4, 283.

Porter, K. R. (1954) Proceed. of Internat. Conf. Electr. Micros., London, p. 539. Ed. by R. Ross, 1956.

(1957) Harvey Lectures 51, 175.

(1954) f. Histochem. Cytochem. 2, 346.

Price, D. (Editor) Dynamics of Proliferating Tissues. The University of Chicago Press, Chicago, 1958.

Pringle, J. W. S. (1951) Behaviour 3, 174.

Pryor, M. G. M. (1940) Proc. Roy. Soc. B128, 378, 393.

Puchtler, H. and Leblond, C. P. (1958) Amer. F. Anat. 102, 1.

Puck, T. T. in Cellular Biology, Nucleic Acids and Viruses Special Publ. N. Y. Acad. Sci. Vol. 5, p. 293, 1957.

Rabinovitz, M. and Olson, M. E. (1957) Fed. Proc. 16, 235.

RANDALl, J. T. (Editor) Nature and Structure of Collagen. Butterworths, 1953.

Ranvier, E. (1879) C. R. Acad. Sci., Paris 88, 1361.

Rashevsky, N. Mathematical Biophysics. Revised edition, University of Chicago Press, 1948.

Rawles, M. (1947) Physiol. Rev. 28, 383.

Reed, R. and Rudall, K. M. (1948) Biochim. biophys. Acta 2, 7.

ReEs, A. L. G. (1951) F. phys. coll. Chem. 55, 1340.

Reeve, E. C. R. and Huxley, J. S. in Essays on Growth and Form, p. 121. Ed. by W. E. Le Gros Clark and P. B. Medawar, Clarendon Press, Oxford, 1945.

Reinberg, A. and Ghata, J. Rythmes et Cycles Biologiques. Presses Universitaires de France, Paris, 1957.

Rich, A. and CRICK, F. H. C. (1955) Nature, Lond. 176, 915.

Richards, A. G. The Integument of Arthropods. University of Minnesota Press, Minneapolis, 1951.

Richards, O. W. and Kavanagh, A. J. in Essays on Growth and Form, p. 188. Ed. by W. E. Le Gros Clark and P. B. Medawar, Clarendon Press, Oxford, 1945.

Robertson, J. D. (1956) F. biophys. biochem. Cytol. 2, 381; (1957) Ibid. 3, 1043; (1958) Ibid. 4, 349.

- (1957) Biochem. Society Symposium, No. 16, p. 1. Ed. by E. M. Crook, Cambridge University Press, 1959.

Robinson, C. The Nature and Structure of Collagen. Butterworths, London, 1953.

- and Ambrose, E. J. (1952) Trans. Faraday Soc. 48, 854.

Rogers, G. E. (1953) Quart. F. Micr. Sci. 94, 253.

- (1959a) Ann. N. Y. Acad. Sci. 83, 378, 408.

- (1959b) F. Ultrastr. Res. 2, 309.

Romer, A. S. The Vertebrate Body, 2nd Ed. Saunders, Philadelphia, 1955.

Rose, S. M. (1952) Amer. Nat. 86, 337.

Rothman, S. Physiology and Biochemistry of the Skin. University of Chicago Press, 1954.

Rougvie, M. A. Ph.D. Thesis, M. I. T., 1954, cited by A. M. Woodin (1955) Nature, Lond. 176, 1117. 
Rudall, K. M. Ph.D. Thesis, Leeds, 1936.

- (1941) Proc. Leeds phil. lit. Soc. 4, 13.

in Fibrous Proteins. Symph. Soc. Dyers and Col. Chorley \& Pickersgill,

Leeds, p. 15, 1946.

- (1947) Biochem. biophys. Acta 1, 549.

- (1952) Advanc. Protein Chem. 7, 253.

- (1953) (Discussion) Proc. Roy. Soc. B141, 39.

- (1955) Symp. Soc. exp. Biol. 9, 49.

- (1955) Proc. Int. Wool Text. Conf. Austr. Vol. F., p. 176; C.S.I.R.O., Melbourne, 1956.

- (1955-6) Lect. Sci. Basis Med. V, 217. Athlone Press, London, 1956.

Ryder, M. L. (1955) Proc. Wool Text. Conf. Austr. Vol. F., p. 63, 1956.

— (1956) F. Agric. Sci. 47, 129.

- (1957) \%. Agric. Sci. 49, 275.

- in The Biology of Hair Growth. Ch. 14, p. 305, Academic Press, New York, 1958.

_- (1959) Quart. F. Microsc. Sci. 100, 1.

SAlECKer, J. (1944) Morphol. Fahrb. 88, 225.

Schellman, J. A. (1955) C. R. Carlsberg. Sèr. chim. 29, 230.

Scheving, L. E. (1959) Anat. Rec. 135, 7.

Sснмірт, W. J. Die Bausteine des Tierskörpers im polarisierten Lichte. Frederich

Cohen, Bonn, 1924.

- (1925) Mikrokosmos 19, 65.

Schmitt, F. C., Gross, J. and Highberger, J. H. (1955) Symp. Soc. exp. Biol. Fibrous Proteins 9, 148.

Sснмітт, F. O. (1941) Growth 5, 1.

- Advanc. Protein Chem. 1, 25. Ed. by M. L. Anson and J. T. Edsall, Academic Press, New York, 1944.

_- (1957) F. Cell Comp. Physiol. 49 (Suppl.), 85.

- (1958) Proc. 4th Int. Cong. Electr. Micros. Vol. 2, p. 1. Springer-Verlag, Berlin, 1960.

- and BeAr, R. S. (1939) Biol. Rev. 14, 27.

Schneider, K. C., Lehrbuch der vergleichen Histologie der Tiere. Fischer, Jena, 1902. Schor, P. (1958) Ph.D. Thesis, Michigan, 1958; (1958) Diss. Abst. 19, No. 6, 1189. Schroeder, W. A., Kay, L. M., Lewis, B. and Munger, N. (1955) F. Amer. chem. Soc. 77, 3901.

Schulz, H. and DE PAOla, D. (1958) Z. f. Zellforsch. 49, 125.

Scott, D. B. (1955) Ann. N. Y. Acad. Sci. 60, 575.

van Scott, E. J. and Flesch, P. (1954) Arch. Derm. Syph. 70, 141.

Selby, C. C. (1955) J. biophys. biochem. Cytol. 1, 429.

- (1957) f. Invest. Dermatol. 29, 131.

Setala, K., Merenmies, L., Stjernvall, L. and Nyholm, M. (1960) Ұ. Nat. Cancer Inst. 24, 329.

Siekevitz, P. (1952) F. Biol. Chem. 195, 549.

— and Palade, G. E. (1958) F. biophys. biochem. Cytol. 4, 557.

Sikorski, J. (1958) Proc. 4th Int. Congr. Elec. Micros. Berlin. Springer-Verlag, Berlin, 1960.

— and Simpson, W. S. (1959) F. Roy. micros. Soc. 78, 35. 
Simкin, J. L. and Work, T. S. The Biological Replication of Macromolecules, p. 164, Cambridge, 1958; (1958) Symp. Soc. exp. Biol. 12, 164.

(1959) Ann. rev. Biochem. 28, 145.

Simmonds, D. H. (1954) Austr. F. biol. Sci. 7, 98.

- (1955) Austr. F. biol. Sci. 8, 114.

(1958) Text. Res. F. 28, 314.

Simmonds, D. H. and Stell, I. G. (1955) Proc. Int. Wool Text. Conf. Austr.

Vol. C., 75; C.S.I.R.O., Melbourne, 1956.

Sjöstrand, F. S. and Hanzon, V. (1954) Exp. Cell Res. 7, 393.

- Internat. Rev. Cytol. 5, 455. Eds. Bourne, G. H. and Danielli, J. F., New York, Academic Press, 1956.

Skertchley, A. and Woods, H. J. (1960) f. Text. Inst. 51, T517.

Slen, S. B. (1958) Nature, Lond. 182, 1322.

Sock, N. W. and Morales, M. F. (1945) Bull. Math. Biophys. 7, 15.

Speakman, J. B. (1928) Proc. Roy. Soc. B103, 377.

- (1931) Proc. Roy. Soc. A132, 167.

— and Hirst, M. (1933) Trans. Faraday Soc. 29, 148.

— (1934) F. Soc. Dyers Col. 50, 34.

- (1936) Nature, Lond. 138, 327.

- (1947) अ. Text. Inst. 38, T102.

- and Sтотт, E. (1931) J. Text. Inst. 22T, 339.

- (1955) Symp. Soc. experim. Biol. 9, 169.

Spearman, R. I. and Barnicot, N. A. (1960) Amer. F. Phys. Anthrop. 18, 91.

Spier, H. W. and van Caneghem, P. (1957) Arch. klin. u. exptl. Dermatol. 202, 120.

Stainsby, G. (Editor) Recent Advances in Gelatin and Glue Research. Pergamon Press, New York, 1958.

Stephenson, M. L., Hecht, L. I., Littlefield, J. W., Loftfield, R. B. and Zamecnik, P. C. Subcellular Particles. Ed. by T. Hayashi, Ronald Press, New York, 1959.

Stoeckenius, W. (1959) F. biophys. biochem. Cytol. 5, 491.

Stoves, J. L. (1942) Analyst 67, 385.

- (1943) Proc. Leeds phil. lit. Soc. 4, 84.

- (1947) f. Soc. Dyers Col. 63, 65.

- in Fibrous Proteins, Symp. Soc. Dyers and Col. Chorley \& Pickersgill, Leeds, p. $58,1946$.

Strangeways, D. H. (1931) Arch. exp. Zellforsch. 11, 344.

Strong, R. M. (1902) Bull. Mus. Comp. Zool. Harv. 40, 147.

Sutherland, G. B. B. M. (1952) Advanc. Protein Chem. 7, 291.

Swanbeck, G. (1959) F. Ultrastr. Res. 3, 51.

Swann, M. M. (1957) Cancer Res. 17, 727; (1958) Ibid. 18, 1118.

Sylven, B. (1941) Acta Chirurg. Scand. 86, Suppl. 66, p. 1.

- (1950) Exper. Cell Res. 1, 582.

TAYLOR, H. S. (1941) Science 93, 465 (Report).

Thomas, J. A. (Editor) Les Facteurs de la Croissance Cellulaire. Masson, Paris, 1956.

Thompson, D'ARcy W., On Growth and Form, 2nd ed. Cambridge University Press, 1942.

Thompson, E. O. P. (1957) Austr. F. biol. Sci. 10, 225.

__ and O'Donnell, I. J. (1959) Austr. F. biol. Sci. 12, 282. 
ThUringer, J. M. (1924) Anat. Rec. 28, 31.

Tristram, G. R. The Proteins. 1A, p. 220. Ed. by H. Neurath and K. Bailey, Academic Press, New York, 1953.

TURner, C. D. General Endocrinology. Saunders, Philadelphia and London, 1960.

UnNA, P. G. Histochemie der Haut. F. Deuticke, Leipzig, 1928.

Vigneaud, du V. Proc. XI Intern. Cong. Pure and App. Chem., 1947.

Waddington, C. H. (1948) Symp. Soc. exp. Biol. 2, 145.

- The Epigenetics of Birds. Cambridge University Press, 1952.

- Principles of Embryology. Allen \& Unwin, London, 1956.

Waldschmidt-Leitz, E. and Zeiss, D. (1955) Z. physiol. Chem. 300, 49.

Ward, W., High, L. M. and Lundgren, H. P. (1946) F. Polymer Res. 1, 22.

- and Lundgren, H. P. (1954) Advanc. Protein Chem. 9, 243.

Warwicker, J. O. (1954) Acta Cryst., Camb. 7, 565.

- (1959) Nature, Lond. 184, BA25 (Rep. Conf. Inst. Phys. Leeds).

Watson, J. D. and Crick, F. H. C. (1953) Nature, Lond. 171, 737.

Watson, M. L. (1954) Biochim. biophys. Acta 15, 475.

— (1960) F. biophys. biochem. Cytol. 7, 489.

- and Avery, J. K. (1954) Amer. F. Anat. 95, 109.

Waugh, D. F. (1954) Advanc. Protein Res. 9, 325.

Weiss, L. (1960) Int. Rev. Cytol. 9, 187. Ed. by G. H. Bourne and J. F. Danielli. Weiss, P. (1933) Amer. Nat. 67, 322.

- (1945) F. exp. Zool. 100, 353.

- (1950) Quart. Rev. Biol. 25, 177.

- (1958) Int. Rev. Cytol. 7, 391. Ed. by G. H. Bourne and J. F. Danielli.

- and Ferris, W. (1954) Proc. Nat. Acad. Sci., Wash. 40, 528.

- and JAmes, R. (1955) Exp. Cell Res. Suppl. 3, 381.

- Specificity in Growth Control. Ch. 10, p. 195. Ed. by E. G. Butler, Princeton University Press, 1955.

- and Kavenau, J. L. (1957) F. gen. Physiol. 41, 1.

Whewell, C. S. and Woods, H. J. in Fibrous Proteins. Symp. Soc. Dyers Col. p. 50, Chorley \& Pickersgill, Leeds, 1946.

Whiteley, H. T. (1958) \%. Anat. 92, 593.

Wildman, A. B. (1955) Proc. Int. Wool Text. Conf. Austr. Vol. F., p. 157; C.S.I.R.O., Melbourne, 1956.

- (1932) Proc. Zool. Soc. Lond. 2, 257.

- and Manby, J. (1938) Trans. Roy. Soc., Edinb. 59, 333.

Wilkins, M. H. F., Zubay, G. and Wilson, H. R. (1959) f. molec. Biol. 1, 179.

Willmer, E. N. Tissue Culture. Methuen monographs. Revised, Methuen, London, 1954.

Wislocki, G. B. (1951) Anat. Rec. 109, 388.

Wolbach, S. B. (1951) Ann. N. Y. Acad. Sci. 53, 517.

Woodin, A. M. (1954a) Nature, Lond. 173, 823.

- (1954b) Biochem. F. 57, 99.

- (1955) Nature, Lond. 176, 1117.

- (1956) Biochem. F. 63, 576. 
Woods, E. F. (1952) Austr. F. Sci. Res. 5A, 555.

- (1959) Austr. F. biol. Sci. 12, 96.

(1959) Austr. F. chem. 12, 497.

Woods, H. J. (1938) Proc. Roy. Soc. B166, 76.

— (1946) F. Colloid Sci. 1, 407.

Yakel, H. L. (1953) Acta Cryst., Camb. 6, 724.

Young, J. Z. (1950) The Life of Vertebrates. Oxford University Press, 1950.

ZAHN, H. (1947) Naturforsch. 26, 104.

- (1947) Z. f. Naturforsch. 26, 286.

(1949) Kolloid-Z. 113, 157.

Zamecnik, P. C. and Keller, E. B. (1954) F. Biol. Chem. 209, 337.

- Keller, E. B., Littlefield, J. W., Hoagland, M. B. and Loftfield, R. B. (1956) f. cell. comp. Physiol. 47, Suppl. 1, 81.

ZuBAY, G. (1959) F. molec Biol. 1, 186.

Zwilling, E. (1955) भ. exp. Zool. 128, 423. 


\section{Author Index}

Abercrombie, M. 85, 140

Alexander, P. 163, 191, 234, 237, $238,239,247,248,249,252,259$

ALFERT, M. 264

Allfrey, V. G. 264

Ambrose, E. J. $\quad 85,176,196,197,199$, 208

ARNDT, U. W. 194

ARTHUR, R. P. 147

Astbury, W. T. 2, 11 et seq., 27, 121, $123,165,170,172,174,175,180,183$, $189,191,192,193,200,201,203$, 206,246, 254, 257, 258

Auber, L. $73,100,157,159,210,219$, $226,227,268,274,275$

BACHRA, B. N. 131

BAHR, G. F. 35, 36 (Fig. 17), 37

BaIley, K. 121, 200

BAKER, J. R. 37, 55, 115

BAKER, R. V. 281

BALDWIN, E. 50

BAMFORD, C. H. $120,180,183,192$, 197, 199

BARNES, R. J. 10, 191, 213

BARnicot, N. A. 274, 279, 280, 282

BARRNETT, R. J. 29, 49, 51, 217

Battistone, G. C. 78

BAYButT, R. B. 181

BEAR, R. S. 127, 166, 167, 168, 206, 207, 213

Beighton, E. 201, 203

Bell, F. O. 13 et seq., 175, 176

Bellamy, L. T. 198

BENDIT, E. G. 192

BenNeTt, H. S. 29

Bergen, von, W. 7

BergmanN, M. 116

Bern, H. A. 232, 264

BERNAL, J. D. 130

BERNHARD, W. $46,47,111$

BiedermanN, W. $80,214,228$

Billingham, R. E. $62,276,277$

Birbeck, M. S. C. $86,92,99$ (Fig. 44d), $112,114,168,224,227,262,267,275$ $279,280,281,282$
Blackburn, S. 163

BlaIR, S. M. 264

BLASCHKo, H. 281

BLOCK, B. 280

BLock, R. J. 29, 31, 78

BLOOM, W. 80

BOEDTKER, H. 127

BOLLIGER, A. 220, 263

Bolling, D. 29, 31

Borsook, H. 116

BORUM, K. 276

BRACHET, J. 109

Bradfield, J. R. G. 39, 136, 221

BRANSON, H. R. 176 et seq.

Braun-Falco, O. 113, 210, 222

Brody, I. 225 (Fig. 98), 229, 247, 248

Broussy, J. $\quad 30,107$

Brown, A. E. 252, 254

Brown, C. H. 20, 30

Brown, L. 180,183

BRUNET, P. 276, 280

Bull, H. B. 257

Bulliard, H. 64, 217, 231

Bullough, W. S. 134, 135, 136, 144 $148,149,221$

Bunn, C. W. 5, 14

Burge, R. E. 187

Burgos, M. H. 145

Burnet, G. W. 78

Burns, M. 150

Burrows, H. 144

Burt, N. 180

Burte, H. 193, 250

Butcher, E. O. 151,152

CAIRNS, J. M. 60,61

Calvery, H. O. 30

Caneghem, van, P. 263

Carruthers, C. 221

CARTER, H. B. 75,78 (Fig. 35), 150

Cassperson, T. O. 109

Cauna, N. 273

Champetier, C. $30,107,214$

Charles, A. 95, 131

Chase, H. B. $62,64,151,152$

Cherry, C. P. 63 
Chèvremont, M. 29, 217

Clark, W. E. Le Gros 69

ClaARKE, W. H. 75, 150

Clarkson, H. 150

Claude, A. 115

Cochran, W. 176

COE, A. B. 275

CoHEN, 127

Collett, A. 88

Colvin, J. R. 33

Coman, C. R. 85

ConsDon, R. 234

Corey, R. B. 172, 176 et seq., 183

Corfield, M. C. 7, 238, 239, 261

Cowdry, E. V. 80

Crane, H. R. 128

CRICK, F. H. C. $2,109,116,119,177$, 183

Cruise, A. J. 24

Cuckow, F. W. 279

Dalton, A. J. 46,115

DALY, M. M. 264

DAN, K. 30

DANFORTH, C. H. 72,75

Danielli, J. F. 37, 38 (Fig. 18), 47, 93,222

DARMON, S. E. 198

DAs, D. B. 240

Davies, H. R. 104

Davson, H. 37

DAY, M. F. 124, 130

DERKSEN, J. C. 214, 235

Dickinson, S. 200

Donohue, J. 182, 194

DotY, P. 127

DRY, F. W. 156,159

DURWARD, A. 137, 152, 232

DUSENBURY, J. H. 275

EARLAND, C. 239,244

EBLING, F. J. 137, 155

ECKER, E. E. 119

EISEN, A. Z. 230

Elliotr, A. 120, 180, 183, 193, 196, 197, 199, 208

ELLIS, W. J. 236

ELLSWORTH, F. F. 272

ELÖD, E. 252, 257, 259, 260

ENGSTRÖM, A. 230

'EsPinASSE, P. G. 104
FABER, J. 90

FARRANT, J. L. 128, 168, 170, 246, 261

FAurÉ-Fremiet, E. 30,107

FAWCETT, D. W. $39,40,42,83$

FeLIX, M. D. 46, 115

FELL, H. B. 63, 107, 220, 222, 264

FERRIS, W. $86,90,130$

Feughelman, M. 176

Fischer, A. 56

FitzPATRICK, T. B. 276, 280, 282

Fleischhauer, K. 77

FlesCh, P. 230

FLORKIN, M. 26

Fox, S. W. 33

Fraenkel, G. 20

Fraenkel-Conrat, H. 116

Fraser, A. S. $149,150,151,159$

Fraser, R. D. B. 170, 188, 194, 200, 209, 274

FREDERIC, J. 29

FREY-WyssLiNG, A. 3

Garnier, Ch. 47

GAUTIER, A. 83, 263

Geiger, W. B. 252, 254, 266

Gelfant, S. 136

Geren, Ben, B. 47

Ghata, J. 137

Gillespie, J. M. 219, 238, 241, 244

Giroud, A. 64, 214, 217, 231

Glinos, A. D. 140

GlüCKSMANN, A. 63, 94, 276

GODDARD, D. R. 236

Godin, C. 163

Gordon, A. H. 234

Gordon, M. 276

GralÉN, N. 236

Grassé, P. P. 46

Gray, E. G. 19

GRIMSTONE, A. V. 39

Gross, J. 127

Gross, R. 220, 263

GRÜNEBERG, H. 276

Guenin, H. A. 83, 263

Gustavson, K. H. 19

Gutman, M. 257

HAAN, DE, R. L. 85

Hackman, R. H. 20 
HadDOW, A. 151

HADŽI, J. 26

Haguenau, F. 46, 47, 111

Hale, H. P. 107

Hall, C. E. 5

Halsey, G. 193,250

HANBY, W. E. $76,120,176,183,198$, 199

Hanson, J. 82

HaNzon, V. 111,112

HAPPEY, F. 120

HaRdy, J. A. 228

HARDY, M. H. $56,78,81,96,112$, 137,218

HARDY, W. E. 180

HARKNESS, D. R. 264

HARRAP, B. S. 289

HARRIS, M. 252, 254

HarVey, E. N. 38,47

HASELBACH, C. 258

HaURowitz, F. 119

Hausman, L. A. 71

Heaysman, J. E. M. 85

Неснт, L. I. 118

HENDLER, R. H. 118

HERGERSBERG, $\mathrm{H} . \quad 51$

Heringa, G. C. 214

Hermodsson, L. H. 112

Hess, A. 19,78

Heyningen, van, W. E. 135

High, L. M. 207

HildemanN, W. H. 60

HiNGLAIS-GUILLARD, N. 62,264

Hirst, M. 250

Hodge, A. J. 126, 127, 168, 170

HOLFRETER, J. 84, 90

Hoagland, M. B. $111,117,118$

Hooper, C. E. S. 135

HORIO, M. 273

Horstmann, E. 69, 80, 82, 90, 263

Hosker, A. 103 (Fig. 46), 104, 105 (Fig. 47)

HotTA, K. 59

Hudson, R. F. 191, 234, 239, 247, 249

Huggins, M. L. 176, 178, 181

Hughes, T. E. 30

HuXLEY, H. E. 182

HuXley, J. 160

Hyman, L. H. 74
INGRAM, V. M. 34

JACKSON, S. F. $86,127,131$

JACOBSEN, C. F. 234

JAMES, R. 63

JARRETT, A. 228, 231

JEFFREY, G. M. $24,168,170$

JoHnson, E. 89 (Fig. 40), 115

JoHNson, M. 136

JONES, B. M. 279

JoNes, C. B. 236, 237, 243, 272

JuHN, M. 104

Kavanagh, A. J. 160

KavenaU, J. L. 138,140

KAY, L. M. $8,33,180$

KEECH, M. K. 24

KelleR, E. B. 116,120

Kendrew, J. C. 170,194

Kennaway, E. 275

KERR, M. F. 163

KNOOP, A. 263

KoNDO, T. 273

Kreautzer, F. L. 140

KrimM, S. 33, 163, 208

KRishNaM, G. 30

Kukita, A. J. 276,280

LAFON, M. 30

LAGERMALM, G. 261, 267

LASNITZKI, I. 63,64

LAURENCE, E. B. $136,137,149$

LAXER, G. 279

LEACH, S. J. 195

LEBLOND, C. P. $43,54,64,82,84,133$

LEE, G. R. 163

LEES, K. 272

LehmanN, E. 72,265

Lennox, F. G. 241 et seq., 244

Lillite, R. F. 96, 104, 135

LINDBERG, J. 261, 265, 267, 268

LINDERSTROM-LANG, K. 200, 234

LindLey, H. $189,190,244,274$

LinDSTROM, B. 230

LitTLEFIELD, J. W. 111, 118, 120

LITVAC, A. 56

Lоснте, Тн. 71

LoftField, R. B. $111,118,120$

LONGLEY, J. B. 107

LORAND, L. 126 
LOTMAR, W. 24

Low, B. W. 181

LuCAS, F. 180

LUDFORD, R. J. 275

LUFT, J. H. 35

LUNDGREN, H. P. 8, 9, 161, 162, 207, $235,238,243,249,272$

LYNE, A. G. 78

MACARTHUR, I. 166, 170, 182, 183

McDonough, E. G. 254

McLoughlin, C. B. 40,60

MacRae, T. P. 170, 188, 194, 200, 209

Malcolm, B. R. 198

Manogue, B. 267

Manton, I. 39

MARK, H. 172,180

MARSH, R. E. 172

Marston, H. 159, 218, 232

Martin, A. J. P. 234

MARWICK, T. C. $24,170,206,257$

Mason, H. S. 280,281

Matoltsy, A. G. 228, 231, 261, 271

Maximov, A. A. 80

MaZia, D. 30

Меснам, D. K. 7, 236, 243, 272

MEDAK, H. 135

MfDawar, P. B. 276, 277

Meijere, DE 75, 76 (Fig. 33)

Mellanby, E. 63, 220

Menkart, J. 275

Mercer, E. H. 30, 38, 47, 49, 86, 90, $92,99,104,107,112,114,121,124$, $128,129,130,156,166,168,170$, $191,200,209,211,216,224,227$, $230,235,237,238,246,248,259$, $261,262,263,267,268,271,273$, 280

MEYER, J. 135

MEYER, K. 54, 58, 61

MeYeR, K. H. 172, 180, 258

Michaelis, L. 236

MiddlebrooK, W. R. 126, 163

Mirsky, A. E. 264

MISzURSKI, B. 56, 57

MizeLL, L. R. 294

Moberger, G. 36

MOFFITT, W. 195
MoHN, M. P. 135

Montagna, W. 57, 59, 60, 62, 78, 81, $82,96,100,137,210,219,221,222$, 228,282

Morales, M. F. 140

Moran, T. 107

Moscona, A. 44, 84, 85

Moss, M. S. 267

MÚlLER, C. 267

MUNGER, N. 180

Nageotte, J. 127

NAY, T. 151

Neurath, H. 175

Nieuwkoop, P. D. 90

Nilsson, O. 62

NiU, M. C. 276

Noback, C. R. 75, 76 (Fig. 33)

NORRIS, M. H. 150, 156

Odland, G. F. 219, 263

O'DonNELl, I. J. 219, 235, 238, 240, 243, 244

Olofsson, B. 236

Olson, M. E. 117

Oparin, A. I. 28

Osawa, S. 264

OSTER, G. 5

OtToson, D. 90

Palade, G. E. $35,46,81,111,114$, 115,118

Parker, K. D. 199, 201, 208

Patterson, W. I. 252

Pauling, L. 128, 172, 176 et seq., 181, 183

Pautard, F. G. E. 20,79

PEACOCK, N. 200

Pearse, A. G. E. $29,54,80$

Pelc, S. R. 220, 264

Perutz, M. F. 2, 165, 182, 194, 202

Peters, L. 193, 176, 250

Peters, R. A. 114

Philip, B. 261, 267, 268

Picken, L. E. R. 24

Pillai, P. A. 83, 263

Pillemer, L. 119

Pinkus, F. 77

PINkUs, H. 148 
Policard, A. 88

Pollister, A. W. 5

Porter, K. R. 39, 47, 48, 86, 90, 93, $94,114,168$

Price, D. 135,143

Puchler, H. 43

Puck, T. T. 57

Queveno. W. C. 221

Rabinovitz, M. 117

RANDALL, J. T. 127

RANVIER, E. 95

RASHEVSKY, N. 140

Rawles, M. 276

REED, R. 124

ReEs, A. L. G. 126, 168, 170, 246 261

REEVE, E. C. R. 160

REINBERG, A. 137

RichaRDS, A. G. 27

RICHARDS, O. W. 160

RILEY, D. P. 194

RoBB 160

RoBertson, J. D. $38,41,47$

RoBinson, C. 127,199

Robson, A. 7, 238, 261

ROE, E. 151

Rogers, G. E. $97,168,170,186,188$, $221,226,230,231,236,237,247,248$, $262,268,274,275$

ROMER, A. S. 49

Rose, S. M. 92, 111, 142

Rothman, S. 59, 136, 222, 230

Rougvie, M. A. 207

Rudall, K. M. 11, 19, 20, 21, 24, 51, $67,72,73,104,106,121,124,126$, $137,150,151,152,157,166,200$, $204,205,214,217,218,231,232$, $233,235,248,260,265,275$

Rugo, H. J. 166, 167, 206, 207

RYDER, M. L. $137,150,219,221,232$, 23.3

SALECKer, J. 90

SAUNDERS, J. W. 60,61

Scheving, L. E. 135

Schmidt, W. J. 5, 10, 43, 211, 213, 226

Sснмiтt, F. O. 2, 100, 127, 213

SCHNEIDER, K. C. 43
Schor, P. 33, 163, 166, 169 (Table 10), 208

SCHROEDER, W. A. $8,33,180$

Schulz, H. 48, 145

ScotT, D. B. 78

ScotT, vaN, E. J. 230

SeIJI, M. 281

Selby, C. C. 86,90

Seligman, A. M. 217

SETÄLÄ, K. 94

Shaw, J. T. B. 180

SHORT, B. F. 149,150

Siekevitz, P. $115,116,117,118$

SikoRSKI, J. 168, 170, 225, 265

Simkin, J. L. 111, 118

Simmonds, D. H. 7, 32, 219, 241, 275

SiMPSON, W. S. 225

Sjöstrand, F. S. $37,47,48,90,111$

SKERTCHLEY, A. 176

SKINNER, B. 238, 261

Slen, S. B. 149

SMIDDY, M. B. 95, 131

Sмith, L. F. 163, 239

SMITH, S. G. 180

Sobel, A. E. 131

Sock, N. W. 140

Speakman, J. B. 73, 172, 193, 234. 240, 249 et seq., 252, 260

Spearman, R. I. 228, 231, 274

SPELley, A. 147

SPIER, H. W. 263

Stainsby, G. 127

Stanford, J. W. 131

Stell, I. G. 241

STENSTRÖM, S. 90

Stephenson, M. L. 118

Stoeckenius, W. $38,47,48$

STOREY, W. F. 133

Sтотт, E. 73

Stoves, J. L. 72, 257, 265

Strangeways, D. H. 56

StREET, A. 172, 191

Strong, R. M. 104

Sutherland, G. B. B. M. 198

SvaEtichin, G. 90

SWANBECK, G. 231

SwANN, M. M. 140

SylvÉN, B. 221, 233

SYNGE, R. L. M. 234 
TAYLOR, H. S. 181

Thomas, J. A. 134

Thompson, D. W. 67

Thompson, E. O. P. 219, 240, 243

Thuringer, J. M. 82

Timmis, G. M. 151

Tristram, G. R. 6 (Fig. 2), 33, 161

TrotTer, I. F. 180,183

TURNER, C. D. 22

UNNA, P. G. 271

VAND, V. 177

VICKERY, H. B. 29

Vigneaud, DU, V. 232

WADDINGTON, C. H. 60, 85, 104, 143

WALDSCHMIDT-LEITZ, E. 180

WARD, W. $8,9,161,162,207,235$, $238,243,249,272$

WARWICKER, J. O. 180,192

Watson, J. D. 109, 112

Watson, M. L. 48, 108, 112

WAUGH, D. F. 128

Weibull, C. 201, 203

WEIDINGER, A. 214

WeInManN, J. P. 135

WeIss, L. 41
WeIss, P. $\quad 39,60,63,83,84,86,90,93$, $130,138,140$

WELLS, J. R. 119

Whewell, C. S. 258, 259, 279

Whiteley, H. J. 116

Wildman, A. B. $71,76,157,159$

WiLkins, M. H. F. 110

Willmer, E. N. 44, 57

Wilson, H. R. 110

Wiseman, A. 244

WisLOCKI, G. B. 84

Woernley, D. L. 286

Wolback, S. B. 276

Woodin, A. M. 163, 207, 238

Woons, E. F. $196,219,235,238,243$, 244

Woons, H. J. $\quad 72,168,170,172,174$, $175,176,183,191,192,193,246$, $255,257,258,259,260$

WORK, T. S. 111,118

YAKEL, H. L. 183

Young, J. Z. 49

ZAHN, H. $252,257,259,260$

ZAMECNIK, P. C. $111,116,117,118$

ZEISS, D. 180

Zubay, G. 110, 201

ZWILLING, E. 60

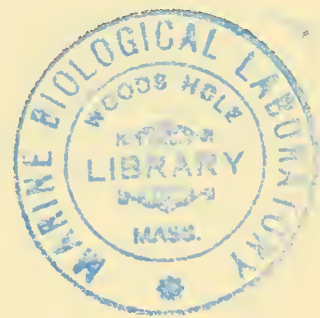




\section{Subject Index}

Acid mucopolysaccharides in hair follicle 220 (Fig. 97), 221

ACTH 54

Adaptation of epidermal thickenings 66

Adenosinetriphosphate (ATP) and mitochondria 116

Aggregation of tropocollogen 128 (Fig. 55)

Allometric growth 159

Allwörden reaction 267 (Fig. 111)

Amide bond, structure of 7 (Fig. 73), 176

Amino acid composition of low S extract of wool 237 ('Table 14)

- - content of fibrous proteins (silk, collagen and wool) 6 (Table 3)

Amphibians, keratinization in 51

Antigenicity of keratins 119

Antlers 67

Arthropodin 20

Astbury-Bell model for $\alpha$-keratin 176 (Fig. 72)

Avian secreted keratins 30,107

Bacterial flagella, protein of 2, 201 Basal layer cells, division of 82

- of keratinizing tissues 80

- membranes and epithelia 53, 90

- in electron microscope 88 (Fig. 39)

Beaks and bills 66

Benzpyrene on epidermis 275

— in skin 94

Biochemical evolution 24, 26, 49

Biochemistry of protein synthesis 115 et seq.

Birefringence 10 (Table 4)

- and stability 21

- changes on stretching hairs 2, 191

— in hair follicle 213 (Fig. 91)

- in swollen cuticle 226

- intrinsic and form 211, 213

- of Stratum corneum 230

Bombyx mori, synthesis of silk by 121

- silk 180

- molecular structure of 179
Bonds consolidating keratins 21, 27, $234,245,249$

Bragg's Law 12, 13

Calcium salts and collagen 20,21, 130

Carcinogenesis tests using mouse skin 275

Carcinogenic hydrocarbons on skin 94,275

Cell adhesion and cell membranes 40 , 84,260

- - in basal layer 82,83 et seq.

- contacts 40 et seq. (Fig. 20)

- during differentiation 84

- in hair follicle 92 (Fig. 41)

- membranes as $\beta$-keratose 43,260

- in keratinized tissues 238

— of basal layer cells 83

- surface 37 et seq.

Cellular adhesion during differentiation $84,90,99$

- in cancer cells 85

- in embryo 84

- behaviour in cine films 85

Cetyl sulphonic acid extract (CSA) 244

- of wool 245 (Table 19)

Chain configuration ( $\alpha$ or $\beta$ ) from infrared spectra 197 et seq.

Chemical difference between $0-$ and p-type keratin 275

- modifications during keratinization (other than cystine formation) 219

- reactivity and chemical composition of keratins 161, 162 (Table 7)

- structure of keratins, present status 161 et seq.

Chitin 20, 24

Chlorine peroxide as disulphide bond oxidant 240

Chrysopa egg stalk 201, 204

Cilia and flagella 39

Citrulline in inner root sheath 226

— in medulla 270

— in trichohyalin 97

Classification of hairs 72 
Claws 69

- cutting edge and uneven keratinization 273

" Club" hair 89 (Fig. 40)

Coiled coils and $\alpha$-helices 183, 184 (Fig. 77)

Collagen 2, 16, 19, 31, 53

- appearance in electron microscope $19,21,168$

- as a secreted fibre 121

- fibre formation of 127

- - heat contraction of 256

- in insects 19

- meshworks 130

- molecular structure of 127

Colleterial glands in insects, proteins of 5,204

- structure of 205 (Fig. 87)

Comparative cytology of cells forming protein 113 (Fig. 50)

Competition, as control factor in growth 149,159

Components of keratinized tissue revealed by chemical extraction 271

Composition and properties of keratoses 239 ('Table 15)

- of rabbit fur and medulla 270 (Table 20)

Concentric membranes, whorls, myelinic forms in cytoplasm 46

Configuration of polypeptides in synthesis 119

Contact inhibition of cell movement 85

Control diagram of hair growth (Chase) 152, 153 (Fig. 63)

- of growth by inhibitors 140 et seq.

Cortex of hair 71, 72, 101

- electron microscopy of 101, 211 et seq., 223

Cortical cells and elasticity of hairs 174

- in hair follicle 4, 223

- of hair and wool 72

- of wool, extensibility 174

Cortisone 54

Crimp, and bilateral structure of wool 273

- formation, theory of 157, 158 (Figs. 66, 67)
Crimp, in wool 156 et seq.

Cross-linkages of protein(s) 19 et seq.

- reformation by alkylation 3, 252

Cross-linking of epidermin (prekeratin) 235

Cross $\beta$-pattern 200 et seq. (Fig. 84)

- explanation of 201

- from bacterial flagella 202

- from insect egg stalks 201

- from muscle 201

Crystalline/amorphous ratio 191

Crystallites, stabilizing effect of 19, 255

- and supercontraction 256

- and X-ray diffraction 12, 256

Crystallographic analysis of keratins 176 et seq.

Curls from curved follicles 156

Cuticle cells, in keratinization 224

- - of hair 72

_ - fine structure of 225 (Plate 20A)

- - action of osmium tetroxide 225

- of hair 71, 72, 98, 99, 265

- extensibility of 265

- structure 266 (Fig. 110)

Cuticular keratin, characteristics 101, 225,265

Cybernetics 145, 157, 158, 159

Cycles in mouse and human skin 89 (Fig. 40), 96, 136

Cyclic activity, generated by feed-back, 141, 145 (Fig. 60)

- changes in vaginal epithelium 135, 136

- growth (rhythms) in feather follicle, 106

Cyclostomes, and mucinogenic glands 59

- teeth of 75

Cystine 21, 29, 214 et seq., 230

- cross-linkages, location of 234, 245 et seq., 252

- - absent from crystallites 246

- formation in follicle 232, 245

- in cuticular keratin 226

- reaction with osmium tetroxide 35,36

Cysts, keratinized 275

Cytology, of cells synthesizing protein 108 et seq., 110 (Fig. 49) 
Cytology of melanocyte 280

Cytoplasmic structures 45 et seq.

Cytoskeleton 114

Degrees of keratinization, 64, 68, 272

- method to estimate 272

Density and crystallinity 194

Dental keratin 78, 108

Dentritic cells, 277, 278

Dermal changes during hair growth 152

Dermis 53

- configuration of 55,81

Dermoepidermal junction 53, 86, 96

- formation of 88

Desmosomes (dermo-epidermal) 41, 42 (Fig. 21), 93

- composition 84

— in light microscope 83

- of basal layer cells 83

Desoxyribonucleic acid (DNA) 54, $80,109,177$

— in hair follicle 219,220 (Fig. 97)

Desquamation of epidermal cells 22, 134, 231

Deuterium exchange 200

Development of stability in hair follicle 214, 215 (Fig. 92), 216 (Figs. 93 and 94)

Dichroic ratios and amorphous phase 198, 199

- after deuterium substitution 199

— in infra-red 196

Dichroism of infra-red absorption and structure of $\alpha$-keratin 176, 196 et seq.

Differentia in cytoplasm 48

Differentiated epidermal tissues 94 et seq.

Differentiation 44

— of epidermal cells $60,90 \mathrm{et} \mathrm{seq}$.

- of surface organelles 44

Difficulties in defining keratins 30 et seq.

Diffusion of pigment from grafts 278

Digital tips 69

Directional friction, and the hair cuticle 73

— and felting 73

Disintegrated wool, electron microscopy of 246
Dispersion of optical rotation and chain configuration 195, 198

Distribution of disulphide groups in hair follicle 218, 220 (Fig. 97)

- of fibre types in vertebrates 23 , 25 (Fig. 12)

- of fundamental fibre types 22 et seq.

Disulphide bonds, and elasticity 252

- and strength of wool fibres 254 (Fig. 107)

- content of stratum corneum 230

Diurnal cycle(s) 136

- absence of in hair follicle 137

- of mitosis in epidermis 148

Dopa 280

Drude's equation 195

Earthworm cuticle 124,130

Ecdysis 22, 160

— horns and scales 67

Egg case of mantids 126

- shell membranes 107 (Plate 18B)

Elastic behaviour of wool and hair 172

- properties, and the structure of hair 172 et seq.

- of wool and humidity 250 (Fig. 103)

- - and temperature 251 (Fig. 104)

- - and $\mathrm{pH} 253$ (Fig. 105)

Electron microscopy and cytology 34 et seq.

- methods 35

- of cuticle 265 (Plate 20A)

- of fibrous keratin 246 (Plates 12, $13,14 \mathrm{~A}, 15,16)$

- of skin 94, 223 (Plates, 4B, 7, 8, $9,17,22)$

"Eleidin " 95 (footnote)

Embryonic cells, generalized structure of 81,82

Enamel of teeth, keratin in 78,108

End groups in keratins 161, 162

(Table 8), 163

- methods 163

Endocuticle of hair 265

Endoplasmic reticulum 46, 114

- nomenclature 48 (Fig. 23)

Entropy and fibre extension 257

— and molecular configuration 255 
Enzymatic digestion of cuticle 266 (Fig. 110)

- of hair 271

- of reduced wool 254

Epicuticle of hairs 265, 267

Epidermal family of cells 57 et seq. (Fig. 26)

- fibrils, organization of 131,132 , 229 (Plate 17)

- glands 57 et seq.

- growth 133 et seq.

- - control of 146 et seq.

- - effect of inhibitors 147

- - stimulated by stripping, blood supply and Thorium-X radiation 149

- keratin, fine structure of 228 et seq. (Plate 17)

- pigments 276 (Table 21)

- thickenings 66, 134

Epidermin and reduced keratins 238

- preparation and properties 235

- sedimentation analysis of 236

Epidermis 55 (Fig. 25)

- fine structure of 94

- - of keratinizing layers 228, 229 (Fig. 99)

— idealized form 56

- local control of morphogenesis 96, 146

Epithelia, differentiation of 90 et seq.

- nutrition of 55,56

Ergastoplasm 46, 48 (Table 23) (Plate 10A)

Exocuticle of hair 265

Extensibility of feather 24, 257

— of hairs 174, 175

- - molecular basis 172,175

Extracellular keratins $\quad 30,107$

Feather(s) 69, 70 (Fig. 30)

- amino acids in 8 (Table 2)

- and scales, homologies 69

- cells, fine structure 104 (Plate 12A)

- cyclic polypeptides 163

- extracts 238, 243

- fibrils 224

- follicle 101 et seq., 102 (Fig. 45), 103 (Fig. 46), 105 (Fig. 47)

- keratin 33, 34
Feather(s), keratin, evolution 24,25

- - solutions 207

- - structure of 205 et seq., 207

(Fig. 88), 209 (Fig. 89)

- - X-ray diffraction data 169

(Table 10), 171 (Table 11)

- monomeric unit of 163,164

- surface cells 73

- thiol groups in follicle of 218 (Fig. 96)

Feed-back, and oscillations 141, 142

- control of growth 140

- control in follicle 152, 153 (Fig.

63), 158,159

- controls 141

- intracellular 141

Felting 73

Feulgen test 54, 220 (Fig. 97b)

Fibre forming properties of extracted keratins 244

Fibrillar structures of epidermis 230 (Plates 17 and 22)

Fibrils of fibrous keratin 187

- formation and X-ray pattern 223

- internal structure 187, 188 (Fig. 81) (Plates 15 and 16)

Fibrinogen 121

Fibrinogen-fibrin system 126

Fibroblasts in dermis 92 (Plate 23B)

Fibrogenesis 125 et seq.

Fibrous texture 123

Filament-plus-matrix model 193, 247 (Fig. 102), 248

Filaments, and fibrils in hair follicle 223 (Plates 13, 15 and 16)

- in $\alpha$-proteins found electron microscopically, 185

$\alpha$-Filaments 183

- and $\alpha$-fibrils, definition 186 (Fig. 80), 187

— characteristics 248

- structure 186

- - organization into larger units 187

Fine histology of hair follicle 95, 223 et seq.

- structure of cells 34 et seq.

Flagella of bacteria (X-ray pattern) 187, 202 (Fig. 86), 203

Flagellin 203 
Fleece mosaics 147

Fluorescent dyes and keratinization 228,231

Folding of polypeptide chains 172

- elasticity of 175

Follicular activity $89,96,151$

- nutrition 232

Gizzard, keratin of 107,108

- of birds 30, Plates 18 and 19

Glandular cells, cytology of 109 et seq., 110 (Fig. 49), (Plate 10A)

Glycogen in epidermis 136, 221

- in outer root-sheath of hair follicle 137,221

- - and growth 221

Golgi apparatus 45 (Fig. 22e), 46, 115

- in basal layer cells 81,82

- in cuticle cells in follicle 224

Grafting of epidermal tissues 60 et seq.

— of pigmented skin 278

- of skin, effect on hair growth 152 , 155

Granular layer of epidermis 95 et seq., 228 et seq. (Plates 22 and 23A)

Growth, factors influencing $134 \mathrm{et} \mathrm{seq}$.

- general theories of 138 et seq.

- limiting factors in 140

- of epidermal structures 133

- theory of Weiss and Kavenau 140 et seq. (Fig. 57 and 58)

- waves, explanation in terms of accumulation of inhibitors 154 (Fig. 64)

- - as graded response to stimulant 155

- - in hair 151 et seq.

Hair(s) 71 et seq. (Fig. 31)

— cuticle, development of 98 (Fig. 43)

- - fine structure in follicle 224 (Plate 20A)

- - structure of 265 et seq.

- cycles 96, 150 et seq.

— — in rat 89 (Fig. 40)

- elastic properties 249 et seq. (Figs. 103-107)

- follicle 72

- - diagrams of histochemical reactions in 211 et seq.
Hair(s), follicle, fine structure 95 et seq., 223 et seq. (Plates 11, 12, 13, 14A, 15)

- - groups 75 et seq. (Figs. 33-35)

- - in tissue culture 96

- human, long spacings from 167

- waving treatments 254

Heat-moisture treatment (Bear and Rugo) 167

- of feather 206

Helical, aggregation 128

- configurations, the $\alpha$-helix 181

- content by optical methods 194 et seq.

_ - of various proteins 195

- - of wool extracts 196

- structures 176 et seq.

$\alpha$-Helices 181 et seq. (Figs. 75, 76)

- extensibility 188, 189 (Fig. 82)

- $\alpha$-filament and electron microscopy 185, 186 (Fig. 79)

- in synthetic polypeptides 183

$\beta$-Helices 209 (Fig. 89)

Henle's Layer during hair growth 224

- in hair follicle 96, 97 (Fig. 42), 226 (Plates 20 and 21)

- properties of 227

Histology of hair and elastic behaviour 174

- of vertebrate epidermis 53 et seq., 80 et seq., 228 et seq.

Holocrine glands 59

Homogenization of tissues 115 et seq. Hoofs 69, 273

Hooke's law region in stress-strain curve of hair 173

Horns 67, 68 (Fig. 29)

- and size of animal 160

- keratinization 2, 217, 231 (Fig. 95)

Huxley layer in hair follicle 97 (Fig. 42), 100

Hyaluronidase 54

Hydrogen bonds, and elastic properties 254

- and infra-red spectra 197

- and protein structure 178

- stabilization by 234

Hydroxyproline, in collagens 6

- in tooth enamel keratin 78 
Ichthyosis vulgaris 64

Inaccessibility and crystallinity 191

Infra-red spectra, and chain configuration 197, 198 (Table 12)

- and structure 196

- of feather 208

—of keratins 197 (Fig. 83)

Inhibitors of growth 140 et seq.

- as hormones 143

- and growth hormones as mitotic controls 148 (Fig. 61), 149

Inner root sheath of hair follicle, fine structure of 95 et seq., 226 et seq.

Insect proteins ( $\alpha$ and $\beta$ types) 203 et seq.

Insulin (fibrous) 29, 128

Interaction between dermis and epidermis 61 (Fig. 27), 86 et seq., 87 (Fig. 38), 88 (Fig. 39)

Intercellular adhesion 40, 44, 81, 84 et seq., 90 et seq., 99 et seq., 98 (Fig. 43), 99 (Fig. 44)

-- and differentiation $60,61,90$, 99

- apparatus during keratinization 263

- "bridges" (desmosomes) 42, 83, 94,260

- cements 85

- in electron microscope 86

- contacts in hair follicle 99 (Fig. 44), 100

Interdigitation of membranes 43, 263

Interfollicular competition in sheep 149,150

Internal energy and entropy 257

Intracellular differentiation in hair bulb 100

Invertebrates, keratins of 30

- tanning in 27

Kerateines and keratoses 241 et seq., 244

- - compositions compared 245

(Table 19)

Keratin, A and B (Unna) 2, 271

- chemical analyses of 6 (Fig. 2), 7 (Table 1) 8 (Table 2), 9 ('Table 3), 161 (Tables 7, 8), 241 (Fig, 100), 242 (Table 17), 243 (Table 18), 245 (Table 19)
Keratin definition of 2, 21, 27 et seq.

— hard 64 et seq., 210

- hard and soft 64 et seq., 65 (Table 5), 134, 228, 272 et seq.

Keratin, hard and soft, in claws and hoofs 69,273

- histological tests for 29

- in hair cuticle cells 101 (Plate 20), 265

- in tooth enamel 78,108

— " pearls" 275

$\alpha$-Keratin pattern (Macarthur) 166

(Fig. 68)

- wide angle 15,170 et seq.

- characteristic $1.5 \AA$ spacing of 15 , 176,182

$\beta$-Keratin, as a pleated sheet 179,180

_ in feather follicle 69,104

- in supercontracted fibres 258

Keratinization in bird skin 63, 231

- in curved follicles 157

— in skin and scales of rodents 231

- in hair follicle 210 et seq.

- not a degenerative phenomenon 56

- process 210 et seq.

- - definition of effects 210

- zone 55 et seq. (Fig. 25), 56, 157

(Fig. 66), 211, 223

Keratinized cysts and epidermal tumours 6, 275

Keratohyalin 65 (Table 5), 95, 228 et seq.

- and trichohyalin 226, 227

- conversion into fibrils 230 et seq. (Plate 21)

- granules in stratum granulosum 228

Keratoses ( $\alpha$ and $\gamma$ ) 237, 238, 240 (Table 16)

$\mathrm{k}, \mathrm{m}, \mathrm{f}$. . proteins 15,19

Krebs cycle intermediates and mitosis 136

Lanthionine 237

Levels of organization 3, 4 (Fig. 1), 10, 123 et seq., 125 (Fig. 54)

Lillie and Juhn's hypothesis 105

(Fig. 47)

Limulus 30 
Lipids in keratinizing tissues 59, 222 Liver, growth after hepatectomy 140 Location of cystine residues 245 et seq. Logistic law of growth 139

Long spacings 11,165 et seq.

- and short spacings, independence of 166

- in electron microscope 19,167

- theories of origin 170

Low angle diffraction patterns 11, 165 et seq.,

—of keratins $166 \quad$ (Fig. 68), 168 (Fig. 69)

Macrofibrils in fibrous keratin 123, 125, 187, 186 (Fig. 80), 224 (Plates $13,14 \mathrm{~A}, 15,16)$

Macromolecules and biology 1

Malpighian layers and cells 57,80 et seq.

Mammary glands 59

Matrix, in electron micrographs 223, 224

- of fibrous keratin ( $\gamma$-component) 248

- plus filament model and X-ray scattering 4, 193

- protein ( $\gamma$-keratin) as interfilamental cement 188, 223, 246 et seq.

Mechanical trauma as growth stimulant 149

Mechanochemical phenomena 249 et seq.

Medulla of hair $71,73,97,268$ et seq.

Melanin 276 et seq.

Melanization, chemistry of 279,280 , 281

Melanocyte(s) 27

- activity and hair cycle 282

- and keratinizing cells 278

- and pigmentation 276 et seq.

— in hair follicle 97 (Fig. 42), 100

Membraneous systems of cytoplasm, definition 46

- nomenclature of 47,48 (Fig. 23) (Plates 4, 5, 6, 7, 10)

Membrane(s) adhesion and morphogenesis 84 et seq.

- - in hair follicle 97 (Fig. 42), 98 (Fig. 43), 99
Membrane(s) as tubules derived from cuticle of hair and wool 267 et seq.

- chemical character 261

- enclosed particulates 45

- in keratinized tissues 260

- morphology 262

Metabolic enzymes in keratinizing tissues 221

Metachromasy 54, 221

Metaplasia 62, 63, 233

- cyclic 144 (Fig. 59)

Microbodies in keratinizing tissues 233 (Plates 22, 23A)

Microsomes 111 et seq.

Mitochondria, definition 45

- in basal layer cells 81

Mitochondrial enzymes 115, 116, 221.

Mitosis in basal layer 82, 135 et seq.

Mitotic apparatus 30

Modulations 63

Molecular, and macromolecular structures $4,119,123,161$

- configuration in supercontracted state 258 et seq.

- macromolecular and histological units of fibrous keratin 186 (Fig. 80)

- structure of degraded keratins 163 et seq.

Mouse, diurnal cycles in epidermis 136

- ear epidermis, mitosis control in 149

- skin 94

Mucinogenic cells, cyclic changes in 145

Mucopolysaccharides 54, 58, 61, 84, 221

Muscle, X-ray pattern of 15,168

Myelinic forms 46

Myosin (actomyosin) 15, 121, 168

Nails, nature of 69

- thiol reaction in 217 (Fig. 95)

Neurokeratin 79

Non-crystalline fraction 190 et seq.

- molecular structure in 192

— in silk 192

Non-keratinous residues 270 et seq.

—as $\beta$-keratoses 238 
Non-keratinous residues, morphology of 266 (Fig. 110), 268( Fig. 112)

Nuclear membrane 48,112

— in keratin cells (Plate 4C), 80, 81 (Fig. 36)

- pores 48, 112 (Plate 4C)

Nuclei, fate of in keratinizing tissues 4, 263

Nucleic acids 109

- distribution in hair follicle 112, 219, 220 (Fig. 97)

Nucleoli 80, 81 (Fig. 36), (Plates 7, 8)

Nucleus $48,80,81$ (Plates $7,8,12,13$ )

- and protein synthesis 109 et seq., 110 (Fig. 49), 264

- pores Plate $4 \mathrm{C}$

Oestrogens and cell division in epidermis 134

Optical rotation and chain configuration 194 et seq.

Orders of magnitude 3,4 (Table 1 )

Organization of fibrous tissues 129 et seq.

Orientation in hair follicle by X-ray diffraction 214

- - origin of 131

— of fibrils in epidermis 231 (Plates 17, 22)

- of fibrous keratin in hair follicle 210 (Fig. 90), 211

Ovokeratin 30, 107 (Plate 18B)

Ortho- and paracortex of wool fibres 268 (Fig. 112), 273

Osmium tetroxide, fixation by 35,36

(Fig. 17), 112

- reaction with cystine $35,36,247$

(Fig. 102)

Osmoregulation and cuticles 50 (Fig. 24)

Oxidation of keratins to keratoses, etc. 238 et seq.

- as histochemical test 29

Particle covered membranes (endoplasmic reticulum) 46

- in secretory cells 111 (Plate 10A)

PAS test 54

— on hair follicle 220 (Fig. 97), 221
Patterns of hair growth and control 150 et seq.

Peptides from silk 180

- structures of 7,176

Peracetic acid as reagent for oxidizing keratins 238

Periodic changes in plumage and pelt 144

- growth, and cyclic activity 143 et seq.

- of feathers 156 et seq.

Peritrophic membrane 124

Phenolases 280

Pheomelanin 276, 282

Phosphatases 222

Phospholipid membranes 35, 46, 49 et seq.

- myelinic forms 46

Phylogeny, and fibre type 22, 25

(Fig. 13)

- and keratinization 22, 49 et seq.

— of hair 72, 73

Physicochemical properties and keratinization 249 et seq.

Pigment granules 276 et seq.

- chemical stability of 278

- internal structure of size 279 (Fig. 115)

Pigmentation 276

Pilosebaceous unit 60,77

Plasma membrane 37 et seq.

- definition of 41

Platypus hair 67,77

Pleated sheet configurations 179, 180 (Fig. 74)

Polymerization of keratin 234

Polypeptides, synthetic and $\alpha$-structure 183

Porcupine quill, and $1 \cdot 5 \AA$ spacing 182

- fine structure 247, 248

- X-ray pattern of 167 (Table 9)

Precursors of keratin 126, 211

Prekeratin, properties of in germinal layers $211 \mathrm{et} \mathrm{seq.}$

Prekeratins 235

Prickle cells and desmosomes 94

Primary and seconday hair follicles 72, 75, 78 (Fig. 35), 150

Proliferating tissues 133 
Proline, and chain configuration 193 196

— in feathers 163

Pronghorns 67

Prosthetic groups, absent in keratins 161

Protein(s), corpuscular and structural 2

- fibre synthesis, classification of 122

(Fig. 52)

- synthesis, cytology of 108, 116, 120,121

- - in epidermal systems 108 et seq., 131

— - in hair cortex 112

- - synthesis theories of 109 et seq. $\alpha$-proteins other than keratins 202 et seq.

Proteus vulgaris and Bacillus subtilis, X-ray pattern of flagella 3, 202

Protofibrils 125

Pycnosis of nuclei 4, 263

Radio sulphur, incorporation into nuclei in keratinizing cells 220

Rana, tail epithelium 90 et seq.

Rat and mouse hair, zig-zags in 156

Reduction of wool 236 et seq.

Regenerated keratins 166, 237, 238

Relation between $\alpha$-helices, $\alpha$-filaments and fibrils 186 (Figs. 79, 80)

Relaxation of hairs and wool fibres 255

Renewal time(s) 134

_ for epidermal tissues 135 ('Table 6)

Residues remaining after chemical extraction of keratins 270

Resistant membranes in keratinized tissues 262, 270

Retaining cells, definition 112, 114, 120

- comparison with secreting cells 113 (Fig. 50)

Reticulin 53

Rhythmic activity, generated by feedback 141, 145 (Fig. 60)

- changes in shape and composition of crimped wool fibres 156

Ribonucleic acid (RNA), and protein synthesis 109 et seq.
Ribonucleic acid granules, fate of in keratinizing tissues 264

- in basal layer cells 80,81

- - as ribosomes 81

— in hair follicle 219, 220 (Fig. 97)

Ribosomes (RNA) (particles) 46, 111, 112

— in basal layer cells 81

- in hair cortex 112, (Plate 11)

Rubber-like properties, of hairs 255 , 256

- of reduced hairs 258

Salt bridges, stabilization by 234

- linkages and elasticity 251, 253 (Fig. 106)

Scale-hair-gland complex 77 (Fig. 34), 78

Scale(s) 66, 67 (Fig. 28)

- embryology of 67

- of fish 66,75

— of snakes 106

- (reptilian) structure 67 (Fig. 28)

- types of keratin in 67,104

Schmitt's hypothesis for development of columnar epithelia 99 (Fig. 44), 100

Sebaceous glands 59

Secondary and tertiary structures in proteins 119

Secreted keratins in birds 79, 107 (Plates 18 and 19)

Secretion of proteins 112 et seq.

Secretory cells, cytology of 110 (Fig. 49), 113, 114

Setting of hairs 259,260

Seven-stranded cable for $\alpha$-proteins 5 , 184, (Fig. 78)

Shrinkage of woollens 73

Sickle tips of primary fibres 150,159

Silk and $\beta$-keratin patterns compared 172

- fibroin 121

- formation of fibrils 129

- structure of 179,180

- glands 203

Soft keratinization 64, 95, 97, 228 et seq.

Soluble derivatives of keratinized tissues 236 et seq. 
Soluble products, by oxidation of keratins 238 et seq.

- by reduction of keratins 240 et seq.

- of partial keratinization 233

Specializations of opposed surfaces 40 et seq.

Specialized appendages of epidermis 66 et seq.

Specific rotation (definition) 195

Spherulites of insulin 130

Spirals and whorls of fibrils in hair cortex 224 (Plate 14A)

Stability, histochemical tests for in hair follicle 214 et seq.

Stabilization 19 et seq., 24, 51, 241 et seq.

Stratum corneum, constituents of 228 - structure of 225 (Fig. 98), (Plate 17), 229 (Fig. 99)

- granulosum 95, 228, 229 (Fig. 99) (Plate 23A)

- lucidum 95, 230 (Plate 22)

Stress-strain curve(s), for hair 172 et seq., 249 et seq.

— for wool, interpretation of 193

- interpretation of by Astbury and Woods 174

Stripping of epidermis as growth stimulant 148

Structural colours 276

Structure of residues remaining after extraction of keratins 271

$\alpha$-Structure, structural proposals

Sulphide solutions of wool 236

Sulphur, content of keratoses ('Table 15)

- content of skin 230

— - and nuclear synthesis 264

- level of entry into follicle 232 et seq.

Sulphydril reagent (Bennett) 29

- reactions in, hair follicle 220 (Fig. 97c)

- - claws and horn 217 (Fig. 95)

Supercontraction 255-8

- in copper solutions 259

- in lithium bromide 259

- in phenol, etc. 259

Supermolecular organization of fibrous tissues 123

Surface " repertoire" of cells 62
Surface specializations of cells and tissue formation 26 (Fig. 14), 86, 87 (Fig. 38), 90 et seq., 97 et seq.

Symphysodon discus and mucin secretion 60

Synthesis of fibres, steps in 125 (Fig. 54)

- cytoplasmic pattern of 121

- keratin in hair bulb 101 et seq., 223 et seq. (Plates 11, 12)

- melanins and melanoproteins 281 (Fig. 116)

- peptide bonds 116 et seq.

- proteins 93 et seq., 115 et seq.

- - in fibre forming systems 121

_ - in hair follicle 96 et seq. (Fig. 42)

- - in retaining cells 120 et seq.

_ - in secreting cells 116

Synthetic polypeptides, compared with $\alpha$-proteins 183

$-\alpha-\beta$ transformation in 199

Tactoids and fibres 130

Tanning 19 et seq., 27

- and resistant membranes in keratinized tissues 262

Teeth and hair 74

- development of 74 (Fig. 32)

- - keratin in enamel of 78,108

Theories of growth 138 et seq.

Thickness of epidermis 66,134

Thioglycollic acid as reducing agent 236, 240

Thiol (SH), and disulphide groups in hair follicle 217 et seq., 220 (Fig. 97c)

- reactions, in horns, nails and claws 217 (Fig. 95)

- — in feather 218 (Fig. 96)

Thyroxin, metamorphosis and keratinization 52

Tissue culture, and differentiation 44

- keratinization in $56,57,63,64$

- types of cells in 57

Tonofibrils 43,94

"TPA" stain, after Leblond et al. 43

Transference of pigment to keratinizing cells 278 
$\alpha-\beta$ transformation, and fibre extension 172,173

— and the $\alpha$-helix 188

$\alpha-\beta$ transformation, proposals of Ambrose, Huggins, Hanby and Zahn 176

Trichohyalin 97, 226 et seq., 230

- an $\alpha$-protein 230

- fine structure of 226

- in inner root sheath 100

- properties of $226 \mathrm{et} \mathrm{seq}$.

- transformation in to fibrils 100 (Plate 21), 226

Trio groups of hair follicles 76 (Fig. 33), 78 (Fig. 35)

Triturus 51

Tropocollagen and collagen 127

Trypsin, action of on hair follicle 217

Tryptic digestion of hair 260

Tumours, epidermal $62,63,274$

Tussah silk 180

Types of fibrous proteins 5

- of fibrous texture 124 (Fig. 53)

- of keratin in feather follicle 106 (Fig. 48)

Tyrosine, as precursor of melanin 280

- residues in fibroin 180

Uneven keratinization and its histology 272 et seq.

- and function of tissue 273

Unguis and subunguis 68, 273

Unoriented $\alpha$-helices, X-ray pattern from 194

Urea, action of saturated solution on hair follicle 216 (Fig. 93), 217

— as solvent for prekeratins 235

— swelling of follicle by 216 (Fig. 94), 235

Uropygial gland in birds 59

Variable amino acid composition of keratins 31 et seq.

- of wool 32 (Fig. 15)

Vascular supply to follicle 152,232

Vertebrates, tanning in
Vitamin A, action on hard keratin in rat scales 231

- up metaplaasia of epidermal transplants 63 et seq.

- D 59,60

Warts and corns 64,66

Water content of hair bulb 222

Wide angle diffraction patterns 11, 13 et seq.

- interpretation of 170 et seq.

Wool, amino acids in 7 (Table 1), 9 (Table 3)

- fibre follicle and asymmetrical fibre 274 (Fig. 114)

_ follicle and crimp 157, 158 (Fig. $66,67), 273$

- root extract 237 (Table 14)

Work index $(30 \%)$, definition of 250

- to stretch wool fibres as function of $\mathrm{pH} 253$ (Fig. 106)

Wound "hormones" 149

X-ray diffraction, and molecular structure of keratins 164 et seq.

- methods 11 (Fig. 3), 24, 165 et seq.

- patterns, of fibres 13 et seq., 164 et seq.

- - idealized type 13 (Fig. 5)

- - of supercontracted fibres 256 (Fig. 109), 257

- and chemical reactions 193

- of bacterial flagella 2 (Fig. 86), 201

- of collagen 16 (Fig. 8)

_ of cuticle 265

- of egg case protein 204 (Plate 3)

- of feather 16 (Plate 2A), 169, 205

- of scales, etc. 206

- $\alpha$-type 14 (Fig. 6), 17 (Fig. 9) (Plate 1A, 3), 165 et seq.

- $\beta$-type 15 (Fig. 7), 18 (Fig. 10), (Plate 1B), 205

- study of hair follicle 214 (Fig. 91)

Xenopus, tail epithelium 87 (Fig. 38), 90 et seq.

Zig-zags, curls and crimps 156 et seq. 







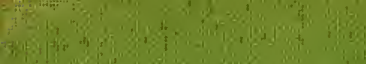
(3) 20

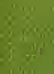

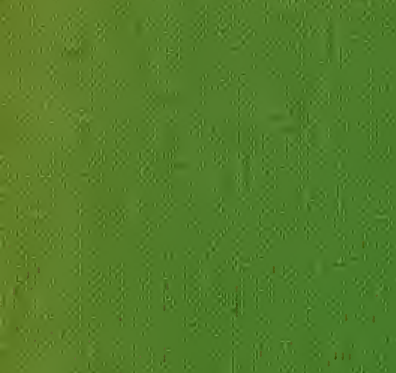

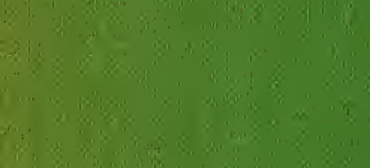

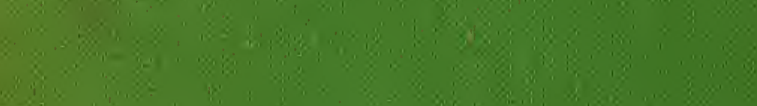

